\title{
FORWARD-BACKWARD ASYMMETRY OF TOP QUARK PAIR PRODUCTION AT THE FERMILAB TEVATRON
}

\author{
A Dissertation \\ by \\ ZIQING HONG
}

\begin{abstract}
Submitted to the Office of Graduate and Professional Studies of Texas A\&M University in partial fulfillment of the requirements for the degree of

DOCTOR OF PHILOSOPHY
\end{abstract}

\begin{abstract}
Chair of Committee, David Toback
Committee Members, Guy Almes Bhaskar Dutta Ricardo Eusebi

Head of Department, George R. Welch
\end{abstract}

December 2015

Major Subject: Physics

Copyright 2015 Ziqing Hong 


\begin{abstract}
This dissertation presents the final measurements of the forward-backward asymmetry $\left(A_{\mathrm{FB}}\right)$ of top quark-antiquark pair events $(t \bar{t})$ at the Collider Detector at Fermilab (CDF) experiment. The $t \bar{t}$ events are produced in proton-anti-proton collisions with a center of mass energy of $1.96 \mathrm{TeV}$ during the Run II of the Fermilab Tevatron. The measurements are performed with the full CDF Run II data $\left(9.1 \mathrm{fb}^{-1}\right)$ in the final state that contain two charged leptons (electrons or muons, the dilepton final state), and are designed to confirm or deny the evidence-level excess in the $A_{\mathrm{FB}}$ measurements in the final state with a single lepton and hadronic jets (lepton+jets final state) as well as the excess in the preliminary measurements in the dilepton final state with the first half of the CDF Run II data. New measurements include the leptonic $A_{\mathrm{FB}}\left(A_{\mathrm{FB}}^{\ell}\right)$, the lepton-pair $A_{\mathrm{FB}}\left(A_{\mathrm{FB}}^{\ell \ell}\right)$ and the reconstructed top $A_{\mathrm{FB}}$ $\left(A_{\mathrm{FB}}^{t \bar{t}}\right)$. Each are combined with the previous results from the lepton + jets final state measured at the CDF experiment. The inclusive $A_{\mathrm{FB}}^{\ell}, A_{\mathrm{FB}}^{\ell \ell}$, and $A_{\mathrm{FB}}^{t \bar{t}}$ measured in the dilepton final state are $0.072 \pm 0.060,0.076 \pm 0.081$, and $0.12 \pm 0.13$, to be compared with the Standard Model (SM) predictions of $0.038 \pm 0.003,0.048 \pm 0.004$, and $0.010 \pm 0.006$, respectively. The CDF combination of $A_{\mathrm{FB}}^{\ell}$ and $A_{\mathrm{FB}}^{t \bar{t}}$ are $0.090_{-0.026}^{+0.028}$, and $0.160 \pm 0.045$, respectively. The overall results are consistent with the SM predictions.
\end{abstract}




\section{ACKNOWLEDGEMENTS}

As in almost all cases in experimental high energy physics, the work in this dissertation would never finish without the hard work, help and support from a lot of people. I cannot write down all the names, but would like to present my deepest thanks to all physicist, technicians, engineers, and all other people who have built, maintained, and operated the Fermilab Tevatron and the CDF detector.

I joined the CDF collaboration knowing little about high energy physics experiments. The kind people in the collaboration tirelessly taught me an awful lot of knowledge I cannot learn elsewhere. I would like to thank the whole collaboration for training me into the person I am now.

The analyses in this dissertation are based on the tremendous amount of work in the CDF Top Dilepton Group. Without the previous work, these analyses could not have started, let alone finished. I am in debt to all members of the CDF Top Dilepton

Group. I would like to offer my special thanks to Yen-Chu Chen, the convener of the CDF Top Dilepton Group, who has been providing me with endless support, from teaching me basic linux skills to a lot of useful and interesting physics discussions. Also, I learn a tremendous amount of skills from the convener of the CDF TopBSM Group, Jon S. Wilson, who later joined A\&M and moved to College Station about the same time as I did. Without Jon, these analyses would not be as sensitive and robust as they are now.

I would not join CDF or get into the business of top quark physics without the guidance of Dave Toback, who has been my advisor, my mentor as well as my friend. I learned almost all my understanding of high energy physics from him. More importantly, I learned the way to become a scientist. I am heavily influenced by his 
attitude of relentlessly pursuing physics goals no matter how hard it is and staying focused on solving the most important problem. I am proud of being his student.

I am grateful to Bhaskar Dutta, Ricardo Eusebi, and Guy Almes for serving on my committee. I also enjoyed working with Ricardo on CMS for my service work, and I am especially thankful to the Brazos cluster administration team led by Guy.

During my stay at the CDF trailers during 2012-2013, I enjoyed the company of the A\&M CDF group every day, including Daniel Cruz, Jason Nett, and Vaikunth Thukral. I also enjoyed the nights spent in the Fermilab User's Center, with the tireless ping-pong buddy, Augustyn, and the "Fermi 4 idiots", Zhen Hu, Ao Liu, and Zijun Xu. After moving back to College Station in 2014, I benefited a lot from the numerous good conversation at the "C-experiment coffee group", with Andy Kubik and Jon S. Wilson. In addition, I would not survive the last five years without the support of the "Aurora Court Gang"; I'm not going to write down names as you know who you are.

Finally, I want to thank my family, especially my wife. They have always been there for me. 
TABLE OF CONTENTS

Page

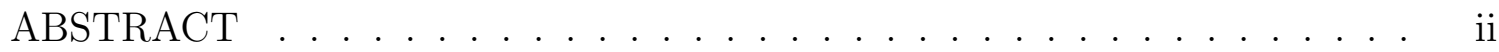

ACKNOWLEDGEMENTS . . . . . . . . . . . . . . . iii

TABLE OF CONTENTS . . . . . . . . . . . . . . . . . . v v

LIST OF FIGURES . . . . . . . . . . . . . . . . . . . . . . viii

LIST OF TABLES . . . . . . . . . . . . . . . . . . . . . . xix

1. INTRODUCTION . . . . . . . . . . . . . . . . . . . 1

1.1 Overview of the Standard Model of Particle Physics . . . . . . . . . . 4

1.2 Properties, Production and Decays of the Top Quark . . . . . . . . . 5

1.2 .1 Top-quark Mass, Lifetime, and Production . . . . . . . . . . . 5

1.2 .2 Top-quark Decays . . . . . . . . . . . . . . . . 7

1.3 Top-Quark Pair Production Forward-Backward Asymmetry . . . . . 10

1.3 .1 Historical Remarks . . . . . . . . . . . . . . . . . 10

1.3.2 Definition of the Forward-Backward Asymmetry Observables . 16

1.3.3 $\quad$ Theoretical Predictions with SM . . . . . . . . . . . . . 20

1.3 .4 Predictions with Physics beyond the SM . . . . . . . . . . . . 24

1.3 .5 Experimental Status . . . . . . . . . . . . . . . 26

1.4 Outline of the Dissertation . . . . . . . . . . . . . . . . . . 30

2. EXPERIMENTAL TOOLS AND EVENT SELECTION . . . . . . . . . . 33

2.1 The Fermilab Tevatron . . . . . . . . . . . . . . . . . . . . . . . . . 33

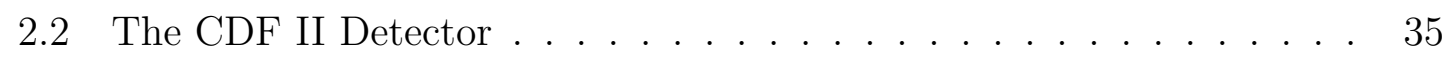

2.3 Identification of the Reconstructed Objects . . . . . . . . . . . . . . . 40

2.4 Event Selection . . . . . . . . . . . . . . . . . . . . 44

3. SIGNAL AND BACKGROUND MODELING . . . . . . . . . . . . . . . . 52

$3.1 \quad$ Top-pair Production Signal with Different Physics Models . . . . . . 53

3.2 Background Modeling. . . . . . . . . . . . . . . . . . 54

3.3 Validation of the Signal and Background Modeling . . . . . . . . . . 58 
4. MEASUREMENT OF THE FORWARD-BACKWARD ASYMMETRY OF LEPTONS FROM TOP-QUARK PAIRS . . . . . . . . . . . . . . 67

4.1 Leptonic $A_{\mathrm{FB}}$ Measurement Methodology . . . . . . . . . . . . 68

4.2 Validation of the Measurement Methodology . . . . . . . . . . . 75

4.2 .1 Validation at Generator Level . . . . . . . . . . . . . . . 75

4.2 .2 Validation with Detector Effects . . . . . . . . . . . . 76

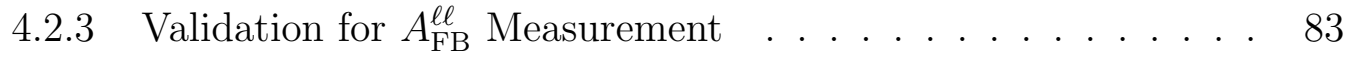

$4.3 \quad$ Measurement of the Leptonic $A_{\mathrm{FB}}$ from Data . . . . . . . . . . . . 90

$4.3 .1 \quad$ Measurement of the $A_{\mathrm{FB}}^{\ell}$ and Uncertainty Estimation . . . . . 90

$4.3 .2 \quad$ Measurement of the $A_{\mathrm{FB}}^{\ell \ell}$ and Uncertainty Estimation . . . . . 101

5. RECONSTRUCTION OF THE MOMENTA OF TOP-QUARK PAIRS . . 107

5.1 Top Reconstruction Basics . . . . . . . . . . . . . . . . . . . . . . . . 109

5.2 Detector Resolution and Physics Assumptions for the Reconstruction 112

5.3 Likelihood for Possible Solutions . . . . . . . . . . . . . . . . 117

5.4 Full Probability Reconstruction . . . . . . . . . . . . . . . . . . 121

6. MEASUREMENT OF THE FORWARD-BACKWARD ASYMMETRY OF TOP-QUARK PAIRS $\ldots \ldots \ldots \ldots \ldots \ldots \ldots$

6.1 Top $A_{\mathrm{FB}}$ Measurement Methodology $\ldots \ldots \ldots \ldots$

6.1.1 Reweighting the POWHEG MC to Create the $A_{\mathrm{FB}}^{t t}$ Methodology Testing Samples . . . . . . . . . . . . . . . . . . . 134

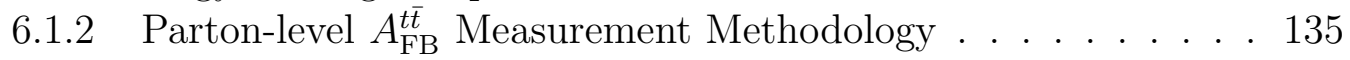

6.1 .3 Compound Poisson Statistics $\ldots \ldots$. . . . . . . . . . . . 140

6.1 .4 Parameters and Signal-Only Uncertainty Expectations .... 145

6.1.5 Adding Backgrounds to the Methodology . . . . . . . . . . . 148

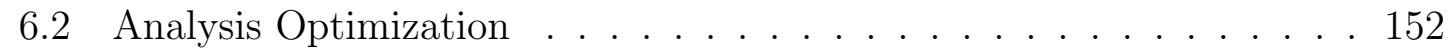

6.2 .1 Max Likelihood vs. Full Probability and Weighting . . . . . 156

6.2 .2 Optimization Based on Tunable Parameters . . . . . . . . . . 157

$6.2 .3 \quad$ Final Optimization Values and Results . . . . . . . . . . . 163

6.3 Validation of the Measurement Methodology . . . . . . . . . . . . . . 164

6.3.1 Additional Validation of Signal and Background Modeling . . 166

6.3.2 Validation of the Inclusive $A_{\mathrm{FB}}^{t t}$ Measurement Methodology . . 169

6.3.3 Validation of the $A_{\mathrm{FB}}^{t t}$ vs. $\left|\Delta y_{t}\right|$ Measurement Methodology. .175

$6.4 \quad$ Final Systematic Uncertainties and Measurement of the Top $A_{\mathrm{FB}}$ from

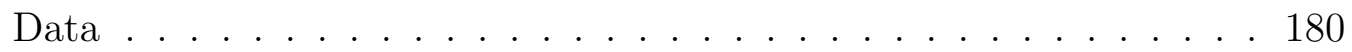

6.4.1 Measurement of the Inclusive $A_{\mathrm{FB}}^{t \bar{t}}$ and Uncertainty Estimation 180

\begin{tabular}{|cc|c|c|}
\hline 6.4 .2 & Measurement of $A_{\mathrm{FB}}^{t t}$ vs $\left|\Delta y_{t}\right|$ and Uncertainty Estimation & $\ldots$ & 183
\end{tabular}

7. COMBINATION WITH PREVIOUS MEASUREMENTS $\ldots . . \ldots . .188$ 
7.1 Outline of the BLUE Methodology and the AIB Technique . . . . . . 188

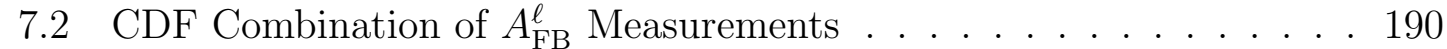

7.3 CDF Combination of Inclusive $A_{\mathrm{FB}}^{t t}$ Measurements . . . . . . . . . 194

7.4 CDF Combination of the Differential $A_{\mathrm{FB}}^{t t}$ vs. $\left|\Delta y_{t}\right| \mid \ldots \ldots . . .198$

8. CONCLUSIONS . . . . . . . . . . . . . . . . . . . . . . . 204

REFERENCES . . . . . . . . . . . . . . . . . . . . . . . . 210

APPENDIX A. LEPTON IDENTIFICATION REQUIREMENTS. . . . . . . . 216

APPENDIX B. ESTIMATION OF THE DRELL-YAN BACKGROUNDS IN

THE DILEPTON+DIJET $+E_{T}$ FINAL STATE $\ldots \ldots \ldots \ldots . \ldots .221$

B.1 Motivation for a Sophisticated DY Estimation Method . . . . . . . . 222

B.2 The Data-MC Hybrid Estimation Method for the $e e$ and $\mu \mu$ Final States 223

B.3 Scale Factors for the $e \mu$ and $\tau \tau$ Final States . . . . . . . . . . . 232

B.4 Performance of the New DY Estimation Method . . . . . . . . . . . 237

APPENDIX C. BACKGROUND AND SIGNAL VALIDATION IN THE 0-

JET AND 1-JET CONTROL REGIONS . . . . . . . . . . . . . . . . . . 239

APPENDIX D. BACKGROUND AND SIGNAL VALIDATION WITH $B-$

TAGGING REQUIREMENT … . . . . . . . . . . . . . . . . 244

APPENDIX E. A DESCRIPTION OF WHY THE METHODOLOGY OF $A_{\mathrm{FB}}^{\ell}$ MEASUREMENT WORKS . . . . . . . . . . . . . 252

E.1 Single and Double Gaussian Modeling. . . . . . . . . . . . . . . . 252

E.2 Comparing the Sensitivity of the $a \cdot \tanh$, Single-Gaussian and DoubleGaussian Models . . . . . . . . . . . . . . . . 262

E.3 Conclusions of $A_{\mathrm{FB}}^{\ell}$ Measurement Methodology Study . . . . . . . 266

APPENDIX F. CORRELATION BETWEEN TWO LEPTONS IN EACH

EVENT . . . . . . . . . . . . . . . . . . . . . . 269 


\section{LIST OF FIGURES}

FIGURE

1.1 The $t \bar{t}$ pair production and decay in a proton-antiproton collision. The proton direction is denoted as the "forward" direction while the antiproton direction is denoted as the "backward" direction. . . . . . 3

1.2 Summary of the particles in the standard model [26]. . . . . . . . . . 5

1.3 Dominant Feynman diagrams of $t \bar{t}$ pair production in the SM with quark-antiquark annihilation process (a) and gluon-gluon fusion process (b) [32].

1.4 Feynman diagrams of $t \bar{t}$ pair production with proton-antiproton collisions, and decaying into the all-jets (a), lepton+jets (b), and dilepton

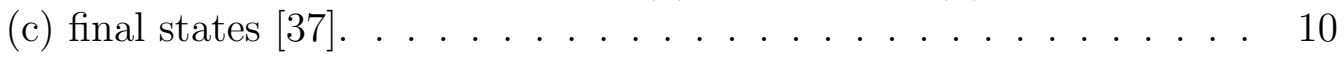

1.5 The $t \bar{t}$ branching fractions. The branching fraction of the "all-jets", the "lepton+jets", and the "dilepton" final states are 46\%, 30\%, and $5 \%$, respectively. The final states with a $\tau$ lepton are not explicitly used, but the scenarios where the $\tau$ lepton decays leptonically into an electron or a muon are included implicitly in the corresponding lepton+jets or dilepton final states [37].]. . . . . . . . . . . . . . . 11

1.6 The leading order (a) and next-to-leading order (b) Feynman diagrams of the $t t$ pair production with quark-antiquark annihilation process [19]. 12

1.7 History of top $A_{\mathrm{FB}}$ SM predictions and results from CDF and D0 [44]. 15

1.8 Example Feynman diagrams of the $t \bar{t}$ pair production via hypothetical new particles beyond the SM, including the axigluons via a $s$-channel production (a) and the $Z^{\prime}$ bosons via a $t$-channel production (b). . . . 25

2.1 The accelerator chain of Fermilab Tevatron, including the location of the CDF and D0 experiments [72]. . . . . . . . . . . . . . . . . . . . . 34

2.2 A cutaway view of the CDF II detector with quadrant cut to expose the different subdetectors [80]. . . . . . . . . . . . . . . . 38 
2.3 The elevation view of CDF II detector so we can see the inner detectors more clearly. Only half of the detector is shown [66]. . . . . . . . . . 39

2.4 The signature responses in the detector from various objects [84]. . . 41

3.1 The comparison of the observed distributions with the expectation, with individual background components specified. The distributions of (a) $\not_{T}$, (b) $\mathrm{m}_{\mathrm{ll}}$, (c) $p_{T}$ of both leptons, (d) $p_{T}$ of the dilepton system, (e) $\mathrm{H}_{\mathrm{T}}$, and (f) jet multiplicity are shown. $\ldots \ldots \ldots 60$

3.2 The same plots as Fig. 33.1, but with (a) leading jet $E_{\mathrm{T}}$, (b) secondary jet $E_{\mathrm{T}}$, (c) positive lepton $q_{\ell} \eta_{\ell}$, (d) negative lepton $q_{\ell} \eta_{\ell}$, (e) $q_{\ell} \eta_{\ell}$ of both leptons, and (f) $\Delta \eta \ldots \ldots \ldots \ldots \ldots \ldots$

3.3 The same as Fig. 3.1 , but where all the backgrounds are combined. . 62

3.4 The same as Fig. 3.2 , but where all the backgrounds are combined. . 63

3.5 The comparison of the expected distribution from the POWHEG simulation with the observation after subtracting off the contributions from background processes. The distributions of (a) $\not_{T}$, (b) $\mathrm{m}_{11}$, (c) $p_{T}$ of both leptons, (d) $p_{T}$ of the dilepton system, (e) $\mathrm{H}_{\mathrm{T}}$, and (f) jet

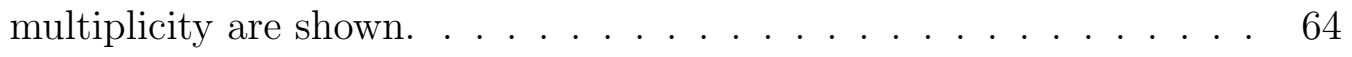

3.6 The same plots as Fig. 3.5 , but with (a) leading jet $E_{\mathrm{T}}$, (b) secondary jet $E_{\mathrm{T}},(\mathrm{c})$ positive lepton $q_{\ell} \eta_{\ell},(\mathrm{d})$ negative lepton $q_{\ell} \eta_{\ell}$, (e) $q_{\ell} \eta_{\ell}$ of both leptons, and (f) $\Delta \eta \ldots \ldots \ldots \ldots \ldots \ldots \ldots \ldots$

4.1 The $q_{\ell} \eta_{\ell}$ distribution of charged leptons produced from $t \bar{t}$ cascade decay from simulations with various physics models at parton level, before any selection requirements. . . . . . . . . . . . . 70

4.2 The $\mathcal{S}\left(q_{\ell} \eta_{\ell}\right)$ term (a) and the $\mathcal{A}\left(q_{\ell} \eta_{\ell}\right)$ term (b) of the $q_{\ell} \eta_{\ell}$ distribution from various physics models. The lines in (b) correspond to the best fits from the $a \cdot \tanh$ model. $\ldots \ldots \ldots \ldots \ldots$. . . . . . . 71

$4.3 \quad$ A comparison between the predicted $A_{\mathrm{FB}}^{\ell}$ from simulations and the $A_{\mathrm{FB}}^{\ell}$ as measured using the $a \cdot \tanh$ parametrization with parton level information from $\left|q_{\ell} \eta_{\ell}\right|<2.0$. The dashed line indicates the location of the equal values, while the points are superimposed at their measured locations. All the points lie along the line within uncertainties. . . . . 76

4.4 The distribution of the asymmetric part of the bin-by-bin acceptance correction, $\mathcal{A}^{C}\left(q_{\ell} \eta_{\ell}\right)$, as estimated with the POWHEG $t \bar{t}$ MC. $\ldots . .79$ 
4.5 A comparison of the binned result, along with the $a \cdot \tanh$ fit, on the asymmetric part of the $q_{\ell} \eta_{\ell}$ distribution as estimated from the various MC samples. We plot both the generator level (hepg) and reconstructed level (reco) on top of each other. The fit parameters at generator level are slightly different from the number shown in Table 4 4.1 due to different binning. In all cases, the variation in the fit results is small compared to the dominant systematic uncertainty. . . 81

4.6 The asymmetric part of the $q_{\ell} \eta_{\ell}$ distribution for the six physics models at reconstructed level, with their corresponding $a \cdot$ tanh fits. . . . . . 82

4.7 Truth level $A_{\mathrm{FB}}^{\ell}$ vs. the measured value of $A_{\mathrm{FB}}^{\ell}$. To measure $A_{\mathrm{FB}}^{\ell}$ we use the $a \cdot \tanh$ fit on the asymmetric part of $q_{\ell} \eta_{\ell}$ distribution at reconstructed level with symmetric part from POWHEG tt MC. . . . . 82

4.8 The same results as in Fig. $4.1,|4.2 \mathrm{a}| ,4.2 \mathrm{~b}$, but using $\Delta \eta$ instead of $q_{\ell} \eta_{\ell} .84$

4.9 The same results as in 4.4 and Fig. 4.6 , but using $\Delta \eta$ instead of $q_{\ell} \eta_{\ell}$. $\quad 85$

4.10 The same as Fig. 4.5 , but using $\Delta \eta$ instead of $q_{\ell} \eta_{\ell}$. . . . . . . . . . 87

\begin{tabular}{|l|l|l|l|l|}
\hline 4.11 The same results as in Fig. & 4.3 & and & 4.7 & but with $A_{\mathrm{FB}}^{\ell \ell}$ (labelled as \\
\hline
\end{tabular} $\mathrm{A}_{\mathrm{FB}}^{\Delta \eta}$ in the figures). . . . . . . . . . . . . . 88

4.12 The symmetric part of the $q_{\ell} \eta_{\ell}$ distribution from data, after background subtraction, with the expectations from POWHEG overlaid. . . 93

4.13 The asymmetric part of $q_{\ell} \eta_{\ell}$ distribution from data after background subtraction. The green line shows the expectations from POWHEG. . . 93

4.14 The results of pseudoexperiments to estimate the uncertainty due to background fluctuation. Fig. 4 .14a $\mid$ shows the $A_{\mathrm{FB}}^{\ell}$ obtained by only fluctuating signal, and Fig. $4.14 \mathrm{~b}$ shows the $A_{\mathrm{FB}}^{\ell}$ obtained by fluctuating both signal and backgrounds. . . . . . . . . . . . . . . . . . 94

4.15 The $a \cdot \tanh$ fits for the positive and negative leptons separately. . . . $\quad 99$

4.16 The $a \cdot \tanh$ fits for $e e, \mu \mu$, and $e \mu$ final states separately. . . . . . . . 100

4.17 The $a \cdot \tanh$ fit for the $A_{\mathrm{FB}}^{\ell}$ data but with the additional requirement of at least one $b$-tagged jet. . . . . . . . . . . . . . 101

4.18 The symmetric and asymmetric parts of $\Delta \eta$. . . . . . . . . . . . . 105 
4.19 The $a \cdot \tanh$ fit for the $A_{\mathrm{FB}}^{\ell \ell}$ data but with the additional requirement of at least one b-tagged jet. . . . . . . . . . . . . . . . 106

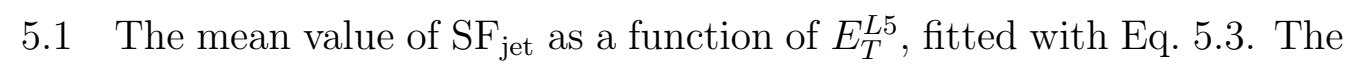
best fit values, summarized in Table 5.1 , are derived from the NLO MC sample (POWHEG), and are used to correct the jets for use in the final fit function. . . . . . . . . . . . . . . . . . . . 113

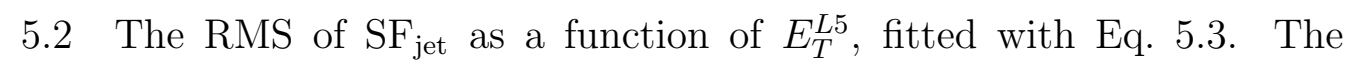
best fit values are summarized in Table 5.1 , and will be used in the final likelihood reconstruction. These are derived from the NLO MC sample (POWHEG). . . . . . . . . . . . . . . . . . . . . . . 114

5.3 The RMS of $E_{x}$ and $E_{y}$ as a function of $E_{T}^{L 5}\left(\right.$ jet1) $+E_{T}^{L 5}$ (jet2), fitted with Eq. 5.3. The best fit values are summarized in Table|5.1| These are derived from the NLO MC sample (POWHEG). . . . . . . . . . . . 115

5.4 The templates of $p_{z, t \bar{t}}, p_{T, t \bar{t}}$ and $m_{t \bar{t}}$ derived from the POWHEG MC at the generator level for the sample of reconstructed events that pass the baseline event selection criteria. Note that the functional forms are designed purely to get a good estimate of the shape, not to be used in any determination of each parameter. . . . . . . . . . . . 116

5.5 The momentum of the neutrino in the $\ell+b$ rest frame. The neutrino momentum can be determined in this frame up to the azimuthal angle $\phi$, which is a free parameter and is used as an input to the final likelihood fit. . . . . . . . . . . . . . . . 120

5.6 The posterior distribution of $\mathrm{jd}_{1}$ and $\mathrm{jd}_{2}$ for one example event from the POWHEG MC. Based on the generator-level information the left column is for the correct lepton-jet pairing and the right column is the incorrect pairing. The red vertical lines show the true values of the parameter. . . . . . . . . . . . . . . . . . 124

5.7 The same as Fig. 5.6, but for $\phi_{1}$ and $\phi_{2}$ for one example event from the POWHEG MC. Based on the generator-level information the left column is for the correct lepton-jet pairing and the right column is the incorrect pairing. Since the two $\phi$ parameters are defined in the $\ell+b$ rest frame, which depends on each hypothesis of the jd parameter, we cannot plot the true value on those plots. . . . . . . . . . . . . . . 125

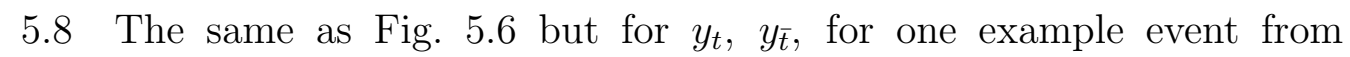
POWHEG MC. Again, the left column is the correct lepton-jet pairing and the red vertical lines show the true values of the parameter. . 126 
5.9 The same as Fig. 5.6 but for $\Delta y_{t}$ and $y_{t}$ vs. $y_{\bar{t}}$, for one example event from POWHEG MC. Again, the left column is the correct lepton-jet pairing and the red vertical lines and the green cross show the true values of the parameter. . . . . . . . . . . . . . . . . 127

5.10 The scatter plot of the log-likelihood vs. $\mathrm{jd}_{1}$ and $\mathrm{jd}_{2}$ for the same event as Fig. 5.6 . Again, the left column is the correct lepton-jet pairing, the red vertical lines show the true values of the parameter, and the green dashed horizontal lines show the maximum log-likelihood value. 128

5.11 The same as Fig. 5.10, but for $\phi_{1}$ and $\phi_{2}$. Again, the left column is the correct lepton-jet pairing, and the green dashed horizontal lines show the maximum log-likelihood value. Since the two $\phi$ parameters are defined in the $\ell+b$ rest frame, which depends on each hypothesis of the jd parameter, we cannot plot the true value on those plots. . . 129

5.12 The same as Fig. 5.10 , but for $y_{t}, y_{\bar{t}}$, and $\Delta y_{t}$. The true values for $y_{t}$ and $\Delta y_{t}$ in the wrong jet assignment column are outside the range of the figure. . . . . . . . . . . . . . . . . . . . . 130

5.13 Stacking the full probability distribution of $\Delta y($ reco $)-\Delta y($ hepg $)$ for each event from the POWHEG sample, normalizing each event to unity, which gives an overall normalization to the size of the POWHEG sample. Note that this plot is made only with the baseline cuts described in Sec. 2 , and the two lepton-jet pairings in each event are weighted according to Eq. 5.10 . A final version after the optimization will be shown in Sec. 6.3 in Fig. 6.24. . . . . . . . . . . . . . . . . . . . . . . 131

6.1 Decomposition of the top quark differential cross section $\left(\frac{d \sigma}{d \cos \theta^{*}}\right)$ in terms of Legendre polynomials, taken from Ref. [57]. . . . . . . . . . 134

6.2 Comparison of the $\cos \theta^{*}$ and $\Delta y_{t}$ distributions between the nominal POWHEG sample $\left(A_{\mathrm{FB}}^{t \bar{t}}=0.052\right.$, blue $)$ and the reweighted POWHEG sample with extra $a_{1}=0.25$, corresponding to $A_{\mathrm{FB}}^{t t}=0.177$ (red). Note that $\cos \theta^{*}, \Delta y_{t}$ and $m_{t \bar{t}}$ are correlated. . . . . . . . . . . . . . 136

6.3 The same as Fig. 6.2 , but with $m_{t \bar{t}}$. Note that $\cos \theta^{*}, \Delta y_{t}$ and $m_{t \bar{t}}$ are correlated. . . . . . . . . . . . . . 137

6.4 The value of $A_{\mathrm{FB}}^{t \bar{t}}$ as a function of extra $a_{1}$ used in reweighting POWHEG MC sample. . . . . . . . . . . . . . . . 138 
6.5 The bin-weighted $\Delta y_{t}$ distribution (a) from the nominal POWHEG at generator level before any event selection and (b) after reconstruction and baseline event selection. . . . . . . . . . . . . . . . . . . 138

6.6 The value of $\operatorname{Eff}[p]\left(A_{\mathrm{FB}}^{t \bar{t}}\right)$ as a function of $A_{\mathrm{FB}}^{t \bar{t}}$ overlaid with a linear fit, shown in the four $\Delta y_{t}$ bins. Note that all the points in each of the figures are correlated. A final version after the optimization is shown in Fig. $6.25 \mid$ and the fits are summarized in Fig. 6.26. . . . . . . . . . 141

6.7 The detector smearing matrix with nominal POWHEG MC, which is the Det $[p][r]$ term in our unfolding method. The final version is in Fig. 6.24b. . . . . . . . . . . . . . . . . . . . . 142

6.8 Row-slice of Fig. 6.7, with extra $a_{1}=-0.3,0$ and 0.5 . The variations among the three samples are tiny. This shows that we do not need $\operatorname{Det}[p][r]$ to be a function of $A_{\mathrm{FB}}^{t t}$, so we take the nominal POWHEG result. . . . . . . . . . . . . . . . . 143

6.9 The posterior distribution of of the inclusive value of $A_{\mathrm{FB}}^{t \bar{t}}$ with the full set of reconstructed events from the POWHEG MC sample. The $A_{\mathrm{FB}}^{t t}$ is extracted with a Gaussian fit to the central region of this distribution. The result $A_{\mathrm{FB}}^{t \bar{t}}=0.053 \pm 0.013$ is consistent with the generator-level value of $0.0524 . \ldots \ldots \ldots \ldots 147$

6.10 The mean values of the inclusive $A_{\mathrm{FB}}^{t \bar{t}}$ measurements using pseudoexperiments(a), the expected statistical uncertainty (b) and the pull (c) from each pseudoexperiment with signal only. The pull distribution looks good. The expected signal-only statistical uncertainty is 0.107 , which is derived from the RMS of (a). . . . . . . . . . . . . . . . . . 149

6.11 Posterior distributions of $A_{\mathrm{FB}}^{t \bar{t}}$ in the two $\left|\Delta y_{t}\right|$ bins with signal only, with Gaussian fits to extract their central values and uncertainties.] . 149

6.12 Results of the pseudoexperiments for $A_{\mathrm{FB}}^{t \bar{t}}\left(\left|\Delta y_{t}\right|<0.5\right)$ (LHS) and $A_{\mathrm{FB}}^{t t}\left(\left|\Delta y_{t}\right|>0.5\right)(\mathrm{RHS})$, with signal only. The pull distributions look good. The expected statistical uncertainty with signal only is 0.28 for $A_{\mathrm{FB}}^{t \bar{t}}\left(\left|\Delta y_{t}\right|<0.5\right)$ and 0.13 for $A_{\mathrm{FB}}^{t \bar{t}}\left(\left|\Delta y_{t}\right|>0.5\right) . \ldots . . . . . .150$

6.13 The same as Fig. 6.10 , but where we have included the backgrounds. The mean values of the inclusive values of $A_{\mathrm{FB}}^{t t}$ as estimated using pseudoexperiments (a), the expected statistical uncertainty (b) and the pull (c). The pull distribution looks good. The expected sig-

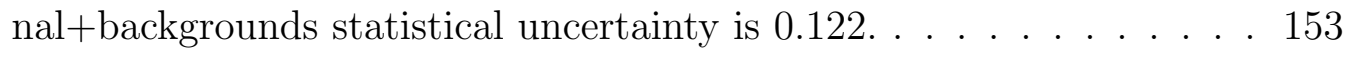


6.14 The same as Fig. 6.12 , but with signal+background. The pull distributions look good. The expected total statistical uncertainties with signal + backgrounds are 0.34 for $A_{\mathrm{FB}}^{t \bar{t}}\left(\left|\Delta y_{t}\right|<0.5\right)$ and 0.16 for $A_{\mathrm{FB}}^{t t}\left(\left|\Delta y_{t}\right|>0.5\right) . \ldots \ldots \ldots \ldots \ldots \ldots 154$

6.15 The $\mathrm{jd}_{\text {peak }}$ distributions for the correct and the incorrect lepton-jet pairings for the two jets. . . . . . . . . . . . . . . . . 159

6.16 The contour plots for $\mathrm{jd}_{1, \text { peak }}$ vs. $\mathrm{jd}_{2, \text { peak }}$ for the correct and the incorrect lepton-jet pairings. . . . . . . . . . . . . . . . . . . . . 159

6.17 Distribution of the reconstructed jet charge, $Q_{\text {jet }}$, for jets originating from $b$ and $b$ quarks as measured in our POWHEG sample passing all the dilepton selection criteria. . . . . . . . . . . . . . . . . . . 160

6.18 Distribution of $m_{l b}^{2}$ for the correct and incorrect lepton-jet pairings taken from the POWHEG sample. The blue curve is from the correct lepton-jet pairing, and the red curve is from the incorrect lepton-jet pairing. . . . . . . . . . . . . . . . 162

6.19 The distributions for $\Delta R_{\min }$ (lepton, jet) of well reconstructed $t \bar{t}$ events, badly reconstructed $t \bar{t}$ events, and the various backgrounds. The vertical dot-dashed line shows $\Delta R_{\min }$ (lepton, jet) $=0.2$, which is the optimal value determined in Sec. 6.2.3. . . . . . . . . . . . . . 163

6.20 The expected statistical uncertainty with signal only (brown dotdashed line), the total statistical uncertainty (with signal + background, blue dashed line), and the total statistical + background systematic uncertainty (the expected uncertainty in the text, green solid line), as functions of various cut and weight values. The optimum values are based on the minimum point of the green line, as marked with the red arrows on the plots, and summarized in Table 6.2 . All other values are held at their optimal values for each plot. The expected uncertainty, after optimization, is 0.122. . . . . . . . . . . . . 165

6.21 Kinematic variables from data compared to predictions after all cuts in Tables $\mid 2.1$ and 6.2 . . . . . . . . . . . . . . . . . . . . . 167

6.22 The same as Fig. 6.21 , but with additional variables. . . . . . . . . . 168

6.23 The final $\Delta y_{t}$ distribution from data compared with the SM expectations after all the event selection requirements and event reconstruction algorithms. . . . . . . . . . . . . . . . . . . . . 169 
6.24 The same figures as Figs. 5.13 and 6.7, but after optimization. The numbers from (b) are summarized in Table 6.4 , which corresponds to

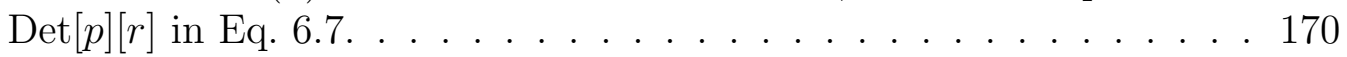

6.25 The same figures as Fig. 6.6 , but after the optimization. The best fit values are summarized in Table. 6.5 and shown in Fig. 6.26, and which corresponds to $\operatorname{Eff}[p]\left(A_{\mathrm{FB}}^{t \bar{t}}\right)$ in Eq. 6.7. . . . . . . . . . . . . . . . . . . 172

6.26 The best fit functions in Fig. 6.25. The best fit values are summarized in Table. 6.5, which corresponds to $\operatorname{Eff}[p]\left(A_{\mathrm{FB}}^{t t}\right)$ in Eq. 6.7

6.27 Inclusive $A_{\mathrm{FB}}^{t \bar{t}}$ measurement results of the pseudoexperiments with the optimal cuts and weights, with signal only (LHS) and with signal+backgrounds (RHS) for the measurement of the inclusive $A_{\mathrm{FB}}^{t t}$. The pull distributions look good. The expected statistical uncertainty with signal only is 0.106 , and that with signal+backgrounds is 0.114 . These can be compared to Fig. 6.10| and $|6.13|$. . . . . . . . . . . . . 173

6.28 A comparison of the $A_{\mathrm{FB}}^{t \bar{t}}$ measured with the unfolding algorithm compared with the $A_{\mathrm{FB}}^{t t}$ generated, for the reweighted POWHEG MC samples, with $-0.1<A_{\mathrm{FB}}^{t t}<0.3$. No bias observed. We note that all the

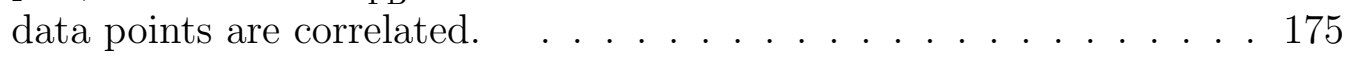

6.29 A comparison of the inclusive $A_{\mathrm{FB}}^{t \bar{t}}$ measured with the unfolding algorithm with the $A_{\mathrm{FB}}^{t t}$ generated, for the reweighted POWHEG MC samples and benchmark BSM scenarios. We take the difference between the generated $A_{\mathrm{FB}}^{t \bar{t}}$ and the measured $A_{\mathrm{FB}}^{t \bar{t}}$ with PYTHIA MC (0.02) as an unfolding systematic uncertainty. . . . . . . . . . . 176

\begin{tabular}{|l|l|l|l|}
\hline 6.30 & The same as Fig. & 6.12 & but after the final optimization requirements.
\end{tabular} The pull distributions look good. The expected statistical uncertainties with signal only are 0.27 for $A_{\mathrm{FB}}^{t \bar{t}}\left(\left|\Delta y_{t}\right|<0.5\right)$ and 0.13 for $A_{\mathrm{FB}}^{t t}\left(\left|\Delta y_{t}\right|<0.5\right) \mid \ldots \ldots \ldots \ldots \ldots \ldots 177$

6.31 The same as Fig. 6.14 , but after the final optimization requirements. The pull distributions look good. The expected statistical uncertainties with signal+backgrounds are 0.30 for $A_{\mathrm{FB}}^{t \bar{t}}\left(\left|\Delta y_{t}\right|<0.5\right)$ and 0.14 for $A_{\mathrm{FB}}^{t t}\left(\left|\Delta y_{t}\right|>0.5\right) . \ldots \ldots \ldots \ldots \ldots \ldots$ 
6.32 A comparison of the differential $A_{\mathrm{FB}}^{t \bar{t}}$ measured with the unfolding algorithm with the $A_{\mathrm{FB}}^{t t}$ generated for the reweighted POWHEG MC samples. The two values are consistent with each other, but since there is some deviation which is in the worst case negligible compared with the expected statistical uncertainties (0.30 and 0.15$)$, we take the deviations as systematic uncertainties. . . . . . . . . . . . . 179

6.33 Posterior probability density of the inclusive $A_{\mathrm{FB}}^{t \bar{t}}$. A Gaussian function is fitted to the center of the distribution to extract the result. . . 183

6.34 Posterior probability density distributions of $A_{\mathrm{FB}}^{t \bar{t}}\left(\left|\Delta y_{t}\right|<0.5\right)$ and $A_{\mathrm{FB}}^{t t}\left(\left|\Delta y_{t}\right|>0.5\right)$ from data. Gaussian functions are fitted to the center of the distributions to extract the results. . . . . . . . . . . . . 186

6.35 The 2D posterior probability density distribution of $A_{\mathrm{FB}}^{t \bar{t}}\left(\left|\Delta y_{t}\right|<0.5\right)$ vs. $A_{\mathrm{FB}}^{t t}\left(\left|\Delta y_{t}\right|>0.5\right)$ from the data, with the result from the POWHEG simulation overlaid. The correlation of the two results is measured to be -0.44 . . . . . . . . . . . . . . . . . 186

6.36 Comparison of $A_{\mathrm{FB}}^{t \bar{t}}$ vs. $\left|\Delta y_{t}\right|$ measurements of this measurement and other Tevatron measurements as well as the NNLO SM predictions. The linear fit for the dilepton measurement is also plotted in (b). . . 187

7.1 The correlation matrix of the differential $A_{\mathrm{FB}}^{t \bar{t}}$ in the lepton+jets and dilepton analyses. . . . . . . . . . . . . . . . . 200

7.2 A comparison of the data points from the dilepton $A_{\mathrm{FB}}^{t \bar{t}}$ vs. $\left|\Delta y_{t}\right|$ result along with the slope from the CDF combination and other Tevatron measurements as well as the NNLO SM prediction [46, 48].] . . . . . . 203

8.1 Comparison of the inclusive $A_{\mathrm{FB}}^{\ell}$ measurements with lepton+jets and dilepton final states from CDF [43], D0 [59, 61], and the NLO [42] SM calculations, as well as the CDF combination. . . . . . . . . . . 206

8.2 Comparison of the inclusive $A_{\mathrm{FB}}^{t \bar{t}}$ measurements with lepton+jets and dilepton final states from CDF [20], D0 [60, 62], the NLO [42] and the NNLO [46] SM calculations, as well as the CDF combination. . . . . 207

8.3 Comparison of all inclusive top $A_{\mathrm{FB}}$ results from the Tevatron with the NLO and NNLO SM predictions. . . . . . . . . . . . . . . . . . . 208 
8.4 Comparison of the individual and combined slope $\alpha$ values of $A_{\mathrm{FB}}^{t \bar{t}}$ vs. $\left|\Delta y_{t}\right|$ from CDF [20], along with the corresponding result in the lepton+jets final state from D0 [60], and the SM calculation at NNLO [46, 48]. . . . . . . . . . . . . . . . . . . . . . . . . . . . . . . . . . . . . 209

B.1 The $E_{T}$ distribution of DY $\rightarrow \tau \tau$ in the signal region as estimated from the POWHEG MC. . . . . . . . . . . . . . . 237

C.1 The distributions of $q_{\ell} \eta_{\ell}$ and $\Delta \eta$ in the 0 and 1 jet control regions. . 242

D.1 The same plots as Fig. 3.1 , but with at least one SECVTX $b$-tagged jet.246

D.2 The same plots as Fig. 3.2 , but with at least one SECVTX b-tagged jet.247

D.3 The same plots as Fig. 3.3 , but with at least one SECVTX b-tagged jet.248

D.4 The same plots as Fig. 3 3.4, but with at least one SECVTX b-tagged jet.249

D.5 The same plots as Fig. 3.5 , but with at least one SECVTX b-tagged jet.250

D.6 The same plots as Fig. 3 3.6, but with at least one SECVTX b-tagged jet.251

E.1 The $q_{\ell} \eta_{\ell}$ distribution from the POWHEG $t \bar{t}$ sample at parton level, with a fit to a single Gaussian function in the region $\left|q_{\ell} \eta_{\ell}\right|<1.4$ (indicated by the dashed lines). Note that the agreement is not good

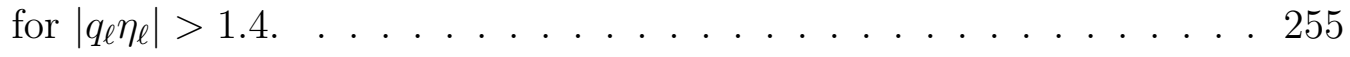

E.2 The $q_{\ell} \eta_{\ell}$ distribution from the POWHEG $t \bar{t}$ sample at parton level, overlaid with the double-Gaussian fit. Note that both the tails and the central part of the distribution are well described. . . . . . . . . . 256

E.3 $\quad$ Fit parameters from our benchmark samples as a function of $A_{\mathrm{FB}}^{\ell}$. . . 257

E.4 With the double-Gaussian modeling, and constraining the two $\sigma$ values and the $r$ to the best estimated values from the benchmark simulations, $A_{\mathrm{FB}}^{\ell}$ appears to be linear as a function of the mean of the double-Gaussian function in the small $A_{\mathrm{FB}}^{\ell}$ region. In a larger region, $A_{\mathrm{FB}}^{\ell}$ asymptotes to $\pm 1 . \ldots \ldots \ldots \ldots . \ldots . \ldots . \ldots 258$

E.5 The $\mathcal{S}\left(q_{\ell} \eta_{\ell}\right)$ term and the $\mathcal{A}\left(q_{\ell} \eta_{\ell}\right)$ term from the double-Gaussian model, with the $\mu$ parameter varied. . . . . . . . . . . 260 
E.6 Figures showing the differential contribution to the total asymmetry as a function of $q_{\ell} \eta_{\ell}$ using the double-Gaussian model. This is estimated using the $\mathcal{S}\left(q_{\ell} \eta_{\ell}\right)$ term times the $\mathcal{A}\left(q_{\ell} \eta_{\ell}\right)$ term, with the $\mu$ parameter varied, with different overall normalizations. (a) The curves are normalized so that $\int \mathcal{S}\left(q_{\ell} \eta_{\ell}\right) \mathrm{d} q_{\ell} \eta_{\ell}=1$ as in Eq. E.6. In this case, the areas under the curves give the inclusive asymmetry. (b) The curves are normalized to $\int \mathcal{S}\left(q_{\ell} \eta_{\ell}\right) \cdot \mathcal{A}\left(q_{\ell} \eta_{\ell}\right) \mathrm{d} q_{\ell} \eta_{\ell}=1$ as in Eq. E.7. In this case, we can see that the differential contribution to the asymmetry as a function of $q_{\ell} \eta_{\ell}$ is largely independent of the value of $\mu$ for small values of $\mu$. . . . . . . . . . . . . . . . . . . . . 261

E.7 Fraction of $A_{\mathrm{FB}}^{\ell}$ within a certain $q_{\ell} \eta_{\ell}$ coverage. The vertical lines show $q_{\ell} \eta_{\ell}=1.25,1.5$ and 2.0 corresponding to the typical detector coverages at CDF and D0. The numbers are given in Table|E.1. The horizontal line indicates that the fraction asymptotes to one as the $q_{\ell} \eta_{\ell}$ coverage goes to infinity. . . . . . . . . . . . . . . . 262

E.8 Comparison among the $a \cdot \tanh$ model, the single-Gaussian model, the double-Gaussian model and the POWHEG simulation. (a) shows the best fits of the $\mathcal{A}\left(q_{\ell} \eta_{\ell}\right)$ distribution (done only using events with $\left|q_{\ell} \eta_{\ell}\right|<2.0$ ), while (b) shows the differential contribution to the $A_{\mathrm{FB}}^{\ell}$ as a function of $q_{\ell} \eta_{\ell}$ from different models. . . . . . . . . . . . . . 264

E.9 Best fit of double-Gaussian model to the $\mathcal{A}\left(q_{\ell} \eta_{\ell}\right)$ distribution for various $t t$ samples at generator level. This figure can be compared directly to Fig. $4.2 \mathrm{~b} \mid$ where we fit the same data, but using the $a \cdot$ tanh function.265

F.1 The $q_{\ell} \eta_{\ell}$ distribution for positive and negative leptons from Powheg MC at generator level. . . . . . . . . . . . . . . . . . . . 270

F.2 $\quad$ Pseudoexperiments to test correlation between two leptons in an event.272 


\section{LIST OF TABLES}

TABLE

Page

1.1 Comparison of the three $A_{\mathrm{FB}}$ observables measured in this dissertation. 21

1.2 The SM predictions of $A_{\mathrm{FB}}^{t t}, A_{\mathrm{FB}}^{\ell}$, and $A_{\mathrm{FB}}^{\ell \ell}$ as well as with some scenarios that include particles beyond the SM (denoted as BSM). The uncertainties for the calculations in the top three rows correspond to the uncertainties due to theoretical calculation techniques, while other uncertainties are due to the size of the simulations. The models with physics beyond the SM are generated with MADGRAPH [58], which is a LO MC generator. . . . . . . . . . . . . . . . . . 27

1.3 Summary of the top-quark $A_{\mathrm{FB}}$ results from the CDF and D0 collaborations which are now completed [46, 42, 20, 43, 59, 60, 61]. Note that while many of the results presented in this dissertation were published before some of the results listed here, we have listed them all for context, but left the answers from this work for Sec. 8 . The lepton+jets final state is denoted as $\mathrm{L}+\mathrm{J}$, and the dilepton final state is denoted as DIL. . . . . . . . . . . . . . . . . . . . . . . . . . 29

2.1 The top dilepton event selection. We refer the reader to Ref. [67] for

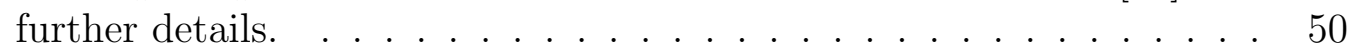

3.1 A description of the simulated $t \bar{t}$ samples. The "Generator" column lists the name of the program used to calculate the main Feynman diagram, while the "Parton shower" column lists the generator to model the hadronization of the final state particles. The column "Luminosity profile" lists the instantaneous luminosity profile used to model the number of multiple collisions in one event; "Full" indicates a profile corresponding to the full Run II periods of CDF is used, while "Partial" indicates a profile matching CDF Run II up to the end of year 2008 is used. For the BSM samples, the mass of the hypothetical particle is denoted as " $m$ ", and its width is denoted as " $[" . . . .55$

$3.2 \quad$ A table of background MC samples. . . . . . . . . . . . . 57 
3.3 Table of the expected number of events in data with the observed, passing all event selections in Table $\mid 2.1$, listed by lepton flavors. . . . 59

4.1 Comparison between the generated $A_{\mathrm{FB}}^{\ell}$ and the results of the measurement methodology at the generator level from the various MC models. No noticeable bias is observed. . . . . . . . . . . . . . . . 77

4.2 The binning choice of the $q_{\ell} \eta_{\ell}$ distribution used in the measurement of $A_{\mathrm{FB}}^{\ell}$, along with the expected number of events from $t \bar{t}+$ backgrounds,

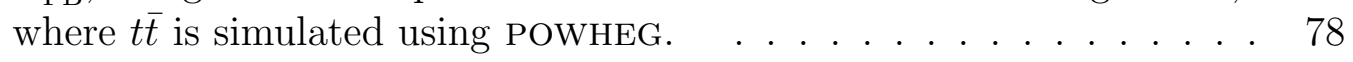

4.3 Comparison between the generated $A_{\mathrm{FB}}^{\ell}$ and the results of the measurement methodology from the various MC models, using the sample of reconstructed events that pass all the event selection requirements in Table 2.1. No noticeable bias is observed. . . . . . . . . . . . . . . 80

4.4 The binning choice of the $\Delta \eta$ distribution used in measurement of $A_{\mathrm{FB}}^{\ell \ell}$, along with the expected number of events from $t \bar{t}+$ backgrounds, where $t \bar{t}$ is simulated using POWHEG. . . . . . . . . . . . . . . . 86

\begin{tabular}{|ll|l|l|}
\hline 4.5 & The same as Table & 4.1 & but with $\Delta \eta$ and $A_{\mathrm{FB}}^{\ell \ell}$ instead of $q_{\ell} \eta_{\ell}$ and $A_{\mathrm{FB}}^{\ell}$
\end{tabular} 89

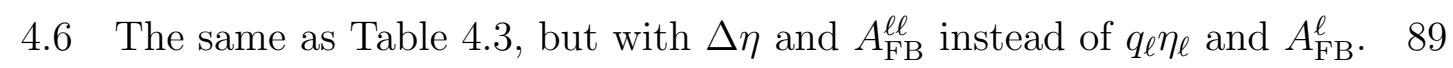

4.7 The uncorrected $A_{\mathrm{FB}}^{\ell}$, with a straight counting of the numbers of the forward and the backward leptons, from the data only and the data after background subtraction, together with the expectation from the POWHEG $t \bar{t}$ MC, which is with NLO SM with QCD corrections but without electroweak corrections. . . . . . . . . . . . . . . 91

4.8 A comparison of the number of leptons in each $q_{\ell} \eta_{\ell}$ range, bin-bybin, before and after the background subtraction. Note that the $t t$ contributions are estimated using the POWHEG MC. . . . . . . . . . . 92

4.9 Table of uncertainties for the $A_{\mathrm{FB}}^{\ell}$ measurement. . . . . . . . . . . . . . 97

4.10 The extrapolated $A_{\mathrm{FB}}^{\ell}$ results in different lepton categories. The uncertainties are statistical only. . . . . . . . . . . . . . . . . . . 98

4.11 A comparison of the number of leptons in each $\Delta \eta$ range bin-by-bin before and after the background subtraction. Note that the $t \bar{t}$ contributions are estimated using the POWHEG MC. . . . . . . . . . . . . . 103 
4.12 The uncorrected $A_{\mathrm{FB}}^{\ell \ell}$, with a straight counting of the numbers of the forward and the backward leptons, from the data only and the data after background subtraction, together with the expectation from the POWHEG $t t$ MC, which is with NLO SM with QCD corrections but without electroweak corrections. . . . . . . . . . . . . . . . . . . 104

4.13 Table of uncertainties for the $A_{\mathrm{FB}}^{\ell \ell}$ measurement. . . . . . . . . . . . . 104

5.1 Best fit values for the mean values of $\mathrm{SF}_{\text {jet }}\left(\overline{\mathrm{SF}_{\text {jet }}}\right)$ and the resolutions $(\sigma)$ of $\mathrm{SF}_{\text {jet }}$ and $\not_{x, y}\left(\sigma_{\mathrm{SF}_{\mathrm{jet}}}\right.$ and $\sigma_{\not_{x, y}}$, respectively $)$. These are derived from the NLO MC sample (POWHEG). . . . . . . . . . . . . . . . . 113

5.2 The fit functions and the best fit values of templates $p_{z, t \bar{t}, p_{T, t \bar{t}} \text { and }}$ $m_{t \bar{t}}$ derived from the POWHEG MC at the generator level. . . . . . . . 117

6.1 The expected total statistical uncertainty, background systematic uncertainty and the sum of the two in quadrature (the expected uncertainty), for the choices of the max-likelihood solution or full probability, and the choice of the more likely lepton-jet pairing (L-J pairing) or weighting both. The best set of choices is with the full probability and weighting both lepton+jets pairings. . . . . . . . . . . . . . . 157

6.2 Summary of the optimum cut and weight values used in the final

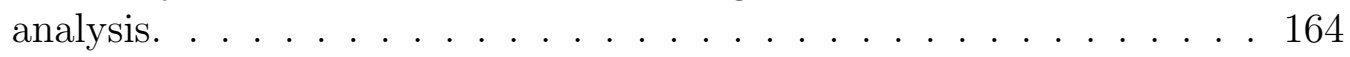

6.3 Expected number of events passing all top dilepton event selections and top reconstruction quality selections, along with the observed number of events in data. . . . . . . . . . . . . . . . . . . . 166

6.4 Summary of the numbers in Fig. $6.24 \mathrm{~b}$. This is Det $[p][r]$ in Eq. 6.7 , The uncertainties in the table are the statistical uncertainties due to the limit size of the MC sample. . . . . . . . . . . . . . . . . . . . . . 171

6.5 Summary of the efficiencies in each $\left|\Delta y_{t}\right|$ bin as a linear function of $A_{\mathrm{FB}}^{t t}$ taken from Fig. 6.25 , after the optimization. This is $\operatorname{Eff}[p]\left(A_{\mathrm{FB}}^{t t}\right)$ in Eq. 6.7. The uncertainties in the table are the statistical uncertainties caused by the limited size of the MC sample. . . . . . . . . . . . . . . 174

6.6 Table of uncertainties for the $A_{\mathrm{FB}}^{t \bar{t}}$ measurement. . . . . . . . . . . . . 182

6.7 Table of uncertainties for the $A_{\mathrm{FB}}^{t \bar{t}}\left(\left|\Delta y_{t}\right|<0.5\right)$ and $A_{\mathrm{FB}}^{t \bar{t}}\left(\left|\Delta y_{t}\right|>0.5\right)$ measurements. . . . . . . . . . . . . . . . . . . . . . . 184

6.8 Covariance matrix of total uncertainty for differential $A_{\mathrm{FB}}^{t \bar{t}}$ vs. $\left|\Delta y_{t}\right| . .185$ 
7.1 Table of uncertainties for $A_{\mathrm{FB}}^{\ell}$ measurement in the lepton+jets $(\mathrm{L}+\mathrm{J})$ and the dilepton (DIL) final state. In the column of correlation, "0" indicates no correlation and "1" indicates fully positive correlation. . 191

7.2 Covariance matrices of the two measurements of the $A_{\mathrm{FB}}^{\ell} . \ldots . . . \quad 194$

7.3 Table of uncertainties for the inclusive $A_{\mathrm{FB}}^{t \bar{t}}$ measurement in the lepton + jets $(\mathrm{L}+\mathrm{J})$ and the dilepton (DIL) final state. In the column of correlation, " 0 " indicates no correlation and " 1 " indicates fully positive correlation. . . . . . . . . . . . . . . . 195

7.4 Covariance matrix of total uncertainty for the lepton+jets and dilepton differential $A_{\mathrm{FB}}^{t t}$ combination. $\ldots \ldots \ldots . \ldots . \ldots 199$

7.5 The bin centroids, the differential $A_{\mathrm{FB}}^{t \bar{t}}$ measurements, and the eigenvalues and eigenvectors of the covariance matrix in the $A_{\mathrm{FB}}^{t t}$ vs. $\left|\Delta y_{t}\right|$ measurements. Everything needed to do the simultaneous linear fit is contained in this table. . . . . . . . . . . . . . . . . . . . 201

A.1 The requirements used to select electrons in the Central ElectroMagnetic calorimeter (CEM). Tight CEM electrons ("CEM") are required to pass all the criteria. Nonisolated CEM electrons ("nisoCEM") pass all of the cuts except for Isolation (marked with an ${ }^{*}$ ). Loose, but isolated, CEM electrons ("looseCEM") are required to pass only the criteria above the line. Nonisolated loose CEM electrons (nisolooseCEM) pass all of the looseCEM cuts except isolation. We refer to Ref. [83] for the technical terms quoted in these tables as well as more details regarding the identification requirements. . . . . . . . . . . . . 218

A.2 The requirements to identify electrons in the plug electromagnetic calorimeter (PEM, and the electrons are identified with tracking package named PHENIX, thus denoted as "PHX"). All the PHX electrons belong to the tight category. We refer to Ref. [83] for the technical terms quoted in these tables as well as more details regarding the identification requirements. . . . . . . . . . . . . . . . 219

A.3 The muon identification selection requirements for candidates in the CMUP systems. Tight muons must pass all the requirements in the table. Note that loose muons "CMU" ("CMP") are defined by not requiring the requirement below the line on the "CMP" ("CMU") counterpart. We refer to Ref. [83] for the technical terms quoted in these tables as well as more details regarding the identification requirements. .................... 219 
A.4 The muon identification selection requirements for candidates in the CMX systems. All CMX muons fall in the tight category. We refer to Ref. [83] for the technical terms quoted in these tables as well as more details regarding the identification requirements. . . . . . . . . . . . . 220

B.1 Definition of signal and control regions for DY estimation. Note that this table is only for the events in the $e e$ and $\mu \mu$ final states (not for the $e \mu$ final state). The events in all regions defined here are after all the "minimal requirements" in Table 2.1 except the requirements on METSig and $\mathrm{m}_{1 l}$. The estimations in Regions 1, 2, and 4, after applying the efficiency for the "signal requirements", are the final estimations in the signal region of this analysis. Note that there are six different versions of this table, one for each of the $e e$ and $\mu \mu$ final states, and each with a jet multiplicity of 0,1 , and $\geq 2$. . . . . . . . . 225

B.2 Inputs to Eq. B.2a for Region $1+3$ and for use in the calculation of the number of DY $\rightarrow e e / \mu \mu$ events in Region $2+4$. The $N^{\text {Data }}$ numbers are obtained from data, while the $N^{\text {Non-DY }}$ and $N^{\text {DYMC }}$ numbers are obtained from simulations of all the backgrounds and signal simulation (with the contribution from $W+$ jets estimated from data) as described in Chapter 3 . The row of $N_{\text {Region } 2+4}^{\text {DYEstimation presents the output }}$ of Eq.|B.2a| The uncertainties are statistical only. . . . . . . . . . . . 229

\begin{tabular}{|ll|l|l|l}
\hline B.3 Inputs to Eqn. & B.2b for Region 3 and for use in the calculation of
\end{tabular} the number of DY $\rightarrow e e / \mu \mu$ events in Region 1 . The $N^{\text {Data }}$ numbers are obtained from data, while the $N^{\text {Non-DY }}$ and $N^{\text {DYMC }}$ numbers are obtained from simulations of all the backgrounds and signal simulation (with the contribution from $W+$ jets estimated from data) as described in Chapter 3 . The row of $N_{\text {Region } 1}^{\text {DY Estimation presents the output }}$ of Eq. B.2b. The uncertainties are statistical only. . . . . . . . . . . . 230

B.4 Efficiencies of the signal requirements with $\geq 2$ jets $\left(\epsilon^{\text {Signal cut }}\right)$. This is the efficiencies for the opposite charged leptons and $H_{T}>200 \mathrm{GeV}$ requirements, to be applied to the outputs with $\geq 2$ jets in Tables $|\mathrm{B} .2|$ and|B.3 $\mid$ using Eq.|B.3. . . . . . . . . . . . . . . . . . . . . . . . . 231

B.5 The calculation of the scale factor for the DY overall normalization $\left(\mathrm{SF}_{\mathrm{DY}}\right)$ with Eq. B.5. The bottom line shows the average of the scale factors in the $e e$ and $\mu \mu$ final states in each jet multiplicity, and will be applied to the DY $\rightarrow e e / \mu \mu \rightarrow e_{\text {fake }} \mu$ and DY $\rightarrow \tau \tau$ estimations as shown in Table|B.7]. . . . . . . . . . . . . . . . . . . 234 
B.6 The calculation of the scale factor for DY $E_{T}$ distribution $\left(\mathrm{SF}_{\mathrm{DY}} E_{T}\right)$ with Eq. B.7. The bottom line shows the average of the scale factors in the $e e$ and $\mu \mu$ final states in each jet multiplicity, and will be applied to the DY $\rightarrow e e / \mu \mu \rightarrow e_{\text {fake }} \mu$ estimations as shown in Table|B.7. The differences between the scale factors in the $e e$ and $\mu \mu$ categories are taken as systematic uncertainties. . . . . . . . . . . . . . 236

B.7 The DY expectation comparison using the MC estimation method with the scale factors using Eq. B.4 (the "MC SF Pred." column) and the "Data-MC Hybrid method" (the "Hybrid Pred." column). The last two columns agree with each other when both entries are available. We use the expectation numbers in the last column when they exist, and the numbers in the next-to-last column otherwise. . . 238

C.1 Table of expected number of events in data with the observed number of events, passing the minimal cuts in Table 2.1 with 0 jets with $E_{\mathrm{T}}>$ $15 \mathrm{GeV}$ within $|\eta|<2.5$, listed by lepton flavors. . . . . . . . . . . . . 240

C.2 Table of expected number of events in data with the observed number of events, passing the minimal cuts in Table 2.1 with 1 jet with $E_{\mathrm{T}}>$ $15 \mathrm{GeV}$ within $|\eta|<2.5$, listed by lepton flavors. . . . . . . . . . . . . 241

C.3 The uncorrected $A_{\mathrm{FB}}^{\ell}$ and $A_{\mathrm{FB}}^{\ell \ell}$ calculated by counting the number of events in the forward and backward regions from data and SM expectation in the 0 and 1 jet control regions. The uncertainties are statistical only. . . . . . . . . . . . . . . . . . . . 243

D.1 Comparing the expected number of events in data passing all requirements in Table 2.1 with at least one tight SECVTX $b$-tag with the observed number of events passing all event selection, listed by lepton flavors. . . . . . . . . . . . . . . . . . . 245

E.1 Fraction of $A_{\mathrm{FB}}^{\ell}$ within typical $q_{\ell} \eta_{\ell}$ coverage at CDF and D0. . . . . . 259

E.2 Results of pseudoexperiments using the different methods to reproduce $A_{\mathrm{FB}}^{\ell}$ of the POWHEG simulation (0.0236), but only using events with $\left|q_{\ell} \eta_{\ell}\right|<2.0$. Note that the uncertainties listed are statistical only and are due to the size of the simulated data sample. . . . . . . . . . . 266 
E.3 Comparison of the predicted $A_{\mathrm{FB}}^{\ell}$ values and the corresponding measured $A_{\mathrm{FB}}^{\ell}$ values with the $a \cdot \tanh$ model and the double-Gaussian model. The uncertainties are statistical only and are always small compared to the expected statistical uncertainty in data collected by the CDF and D0 experiments. . . . . . . . . . . . . . . 267

E.4 Hyperbolic tangent fit on generator level asymmetric part. . . . . . . 267 


\section{INTRODUCTION}

The standard model (SM) of particle physics [1, 2, 3, provides the current best understanding of the known fundamental particles in nature as well as their interactions. Despite its great success in describing almost all of the phenomena of the elementary particles, it suffers from a number of problems. For example, it fails to explain the presence of dark matter [4], which is estimated to constitute $85 \%$ of the total matter in the universe. This calls for careful experimental examination of the SM and following up on any hints that might lead to evidence for physics beyond the SM.

One important category of the experimental examinations of the SM is the measurement of the properties of the top quark, the last quark discovered in the SM, and its interactions in high energy collisions. All these properties, including its mass, lifetime, and production cross section, serve as input parameters to the SM; if new phenomena or deviations from SM predictions are observed during measurements, this could indicate first hints of physics beyond the SM (BSM) [5], or even the existence of new fundamental particles.

The top quark is the heaviest known fundamental particle and was first observed by the CDF and D0 experiments at the Fermilab Tevatron collider in 1995 [6, 7], a proton-antiproton collider running with a center-of-mass energy of $1.8 \mathrm{TeV}$ (1800 times the proton mass in energy) at the time of the discovery. Ever since its discovery, people have been probing the properties of this particle, both at the Tevatron at Fermilab and at the Large Hadron Collider (LHC) [8] at CERN, which is a protonproton collider running at $\sqrt{s}=7,8$, and $13 \mathrm{TeV}$.

While most of the measurements yield results consistent with the SM predic- 
tions [9], one set of measurements generated a lot of interest. This set of measurements studied high energy proton-antiproton collisions at the Fermilab Tevatron which can produce pairs of top quarks (actually a top quark and a top antiquark, often denoted as $t \bar{t}$ ), and considers the probability of the outgoing top quark going in the direction of the proton (the "forward" direction) versus the direction of the antiproton (the "backward" direction), as shown in Fig. 1.1. The difference between the two probabilities is denoted as the "forward-backward asymmetry" $\left(A_{\mathrm{FB}}\right)$. The simplest calculation (with only the Leading Order Feynman diagrams) with the SM predicts equal number of top quarks produced going in the forward and backward directions. Thus, there would be zero asymmetry. If more top quarks are observed to go in the forward direction, we would have a positive asymmetry. Detailed calculations (taking into account higher order diagrams) of the SM predict a small, but measurable asymmetry at the Tevatron. This makes it unique because at the LHC, despite running at higher energies and producing a lot more $t \bar{t}$ pairs, the collisions are between protons and protons and are symmetric along the beamline direction, therefore there is no asymmetry expected at any order.

Early calculations, shortly after the discovery of the top quark but before any measurements were done, suggested a measurable asymmetry at about about the 5-6\% level [10, 11, 12, 13, 14]. The first measurements from both the CDF and the D0 collaborations, from the second major run of the Tevatron using a center-ofmass energy of $1.96 \mathrm{TeV}$, were published in 2008 [15, 16] and both showed results consistently higher than the prediction, around $\sim 20 \%$, yet still agreed with the SM prediction within statistics due to large uncertainties (within $2 \sigma$ ). This spurred a huge amount of activity, including refining the predictions and adding more data to the measurements as it became available. By 2012, when the work presented in this dissertation began, the deviation had become statistically significant at the 


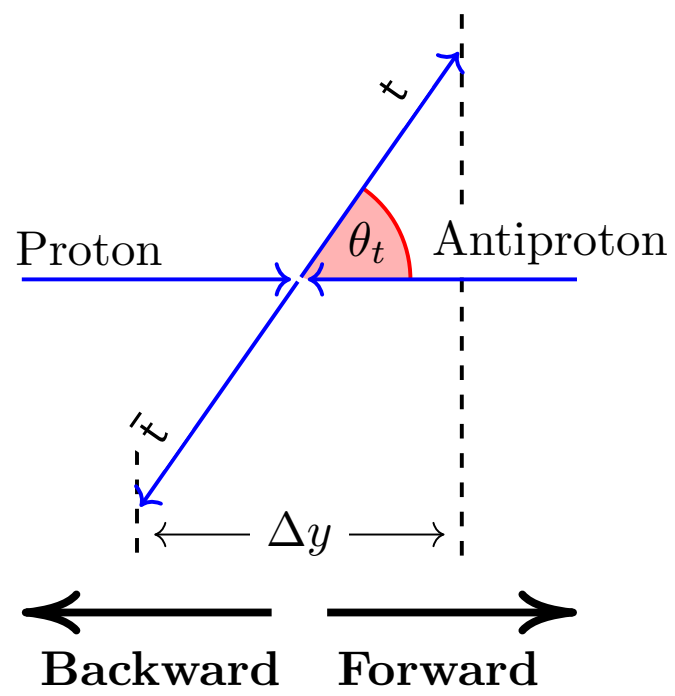

Figure 1.1: The $t \bar{t}$ pair production and decay in a proton-antiproton collision. The proton direction is denoted as the "forward" direction while the antiproton direction is denoted as the "backward" direction.

$\sim 3 \sigma$ level [19, 17, 20, 18]. This deviation was incredibly exciting to the particle physics community, and generated many talks and papers, because, if confirmed, it might suggest a hint of the existence of a new fundamental particle in nature [21]. The analyses presented in this dissertation are designed to either confirm or deny the deviations between the experimental results and the SM predictions with independent observables.

In this section we provide a brief summary of the SM of particle physics with an emphasis on the top quark, and explain why and how we will do this particular set of measurements. We also put the current analysis in its historical context, give some theoretical remarks about what the predictions from the SM are, as well as what the predictions would be if there were new fundamental particles in nature. In addition, we provide a summary of the experimental status of other results that have come forward during the time these analyses were in progress. This section ends with an 
outline of the dissertation.

\subsection{Overview of the Standard Model of Particle Physics}

The SM of particle physics serves as a "periodic table" for the fundamental particles, as shown in Fig. 1.2, and well describes the three fundamental forces, the strong, the week, and the electromagnetic forces. (The gravitational force is not yet included in the SM.) The fundamental particles are categorized into quarks, leptons, gauge bosons, and the Higgs boson. The quarks and the leptons are fermions, with spin $1 / 2$, and act as the building blocks of matter. For example, three quarks (two up quarks and one down quark) make up the proton; the electron is a lepton and is the other fundamental building block of the atoms that fill the universe. There are six flavors of quarks, and six flavors of leptons, grouped into three generations. The corresponding particles among the three generations have the same charge and spin numbers, but different masses. The quarks are not stable by themselves. All quarks but the top quark can form hadrons (stable or semi-stable particles consisting of quarks) [9]. Top quarks decay immediately after their production due to an extremely short lifetime [9].

The gauge bosons and the Higgs boson have integer spins (0 or 1). The gluons are the carriers of strong force; the photons are the carriers of the electromagnetic force; and the $W$ and $Z$ bosons are the carriers of the weak force. The Higgs mechanism [22] is responsible for the masses of the particles, and predicts the last particle of the SM, the Higgs boson, which was observed in 2012 at the Tevatron and the LHC [23, 24, 25]. The quarks are subject to the strong, weak and electromagnetic forces, while the leptons are subject to only the weak and electromagnetic forces. A detailed review of the SM can be found in Ref. [9]. 


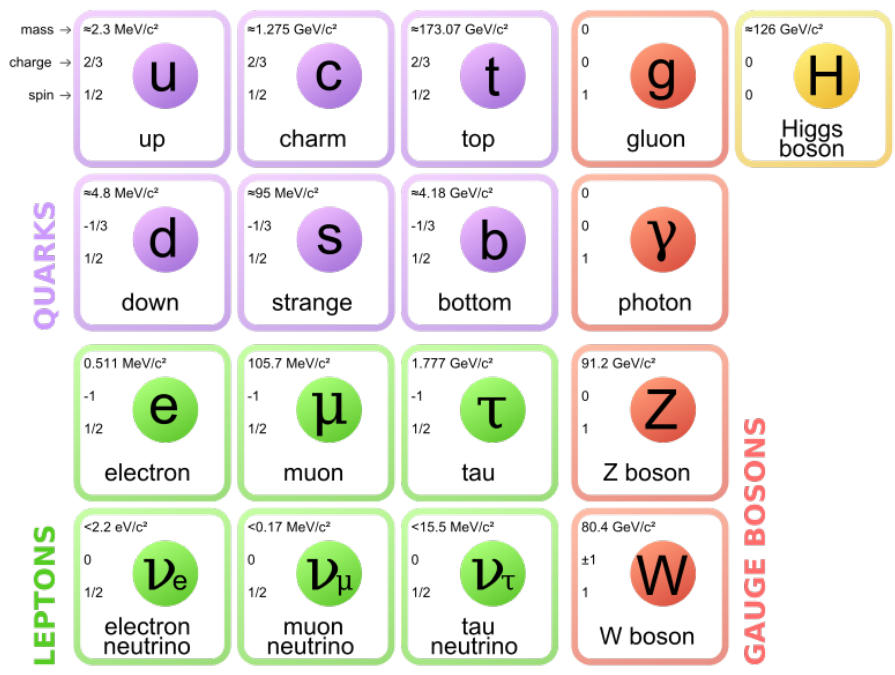

Figure 1.2: Summary of the particles in the standard model [26].

\subsection{Properties, Production and Decays of the Top Quark}

In this section we briefly summarize a selection of the properties of the top quark (its mass and lifetime), how it can be produced via proton-antiproton or protonproton collisions, and how it decays. This will help us understand how to identify top quarks in collisions, as well as how to make predictions for what we expect in our experiment. As detailed in Sec. 2, we call the products of one collision an "event", and an ensemble of events a "sample".

\subsubsection{Top-quark Mass, Lifetime, and Production}

The top quark is the heaviest known elementary particle, and its mass is an important parameter of the SM [27]. It provides a critical input to check if the SM is a self-consistent model [5], which is done with a global fit of the entire SM to all the results of the measurement of the SM parameters. The most precise measurement of the top-quark mass to date is the Tevatron combination [28, with a result of $m_{\text {top }}=174.34 \pm 0.64 \mathrm{GeV}$. 
The lifetime of top quark is another feature that makes the top quark special. Because its lifetime is shorter than the typical hadronization time scale, it does not form any bound state (stable or semi-stable composite particles) with other quarks, but rather decays immediately after its production [29]. This provides a unique opportunity to study a "bare" quark. The CDF collaboration performed a direct measurement of the top quark width, which is directly related to the topquark lifetime, with the full CDF Run II dataset resulting in a result of $\tau^{\text {top }}=$ $2.98_{-1.35}^{+3.00} \times 10^{-25} \mathrm{~s}[30]$. The D0 collaboration measured the top-quark lifetime in an indirect way, yielding a result of $\tau^{\text {top }}=3.29_{-0.63}^{+0.90} \times 10^{-25} \mathrm{~s}[31$. These results verified that the top-quark lifetime is shorter than the typical hadronization time scale $\left(\mathrm{O}\left(10^{-24} \mathrm{~s}\right)\right)$.

While the mass of the top quark is an inherent property, the production of top quarks in high energy collisions reveals information about the interactions between top quarks and protons (actually the quarks and gluons inside the protons), and potentially other mediators. Large deviations between the measured cross section and the expected value would suggest that new particles or interactions beyond the SM exist in the process. The expectations from the SM are that top quarks can be produced via both strong interactions and electroweak interactions at the Tevatron and the LHC. The strong production of top quarks is dominated by $t \bar{t}$ pair production, in particular the quark-antiquark annihilation process at the Tevatron (85\%, as shown in Fig. 1.3a) and the gluon-gluon fusion process at the LHC (90\%, as shown in Fig. 1.3b [9]. The electroweak interactions typically produce a single top quark. The $t$-channel diagram dominates at both the Tevatron and the LHC, while the contributions from the $s$-channel and the $t W$-channel at both places have been observed to be small [9]. We will focus on $t \bar{t}$ pair production in this dissertation, since this is the production mode where the aforementioned forward-backward asymmetry 
observables are based on, and where the initial discrepancy was observed [10, 15, 19, 17, 20, 16, 18]. We refer the reader to Ref. [9] for further details about the electroweak production of top quarks, and note that the ability to measure an asymmetry with them does not yet exist.

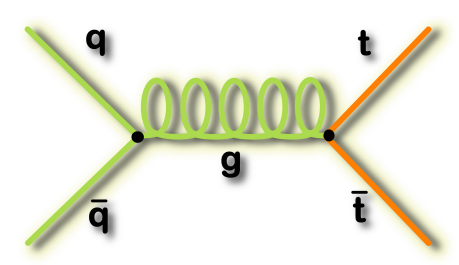

(a)

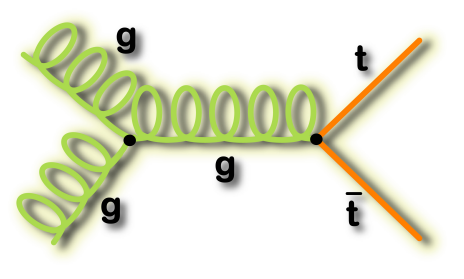

(b)

Figure 1.3: Dominant Feynman diagrams of $t \bar{t}$ pair production in the SM with quarkantiquark annihilation process (a) and gluon-gluon fusion process (b) [32].

The inclusive production cross section of $t \bar{t}$ at the Tevatron, integrating over all known processes, has been measured by the CDF and the D0 collaborations. At a center-of-mass energy of $1.96 \mathrm{TeV}$, the most precise measurement is $7.60 \pm$ $0.41 \mathrm{pb}$ [33], which is in agreement with the SM calculation of $7.35_{-0.24}^{+0.20} \mathrm{pb}$ at nextto-next-to-leading order with additional corrections from gluon contributions [34, 35]. Results at the LHC also show consistency with SM predictions [9]. We note here that the measurement of the forward-backward asymmetry, which is the main analysis described in this dissertation, is essentially a more sophisticated measurement of the production mechanisms as it probes the cross section of $t \bar{t}$ as a function of the angle between the beam line and the top-quark outgoing direction.

\subsubsection{Top-quark Decays}

Due to its large mass (being much heavier than the $W$ boson), the top quark is the only quark that can decay (and dominantly does decay) into a real (on shell) $W$ boson 
and a down-type quark ( $d, s$, and $b$ quark). In addition to decaying before forming any hadron due to its short lifetime, the probability that a top quark decays into the down-type quark in the same generation (a $b$ quark) instead of other down-type quarks is predicted and observed to be close to unity [9], indicating that top quark decays almost exclusively to a $W$ boson and a bottom (b) quark. Sequentially, the $W$ boson either decays leptonically into a charged lepton and a neutrino $\left(W^{+} \rightarrow \ell^{+} \nu\right.$ and its charge conjugate, about $\frac{1}{3}$ of the time) or hadronically into two light quarks $\left(W^{+} \rightarrow q \overline{q^{\prime}}\right.$ and its charge conjugate, where $q \overline{q^{\prime}}$ is dominated by $u \bar{d}$ and $c \bar{s}$, about $\frac{2}{3}$ of the time). The light quarks and the $b$ quark then "hadronize" after their production by pulling quark-antiquark pairs out of the vacuum and recombining into hadrons until they reach a state where there is no free quark. The newly formed hadrons, together with their cascade decay products, form a spray of particles within a narrow cone following the original direction of the parent quark, which is referred to as a "jet" 36].

Depending on the $W$-boson decay modes, $t \bar{t}$ events have multiple final states, as summarized in Figs. 1.4 and 1.5. Three major categories are:

1. The final state where both $W$ bosons decay hadronically $\left(t \bar{t} \rightarrow W^{+} W^{-} b \bar{b} \rightarrow\right.$ $q \overline{q^{\prime}} q \overline{q^{\prime}} b \bar{b} \rightarrow$ all jets, as shown in Fig. 1.4a) is denoted as the "all-jets" final state. This final state features the largest branching fraction (46\%), so it has the largest expected number of events, but also the largest number of backgrounds, other SM processes that mimic the same final state. Also, due to the large uncertainty in the jet energy measurement and the difficulty in determining the charge of the jets, it is hard to reconstruct the top momenta in this final state, and almost impossible to distinguish the top quark from the top antiquark. Thus, no $A_{\mathrm{FB}}$ measurement is performed in this final state. 
2. The final state where one of the $W$ boson decays leptonically and the other $W$ boson decays hadronically $(t \bar{t} \rightarrow \ell \nu q \bar{q} b \bar{b}$, as shown in Fig. 1.4b) is denoted as the "lepton+jets" final state. This final state has a decent branching fraction $(30 \%)$, and the charged lepton in the final state provides distinguishing power between the top quark and the top antiquark. The backgrounds are limited but substantial. It is because of this balance that it is known as the "golden final state" for the top $A_{\mathrm{FB}}$ measurements. The previous CDF top $A_{\mathrm{FB}}$ measurements are made in this final state.

3. The final state where both $W$ bosons decay leptonically $\left(t \bar{t} \rightarrow \ell^{+} \ell^{\prime-} \nu \overline{\nu^{\prime}} b \bar{b}\right.$, as shown in Fig. 1.4c is denoted as the "dilepton" final state. It has the advantage that there are even fewer SM background processes that produces the same signature of this final state, so any sample of selected events will have a higher expected purity of $t \bar{t}$ events. Also, both charged leptons are measured with high precision, and this allows a new observable, the $A_{\mathrm{FB}}$ of the lepton pairs, to be measured. However, the two neutrinos in the final state escape the CDF detector without leaving a trace, thus it is difficult to reconstruct the momenta (in particular the direction) of the top quark and antiquark from their decay products. In addition, the small branching fraction of this final state $(5 \%)$ also limits the number of events to study, which leads to the large statistical uncertainty in the analyses presented in this dissertation. Taking all these into consideration, this is the second best measurement option for the $A_{\mathrm{FB}}$ observables, and a natural complement to the lepton + jets measurements. We focus on this final state in this dissertation.

We note that while the final states including $\tau$ leptons are not explicitly used in these analyses, the scenarios where the $\tau$ lepton decays leptonically into an electron 


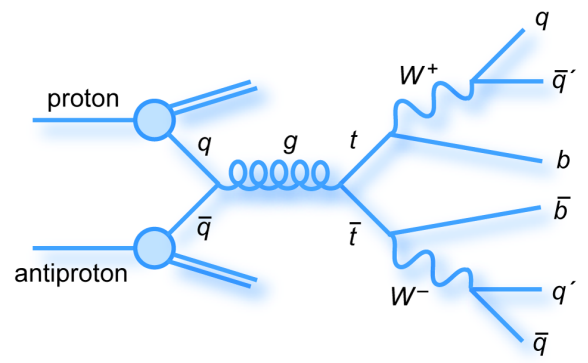

(a)

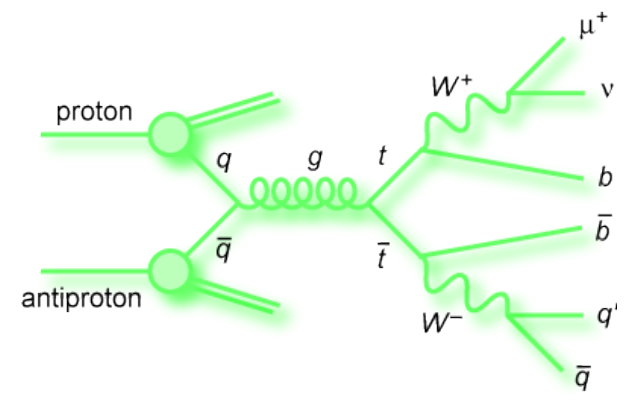

(b)

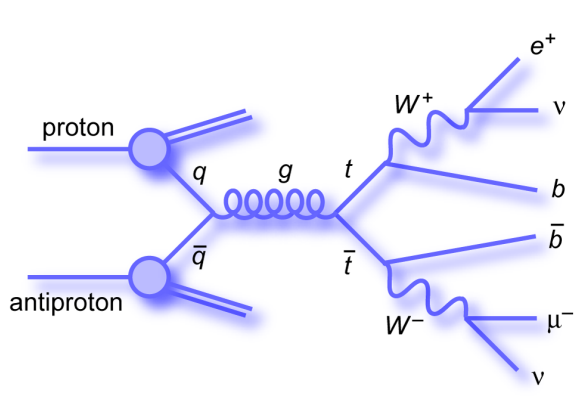

(c)

Figure 1.4: Feynman diagrams of $t \bar{t}$ pair production with proton-antiproton collisions, and decaying into the all-jets (a), lepton+jets (b), and dilepton (c) final states 37 .

or a muon are included implicitly in the corresponding lepton+jets and dilepton final states.

\subsection{Top-Quark Pair Production Forward-Backward Asymmetry}

\subsubsection{Historical Remarks}

In this subsection we present a more detailed historical review of the top-quark pair production forward-backward asymmetry predictions and measurements at the Tevatron to explain the powerful motivation for the analyses in this dissertation. The first SM calculation of top $A_{\mathrm{FB}}$ was made back in 1998, before the start of the second run of Tevatron (Run II, with a center of mass energy of $1.98 \mathrm{TeV}$ ). The calculation was based on the Feynman diagrams shown in Fig. 1.6, which are the 


\section{Top Pair Branching Fractions}

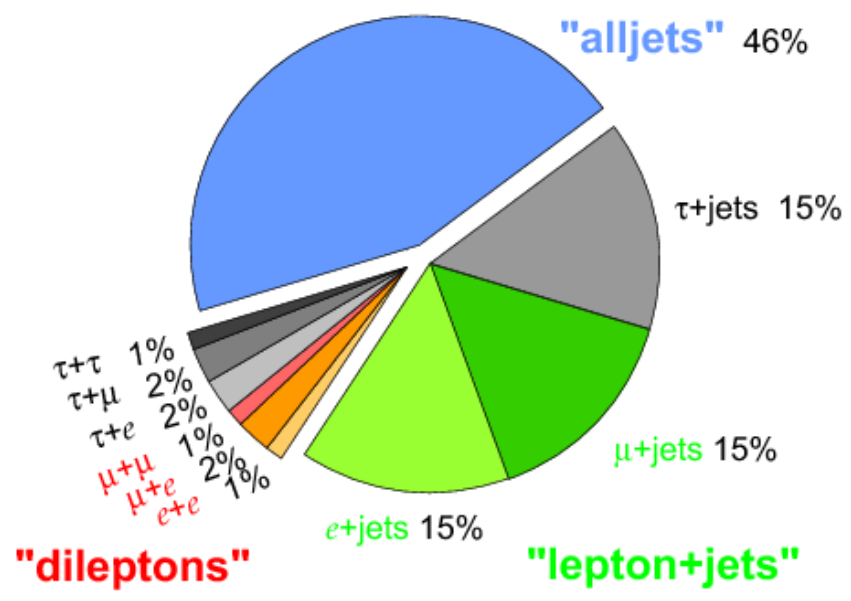

Figure 1.5: The $t \bar{t}$ branching fractions. The branching fraction of the "all-jets", the "lepton+jets", and the "dilepton" final states are 46\%, 30\%, and 5\%, respectively. The final states with a $\tau$ lepton are not explicitly used, but the scenarios where the $\tau$ lepton decays leptonically into an electron or a muon are included implicitly in the corresponding lepton + jets or dilepton final states [37].

Leading Order (LO) diagram as shown on the left, and the Next-to-Leading Order (NLO) contributions on the right [10, 11]. This calculation suggested a small but measurable asymmetry of 5-6\% with proton-antiproton collisions at a center-of-mass energy around $2 \mathrm{TeV}$ [10, 11], as a precise probe of top-quark production.

The first (unpublished) measurement of the top quark $A_{\mathrm{FB}}$ was made in 2006 at $\mathrm{CDF}$ with less than $700 \mathrm{pb}^{-1}$ of data (around $1 / 15$ of the entire dataset) in the lepton+jets final state [38]. This measurement yielded a large asymmetry of $\sim 20 \%$, but still consistent (within $2 \sigma$ ) with the SM predictions due to its large uncertainty. More dedicated calculations of top-quark $A_{\mathrm{FB}}$ for Tevatron Run II quickly followed this measurement, and by 2008 more precise predictions gave values between $4 \%$ and $8 \%$ [13, 14, 12]. Rather than publish the first measurement, the CDF collaboration kept refining the measurement and adding in more data as they became available. 


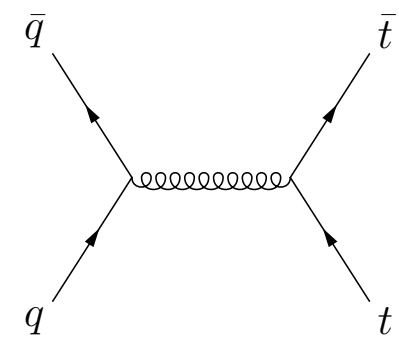

(a)

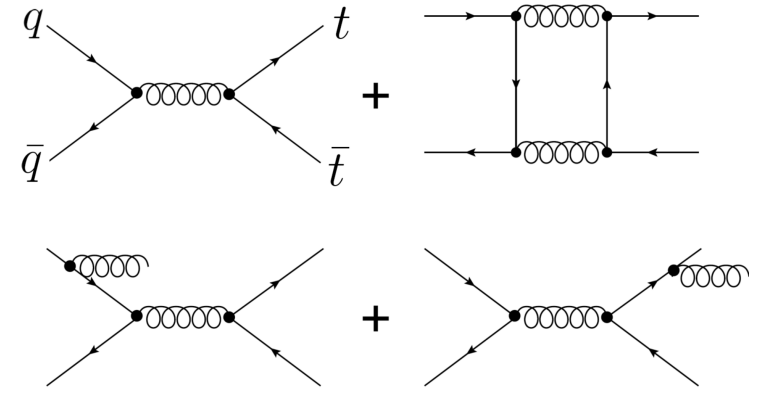

(b)

Figure 1.6: The leading order (a) and next-to-leading order (b) Feynman diagrams of the $t \bar{t}$ pair production with quark-antiquark annihilation process [19].

In $2008 \mathrm{CDF}$ released its first publication with $\sim 2 \mathrm{fb}^{-1}$ of data (1/5 of the final dataset), reporting an asymmetry larger than predicted (again at the 20\% level, still with large uncertainty) [15]. In the same year the D0 collaboration released their first publication of top-quark $A_{\mathrm{FB}}$ with $\sim 1 \mathrm{fb}^{-1}$ of data, confirming a larger-thanexpected asymmetry (also at the 20\% level) [16]. The large asymmetry reported by both collaborations triggered a tremendous amount of interest, followed by over 200 proposals of physics beyond the SM (typically the presence of new particles) that could explain this "anomaly" (Ref. [21] and references therein). By 2011, the CDF collaboration had published their results with the first half of the full dataset $\left(\sim 5 \mathrm{fb}^{-1}\right)$. This set of results included a new measurement of the asymmetry as a function of the invariant mass of the $t \bar{t}$ system, which showed a deviation from the best SM calculation at that time at the $3 \sigma$ level (considered the standard for "evidence" in particle physics) [19]. This observation is particularly exciting since such a deviation as a function of mass is a natural consequence of the existence of a new particle.

To try to understand the deviation between the theoretical calculation and the experimental results, the particle physics field (both on the theoretical side and on the 
experimental side) took three approaches: 1) to make more precise SM calculations, 2) to consider theoretical models beyond the SM that could predict the larger-thanexpected asymmetry but not affect all the other observations that were consistent with SM predictions, and 3) to measure the asymmetry with more data, other final states, other observables, and alternative techniques. On the theoretical side, by the year 2012, the community had produced a enormous number of creative new physics proposals, but perhaps more importantly had made significant progress in the SM calculation techniques, for example by including previously omitted extra effects, such as contributions from the electroweak processes (diagrams other than those in Fig. 1.6, for example with photons included) [42, 39, 40, 41]. We will discuss examples of new particle predictions in Sec. 1.3.4.

In the mean time, on the experimental side, the CDF and D0 collaborations took the third approach and started by measuring the top-quark $A_{\mathrm{FB}}$ with their entire dataset (a doubling of the dataset used by the previous measurement), and in an alternative final state (the dilepton final state). Using more sophisticated techniques (reducing the uncertainties caused by the measurement techniques, as well as measuring differential $A_{\mathrm{FB}}$ values as a function of $\left|\Delta y_{t}\right|$ and $\left.m_{t \bar{t}}\right)$, the result from the CDF collaboration was still significantly larger than the state-of-art SM prediction at that point [20, 42]. At the same time, a preliminary top $A_{\mathrm{FB}}$ measurement in the dilepton final state at CDF using the first half of the full dataset yielded an extremely large asymmetry $((42 \pm 16) \%)$ [17. This anomalous result was the first $A_{\mathrm{FB}}$ measurement in a different final state, and further led the field to seriously consider that this might be a real hint to physics beyond the SM. However, due to the large uncertainty in all the results, no definite conclusion could be drawn. The results from the D0 during the same period of time, with half of the the entire dataset, showed a tantalizingly high asymmetry, around 20\% [18], but still consistent with SM predictions within 
uncertainties. Figure 1.7 shows the evolution of the top $A_{\mathrm{FB}} \mathrm{SM}$ predictions and the published results from the CDF and D0 collaborations around the time this work started.

In an attempt to shed further light on this puzzle, the CDF collaboration pursued an alternative observable that is potentially sensitive to the same phenomenon, namely the forward-backward asymmetry of the charged leptons (electrons or muons) from the top quark decays. This observable is closely related to the original top-quark $A_{\mathrm{FB}}$ due to the fact that the charged leptons tend to follow the direction of their parent top quarks. On the other hand, this observable is a less direct measurement of the production asymmetry of the top-quark pairs, because it is sensitive to new features of the production (the spin of top quarks). On the experimental side, this observable features a simpler measurement technique that is less prone to the uncertainties in the determination of the directions of top quarks (which need to be reconstructed from their decay products). The result was released slightly after the analysis in this dissertation started, and again yields a result significantly higher than the SM calculation [43, 42] (3.8\% predicted vs. $\left(9.4_{-2.9}^{+3.2}\right) \%$ observed).

To quickly summarize, from 2006 to 2012, when the analyses in this dissertation started, both the CDF and D0 collaborations observed a growing significance of the deviation between multiple SM predicted top-quark $A_{\mathrm{FB}}$ observables and the experimental results. If these deviations were confirmed, it could be a real hint to the presence of a new particle. The analyses presented in this dissertation serve as a follow up on the various potential anomalies by measuring all the top $A_{\mathrm{FB}}$ related observables in the dilepton final state with the full CDF dataset. These analyses are designed to use all the updated knowledge accumulated about the $t \bar{t}$ production and decay as well as comparisons to the latest theoretical predictions. The purpose of the work presented in this dissertation is to use novel, state of the art experimental 
techniques on the full CDF top dilepton dataset and perform independent checks regarding the previous excess in the top $A_{\mathrm{FB}}$ measurements, and to combine the results in this final state with the previous results to gain the best determination of the top $A_{\mathrm{FB}}$ observables. Since other work, both calculations from the theoretical community and experimental measurements at D0, was occurring concurrently we will present the full set of results at the very end of this dissertation to give a coherent picture of the current status at the end of 2015.

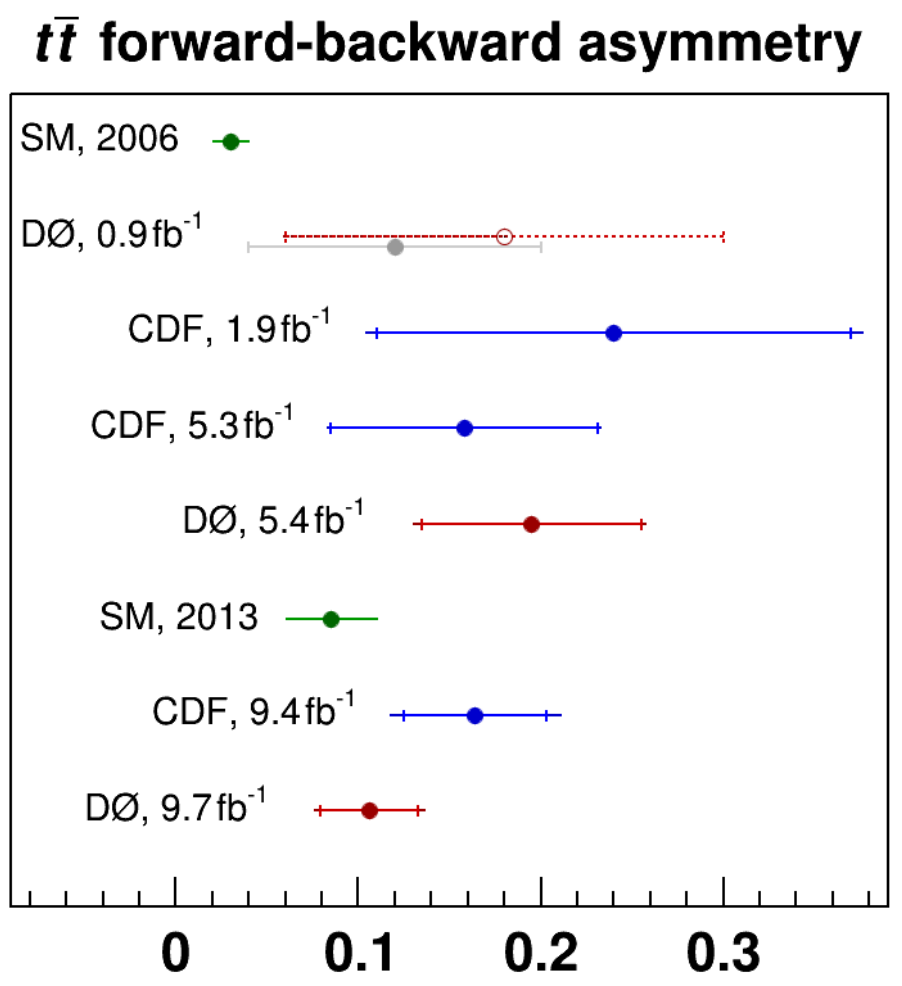

Figure 1.7: History of top $A_{\mathrm{FB}} \mathrm{SM}$ predictions and results from CDF and D0 [44]. 


\subsubsection{Definition of the Forward-Backward Asymmetry Observables}

We will probe the asymmetric behavior of the production and decay of top-quark pairs using three different, but complementary observables. These are 1) the $A_{\mathrm{FB}}$ of the momenta of top quarks, 2) the single lepton $A_{\mathrm{FB}}$, and 3) the lepton-pair $A_{\mathrm{FB}}$. The definitions, advantages and disadvantages of each observable are detailed in the following subsubsections and summarized in Table 1.1 .

\subsubsection{Definition of the $A_{F B}$ of top-quark momenta}

For reasons that will be explained shortly, in this work we will use the following definition of the $A_{\mathrm{FB}}$ of the fully reconstructed $t \bar{t}$ system:

$$
A_{\mathrm{FB}}^{t \bar{t}}=\frac{N\left(\Delta y_{t}>0\right)-N\left(\Delta y_{t}<0\right)}{N\left(\Delta y_{t}>0\right)+N\left(\Delta y_{t}<0\right)}
$$

where $N$ is the number of events, $y$ is the rapidity of the top quark or antiquark, and $\Delta y_{t}=y_{t}-y_{\bar{t}}$. The rapidity $(y)$ of a particle (a top quark in this scenario) is defined as $y=\frac{1}{2} \ln \frac{E+p_{z}}{E-p_{z}}$, where $E$ is the energy of the particle, and $p_{z}$ is the momentum of the particle along the proton-antiproton beam axis ( $z$ component). This observable

will be referred as the "reconstructed top $A_{\mathrm{FB}}$ " (or simply $A_{\mathrm{FB}}^{t \bar{t}}$ ), since the momenta, or the rapidities, of top quarks need to be reconstructed from their decay products.

The rapidity $y$ quantifies the direction the top-quark or antiquark is moving. The case $y=0$ indicates the quark is moving perpendicular to the beam line; positive $y$ is in the forward direction and negative $y$ is in the negative direction; the more positive (negative) the rapidity, the smaller the angle between the momentum of the quark and the forward (backward) direction. In this sense we can clearly see how the value of $y$ is directly related to the definition of forward and backward. We choose the variable $\Delta y_{t}$ instead of the top-quark outgoing angle $\theta$ or the rapidity 
$y$ of either the top quark or antiquark for a number of reasons. The first is that the variable $\Delta y_{t}$ gauges the relative direction between the top-quark and the topantiquark, so that the asymmetry is properly accounted in the situation where both top quarks and antiquarks have the same sign of $y$ (due to the initial quark or gluon pairs being heavily boosted). It also has a larger predicted $A_{\mathrm{FB}}$ than the one using just the $y$ of the quarks along, which makes it easier to measure. Also, $\Delta y_{t}$ has the advantage of being invariant under Lorentz boosts along the beamline (the rapidity itself is not, nor is the angle $\theta$ ), so that this observable measured in the frame of the laboratory ( $p \bar{p}$ rest frame) can be interpreted easily in any frame moving parallel to the beamline (e.g. frames where $q \bar{q}$ or $t \bar{t}$ only moves perpendicular to the beamline). Finally, comparing to the angle $\theta, \Delta y_{t}$ is less sensitive to the transverse momentum of the $t \bar{t}$ system, whereas $\theta$ needs to be measured in the $t \bar{t}$ rest frame, which is subject to the potential bias when the modeling of $p_{T, t \bar{t}}$ is not sufficiently good (details will be discussed in Sec. 5). This observable defined with $\Delta y_{t}$ is a thus a better, while still direct, probe of the production of the top quark than using just $\theta$ or $y$, and is therefore the variable used in the initial measurements which were inconsistent with SM predictions.

Comparing to the other two leptonic observables described below, it has the advantage that the expected magnitude of the value from the SM is larger for this asymmetry, thus it's a more significant effect to measure relative to zero. However, since top quarks decay immediately after their production, the momenta of the top quarks need to be reconstructed from their decay products. This is a complicated procedure, and the directions of the top-quarks determined from the reconstruction come with non-negligible resolutions. This introduces systematic uncertainties and potential biases into the measurement.

We note that in addition to the inclusive asymmetry values, measurements of the 
differential $A_{\mathrm{FB}}^{t \bar{t}}$ as a function of both the invariant mass of the $t \bar{t}$ system $\left(m_{t \bar{t}}\right)$ and $\left|\Delta y_{t}\right|$ can help probe the interactions in important ways. Specifically, the asymmetry is expected to rise as a function of $m_{t \bar{t}}$ or $\left|\Delta y_{t}\right|$ in the SM [10, 19]. This occurs because the contribution from quark-antiquark annihilation production (which is asymmetric) is expected to be larger in high $m_{t \bar{t}}$ and high $\left|\Delta y_{t}\right|$ regions. While as the gluons tend to have lower energy, the gluon-gluon production (which is symmetric) tend to produce $t \bar{t}$ with low invariance mass, and low $\left|\Delta y_{t}\right|$ due to the kinematic correlation between them. In addition, certain hypothetical new particles are expected to affect the asymmetry in certain kinematical regions significantly, thus alter the trend of $A_{\mathrm{FB}}$ vs. $m_{t \bar{t}}$ or $\left|\Delta y_{t}\right|$ [45], but might not have as big an impact on the inclusive value of $A_{\mathrm{FB}}$. Thus, the differential measurement of $A_{\mathrm{FB}}$ can be more sensitive to the presence of certain new particles than the inclusive value and thus provides important complementary information.

\subsubsection{Definition of the single lepton $A_{F B}$}

The single lepton $A_{\mathrm{FB}}\left(A_{\mathrm{FB}}^{\ell}\right)$ is defined as

$$
A_{\mathrm{FB}}^{\ell}=\frac{N\left(q_{\ell} \eta_{\ell}>0\right)-N\left(q_{\ell} \eta_{\ell}<0\right)}{N\left(q_{\ell} \eta_{\ell}>0\right)+N\left(q_{\ell} \eta_{\ell}<0\right)},
$$

where $N$ is the number of charged leptons (summed over all events), $q_{\ell}$ is the electric charge of the charged leptons from top cascade decays, and $\eta_{\ell}$ is the pseudorapidity of the charged leptons. The pseudorapidity is the rapidity in the limit of zero particle mass, and is invariant under Lorentz boosts along the beamline. Making the reasonable assumption of charge-parity symmetry $(\mathrm{CP})$ conservation, for leptons of opposite electric charge, the effects on the lepton pseudorapidity from both the top-quark asymmetry (even with a possible polarization) are equal in magnitude but opposite in sign [43]. Thus, including the charge of the lepton naturally merges both 
the positive and the negative leptons into a single observable, but effectively doubles the size of data.

This observable has advantages and disadvantages. On the one hand, the charged leptons tend to follow the direction of their parent top quarks, making the direction of the charged lepton a good, albeit imperfect, measurement of the top direction, and the direction of the charged leptons are much more precisely measured than the direction of the top quarks themselves. On the other hand, this observable is not only dependent on the production diagrams, but on the top-quark decay process, which is sensitive to the spin of the produced top quarks. Then again, if any new intermediate state (new particles) couples only to top quarks with specific spin states, it can affect the $A_{\mathrm{FB}}^{t \bar{t}}$ and $A_{\mathrm{FB}}^{\ell}$ observables in different ways, making both measurements valuable in their own right. Experimentally, this observable only requires one charged lepton in the final state, and can be measured in both the lepton+jets and the dilepton final state (with two measurements per event in the dilepton final state). In terms of absolute magnitude of the asymmetry, the value of $A_{\mathrm{FB}}^{\ell}$ is "diluted" by a factor of $\sim 2$ in the scenario of SM-like top-quark decays (which is approximately isotropic in the rest frame of the top quark in the scenario no spin), thus this is an even more subtle effect to measure.

\subsubsection{Definition of the lepton pair $A_{F B}$}

The lepton pair $A_{\mathrm{FB}}\left(A_{\mathrm{FB}}^{\ell \ell}\right)$ is defined in a way that is similar to the definition of the reconstructed $A_{\mathrm{FB}}$ as

$$
A_{\mathrm{FB}}^{\ell \ell}=\frac{N(\Delta \eta>0)-N(\Delta \eta<0)}{N(\Delta \eta>0)+N(\Delta \eta<0)},
$$

where $\Delta \eta=\eta_{\ell^{+}}-\eta_{\ell^{-}}$. The expected $A_{\mathrm{FB}}^{\ell \ell}$ is typically larger than the expectation of $A_{\mathrm{FB}}^{\ell}$ for kinematic reasons [42], which indicates that the asymmetry of $\Delta \eta$ is a more 
significant effect. However, this observable requires two charged leptons in the final state, thus can only be measured in the dilepton final state. Despite the expected large uncertainty due to the limited number of events accessible, it provides extra information if new physics were present in this process for the same reasons as in the single lepton case.

\subsubsection{Theoretical Predictions with SM}

With the definitions of the $A_{\mathrm{FB}}$ observables specified in Sec. 1.3.2, we start our discussion by listing the theoretical predictions of each observable from the SM; BSM predictions will be given in Sec. 1.3.4. Since the top quark pairs are expected to be predominately produced via strong interactions, they are described primarily by quantum chromodynamics (QCD), the part in the SM that describes the strong interactions. At leading order (LO) as shown in Fig. 1.6a, QCD predicts no net $A_{\mathrm{FB}}$ in $t \bar{t}$ production. All asymmetric effects come from higher order QCD contributions [10] as well as contributions from electroweak interactions [42]. At next-to-leading order (NLO) as shown in Fig. 1.6b, the asymmetry arises from the interplay between the two sets of interferences among diagrams: the interference between the "Born" diagram (the leading order $s$-channel diagram as shown in Fig. 1.6a) and the "box" diagram (the upper row of Fig. 1.6b and the interference between the initial- and final-state radiation diagrams (the lower row of Fig. 1.6b). The interference between the Born diagram and the box diagram gives rise to a positive asymmetry, while the interference between the initial- and final-state radiation diagrams gives rise to a negative asymmetry. These two opposite-signed effects, convoluted with the expected weights from the SM, result in a small net asymmetry, as well as an asymmetry that varies as a function of $m_{t \bar{t}}$ and $\left|\Delta y_{t}\right|$.

There are many different approaches in estimating the NLO SM prediction of the 


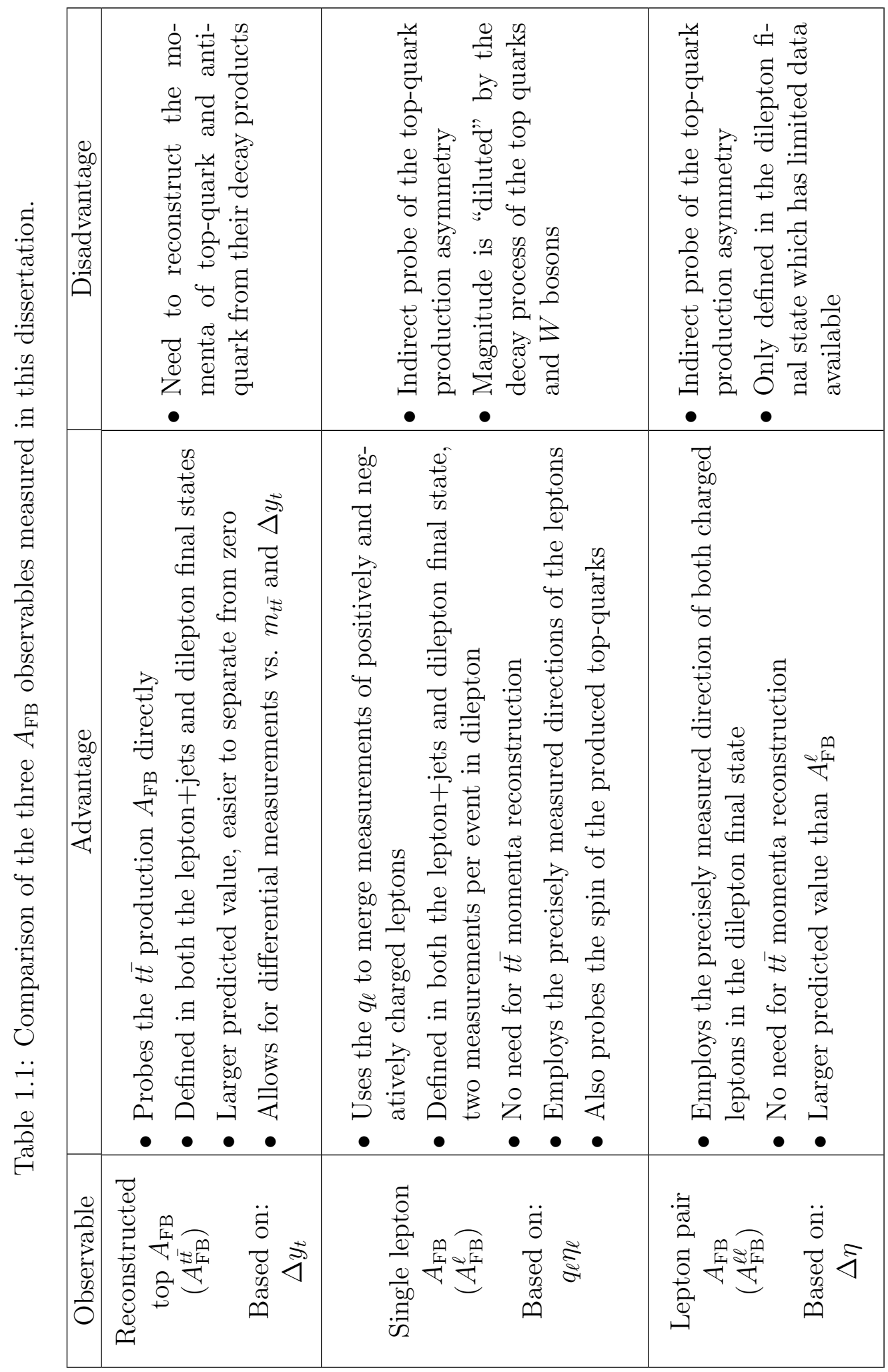


inclusive value of $A_{\mathrm{FB}}^{t \bar{t}}$, and current predictions range from 0.05 to 0.125 , as discussed in Ref. 21] and references therein. The NLO SM calculation we compare to (we will refer to it as our baseline NLO method) yields $A_{\mathrm{FB}}^{t \bar{t}}=0.088 \pm 0.006$ [42], as it contains the QCD calculation at NLO with the LO electroweak contributions. Higher order calculations are only available recently: a next-to-next-leading order (NNLO) calculation yields $A_{\mathrm{FB}}^{t \bar{t}}=0.095 \pm 0.007$ [46], and $A_{\mathrm{FB}}^{t \bar{t}}=0.100 \pm 0.006$ after adding in soft-gluon corrections (NNLO+NNLL) [47]. For the differential measurements of $A_{\mathrm{FB}}^{t \bar{t}}$ as a function of $m_{t \bar{t}}$ and $\left|\Delta y_{t}\right|$, both results are well described (and quantified) with linear functions (although there is no a priori reason for them to be linear). An NNLO calculation estimates the slope of $A_{\mathrm{FB}}^{t \bar{t}}$ vs. $\left|\Delta y_{t}\right|$ to be $0.114_{-0.012}^{+0.005}$ [46, 48]. On the other hand, there is no NNLO prediction of the slope of $A_{\mathrm{FB}}^{t \bar{t}}$ vs. $m_{t \bar{t}}$; an NLO prediction yields a slope of $(3.4 \pm 1.2) \times 10^{-4}\left(\mathrm{GeV} / \mathrm{c}^{2}\right)^{-1}$ [19].

For the leptonic observables, no NNLO or beyond calculations are current available. The baseline NLO SM calculation yields $A_{\mathrm{FB}}^{\ell}=0.038 \pm 0.003$, and $A_{\mathrm{FB}}^{\ell \ell}=$ $0.048 \pm 0.004$ [42]. A special prediction for the $A_{\mathrm{FB}}^{\ell}$ is made based on the relationship between the $A_{\mathrm{FB}}^{t \bar{t}}$ and the $A_{\mathrm{FB}}^{\ell}$ assuming the SM-like top-quark decay process $\left(A_{\mathrm{FB}}^{\ell} / A_{\mathrm{FB}}^{t \bar{t}} \sim 0.46\right)$. If the genuine value of $A_{\mathrm{FB}}^{t \bar{t}}$ would be that measured by the CDF collaboration in the lepton+jets final state [20], the predicted value for $A_{\mathrm{FB}}^{\ell}$ for top quarks decaying according to the SM would rise from 0.038 to $0.070<A_{\mathrm{FB}}^{\ell}<0.076[43]$.

We note here that comparing with the initial prediction around 5-6\% in 1998 [10], the SM prediction of $A_{\mathrm{FB}}^{t \bar{t}}$ itself has increased to $10 \%$. The original experimental "anomaly", the difference between the observation and the SM expectation, is reduced due to the refinement of SM calculations. While the "significance" of the anomaly is clearly reduced (which lessens the excitement), the field can take pride in the advancement of the SM calculations because of the "observation" of the anomaly 
in the top-quark $A_{\mathrm{FB}}$ observables. Looking forward, we will always compare to the best known values for each observable.

Besides the SM calculations mentioned above, we use computer programs that perform calculations of particle interactions and decay with Monte Carlo (MC) techniques to simulate the detailed properties of the $t \bar{t}$ production and decay in an event-by-event way. These programs are referred as "MC event generators" (or simply "generators"). A set of events simulated with a specific hypothesized underlying physical scenario (with a specific generator) is referred to as a sample. The simulations with MC event generators have advantages and disadvantages. On the one hand they are usually not as precise as the dedicated calculations mentioned above due to limitations in the MC generators; the best available generators can calculate the SM scenario to NLO, and the new particle scenarios only to LO. On the other hand, these simulations are extremely useful as they mimic the collisions happening in the detector so that we can take into account important experimental effects. As described in further detail in Sec. 3, in addition to simulating the collisions and their final state particles, we can simulate the process the final state quarks undergo as they develop into jets, as well as the interaction between the particles and the detector. In this way, we study the behavior of certain physical scenarios as if we observe them in the detector. This allows us to develop and validate methodologies to correct for the detector effects and measure the underlying physical observables.

We employ a variety of MC event generators to simulate scenarios with the SM both at LO and at NLO. Different generators are used for different purposes. We use the MC sample generated by the program known as POWHEG [49] as our benchmark as it has the most precise SM simulation at NLO. Samples from SM calculations at LO generated by other programs, which go by the names of Pythia [50], Alpgen [51, and HeRwig [52], are used for cross checks. The differences among the three LO SM 
event generators are subtle: the PYTHIA generator doesn't take particle spins into consideration, while the other two do; also, the HERWIG generator uses a different model to simulate the hadronization of quarks from PYTHIA; the ALPGEN generator does not perform parton hadronization, but it can be set up to pass the simulation results to either HERWIG or PYTHIA for that. For these reasons, we use all three to investigate if these subtle differences have significant impact on the measurements as they could indicate systematic biases in the measurement.

\subsubsection{Predictions with Physics beyond the SM}

A variety of samples with scenarios containing particles beyond the SM have also been simulated with MC event generators. We have mainly explored two categories of scenarios $s$-channel production of axigluons (Fig. 1.8a) and $t$-channel production of $Z^{\prime}$ bosons (Fig. $1.8 \mathrm{~b}$ ). These two are the simplest extensions to the $\mathrm{SM}$ that can alter the various observables significantly from SM-only predictions.

Axigluons are hypothesized bosons which are similar to SM gluons, but are massive. The scenarios with axigluons alter the top $A_{\mathrm{FB}}$ due to the interference between the $s$-channel "Born" diagrams (Fig. 1.6a and Fig. 1.8a). The width $(\Gamma)$ of the axigluons are chosen to be wide so that they are not excluded by the null results from existing searches for di-jet resonances. Since we cannot exhaust all possible configurations, we picked three axigluon masses (below the $t \bar{t}$ production threshold, around the threshold, and above the energy reach of the Tevatron but will produce off-shell effects) as our new physics scenarios.

The $Z^{\prime}$ bosons are hypothesized particles that share similar properties with the $Z$ bosons, but have a different mass. The scenarios with $Z^{\prime}$ bosons alter the top $A_{\mathrm{FB}}$ via a scattering between the quarks in the protons and the antiquarks in the antiprotons. 


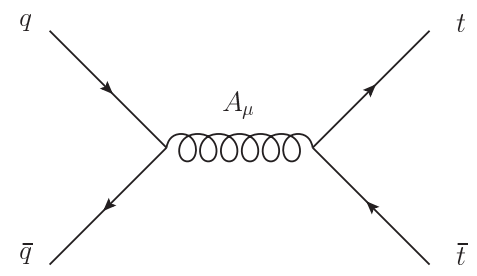

(a)

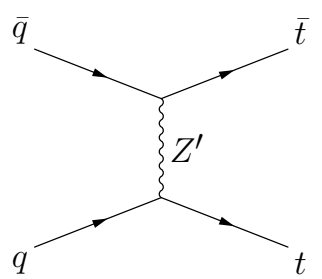

(b)

Figure 1.8: Example Feynman diagrams of the $t \bar{t}$ pair production via hypothetical new particles beyond the SM, including the axigluons via a $s$-channel production (a) and the $Z^{\prime}$ bosons via a $t$-channel production (b).

The selection of the model parameters (e.g. the particle masses and couplings) are specifically tuned so that they don't produce expectations conflicting with the existing experimental results in, for example, the $t \bar{t}$ production cross section [33]. We note that some of the scenarios originally selected for use in the analysis in 2011 are now disfavored by other experiments, but are included here for completeness. The detailed list of the scenarios we considered with particles beyond the SM is described below, with the predicted $A_{\mathrm{FB}}$ values (along with those with the SM scenarios) summarized in Table 1.2 :

1. The scenario with a $200 \mathrm{GeV} / \mathrm{c}^{2}$ mass axigluon with different types of couplings [53]: Three types of couplings are considered: 1) the scenario where the axigluons only interact with the left-handed top quarks and right-handed top antiquarks (left-handed coupling); 2) the scenario where the axigluons only interact with the right-handed top quarks and left-handed top antiquarks (righthanded coupling); 3) the scenario where the axigluon interacts in an opposite way with the left-handed top quarks and the right-handed top quarks (pure axial couplings). Since this axigluon is light, below the production threshold of the $t \bar{t}$ process $(\sim 350 \mathrm{GeV})$, it doesn't affect the $t \bar{t}$ production cross section in 
a way that would be measurable with current experimental uncertainties. The different couplings produce the same value of $A_{\mathrm{FB}}^{t \bar{t}}$, but very different values of $A_{\mathrm{FB}}^{\ell}$ and $A_{\mathrm{FB}}^{\ell \ell}$.

2. The scenario with an axigluon with a mass of $425 \mathrm{GeV} / \mathrm{c}^{2}$ which is near the $t \bar{t}$ production threshold with an axial coupling [54]: This model is different than the above model as it would produce a different sign of $A_{\mathrm{FB}}^{t \bar{t}}$ below and above the mass of the axigluon.

3. Two scenarios with a heavy axigluon with an axial coupling and mass of 1.8 or $2.0 \mathrm{TeV} / \mathrm{c}^{2}$ [19]: These heavy masses were chosen so that the axigluons do not affect the $t \bar{t}$ production cross section much, but predict a large $A_{\mathrm{FB}}^{t \bar{t}}$ that matches the results from the lepton+jets final state at CDF. However they are recently disfavored by the LHC results [55] using other techniques.

4. The scenario with a $t$-channel $Z^{\prime}$ boson with a mass of $200 \mathrm{GeV} / \mathrm{c}^{2}$ [56]: These models are chosen because they predict an $A_{\mathrm{FB}}^{t \bar{t}}$ that matches the larger-than$\mathrm{SM}$ results from the lepton+jets final state at CDF. However, we note that they are disfavored now by a detailed study of the $t \bar{t}$ differential cross section from the lepton+jets final state from the CDF collaboration [57].

\subsubsection{Experimental Status}

The historical evolution of the experimental status before the start of this work was summarized in Sec. 1.3.1, and the current state of the art of SM predictions (which did change significantly in the last few years) were summarized in Sec. 1.3.3. In this section, we summarize the current status of the other experimental results that occurred during the time of this work to lay out the final context for our results. 


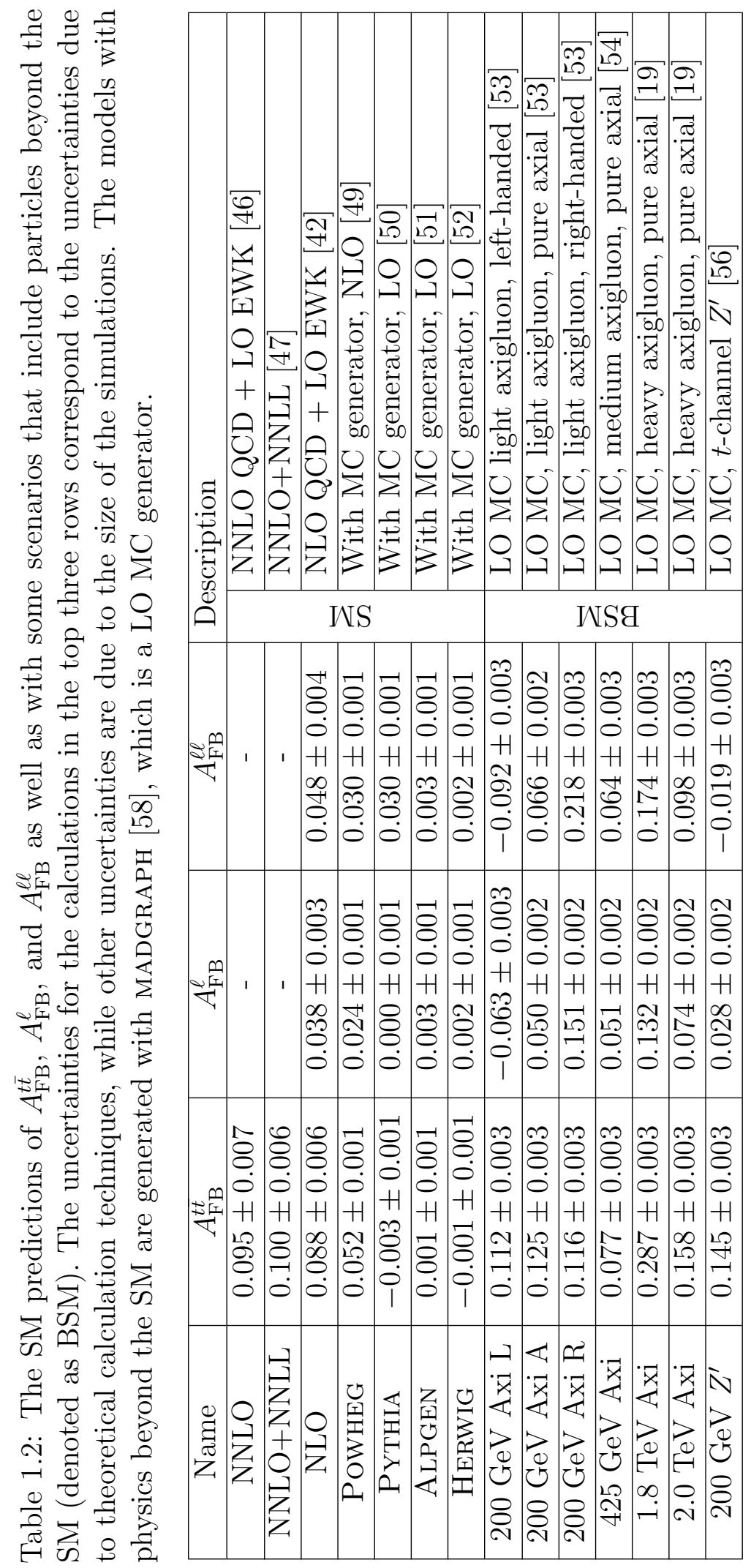


As stated earlier, the measurement of the inclusive $A_{\mathrm{FB}}^{t \bar{t}}$ can be made in the $t \bar{t} \rightarrow \ell \nu q \bar{q} b \bar{b}$ (lepton+jets) final state and the $t \bar{t} \rightarrow \ell^{+} \ell^{\prime-} \nu \overline{\nu^{\prime}} b \bar{b}$ (dilepton) final state. During the same time period of this work, both the CDF and the D0 collaborations released their final measurements of the three top $A_{\mathrm{FB}}$ observables with all the data both collaborations collected. These include the measurement of the inclusive reconstructed top $A_{\mathrm{FB}}\left(A_{\mathrm{FB}}^{t \bar{t}}\right)$, the differential measurement of $A_{\mathrm{FB}}^{t \bar{t}}$ as functions of $m_{t \bar{t}}$ and $\Delta y_{t}$, as well as both leptonic observables. They are summarized in Table 1.3 and discussed below.

The inclusive $A_{\mathrm{FB}}^{t \bar{t}}$ results are shown at the bottom of Fig. 1.7. With the CDF data corresponding to $9.4 \mathrm{fb}^{-1}$ of integrated luminosity, the measurement in the lepton+jets final state [20] yields a value of $0.164 \pm 0.047$, which is consistent with the NNLO SM prediction of $0.095 \pm 0.007$ within $1.5 \sigma$. The same measurements with D0 data corresponding to $9.7 \mathrm{fb}^{-1}$ of integrated luminosity in the lepton+jets [60] and dilepton final state 62] show results of $0.106 \pm 0.030$ and $0.180 \pm 0.086$, which are consistent with the NNLO SM prediction. On the other hand, the $A_{\mathrm{FB}}^{t \bar{t}}$ measurement in the dilepton final state with the first half of the CDF data (the predecessor to this analysis) yields a surprisingly high result of $0.42 \pm 0.16$ [17]. However, because of the large uncertainty, it is only $2 \sigma$ higher than the NNLO SM prediction.

The measurements in the lepton+jets final states also reported the differential $A_{\mathrm{FB}}^{t \bar{t}}$ results as functions of $\left|\Delta y_{t}\right|$ and $m_{t \bar{t}}$. The differential $A_{\mathrm{FB}}^{t \bar{t}}$ as a function of $\left|\Delta y_{t}\right|$ measurement by CDF in the lepton+jets final state [20] yields a slope of $0.253 \pm$ 0.062 , which is $2.2 \sigma$ higher than the NNLO SM prediction of $0.114_{-0.012}^{+0.005}$. The same measurement carried out at D0 in the lepton+jets final state 60] yields a slope of $0.154 \pm 0.043$ which is consistent with the NNLO SM prediction. We note that the final differential $A_{\mathrm{FB}}^{t \bar{t}}$ as a function of $m_{t \bar{t}}$ measured at CDF of $15.5 \pm 4.8 \times$ $10^{-4}\left(\mathrm{GeV} / \mathrm{c}^{2}\right)^{-1}$ shows a $2 \sigma$ deviation from the NLO SM prediction of $3.4 \pm 1.2 \times$ 


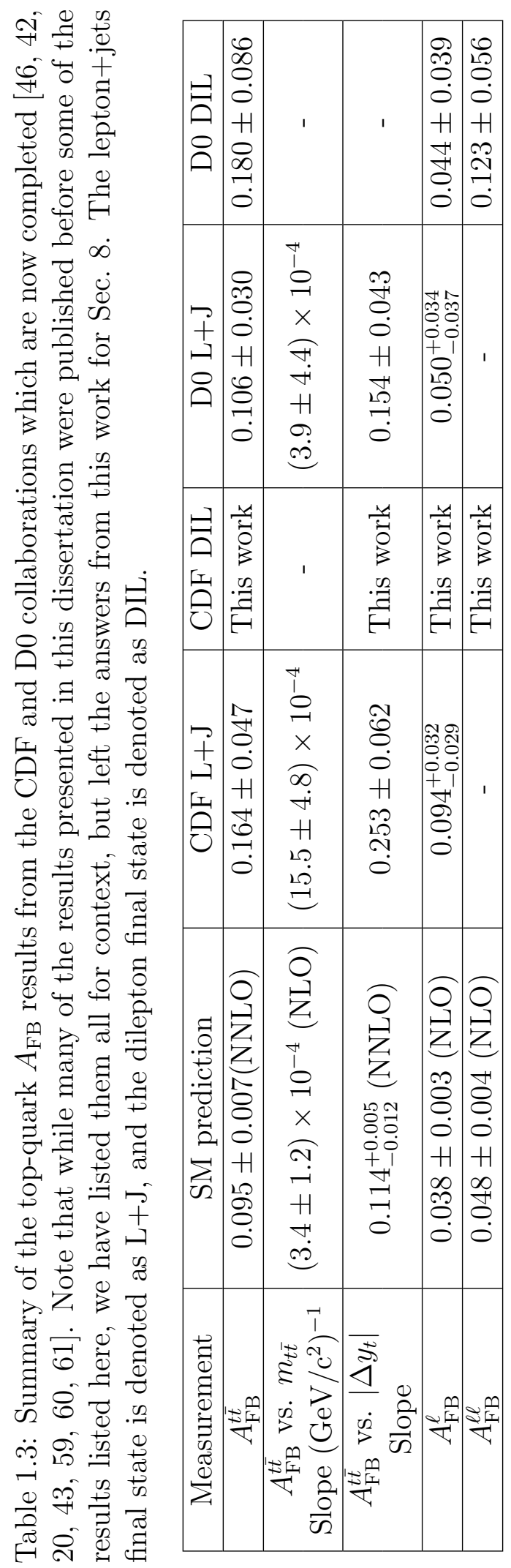


$10^{-4}\left(\mathrm{GeV} / \mathrm{c}^{2}\right)^{-1}$, while the measurement at D0 of $3.9 \pm 4.4 \times 10^{-4}\left(\mathrm{GeV} / \mathrm{c}^{2}\right)^{-1}$ shows consistency.

For the leptonic $A_{\mathrm{FB}}$ observables, the measurements of $A_{\mathrm{FB}}^{\ell}$ in the lepton+jets final state by the CDF collaboration and in the lepton+jets and dilepton final state by the D0 collaboration yielded $0.094_{-0.029}^{+0.032}$ [43] and $0.047 \pm 0.027$ [61, 59], respectively. Again, the result by the CDF collaboration shows mild tension with the NLO SM prediction of $0.038 \pm 0.003$ while the result from D0 shows consistency. A measurement of $A_{\mathrm{FB}}^{\ell \ell}$ by the $\mathrm{D} 0$ collaboration in the dilepton final state is $A_{\mathrm{FB}}^{\ell \ell}=0.123 \pm 0.056$ [61], consistent with the NLO SM prediction of $0.048 \pm 0.004$. A figure summarizing all the top $A_{\mathrm{FB}}$ results at the Tevatron, including the results presented in this dissertation, can be found in Sec. 8 .

It is worth mentioning one more relevant result before the description of our analysis and results. A more detailed study of the production cross section of the $t \bar{t}$ system as a function of the outgoing angle of the top quark in the $t \bar{t}$ rest frame $\left(\theta^{*}\right)$ was performed in the lepton+jets final state at CDF [57]. The differential cross section $\frac{d \sigma}{d \cos \theta^{*}}$ was measured and the results were decomposed in terms of Legendre polynomials. It was determined that the "excess" in the $A_{\mathrm{FB}}^{t \bar{t}}$ measurement in this channel is due to the coefficient of the first order Legendre polynomial, which is the term linearly dependent on $\cos \theta^{*}$. This result is used later in the $A_{\mathrm{FB}}^{t \bar{t}}$ measurement in Sec. 6 to motivate an ad hoc procedure to explore variations in $A_{\mathrm{FB}}^{t \bar{t}}$ in a more model independent way without causing significant changes to other kinematic distributions.

\subsection{Outline of the Dissertation}

At the time this work started, the top $A_{\mathrm{FB}}$ measurements in the lepton+jets final state with the full CDF dataset were approaching their completion, indicating both 
the inclusive and differential results different from the SM prediction at the $2 \sigma$ level. At the same time, the top $A_{\mathrm{FB}}$ measured in the dilepton final state with the first half of the CDF dataset observed a result that showed surprisingly large asymmetry, but with large statistical uncertainties. These all indicated potential hints for physics beyond the SM, perhaps suggesting the presence of new particles (although the SM predictions are now larger than they were then). The measurements and new techniques presented in this dissertation are designed to follow up on these hints by measuring all the top $A_{\mathrm{FB}}$ observables in the dilepton final state with the full CDF dataset. The final results will be presented, along with the updated theory results and the set of results from the D0 collaboration, which helps bring this story to a close. All the results presented here are either already published or are in process of the final submission, thus the dissertation itself is partially historical in approach.

The structure of this dissertation is as follows: Since many of the experimental details and basic techniques that these results build upon have been established for over a decade, in Sec. 2 we will only very briefly summary the experimental tools used for this analysis, namely the Fermilab Accelerator Complex, the Tevatron Collider, and the CDF II detector. In this section we also mention briefly the way that the physical objects produced in collisions and decays of top quarks (electrons, muons, jets, etc.) are reconstructed based on the outputs from the detector. The event selection criteria used in this analysis to select out a set of events which are dominantly $(\sim 70 \%)$ from the $t \bar{t} \rightarrow \ell^{+} \ell^{\prime-} \nu \overline{\nu^{\prime}} b \bar{b}$ process is also described in Sec. 2 . Section 3 describes the estimation and validation of the sources of events that compose the dataset that survive the event selection criteria listed in Sec. 2. Section 4 describes the measurement of $A_{\mathrm{FB}}^{\ell}$ and $A_{\mathrm{FB}}^{\ell \ell}$ in the dilepton channel at CDF as this is a simpler measurement (and because it was done first). It also details many of the new techniques that were first developed during the analysis process (with even more 
novel understanding of the phenomenological aspects of the methodology placed in Appendix E. Since the measurement of the $A_{\mathrm{FB}}^{t \bar{t}}$ requires the reconstruction of the momenta of the $t \bar{t}$ events, which is a complicated and difficult process, Sec. 5 details the algorithm to reconstruct the momenta of the top quarks and antiquarks from their decay products, which are used for the $A_{\mathrm{FB}}^{t \bar{t}}$ measurement. Section 6 describes the measurement of the inclusive $A_{\mathrm{FB}}^{t \bar{t}}$ and the measurement of the $A_{\mathrm{FB}}^{t \bar{t}}$ vs. $\left|\Delta y_{t}\right|$ in the dilepton channel at CDF; the measurement of $A_{\mathrm{FB}}^{t \bar{t}}$ vs. $m_{t \bar{t}}$ is not performed due to the limited statistics in the dilepton final state. Section 7 describes the combination of the top $A_{\mathrm{FB}}$ observables in both the lepton+jets and the dilepton final states at CDF. The final conclusions, including the current state of affairs in the field, are given in Sec. 8 , 


\section{EXPERIMENTAL TOOLS AND EVENT SELECTION}

In this section we provide a very brief summary of the experimental tools used for the measurements in this dissertation and the criteria we apply to select events that are consistent with the production and decay of $t \bar{t}$ in the dilepton final state. The experimental tools include the Fermilab Tevatron particle accelerator and the CDF II detector, which have been in existence since the 1980's and are well documented elsewhere. For more details we refer the reader to extensive documentation in the literature. See for example, Refs. [63, 64, 66, 65]. Since the Tevatron delivers more than a million collisions per second in the center of the detector, we apply a set of event selection criteria (cuts) to obtain a sample of events which are predominantly from the production and decay of $t \bar{t}$ events in the dilepton final state (the "signal" process in this dissertation), and to reduce the contributions from other SM processes that mimic the signature ("background" processes). The description of the event selection criteria is again a very brief summary of the detailed work of the CDF collaboration done over more than twenty years, and which has been used in multiple analyses to measure the top quark and its properties over the last decade. We refer to Ref. [67] for more details. We note that in the measurement of $A_{\mathrm{FB}}^{t \bar{t}}$ with top reconstruction in Sec. 6, we will introduce additional requirements based on top reconstruction to further improve the sensitivity of that measurement.

\subsection{The Fermilab Tevatron}

The Tevatron is located at the Fermi National Accelerator Laboratory (Fermilab) in Batavia, Illinois which is just west of Chicago. It is a circular particle accelerator colliding high energy protons against antiprotons. Figure 2.1 shows the accelerator chain of the Fermilab Tevatron. The accelerating process starts by periodically (every 


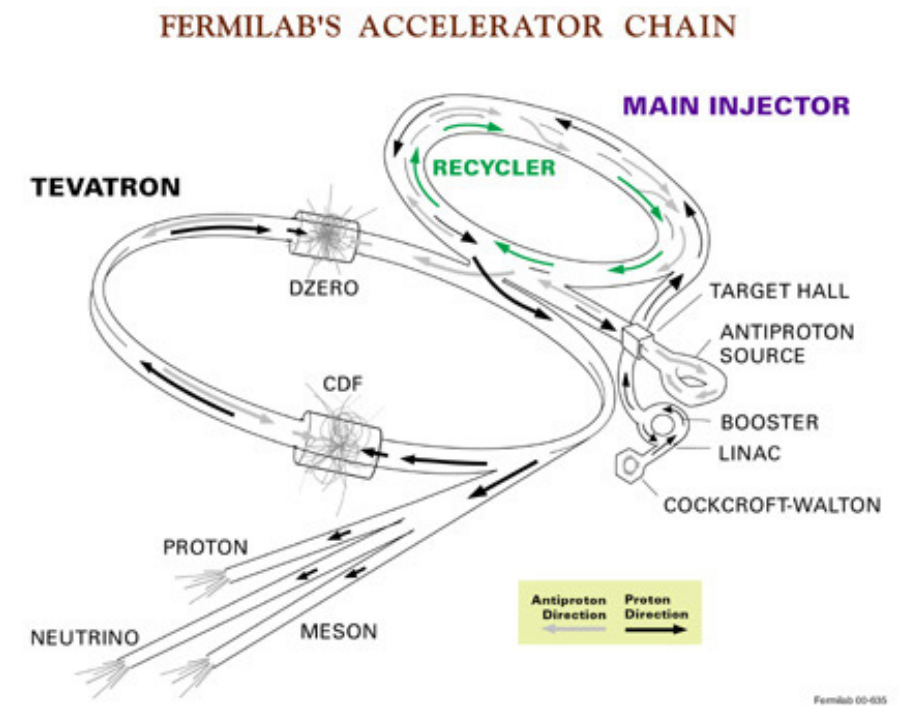

Figure 2.1: The accelerator chain of Fermilab Tevatron, including the location of the CDF and D0 experiments 72 .

$66 \mathrm{~ms}$ ) ionizing small amounts of compressed hydrogen gas and extracting negativelycharged hydrogen ions $\left(H^{-}\right)$to form a stream of $H^{-}$ions $\left(H^{-}\right.$beam). The $H^{-}$ beam is accelerated by the Cockcroft-Walton preaccelerator (to $750 \mathrm{keV}$ ) 68] and the Linear Accelerator (Linac) (to $400 \mathrm{MeV}$ ) [69]. During the acceleration in the Linac, the beam is separated into discrete packets of particles (bunches). After being accelerated by the Linac, the electrons from the $H^{-}$ions are stripped off to form $H^{+}$ions (or protons) [68]. The proton beams are then further accelerated by a series of accelerators, namely the Booster (to $8 \mathrm{GeV}$ ) [70], the Main Injector (to $150 \mathrm{GeV}$ ) [71] and finally the Tevatron [64, to an energy of $980 \mathrm{GeV}$.

The antiprotons are produced by directing a proton beam from the Main Injector to a nickel alloy target, and then using a magnet system to direct all the antiprotons created in the collision in a specific direction. This set of particles, at an energy of about $8 \mathrm{GeV}$, is passed to the Debuncher [73] which reduces their momentum spread so that they can be efficiently bunched, transferred and accelerated, and accumulated 
in the Accumulator. The antiprotons are then transferred to the Recycler [73] in the Main Injector when enough of them are collected, and are accelerated to $150 \mathrm{GeV}$. The antiprotons are ultimately transferred to the Tevatron for final acceleration, also to an energy of $980 \mathrm{GeV}[73]$.

The protons and antiprotons circulate in opposite directions along the Tevatron ring in 36 bunches. The beams are focused at two points for collisions, where two general purpose detectors, named CDF and D0, are located with the collision points at the center of each detector. The bunches of protons and anti-protons pass through each other (called a bunch crossing) every 396 ns, at nearly the center of the detector, and with a Gaussian distribution along the beam line with a width of $\sim 24 \mathrm{~cm}$. Each bunch crossing is labeled as an "event", and there are often multiple collisions per event. The density of the proton or anti-proton in a bunch is proportional to the "instantaneous luminosity" of the collisions, and thus proportional to the number of collisions in the event. The instantaneous luminosity kept increasing during the Tevatron Run II, with the highest average collisions per event peaking around seven; this was achieved at the end of Run II. During the Tevatron Run II between Mar. 2001 and Sep. 2011, the Tevatron delivered proton-antiproton $(p \bar{p})$ collisions at the center of mass energy of $\sqrt{s}=1.96 \mathrm{TeV}$ with an integrated luminosity of $11.8 \mathrm{fb}^{-1}$

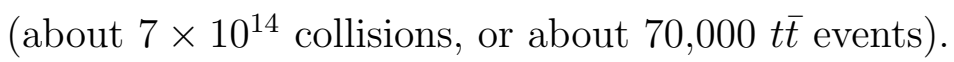

\subsection{The CDF II Detector}

The CDF II detector, shown in Figs. 2.2 and 2.3, is a general purpose detector located at one of the two collision points at the Tevatron. We define the beam axis as the $z$-axis of our coordinate system, and call the proton direction as the positive direction, or "forward", while the antiproton direction is denoted as the negative direction, or "backward". The CDF detector is azimuthally symmetric around the 
beam line and forward-backward symmetric. It is a cylinder-shape detector with a "barrel" portion in the center and two endcap (plug) portions at both ends. From inside (the beamline) to outside (along the cylindrical radius direction) the detector consists of a tracking system to measure the trajectory 4-momentum of the charged particles [74, 75], an electromagnetic calorimeter to measure the energy of the electrons and the photons [76], a hadronic calorimeter to measure the energy of hadrons and jets (for example from the hadronization of the light quarks and $b$-quarks from the top-quark decays) [77], and muon systems that measure the properties of the muons [78, 79]. The tracking system is contained in a superconducting solenoid, providing a $1.4 \mathrm{~T}$ magnetic field parallel to the beam axis which allows us to determine the electric charges of particles, as well as their 4-momenta.

We will refer to two different cylindrical coordinate systems. The first is with the origin at the center of the detector when referring to the detector structure, and a second which places the origin where the highest-energy collision happens during each bunch crossing (denoted as the "primary vertex" as explained in Sec. 2.3) when referring to the kinematic variables of an event. Since the $z$-axis is defined as pointing in the direction of the proton beam, we define $\theta$ and $\phi$ to represent the polar and azimuthal angles, respectively. Since the rest frame of the incoming quarks or gluons is not measurable (within the proton each quark/gluon carries a variable fraction of the proton momentum), but overwhelmingly does move along the beamline, we define two parameters similar to $\theta$ : rapidity and pseudorapidity. These two variables are useful as they are invariant under a boost along the beamline. (Actually the difference between two rapidities is invariant under boosts along beamline, while a single rapidity is not.) Rapidity is defined as $y=\frac{1}{2} \ln \frac{E+p_{z}}{E-p_{z}}$, where $E$ is the energy of the particle, and $p_{z}$ is the longitudinal momentum in the direction of the beamline defined as $p \cos \theta$. We use rapidity to quantify the direction the particle 
goes: $y=0$ indicates that the particle is going perpendicular to the beam line; the larger a positive (negative) $y$ is, the more the particle is going in the forward (backward) direction. Pseudorapidity, $\eta$, is the rapidity in the limit of zero particle mass. Since the relation between $\eta$ and $\theta$ is monotonic, we also use $\eta$ to refer to the polar angle of a detector component, in which case the expression can be simplified as $\eta=-\ln \tan (\theta / 2)$. The transverse momentum $p_{T}$ (transverse energy $E_{T}$ ) is defined to be $p \sin \theta(E \sin \theta)$. We note that for the final state particles whose masses are small compared to their kinematic energy, we use $p_{T}$ and $E_{T}$ interchangeably (although $p$ is usually used when we measure quantities from the tracking chamber, and $E$ is used when we measure quantities from the calorimeter). In general all kinematic quantities are measured in the lab frame, but calculated using the collision position (as measured by the primary vertex of the event). However, there are some variables, typically detector quantities, which are measured in a coordinate system that is determined by the center of the detector. We will point these out explicitly when they arise.

Of the $11.8 \mathrm{fb}^{-1}$ data the Tevatron delivered, the CDF II detector acquired $9.9 \mathrm{fb}^{-1}$ of them that are good for physics studies. Within these data, a list of data-taking periods (runs) during which the detector is in a stable and good working status is selected as the "good run list". The good run list used by the analyses in this dissertation is standard at $\mathrm{CDF}$ and corresponds to an integrated luminosity of $9.1 \mathrm{fb}^{-1}$, and is described in detail in Ref. [67]. We note that only a subset of these runs have the inner layer of the tracking system, the silicon detector, in a fully functional status. When the information from the silicon detector is used in studies to validate our data (for identifying secondary vertices from jets originating from $b$-quarks), a subset of the good run list is used, which corresponds to an integrated luminosity of $8.7 \mathrm{fb}^{-1}$. This will be described in more detail in Sec. 3 as the sample 


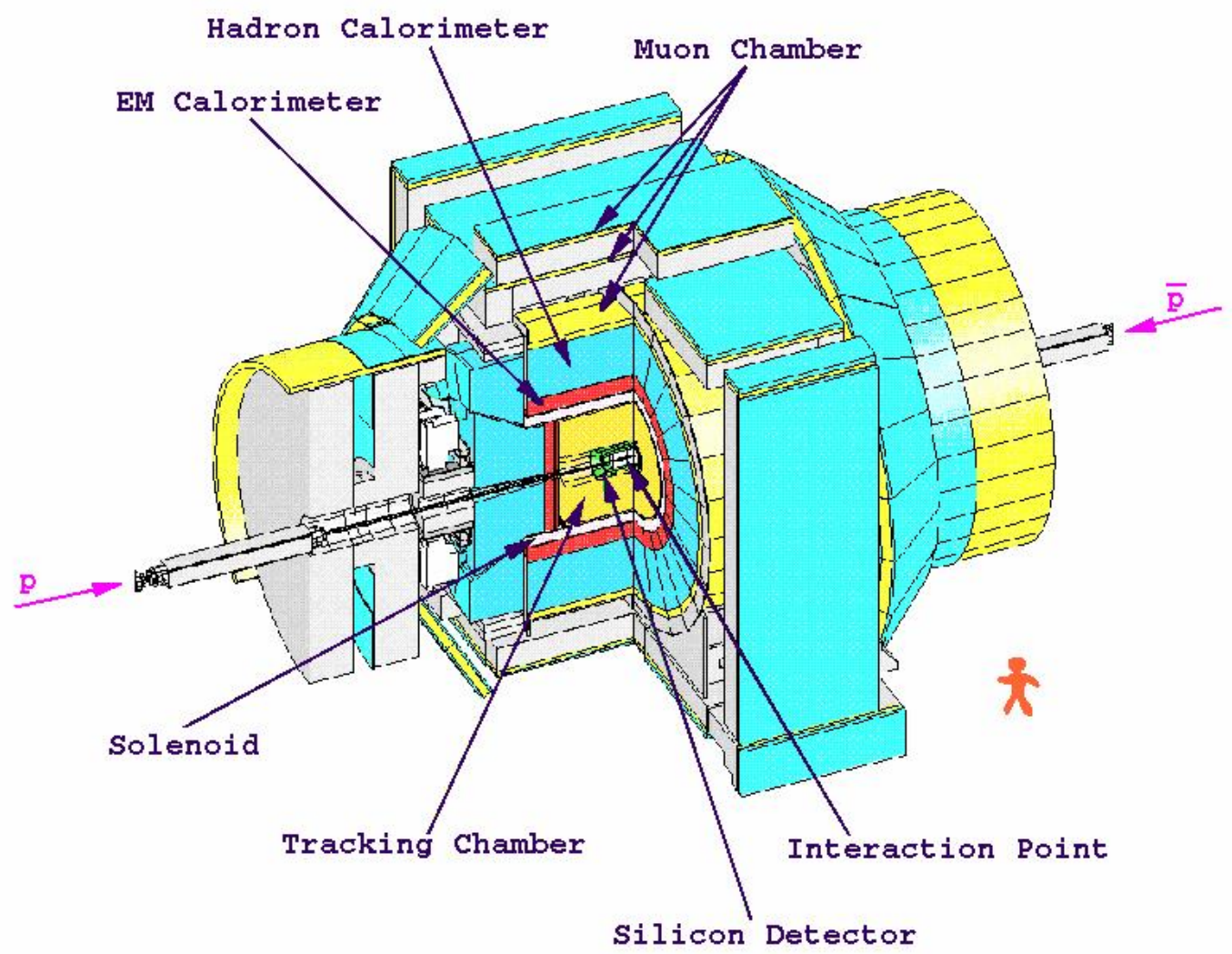

Figure 2.2: A cutaway view of the CDF II detector with quadrant cut to expose the different subdetectors [80]. 


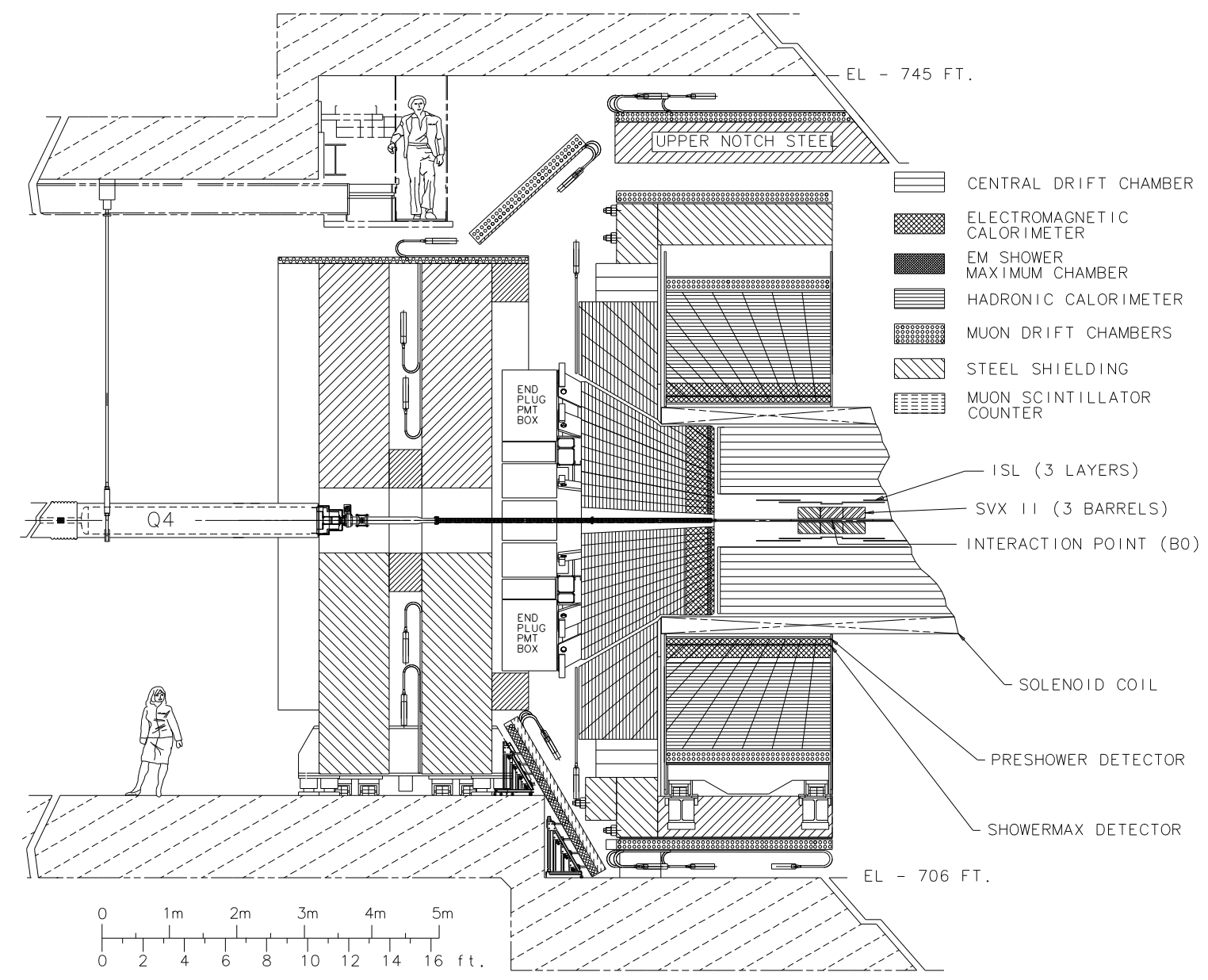

Figure 2.3: The elevation view of CDF II detector so we can see the inner detectors more clearly. Only half of the detector is shown [66]. 
with the requirement of a secondary vertex is not used in the main analyses, but is useful to confirm the robustness of the measurements with a sample that is expected to have a higher purity of top quarks, but of a smaller size due to the inefficient b-quark identification algorithm.

\subsection{Identification of the Reconstructed Objects}

The CDF II detector is used to identify and reconstruct the properties of the final state objects, i.e., electrons, muons, collimated clusters of hadrons (jets), as well as the imbalance of $E_{\mathrm{T}}\left(E_{T}\right)$ which is caused by undetectable particles such as neutrinos. The object identification and reconstruction techniques have been used in hundreds of analyses published by the collaboration, so we will not describe them in detail here, but point the reader to Refs. [83, 81, 82].

The main idea of the object identification is to look for an expected set of detector responses from a specific object. For example an electron will leave a charged particle trajectory through the tracking chamber, then deposit almost all of its energy in the electromagnetic calorimeter. The signature responses in the detector from various objects are summarized in Fig. 2.4. We apply a set of selection criteria for the identification of each object. If a group of responses in the detector passes the criteria, we "reconstruct" a candidate object from this group of responses, and measure the corresponding parameters of the object, for example its 4-momentum. We define the purity of a sample of objects as the fraction of the objects in the sample surviving the requirements that are originated from the real corresponding objects (instead of some other objects mimicing the signature); while the efficiency of the requirements is defined as the fraction of real objects that pass the requirements. The same definitions of purity and efficiency are also applicable for the event selection criteria in Sec. 2.4. The object identification criteria (as well as the event selection criteria) 
are designed for an optimal balance between purity and efficiency.

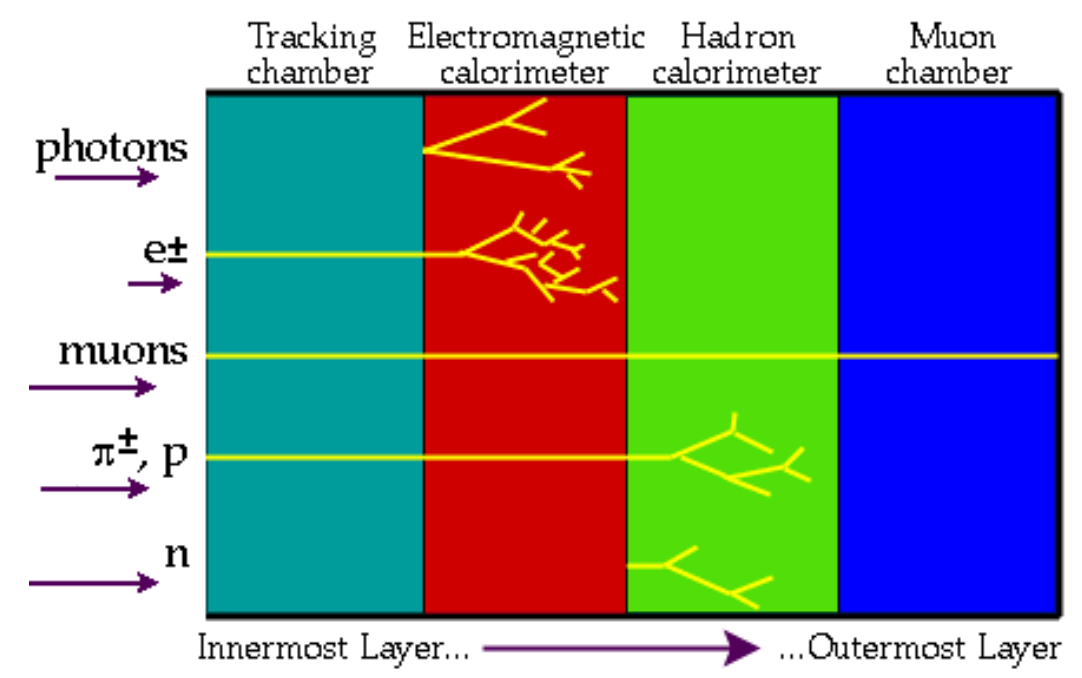

Figure 2.4: The signature responses in the detector from various objects [84].

Here we very briefly summarize the object identification techniques that are crucial to this analysis. Charged particles that traverse the detector are identified as curved trajectories (tracks) in the tracking chamber, and are reconstructed with a silicon microstrip detector and/or a large open-cell drift chamber in the $1.4 \mathrm{~T}$ solenoidal magnetic field. The position of any collision is reconstructed by grouping the projections of tracks to the beam line [82] which we will refer to as a vertex; the vertex from which the $t \bar{t}$ event originates is referred to as the primary vertex. In the presence of a particle with a relatively long lifetime $\left(O\left(10^{-12} \mathrm{~s}\right)\right)$, a particle might travel a fraction of a millimeter or longer before it decays, resulting in a bunch of particles originating from a position away from the beam line. In this situation, a "secondary vertex" can be reconstructed from the tracks that is significantly away from the beam line. The existence of a secondary vertex is used for identifying jets from $b$-quark hadronization, which is further described below. 
Electrons are identified by matching, high momentum charged-particle tracks to a localized deposit of a large amount of energy in the electromagnetic calorimeter. Muons travel all the way through the tracking chamber, calorimeters and steel shielding and eventually hit the muon detectors, and are identified by matching tracks to hits in the muon detectors. A series of identification requirements are imposed to select electrons or muons candidates. These requirements are selected so that the majority of the objects passing the requirements originate from real electrons and muons from $W$ boson decays, and only a tiny fraction of the identified objects are from a different particle or a cluster of particles (for example a spray of particles from a jet) that mimic the features of an electron or a muon. We will refer to objects that pass all the electron or muon identification requirements, but are not from real electrons or muons (regardless of their source), as "fakes". The identification requirements for electrons and muons have been standardized at CDF for many years and are summarized in Appendix A and detailed in Ref. [83].

For the identification of the leptons, to help obtain a better balance between the purity and the efficiency in selecting events in the dilepton final state, we define two sets of categories: 1) a "tight" category vs. a "loose" category, and 2) an "isolated" category vs. a "non-isolated" category. The detailed definitions of these categories are summarized in Appendix A. A lepton is described as being a "tight" lepton if it satisfies all the object identification criteria, thus the sample of tight leptons is expected to have a higher purity, but a lower selection efficiency. A lepton not passing all the criteria (fails the tight lepton identification) but passing a specific subset of them is described as a "loose" lepton, thus a sample of loose leptons is expected to have a lower purity but higher efficiency, which also indicates that more real lepton events passing the criteria.

Since the leptons from the decay of a $W$ boson in $t \bar{t}$ events are likely to be far, 
in the $\eta-\phi$ space, from all the other final state objects, we can use this tendency to further refine the event selection process. Specifically, we consider the amount of energy around a lepton and refer to this as the amount of isolation energy, and refer to leptons which have a small amount of energy nearby as being "isolated" [83]. In both the tight and loose lepton categories, the leptons are further categorized into isolated leptons (for those which pass the isolation requirement) or non-isolated leptons (for those which fail). The isolated leptons are expected to have higher purity than the non-isolated ones, since the non-isolated leptons are likely to be from a cascade leptonic decay of a hadron in a jet, whereas leptons from the decay of a $W$ boson are not expected to have any energy nearby, and are thus expected to be isolated. We note here that in the event selection criteria described in Sec. 2.4, we require one electron or muon to be both tight and isolated, and allow the second electron or muon to be from either category (tight or loose, isolated or non-isolated), for a balance of high expected signal purity and overall selection efficiency.

Since the decay products of final state quarks and gluons are a spray of collimated hadrons, we reconstruct them as jets with the standard JETCLU cone algorithm [81] by grouping all the energy deposited in the calorimeter in a cone with a radius of 0.4 , where the radius of a cone is defined as $\Delta R=\sqrt{(\Delta \eta)^{2}+(\Delta \phi)^{2}}$. Since the detector is not perfect, and responds differently to each type of jet, a variety of corrections can be applied to the measurement of the energy of jets so that the observed jet energy better reflects the original parton (quark or gluon) energy better.

The jets from $b$-quark hadronization can be identified (b-tagged) based on the presence of a secondary vertex due to the finite lifetime of the $B$ hadrons. This is done with an algorithm known as the SECVTX algorithm [82. Jets from $b$-quarks have a $\sim 50 \%$ chance of being identified using this technique, while only $\sim 1 \%$ of light quark/gluon jets would fake the criteria due to resolution effects. Note that this is a 
very powerful technique, and is used in many analyses when high purity is needed. However, in our analysis we need higher efficiency so we will not require $b$-tagged jets in our final event selection criteria, but we will select a sample of events with a $b$-tagged jets for studies to confirm our understandings of the final sample.

In the scenario where one or more particles (mostly neutrinos) leave the detector without being detected, the transverse momentum observed in the detector does not match that before the collision. Since the initial state has essentially zero transverse momentum (less than a few $\mathrm{GeV}$ ), this results in a net transverse momentum leftover, denoted as the imbalanced transverse momentum (imbalanced transverse energy, or the "missing transverse energy" in the limit of negligible particle mass, which is a good assumption for the final state particles involved in this analysis). The missing

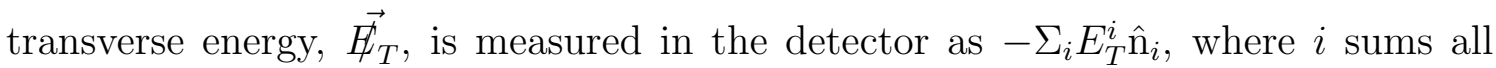
the transverse energies everywhere in the detector, in particular all the segments (towers) of the electromagnetic and hadronic calorimeters with $|\eta|<3.6, \hat{\mathrm{n}}_{i}$ is a unit vector perpendicular to the beam axis and pointing at the $i$ th calorimeter tower. In the calculation of $E_{T}$, any energy deposited by muons in the calorimeter is removed from the sum since they deposit only a few percent of their energy in the calorimeter; and the transverse momentom of any muon, as measured by the tracking chamber, is added back in. The scalar quantity $\ddot{E}_{T}$ is the magnitude of ${\overrightarrow{E_{T}}}_{T}$.

\subsection{Event Selection}

Since collisions happen in the center of the detector every 396 ns (corresponding to a frequency of $2.5 \mathrm{MHz}$ ), an enormous amount of data is created every second. Due to the limit on the speed at which data can be written to long-term storage for later analysis $(\sim 23 \mathrm{MB} / \mathrm{s})$ and the large amount of data per event, the frequency of event recording cannot exceed $\sim 100 \mathrm{~Hz}$. Dedicated online selection (trigger) and 
data acquisition systems are designed to accommodate the large data volume by only recording collisions of interest based on preliminary information from tracking, calorimetry and muon systems in real time [85, 86]. The analyses presented in this dissertation use events that are selected by the trigger system based on the presence of a high-momentum $\left(p_{T}>18 \mathrm{GeV}\right)$ electron or muon detected by the barrel part $(|\eta|<1.1)$ of the detector (the central high $p_{T}$ electron and muon triggers) [83].

After the events passing the online event selection and are recorded by the longterm storage, we further apply a set of offline event selection criteria to obtain a sample that is enriched by our signal events with $t \bar{t}$ production and decay in the dilepton final state. The event selection used in the analyses presented in this dissertation (referred as the "baseline" event selection) is the standard Top Dilepton event selection with minor modifications. These criteria were used in the measurement of the $t \bar{t}$ cross section in the dilepton final state, and more details can be found in Ref. [67]. Below we list the key features of this event selection criteria, as well as the motivations of them.

Since there is no way to tell in data whether an individual event is from the signal process or from any background processes, we employ a set of event selection criteria to select a ensemble of events in which a large portion of the sample is from the signal process. This also allows for robust estimations of the contributions from each of the signal and background processes. The estimations of the contributions are described in Sec. 3, but we note the major background processes and their signatures here to describe the motivation of the event selection criteria.

An example Feynman diagram of the top pair production and decay in the dilepton final state $\left(t \bar{t} \rightarrow \ell^{+} \ell^{\prime-} \nu \overline{\nu^{\prime}} b \bar{b}\right)$ is shown in Fig. 1.4c. The key signature of the signal process is the presence of two high-energy oppositely charged leptons, two or more jets from $b$-quarks, and large $E_{T}^{t}$ from the two neutrinos (dilepton+dijet+ $\left.E_{T}^{\prime}\right)$. 
The background processes include SM production of $W$ bosons, $Z / \gamma^{*}, J / \psi, \Upsilon$, and diboson $(W W, W Z, Z Z$, and $W \gamma)$ processes, with associated production of extra jets. Below we list each background processes, and the way they mimic the signal. After that we will list the event selection requirements that aim to reject each:

- The production of a $W$ boson in association with three or more jets ( $W+$ jets) where one of the jets fakes a lepton $\left(W \mathrm{jjj} \rightarrow \ell \nu \mathrm{j}_{\text {fakej }} \rightarrow \ell \ell_{\text {fakejj }}+E_{T}\right)$ : This process can mimic the dilepton+dijet $+E_{T}$ signature when the $W$ boson decays to a charged lepton and a neutrino (causing real $\mathscr{E}_{T}$ ), and one jet is misidentified as a charged lepton. Despite the small probability of a jet being mis-identified as a charged lepton, this is one of the major backgrounds due to the huge production cross section of this process (two orders of magnitude larger than the $t \bar{t}$ production cross section).

- The production of a $Z$ boson or a virtual photon (Drell-Yan process, denoted as DY or $\left.Z / \gamma^{*}\right)$ in association with two or more jets (DY+jets, or $Z / \gamma^{*}+$ jets): We separate the $Z / \gamma^{*}$ production into two categories, since they mimic the signal in different ways: 1) where the $Z / \gamma^{*}$ decays to a pair of electrons or muons, and 2) where the $Z / \gamma^{*}$ decays to a pair of $\tau$ leptons.

1. The situations where the $Z / \gamma^{*}$ decays to a pair of electrons or muons $\left(Z / \gamma^{*} \rightarrow e^{+} e^{-} / \mu^{+} \mu^{-}\right)$can mimic the signal when an instrumental mismeasurement of energy occurs, resulting in fake $E_{T}^{\prime}\left(Z / \gamma^{*} \mathrm{jj} \rightarrow \ell^{+} \ell^{-} \mathrm{jj} \rightarrow\right.$ $\left.\ell^{+} \ell^{-} \mathrm{jj}+E_{T, \text { fake }}\right)$.

2. When the $Z / \gamma^{*}$ decays to a pair of $\tau$ leptons $\left(Z / \gamma^{*} \rightarrow \tau^{+} \tau^{-}\right)$, and the $\tau$ leptons decay leptonically into electrons, muons and neutrinos $\left(Z / \gamma^{*} \mathrm{jj} \rightarrow\right.$ $\left.\tau \tau \mathrm{jj} \rightarrow \ell^{+} \ell^{-} \nu \nu \nu \nu \mathrm{jj} \rightarrow \ell^{+} \ell^{-} \mathrm{jj}+E_{T}\right)$, this background processes features the same dilepton + dijet $+\not_{T}^{\prime}$ signature as the signal. 
- Standard Model diboson production, including the $W W, W Z, Z Z$, and $W \gamma$ productions in associated with jets: Among these, the $W W$ process is expected to have the largest contribution. The $W W$ process can mimic the dilepton $+\operatorname{dijet}+\not_{T}$ signature when both $W$ bosons decay leptonically.

- Low-mass dilepton resonance $(J / \psi, \Upsilon$, etc.) production in association with jets: Similar to the $Z / \gamma^{*} \rightarrow e e / \mu \mu$ category, this category of events can mimic the dilepton + dijet $+E_{T}$ signature when an instrumental mis-measurement of energy occurs, resulting in fake $\ddot{H}_{T}$.

- The top-pair production in the lepton+jets final state, when one jet is misidentified as a charged lepton: Since this category is actually from $t \bar{t}$ production, events in this category could be interpreted as signals in certain measurements. However, since one of the the charged leptons from these events is faked by a jet (usually a $b$-quark jet), they behave differently from the $t \bar{t}$ dilepton events in the analyses in this dissertation, thus we consider them as backgrounds.

Below we list the key features of the event selection criteria which are designed to be efficient for our signal while rejecting the majority of the backgrounds. Before going into that, we note that it will be useful to have a number of control regions where the contribution from the signal does not dominate, so that we can test the methods to estimate the contributions from each background process. For this purpose, we group our baseline event selection criteria into two phases: we define a set of "minimal" cuts that selects events with dilepton $+\not_{T}$ signature, and then a set of "signal" cuts to further select opposite-charged dilepton events with two or more jets as well as other kinematic cuts that have a high efficiency for signal, but are targeted to reject the backgrounds described above. Events passing the minimal cuts but with zero or one jet fall into our "0-jet" and "1-jet" control regions, respectively. 
The set of requirements are summarized in Table 2.1, and described below. The minimal requirements include:

- Exactly two high energy $\left(E_{\mathrm{T}}>20 \mathrm{GeV}\right)$ charged leptons consistent with the dilepton final state. At least one of the two charged leptons is required to pass the tight lepton and isolated lepton identification criteria to enhance the purity of the sample, but the second is only required to pass the loose requirement and is not required to be isolated to retain high selection efficiency. This requirement is especially helpful against $W+$ jets backgrounds. It has been found that the requirement of a second lepton is needed, but that only looser, more efficient, requirements are needed since the backgrounds are small enough. Any event with more than two charged leptons are rejected.

- Large $E_{T}\left(E_{T}>25 \mathrm{GeV}\right)$ that is consistent with two high energy neutrinos from $W$-boson decay leaving the detector. This is helpful in rejecting events like $Z / \gamma^{*} \rightarrow e e / \mu \mu, J / \psi$, or $\Upsilon$, which have no physical source of $\mathbb{E}_{T}$ in the final state.

- Extra large $E_{T}\left(E_{T}^{\prime}>50 \mathrm{GeV}\right)$ when the direction of $E_{T}^{\prime}$ is within $20^{\circ}$ of any lepton or jet, to reject events whose $\mathscr{H}_{T}$ originates from instrumental effects, or $E_{T}^{\prime}$ produced as part of the decay of a $\tau$ lepton. This is particularly helpful in rejecting processes with $Z / \gamma^{*} \rightarrow \tau \tau$.

- The parameter $\not_{T}$-significance is defined as METSig $=\frac{E_{T}}{\sqrt{E_{T}^{\text {sum }}}}$, where $E_{T}^{\text {sum }}$ is the scaler sum of the $E_{T}$ of all the charged leptons and jets in an event. This parameter quantifies the likelihood that the $\boldsymbol{H}_{T}$ is from a real physical source (e.g. neutrino leaving the detector) rather than instrumental mismeasurement. This variable is motivated by the fact that as more energy that is deposited in 
the calorimeter, the probability for getting a large absolute value of the $\mathscr{E}_{T}$ rises significantly. The resolution of $E_{T}$ measurement is proportional to $\sqrt{E_{T}^{\text {sum }}}$, so the variable METSig effectively quantifies how many standard deviations the measured $E_{T}$ is away from zero. We require METSig $>4 \sqrt{\mathrm{GeV}}$ for di-electron and di-muon events, but only for events where it is needed, specifically when the invariant mass of the two charged leptons $\left(\mathrm{m}_{11}\right)$ is consistent with the $Z$ boson mass $\left(76 \mathrm{GeV} / \mathrm{c}^{2}<\mathrm{m}_{\mathrm{ll}}<106 \mathrm{GeV} / \mathrm{c}^{2}\right)$ to reject contamination from $Z / \gamma^{*}$ production in associated with jets.

- To reduce the sample contamination from low mass dilepton resonances from $J / \psi$ and $\Upsilon$ sources, we require a minimal value of $\mathrm{m}_{l l}$. In analyses that predate this analysis, this requirement was set at $5 \mathrm{GeV}$ [67]. Since we only have $Z / \gamma^{*}+$ jets simulations with the $\mathrm{m}_{\mathrm{ll}}>8 \mathrm{GeV}$, we have raised the dilepton invariant mass cut to $10 \mathrm{GeV}$ to avoid potential mismodelling in the low dilepton invariant mass region. This modification in the event selection criteria has a minor effect on the analysis.

To separate events from the control samples and the final signal samples, we include the following additional requirements:

- The two leptons (electrons or muons) have opposite electric charges. This is a signature of $t \bar{t} \rightarrow \ell^{+} \ell^{\prime-} \nu \overline{\nu^{\prime}} b \bar{b}$ decay and helps reject against $W+$ jets events where the charge of the fake lepton is not necessarily opposite in charge from the leptons from the $W$-boson decay.

- Two or more high energy jets with $E_{\mathrm{T}}>15 \mathrm{GeV}$ and $|\eta|<2.5$. This is helpful in rejecting Diboson + jets and $Z / \gamma^{*}+$ jets events since all jets in those backgrounds are typically at low energy, or have large $|\eta|$, as they are from 
Table 2.1: The top dilepton event selection. We refer the reader to Ref. [67] for further details.

\begin{tabular}{|c|c|}
\hline \multirow{4}{*}{ 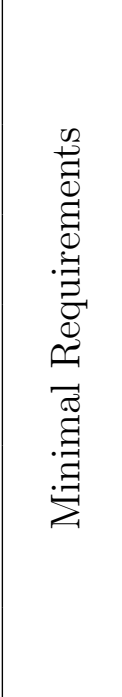 } & $\begin{array}{l}\text { Exactly two leptons with } E_{\mathrm{T}}>20 \mathrm{GeV} \text { and passing standard } \\
\text { identification requirements }[83] \\
\text {-At least one central lepton that pass the online selection } \\
\text {-At least one tight and isolated lepton }\end{array}$ \\
\hline & $\begin{array}{l}E_{T}>25 \mathrm{GeV} \text {, but } \not_{T}>50 \mathrm{GeV} \text { when there is any lepton or jet } \\
\text { within } 20^{\circ} \text { of the direction of } \mathscr{H}_{T}^{\prime}\end{array}$ \\
\hline & $\begin{array}{l}\operatorname{METSig}\left(=\frac{E_{T}^{\prime}}{\sqrt{E_{T}^{\text {sum }}}}\right)>4 \sqrt{\mathrm{GeV}} \text { for } e \text { and } \mu \mu \text { events when } \\
76 \mathrm{GeV} / \mathrm{c}^{2}<\mathrm{m}_{1 \mathrm{l}}<106 \mathrm{GeV} / \mathrm{c}^{2}\end{array}$ \\
\hline & $m_{l l}>10 \mathrm{GeV} / \mathrm{c}^{2}$ \\
\hline \multirow{3}{*}{ 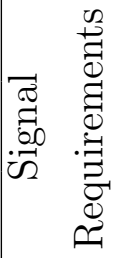 } & Two or more jets with $E_{\mathrm{T}}>15 \mathrm{GeV}$ within $|\eta|<2.5$ \\
\hline & $\mathrm{H}_{\mathrm{T}}>200 \mathrm{GeV}$ \\
\hline & Two oppositely charged leptons \\
\hline
\end{tabular}

initial- and/or final-state radiations, and the $b$-quark jets from the top quark decays are expected to be very energetic.

- The summed transverse energy over all the particles in an event $\left(H_{T}\right)$ satisfying $H_{T}>200 \mathrm{GeV}$. The typical $t \bar{t}$ process has at least twice the top mass $(2 \times$ $175 \mathrm{GeV}=350 \mathrm{GeV}$ ) produced in the collision, so that there are a lot of high energy final state objects. This is not usually the case most of the backgrounds.

We note that in the measurement of $A_{\mathrm{FB}}^{t \bar{t}}$ with top reconstruction in Sec. 6, we will introduce more event selection requirements based on top reconstruction to further improve the sensitivity of that measurement.

With our dataset defined we move next to the modeling of the signal and the 
backgrounds in Sec. 3 . 


\section{SIGNAL AND BACKGROUND MODELING}

Even after the set of dedicated event selection criteria described in Sec. 2, the data are still a mixture of events from a variety of sources, including events from the

process of interest $\left(t \bar{t} \rightarrow \ell^{+} \ell^{\prime-} \nu \overline{\nu^{\prime}} b \bar{b}\right.$, signals) as well as events from other processes (backgrounds). In order to properly measure the important physics parameters of interest, we need to model both the signal (with different model parameters) as well as the backgrounds. We note that we use multiple signal models as it allows us to test the behavior of the signal events under different physical scenarios (different potential values of the $A_{\mathrm{FB}}$ ), and to develop and validate the methodology used to perform the measurements to determine the correct $A_{\mathrm{FB}}$ from what we observe from the detector. In this section we describe our various $t \bar{t}$ signal models as well as the modeling of each of the background sources. The signal and background modeling methodology is very similar to that used in the $t \bar{t}$ cross section measurement in the same final state [67], with a few minor updates, thus we will only summarize it here, but give more details in Appendix B when needed.

We begin this section with a description of the various $t \bar{t}$ production models, including hypotheses with LO and NLO SM, as well as with scenarios with physics beyond the SM (BSM) in Sec. 3.1. In Sec. 3.2 we describe the background modeling methodology used in this analysis, with an emphasis on the improvements we have made relative to previous measurements. Note that some of the details of the improvements are summarized in Appendix B to maintain the flow the the main text. In Sec. 3.3 we present the data validation with the baseline event selection criteria. A set of validation checks, using events with the 0-jet and 1-jet control regions, and separately with the subset of the events with an additional $b$-tagging 
requirement are summarized in Appendices $\mathrm{C}$ and $\mathrm{D}$, respectively. We note that the data validation for the measurement of $A_{\mathrm{FB}}^{t \bar{t}}$, after additional requirements based on top reconstruction quality, will be presented in Sec. 6 .

\subsection{Top-pair Production Signal with Different Physics Models}

We listed the SM and BSM models we employ to simulate $t \bar{t}$ production and decay in Table 1.2. The detailed kinematic properties of each scenario are simulated using Monte Carlo (MC) event generators. The event generators use calculations based on the Feynman diagrams to create an ensemble of collision events (a sample) that mimic the events we would see in the detector if nature were described by the corresponding Feynman diagram. The generators include ALPGEN [51], HERWIG [52], MAdGRAPH [58], POWHEG [49], and Pythia [50]. The hadronization of the outgoing quarks, the process during which the quarks pull apart and recombine quark-antiquark pairs from the vacuum until all particles are in a color-neutral state (parton showering), is also calculated using MC techniques by the HERWIG or PYTHIA programs. We also use the HERWIG or PYTHIA programs to add in initialand final-state radiation to simulate the extra jets in the process. Since the Tevatron collides a bunch of protons against a bunch of antiprotons, there could be more than one collision happening during each bunch crossing. The number of extra collisions besides the highest energy collision we are interested in is closely related to the instantaneous luminosity, which reflects how many particles are in a bunch of proton/antiproton. The extra collisions are simulated with the PYTHIA event generator, with the number of extra collisions per event configured to match the profile of the instantaneous luminosity in the actual data taking periods. The simulation of the detector response to the generated events, both the primary collision and extra interactions, is accomplished with a GEANT-based [87] package named CDFSIM [88]. 
After the simulation of the detector responses, the samples are passed through the same object reconstruction and identification algorithms as data.

In addition to the $A_{\mathrm{FB}}$ values for the SM and BSM models we listed in Table 1.2 , here we list additional details about each sample in Table 3.1. We note that unless specified otherwise, the POWHEG sample is used throughout this dissertation as our default sample for signal modeling.

\subsection{Background Modeling}

For modeling the background contributions, we use a combination of data-based and MC-based techniques. We need to both estimate the number of events as well as model the kinematic properties for each background component in our data. We mostly follow the same procedure that was used in the $t \bar{t}$ cross section measurement in the same final state [67], but introduce a number of improvements that will be enumerated below.

In Table 3.2 we list background MC samples with their corresponding cross section, along with the generator used to create each. We note that instead of using the cross section predicted by the generator, we use standalone calculations of the cross sections for $W W, W Z$, and $Z Z$ processes at NLO. For cross sections of $W \gamma$ and DY processes, which are calculated at LO, a set of K-factors, which are estimates of the multiplicative corrections on the cross sections for NLO effects not accounted in the corresponding LO calculations, are derived elsewhere and used with the generator calculated cross sections [67]. The K-factors are included in the table. The final cross sections we used are the cross sections listed multiplied by the corresponding K-factors, so all background calculations of the event rates correspond to the SM predictions at NLO. We next outline the key points of the background estimation procedure: 


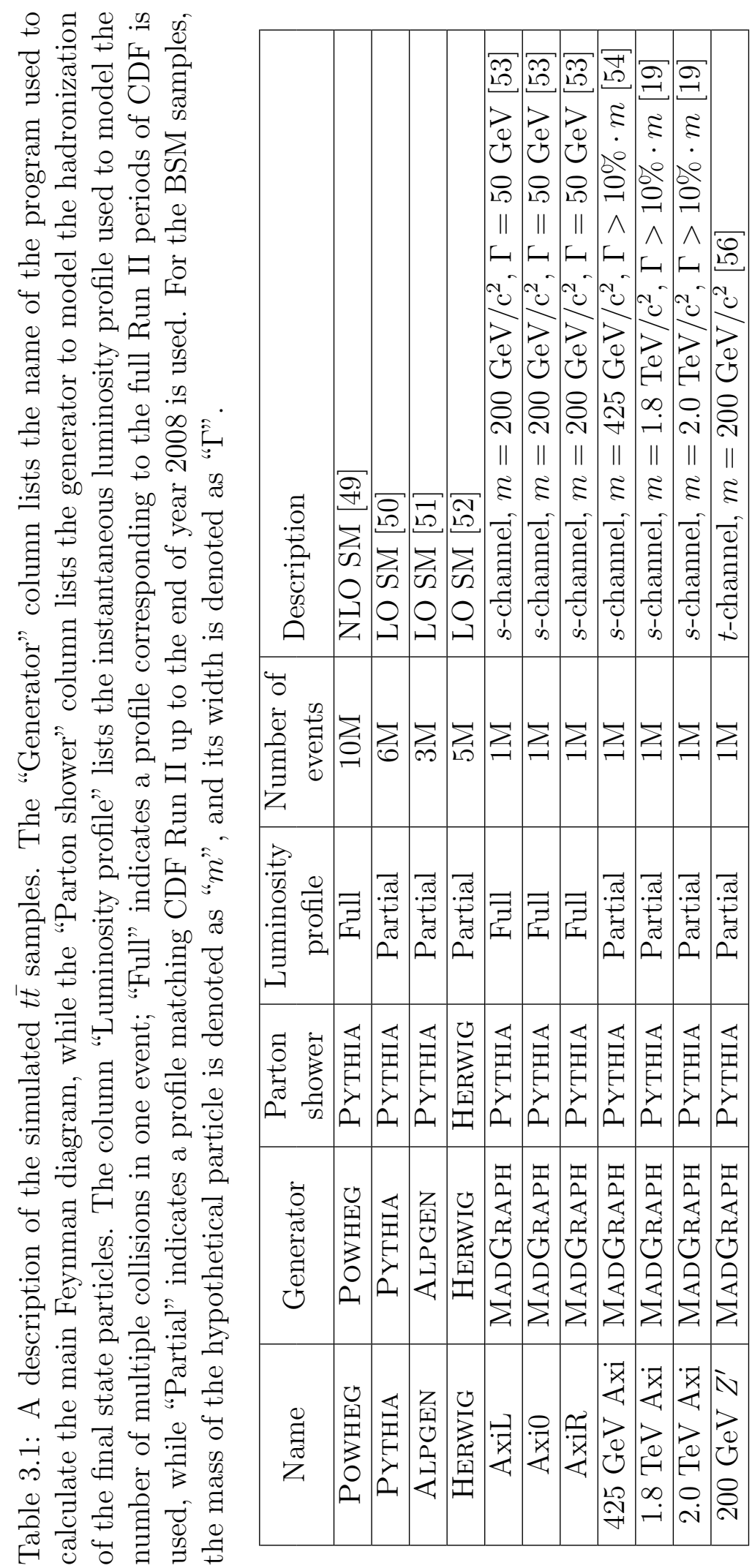


- The diboson $(W W, W Z, Z Z$ and $W \gamma)$ backgrounds are estimated by normalizing the corresponding MC samples to the integrated luminosity to data with the cross-sections (and K-factors) listed in Table 3.2. While each simulation is at LO, we use the PYTHIA MC to add initial- and final-state radiation to simulate the extra jets.

- The $W+$ jets background is estimated with a data-driven method, based on a series of probabilities a jet being mis-identified as a lepton, derived from a separate data set. This procedure has been used for many years and is described in detail in Ref. [67].

- The DY $\rightarrow e e / \mu \mu$ and DY $\rightarrow \tau \tau$ backgrounds are estimated with a data-MC hybrid method, with details described in Appendix B. The method we use is an improvement over the previous method used in Ref. 67.

- Each MC sample is corrected for the trigger efficiency, the object identification efficiency, the vertex $z_{0}$ reconstruction efficiency, the fake charge rates for electrons in the plug detector, and normalized to the integrated luminosity from the corresponding triggers. These details are described in Ref. [67].

While we mostly follow the same procedure as the $t \bar{t}$ cross section measurement, we have made the following improvements since the previous analyses:

- We consider $t \bar{t}$ events in the lepton+jets final state faking the dilepton final state to be a background to the analysis. To do this, we added a background category denoted as " $t \bar{t}$ non-dilepton", and estimate it by normalizing the POWHeg $t \bar{t}$ MC samples to the integrated luminosity to data with the theoretical cross section of $7.4 \mathrm{pb}$ [33]. 
Table 3.2: A table of background MC samples.

\begin{tabular}{|c|c|c|c|c|}
\hline & Process & $\begin{array}{c}\text { Cross Section } \\
(\mathrm{pb})\end{array}$ & $\begin{array}{c}\mathrm{K}- \\
\text { factor }\end{array}$ & Generator \\
\hline & $W W$ & $11.34 \pm 0.68$ & N.A. & PYTHIA \\
\hline & $W Z$ & $3.47 \pm 0.21$ & N.A. & PYTHIA \\
\hline & $Z Z$ & $3.62 \pm 0.22$ & N.A. & PYTHIA \\
\hline & $W(\rightarrow e \nu) \gamma$ & $32 \pm 3.2$ & 1.36 & BAUR [89] \\
\hline & $W(\rightarrow \mu \nu) \gamma$ & $32 \pm 3.2$ & 1.34 & BAUR \\
\hline & $75 \mathrm{GeV}<\mathrm{m}_{\mathrm{ll}}<105 \mathrm{GeV}$, ee+0p & 157 & 1.4 & ALPGEN \\
\hline & $75 \mathrm{GeV}<\mathrm{m}_{\mathrm{ll}}<105 \mathrm{GeV}$, ee $+1 \mathrm{p}$ & 21.5 & 1.4 & ALPGEN \\
\hline & $75 \mathrm{GeV}<\mathrm{m}_{\mathrm{ll}}<105 \mathrm{GeV}$, ee $+\geq 2 \mathrm{p}$ & 4.14 & 1.4 & ALPGEN \\
\hline & $75 \mathrm{GeV}<\mathrm{m}_{11}<105 \mathrm{GeV}, \mu \mu+0 \mathrm{p}$ & 157 & 1.4 & ALPGEN \\
\hline & $75 \mathrm{GeV}<\mathrm{m}_{11}<105 \mathrm{GeV}, \mu \mu+1 \mathrm{p}$ & 21.6 & 1.4 & ALPGEN \\
\hline & $75 \mathrm{GeV}<\mathrm{m}_{1 \mathrm{l}}<105 \mathrm{GeV}, \mu \mu+\geq 2 \mathrm{p}$ & 4.12 & 1.4 & ALPGEN \\
\hline & $75 \mathrm{GeV}<\mathrm{m}_{1 \mathrm{l}}<105 \mathrm{GeV}, \tau \tau+0 \mathrm{p}$ & 157 & 1.4 & ALPGEN \\
\hline & $75 \mathrm{GeV}<\mathrm{m}_{11}<105 \mathrm{GeV}, \tau \tau+1 \mathrm{p}$ & 21.6 & 1.4 & ALPGEN \\
\hline & $75 \mathrm{GeV}<\mathrm{m}_{1 \mathrm{l}}<105 \mathrm{GeV}, \tau \tau+\geq 2 \mathrm{p}$ & 4.13 & 1.4 & ALPGEN \\
\hline & $20 \mathrm{GeV}<\mathrm{m}_{11}<75 \mathrm{GeV}$, ee+0p & 159 & 1.4 & ALPGEN \\
\hline & $20 \mathrm{GeV}<\mathrm{m}_{11}<75 \mathrm{GeV}$, ee $+1 \mathrm{p}$ & 8.36 & 1.4 & ALPGEN \\
\hline & $20 \mathrm{GeV}<\mathrm{m}_{11}<75 \mathrm{GeV}$, ee $+\geq 2 \mathrm{p}$ & 1.81 & 1.4 & ALPGEN \\
\hline & $20 \mathrm{GeV}<\mathrm{m}_{\mathrm{ll}}<75 \mathrm{GeV}, \mu \mu+0 \mathrm{p}$ & 160 & 1.4 & ALPGEN \\
\hline & $20 \mathrm{GeV}<\mathrm{m}_{l l}<75 \mathrm{GeV}, \mu \mu+1 \mathrm{p}$ & 8.30 & 1.4 & ALPGEN \\
\hline & $20 \mathrm{GeV}<\mathrm{m}_{1 l}<75 \mathrm{GeV}, \mu \mu+\geq 2 \mathrm{p}$ & 1.81 & 1.4 & Alpgen \\
\hline & $20 \mathrm{GeV}<\mathrm{m}_{11}<75 \mathrm{GeV}, \tau \tau+0 \mathrm{p}$ & 160 & 1.4 & ALPGEN \\
\hline & $20 \mathrm{GeV}<\mathrm{m}_{1 \mathrm{l}}<75 \mathrm{GeV}, \tau \tau+1 \mathrm{p}$ & 8.35 & 1.4 & ALPGEN \\
\hline & $20 \mathrm{GeV}<\mathrm{m}_{\mathrm{ll}}<75 \mathrm{GeV}, \tau \tau+\geq 2 \mathrm{p}$ & 1.81 & 1.4 & ALPGEN \\
\hline & $105 \mathrm{GeV}<\mathrm{m}_{\mathrm{ll}}<600 \mathrm{GeV}, \mathrm{ee}+0 \mathrm{p}$ & 4.06 & 1.4 & ALPGEN \\
\hline DY & $105 \mathrm{GeV}<\mathrm{m}_{\mathrm{ll}}<600 \mathrm{GeV}$, ee $+1 \mathrm{p}$ & 0.707 & 1.4 & ALPGEN \\
\hline & $105 \mathrm{GeV}<\mathrm{m}_{1 \mathrm{l}}<600 \mathrm{GeV}$, ee $+\geq 2 \mathrm{p}$ & 0.141 & 1.4 & ALPGEN \\
\hline & $105 \mathrm{GeV}<\mathrm{m}_{11}<600 \mathrm{GeV}, \mu \mu+0 \mathrm{p}$ & 4.06 & 1.4 & ALPGEN \\
\hline & $105 \mathrm{GeV}<\mathrm{m}_{\mathrm{ll}}<600 \mathrm{GeV}, \mu \mu+1 \mathrm{p}$ & 0.702 & 1.4 & ALPGEN \\
\hline & $105 \mathrm{GeV}<\mathrm{m}_{1 \mathrm{l}}<600 \mathrm{GeV}, \mu \mu+\geq 2 \mathrm{p}$ & 0.141 & 1.4 & ALPGEN \\
\hline & $105 \mathrm{GeV}<\mathrm{m}_{11}<600 \mathrm{GeV}, \tau \tau+0 \mathrm{p}$ & 4.06 & 1.4 & ALPGEN \\
\hline & $105 \mathrm{GeV}<\mathrm{m}_{11}<600 \mathrm{GeV}, \tau \tau+1 \mathrm{p}$ & 0.710 & 1.4 & ALPGEN \\
\hline & $105 \mathrm{GeV}<\mathrm{m}_{1 \mathrm{l}}<600 \mathrm{GeV}, \tau \tau+\geq 2 \mathrm{p}$ & 0.140 & 1.4 & ALPGEN \\
\hline & $8 \mathrm{GeV}<\mathrm{m}_{1 \mathrm{ll}}<20 \mathrm{GeV}$, ee+0p & 1514 & 1.4 & ALPGEN \\
\hline & $8 \mathrm{GeV}<\mathrm{m}_{1 \mathrm{l}}<20 \mathrm{GeV}$, ee $+1 \mathrm{p}$ & 19.7 & 1.4 & ALPGEN \\
\hline & $8 \mathrm{GeV}<\mathrm{m}_{\mathrm{ll}}<20 \mathrm{GeV}$, ee $+2 \mathrm{p}$ & 6.98 & 1.4 & Alpgen \\
\hline & $8 \mathrm{GeV}<\mathrm{m}_{11}<20 \mathrm{GeV}, \mu \mu+0 \mathrm{p}$ & 1508 & 1.4 & ALPGEN \\
\hline & $8 \mathrm{GeV}<\mathrm{m}_{\mathrm{ll}}<20 \mathrm{GeV}, \mu \mu+1 \mathrm{p}$ & 19.6 & 1.4 & ALPGEN \\
\hline & $8 \mathrm{GeV}<\mathrm{m}_{1 \mathrm{l}}<20 \mathrm{GeV}, \mu \mu+2 \mathrm{p}$ & 6.96 & 1.4 & ALPGEN \\
\hline
\end{tabular}


- We note that part of the MC samples we use in this analysis now use the luminosity profile corresponding to the full CDF Run II periods. These include the $W W$, the DY, and the $t \bar{t}$ POWHEG samples. Studies indicate that not using the full luminosity profile has a negligible effect on the final analysis results.

- We introduce scale factors for DY to correct for mismodeling of cross-section and $E_{T}$ in MC. The procedure is described in Appendix $B$.

3.3 Validation of the Signal and Background Modeling

In this section we present the validation of the signal and background modeling. We first show the validation with the baseline event selections described in Sec. 2.4; this data set is used for the measurements of the $A_{\mathrm{FB}}^{\ell}$ and $A_{\mathrm{FB}}^{\ell \ell}$. We note that the validation of the data set used for the measurement of $A_{\mathrm{FB}}^{t \bar{t}}$ is shown in Sec. 6 .

We first list the expected number of events from each background component as well as from $t \bar{t}$ dilepton signal compared with the observed numbers in Table 3.3 . listed by lepton categories. The number of observed events is consistent with the expectations.

As a check we compare differential distributions from all sources with data for our final state. These were selected to form a complete list of the properties of the final state objects. Figures 3.1 and 3.2 show a number of distributions, with each individual background components shown separately along with $t \bar{t}$ and overlaid with data. Figures 3.3 and 3.4 combines all background sources into one component. Figures 3.5 and 3.6 shows distributions from $t \bar{t}$ signal MC, overlaid with data after background subtraction. In all cases the data looks well modeled by the expectations.

We note that we also have checked signal and background modeling in a number of different ways. The first is in the 0 -jet and 1-jet control regions. These two control regions do not receive significant contributions from the $t \bar{t}$ dilepton signal, 


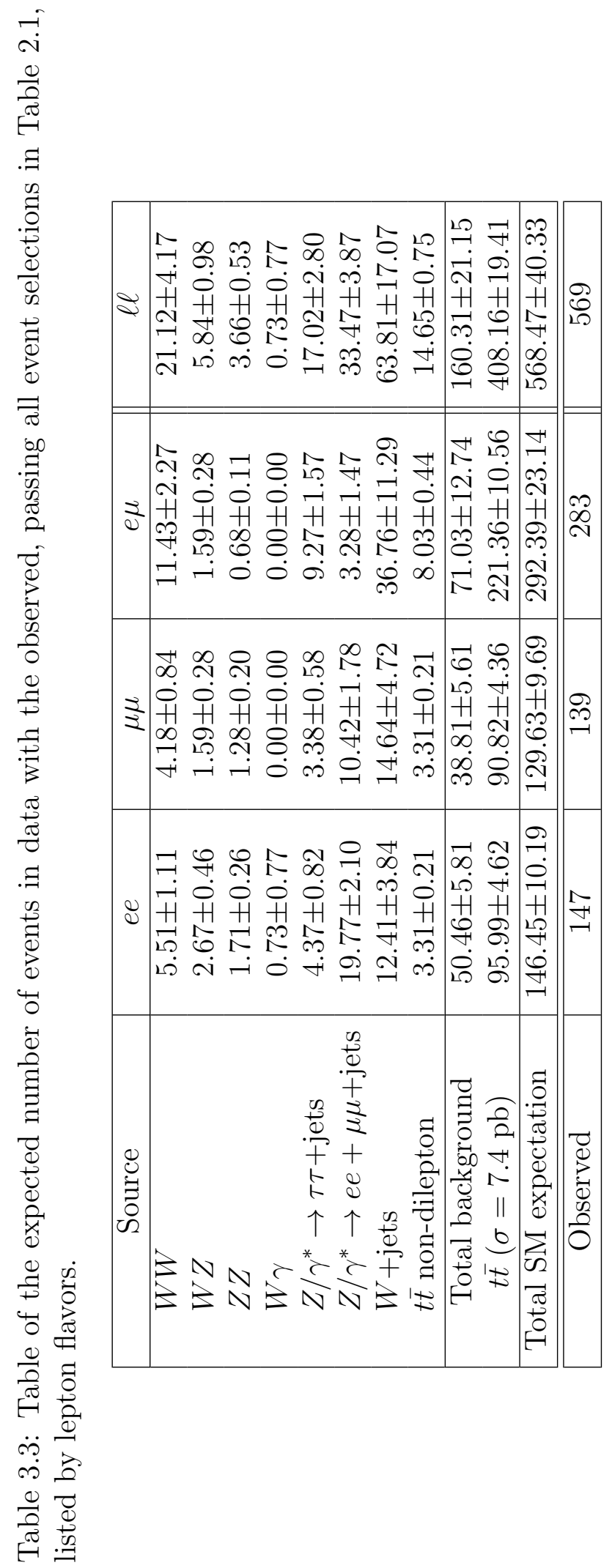



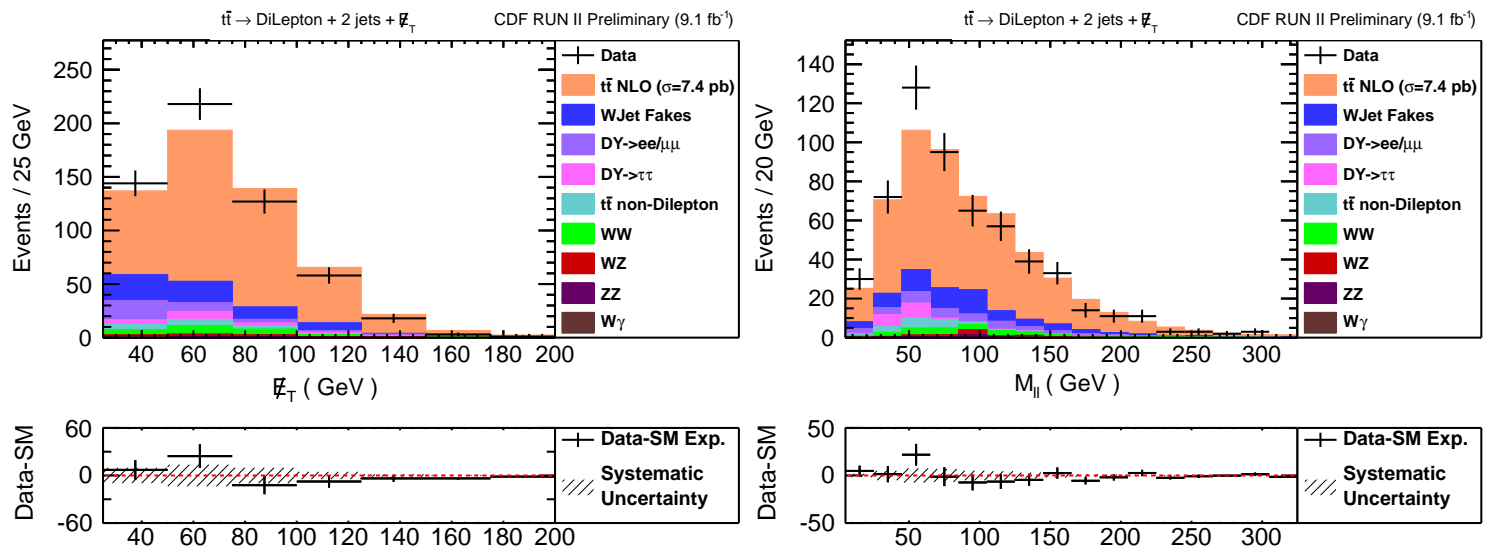

(a) $\ddot{H}_{T}$

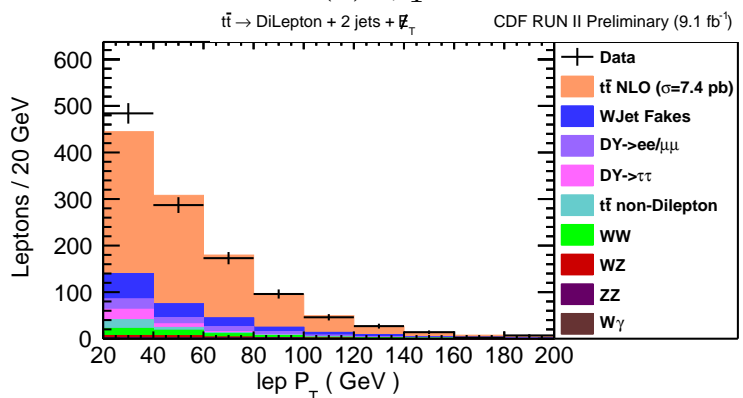

(b) $\mathrm{m}_{11}$
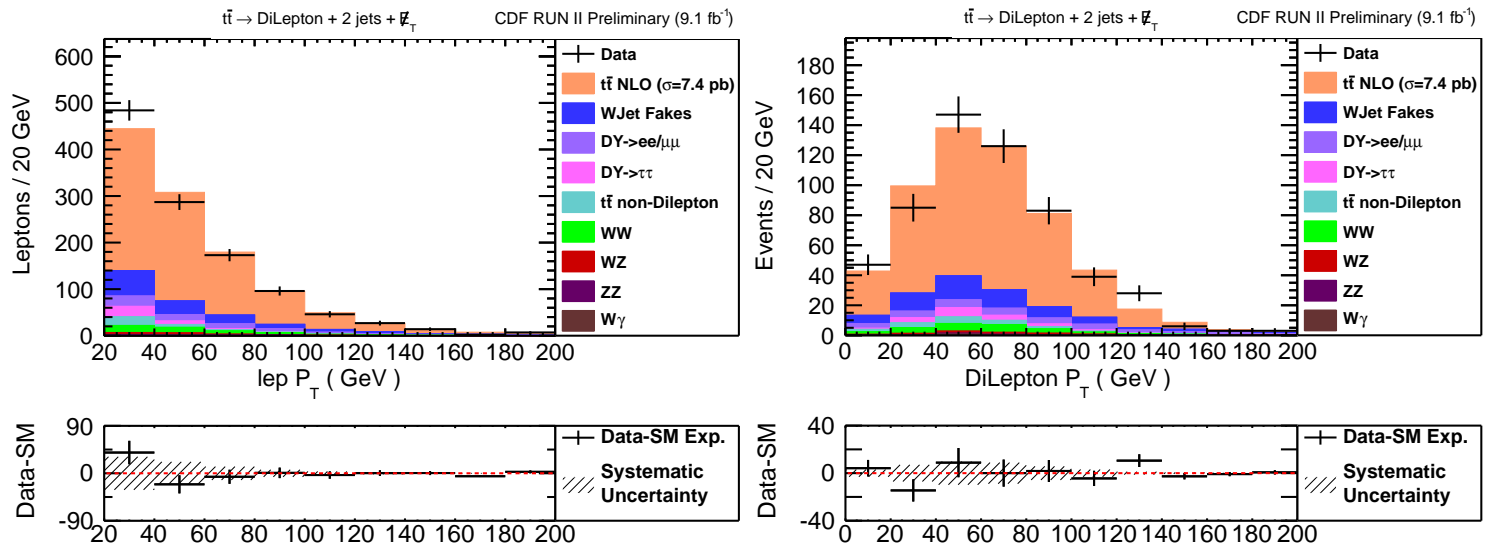

(c) $p_{T}$ for both leptons
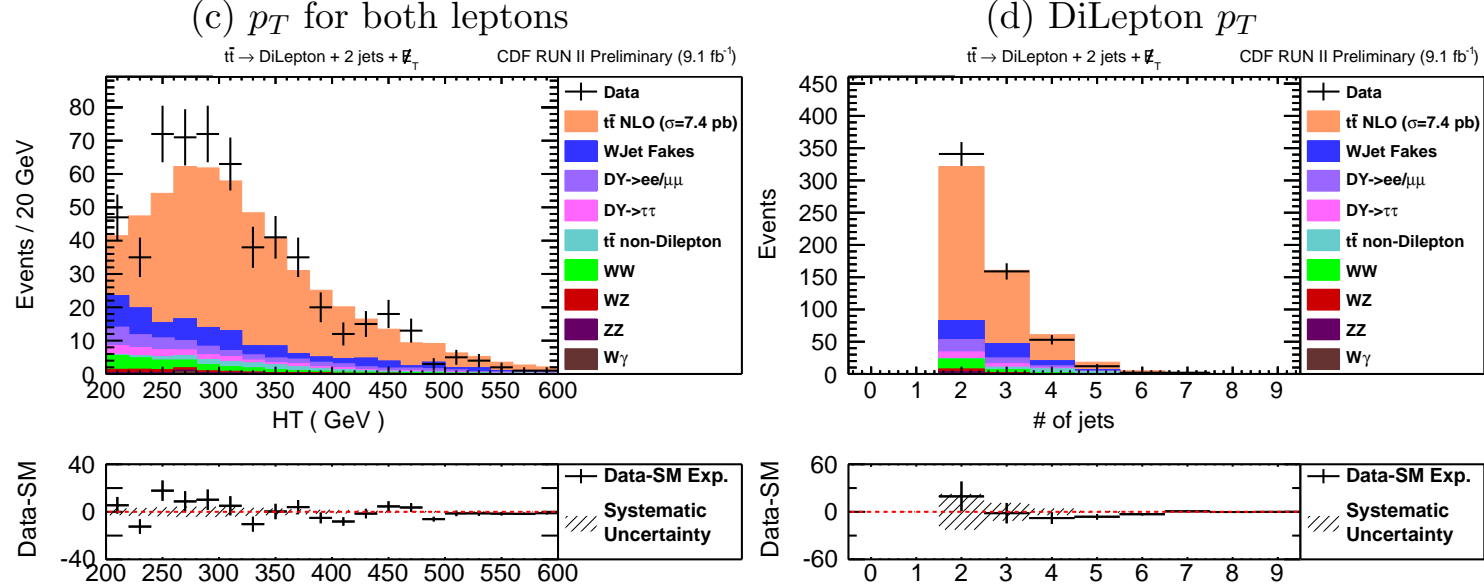

(e) $\mathrm{H}_{\mathrm{T}}$

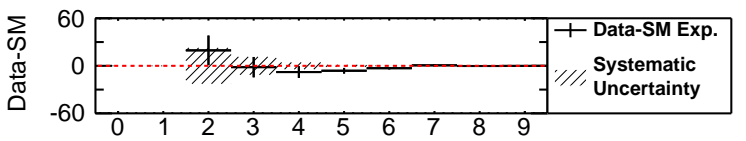

(f) Jet Multiplicity

Figure 3.1: The comparison of the observed distributions with the expectation, with individual background components specified. The distributions of (a) $\not_{T}$, (b) $m_{l l}$, (c) $p_{T}$ of both leptons, (d) $p_{T}$ of the dilepton system, (e) $\mathrm{H}_{\mathrm{T}}$, and (f) jet multiplicity are shown. 

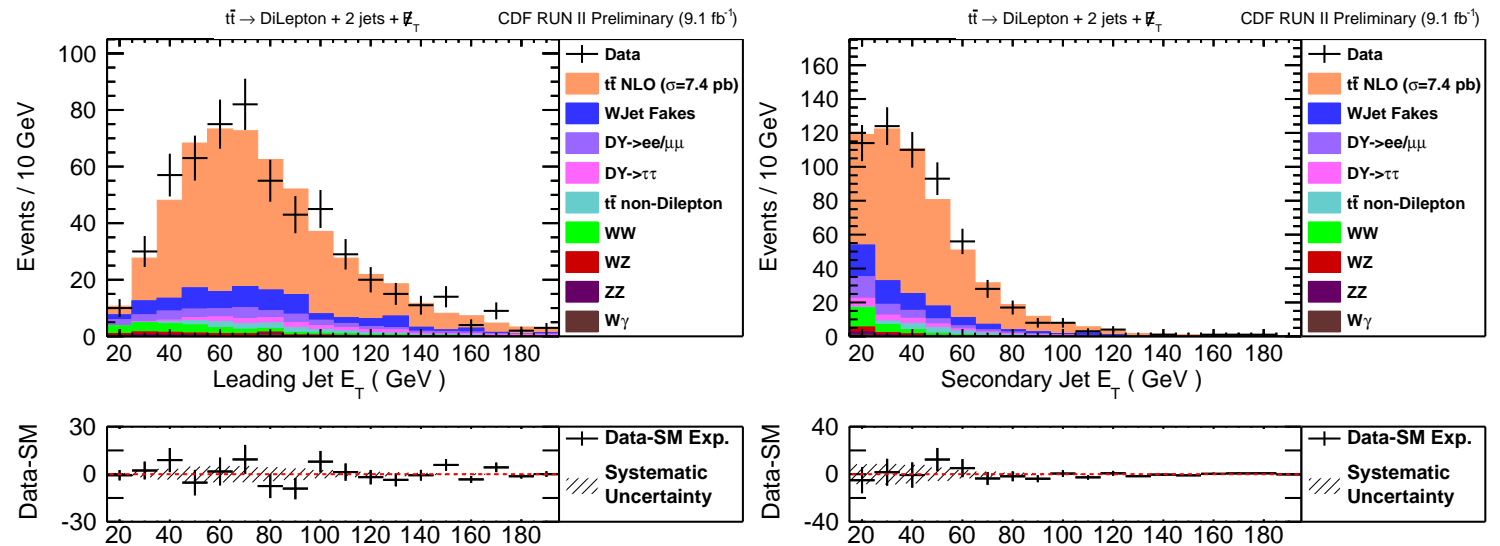

(a) Leading jet $E_{\mathrm{T}}$

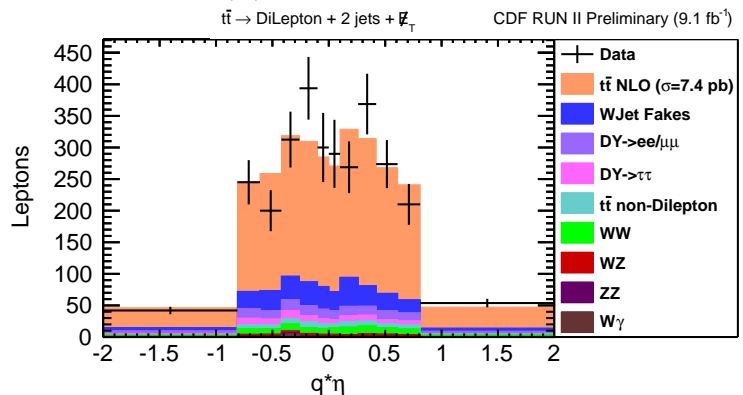

(b) Secondary jet $E_{\mathrm{T}}$
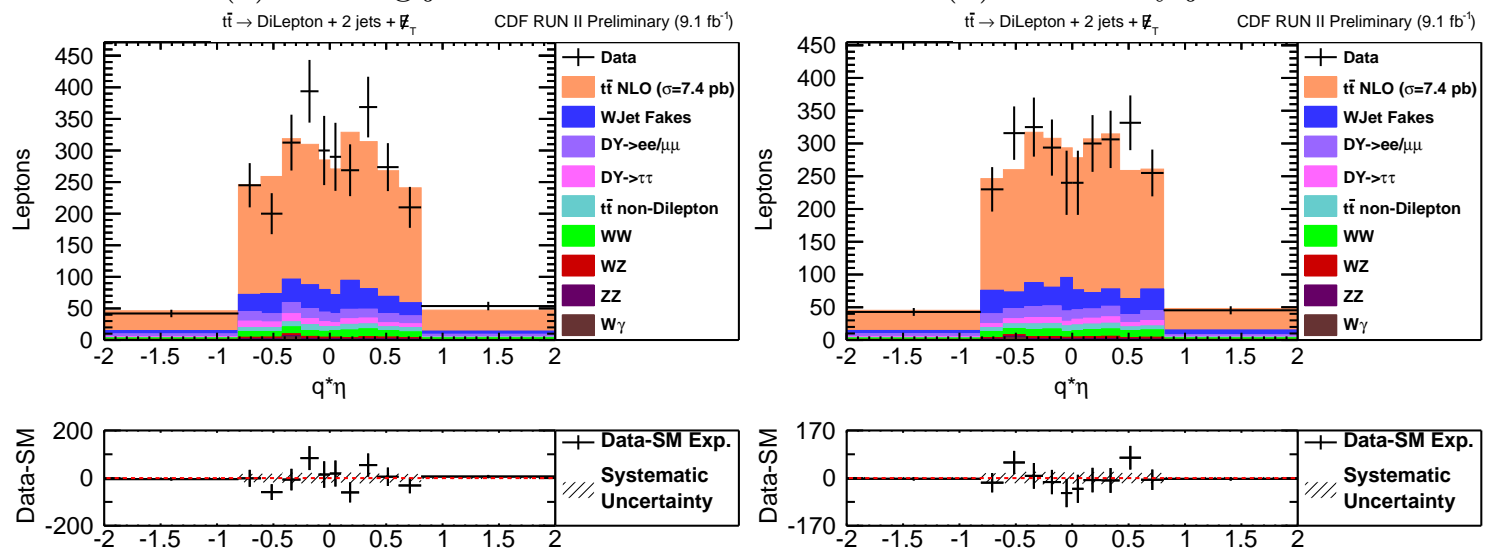

(c) Positive Lepton $q_{\ell} \eta_{\ell}$

(d) Negative Lepton $q_{\ell} \eta_{\ell}$
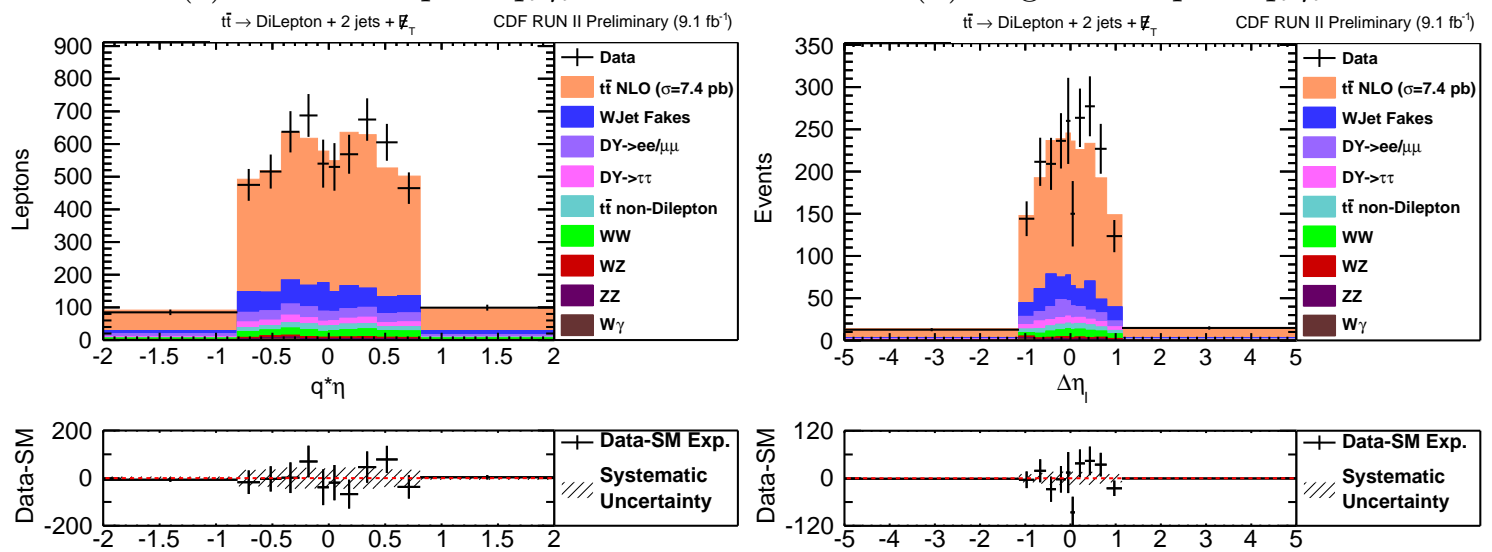

(e) Both Lepton $q_{\ell} \eta_{\ell}$

(f) $\Delta \eta$

Figure 3.2: The same plots as Fig. 3.1, but with (a) leading jet $E_{\mathrm{T}}$, (b) secondary jet $E_{\mathrm{T}}$, (c) positive lepton $q_{\ell} \eta_{\ell}$, (d) negative lepton $q_{\ell} \eta_{\ell}$, (e) $q_{\ell} \eta_{\ell}$ of both leptons, and (f) $\Delta \eta$. 


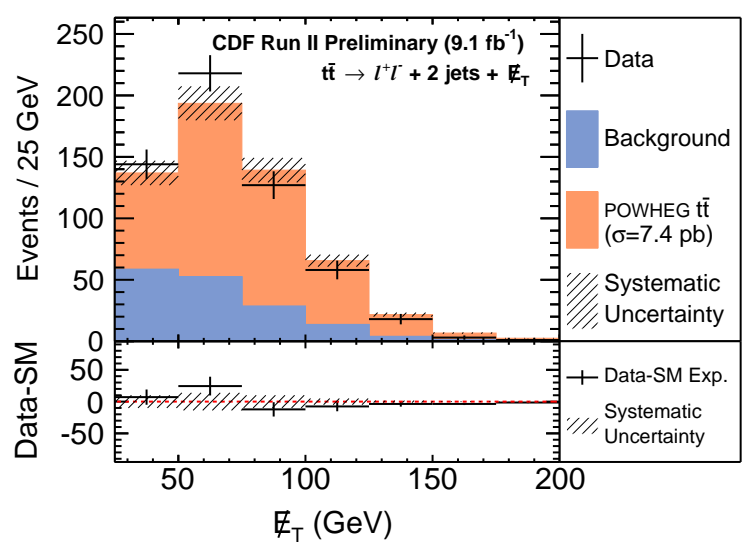

(a) $\not_{T}$

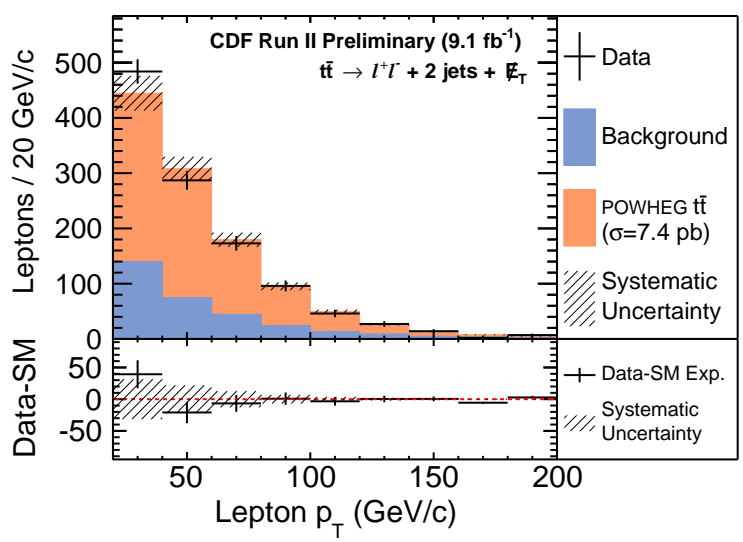

(c) $p_{T}$ for both leptons
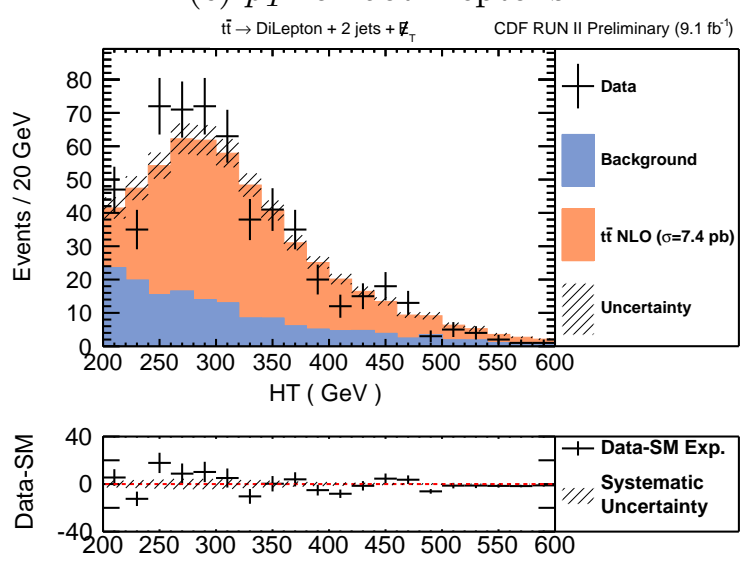

(e) $\mathrm{H}_{\mathrm{T}}$

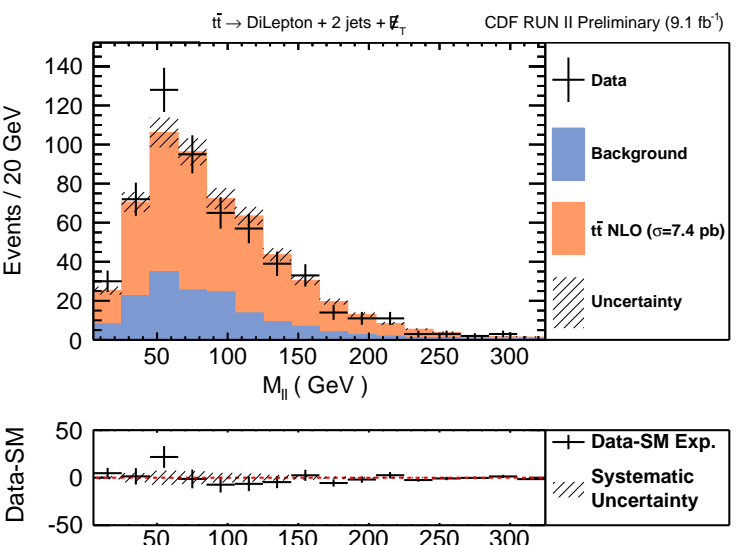

(b) $\mathrm{m}_{11}$

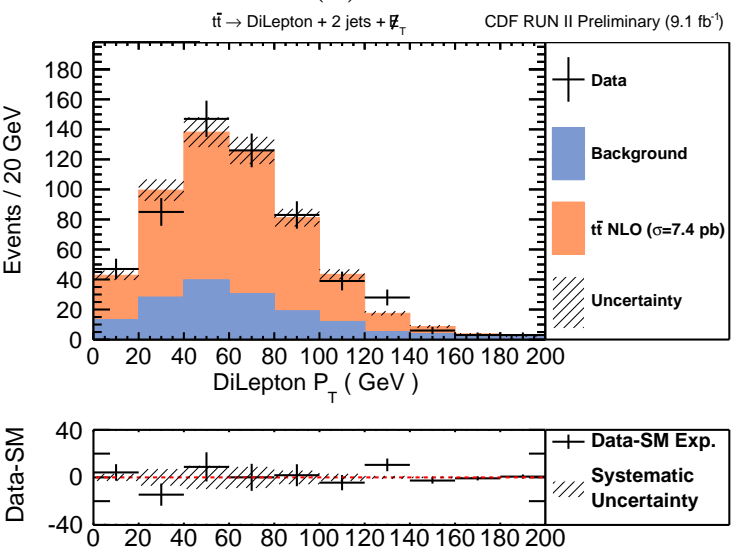

(d) DiLepton $p_{T}$

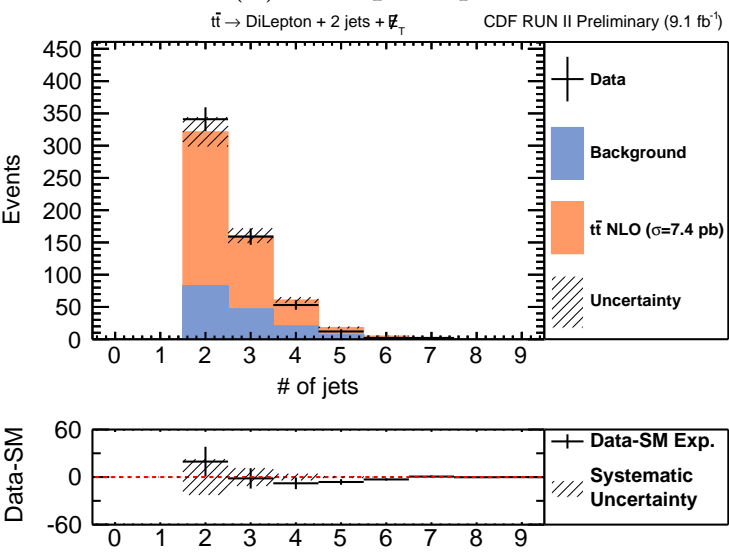

(f) Jet Multiplicity

Figure 3.3: The same as Fig. 3.1, but where all the backgrounds are combined. 

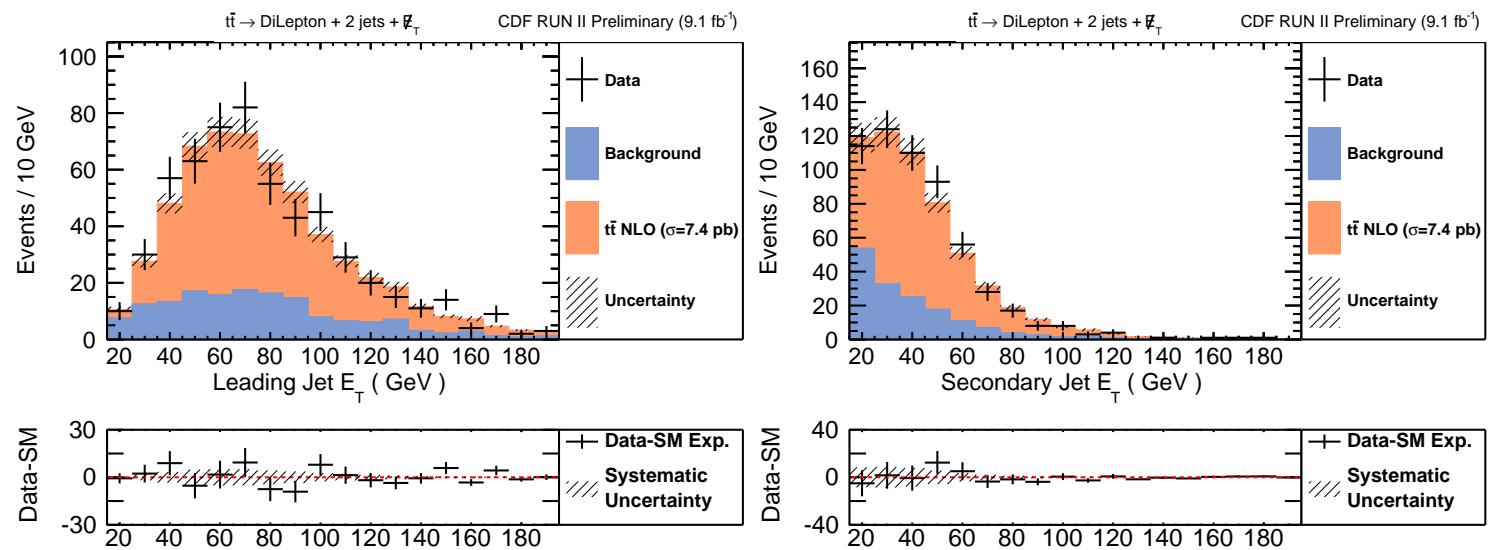

(a) Leading jet $E_{\mathrm{T}}$

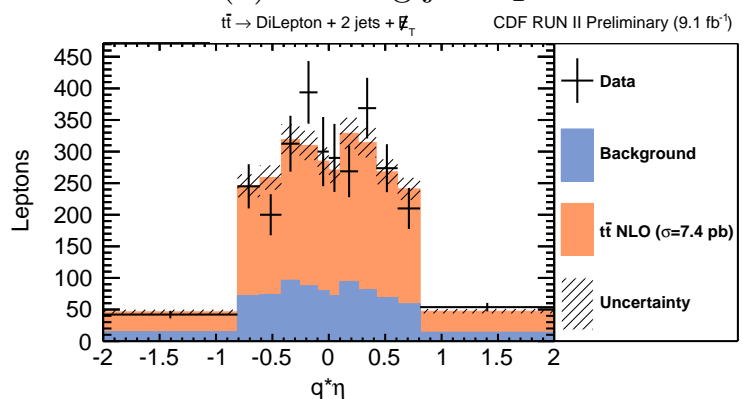

(b) Secondary jet $E_{\mathrm{T}}$
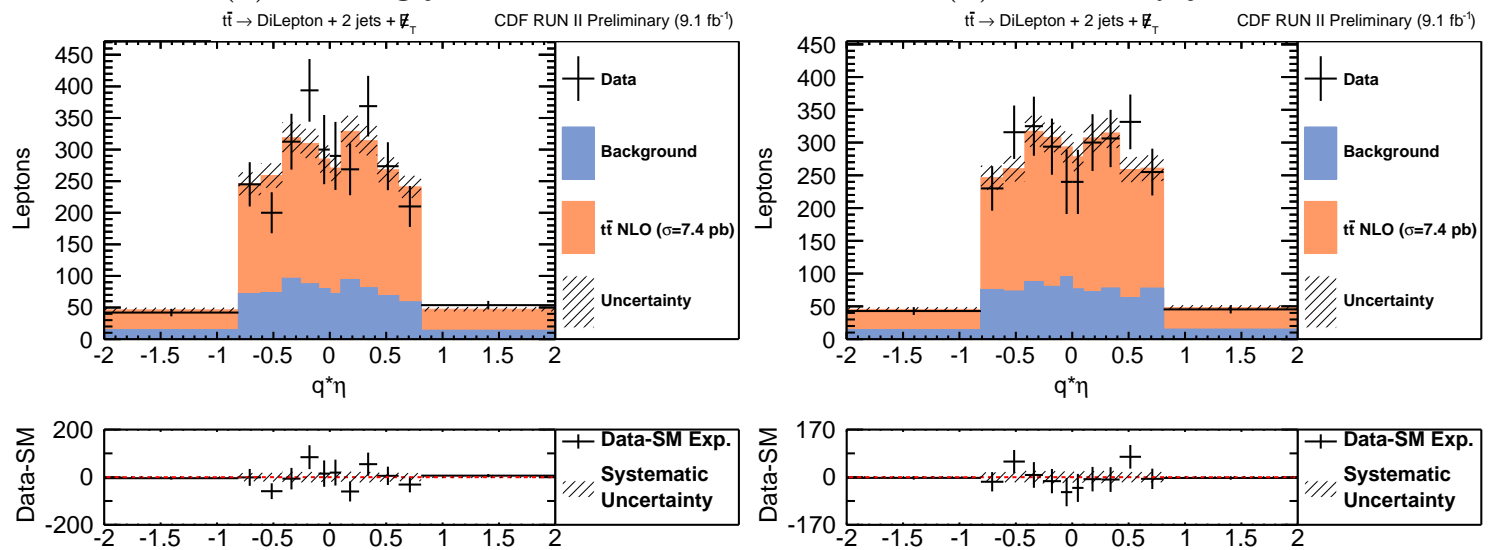

(c) Positive Lepton $q_{\ell} \eta_{\ell}$

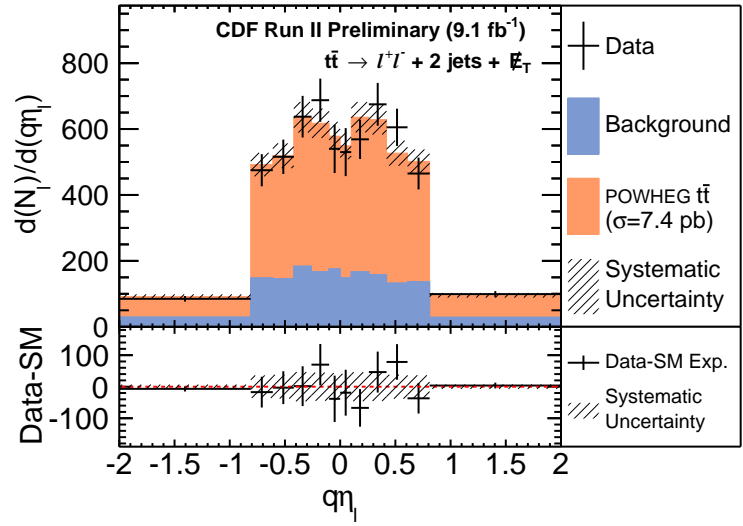

(e) Both Lepton $q_{\ell} \eta_{\ell}$ (d) Negative Lepton $q_{\ell} \eta_{\ell}$

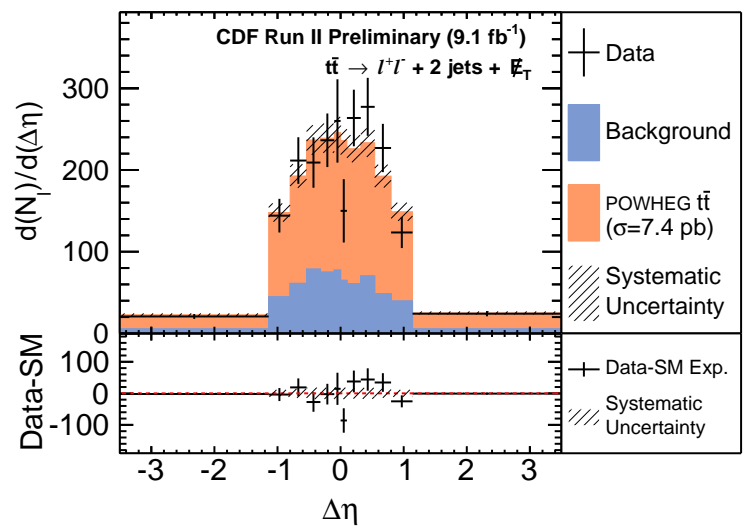

(f) $\Delta \eta$

Figure 3.4: The same as Fig. 3.2, but where all the backgrounds are combined. 

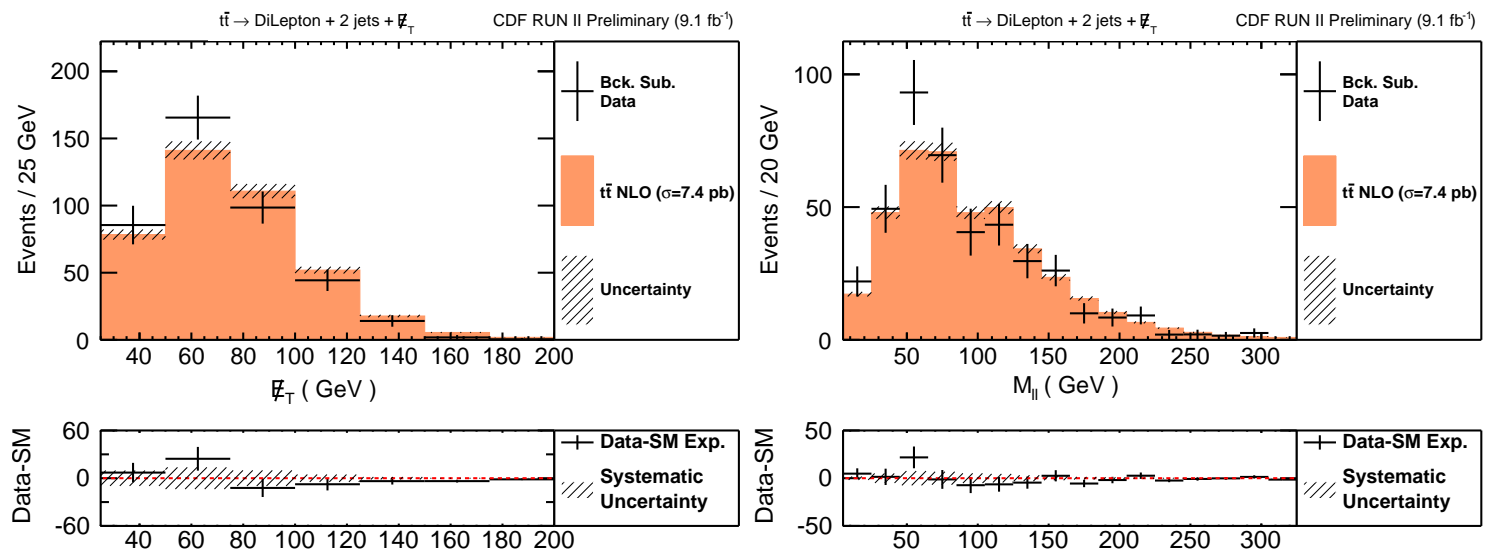

(a) $\not_{T}$

(b) $m_{l l}$
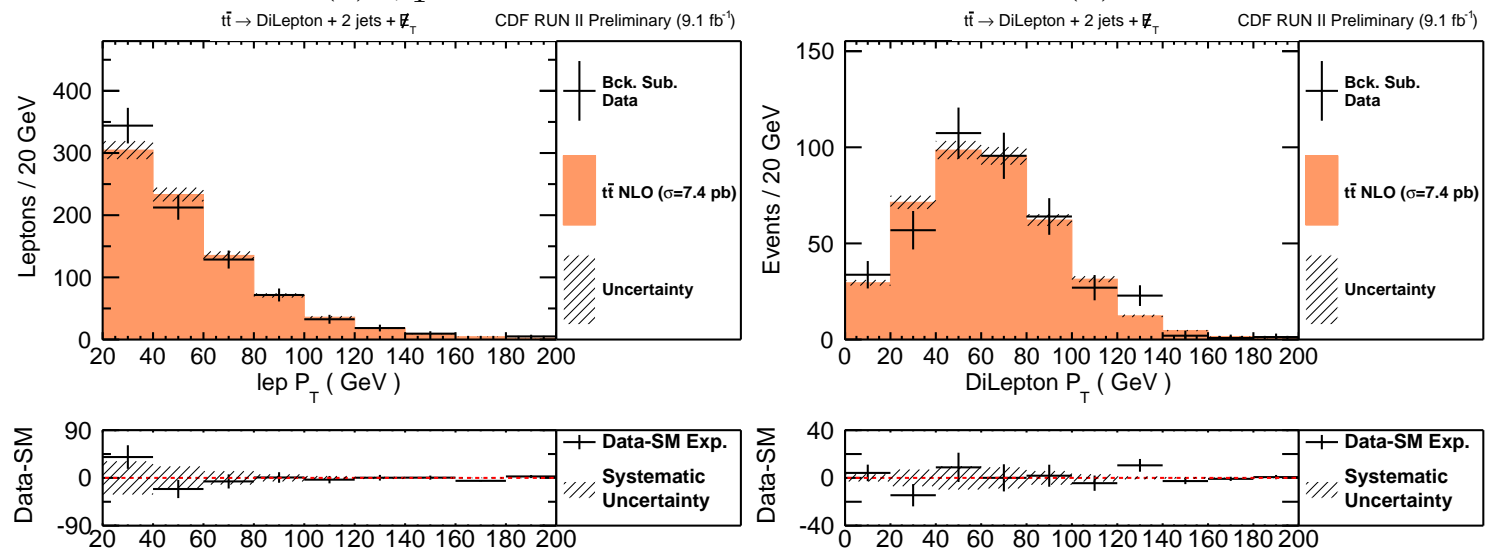

(c) $p_{T}$ for both leptons

(d) DiLepton $p_{T}$
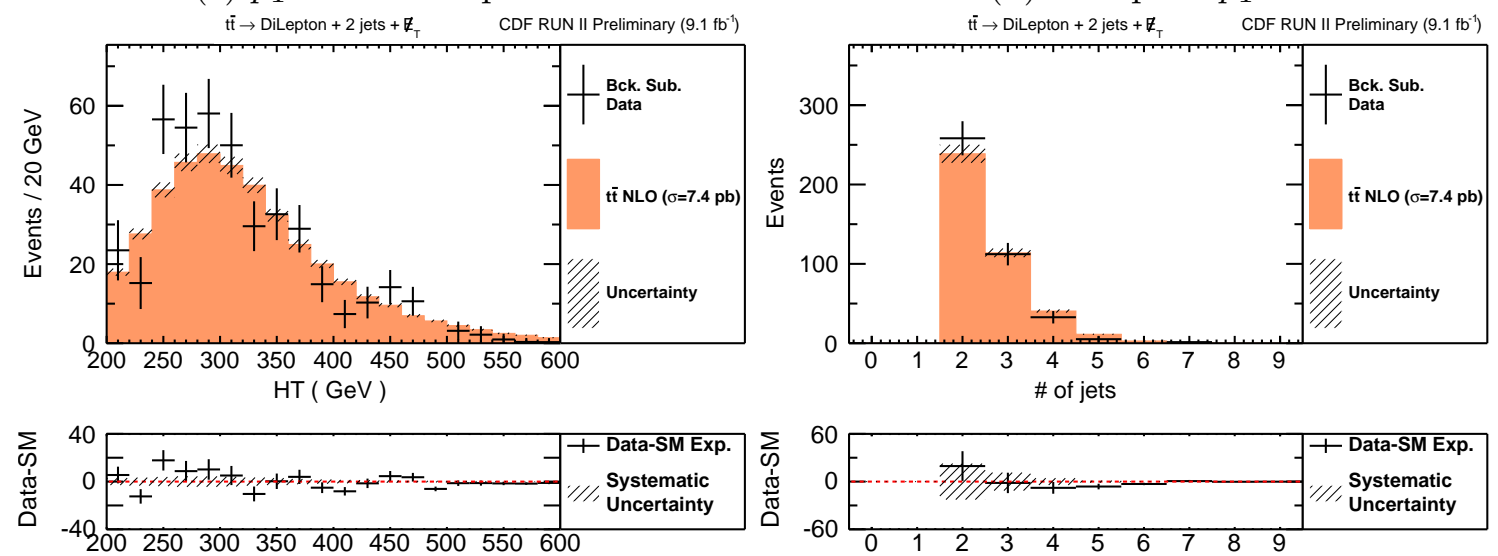

(e) $\mathrm{H}_{\mathrm{T}}$

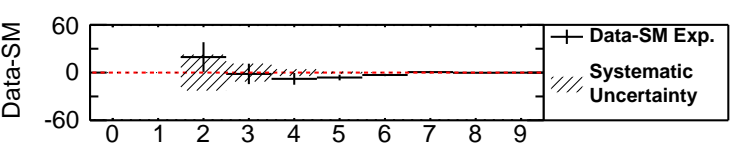

(f) Jet Multiplicity

Figure 3.5: The comparison of the expected distribution from the POWHEG simulation with the observation after subtracting off the contributions from background processes. The distributions of (a) $\not_{T}$, (b) $\mathrm{m}_{\mathrm{ll}}$, (c) $p_{T}$ of both leptons, (d) $p_{T}$ of the dilepton system, (e) $\mathrm{H}_{\mathrm{T}}$, and (f) jet multiplicity are shown. 

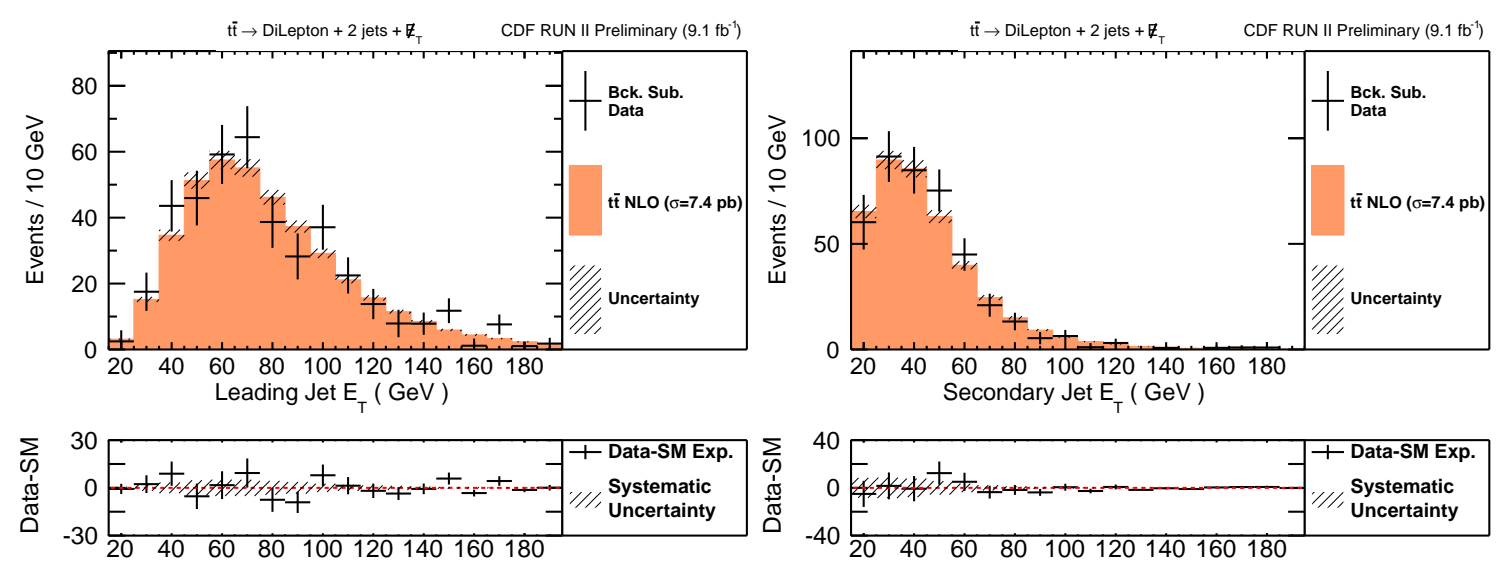

(a) Leading jet $E_{\mathrm{T}}$

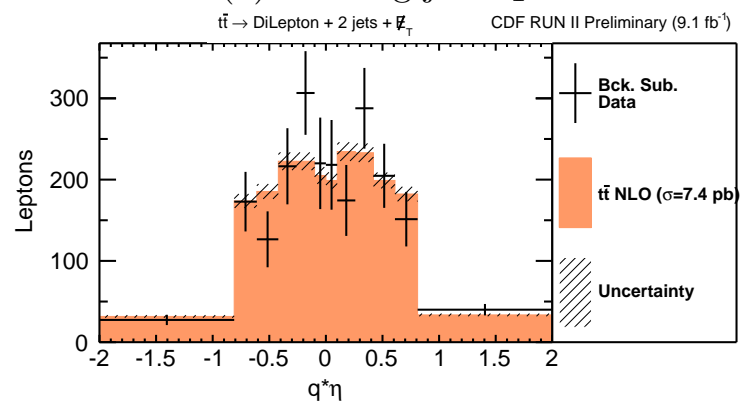

(b) Secondary jet $E_{\mathrm{T}}$
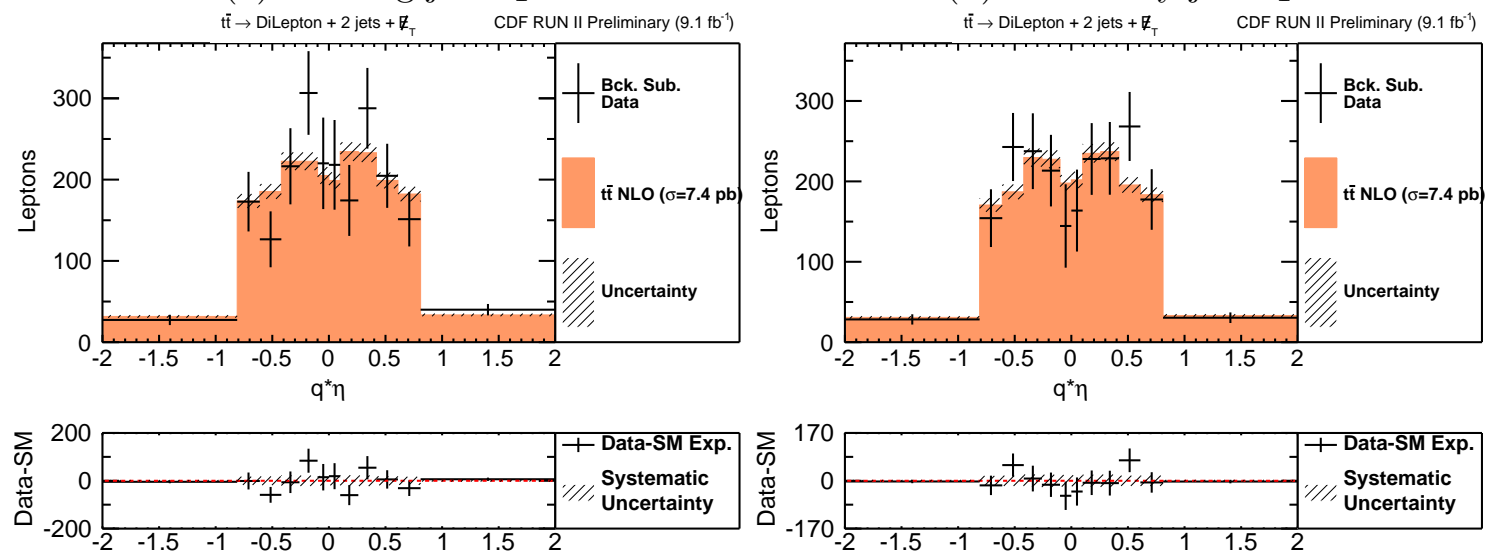

(c) Positive Lepton $q_{\ell} \eta_{\ell}$

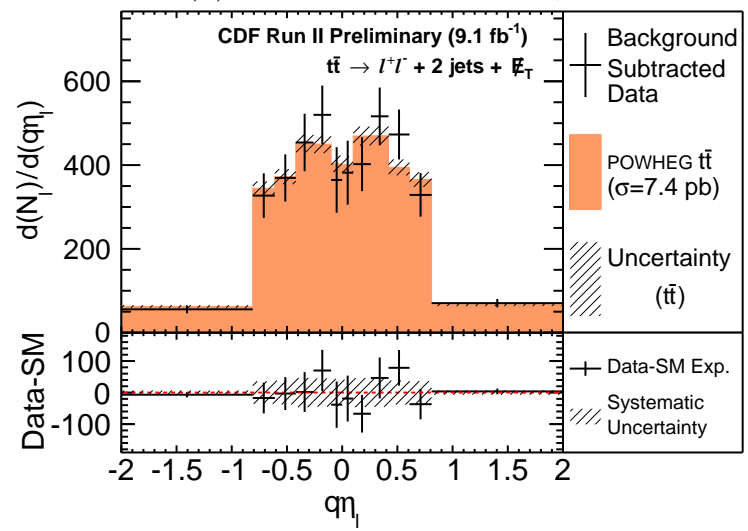

(e) Both Lepton $q_{\ell} \eta_{\ell}$

(d) Negative Lepton $q_{\ell} \eta_{\ell}$

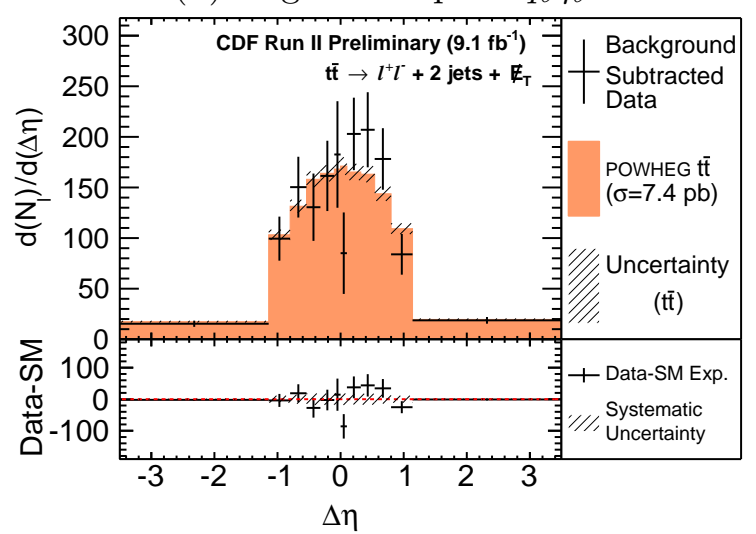

(f) $\Delta \eta$

Figure 3.6: The same plots as Fig. 3.5, but with (a) leading jet $E_{\mathrm{T}}$, (b) secondary jet $E_{\mathrm{T}}$, (c) positive lepton $q_{\ell} \eta_{\ell}$, (d) negative lepton $q_{\ell} \eta_{\ell},(\mathrm{e}) q_{\ell} \eta_{\ell}$ of both leptons, and (f) $\Delta \eta$. 
and are thus ideal for validation of the modeling of the backgrounds. The results are in Appendix C. The observations are consistent with the expectations in both control regions. In addition, we have checked the signal and background modeling in the signal region with an extra requirement of at least one jet being $b$-tagged. This sample is purer than the nominal signal sample without the additional requirement, but has about half of the expected events due to the efficiency of the SECVTX $b$ tagging algorithm $(\sim 50 \%)$ [82. The results are summarized in Appendix D, In all cases, the expectation agree with the observation well, indicating the modeling of the signal and background processes is robust.

Now that we have powerful modeling of the signal and background processes, we move on to describing the measurement methodology of the $A_{\mathrm{FB}}^{\ell}$ and $A_{\mathrm{FB}}^{\ell \ell}$ as well as the measurements themselves in the next section. 


\section{MEASUREMENT OF THE FORWARD-BACKWARD ASYMMETRY OF LEPTONS FROM TOP-QUARK PAIRS*}

With our dataset in hand and our backgrounds well understood, we are now ready to proceed with our measurements of the top $A_{\mathrm{FB}}$ observables. We will first describe the leptonic $A_{\mathrm{FB}}$ measurements, $A_{\mathrm{FB}}^{\ell}$ and $A_{\mathrm{FB}}^{\ell \ell}$ (also denoted as $\mathrm{A}_{\mathrm{FB}}^{\text {lep }}$ and $\mathrm{A}_{\mathrm{FB}}^{\Delta \eta}$ in some figures, respectively) [90], since these two measurements do not involve the reconstruction of the top 4-momenta. We will then introduce the top reconstruction algorithm we use in Sec. 5, and the measurements of $A_{\mathrm{FB}}^{t \bar{t}}$ and $A_{\mathrm{FB}}^{t \bar{t}}$ vs. $\left|\Delta y_{t}\right|$ follow in Sec. 6.

The goal of the $A_{\mathrm{FB}}^{\ell}$ and $A_{\mathrm{FB}}^{\ell \ell}$ measurements is to determine the inclusive partonlevel asymmetries. (Note that we use the terms of "parton-level", "truth-level", "generator-level", and "hepg-level" interchangeably, where "hepg" is an acronym for high-energy-physics generator.) Since we do not reconstruct all the events, we will need a measurement methodology to account for both the limited detector acceptance as well as event selection effects. As explained shortly, the leptonic asymmetry is a subtle effect to measure. The data we will use is the measured $q_{\ell} \eta_{\ell}$ from each lepton in each event (or the $\Delta \eta$ from each event), and we will turn the distribution of this observable into a second distribution (the "asymmetric part" of the $q_{\ell} \eta_{\ell}$ or $\Delta \eta$ distribution, the $\mathcal{A}\left(q_{\ell} \eta_{\ell}\right)$ in Sec. 4.1) which is sensitive to the asymmetry itself.

In this section, we will first describe the distributions of the observables we will consider for our measurement, and the cleaver way we will measure parameters from

\footnotetext{
*Reprinted with permission from "Measurement of the inclusive leptonic asymmetry in topquark pairs that decay to two charged leptons at CDF" by the CDF Collaboration, 2014, Phys. Rev. Lett. 113, 042001, and "Forward-Backward Asymmetry of Leptonic Decays of ttbar at the Fermilab Tevatron" by Z. Hong, R. Edgar, S. Henry, D. Toback, J.S. Wilson, and D. Amidei, 2014, Phys. Rev. D 90, 014040. Copyright [2015] by the American Physical Society.
} 
the distribution that are directly related to the inclusive asymmetry. In particular, the measurement methodology is based on the asymmetric part of the $q_{\ell} \eta_{\ell}$ distribution as described in Sec. 4.1. A detailed validation is presented in Sec. 4.2, first at the generator level, as if we had a perfect detector that reconstructs every event perfectly. Then we will show, using full detector response simulation, that the methodology works as well when the detector and event selection effects are taken into consideration. We note that the methodology, before we began this analysis and first used by in the lepton+jets analysis, was known to work, but it was not fully understood why it worked [43, 91]. A detailed study about why it works, performed during the analysis of the data, is presented in Appendix E and published separately in Ref. [92]. The methodology is then applied to data, and the systematic uncertainties of the measurement are estimated, with the results given in Sec. 4.3 .

\subsection{Leptonic $A_{\mathrm{FB}}$ Measurement Methodology}

The definition of $A_{\mathrm{FB}}^{\ell}$ is given in Eq. 1.2 and can be interpreted as the probability of a lepton going forward minus the probability of a lepton going backward. Due to the limited detector coverage, the imperfect detector acceptance, the finite detector resolution, and contamination from non-t $\bar{t}$ sources, a measurement methodology is needed to retrieve the inclusive parton-level leptonic $A_{\mathrm{FB}}$ from data. To do so, we use the same procedure used in measuring the $A_{\mathrm{FB}}^{\ell}$ in the lepton+jets final state, but present in this section and Appendix E a detailed study and validation of why it works [43, 92]. In this section, we describe an overview of the measurement methodology. Note that while we will be using the same methodology for both $A_{\mathrm{FB}}^{\ell}$ and $A_{\mathrm{FB}}^{\ell \ell}$, our description here will first mention $A_{\mathrm{FB}}^{\ell}$ explicitly. The results for $A_{\mathrm{FB}}^{\ell \ell}$ will be listed after that.

We note before beginning that, as stated in Sec. 11, we will follow the assumption 
of $\mathrm{CP}$ conservation, and use the parameter $q_{\ell} \eta_{\ell}$ to merge both the positively charged and negatively charged leptons into a single observable [43, and will use both leptons in each event for our measurement of $A_{\mathrm{FB}}^{\ell}$. As there is potential concern about the correlation between the leptons, we have studied this issue in detail. As shown in Appendix $\mathrm{F}$, the impact on the measurement is very small compared to the dominant uncertainties. Thus, we use both leptons to enhance statistics. A second issue we mention before continuing is that because we only have $\sim 500$ expected $t \bar{t}$ events in the final sample, the expected statistical uncertainty on the final $A_{\mathrm{FB}}^{\ell}$ result is at the 0.05 level, and is expected to be our major uncertainty. Any uncertainty we discuss throughout this section is compared to this.

For the measurement of $A_{\mathrm{FB}}^{\ell}$ and $A_{\mathrm{FB}}^{\ell \ell}$, we use the NLO SM sample POWHEG as our benchmark SM model, and two additional LO SM samples, ALPGEN and PYTHIA for comparison. We also employ the three $200 \mathrm{GeV}$ axigluon models with left-handed, right-handed and pure axial couplings described in Table 1.2 as our benchmark BSM scenarios. These three axigluon models are particularly interesting as they have the same $A_{\mathrm{FB}}^{t \bar{t}}$ values, which are consistent with the experimental results from the lepton+jets final state, but different couplings so that each has a very different $A_{\mathrm{FB}}^{\ell}$ and $A_{\mathrm{FB}}^{\ell \ell}$ value. The three samples are denoted as "AxiL", "AxiR", and "Axi0" respectively. All six MC samples will be used both to develop and validate our measurement methodology.

Figure 4.1 shows the $q_{\ell} \eta_{\ell}$ distribution at the generator level from each of the six $t \bar{t}$ MC samples. From counting the numbers of forward and backward events, we determine that they span the range of $-6 \%<A_{\mathrm{FB}}^{\ell}<15 \%$, which well-surrounds the SM expectation of $3.8 \%$, as well as the results from the lepton+jets measurement of $9.2 \%$. Despite the significant differences in $A_{\mathrm{FB}}^{\ell}$, the spectra look quite similar visually. Thus, we need a good way to distinguish between the subtle differences. 


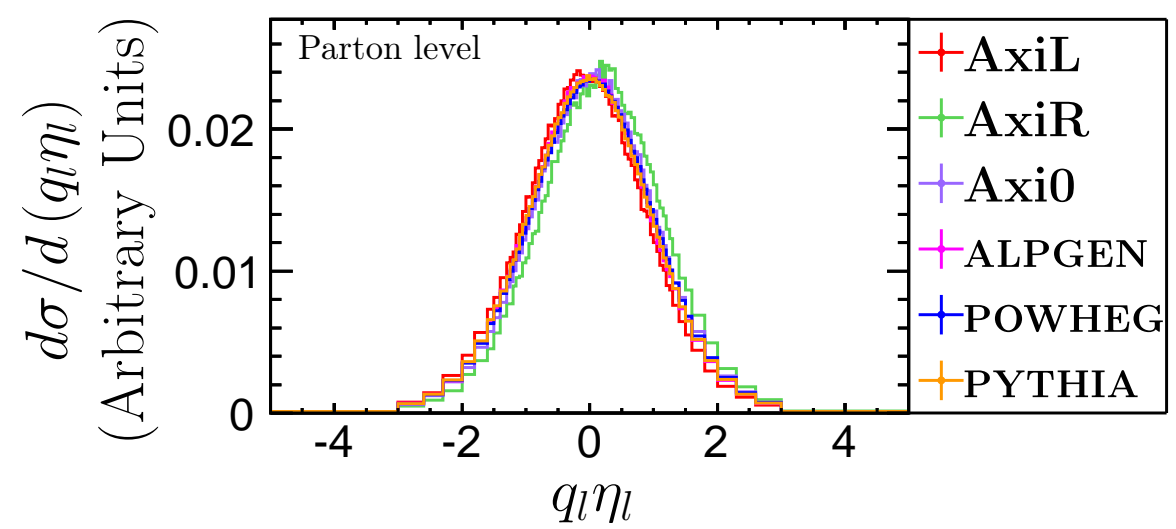

Figure 4.1: The $q_{\ell} \eta_{\ell}$ distribution of charged leptons produced from $t \bar{t}$ cascade decay from simulations with various physics models at parton level, before any selection requirements.

We employ a novel technique which uses a variable that is sensitive to this subtle difference, and was first applied in the CDF measurement of $A_{\mathrm{FB}}^{\ell}$ in the lepton+jets final state [43, 91]. This technique uses a decomposition of the $q_{\ell} \eta_{\ell}$ spectrum into a symmetric part (the $\mathcal{S}\left(q_{\ell} \eta_{\ell}\right)$ term) and an asymmetric part (the $\mathcal{A}\left(q_{\ell} \eta_{\ell}\right)$ term). The decomposition can be done with the following formulas in the range $q_{\ell} \eta_{\ell} \geq 0$ :

$$
\begin{aligned}
\mathcal{S}\left(q_{\ell} \eta_{\ell}\right) & =\frac{\mathcal{N}\left(q_{\ell} \eta_{\ell}\right)+\mathcal{N}\left(-q_{\ell} \eta_{\ell}\right)}{2}, \text { and } \\
\mathcal{A}\left(q_{\ell} \eta_{\ell}\right) & =\frac{\mathcal{N}\left(q_{\ell} \eta_{\ell}\right)-\mathcal{N}\left(-q_{\ell} \eta_{\ell}\right)}{\mathcal{N}\left(q_{\ell} \eta_{\ell}\right)+\mathcal{N}\left(-q_{\ell} \eta_{\ell}\right)}
\end{aligned}
$$

where $\mathcal{N}\left(q_{\ell} \eta_{\ell}\right)$ represents the number of events as a function of $q_{\ell} \eta_{\ell}$. With this decomposition, the $A_{\mathrm{FB}}^{\ell}$ defined in Eq. 1.2 can be rewritten in terms of $\mathcal{S}\left(q_{\ell} \eta_{\ell}\right)$ and $\mathcal{A}\left(q_{\ell} \eta_{\ell}\right)$ as:

$$
A_{\mathrm{FB}}^{\ell}=\frac{\int_{0}^{\infty} \mathrm{d} x[\mathcal{A}(x) \cdot \mathcal{S}(x)]}{\int_{0}^{\infty} \mathrm{d} x^{\prime} \mathcal{S}\left(x^{\prime}\right)} .
$$

The $\mathcal{S}\left(q_{\ell} \eta_{\ell}\right)$ term and the $\mathcal{A}\left(q_{\ell} \eta_{\ell}\right)$ term distributions from the benchmark samples 


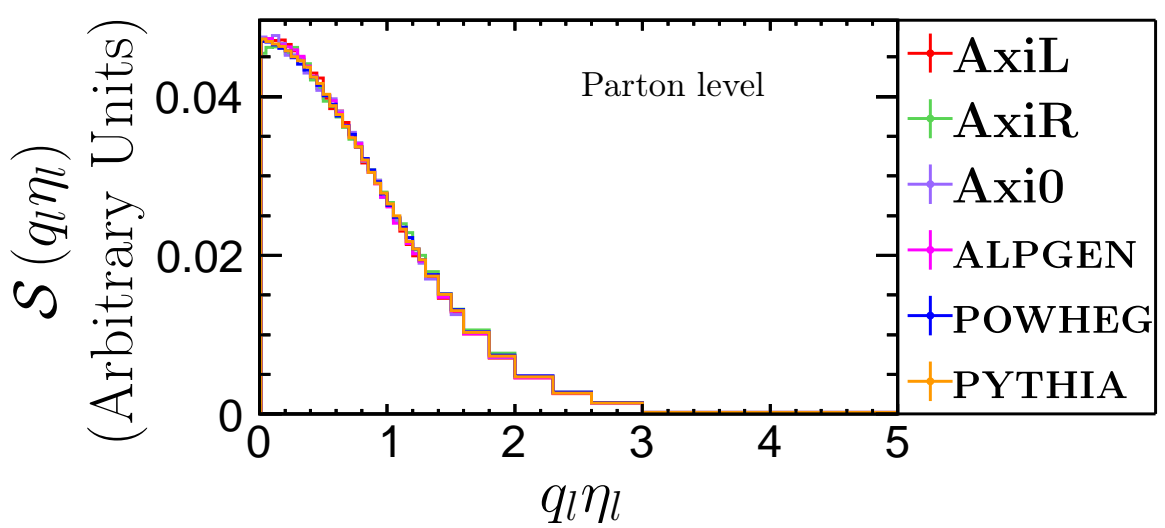

(a)

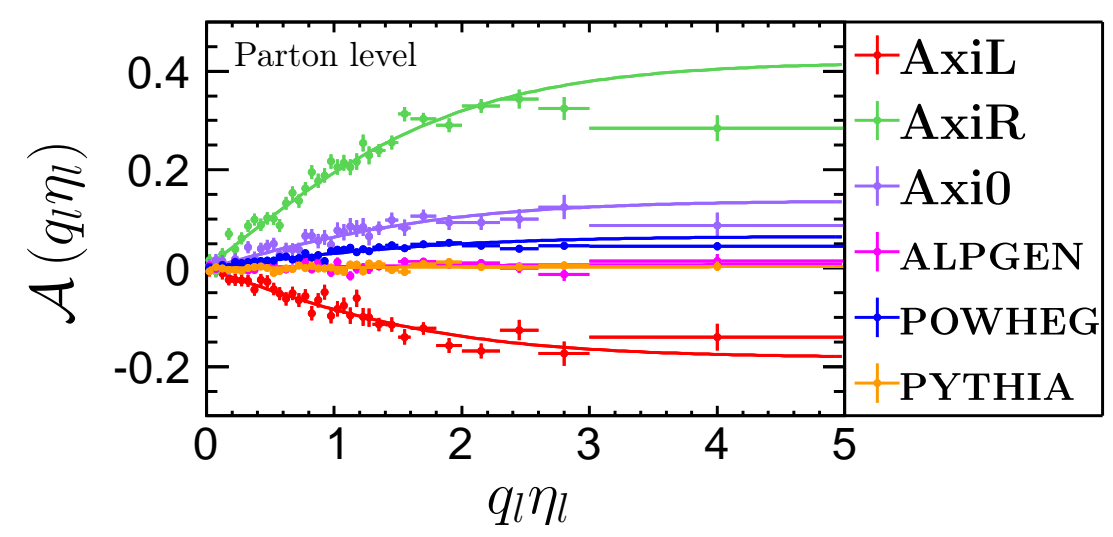

(b)

Figure 4.2: The $\mathcal{S}\left(q_{\ell} \eta_{\ell}\right)$ term (a) and the $\mathcal{A}\left(q_{\ell} \eta_{\ell}\right)$ term (b) of the $q_{\ell} \eta_{\ell}$ distribution from various physics models. The lines in (b) correspond to the best fits from the $a \cdot$ tanh model. 
are shown in Fig. $4.2 \mathrm{a}$ and Fig. $4.2 \mathrm{~b}$, respectively, and show the benefit of the decomposition technique. From Fig. 4.2a we can readily see that since the variation of the $\mathcal{S}\left(q_{\ell} \eta_{\ell}\right)$ term among the various $t \bar{t}$ samples is small, choosing any one of them in the calculation of Eq. 4.2 for the measurement introduces an uncertainty that is tiny compared to the dominant uncertainties. The small differences for $\left|q_{\ell} \eta_{\ell}\right|<0.2$ do not have much effect on the measurement for the reasons described in Appendix $\mathrm{E}$. On the other hand, as shown in Fig. $4.2 \mathrm{~b}$, the $\mathcal{A}\left(q_{\ell} \eta_{\ell}\right)$ term varies significantly from model to model and is thus a strong predictor of $A_{\mathrm{FB}}^{\ell}$. Using trial and error, it has been found that the $\mathcal{A}\left(q_{\ell} \eta_{\ell}\right)$ term is well described in the region $\left|q_{\ell} \eta_{\ell}\right|<2.0 \mathrm{using}$ the functional form of

$$
\mathcal{A}\left(q_{\ell} \eta_{\ell}\right)=a \cdot \tanh \left(\frac{1}{2} q_{\ell} \eta_{\ell}\right)
$$

where $a$ is a free parameter that is directly related to the final asymmetry. Best fits of the simulations to the $a \cdot$ tanh model from Eq. 4.3 are also shown in Fig. 4.2b. (A detailed discussion of why this functional form works is given in Appendix E.)

The previous description of the methodology is based on the generator level quantities. In another word, no detector response is considered yet. Next, we comment on how the detector effects will be included before doing the validation of the method at the generator level. We note that in the formulas below, the superscript "truth" indicates that the quantity is measured at the generator level before any detector response or event selection, and the superscript "reco" indicates that the quantity is measured after the detector response, object identification, and event selection. We omitted the superscript "truth" for the $\mathcal{N}\left(q_{\ell} \eta_{\ell}\right), \mathcal{S}\left(q_{\ell} \eta_{\ell}\right)$, and $\mathcal{A}\left(q_{\ell} \eta_{\ell}\right)$ terms previously in Eqs. 4.1, 4.2, and 4.3 for briefness.

Before starting discussing the detector effects on the measurement, we note that 
since the $\mathcal{S}\left(q_{\ell} \eta_{\ell}\right)$ term does not vary among different models, we will use the $\mathcal{S}\left(q_{\ell} \eta_{\ell}\right)$ term estimated from the POWHEG MC sample at the generator level, before any selection requirement or detector response. Thus, the $\mathcal{S}\left(q_{\ell} \eta_{\ell}\right)$ term we use corresponds to the truth level $\mathcal{S}\left(q_{\ell} \eta_{\ell}\right)$, or $\mathcal{S}^{\text {truth }}\left(q_{\ell} \eta_{\ell}\right)$. On the other hand, the $\mathcal{A}\left(q_{\ell} \eta_{\ell}\right)$ distribution is very sensitive to different $A_{\mathrm{FB}}^{\ell}$ values. We will measure the $\mathcal{A}\left(q_{\ell} \eta_{\ell}\right)$ distribution from data (corresponding to $\mathcal{A}^{\text {reco }}\left(q_{\ell} \eta_{\ell}\right)$ ), which is subject to the effects of the detector response, object identification, and event selection. Because of this, we need to correct for the detector effects on the $\mathcal{A}^{\text {reco }}\left(q_{\ell} \eta_{\ell}\right)$ back to $\mathcal{A}^{\text {truth }}\left(q_{\ell} \eta_{\ell}\right)$, so that we can calculate parton-level inclusive $A_{\mathrm{FB}}^{\ell}$ with Eq. 4.2. Next we will show the corrections only on the $\mathcal{A}^{\text {reco }}\left(q_{\ell} \eta_{\ell}\right)$ distribution.

As will be shown shortly, the definition of the $\mathcal{A}^{\text {reco }}\left(q_{\ell} \eta_{\ell}\right)$ distribution has the advantage that it greatly reduces the effect of the detector response on the measurement. We note that after choosing a reasonable binning (large compared to the detector resolution, which is $\sim 0.04$ ), bin-to-bin migration due to detector response is negligible, so the correction on the detector response is simplified to a bin-by-bin multiplicative correction as a function of $q_{\ell} \eta_{\ell}$. This correction is denoted as the "acceptance correction", which corrects for the fact that we can only measure a fraction of the events that are generated, due to the limited detector coverage and the inefficiency in the event selection. Here we use $C\left(q_{\ell} \eta_{\ell}\right)$ to represent the bin-by-bin acceptance correction, which is the ratio of the number of events in a certain $q_{\ell} \eta_{\ell}$ range at generator level to that after detector simulation, reconstruction, and event selection, as

$$
C\left(q_{\ell} \eta_{\ell}\right)=\frac{\mathcal{N}^{\text {truth }}\left(q_{\ell} \eta_{\ell}\right)}{\mathcal{N}^{\text {reco }}\left(q_{\ell} \eta_{\ell}\right)}
$$

where $\mathcal{N}\left(q_{\ell} \eta_{\ell}\right)$ is the number of events in the corresponding $q_{\ell} \eta_{\ell}$ range.

In the limit of small asymmetries, as proved mathematically in Ref. [91], we can 
use the $\mathcal{S}^{\text {truth }}\left(q_{\ell} \eta_{\ell}\right)$ directly from the generator-level calculations from MC samples, and only the $\mathcal{A}^{\text {reco }}\left(q_{\ell} \eta_{\ell}\right)$ term receives a correction. We find in addition that only the asymmetric part of the acceptance correction affects $A_{\mathrm{FB}}^{\ell}$ acting as an additive correction to $\mathcal{A}\left(q_{\ell} \eta_{\ell}\right)$ as in the formula below with $q_{\ell} \eta_{\ell}>0$ :

$$
\mathcal{A}^{\text {truth }}\left(q_{\ell} \eta_{\ell}\right)=\mathcal{A}^{\text {reco }}\left(q_{\ell} \eta_{\ell}\right)+\mathcal{A}^{C}\left(q_{\ell} \eta_{\ell}\right)
$$

where $\mathcal{A}^{\text {truth }}\left(q_{\ell} \eta_{\ell}\right)$ represents the asymmetric part of the $q_{\ell} \eta_{\ell}$ distribution before the detector response or any event selection (which is what is calculated in Eq. 4.1b), $\mathcal{A}^{\text {reco }}\left(q_{\ell} \eta_{\ell}\right)$ represents the asymmetric part of $q_{\ell} \eta_{\ell}$ distribution after detector simulation and reconstruction, calculated in the region of $q_{\ell} \eta_{\ell}>0$ as

$$
\mathcal{A}^{\text {reco }}\left(q_{\ell} \eta_{\ell}\right)=\frac{\mathcal{N}^{\text {reco }}\left(q_{\ell} \eta_{\ell}\right)-\mathcal{N}^{\text {reco }}\left(-q_{\ell} \eta_{\ell}\right)}{\mathcal{N}^{\text {reco }}\left(q_{\ell} \eta_{\ell}\right)+\mathcal{N}^{\text {reco }}\left(-q_{\ell} \eta_{\ell}\right)}
$$

and $\mathcal{A}^{C}\left(q_{\ell} \eta_{\ell}\right)$ is the asymmetric part of bin-by-bin acceptance correction, which is calculated again when $q_{\ell} \eta_{\ell}>0$ as

$$
\mathcal{A}^{C}\left(q_{\ell} \eta_{\ell}\right)=\frac{C\left(q_{\ell} \eta_{\ell}\right)-C\left(-q_{\ell} \eta_{\ell}\right)}{C\left(q_{\ell} \eta_{\ell}\right)+C\left(-q_{\ell} \eta_{\ell}\right)}
$$

where $\mathcal{A}^{C}$ is found to be small because the CDF detector is designed to be forwardbackward symmetric.

Eventually, we need the term $\mathcal{A}^{\text {truth }}\left(q_{\ell} \eta_{\ell}\right)$, which will be used in Eq. 4.2 to obtain the parton-level inclusive $A_{\mathrm{FB}}^{\ell}$. However, we can only measure $\mathcal{A}^{\text {reco }}\left(q_{\ell} \eta_{\ell}\right)$ in our data. The correction term $\mathcal{A}^{C}\left(q_{\ell} \eta_{\ell}\right)$ is derived from $t \bar{t} \mathrm{MC}$ samples (which will be shown to be consistent with zero) to bring us from $\mathcal{A}^{\text {reco }}\left(q_{\ell} \eta_{\ell}\right)$ to $\mathcal{A}^{\text {truth }}\left(q_{\ell} \eta_{\ell}\right)$.

Considering the features of the decomposition technique described above, the strategy of this method is 
1. Measure $\mathcal{A}^{\text {reco }}\left(q_{\ell} \eta_{\ell}\right)$ in the data,

2. Estimate the correction term $\mathcal{A}^{C}\left(q_{\ell} \eta_{\ell}\right)$ from $t \bar{t} \mathrm{MC}$ samples,

3. Use Eq. 4.5 to get $\mathcal{A}^{\text {truth }}\left(q_{\ell} \eta_{\ell}\right)$,

4. Fit the $\mathcal{A}^{\text {truth }}\left(q_{\ell} \eta_{\ell}\right)$ distribution with an $a \cdot \tanh$ function, which is our modeling of $\mathcal{A}^{\text {truth }}\left(q_{\ell} \eta_{\ell}\right)$

5. Obtain the $\mathcal{S}\left(q_{\ell} \eta_{\ell}\right)$ term from the best $t \bar{t}$ MC sample at parton level, which corresponds to $\mathcal{S}^{\text {truth }}\left(q_{\ell} \eta_{\ell}\right)$ to be used in Eq. 4.2 .

6. Use the $\mathcal{A}^{\text {truth }}\left(q_{\ell} \eta_{\ell}\right)$ modeled by the $a \cdot \tanh$ fit in (4) and the $\mathcal{S}^{\operatorname{truth}}\left(q_{\ell} \eta_{\ell}\right)$ in (5) to get the parton-level inclusive $A_{\mathrm{FB}}^{\ell}$ with Eq. 4.2 .

Note that since only the asymmetric part of the acceptance correction contributes to the asymmetric part of the $q_{\ell} \eta_{\ell}$ distribution, and we use the symmetric part from generator level $t \bar{t} \mathrm{MC}$, this methodology corrects for the detector response and the limited detector $\eta$ coverage at the same time. In the next section, we will provide the validation of the measurement methodology by using the samples to create pseudodatasets and show that by using the procedure we reproduce the generator level $A_{\mathrm{FB}}^{\ell}$

\subsection{Validation of the Measurement Methodology}

In this section we provide a detailed validation of the measurement methodology at the parton-level and after including the detector effects. In Sec. 4.2 .3 we show the validation of applying the same methodology to the $A_{\mathrm{FB}}^{\ell \ell}$ measurement.

\subsubsection{Validation at Generator Level}

The true value of $A_{\mathrm{FB}}^{\ell}$ for each $\mathrm{MC}$ sample is calculated using the full set of generated events, and counting the numbers of events with $q_{\ell} \eta_{\ell}>0$ and $q_{\ell} \eta_{\ell}<0$. 
This is readily compared with the result using our methodology to determine both the value of $a$ from the $\mathcal{A}\left(q_{\ell} \eta_{\ell}\right)$ distribution (where we only use the events with $\left|q_{\ell} \eta_{\ell}\right|<2.0$ to do a simple simulation of the detector and use the MINUIT package 93 ] to estimate the best values of $a$ and their uncertainties), and then turning the value of $a$ into our best estimate of $A_{\mathrm{FB}}^{\ell}$ using Eqs. 4.2 and 4.3 . The comparison between the predicted $A_{\mathrm{FB}}^{\ell}$ and the $A_{\mathrm{FB}}^{\ell}$ obtained with the measurement methodology is shown in Fig. 4.3 and Table 4.1. The differences are on the order of a fraction of a percent, which is tiny compared to the expected dominant uncertainties at the level of 0.05 .

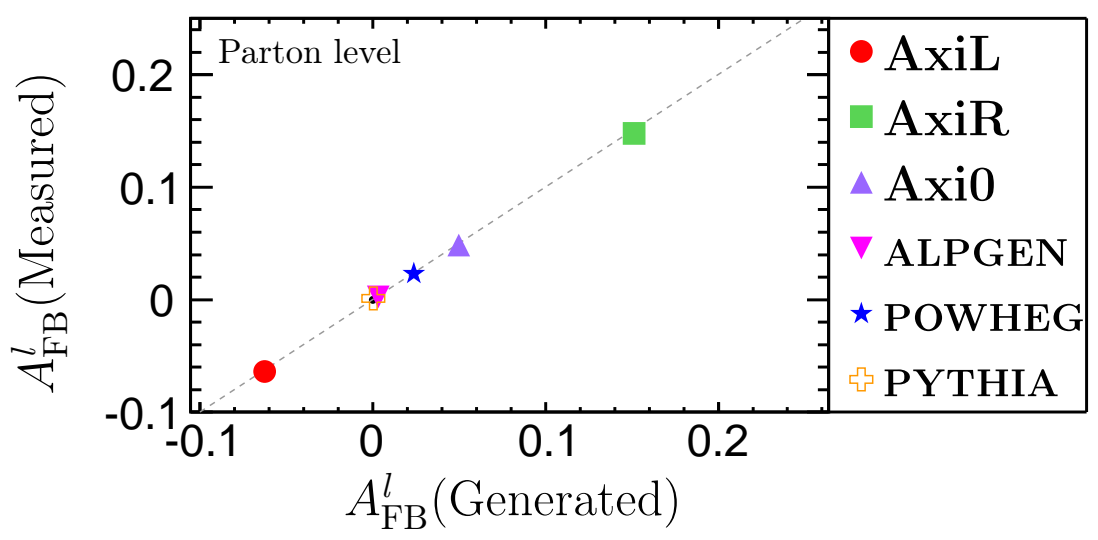

Figure 4.3: A comparison between the predicted $A_{\mathrm{FB}}^{\ell}$ from simulations and the $A_{\mathrm{FB}}^{\ell}$ as measured using the $a \cdot \tanh$ parametrization with parton level information from $\left|q_{\ell} \eta_{\ell}\right|<2.0$. The dashed line indicates the location of the equal values, while the points are superimposed at their measured locations. All the points lie along the line within uncertainties.

\subsubsection{Validation with Detector Effects}

In this subsection we describe the measurement of the $A_{\mathrm{FB}}^{\ell}$ taking the detector and object reconstruction effects into account. Since we have imperfect detector 
Table 4.1: Comparison between the generated $A_{\mathrm{FB}}^{\ell}$ and the results of the measurement methodology at the generator level from the various $\mathrm{MC}$ models. No noticeable bias is observed.

\begin{tabular}{|c|c|c|c|c|}
\hline Model & $\begin{array}{c}A_{\mathrm{FB}}^{\ell} \\
(\text { Generated })\end{array}$ & $\begin{array}{c}A_{\mathrm{FB}}^{\ell} \\
(\text { Gen. level measured })\end{array}$ & Difference & $\begin{array}{c}\text { Fit parameter } \\
(a)\end{array}$ \\
\hline AxiL & $-0.0626 \pm 0.0024$ & $-0.0640 \pm 0.0021$ & 0.0014 & $-0.182 \pm 0.006$ \\
\hline AxiR & $0.1513 \pm 0.0024$ & $0.1477 \pm 0.0020$ & 0.0036 & $0.419 \pm 0.005$ \\
\hline Axi0 & $0.0497 \pm 0.0024$ & $0.0484 \pm 0.0021$ & 0.0013 & $0.137 \pm 0.006$ \\
\hline Alpgen & $0.0032 \pm 0.0013$ & $0.0026 \pm 0.0012$ & 0.0006 & $0.007 \pm 0.003$ \\
\hline Pythia & $0.0001 \pm 0.0008$ & $0.0011 \pm 0.0009$ & -0.0010 & $0.003 \pm 0.002$ \\
\hline Powheg & $0.0236 \pm 0.0007$ & $0.0229 \pm 0.0008$ & 0.0007 & $0.065 \pm 0.002$ \\
\hline
\end{tabular}

resolution and incomplete detector coverage, we use simulated data from all six $t \bar{t}$ MC samples to see if there are any biases in our methodology or if further corrections are needed.

Since this analysis is statistics limited, we choose our binning of $q_{\ell} \eta_{\ell}$ carefully. There are about 1100 leptons (signal and backgrounds together) expected in data. Since the expectation is that the $A_{\mathrm{FB}}$ approaches zero when $q_{\ell} \eta_{\ell}$ goes to zero due to the continuity of the $q_{\ell} \eta_{\ell}$ spectrum, we intentionally choose the first bin to be narrower. In this methodology we are doing a fit on the asymmetric part of the $q_{\ell} \eta_{\ell}$ distribution, thus ideally we want as many bins as possible across the whole $q_{\ell} \eta_{\ell}$ range, but for each bin to have reasonable number of events. To balance the number of bins and the statistics in each bin, we chose to have 6 bins across the whole $\eta$ range. The first bin is chosen to have half as many expected entries, and the rest of the five bins are chosen so that they have roughly the same expected number of entries. The choice of 6 bins is not thoroughly optimized, but we tried the same binning idea with 5 or 7 bins, and they give similar result. Table 4.2 shows the $q_{\ell} \eta_{\ell}$ binning choice we use for measuring $A_{\mathrm{FB}}^{\ell}$.

We next consider the issue of whether the data needs to be corrected for either 
Table 4.2: The binning choice of the $q_{\ell} \eta_{\ell}$ distribution used in the measurement of $A_{\mathrm{FB}}^{\ell}$, along with the expected number of events from $t \bar{t}+$ backgrounds, where $t \bar{t}$ is simulated using POWHEG.

\begin{tabular}{cc}
\hline Bin range & $t \bar{t}+$ backgrounds \\
\hline$(-2,-0.81)$ & $109.1 \pm 7.9$ \\
{$[-0.81,-0.61)$} & $98.4 \pm 7.2$ \\
{$[-0.61,-0.42)$} & $98.6 \pm 7.2$ \\
{$[-0.42,-0.26)$} & $101.7 \pm 7.4$ \\
{$[-0.26,-0.10)$} & $98.8 \pm 7.2$ \\
{$[-0.10,0)$} & $57.8 \pm 4.3$ \\
{$[0,0.10)$} & $54.9 \pm 4.1$ \\
{$[0.10,0.26)$} & $101.7 \pm 7.4$ \\
{$[0.26,0.42)$} & $100.6 \pm 7.2$ \\
{$[0.42,0.61)$} & $100.1 \pm 7.2$ \\
{$[0.61,0,81)$} & $100.4 \pm 7.3$ \\
{$[0.81,2)$} & $113.6 \pm 8.2$ \\
\hline
\end{tabular}

the lepton $\eta$ resolution or the detector acceptance as a function of $\eta$. We note at the beginning, that our studies show that the answer is no, but give more details next to show our evidence for this conclusion. As proved in Ref. 91] and shown in Eq. 4.5. only the asymmetric part of the acceptance correction affects the $A_{\mathrm{FB}}$ measurement. Due to the small branching ratio and acceptance in the TopDilepton channel, the errors on the asymmetric part of acceptance correction are non-negligible even with our biggest signal sample; the POWHEG $t \bar{t}$ sample with $10 \mathrm{M}$ events only produces about $70 \mathrm{k}$ candidate events. Fig. 4.4 shows the asymmetric part of the acceptance correction (defined in Eq. 4.5) estimated with the POWHEG $t \bar{t}$ MC sample. Though the middle 4 bins appear negative, the corrections in all bins are consistent with zero within statistics. In principle, small corrections might be needed, but we don't have the ability to determine the asymmetric part of acceptance correction with precision. In practice, we will proceed using zero correction, show that the impact 
of this assumption is small, and then assign a systematic uncertainty due to this approach.

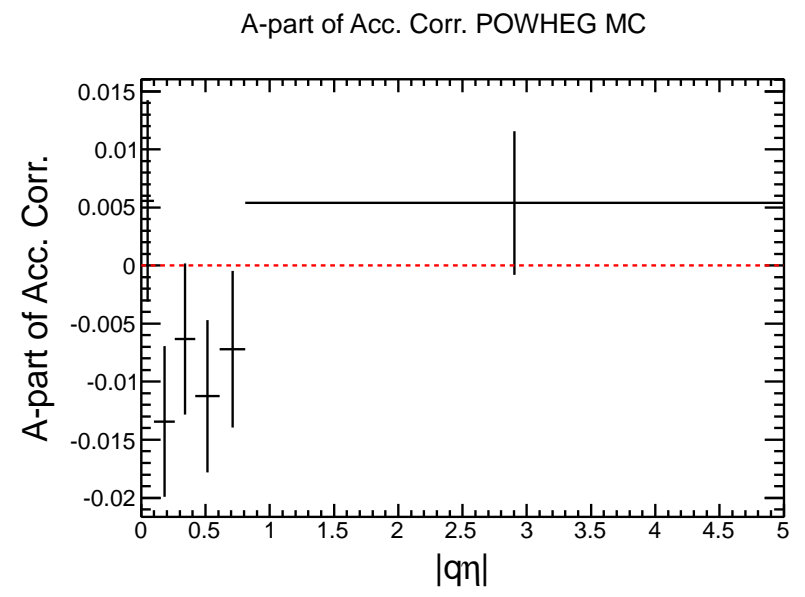

Figure 4.4: The distribution of the asymmetric part of the bin-by-bin acceptance correction, $\mathcal{A}^{C}\left(q_{\ell} \eta_{\ell}\right)$, as estimated with the POWHEG $t \bar{t}$ MC.

We will next show that with zero acceptance correction, we can get back to the truth level $A_{\mathrm{FB}}^{\ell}$ with various $t \bar{t} \mathrm{MC}$ samples within statistics, and that the uncertainty due to this approach is small compared to the dominant systematic uncertainty. With the assumption of zero acceptance correction, we perform the fit with Eq. 4.3 on the $q_{\ell} \eta_{\ell}$ distribution of the sample of events that pass all the selection requirements in Table 2.1 (after full simulation) from each $t \bar{t}$ MC sample. Fig. 4.5 shows the comparison between the fit at generator level and the fit at reconstructed level. The variation in the fit results is small compared to the dominant systematic uncertainty. Fig. 4.6 shows the reconstructed level asymmetric part of $q_{\ell} \eta_{\ell}$ from all samples together with the best $a \cdot \tanh$ fits. Fig. 4.7 and Table 4.3 show the result of the extrapolated $A_{\mathrm{FB}}^{\ell}$ with each of the $t \bar{t}$ models. We note that with the $a \cdot \tanh$ fit 
Table 4.3: Comparison between the generated $A_{\mathrm{FB}}^{\ell}$ and the results of the measurement methodology from the various MC models, using the sample of reconstructed events that pass all the event selection requirements in Table 2.1. No noticeable bias is observed.

\begin{tabular}{|c|c|c|c|c|}
\hline Model & $\begin{array}{c}A_{\mathrm{FB}}^{\ell} \\
(\text { Generated })\end{array}$ & $\begin{array}{c}A_{\mathrm{FB}}^{\ell} \\
(\text { Reco. level measured })\end{array}$ & Difference & $\begin{array}{c}\text { Fit parameter } \\
(a)\end{array}$ \\
\hline AxiL & $-0.0626 \pm 0.002$ & $-0.063 \pm 0.011$ & 0.0001 & $-0.178 \pm 0.032$ \\
\hline AxiR & $0.151 \pm 0.002$ & $0.147 \pm 0.011$ & 0.004 & $0.417 \pm 0.031$ \\
\hline Axi0 & $0.050 \pm 0.002$ & $0.065 \pm 0.011$ & -0.015 & $0.183 \pm 0.031$ \\
\hline ALPGEN & $0.003 \pm 0.001$ & $-0.004 \pm 0.006$ & 0.008 & $-0.012 \pm 0.017$ \\
\hline PYTHIA & $0.0001 \pm 0.0008$ & $-0.005 \pm 0.004$ & 0.005 & $-0.014 \pm 0.011$ \\
\hline POWHEG & $0.024 \pm 0.0007$ & $0.029 \pm 0.003$ & -0.006 & $0.084 \pm 0.009$ \\
\hline
\end{tabular}

on the reconstructed level asymmetric part of $q_{\ell} \eta_{\ell}$ distribution, $\mathcal{A}^{\text {reco }}\left(q_{\ell} \eta_{\ell}\right)$, together with symmetric part from POWHEG $t \bar{t} \mathrm{MC}, \mathcal{S}^{\operatorname{truth}}\left(q_{\ell} \eta_{\ell}\right)$, we get back to the truth level $A_{\mathrm{FB}}^{\ell}$ values with no noticeable bias. The differences are small compared to expected statistical uncertainty around 0.05 .

For concreteness, and to estimate our uncertainties conservatively, we quote the difference from POWHEG sample (0.006) as the systematic uncertainty for the "Asymmetric Modelling" to cover potential biases caused by the $a \cdot \tanh$ fit and the zero acceptance correction assumption. 


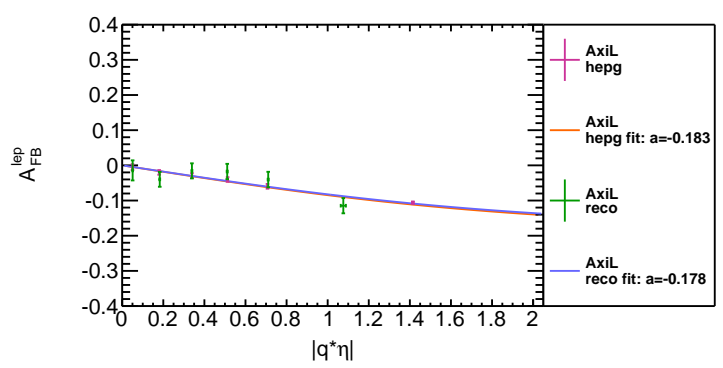

(a) AxiL

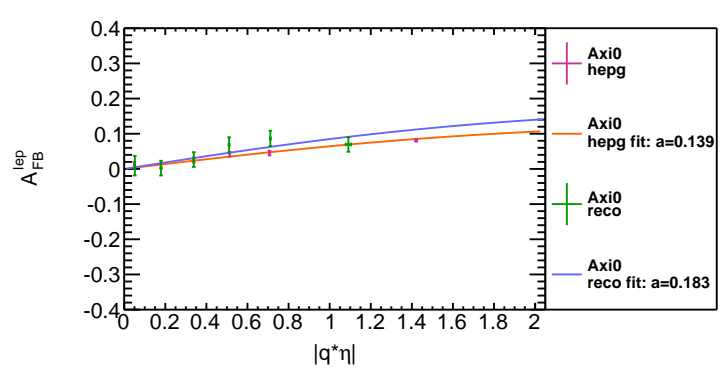

(c) Axi0

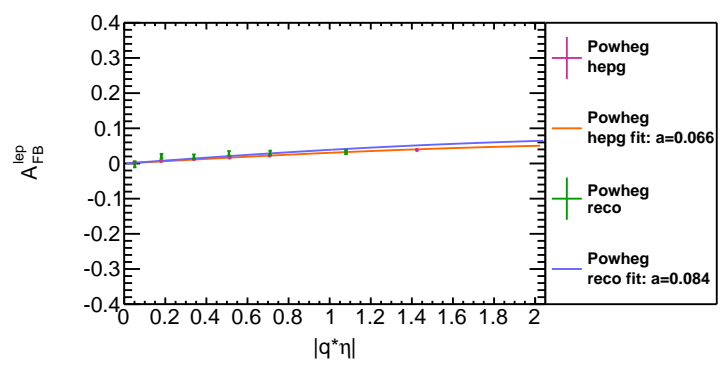

(e) Powheg

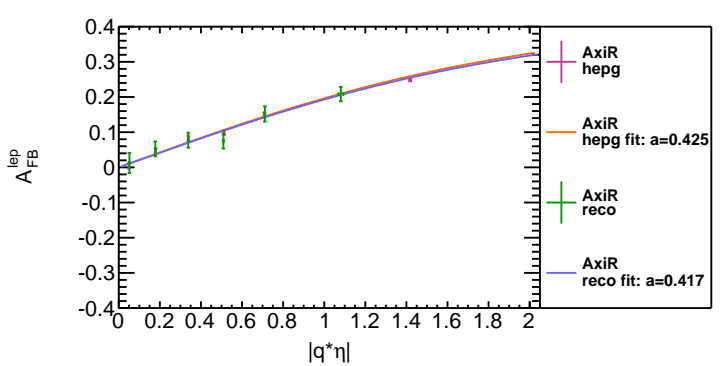

(b) AxiR

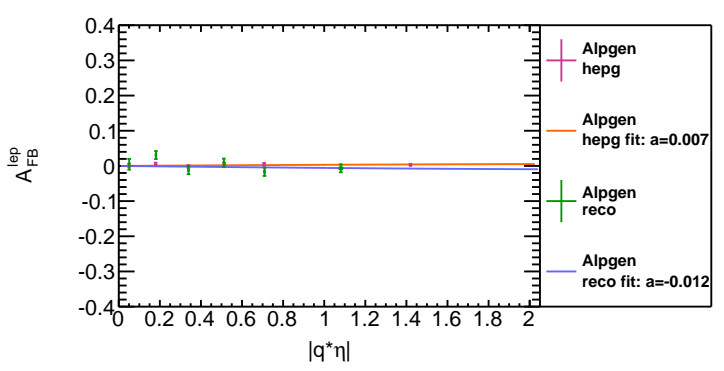

(d) Alpgen

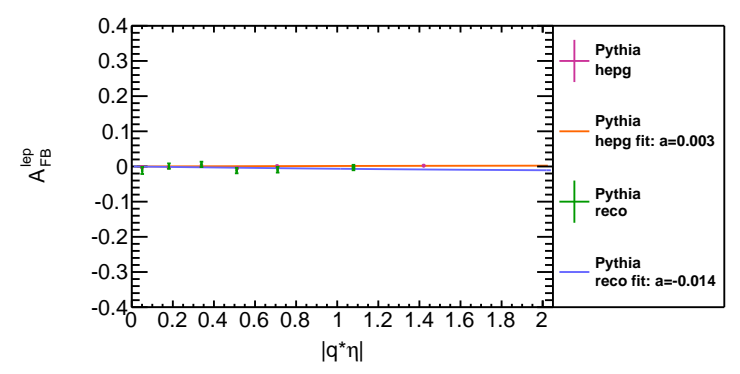

(f) Pythia

Figure 4.5: A comparison of the binned result, along with the $a \cdot \tanh$ fit, on the asymmetric part of the $q_{\ell} \eta_{\ell}$ distribution as estimated from the various MC samples. We plot both the generator level (hepg) and reconstructed level (reco) on top of each other. The fit parameters at generator level are slightly different from the number shown in Table 4.1 due to different binning. In all cases, the variation in the fit results is small compared to the dominant systematic uncertainty. 


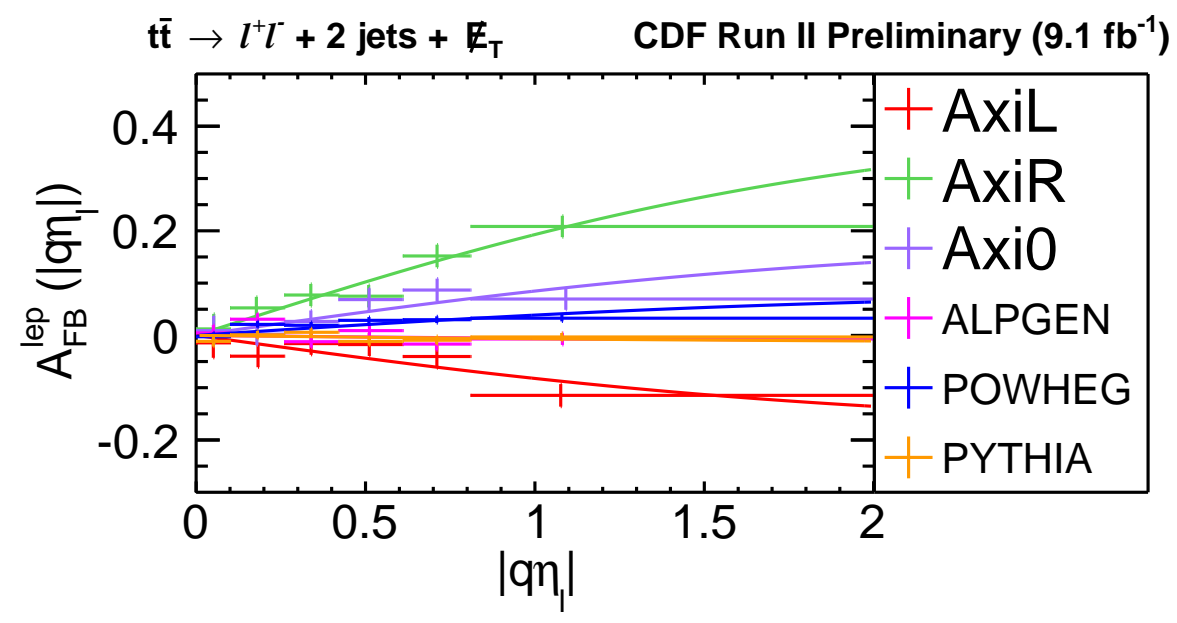

Figure 4.6: The asymmetric part of the $q_{\ell} \eta_{\ell}$ distribution for the six physics models at reconstructed level, with their corresponding $a \cdot \tanh$ fits.

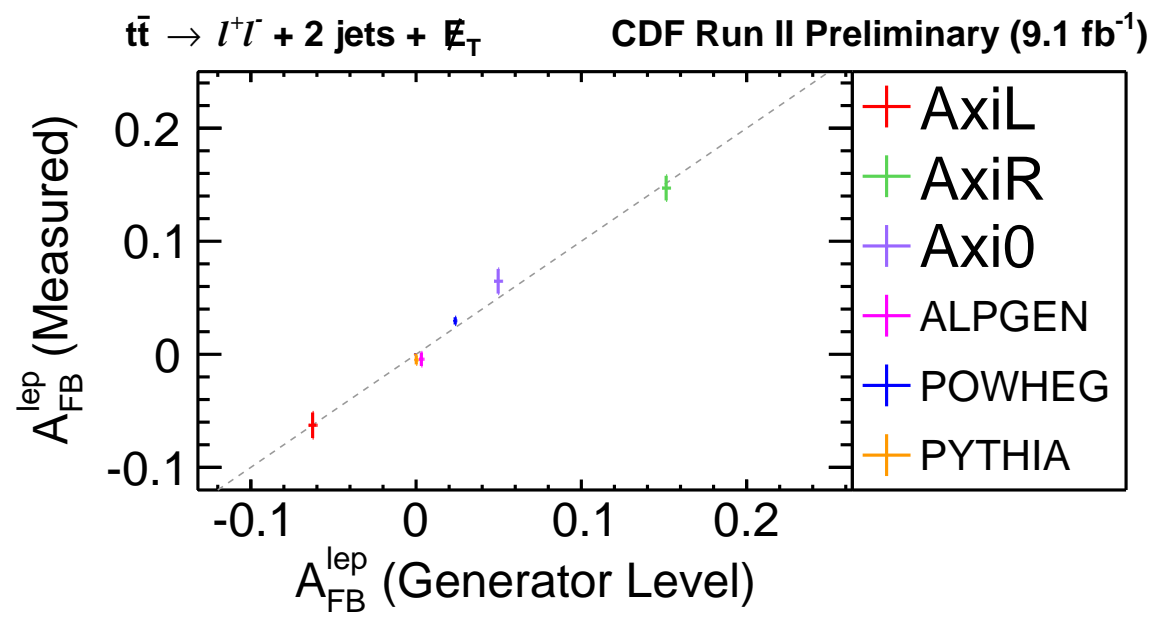

Figure 4.7: Truth level $A_{\mathrm{FB}}^{\ell}$ vs. the measured value of $A_{\mathrm{FB}}^{\ell}$. To measure $A_{\mathrm{FB}}^{\ell}$ we use the $a \cdot \tanh$ fit on the asymmetric part of $q_{\ell} \eta_{\ell}$ distribution at reconstructed level with symmetric part from POWHEG $t \bar{t}$ MC. 


\subsubsection{Validation for $A_{F B}^{\ell \ell}$ Measurement}

With our validation of the methodology on the $A_{\mathrm{FB}}^{\ell}$ measurement now complete, we show that the same approach also works for measuring $A_{\mathrm{FB}}^{\ell \ell}$.

As shown in Table. 4.4, we choose the binning of $\Delta \eta$ distribution in the same manner as described in Sec. 4.2.2 although the number of events per bin is reduced by $50 \%$. . In the same way, we start by looking at the $\Delta \eta$ distribution at generator level from various $t \bar{t} \mathrm{MC}$ samples, and its decomposition into the symmetric and asymmetric parts, together with hyperbolic tangent fit on the asymmetric part. The $a \cdot$ tanh fit is then applied to the asymmetric part of the $\Delta \eta$ distribution after reconstruction, and the asymmetric part of acceptance correction is tested with the POWHEG $t \bar{t}$ MC. The results are shown in Figs. 4.8 and 4.9. Tables 4.5, and 4.6 and Figs. 4.10 and 4.11 show the comparison between the truth level $A_{\mathrm{FB}}^{\ell \ell}$ and the $A_{\mathrm{FB}}^{\ell \ell}$ obtained with $a \cdot \tanh$ fit at generator and reconstructed level.

The $A_{\mathrm{FB}}^{\ell \ell}$ values obtained with the $a \cdot \tanh$ fit after reconstruction from various $t \bar{t}$ samples agree with the truth level $A_{\mathrm{FB}}^{\ell \ell}$ from each of the six test samples within statistics. The difference obtained from the POWHEG $t \bar{t}$ sample is quoted as a systematic uncertainty for the extrapolation methodology for $A_{\mathrm{FB}}^{\ell \ell}$ conservatively. 


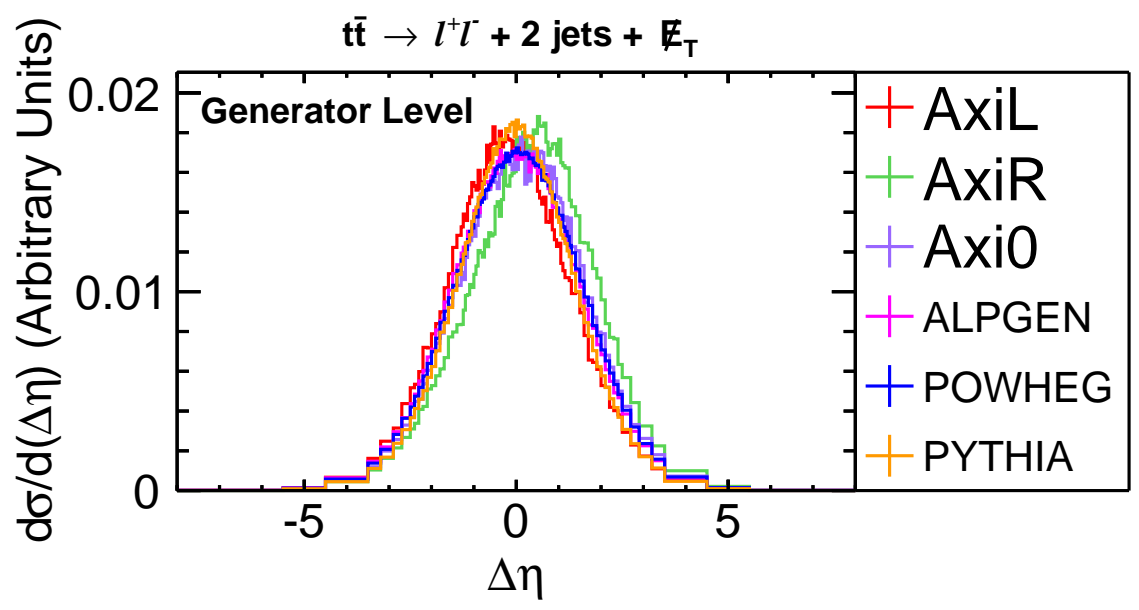

(a)

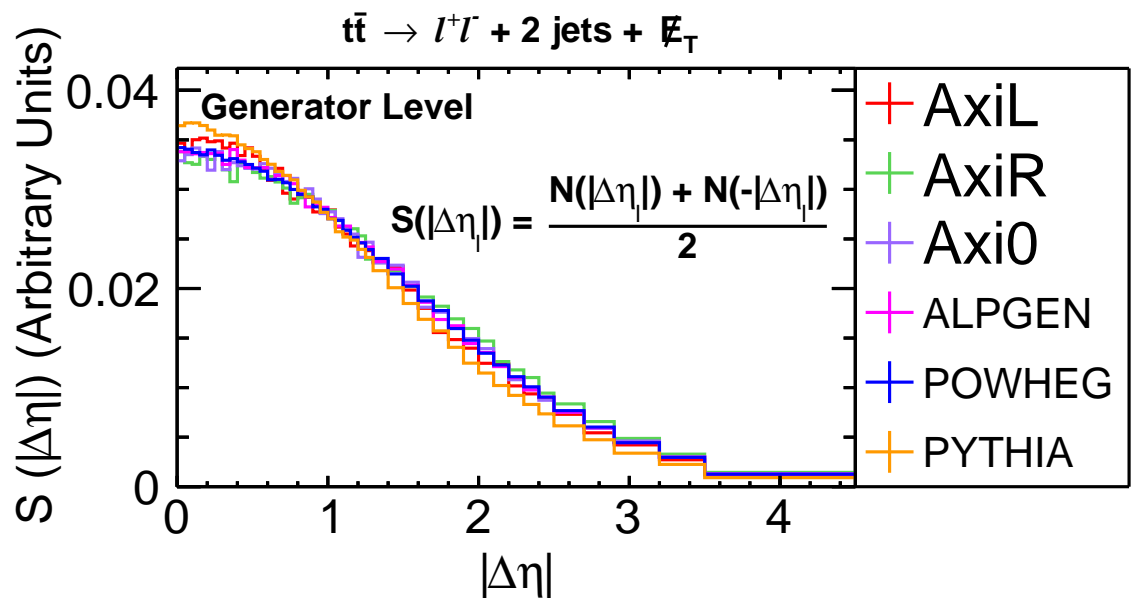

(b)

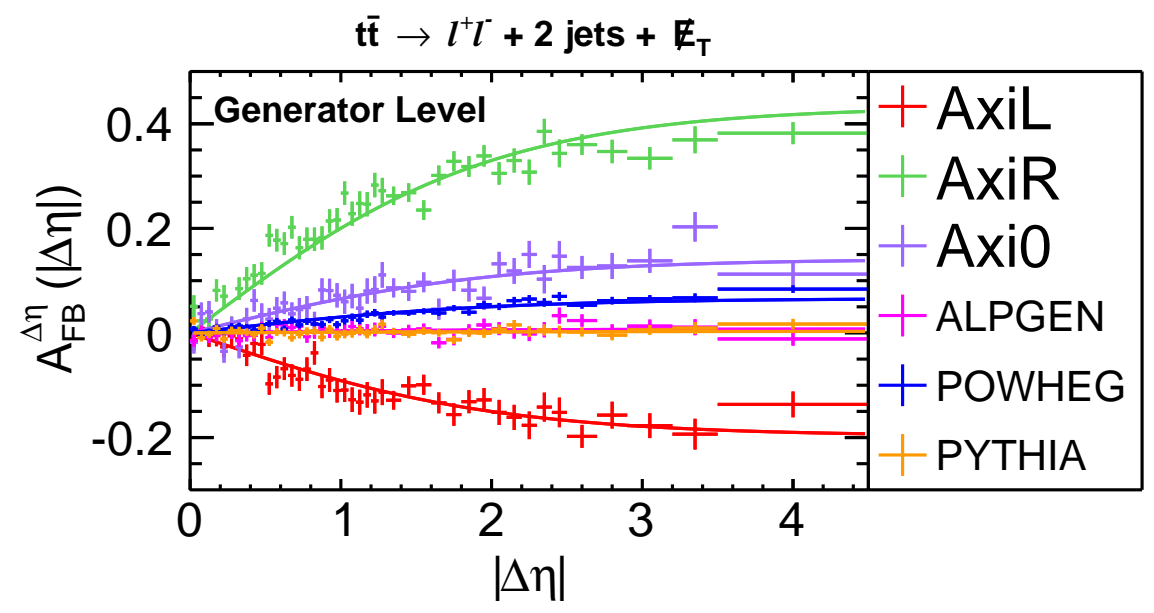

(c)

Figure 4.8: The same results as in Fig. $4.1,4.2 \mathrm{a}, 4.2 \mathrm{~b}$, but using $\Delta \eta$ instead of $q_{\ell} \eta_{\ell}$. 


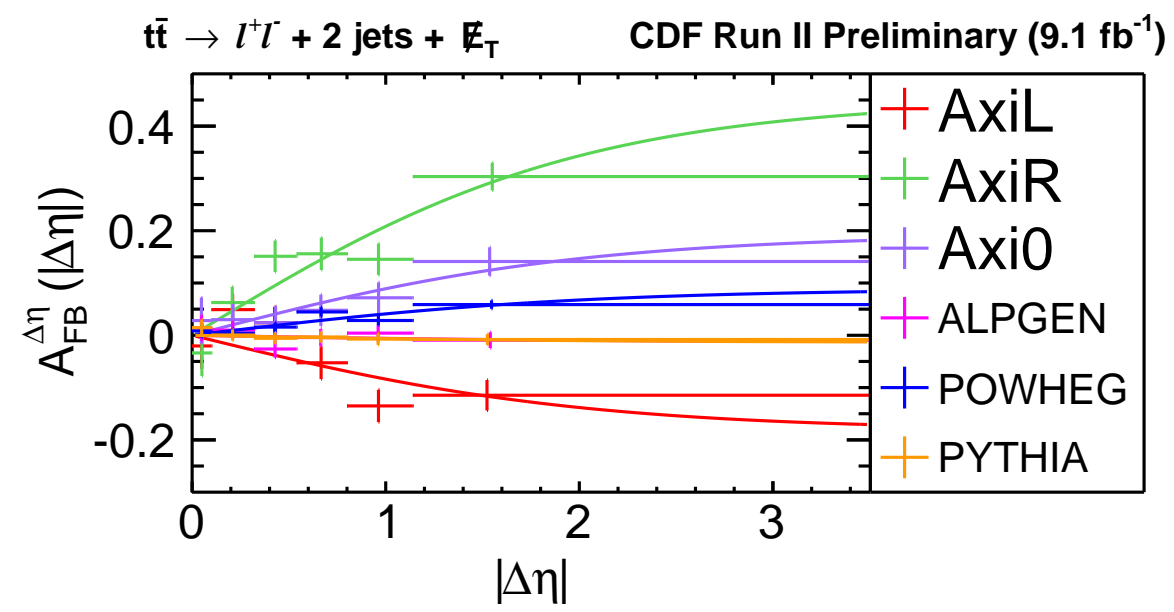

(a)

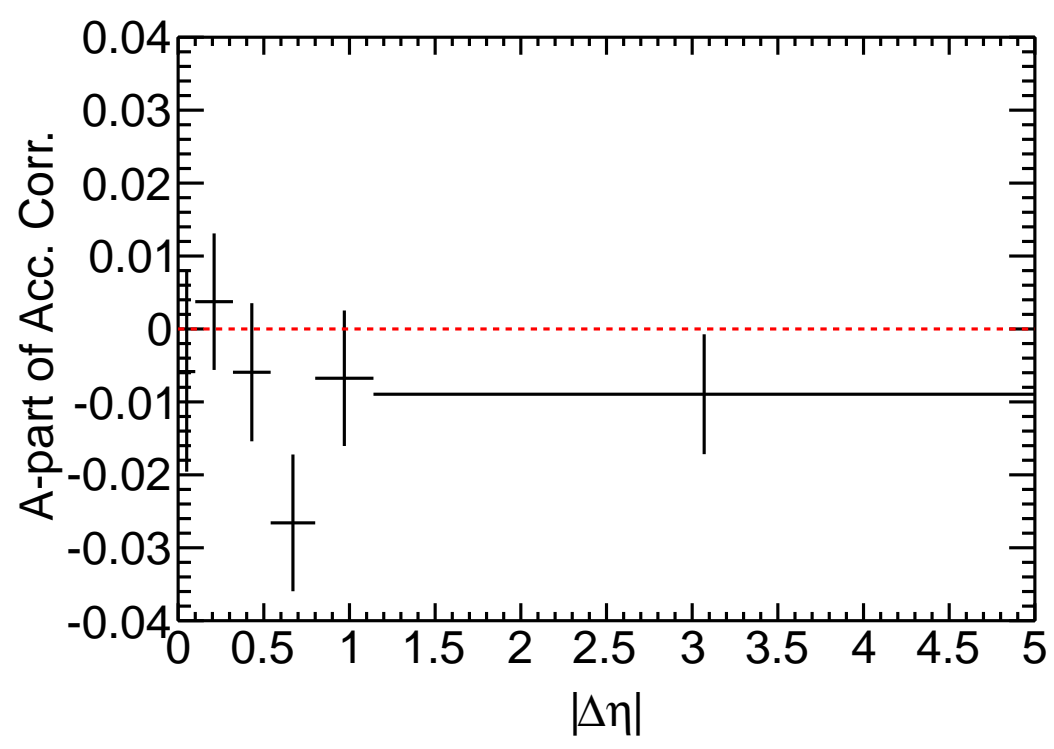

(b)

Figure 4.9: The same results as in 4.4 and Fig. 4.6, but using $\Delta \eta$ instead of $q_{\ell} \eta_{\ell}$. 
Table 4.4: The binning choice of the $\Delta \eta$ distribution used in measurement of $A_{\mathrm{FB}}^{\ell \ell}$, along with the expected number of events from $t \bar{t}+$ backgrounds, where $t \bar{t}$ is simulated using POWHEG.

\begin{tabular}{cc}
\hline Bin range & $t \bar{t}+$ backgrounds \\
\hline$(-3.5,-1.14)$ & $53.9 \pm 3.9$ \\
{$[-1.14,-0.80)$} & $50.3 \pm 3.8$ \\
{$[-0.80,-0.54)$} & $50.1 \pm 3.8$ \\
{$[-0.54,-0.32)$} & $52.1 \pm 4.0$ \\
{$[-0.32,-0.1)$} & $52.6 \pm 3.9$ \\
{$[-0.1,0)$} & $24.6 \pm 1.9$ \\
{$[0,0.1)$} & $23.6 \pm 1.8$ \\
{$[0.1,0.32)$} & $49.7 \pm 3.7$ \\
{$[0.32,0.54)$} & $51.3 \pm 3.8$ \\
{$[0.54,0.8)$} & $50.0 \pm 3.6$ \\
{$[0.8,1.14)$} & $50.6 \pm 3.8$ \\
{$[1.14,3.5)$} & $59.1 \pm 4.3$ \\
\hline
\end{tabular}




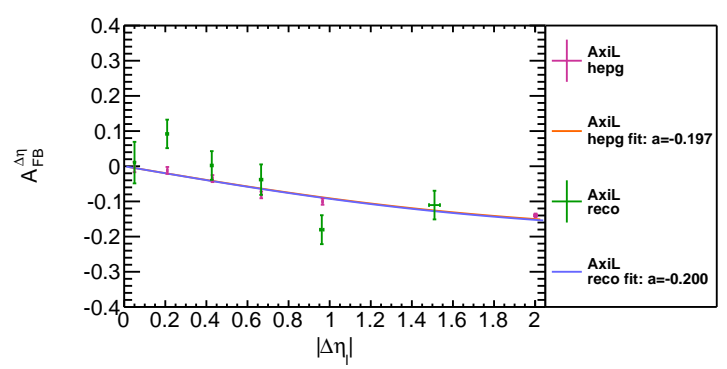

(a) AxiL

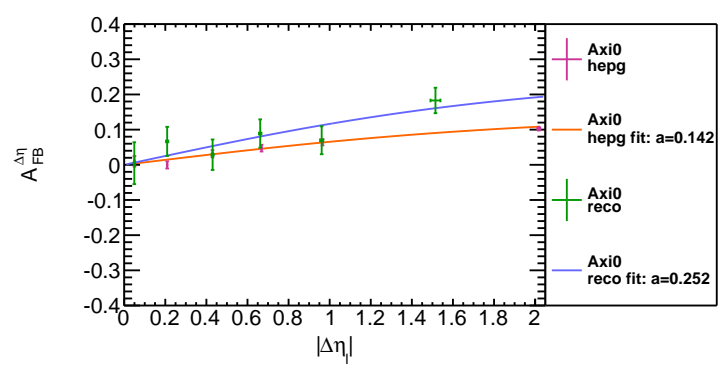

(c) Axi0

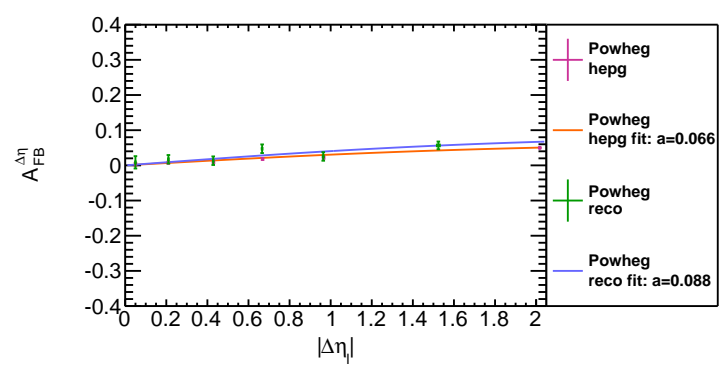

(e) Powheg

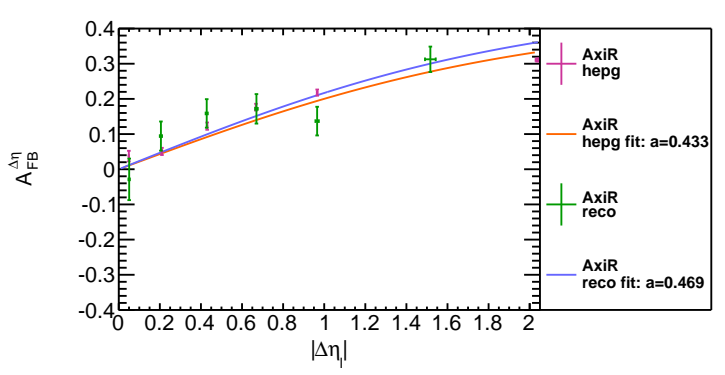

(b) AxiR

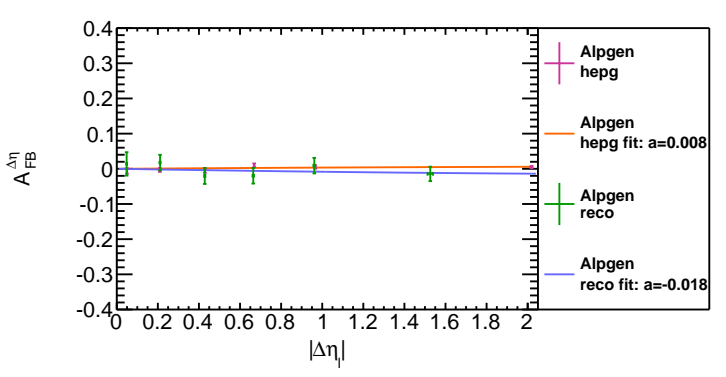

(d) Alpgen

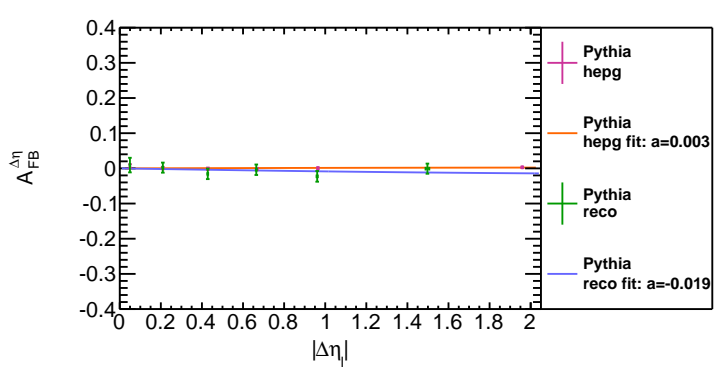

(f) Pythia

Figure 4.10: The same as Fig. 4.5, but using $\Delta \eta$ instead of $q_{\ell} \eta_{\ell}$. 


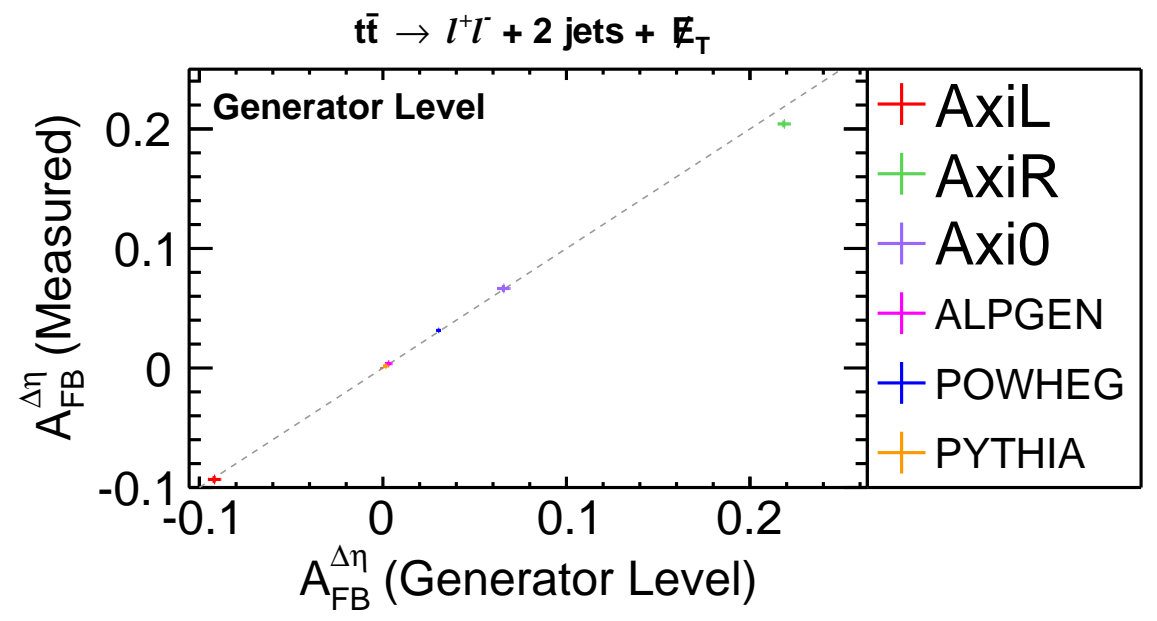

(a)

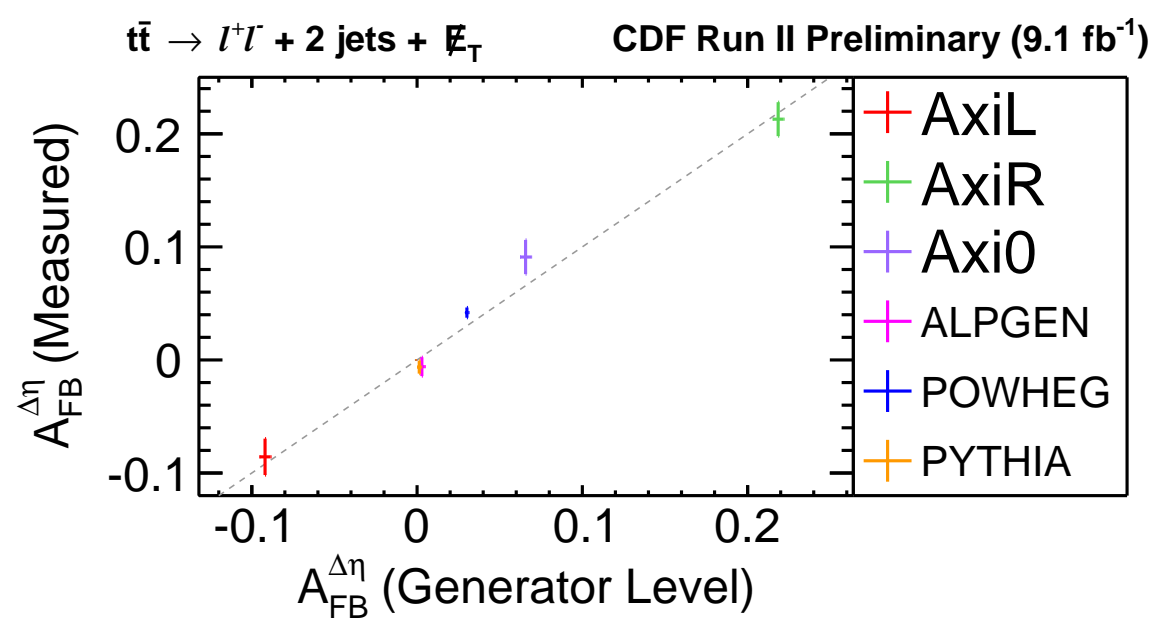

(b)

Figure 4.11: The same results as in Fig. 4.3 and 4.7, but with $A_{\mathrm{FB}}^{\ell \ell}$ (labelled as $\mathrm{A}_{\mathrm{FB}}^{\Delta \eta}$ in the figures). 
Table 4.5: The same as Table 4.1, but with $\Delta \eta$ and $A_{\mathrm{FB}}^{\ell \ell}$ instead of $q_{\ell} \eta_{\ell}$ and $A_{\mathrm{FB}}^{\ell}$.

\begin{tabular}{|c|c|c|c|c|}
\hline Model & $\begin{array}{c}A_{\mathrm{FB}}^{\ell \ell} \\
(\text { Generated })\end{array}$ & $\begin{array}{c}A_{\mathrm{FB}}^{\ell \ell} \\
(\text { Gen. level measured })\end{array}$ & Difference & $\begin{array}{c}\text { Fit parameter } \\
(a)\end{array}$ \\
\hline AxiL & $-0.0920 \pm 0.0034$ & $-0.0933 \pm 0.0030$ & 0.0013 & $-0.197 \pm 0.006$ \\
\hline AxiR & $0.2185 \pm 0.0033$ & $0.2042 \pm 0.0028$ & 0.0143 & $0.432 \pm 0.006$ \\
\hline Axi0 & $0.0656 \pm 0.0034$ & $0.0665 \pm 0.0029$ & -0.0009 & $0.141 \pm 0.006$ \\
\hline ALPGEN & $0.0029 \pm 0.0018$ & $0.0036 \pm 0.0016$ & -0.0007 & $0.008 \pm 0.003$ \\
\hline PYTHIA & $0.0013 \pm 0.0012$ & $0.0015 \pm 0.0012$ & -0.0002 & $0.003 \pm 0.002$ \\
\hline POWHEG & $0.0301 \pm 0.0010$ & $0.0314 \pm 0.0010$ & -0.0013 & $0.066 \pm 0.002$ \\
\hline
\end{tabular}

Table 4.6: The same as Table 4.3 , but with $\Delta \eta$ and $A_{\mathrm{FB}}^{\ell \ell}$ instead of $q_{\ell} \eta_{\ell}$ and $A_{\mathrm{FB}}^{\ell}$.

\begin{tabular}{|c|c|c|c|c|}
\hline Model & $\begin{array}{c}A_{\mathrm{FB}}^{\ell \ell} \\
\text { Generated) }\end{array}$ & $\begin{array}{c}A_{\mathrm{FB}}^{\ell \ell} \\
\text { (Reco. level measured) }\end{array}$ & Difference & $\begin{array}{c}\text { Fit parameter } \\
(a)\end{array}$ \\
\hline AxiL & $-0.092 \pm 0.003$ & $-0.086 \pm 0.016$ & -0.006 & $-0.182 \pm 0.034$ \\
\hline AxiR & $0.218 \pm 0.003$ & $0.215 \pm 0.015$ & 0.003 & $0.454 \pm 0.032$ \\
\hline Axi0 & $0.066 \pm 0.002$ & $0.092 \pm 0.015$ & -0.026 & $0.194 \pm 0.032$ \\
\hline ALPGEN & $0.003 \pm 0.002$ & $-0.006 \pm 0.008$ & 0.009 & $-0.013 \pm 0.018$ \\
\hline PYTHIA & $0.001 \pm 0.001$ & $-0.006 \pm 0.006$ & 0.008 & $-0.013 \pm 0.012$ \\
\hline POWHEG & $0.030 \pm 0.001$ & $0.042 \pm 0.004$ & -0.012 & $0.089 \pm 0.010$ \\
\hline
\end{tabular}




\subsection{Measurement of the Leptonic $A_{\mathrm{FB}}$ from Data}

With the methodology described in the last section well validated, we can take the data, subtract off the backgrounds and extrapolate to get the final measured values of the $A_{\mathrm{FB}}$. We first report the measurement of $A_{\mathrm{FB}}^{\ell}$ with the estimation of its systematic uncertainties, then we show briefly the measurement of $A_{\mathrm{FB}}^{\ell \ell}$ with the same approach, together with its systematic uncertainties.

\subsubsection{Measurement of the $A_{F B}^{\ell}$ and Uncertainty Estimation}

We start by calculating the $A_{\mathrm{FB}}^{\ell}$ with a direct counting method from data only and from data after background subtraction without correcting for the detector effects (uncorrected $A_{\mathrm{FB}}^{\ell}$ ) in Sec. 4.3.1.1, then apply our measurement methodology on the data after background subtraction in Sec. 4.3.1.2 for the parton-level result. The estimation of the systematic uncertainties is described in Sec. 4.3.1.3.

\subsubsection{Measuring raw $A_{F B}^{\ell}$ with a direct counting}

The $q_{\ell} \eta_{\ell}$ distribution from data is shown in Fig. 3.2e, and the distribution after background subtraction is shown in Fig. 3.6e. Table 4.7 lists measured uncorrected $A_{\mathrm{FB}}^{\ell}$, which is done with a straight counting of the numbers of the forward and the backward leptons with no correction for the detector responses, together with the uncorrected $A_{\mathrm{FB}}^{\ell}$ from the POWHEG $t \bar{t}$ MC. While this is interesting comparison, the parton-level inclusive value of the $A_{\mathrm{FB}}^{\ell}$ considers the $q_{\ell} \eta_{\ell}$ distribution and the full measurement methodology described in the previous subsection.

\subsubsection{Parton-level $A_{F B}^{\ell}$}

Table 4.8 gives the number of leptons in each $q_{\ell} \eta_{\ell}$ range both before and after background subtraction, and compares it to the expectations from the POWHEG MC samples. The decomposition of the background-subtracted data into the symmetric 
Table 4.7: The uncorrected $A_{\mathrm{FB}}^{\ell}$, with a straight counting of the numbers of the forward and the backward leptons, from the data only and the data after background subtraction, together with the expectation from the POWHEG $t \bar{t} \mathrm{MC}$, which is with NLO SM with QCD corrections but without electroweak corrections.

\begin{tabular}{cc}
\hline Source & $\begin{array}{c}\text { Uncorrected } \\
A_{\mathrm{FB}}^{\ell}\end{array}$ \\
\hline \hline PowHEG $t \bar{t}$ & $0.024 \pm 0.003$ \\
\hline Data & $0.02 \pm 0.03$ \\
\hline \hline
\end{tabular}

and asymmetric part is performed according to Eq. 4.1, and Fig. 4.12 shows the symmetric part of $q_{\ell} \eta_{\ell}$ distribution from data after background subtraction, along with the expectations from POWHEG. The agreement is good. Fig. 4.13 shows the $a \cdot$ tanh fit on the asymmetric part of data after background subtraction. We find $a=0.21 \pm 0.15$ (stat.). The $A_{\mathrm{FB}}^{\ell}$ retrieved from this fit is

$$
A_{\mathrm{FB}}^{\ell}=0.072 \pm 0.052(\text { stat. })
$$

where the uncertainty is statistical only, derived from the statistical uncertainty on the fit parameter $a$.

\subsubsection{Systematic uncertainties for $A_{F B}^{\ell}$}

The systematic uncertainties are estimated using techniques that are similar to those used in the lepton+jets measurement [43]. As will be seen, the dominant uncertainty on the measurement is the statistical uncertainty, while the dominant systematic uncertainty is from the estimation of the background. The results are summarized in Table 4.9.

To estimate the uncertainty on $A_{\mathrm{FB}}^{\ell}$ from both the uncertainty in the normalization of the backgrounds as well as from the uncertainty in the shape of the background 
Table 4.8: A comparison of the number of leptons in each $q_{\ell} \eta_{\ell}$ range, bin-by-bin, before and after the background subtraction. Note that the $t \bar{t}$ contributions are estimated using the POWHEG MC.

(a) $t \bar{t}+$ backgrounds and data

\begin{tabular}{ccc}
\hline Bin Range & $t \bar{t}+$ backgrounds & Data \\
\hline$(-2,-0.81)$ & $109.1 \pm 7.9$ & 101 \\
{$[-0.81,-0.61)$} & $98.4 \pm 7.2$ & 95 \\
{$[-0.61,-0.42)$} & $98.6 \pm 7.2$ & 98 \\
{$[-0.42,-0.26)$} & $101.7 \pm 7.4$ & 102 \\
{$[-0.26,-0.10)$} & $98.8 \pm 7.2$ & 110 \\
{$[-0.10,0)$} & $57.8 \pm 4.3$ & 54 \\
{$[0,0.10)$} & $54.9 \pm 4.1$ & 53 \\
{$[0.10,0.26)$} & $101.7 \pm 7.4$ & 91 \\
{$[0.26,0.42)$} & $100.6 \pm 7.2$ & 108 \\
{$[0.42,0.61)$} & $100.1 \pm 7.2$ & 115 \\
{$[0.61,0,81)$} & $100.4 \pm 7.3$ & 93 \\
{$[0.81,2)$} & $113.6 \pm 8.2$ & 118 \\
\hline
\end{tabular}

(b) $t \bar{t}$ and data after background subtraction

\begin{tabular}{ccc}
\hline Bin Range & $t \bar{t}$ & Data-backgrounds \\
\hline$(-2,-0.81)$ & $74.4 \pm 3.6$ & $66.3 \pm 11.2$ \\
{$[-0.81,-0.61)$} & $68.9 \pm 3.3$ & $65.4 \pm 10.6$ \\
{$[-0.61,-0.42)$} & $70.8 \pm 3.4$ & $70.2 \pm 10.7$ \\
{$[-0.42,-0.26)$} & $72.4 \pm 3.5$ & $72.6 \pm 11.0$ \\
{$[-0.26,-0.10)$} & $72.0 \pm 3.5$ & $83.2 \pm 11.2$ \\
{$[-0.10,0)$} & $40.3 \pm 2.0$ & $36.4 \pm 7.8$ \\
{$[0,0.10)$} & $40.1 \pm 2.0$ & $38.2 \pm 7.6$ \\
{$[0.10,0.26)$} & $75.1 \pm 3.6$ & $64.4 \pm 10.3$ \\
{$[0.26,0.42)$} & $75.2 \pm 3.6$ & $82.6 \pm 11.0$ \\
{$[0.42,0.61)$} & $75.0 \pm 3.6$ & $89.9 \pm 11.3$ \\
{$[0.61,0,81)$} & $73.1 \pm 3.5$ & $65.8 \pm 10.4$ \\
{$[0.81,2)$} & $79.4 \pm 3.8$ & $83.8 \pm 11.9$ \\
\hline
\end{tabular}




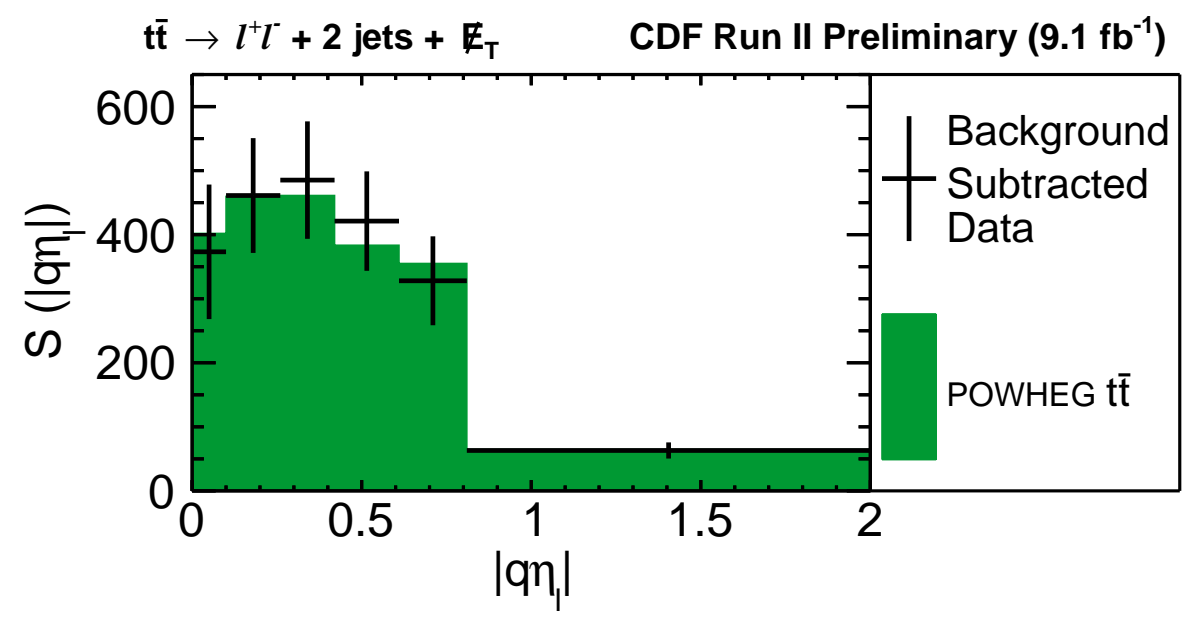

Figure 4.12: The symmetric part of the $q_{\ell} \eta_{\ell}$ distribution from data, after background subtraction, with the expectations from POWHEG overlaid.

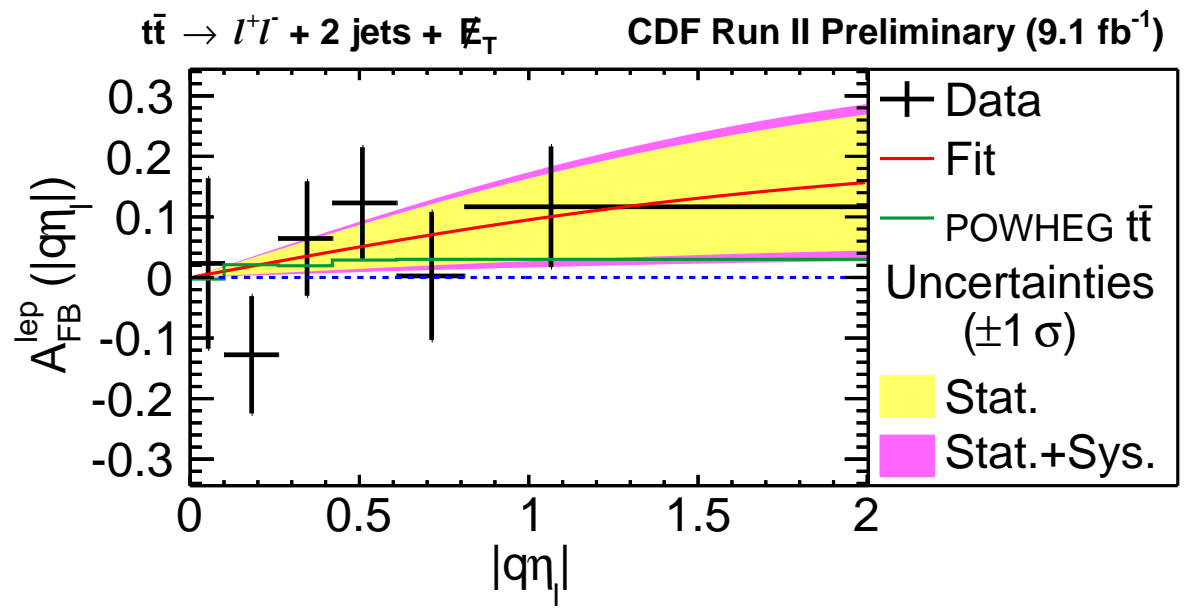

Figure 4.13: The asymmetric part of $q_{\ell} \eta_{\ell}$ distribution from data after background subtraction. The green line shows the expectations from POWHEG. 
$q_{\ell} \eta_{\ell}$ spectra, we generate two sets of pseudoexperiments. For the first set of pseudoexperiments, we add in fluctuations in the $t \bar{t}$ signal only (ignoring the backgrounds) and use this to help estimate the statistical uncertainty solely due to the $t \bar{t}$ signal. To do this, we use the POWHEG $t \bar{t}$ MC as our signal sample to simulate the $q_{\ell} \eta_{\ell}$ distribution in a binning that is the same as for the data. To simulate a pseudoexperiment, we normalize the total number of $t \bar{t}$ events according to the expected $t \bar{t}$ event count and its total uncertainty (statistical and systematic) and fluctuate each bin of the $q_{\ell} \eta_{\ell}$ distribution according to Poission statistics with the expected number of events in that bin as the mean. The fluctuated $q_{\ell} \eta_{\ell}$ distribution, our pseudoexperiment, is then subject to the decomposition and extrapolation procedure to get the fully extrapolated $A_{\mathrm{FB}}^{\ell}$ for each experiment, with the result from $10 \mathrm{k}$ pseudoexperiments shown in Fig. 4.14a. A Gaussian function is fitted to the distribution and we find that the mean is consistent with the expected $A_{\mathrm{FB}}^{\ell}$; the RMS of the distribution, 0.043, represents the statistical uncertainty from signal.

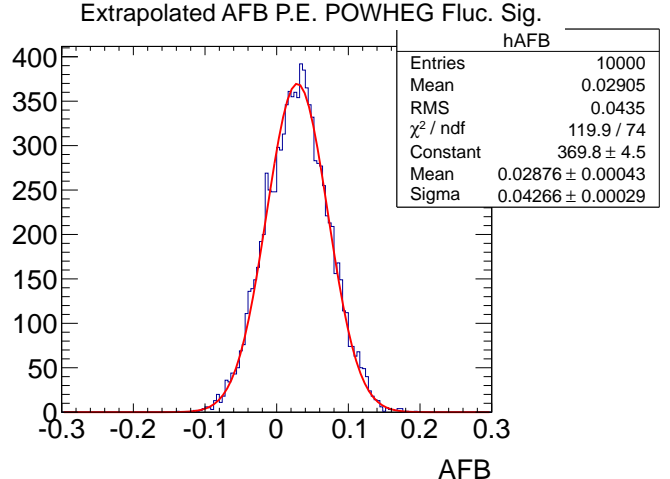

(a)

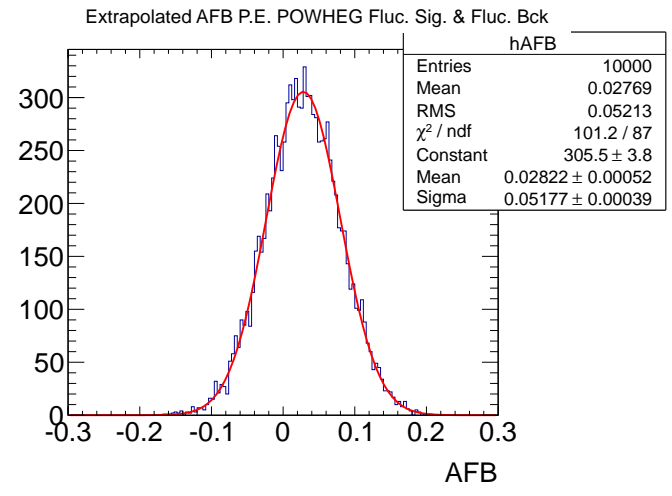

(b)

Figure 4.14: The results of pseudoexperiments to estimate the uncertainty due to background fluctuation. Fig. 4.14a shows the $A_{\mathrm{FB}}^{\ell}$ obtained by only fluctuating signal, and Fig. $4.14 \mathrm{~b}$ shows the $A_{\mathrm{FB}}^{\ell}$ obtained by fluctuating both signal and backgrounds. 
To estimate the uncertainty due to the backgrounds we generate a second set of pseudoexperiments but using both the $t \bar{t}$ signal and each background component. We create the bin-by-bin $q_{\ell} \eta_{\ell}$ distribution according to their mean rate and total rate uncertainties. Each bin of each component is fluctuated according to Poission statistics. The sum of the fluctuated signal and backgrounds is subjected to the nominal background subtraction and $a \cdot \tanh$ fit, then $A_{\mathrm{FB}}^{\ell}$ is retrieved from the $a \cdot \tanh$ fit. The results are shown in Fig. $4.14 \mathrm{~b}$ and a Gaussian function is fitted to the distribution. The mean $A_{\mathrm{FB}}^{\ell}$ is consistent with mean of previous set of pseudoexperiments, and the RMS of 0.052 represents the statistical uncertainty from the signal and background and is consistent with the result given previously. While the difference between the two RMS values in quadrature (0.029) can be thought of as the uncertainty due to the statistical fluctuations of the backgrounds, we take a systematic uncertainty on the normalization and shape of the backgrounds to be equal to this value, and add it in quadrature for the final uncertainty estimate. As previously noted, this is the dominant systematic uncertainty in our measurement.

To get a validation of the estimation of the background systematics, we test the background systematics with another approach, even though we are quoting the previous result as our final estimation of background systematics. Since the $W+$ jets and DY+jet processes are the two leading background components, we estimate the $A_{\mathrm{FB}}^{\ell}$ as if all the backgrounds were from $W+$ jets or $\mathrm{DY}+$ jets. We estimate the $A_{\mathrm{FB}}^{\ell}$ from data by scaling the $W+$ jets background template to the overall background rate and suppress other background template, and get an $A_{\mathrm{FB}}^{\ell}$ of 0.062 . When doing the same process with DY+jets, we obtain an $A_{\mathrm{FB}}^{\ell}$ of 0.106 . Comparing to the nominal $A_{\mathrm{FB}}^{\ell}$ result at 0.072 , the corresponding background systematics is at the level of 0.030. This estimation of background systematics provides the confidence that the background systematics we are quoting is at a reasonable conservative level. 
As mentioned in Sec. 4.2 .2 , we assign the difference between the measured $A_{\mathrm{FB}}^{\ell}$ and the $A_{\mathrm{FB}}^{\ell}$ at truth level from POWHEG MC as the A-part Model systematic uncertainty to cover potential mismodeling introduced by the $a \cdot$ tanh fit and zero acceptance correction. This has a value of 0.006 .

To estimate the systematic uncertainty due to the variation in the symmetric part of $q_{\ell} \eta_{\ell}$ distribution from different physics model, which we call the S-part Model uncertainty, we calculate $A_{\mathrm{FB}}^{\ell}$ using the $\mathcal{S}\left(q_{\ell} \eta_{\ell}\right)$ model from ALPGEN, HERWIG, PYTHIA, AxiL, AxiR, and Axi0, and take the largest difference between these $A_{\mathrm{FB}}^{\ell}$ values and the central value of the measured $A_{\mathrm{FB}}^{\ell}$. We find this to be 0.001 .

Other systematic uncertainties are estimated according to the standard procedures in the top group of the CDF collaboration [20], and are very small in comparison with the dominant uncertainties. We quickly summarize each:

- The jet energy scale (JES) systematic uncertainty is estimated by simultaneously shifting all jet energies up and down $1 \sigma$, and taking the larger difference between shifted $A_{\mathrm{FB}}^{\ell}$ and central value.

- The parton showering systematic uncertainty estimated to be the difference between the $A_{\mathrm{FB}}^{\ell}$ values obtained with ALPGEN MC showered by PYTHIA and ALPGEN MC showered by HERWIG.

- The color reconnection systematic uncertainty is estimated to be the difference between the $A_{\mathrm{FB}}^{\ell}$ values obtained with two $t \bar{t} \mathrm{MC}$ samples with two alternative color reconnection models.

- The initial- and final-state radiation (I/FSR) uncertainty is estimated to be half of the difference between $A_{\mathrm{FB}}^{\ell}$ values obtained with samples with "more" or "less" amounts of I/FSR. 
Table 4.9: Table of uncertainties for the $A_{\mathrm{FB}}^{\ell}$ measurement.

\begin{tabular}{|c|l|}
\hline Source of Uncertainties & Value \\
\hline Backgrounds & 0.029 \\
A-part Model & 0.006 \\
JES & 0.004 \\
S-part Model & 0.001 \\
PDF & 0.0002 \\
Parton Showering & 0.0002 \\
Color Reconnection & 0.00007 \\
IFSR & 0.00008 \\
\hline Total Systematic & 0.030 \\
\hline Statistical & 0.052 \\
\hline \hline Total Uncertainty & 0.060 \\
\hline
\end{tabular}

- The PDF uncertainty is calculated according to standard procedure of the CDF top group by re-weighting the PYTHIA sample corresponding to different PDF models.

Table 4.9 summaries the systematic and statistical uncertainties for $A_{\mathrm{FB}}^{\ell}$. The total systematic uncertainty is 0.03 , which is estimated by adding all the systematics in quadrature, and is dominated by the systematic uncertainty of backgrounds.

\subsubsection{Result of the measurement of $A_{F B}^{\ell}$}

After applying the full measurement methodology to the data, after background subtraction, and taking the systematic uncertainties into account, the parton-level $A_{\mathrm{FB}}^{\ell}$ result is:

$$
A_{\mathrm{FB}}^{\ell}=0.072 \pm 0.052 \text { (stat.) } \pm 0.030 \text { (syst.) }=0.072 \pm 0.060
$$

which is consistent with the NLO SM prediction of $0.038 \pm 0.003$. The comparison between this result and all other results and the SM predictions are summarized in 
Sec. 8 .

\subsubsection{Cross checks for $A_{F B}^{\ell}$}

We performed the same measurement in several subsets of the data as cross checks. The subsets we used are with different lepton categories $(e e, \mu \mu$, and $e \mu)$, with different lepton charges (positive and negative leptons only), and with events with at least one SECVTX $b$-tagged jet.

Table 4.10 lists the measured values of $A_{\mathrm{FB}}^{\ell}$ from positive and negative leptons only, and from the $e e, \mu \mu$ and $e \mu$ categories separately. The plots for the fits can be found in Figs. 4.15 and 4.16. All sub-categories are consistent with each other within statistics.

Table 4.10: The extrapolated $A_{\mathrm{FB}}^{\ell}$ results in different lepton categories. The uncertainties are statistical only.

\begin{tabular}{cc}
\hline Lepton Category & Extrapolated $A_{\mathrm{FB}}^{\ell}$ \\
\hline \hline All & $0.072 \pm 0.052$ \\
\hline $\mathrm{ee}$ & $0.128 \pm 0.101$ \\
$\mu \mu$ & $0.075 \pm 0.117$ \\
$\mathrm{e} \mu$ & $0.044 \pm 0.070$ \\
\hline Positive Lepton & $0.099 \pm 0.073$ \\
Negative Lepton & $0.043 \pm 0.070$ \\
\hline
\end{tabular}

A separate check is done with a subset of the data with at least one tight SECVTX $b$-tagged jet. This subset of data has a higher purity, but lower statistics makes it not worth making this the final result since it is expected to have an overall worse sensitivity as we are statistics limited. Figure 4.17 shows the fit with this subset of data. The result is

$$
A_{\mathrm{FB}}^{\ell}(b \text {-tag) }=0.105 \pm 0.062 \text { (stat.). }
$$




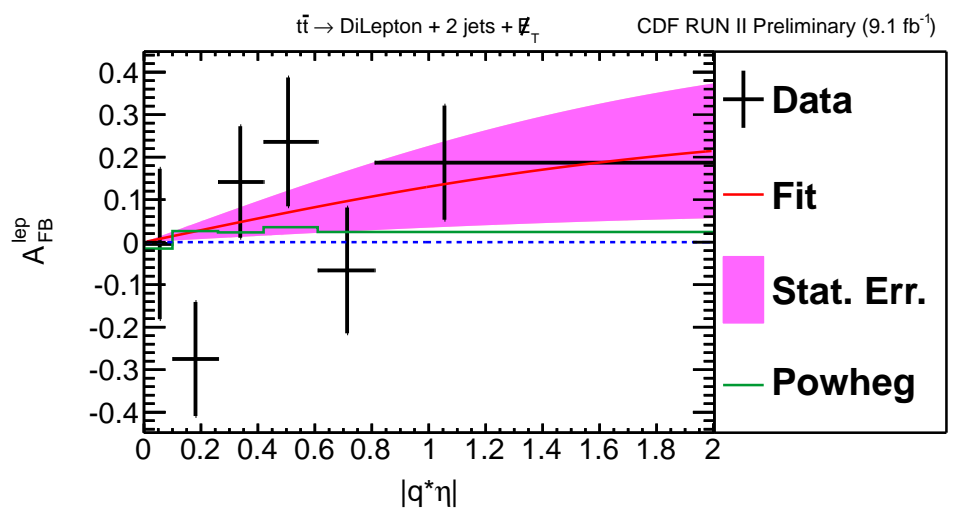

(a) Positive lepton

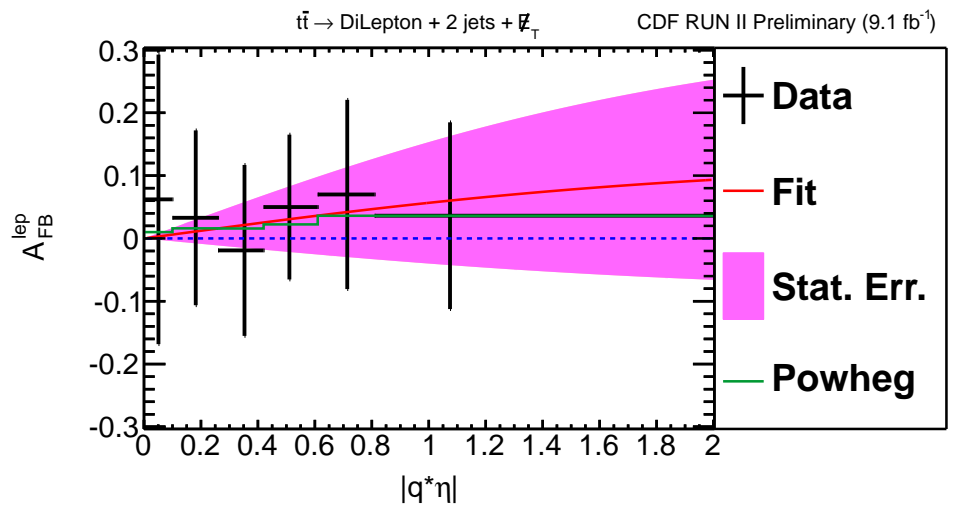

(b) Negative lepton

Figure 4.15: The $a \cdot \tanh$ fits for the positive and negative leptons separately. 


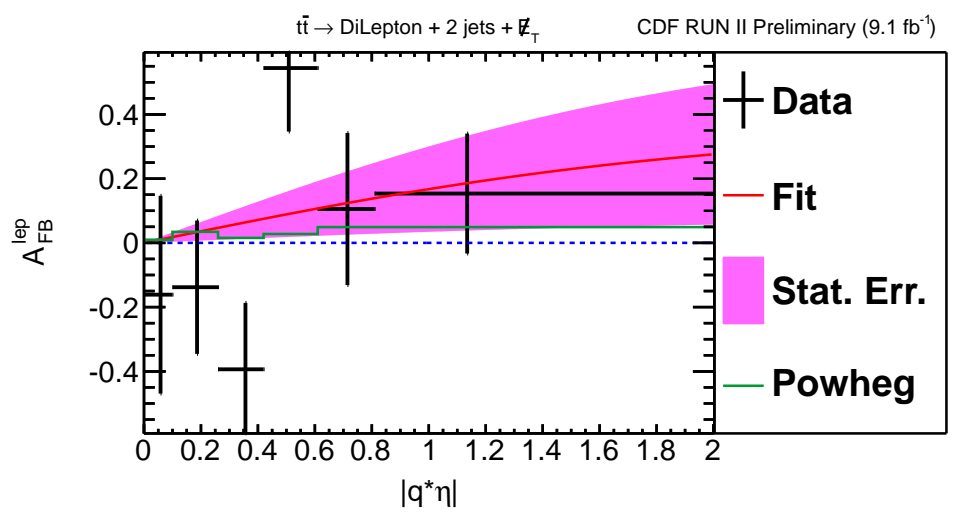

(a) $e e$

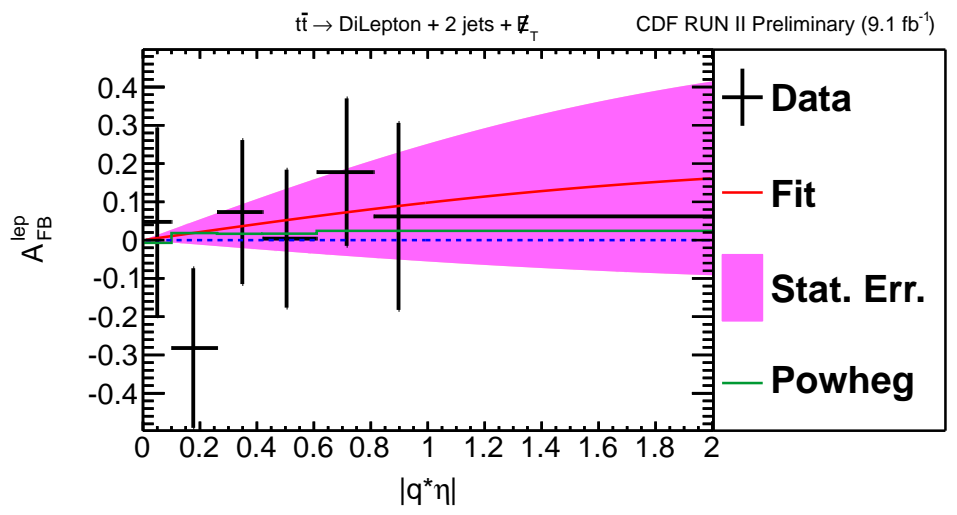

(b) $\mu \mu$

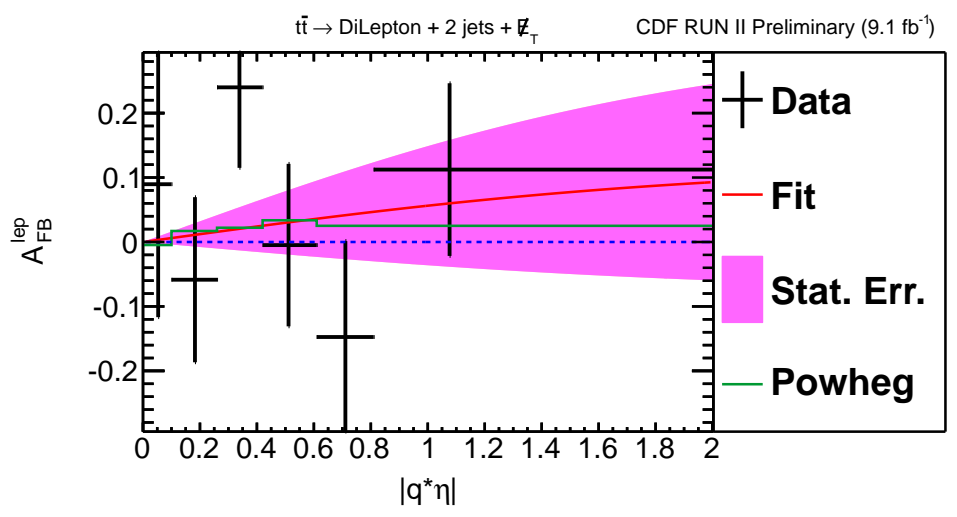

(c) $e \mu$

Figure 4.16: The $a \cdot \tanh$ fits for $e e, \mu \mu$, and $e \mu$ final states separately. 
The result is consistent with the result without the extra cut as well as the NLO SM prediction.

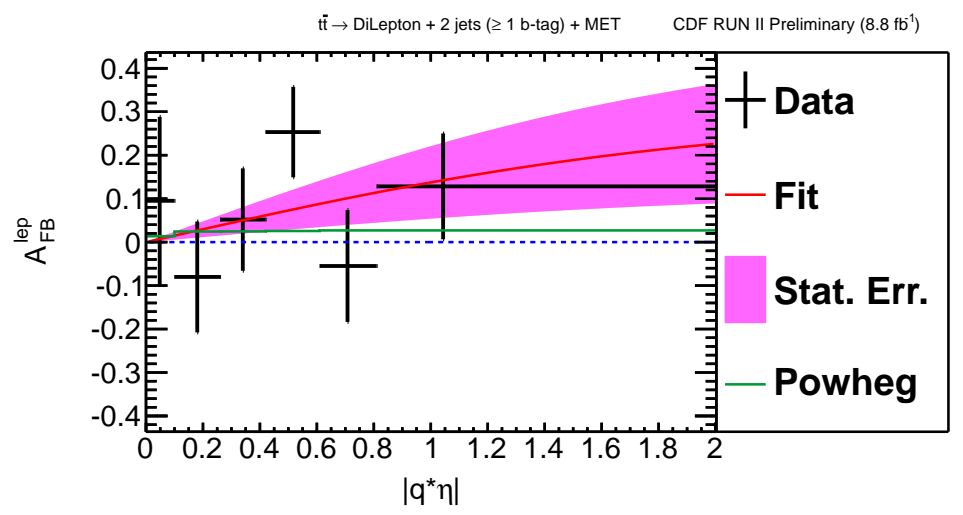

Figure 4.17: The $a \cdot \tanh$ fit for the $A_{\mathrm{FB}}^{\ell}$ data but with the additional requirement of at least one $b$-tagged jet.

\subsubsection{Measurement of the $A_{F B}^{\ell \ell}$ and Uncertainty Estimation}

The same extrapolation method is also applied to the $\Delta \eta$ distribution to extract $A_{\mathrm{FB}}^{\ell \ell}$ (also denoted as $\mathrm{A}_{\mathrm{FB}}^{\Delta \eta}$ in some figures). The distributions of $\Delta \eta$ before and after the background subtraction are shown in Fig. 3.2f and Fig. 3.6f, and the numbers are listed bin-by-bin in Table 4.11. The uncorrected $A_{\mathrm{FB}}^{\ell \ell}$ values, with a straight counting with no corrections for the detector response, from data before and after the background subtraction are listed in Table 4.12, together with the prediction from POWHEG. The symmetric and asymmetric parts of the $\Delta \eta$ distribution are shown in Fig. 4.18 with the $a \cdot \tanh$ fit, yielding $a=0.16 \pm 0.15$ (stat.). The $A_{\mathrm{FB}}^{\ell \ell}$ extracted from this fit is

$$
A_{\mathrm{FB}}^{\ell \ell}=0.076 \pm 0.072 \text { (stat.) } \pm 0.039 \text { (sys.) }=0.076 \pm 0.082,
$$


where we have used the uncertainties listed in Table 4.13 , which are estimated using the same techniques as in Sec. 4.3.1.3. This result is consistent with the NLO SM prediction of $0.040 \pm 0.004$. The comparison between this result and other results and SM predictions are summarized in Sec. 8 .

The cross check with at least one tight SECVTX $b$-tagged jet is also performed with the $A_{\mathrm{FB}}^{\ell \ell}$ measurement. The plot for the fit is shown in Fig. 4.19, The result is

$$
A_{\mathrm{FB}}^{\ell \ell}(b-\mathrm{tag})=0.148 \pm 0.092 \text { (stat.) }
$$

which is consistent with both the result without the extra $b$-tagging requirement and the SM prediction. 
Table 4.11: A comparison of the number of leptons in each $\Delta \eta$ range bin-by-bin before and after the background subtraction. Note that the $t \bar{t}$ contributions are estimated using the POWHEG MC.

(a) $t \bar{t}+$ backgrounds and data

\begin{tabular}{ccc}
\hline Bin Range & $t \bar{t}+$ backgrounds & Data \\
\hline$(-3.5,-1.14)$ & $53.9 \pm 3.9$ & 49 \\
{$[-1.14,-0.80)$} & $50.3 \pm 3.8$ & 49 \\
{$[-0.80,-0.54)$} & $50.1 \pm 3.8$ & 55 \\
{$[-0.54,-0.32)$} & $52.1 \pm 4.0$ & 46 \\
{$[-0.32,-0.1)$} & $52.6 \pm 3.9$ & 52 \\
{$[-0.1,0)$} & $24.6 \pm 1.9$ & 26 \\
{$[0,0.1)$} & $23.6 \pm 1.8$ & 15 \\
{$[0.1,0.32)$} & $49.7 \pm 3.7$ & 58 \\
{$[0.32,0.54)$} & $51.3 \pm 3.8$ & 61 \\
{$[0.54,0.8)$} & $50.0 \pm 3.6$ & 59 \\
{$[0.8,1.14)$} & $50.6 \pm 3.8$ & 42 \\
{$[1.14,3.5)$} & $59.1 \pm 4.3$ & 57 \\
\hline
\end{tabular}

(b) $t \bar{t}$ and data after background subtraction.

\begin{tabular}{ccc}
\hline Bin Range & $t \bar{t}$ & Data-backgrounds \\
\hline$(-3.5,-1.14)$ & $41.1 \pm 2.0$ & $36.3 \pm 7.2$ \\
{$[-1.14,-0.80)$} & $35.1 \pm 1.7$ & $33.8 \pm 7.4$ \\
{$[-0.80,-0.54)$} & $34.2 \pm 1.7$ & $39.1 \pm 7.8$ \\
{$[-0.54,-0.32)$} & $34.8 \pm 1.7$ & $28.7 \pm 7.3$ \\
{$[-0.32,-0.1)$} & $36.1 \pm 1.8$ & $35.5 \pm 7.6$ \\
{$[-0.1,0)$} & $16.8 \pm 0.8$ & $18.3 \pm 5.2$ \\
{$[0,0.1)$} & $17.1 \pm 0.9$ & $8.5 \pm 4.0$ \\
{$[0.1,0.32)$} & $36.4 \pm 1.8$ & $44.6 \pm 7.9$ \\
{$[0.32,0.54)$} & $35.9 \pm 1.8$ & $45.5 \pm 8.1$ \\
{$[0.54,0.8)$} & $37.4 \pm 1.8$ & $46.3 \pm 7.9$ \\
{$[0.8,1.14)$} & $37.2 \pm 1.8$ & $28.5 \pm 6.8$ \\
{$[1.14,3.5)$} & $46.3 \pm 2.2$ & $44.2 \pm 7.8$ \\
\hline
\end{tabular}


Table 4.12: The uncorrected $A_{\mathrm{FB}}^{\ell \ell}$, with a straight counting of the numbers of the forward and the backward leptons, from the data only and the data after background subtraction, together with the expectation from the POWHEG $t \bar{t} \mathrm{MC}$, which is with NLO SM with QCD corrections but without electroweak corrections.

\begin{tabular}{cc}
\hline Source & $\begin{array}{c}\text { Uncorrected } \\
A_{\mathrm{FB}}^{\ell \ell}\end{array}$ \\
\hline \hline PowHEG $t \bar{t}$ & $0.030 \pm 0.004$ \\
\hline Data & $0.03 \pm 0.04$ \\
\hline Background Subtracted Data & $0.06 \pm 0.06$ \\
\hline
\end{tabular}

Table 4.13: Table of uncertainties for the $A_{\mathrm{FB}}^{\ell \ell}$ measurement.

\begin{tabular}{|c|l|}
\hline Source of Uncertainties & Value \\
\hline Backgrounds & 0.037 \\
A-part Model & 0.012 \\
JES & 0.003 \\
S-part Model & 0.004 \\
PDF & 0.0001 \\
Parton Showering & 0.00003 \\
Color Reconnection & 0.00004 \\
IFSR & 0.00009 \\
\hline Total Systematic & 0.039 \\
\hline Statistical & 0.072 \\
\hline \hline Total Uncertainty & 0.082 \\
\hline
\end{tabular}




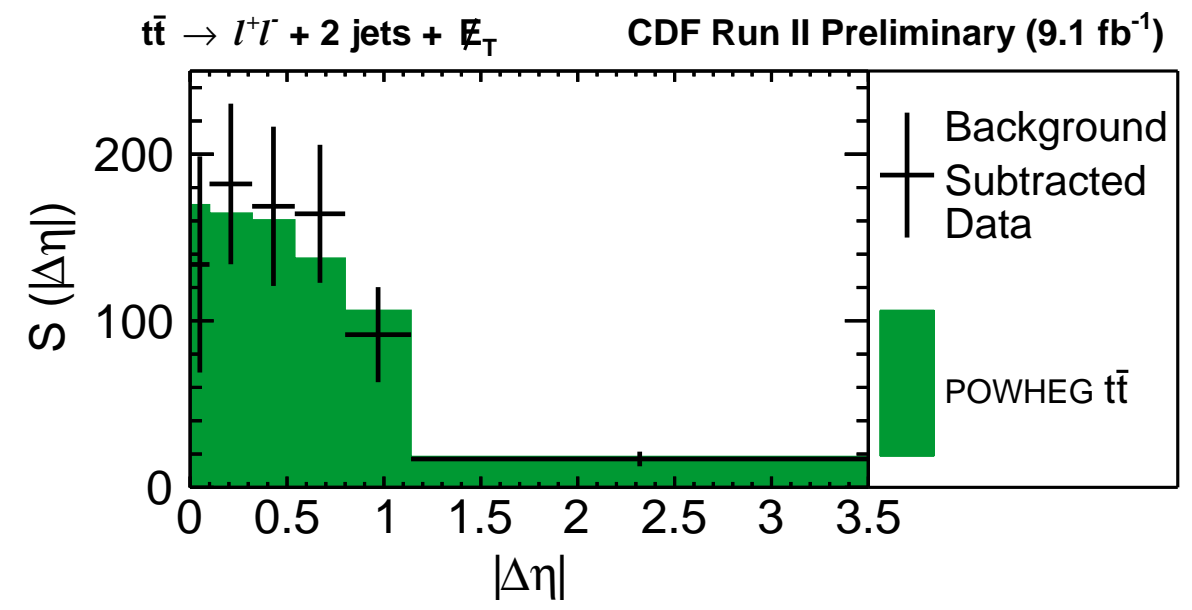

(a) The symmetric part of the $\Delta \eta$ distribution from data after background subtraction with the expectations from POWHEG overlaid.

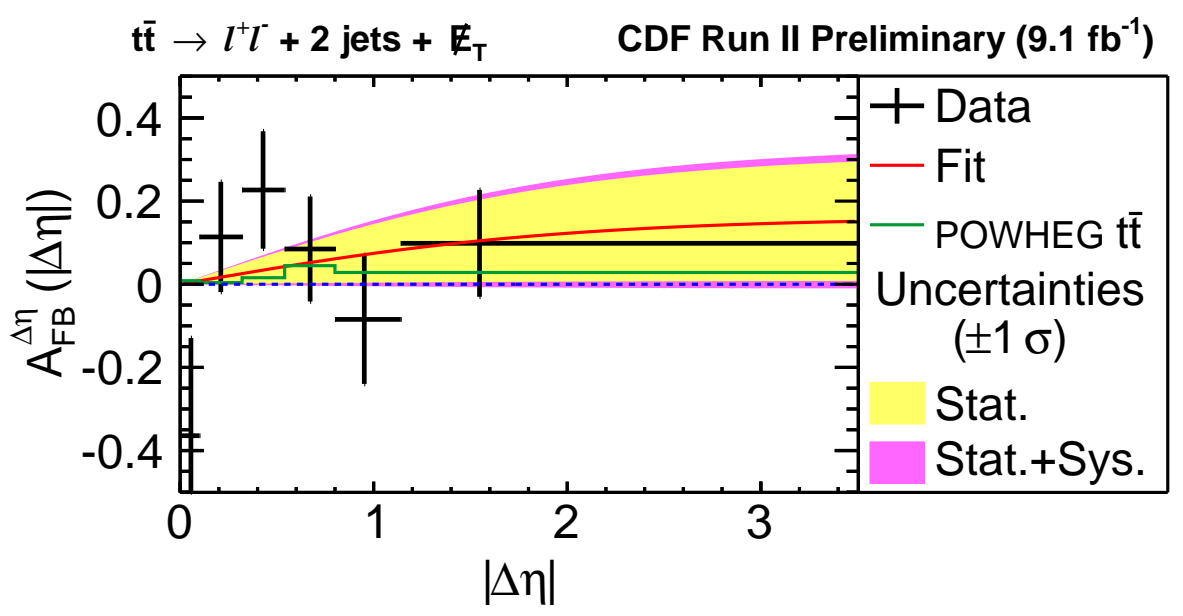

(b) The asymmetric part of the $\Delta \eta$ distribution from data after background subtraction. The green line shows expectation from POWHEG MC.

Figure 4.18: The symmetric and asymmetric parts of $\Delta \eta$. 


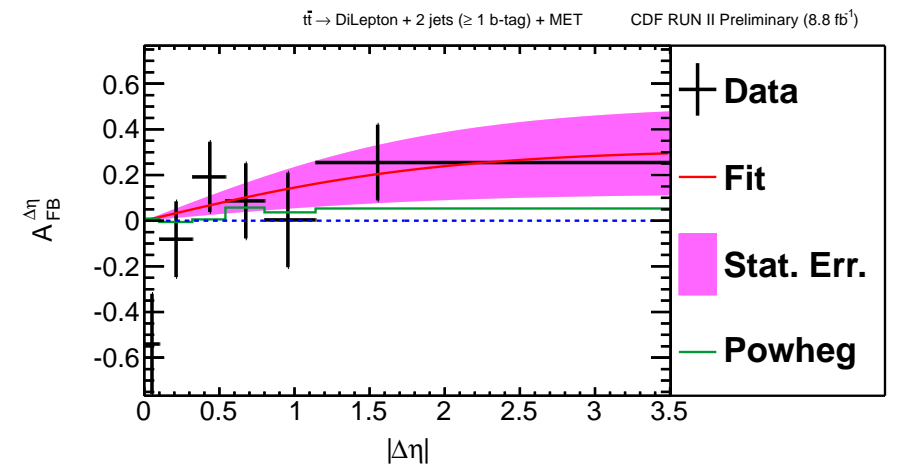

Figure 4.19: The $a \cdot \tanh$ fit for the $A_{\mathrm{FB}}^{\ell \ell}$ data but with the additional requirement of at least one $b$-tagged jet. 


\section{RECONSTRUCTION OF THE MOMENTA OF TOP-QUARK PAIRS}

In this section we describe the algorithm we use to reconstruct the momenta of

the top quark and antiquark in $t \bar{t}$ production for use in the measurement of the $A_{\mathrm{FB}}^{t \bar{t}}$ described in the next section. An example Feynman diagram of a $t \bar{t}$ event in the dilepton final state $\left(t \bar{t} \rightarrow \ell^{+} \ell^{\prime-} \nu \overline{\nu^{\prime}} b \bar{b}\right)$ is shown in Fig. 1.4c. We reconstruct the top quark and antiquark by combining the final-state decay products together to form two $W$ bosons and then two top quarks. This involves pairing each lepton with some portion of the $\not_{T}$ to reconstruct a $W$-boson, and then pairing each reconstructed $W$ with one of the jets to form a top. The primary challenges of the reconstruction technique are 1) to choose the correct lepton-jet pairing, 2) to solve for the neutrino momentum within each pairing, and 3) to determine the best solution when multiple solutions exist.

We follow the lead of previous dilepton analyses, done with $5.1 \mathrm{fb}^{-1}$ of data, which use the method of reconstructing the top kinematics aiming to identify the most probable solution with a likelihood-based algorithm [17]. To make better use of the information, and improve on previous measurements, we make a number of important changes to the reconstruction algorithm:

1. We add back the mass of the $b$-quarks, which was previously assumed to be zero in the reconstruction algorithm.

2. We use the templates of the kinematic variables derived from a NLO MC (POWHEG [49, 94]) instead of those derived from a LO MC (PYTHIA [50]).

3. Instead of taking the most likely solution from each event by as determined from the likelihood term, as in Ref. [17], this analysis uses a Markov-chain 
Monte Carlo [97 (MCMC) to scan the parameter space effectively and extract the full probability distribution of all possible solutions for each event.

4. We include both the track-weighted jet charge [98], the $\Delta R_{\min }$ (lepton, jets), and the $m_{l b}$ [99] information to better select lepton-jet pairings, and to reject badly reconstructed events and backgrounds.

5. We make quality cuts on the reconstruction to reject badly reconstructed lepton-jet pairings and events.

We will explore different combinations of various strategies and chose the best strategy based on the expected uncertainty of $A_{\mathrm{FB}}^{t \bar{t}}$. These will be partially described in this section and partially in Section 6.1.

In the following sections, beginning in Sec. 5.1, we will first describe the basic ideas of the top reconstruction algorithm in the dilepton final state, the energy momentum conservation equations we use to reconstruct the top-quark kinematics, as well as the issues that confront our measurement uncertainties. The effects of the detector resolutions and the physics assumptions are described in Sec. 5.2. These include the details about the $b$-jet energy corrections and the resolutions of the jet $E_{\mathrm{T}}$ and $E_{T}$. In addition, since even with all the measurements we have, we still have an under-constrained system, we must use additional information to help us decide the probability of each of the many potential solutions. Specifically, we use the kinematics of the event for each possible solution, and compare it to the expected kinematics of the $t \bar{t}$ production as predicted by POWHEG; this comparison will be included in the likelihood. The description of the $p_{z, t \bar{t}}, p_{T, t \bar{t}}$ and $m_{t \bar{t}}$ distributions used in the comparison are also shown in Sec. 5.2. In Sec. 5.3 we lay out the rest of the pieces needed for the likelihood equation, and the equation itself. We describe the final implementation in Sec. 5.4. A comparison of the data to expectations 
after the reconstruction is given in Sec.6 after introducing additional event selection requirements based on the reconstruction quality and used in the final minimization of the expected uncertainty of the measurement.

\subsection{Top Reconstruction Basics}

We quickly overview the $t \bar{t}$ event reconstruction which we will use to determine $y_{t}, y_{\bar{t}}$, and $\Delta y_{t}$ event-by-event. A fully measured set of the final state objects from a $t \bar{t} \rightarrow \ell^{+} \ell^{\prime-} \nu \bar{\nu}^{\prime} b \bar{b} \rightarrow \ell^{+} \ell^{-}+$jets $+E_{T}$ event does not yield enough information to uniquely determine the kinematics of the original top and/or anti-top. As such it is an forms an under-constrained system. We can only determine the four-momenta of the top quark and antiquark probabilistically by using the measured values of the four-momenta of all the final state particles as well as some assumptions about the production and decay. As previously mentioned, the primary issues come with the $b / \bar{b}$ ambiguity and the ability to pick the correct solution when multiple solutions exist within a given pairing choice. Within the top reconstruction we assume/use:

1. Four mass constraints in the $t \bar{t}$ system and its decay products: the two $W$ boson masses $\left(m_{W}=80.4 \mathrm{GeV} / \mathrm{c}^{2}\right)$ and the two top-quark masses $\left(m_{t}=\right.$ $\left.172.5 \mathrm{GeV} / \mathrm{c}^{2}\right)[9]$.

2. The two opposite-charged leptons are measured with high precision. We take the measured momenta of the charged leptons as their true values (i.e., negligible uncertainties on magnitudes and directions).

3. We assume the two jets with the largest $E_{\mathrm{T}}$ (and $|\eta|<2.5$ ) come from the hadronization of the $b$ and the $\bar{b}$ quarks, but make no initial assumption about which is which. The directions of the jets are assumed to correctly indicate the directions of their original quarks. However, for the energies, we apply a top- 
specific (b-)jet energy correction after the standard CDF jet energy correction (referred as the Level 5 or L5 correction for technical reasons) 83] so that the mean of the jet $E_{\mathrm{T}}$ matches the $b$-quark $E_{\mathrm{T}}$ [17]. Within the reconstruction procedure we create two "jet deviation (jd)" parameters which allow the jet $E_{\mathrm{T}}$ values to float according to their mean values and resolutions in the fit (as described in Sec. 5.2). We fix the masses of the $b(\bar{b})$ quarks to $4.66 \mathrm{GeV} / \mathrm{c}^{2}$.

4. A charged lepton needs to be paired with a $b / \bar{b}$ quark to form a $t / \bar{t}$ quark (together with the undetected neutrinos). Since there is no perfect separation between jets from the $b$-quarks and jets from the $\bar{b}$-quarks, we consider both lepton-jet pairings in the reconstruction, but will use techniques to reduce the contamination of the measurement from wrong pairings.

5. While the two neutrinos in the final state are not detected, resulting in six unknown variables (assuming massless neutrinos), the sum of the transverse momenta of the two neutrinos produces an imbalanced- $p_{T}$ of the event $\left(\vec{E}_{T}\right)$ which can be used. However, since the two measured components of $\vec{H}_{T}\left(\not_{x}\right.$ and $E_{y}$ ) have large resolutions, the vector sum of the transverse momenta of the neutrinos are allowed to float according to the measured mean values and resolutions of $\not_{x}$ and $\not_{y}$ (which are assumed to be identical). 
With these pieces, we write down the energy momentum conservation equations:

$$
\begin{aligned}
M_{\ell^{+} \nu}^{2} & =\left(E_{\ell^{+}}+E_{\nu}\right)^{2}-\left(\vec{p}_{\ell^{+}}+\vec{p}_{\nu}\right)^{2}=M_{W}^{2} \\
M_{\ell^{-}}^{2} & =\left(E_{\ell^{-}}+E_{\bar{\nu}}\right)^{2}-\left(\vec{p}_{\ell^{-}}+\vec{p}_{\bar{\nu}}\right)^{2}=M_{W}^{2} \\
M_{\ell^{+} \nu b}^{2} & =\left(E_{\ell^{+}}+E_{\nu}+E_{b}\right)^{2}-\left(\vec{p}_{\ell^{+}}+\vec{p}_{\nu}+\vec{p}_{b}\right)^{2}=M_{t}^{2} \\
M_{\ell^{-} \bar{\nu} \bar{b}}^{2} & =\left(E_{\ell^{-}}+E_{\bar{\nu}}+E_{\bar{b}}\right)^{2}-\left(\vec{p}_{\ell^{-}}+\vec{p}_{\bar{\nu}}+\vec{p}_{\bar{b}}\right)^{2}=M_{t}^{2} \\
\left(\vec{p}_{\nu}+\vec{p}_{\bar{\nu}}\right)_{x} & =E_{x} \\
\left(\vec{p}_{\nu}+\vec{p}_{\bar{\nu}}\right)_{y} & =E_{y} .
\end{aligned}
$$

There are ten unknown variables in the $t \bar{t}$ dilepton final state (six variables from the momenta of the neutrinos, two variables characterizing the floating of the jet $E_{\mathrm{T}} \mathrm{S}$ and two variables characterizing the floating of the $\overrightarrow{\vec{H}}_{T}$ ). On the other hand, we have only six constraints from Eq. 5.1 (the two $W$-boson masses, the two top-quark masses, and the constraints from $\not_{x}$ and $\not_{y}$ ). Thus, for each event the variables and constraints form two under-constrained systems with degenerate solutions (from the $b / \bar{b}$ ambiguity) in two four-dimensional parameter spaces. By the time we are done, we calculate the likelihood of the $t \bar{t}$ event kinematics as a function of the 4 continuous parameters (related to 2 jet energy and 2 neutrino $\phi$ solutions) and one discrete (lepton-jet pairing choice) parameter.

In the following section, we will detour a little bit to describe the $b$-jet energy corrections and the resolutions for the jet $E_{\mathrm{T}}$ and $\not_{T}$ and the distributions of $p_{z, t \bar{t}}$, $p_{T, t \bar{t}}$ and $m_{t \bar{t}}$ (we will refer to these as templates), which are key ingredients of the likelihood fit (Sec. 5.3). 


\subsection{Detector Resolution and Physics Assumptions for the Reconstruction}

To include the best information about the $b$-jets in the likelihood, following Ref. [17, we use the jet $E_{\mathrm{T}}$ values after two sequential energy corrections: 1) the Level 5 jet energy corrections mentioned above (yielding the value $E_{T, \mathrm{jet}}^{\mathrm{L} 5}$ ) [81], and 2) a top-specific $b$-jet energy correction so that the mean of the reconstructed jet $E_{\mathrm{T}}$ matches the $E_{\mathrm{T}}$ of the $b$-quark from the top quark decay (as determined in $\mathrm{MC}$, here labeled as $\left.E_{T, b}^{\text {true }}\right)$. The additional scale factor is defined as the mean value of

$$
\mathrm{SF}_{\text {jet }}=\frac{E_{T, b}^{\mathrm{true}}-E_{T, \mathrm{jet}}^{\mathrm{L} 5}}{E_{T, \mathrm{jet}}^{\mathrm{L} 5}}
$$

denoted as $\left(\overline{\mathrm{SF}_{\text {jet }}}\right)$, and is parametrized as a function of $E_{T, \text { jet }}^{\mathrm{L} 5}$ in three sections of $\eta$ ranges $\left|\eta_{\text {jet }}\right|<0.7,0.7<\left|\eta_{\text {jet }}\right|<1.3$ and $1.3<\left|\eta_{\text {jet }}\right|<2.5$. We note that the sample to derive the corrections are made with events from the POWHEG MC with full detector simulation (done with CDFSim [88]) that pass the full set of baseline cuts in Table 2.1, and the requirement that the generator-level $b$-quark appears within a cone of the jet with the size of 0.4 , are used to create the correction terms. The mean values of $\mathrm{SF}_{\text {jet }}$ are shown in Fig. 5.1 and each is fitted with a function of the form of

$$
\exp \left(p_{0}+p_{1} x\right)+p_{2} .
$$

The best fit values are summarized in Table 5.1. We use the corrected jet $E_{\mathrm{T}}$ in our top reconstruction algorithm via

$$
E_{T, \text { jet }}^{\text {corr }}=\mathrm{SF}_{\text {jet }} \cdot E_{T, \text { jet }}^{L 5}
$$

In addition to the best value of the $E_{\mathrm{T}}$ of the jets, in our likelihood calculation we 


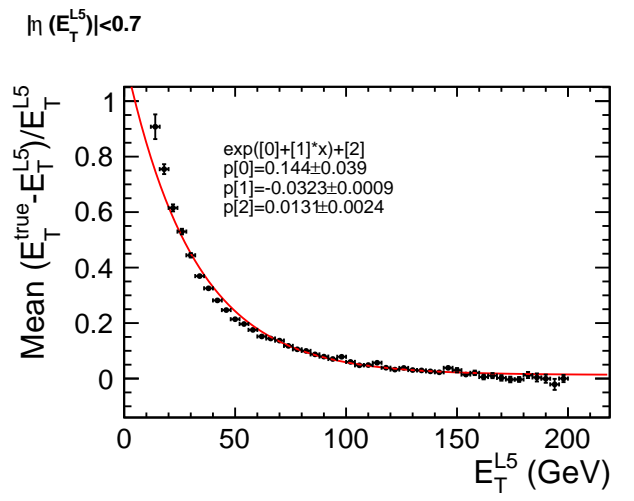

(a) $\left|\eta_{\text {jet }}\right|<0.7$

$1.3<\left|\eta\left(E_{T}^{L 5}\right)\right|<2.5$ $0.7<\ln \left(E_{\mathrm{T}}^{15}\right)<1.3$

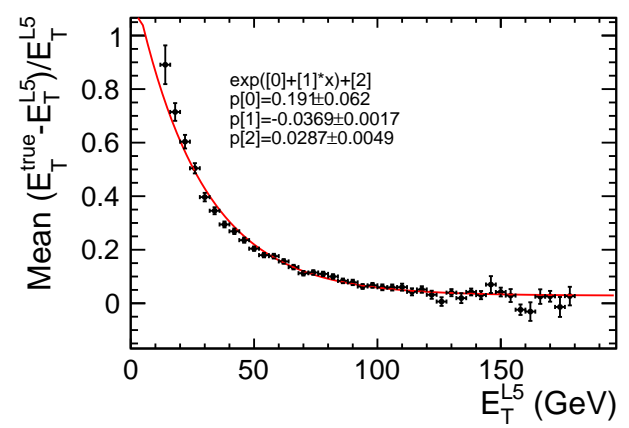

(b) $0.7<\left|\eta_{\text {jet }}\right|<1.3$

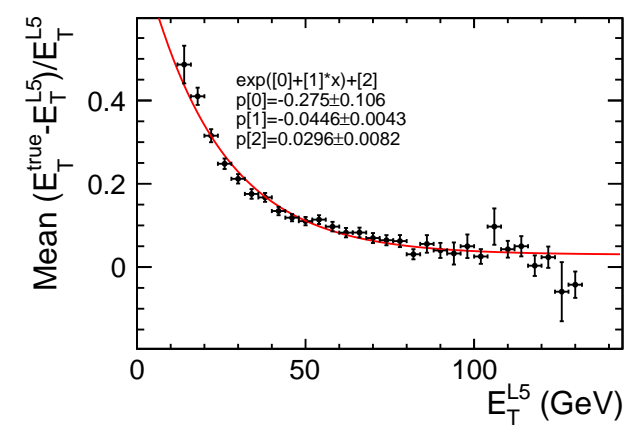

(c) $1.3<\left|\eta_{\text {jet }}\right|<2.5$

Figure 5.1: The mean value of $\mathrm{SF}_{\text {jet }}$ as a function of $E_{T}^{L 5}$, fitted with Eq. 5.3. The best fit values, summarized in Table 5.1, are derived from the NLO MC sample (POWHEG), and are used to correct the jets for use in the final fit function.

Table 5.1: Best fit values for the mean values of $\mathrm{SF}_{\text {jet }}\left(\overline{\mathrm{SF}_{\text {jet }}}\right)$ and the resolutions $(\sigma)$ of $\mathrm{SF}_{\text {jet }}$ and $\not_{x, y}^{\prime}\left(\sigma_{\mathrm{SF}_{\mathrm{jet}}}\right.$ and $\sigma_{E_{x, y}}$, respectively). These are derived from the NLO MC sample (POWHEG).

\begin{tabular}{|c|c|c|c|c|}
\hline \multicolumn{2}{|c|}{} & $p_{0}$ & $p_{1}$ & $p_{2}$ \\
\hline \multirow{2}{*}{$\left|\eta_{\text {jet }}\right|<0.7$} & $\overline{\mathrm{SF}_{\text {jet }}}$ & 0.144 & -0.032 & 0.013 \\
\cline { 2 - 5 } & $\sigma_{\mathrm{SF}_{\text {jet }}}$ & -0.196 & -0.028 & 0.138 \\
\hline \multirow{2}{*}{$0.7<\left|\eta_{\text {jet }}\right|<1.3$} & $\overline{\mathrm{SF}_{\text {jet }}}$ & 0.191 & -0.037 & 0.029 \\
\cline { 2 - 5 } & $\sigma_{\mathrm{SF}_{\text {jet }}}$ & -0.260 & -0.028 & 0.117 \\
\hline \multirow{2}{*}{$1.3<\left|\eta_{\text {jet }}\right|<2.5$} & $\overline{\mathrm{SF}_{\text {jet }}}$ & -0.275 & -0.045 & 0.030 \\
\cline { 2 - 5 } & $\sigma_{\mathrm{SF}_{\text {jet }}}$ & -0.573 & -0.030 & 0.114 \\
\hline \multicolumn{2}{|c|}{$\sigma_{B_{x, y}^{\prime}}$} & -0.381 & 0.007 & 15.032 \\
\hline
\end{tabular}


will need the jet energy resolution. To do this, instead of using the resolution of the jet directly, we use the resolution (RMS) of $\mathrm{SF}_{\text {jet }}$ which is also measured with POwHEG MC sample. The results are shown in Fig. 5.2 and found to be well parametrized as a function of the $E_{T, \text { jet }}^{L 5}$. Using the same functional form as in Eq. 5.3, the best fit values are shown in Fig. 5.2 and also summarized in Table 5.1. The resolution of the jet $E_{\mathrm{T}}$ is calculated as

$$
\sigma_{\text {jet }}=\sigma_{\mathrm{SF}_{\text {jet }}} \cdot E_{T, \text { jet }}^{L 5}
$$

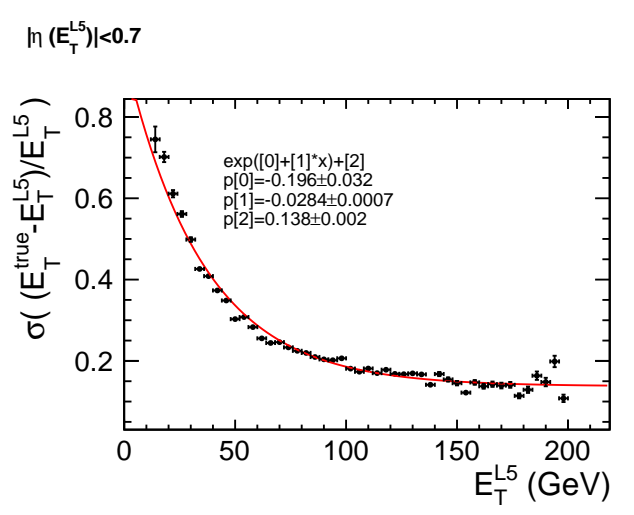

(a) $\left|\eta\left(E_{T}^{L 5}\right)\right|<0.7$
$0.7<\left|\eta\left(\mathrm{E}_{\mathrm{T}}^{\mathrm{L5}}\right)\right|<1.3$

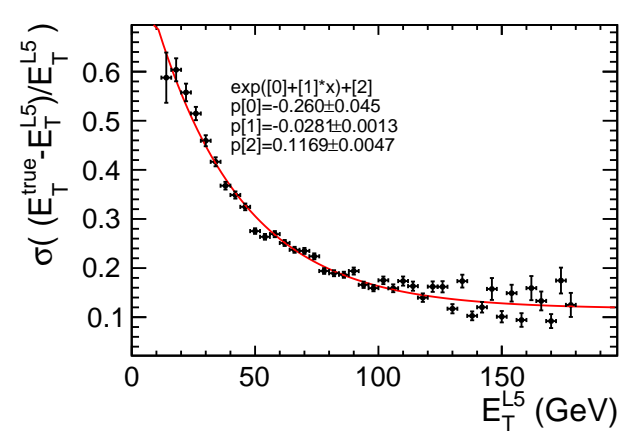

(b) $0.7<\left|\eta\left(E_{T}^{L 5}\right)\right|<1.3$

$1.3<\ln \left(\mathrm{E}_{\mathrm{T}}^{\mathrm{L} .5}\right)<2.5$

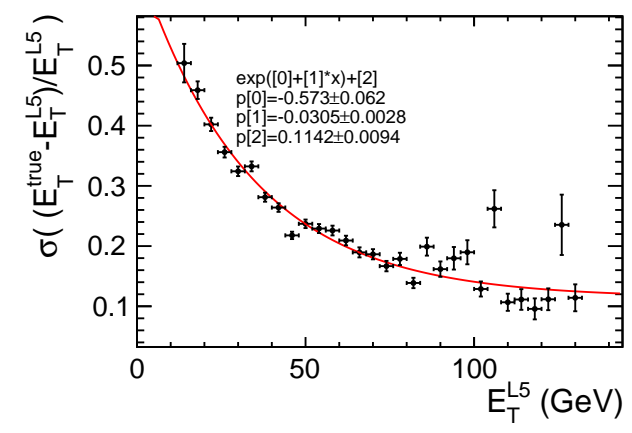

(c) $1.3<\left|\eta\left(E_{T}^{L 5}\right)\right|<2.5$

Figure 5.2: The RMS of $\mathrm{SF}_{\text {jet }}$ as a function of $E_{T}^{L 5}$, fitted with Eq. 5.3. The best fit values are summarized in Table 5.1, and will be used in the final likelihood reconstruction. These are derived from the NLO MC sample (POwHEG). 
Our studies have shown that recalculating the $\vec{E}_{T}$ after the corrections to jet $E_{\mathrm{T}}$ does not improve the resolution so we do not perform this correction. We also find that since the $\mathscr{H}_{T}$, on average, well reproduces the mean true $\mathscr{E}_{T}$, no other correction is used.

The resolution (RMS) of $\mathscr{E}_{x}$ and $\mathscr{H}_{y}\left(\sigma_{E_{x}}\right.$ and $\sigma_{E_{y}^{\prime}}$, respectively, and where we assume the two are equal to each other) are estimated using techniques similar to those described above. The results are shown in Fig. 5.3 and we note that the resolution is well parametrized as a function of the sum of the two jet $E_{\mathrm{T}}$ values at Level-5 $\left(E_{T, \text { jet1 }}^{L 5}+E_{T, \text { jet2 }}^{L 5}\right)$ before the top-specific correction. The histogram is then fitted with Eq. 5.3. The best fit values are again summarized in Table 5.1.

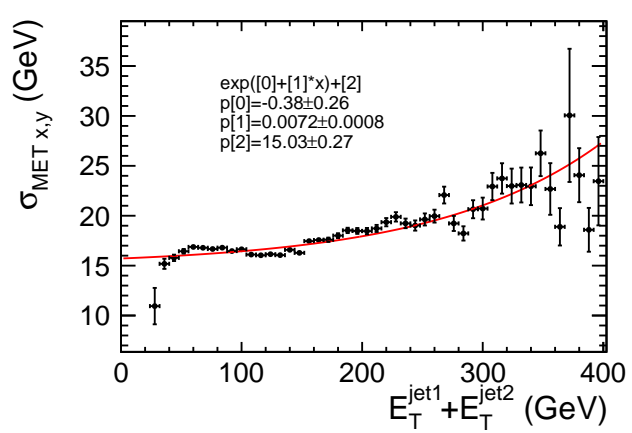

Figure 5.3: The RMS of $E_{x}$ and $E_{y}^{\prime}$ as a function of $E_{T}^{L 5}\left(\right.$ jet1) $+E_{T}^{L 5}$ (jet2), fitted with Eq. 5.3. The best fit values are summarized in Table 5.1. These are derived from the NLO MC sample (POWHEG).

To help distinguish between more-likely reconstruction results of the $t \bar{t}$ and lesslikely ones, we compare the various potential solutions from the fitter to the expected kinematic distributions of $t \bar{t}$ at generator level. We again use the sample of events that pass all the cuts in Table 2.1. Specifically, we compare the the $z$ and transverse 
components of the momentum of the $t \bar{t}$ system as well as the invariant mass of the $t \bar{t}$ system $\left(p_{z, t \bar{t}}, p_{T, t \bar{t}}\right.$ and $\left.m_{t \bar{t}}\right)$. The distributions, or templates as we will refer them, are shown in Fig. 5.4 and each is fit with a custom function that well-approximates the shape. The fit functions and the best fit values are summarized in Table 5.2. Note that in all three cases the parameter $p_{0}$ in an overall normalization factor that is only related to the size of the $\mathrm{MC}$ sample, it thus has no effect on the top reconstruction algorithm.

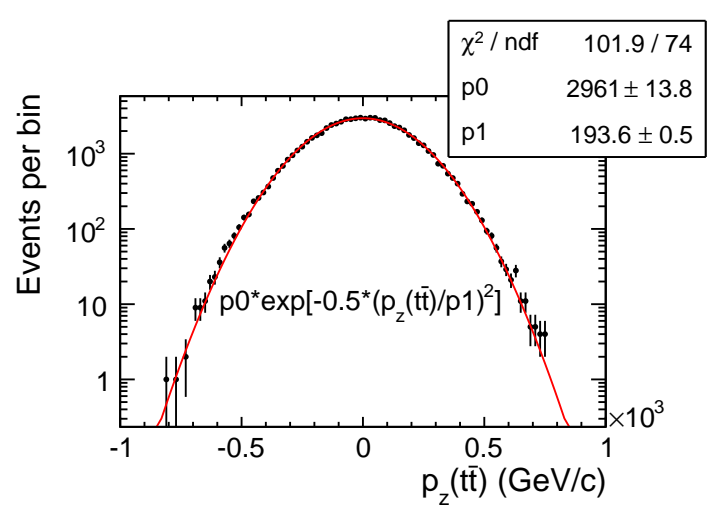

(a) $p_{z, t \bar{t}}$

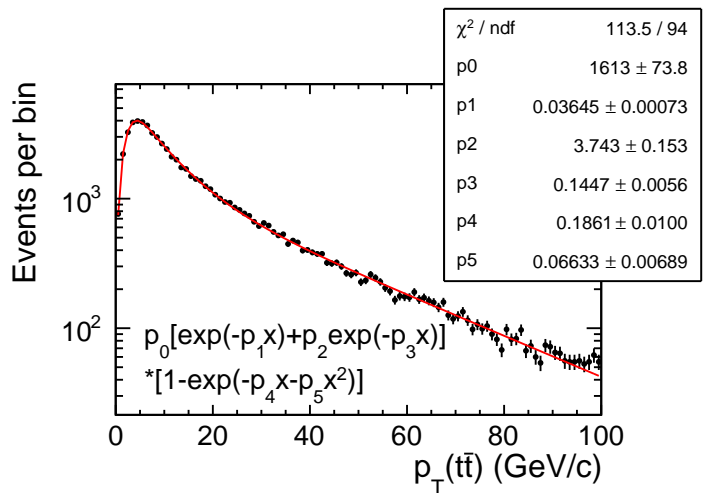

(b) $p_{T, t \bar{t}}$

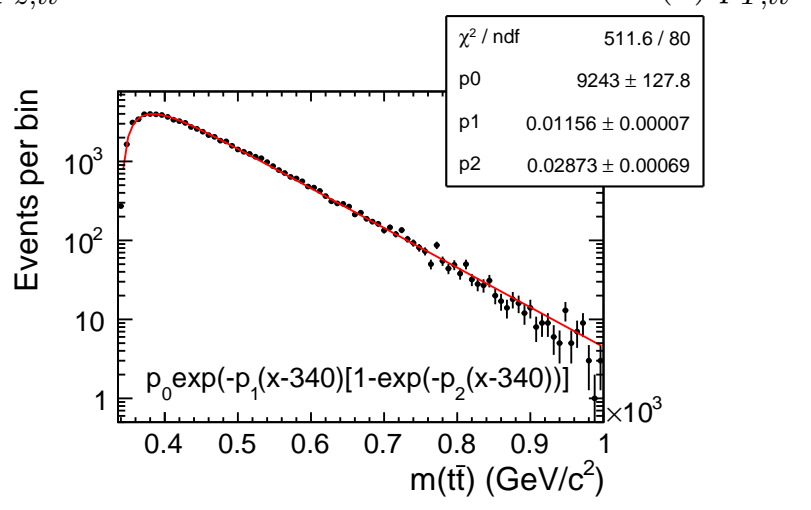

(c) $m_{t \bar{t}}$

Figure 5.4: The templates of $p_{z, t \bar{t}}, p_{T, t \bar{t}}$ and $m_{t \bar{t}}$ derived from the POWHEG MC at the generator level for the sample of reconstructed events that pass the baseline event selection criteria. Note that the functional forms are designed purely to get a good estimate of the shape, not to be used in any determination of each parameter. 
Table 5.2: The fit functions and the best fit values of templates $p_{z, t \bar{t}}, p_{T, t \bar{t}}$ and $m_{t \bar{t}}$ derived from the POWHEG MC at the generator level.

\begin{tabular}{|c|l|l|l|l|}
\hline Quantity & Fit function & \multicolumn{3}{|c|}{ Parameters } \\
\hline \multirow{2}{*}{$p_{z, t \bar{t}}$} & $p_{0} \exp \left[-\frac{1}{2}\left(\frac{x}{p_{1}}\right)^{2}\right]$ & $p_{0}$ & $p_{1}$ \\
& & 2961 & 193.6 \\
\hline \multirow{5}{*}{$p_{T, t \bar{t}}$} & $p_{0}\left[\exp \left(-p_{1} x\right)+p_{2} \exp \left(-p_{3} x\right)\right]$ & $p_{0}$ & $p_{1}$ & $p_{2}$ \\
\cline { 3 - 5 } & $\cdot\left[1-\exp \left(-p_{4} x-p_{5} x^{2}\right)\right]$ & $p_{3}$ & 0.03645 & 3.743 \\
\hline \multirow{2}{*}{$m_{t \bar{t}}$} & $p_{0} \exp \left[-p_{1}(x-340)\right]$ & 0.1447 & 0.1861 & $p_{5}$ \\
& $\cdot\left[1-\exp \left(-p_{2}(x-340)\right)\right]$ & $p_{0}$ & $p_{1}$ & $p_{2}$ \\
& & 9243 & 0.01156 & 0.2873 \\
\hline
\end{tabular}

We note that creating likelihoods for a potential solution using POWHEG MC does introduce a model dependence in the top reconstruction algorithm. The result with this top reconstruction should thus be interpreted with this in mind.

\subsection{Likelihood for Possible Solutions}

In this section we define the four independent variables that will be used to parametrize the likelihood term derived from Eq. 5.1. Even if we do not allow the jet $E_{\mathrm{T}}$ values and $E_{x, y}$ to float, there are up to four solutions to each set of equations due to the quadratic structure of Eq. 5.1. The probability density function of the solution in $\Delta y_{t}$ is then the sum of a couple of delta functions at the positions of each solution. The two lepton-jet pairings further introduce two sets of equations, which leads to two sets of solutions. In practice, as described in the previous sections, we allow the two jet $E_{\mathrm{T}}$ values and $E_{x, y}$ to float, which naturally "smears" the delta functions to peaks with finite widths, or said differently, to a multi-peaked probability distribution in $\Delta y_{t}$ for any given event. Our goal is to determine this multi-peaked probability distribution in $\Delta y_{t}$ on the event-by-event basis. To accomplish this goal more efficiently, we parametrize our likelihood in terms of two values for the $b / \bar{b}$-quark 
jet $E_{\mathrm{T}}$ values, and two for the two neutrinos. We next discuss each.

Due to the large resolution of jet energy measurements, we allow the jet energy to float in the fit. The way this is done in practice is to create a "jet deviation" parameter

$$
\mathrm{jd}=\frac{E_{T, \mathrm{jet}}^{\mathrm{corr}}-E_{T, b}^{\mathrm{fit}}}{\sigma_{\text {jet }}}
$$

for each jet to characterize the difference between the corrected jet $E_{\mathrm{T}}$ and the current guess of the $E_{\mathrm{T}}$ of the $b / \bar{b}$ quark in the fit, $E_{T, b}^{\mathrm{fit}}$. A large jd guess means that to accomplish the current hypothetical kinematics of the $t \bar{t}$ event, the jet $E_{\mathrm{T}}$ needs to be "pulled away" from its measured value, thus it will be assigned with a smaller probability (according to a Gaussian distribution) in the likelihood term we will introduce later in this section.

With each lepton-jet pairing and a specific $E_{\mathrm{T}}$ of the $b(\bar{b})$ quark, for the determination of the neutrino momenta, it is advantageous to work in the rest frame of the $\ell+b$ system as shown in Fig. 5.5. In this case each leg of the system $\left(t \rightarrow W^{+} b \rightarrow \ell^{+} \nu b\right.$ and its charge conjugate) can be straightforwardly determined up to the azimuthal angle $(\phi)$ of the neutrino $(\nu)$ around the lepton momentum as shown in the figure. In that frame, one can solve the energy momentum conservation equations to obtain

$$
\begin{aligned}
\hat{p_{\nu}} & =\frac{m_{t}^{2}-m_{\ell b}^{2}}{2 m_{\ell b}} \\
\hat{p_{\nu}} \cos \hat{\theta} & =\frac{2 \hat{E_{\ell}} \hat{p_{\nu}}-m_{W}^{2}}{2 \hat{p_{\ell}}},
\end{aligned}
$$

where $\hat{p_{\nu}}$ is the magnitude of the momentum of the neutrino in the corresponding $\ell+b$ rest frame, $\hat{E}_{\ell}$ and $\hat{p}_{\ell}$ are the energy and magnitude of the momentum of the charged lepton in the $\ell+b$ rest frame, and $m_{l b}$ is the invariant mass of $\ell+b$. Keep in mind that the momentum of the neutrino in this construction is dependent on the jet 
$E_{\mathrm{T}}$, thus the neutrino information is described with two parameters $(\mathrm{jd}, \phi)$, where jd determines the $b$-quark momentum, the $m_{l b}$ and the rest frame of $\ell+b$, and $\phi$ is our second free parameter for the fit. Note that have not marked the $\phi$ parameter with a hat to simplify the equations which will arise. Also note that the constraint of $|\cos \hat{\theta}|<1$ determines the kinematic allowed parameter space.

After transferring back to the lab frame, the neutrino momentum can be written as $\overrightarrow{p_{\nu}}=\overrightarrow{p_{\nu}}(\mathrm{jd}, \phi)$. The $\not_{x, y}$ of in terms of any neutrino solution is then

$$
\begin{aligned}
& \not_{x}^{{ }_{x}^{\mathrm{fit}}}=\left(\vec{p}_{\nu}\left(\mathrm{jd}_{\nu}, \phi_{\nu}\right)+\vec{p}_{\bar{\nu}}\left(\mathrm{jd}_{\bar{\nu}}, \phi_{\bar{\nu}}\right)\right)_{x} \\
& \underline{E}_{y}{ }^{\mathrm{fit}}=\left(\vec{p}_{\nu}\left(\mathrm{jd}_{\nu}, \phi_{\nu}\right)+\vec{p}_{\bar{\nu}}\left(\mathrm{jd}_{\bar{\nu}}, \phi_{\bar{\nu}}\right)\right)_{y},
\end{aligned}
$$

where the subscript $\nu$ denotes the $\ell+b$ leg with negatively charged $\ell$, and the subscript $\bar{\nu}$ denotes the other leg. The values in Eq. 5.8 are compared to their measured values in the reconstruction, and due to the large resolution of $\not_{x, y}$, we also allow each to float according to their measured values and the corresponding resolutions $\left(\sigma\left(\not_{x, y}\right)\right)$.

Given the above, for each lepton-jet pairing, every possible solution is uniquely determined by a set of four parameters $\left(\mathrm{jd}_{1}, \mathrm{jd}_{2}, \phi_{1}, \phi_{2}\right)$ and the likelihood of the solution can be calculated as the product of the terms as follows:

1. The likelihood of the two hypothetical $b$-quark $E_{\mathrm{T}}$ values matching the corrected jet $E_{\mathrm{T}}$ values.

2. The likelihood of the hypothetical $\ddot{E}_{x, y}$ matching the measured $\not_{x, y}$.

3. The likelihood of the hypothetical fitted $t \bar{t}$ system $\left(p_{z, t \bar{t}}, p_{T, t \bar{t}}\right.$ and $\left.m_{t \bar{t}}\right)$, based on the previous assumptions, matching the a priori templates derived from the POWHEG MC. 


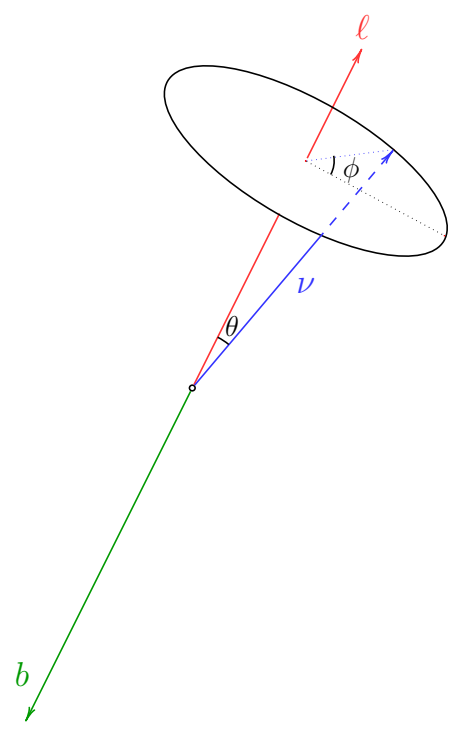

Figure 5.5: The momentum of the neutrino in the $\ell+b$ rest frame. The neutrino momentum can be determined in this frame up to the azimuthal angle $\phi$, which is a free parameter and is used as an input to the final likelihood fit.

The likelihood used in top reconstruction algorithm can be written explicitly as

$$
\begin{aligned}
& \mathcal{L}\left(\phi_{1}, \phi_{2}, \mathrm{jd}_{1}, \mathrm{jd}_{2}\right)=P\left(p_{z, t \bar{t}}\right) \times P\left(p_{T, t \bar{t}}\right) \times P\left(m_{t \bar{t}}\right) \\
& \quad \times \frac{1}{\sigma_{\text {jet} 1}} \exp \left(-\frac{1}{2}\left(\frac{E_{T, \text { jet } 1}^{\mathrm{corr}}-E_{T, b}^{\mathrm{fit}}}{\sigma_{\text {jet} 1}}\right)^{2}\right) \times \frac{1}{\sigma_{\text {jet2 }}} \exp \left(-\frac{1}{2}\left(\frac{E_{T, \mathrm{jet} 2}^{\mathrm{corr}}-E_{T, b}^{\mathrm{fit}}}{\sigma_{\text {jet} 2}}\right)^{2}\right) \\
& \times \frac{1}{\sigma\left(E_{x}\right)} \exp \left(-\frac{1}{2}\left(\frac{E_{x}^{\text {meas }}-E_{x}^{\mathrm{fit}}}{\sigma_{E_{x}^{\prime}}}\right)^{2}\right) \times \frac{1}{\sigma\left(E_{y}\right)} \exp \left(-\frac{1}{2}\left(\frac{E_{y}^{\text {meas }}-E_{y}^{\mathrm{fit}}}{\sigma_{E_{y}}}\right)^{2}\right),
\end{aligned}
$$

where $\mathscr{E}_{x, y}^{\text {meas }}$ are the $x$ and $y$ components of the measured $\vec{H}_{T}$ and $P\left(p_{z, t \bar{t}}\right) P\left(p_{T, t \bar{t}}\right)$ and $P\left(m_{t \bar{t}}\right)$ are the probabilities as determined from the templates of $p_{z, t \bar{t}}, p_{T, t \bar{t}}$ and $m_{t \bar{t}}$. Note that because of our definition of the jd parameter, the middle terms in Eq. 5.9 simplify to $\frac{1}{\sigma_{\text {jet } 1,2}} \exp \left(-\frac{1}{2} \mathrm{jd}_{1,2}^{2}\right)$. 


\subsection{Full Probability Reconstruction}

Each set of the four parameters $\left(\mathrm{jd}_{1}, \mathrm{jd}_{2}, \phi_{1}, \phi_{2}\right)$ in the kinematic allowed region in a lepton-jet pairing represents a possible solution of the $t \bar{t}$ system, and the likelihood of the solution is specified with Eq. 5.9. We employ an MCMC [100] method to effectively scan the four-dimensional parameter space with each of the two lepton-jet pairings, and meanwhile map out the probability distributions of the parameters of interest ( $\Delta y_{t}$ in this analysis) after integrating out all other parameters (marginalization). The package we use for MCMC sampling is the Bayesian Analysis Toolkit (BAT) [97.

To illustrate the probability distribution function for an event, we choose a well measured event from POWHEG MC sample and display the reconstruction results. Figures 5.6, 5.7, 5.8 and 5.9 show the posterior distributions as a function of $\mathrm{jd}_{1}$, $\mathrm{jd}_{2}, \phi_{1}, \phi_{2}$ and corresponding values of $y_{t}, y_{\bar{t}}$ and $\Delta y_{t}$. The two columns represent the two lepton-jet pairings. For this event, based on generator-level information, the left column of plots are from the correct lepton-jet pairing while the right column of plots are from the incorrect pairing. The red vertical lines and the green cross show the position of the true values. Since the two $\phi$ parameters are defined in the $\ell+b$ rest frame, which depends on each hypothesis of the jd parameter, we cannot plot the true value on those plots. Note that in this event the true pairing appears to be well reconstructed (although there are solutions which are not perfect), and the wrong pairing is far from the expectation for being from a top pair.

Visually, good reconstruction usually has the feature that the probability distributions of the jd parameters are peaked around zero, while for wrong lepton-jet pairing or for background events, the reconstruction algorithm sometimes needs to pull the jet $E_{\mathrm{T}}$ far away from the measured values to successfully reconstruct the 
$t \bar{t}$ system. We will take the advantage of this feature later in the optimization process. We can also see multiple peak structure in the posteriors of $\phi$ and $\Delta y_{t}$. This illustrates the multiple solution structure of Eq. 5.1.

Figures 5.10, 5.11 and 5.12 show the scatter plots of the log-likelihood (log of Eq. 5.9 versus the same set of parameters. Again the left column of plots are from the correct lepton-jet pairing while the right column of plots are from the incorrect pairing. Each point in the plot is a possible solution to Eq. 5.1, and the $y$-axis value shows its log-likelihood; the MCMC algorithm is designed to draw more points in the high-likelihood region. The red vertical lines show the position of the true values, the green horizontal lines show the value of the maximum likelihood, and the two "true" $\phi$ parameters are not shown for the reasons mentioned above.

For comparison with the previous analysis, we note that the top reconstruction algorithm used in the analysis with $5.1 \mathrm{fb}^{-1}$ [17] picks the highest point in Figs. 5.10, 5.11 and 5.12 . With our MCMC-based reconstruction algorithm, we have the possibility of choosing between the old method of using the maximum-likelihood solution or using the full probability distribution within each lepton-jet pairing. In the same manner, we have the possibility of either picking the more-likely lepton-jet pairing or weighting the two pairings according to their likelihoods. For the purposes of demonstrating results we will weight the two lepton-jet pairings with the maximum likelihood achieved in each pairing $\left(L_{m a x, \text { even }}\right.$ and $L_{m a x, \text { odd }}$, even for pairing the leading $E_{\mathrm{T}}$ lepton with the leading $E_{\mathrm{T}}$ jet, etc.). The weight of each lepton-jet pairing is determined by

$$
\begin{gathered}
w_{\text {even }}=\frac{L_{\text {max }, \text { even }}}{L_{\text {max }, \text { even }}+L_{\text {max }, \text { odd }}} \\
w_{\text {odd }}=\frac{L_{\text {max }, \text { odd }}}{L_{\text {max }, \text { even }}+L_{\text {max }, \text { odd }}} .
\end{gathered}
$$

We will explore different combinations and chose the better strategy based on the 
expected uncertainty of $A_{\mathrm{FB}}^{t \bar{t}}$. We will improve Eq. 5.10 in Sec. 6.1 using information from an track-momentum-weighted jet charge algorithm [98] to separate $b$-jets from $\bar{b}$-jets.

The difference between the reconstructed $\Delta y$ (denoted as $\Delta y($ reco) $)$ and the generated $\Delta y$ (denoted as $\Delta y$ (hepg), where "hepg" stands for high-energy-physics generator ) for the POWHEG MC sample is shown in Fig. 5.13. This figure characterizes the resolution of the $\Delta y_{t}$ with these choices of the top reconstruction algorithm. We note that this plot is made with only the baseline cuts described in Sec. 2, a final version after the optimization procedure will be shown in Sec.6.3. We also note that the figure is made by stacking the full probability distribution of $\Delta y($ reco $)-\Delta y($ hepg $)$ for each event, normalizing each event to unity, and where the two lepton-jet pairings in each event are weighted according to Eq. 5.10. We note that $61 \%$ of the time the $\Delta y_{t}$ is reconstructed within 0.5 of its true value. We note here that the events in the tails of the distribution in Fig. 5.13 would cause a badly mis-measured $\Delta y$ (with the corresponding probability), which worsens the uncertainties of the parton-level $A_{\mathrm{FB}}^{t \bar{t}}$ measurements quickly, as explained in Sec. 6.1. This motivates the various pieces of the optimization procedure in Sec. 6.1 to reduce the tails of this distribution.

With a procedure to measure $\Delta y_{t}$ for our data, the next step of the analysis is to develop an unfolding procedure to extract the $A_{\mathrm{FB}}^{t \bar{t}}$ information at the parton level from the output of the top reconstruction. After that, an optimization procedure will be performed to minimize the expected uncertainties of the $A_{\mathrm{FB}}^{t \bar{t}}$ measurement. The data will be processed with the optimum configuration and presented in the next section. 

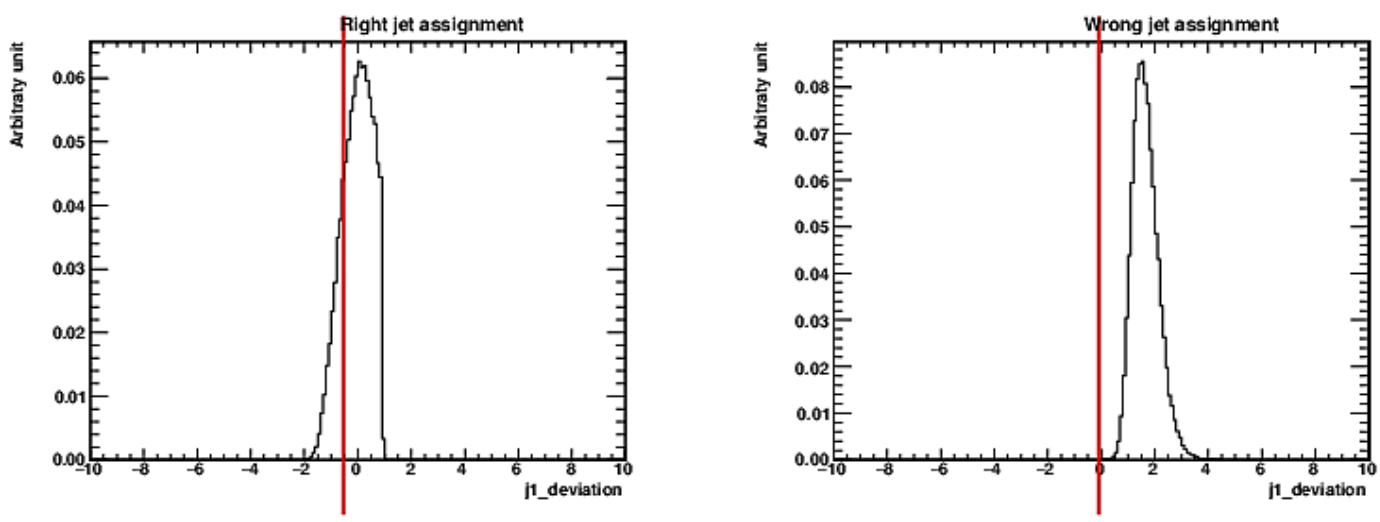

(a) $\mathrm{jd}_{1}$
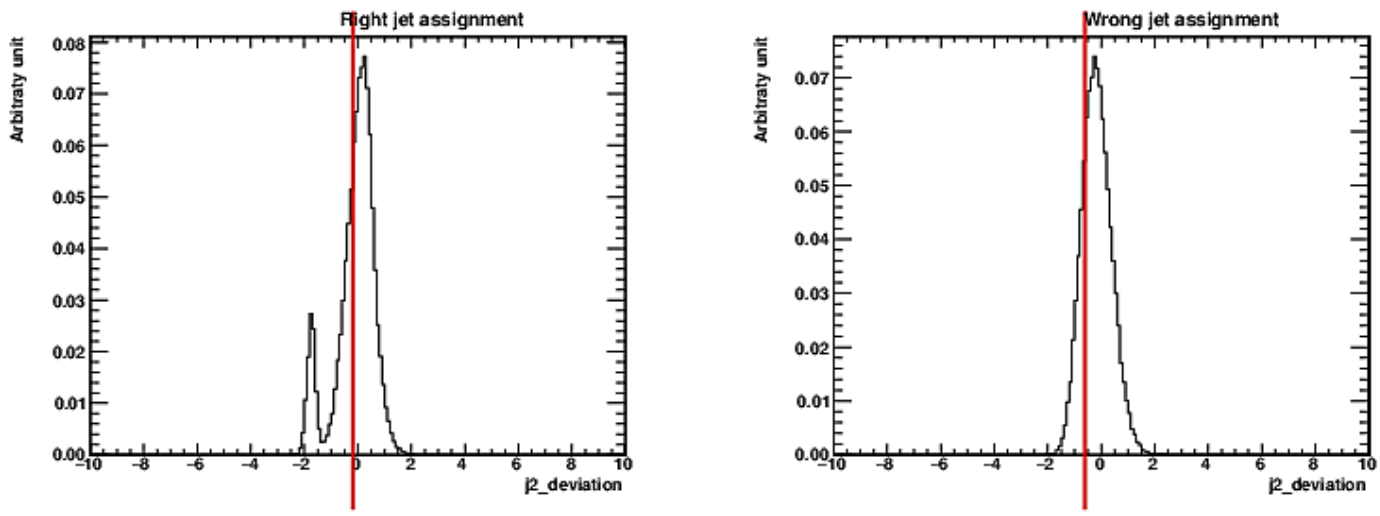

(b) $\mathrm{jd}_{2}$

Figure 5.6: The posterior distribution of $\mathrm{jd}_{1}$ and $\mathrm{jd}_{2}$ for one example event from the POWHEG MC. Based on the generator-level information the left column is for the correct lepton-jet pairing and the right column is the incorrect pairing. The red vertical lines show the true values of the parameter. 

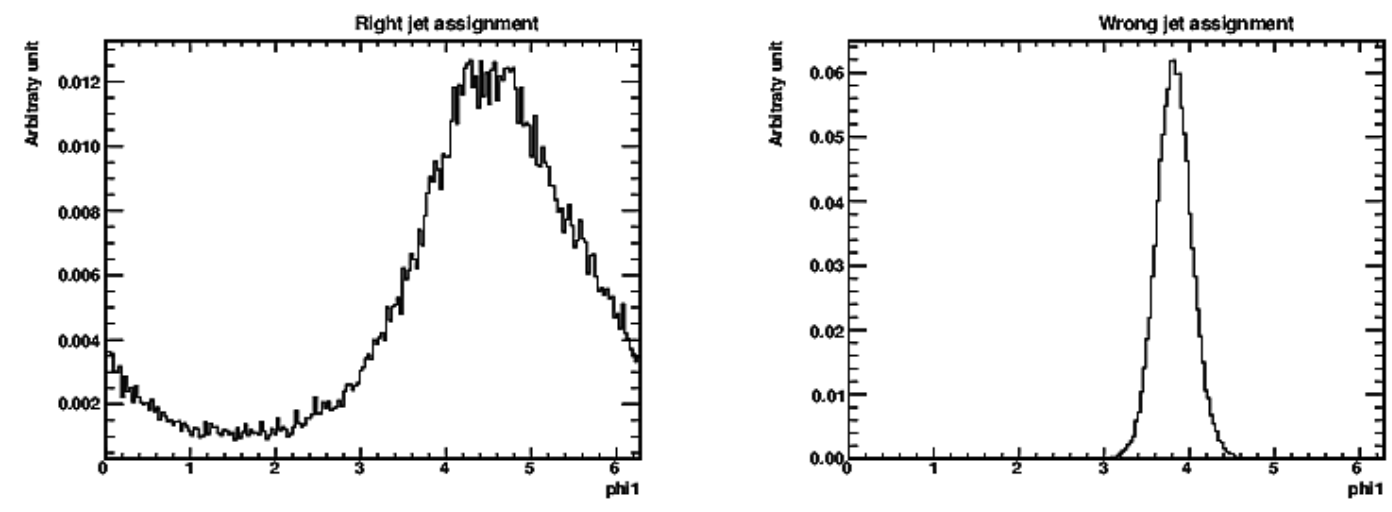

(a) $\phi_{1}$
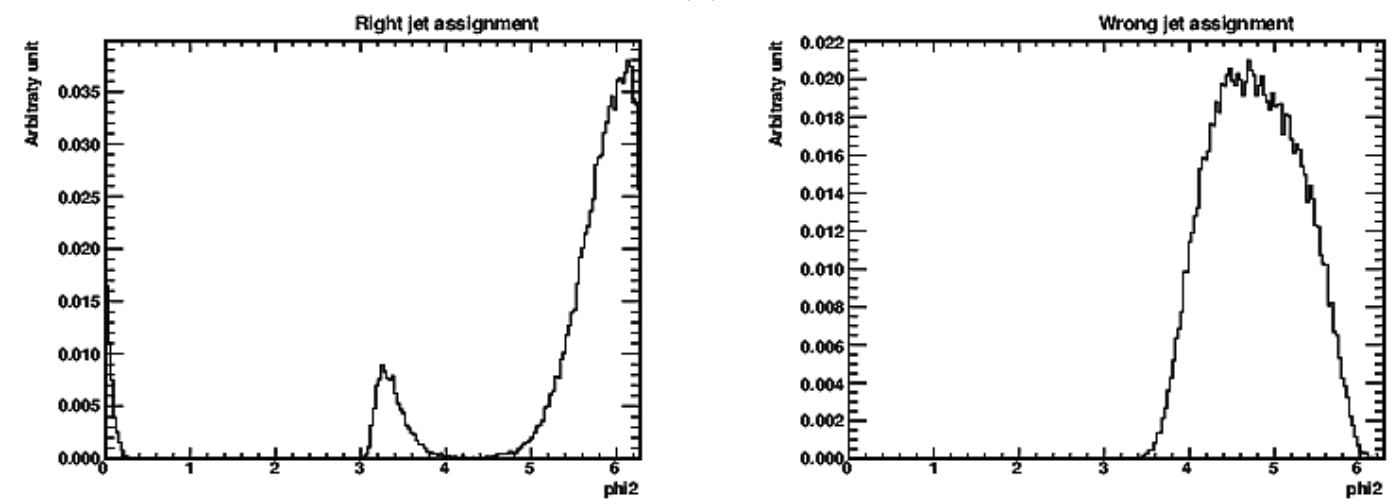

(b) $\phi_{2}$

Figure 5.7: The same as Fig. 5.6, but for $\phi_{1}$ and $\phi_{2}$ for one example event from the POWHEG MC. Based on the generator-level information the left column is for the correct lepton-jet pairing and the right column is the incorrect pairing. Since the two $\phi$ parameters are defined in the $\ell+b$ rest frame, which depends on each hypothesis of the jd parameter, we cannot plot the true value on those plots. 

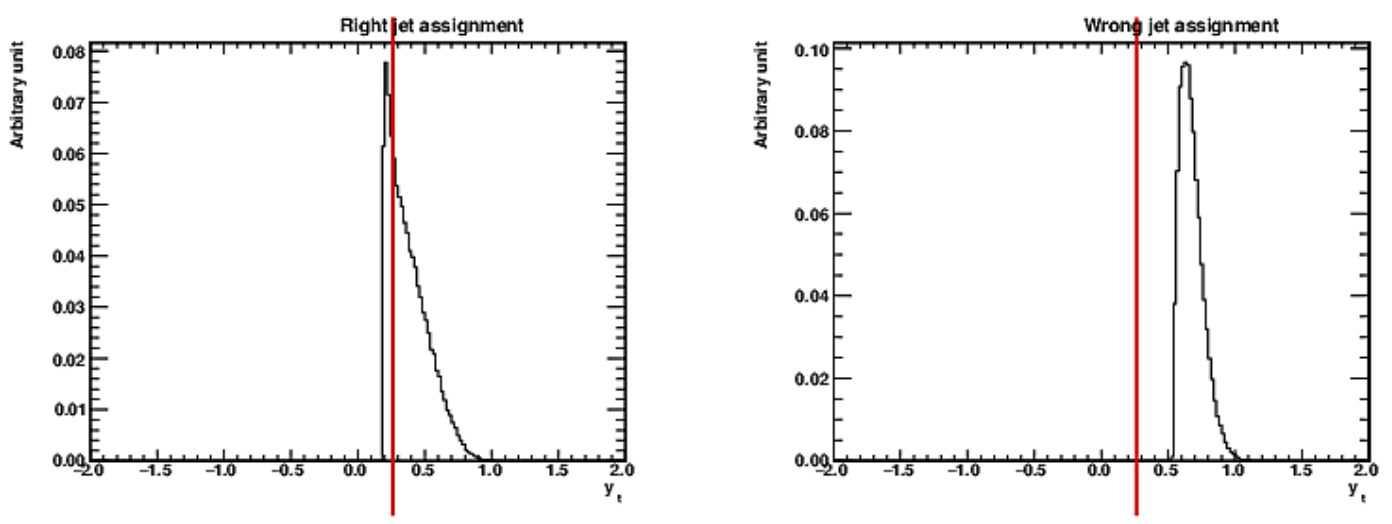

(a) $y_{t}$
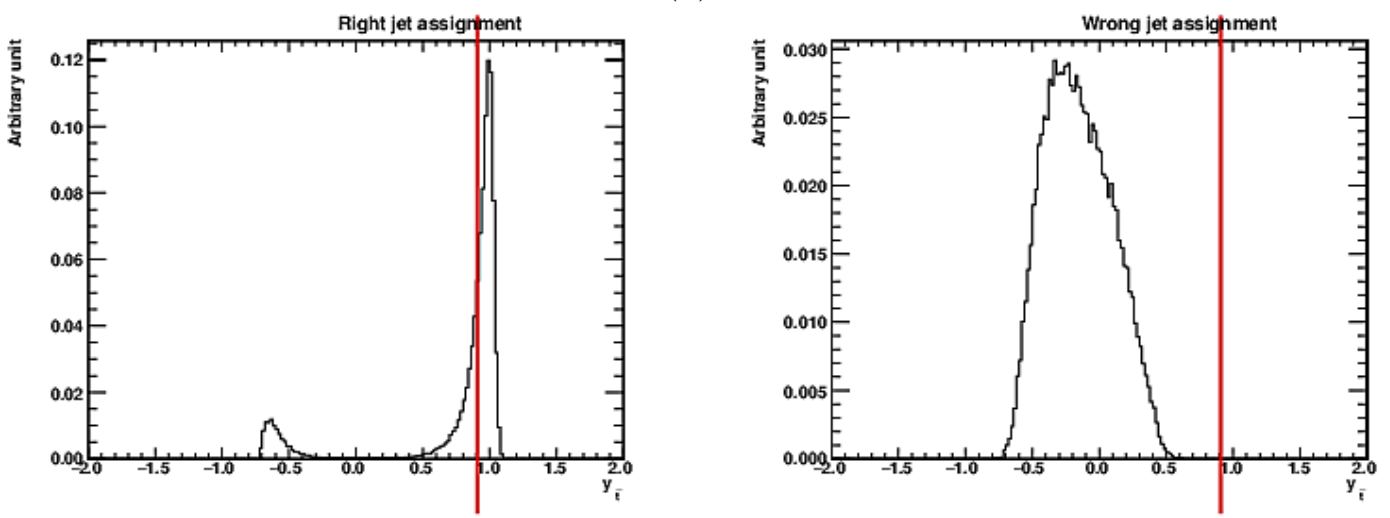

(b) $y_{\bar{t}}$

Figure 5.8: The same as Fig. 5.6 but for $y_{t}, y_{\bar{t}}$, for one example event from POWHEG MC. Again, the left column is the correct lepton-jet pairing and the red vertical lines show the true values of the parameter. 

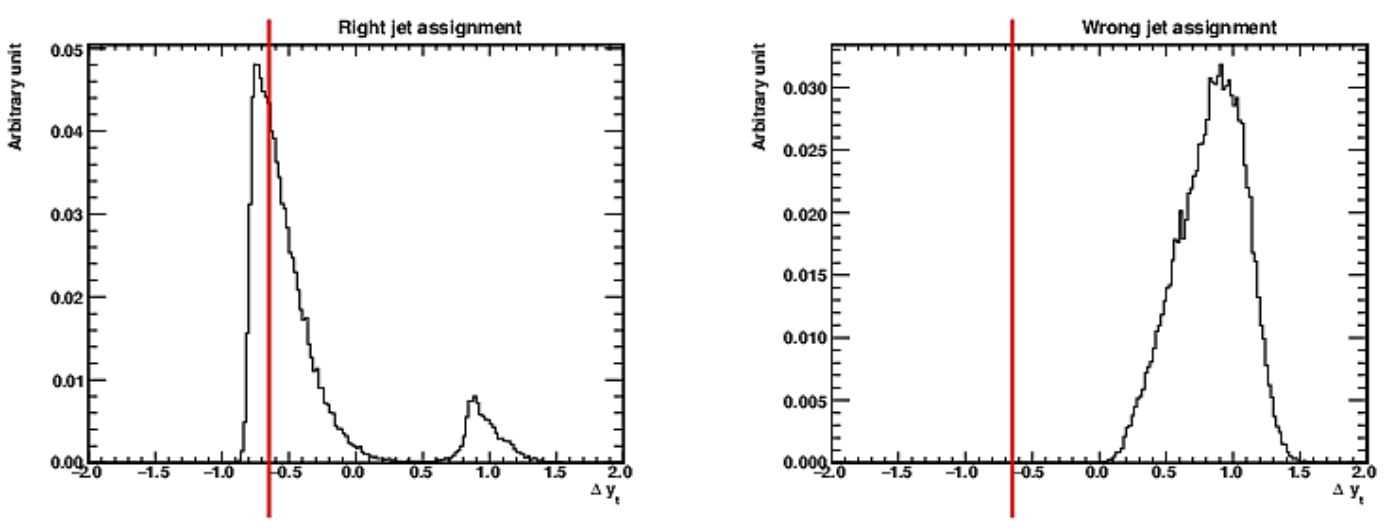

(a) $\Delta y_{t}$
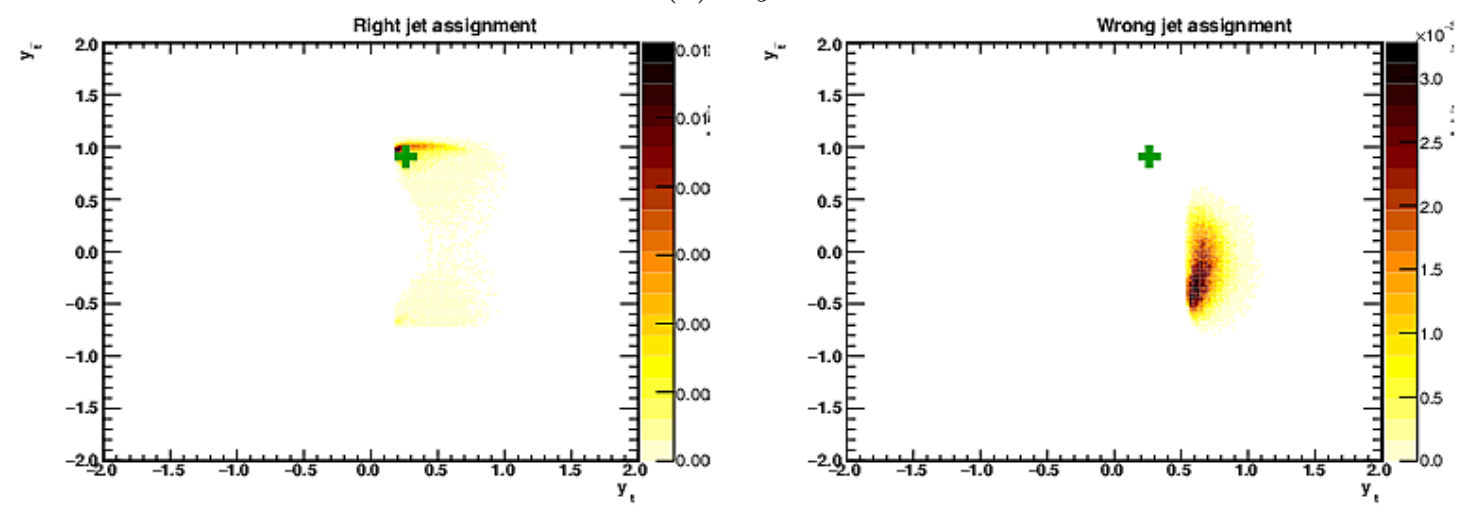

(b) $y_{t}$ vs. $y_{\bar{t}}$

Figure 5.9: The same as Fig. 5.6 but for $\Delta y_{t}$ and $y_{t}$ vs. $y_{\bar{t}}$, for one example event from Powheg MC. Again, the left column is the correct lepton-jet pairing and the red vertical lines and the green cross show the true values of the parameter. 

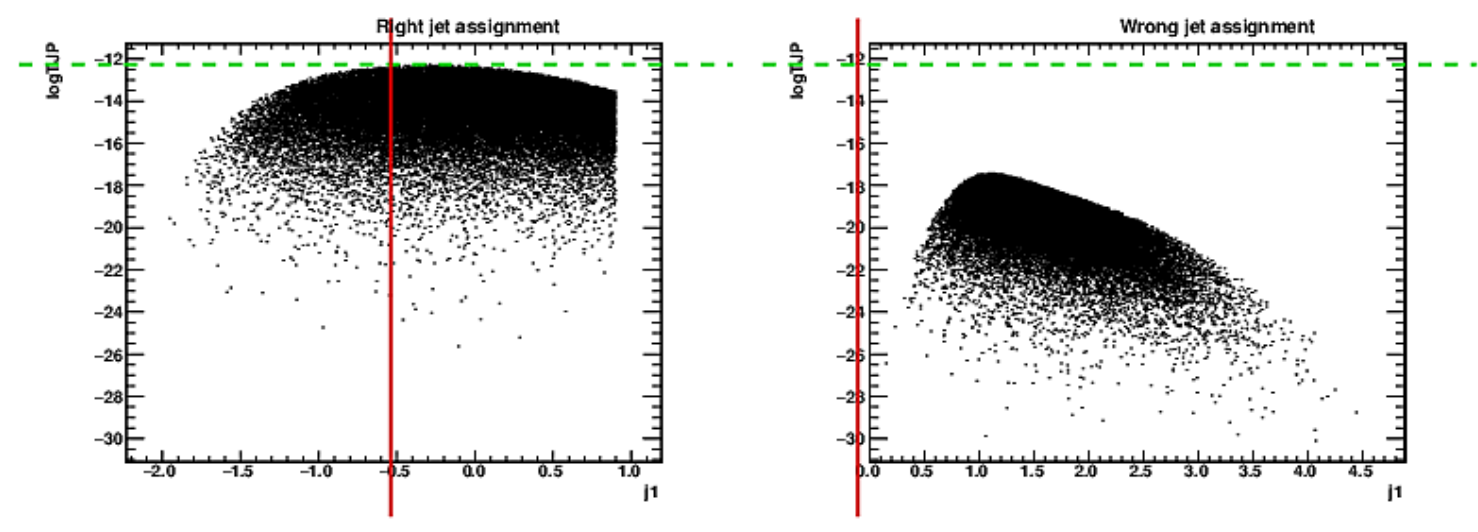

(a) $\mathrm{jd}_{1}$
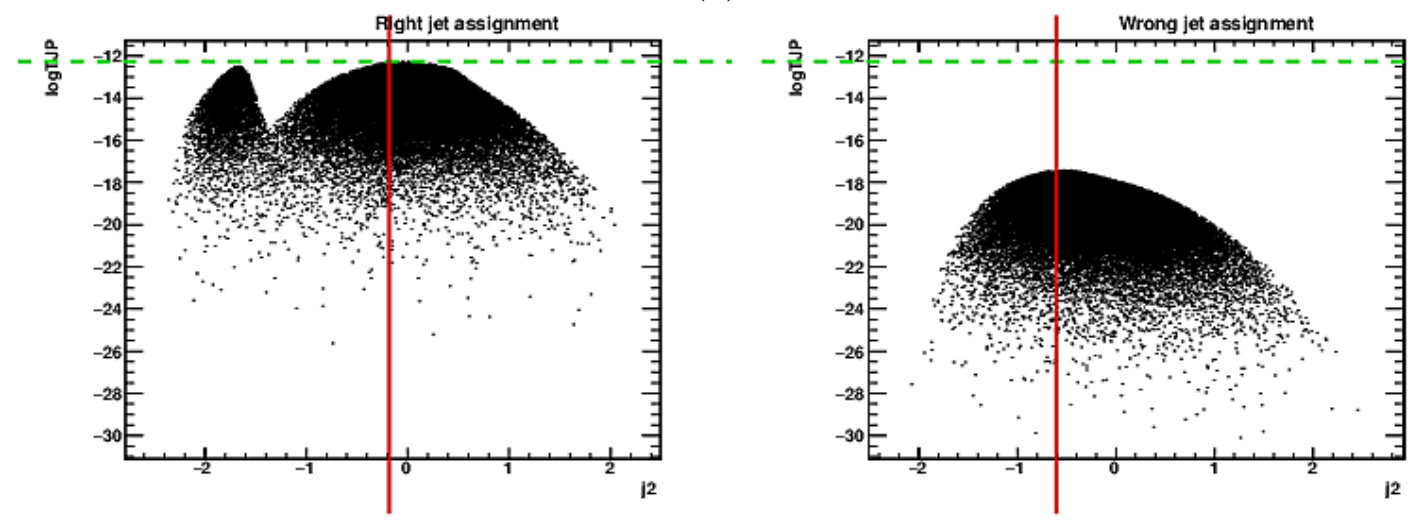

(b) $\mathrm{jd}_{2}$

Figure 5.10: The scatter plot of the log-likelihood vs. $\mathrm{jd}_{1}$ and $\mathrm{jd}_{2}$ for the same event as Fig. 5.6. Again, the left column is the correct lepton-jet pairing, the red vertical lines show the true values of the parameter, and the green dashed horizontal lines show the maximum log-likelihood value. 

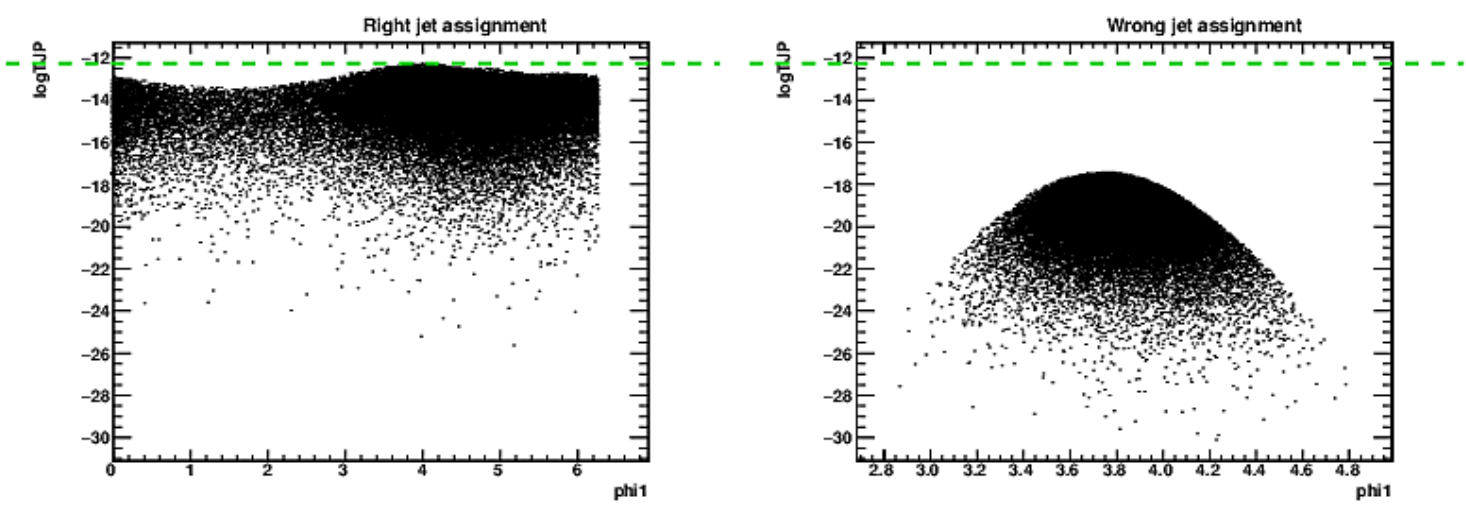

(a) $\phi_{1}$
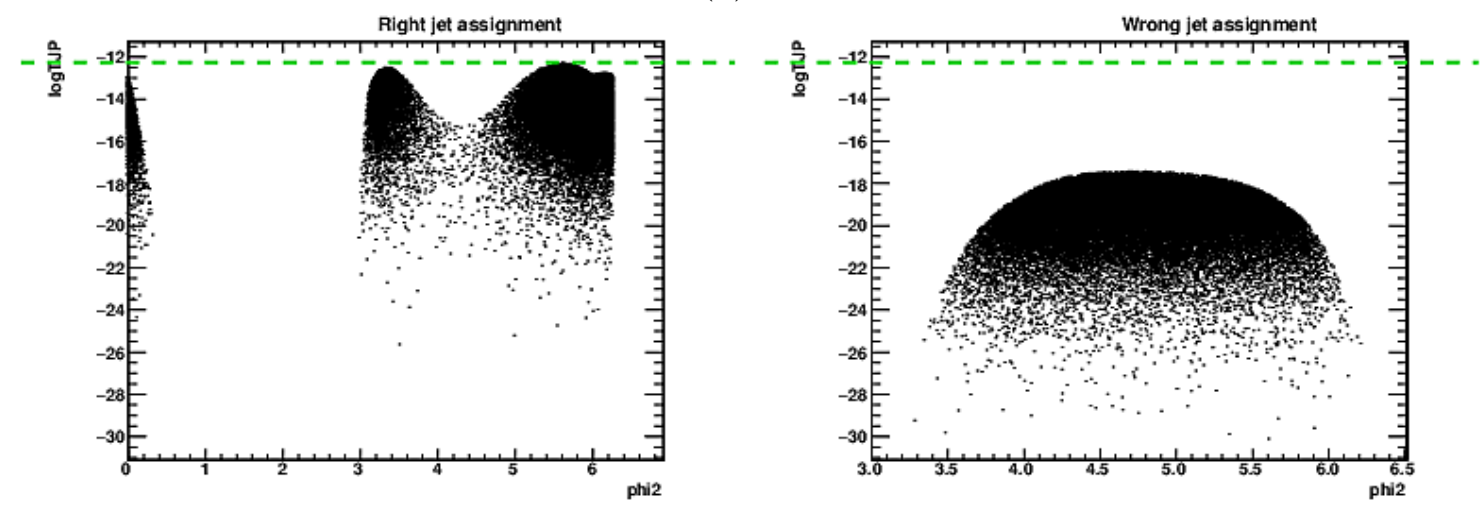

(b) $\phi_{2}$

Figure 5.11: The same as Fig. 5.10, but for $\phi_{1}$ and $\phi_{2}$. Again, the left column is the correct lepton-jet pairing, and the green dashed horizontal lines show the maximum log-likelihood value. Since the two $\phi$ parameters are defined in the $\ell+b$ rest frame, which depends on each hypothesis of the jd parameter, we cannot plot the true value on those plots. 

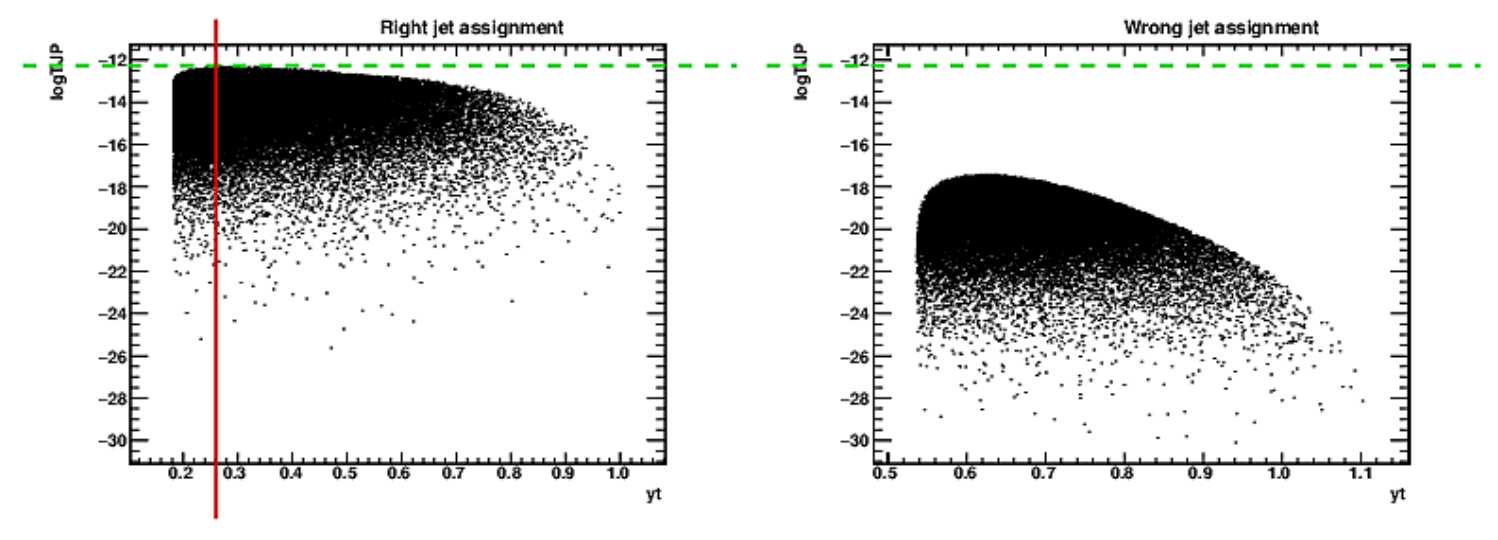

(a) $y_{t}$
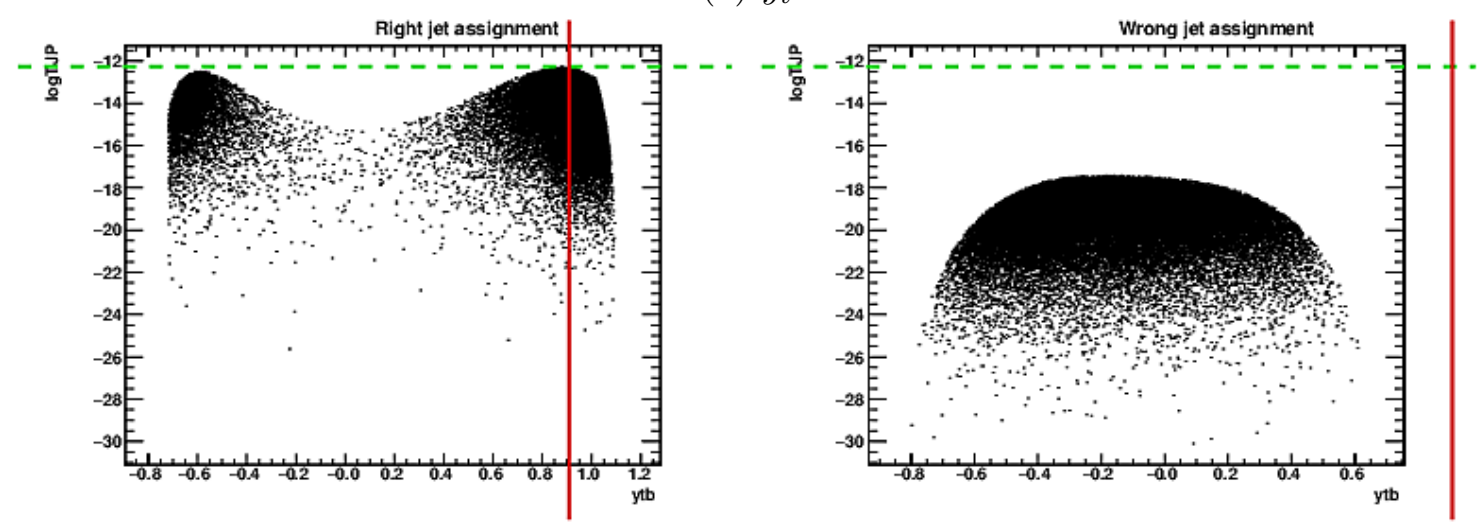

(b) $y_{\bar{t}}$
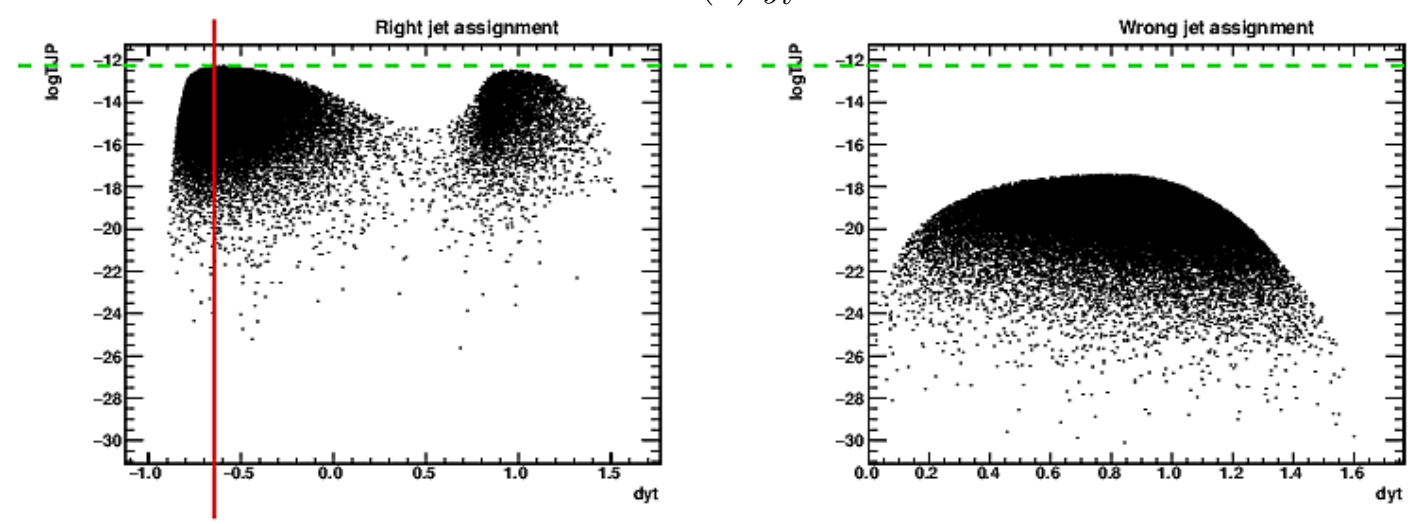

(c) $\Delta y_{t}$

Figure 5.12: The same as Fig. 5.10, but for $y_{t}, y_{\bar{t}}$, and $\Delta y_{t}$. The true values for $y_{t}$ and $\Delta y_{t}$ in the wrong jet assignment column are outside the range of the figure. 


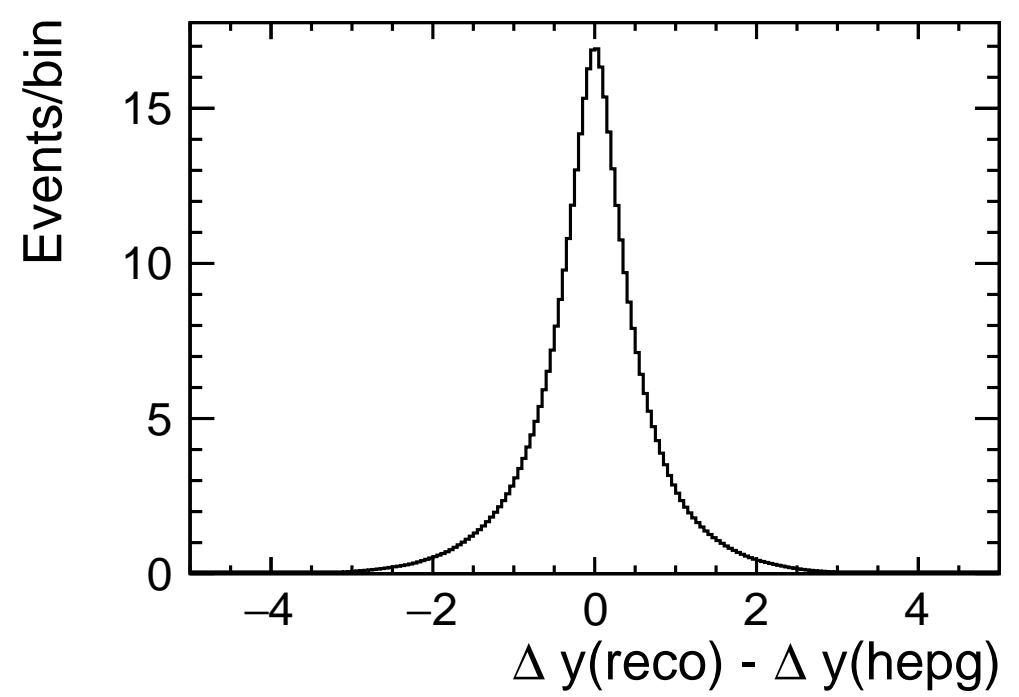

Figure 5.13: Stacking the full probability distribution of $\Delta y$ (reco) $-\Delta y$ (hepg) for each event from the POWHEG sample, normalizing each event to unity, which gives an overall normalization to the size of the POWHEG sample. Note that this plot is made only with the baseline cuts described in Sec. 2, and the two lepton-jet pairings in each event are weighted according to Eq. 5.10. A final version after the optimization will be shown in Sec. 6.3 in Fig. 6.24. 


\section{MEASUREMENT OF THE FORWARD-BACKWARD ASYMMETRY OF TOP-QUARK PAIRS}

With a full measurement of the $\Delta y_{t}$ distribution in hand in this section we describe the measurement of $A_{\mathrm{FB}}^{t \bar{t}}$ in the dilepton final state. In order to obtain partonlevel results so that they can be compared with theoretical calculations and results from other measurements, we introduce a state-of-the-art measurement methodology, described in Sec. 6.1. We perform an optimization of the selection criteria and methodology to reduce the measurement uncertainty in Sec. 6.2 that takes into account a number of new techniques that reflect the field's understanding of how to separate signal from backgrounds, and to maximize the impact of each signal event. The validation of the final methodology is shown in Sec. 6.3. The measurement of $A_{\mathrm{FB}}^{t \bar{t}}$ from data and the estimation of uncertainties are described in Sec. 6.4 . We will first do the inclusive $A_{\mathrm{FB}}^{t \bar{t}}$, then the differential values as a function of $\left|\Delta y_{t}\right|$. We note that due to the limited sample size in the dilepton final state (small number of events in each bin, which will break our statistics model), we cannot perform the measurement of the differential $A_{\mathrm{FB}}^{t \bar{t}}$ as a function of $m_{t \bar{t}}$ in this analysis.

\subsection{Top $A_{\text {FB }}$ Measurement Methodology}

For the same reasons described in Sec. 4, mostly because of the limited acceptance and the efficiency of the detector, the imperfect resolution of the $\Delta y_{t}$ reconstruction and the background contributions, a sophisticated procedure is needed to turn the measured $\Delta y_{t}$ distribution into parton-level $A_{\mathrm{FB}}$ measurements. The procedure we use is to bin the data into four bins of $\Delta y_{t}$, and create a Bayesian model to correct for all the effects and take us back to the true $\Delta y_{t}$ distribution, and then use this to determine $A_{\mathrm{FB}}^{t \bar{t}}$. We will refer to this technique as the "unfolding" procedure. 
To develop and test the unfolding procedure, we need a set of samples for the $t \bar{t}$ signal under different physical hypotheses, which span the possible range of $A_{\mathrm{FB}}^{t \bar{t}}$. We then use the unfolding procedure to measure the underlying $A_{\mathrm{FB}}^{t \bar{t}}$ from each sample to test if the unfolding procedure works as we expect. For example, if we put in a model with a true $A_{\mathrm{FB}}^{t \bar{t}}$ of $10 \%$, we want the measurement methodology to report a result that is consistent with the $10 \%$ asymmetry, based on the observed objects from the detector (or detector simulation). The same is true for $-10 \%$.

While we have a number of models of $t \bar{t}$, as described and used in Sec. 4, we want a better motivated method. Since there is no free parameter in the SM which causes $A_{\mathrm{FB}}^{t \bar{t}}$ to vary, we use a hint suggested by a previous CDF measurement [57] to create a series of samples based on POWHEG, but with different $A_{\mathrm{FB}}^{t \bar{t}}$, to validate an unfolding procedure. We will also check our results with benchmark new physics models but do not expect models of new physics, which have kinematics that are not similar to that of the SM (which is crucial to the reconstruction), to give a result that is unbiased by our methodology.

In this section we will first show the procedure we use to create the reweighted POWHEG samples with $-10 \%<A_{\mathrm{FB}}^{t \bar{t}}<30 \%$ and show some of the sample properties in Sec. 6.1.1. Following that, we will describe the unfolding model in Sec. 6.1.2. In Sec. 6.1 .3 we describe the "compound Poisson" statistics we use in comparing the expectations with the observations. Sections 6.1.4 and 6.1.5 show the implementation of the unfolding model and the pseudoexperiment tests with signal only and with signal+backgrounds. We will perform an optimization of the measurement in Section 6.2. A complete set of validation of the methodology, after the optimization procedure, will be shown in Sec. 6.3 . 


\subsubsection{Reweighting the POWHEG MC to Create the $A_{F B}^{t \bar{t}}$ Methodology Testing Samples}

We start by describing the technique to create our samples with various $A_{\mathrm{FB}}^{t \bar{t}}$ for use in testing and validating our unfolding procedures. The measurement of the top quark differential cross section $\left(\frac{d \sigma}{d \cos \theta^{*}}\right)$ in terms of Legendre polynomials in the lepton+jets final state [57], shown in Fig. 6.1, suggests that the "excess" of $A_{\mathrm{FB}}^{t \bar{t}}$ is due to the coefficient of the first order Legendre polynomials, the $a_{1} \cdot \cos \left(\theta^{*}\right)$ term. All other Legendre moments are measured to be in good agreement with the NLO SM prediction.

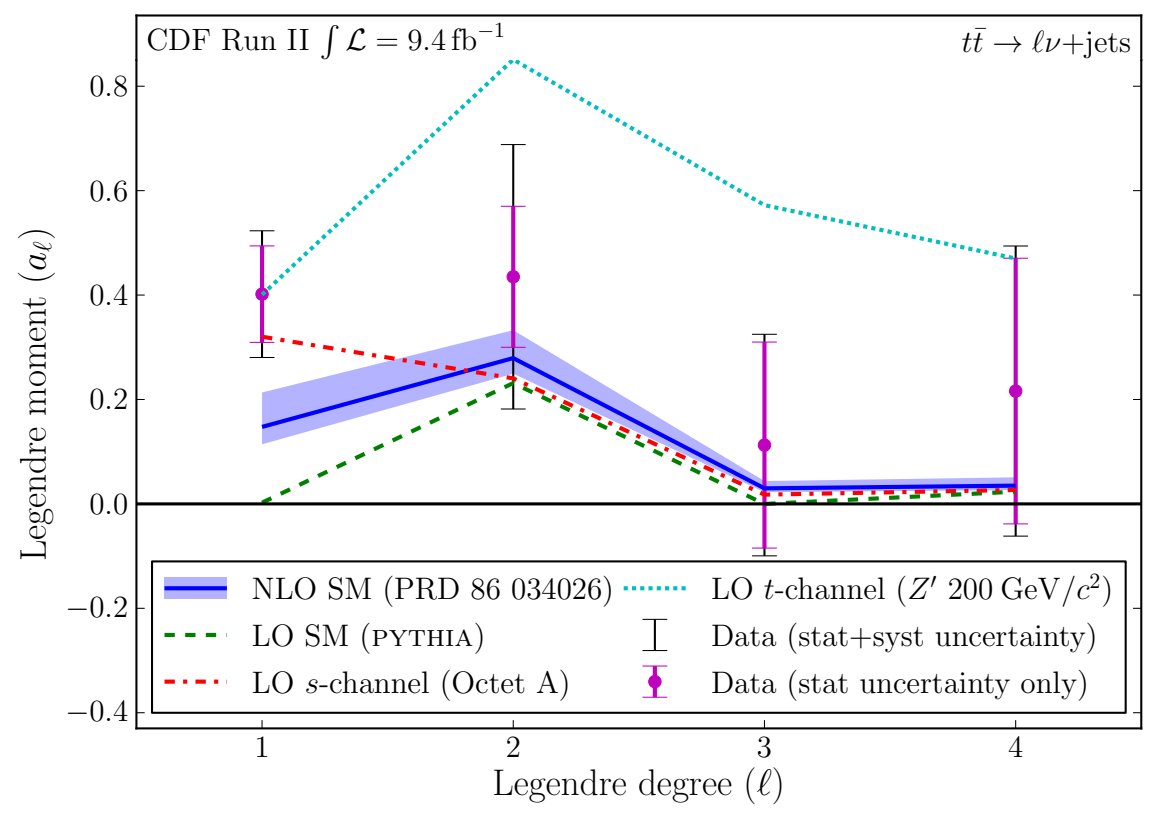

Figure 6.1: Decomposition of the top quark differential cross section $\left(\frac{d \sigma}{d \cos \theta^{*}}\right)$ in terms of Legendre polynomials, taken from Ref. [57.

Motivated by this result, we create a series of MC samples with various $A_{\mathrm{FB}}^{t \bar{t}}$ 
by reweighting the NLO SM MC POWHEG with various extra linear contributions to $\frac{d \sigma}{d \cos \theta^{*}}$. To be specific, we use $-0.3<\operatorname{extra} a_{1}<0.5$ with steps of 0.05 , which corresponds to $-0.1<A_{\mathrm{FB}}^{t \bar{t}}<0.3$. We note that $\cos \theta^{*}, \Delta y_{t}$ and $m_{t \bar{t}}$ are correlated. Figures 6.2 and 6.3 shows a comparison of all three variables for the nominal POWHEG sample (extra $a_{1}=0, A_{\mathrm{FB}}^{t \bar{t}}=0.052$ ) and the reweighted POWHEG sample with extra $a_{1}=0.25$, corresponding to $A_{\mathrm{FB}}^{t \bar{t}}=0.177$. The $\Delta y_{t}$ distribution and $A_{\mathrm{FB}}^{t \bar{t}}$ shift as expected. Figure 6.4 shows the parton-level $A_{\mathrm{FB}}^{t \bar{t}}$ as a function of extra $a_{1}$ used in reweighting the POWHEG MC sample. In the region of interest, $A_{\mathrm{FB}}^{t \bar{t}}$ appears roughly linearly dependent with the extra $a_{1}$.

\subsubsection{Parton-level $A_{F B}^{t \bar{t}}$ Measurement Methodology}

The data we have for use in the unfolding is the $\Delta y_{t}$ distribution. Based on the expectations from the SM we use four bins of $\Delta y_{t}$ at both parton level and after the reconstruction described in Sec. 5, designed to have similar expected number of events per bin. They are $(-\infty,-0.5),(-0.5,0),(0,0.5)$ and $(0.5, \infty)$. The binwidth weighted $\Delta y_{t}$ distributions from POWHEG at generator level before any event selection, and after reconstruction and baseline event selection, are shown in Fig. 6.5.

This binning choice results in two forward bins and two backward bins. The truth level (parton level) inclusive $A_{\mathrm{FB}}^{t \bar{t}}$ is expressed as

$$
A_{\mathrm{FB}}^{t \bar{t}}(\text { incl. })=\frac{\left(N_{\text {parton }}[3]+N_{\text {parton }}[4]-N_{\text {parton }}[1]-N_{\text {parton }}[2]\right)}{\left(N_{\text {parton }}[3]+N_{\text {parton }}[4]+N_{\text {parton }}[1]+N_{\text {parton }}[2]\right)}
$$

where $N_{\text {parton }}[p]$ represents the number of events in the $p$-th bin at parton-level. The differential $A_{\text {FB }}^{t \bar{t}}$ values will be defined later in this subsection with the reparametrization of the unfolding model.

The reweighted PowHEg samples described in Sec. 6.1.1 are used to create and validate our unfolding procedure. There are two primary effects that must be cor- 


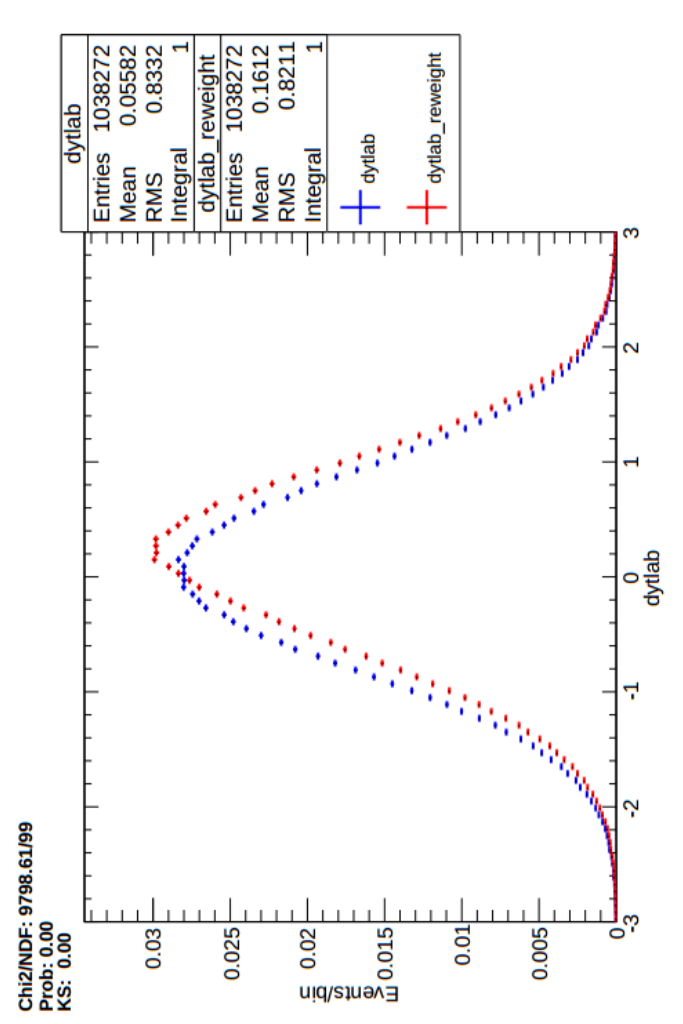

$\underset{\frac{0}{\Xi}}{3}$

*

เి 0

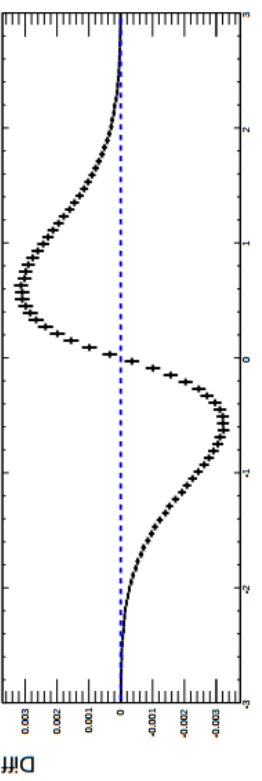

11.

为出

एँ

穿守

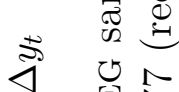

尚重点
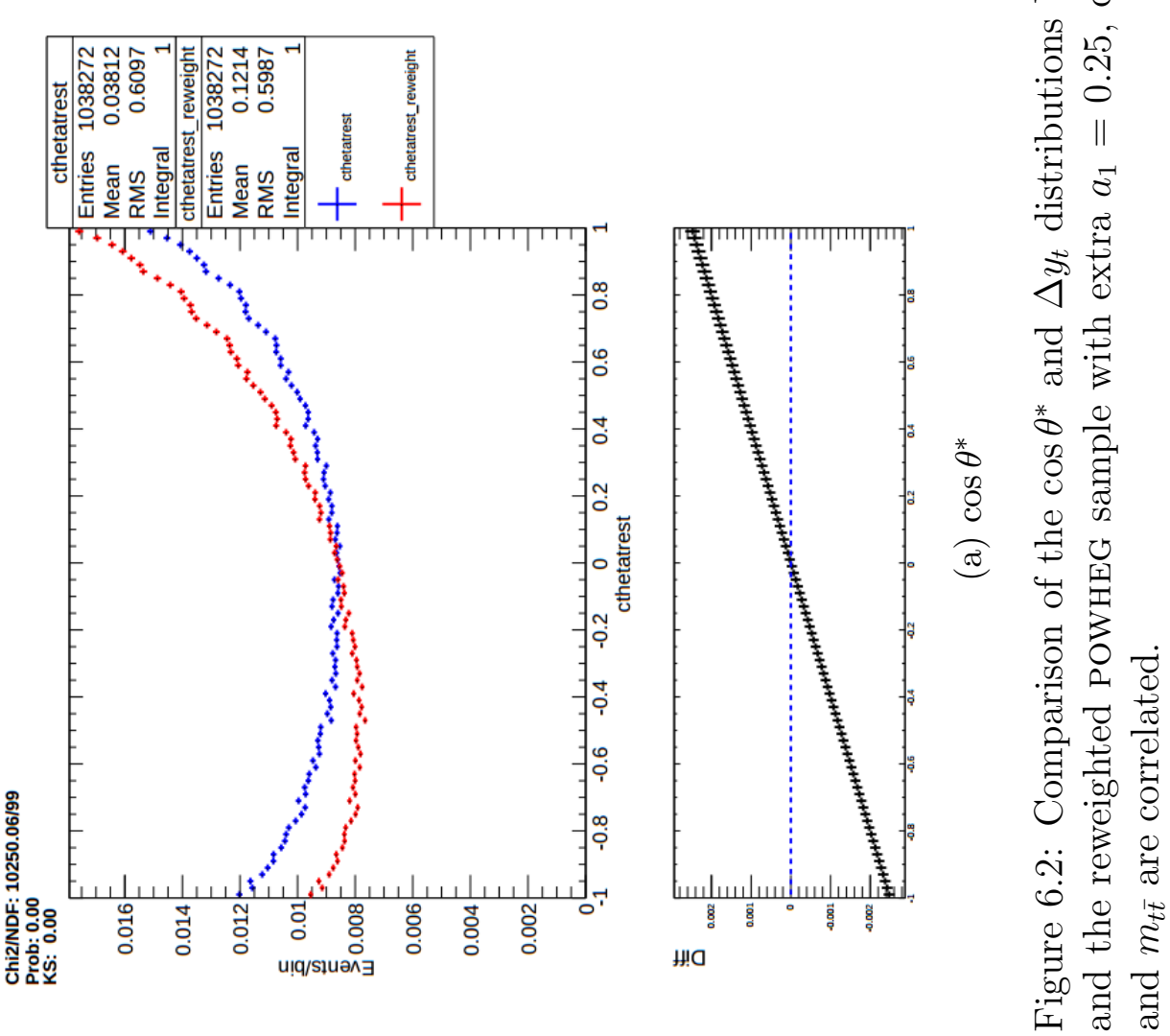

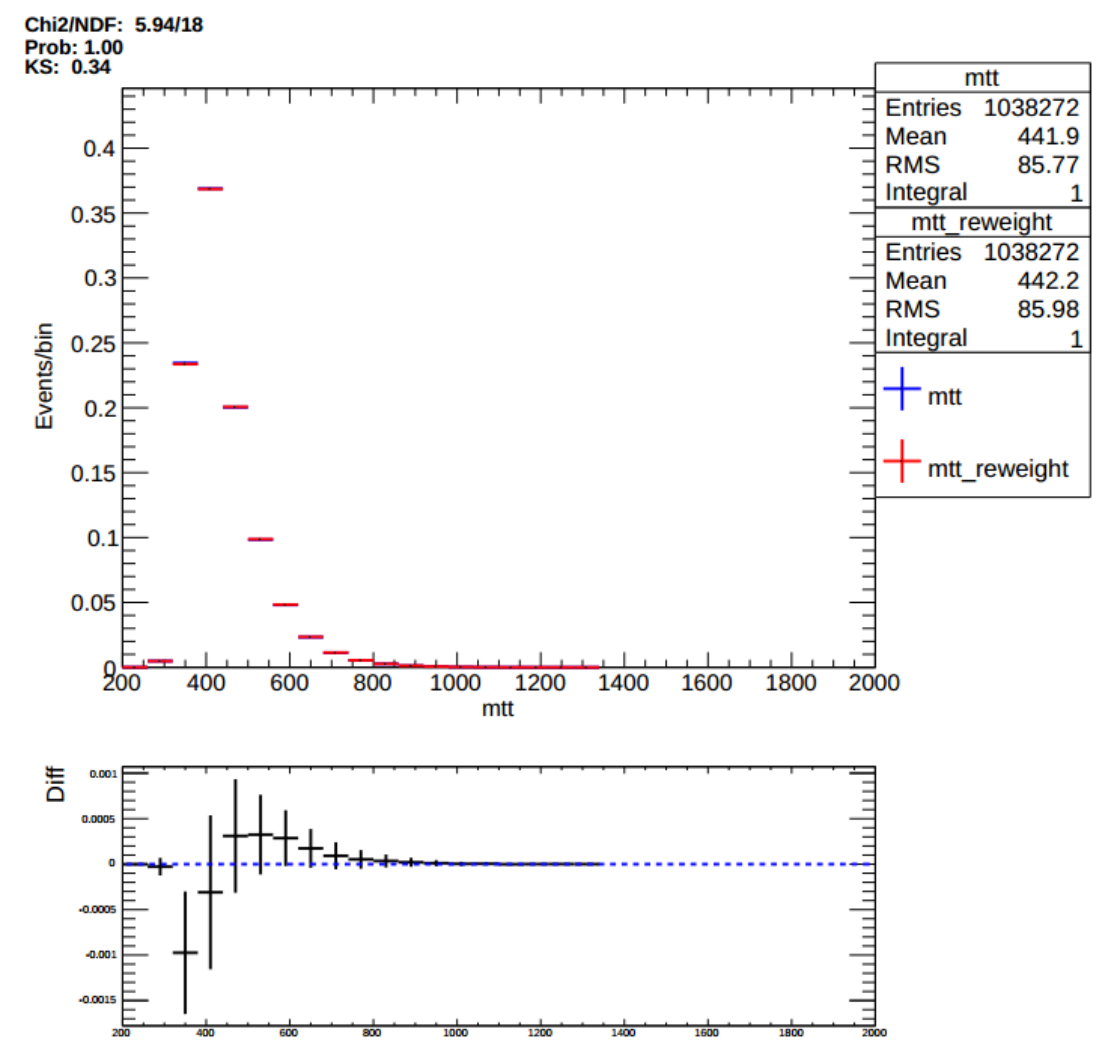

Figure 6.3: The same as Fig. 6.2, but with $m_{t \bar{t}}$. Note that $\cos \theta^{*}, \Delta y_{t}$ and $m_{t \bar{t}}$ are correlated. 


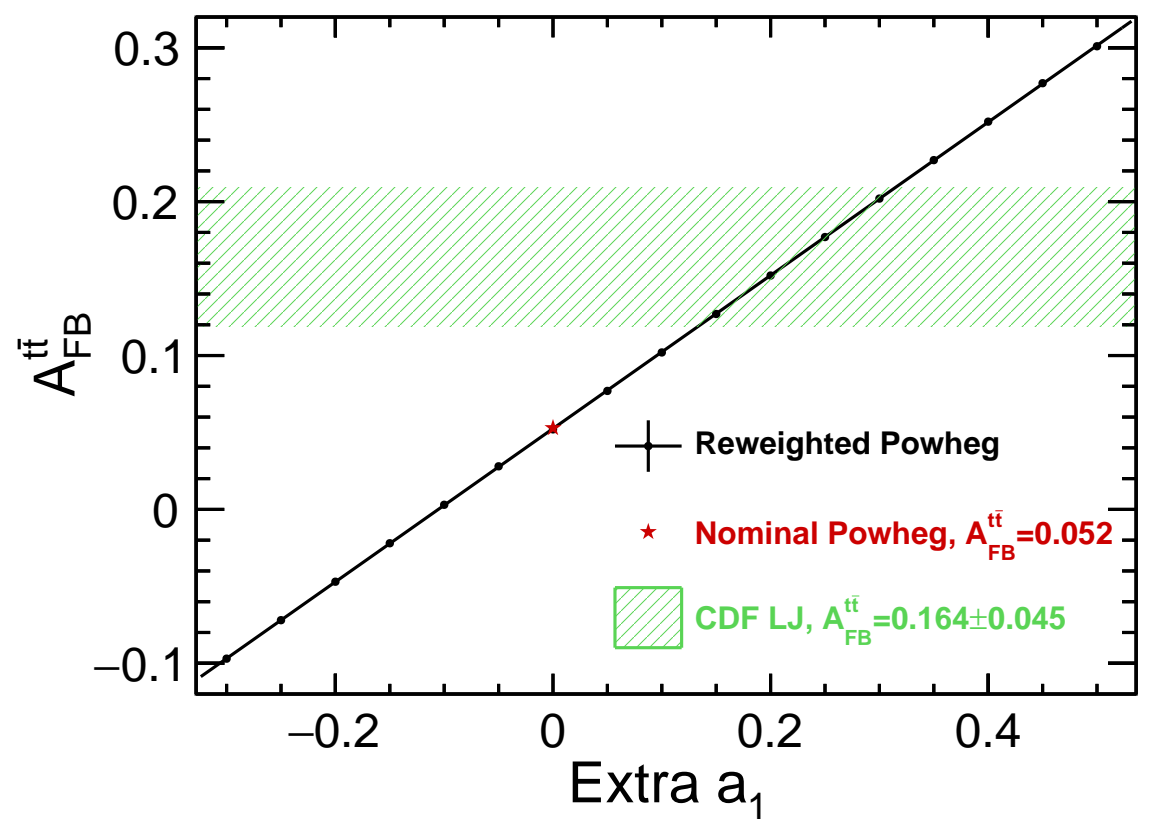

Figure 6.4: The value of $A_{\mathrm{FB}}^{t \bar{t}}$ as a function of extra $a_{1}$ used in reweighting POWHEG MC sample.
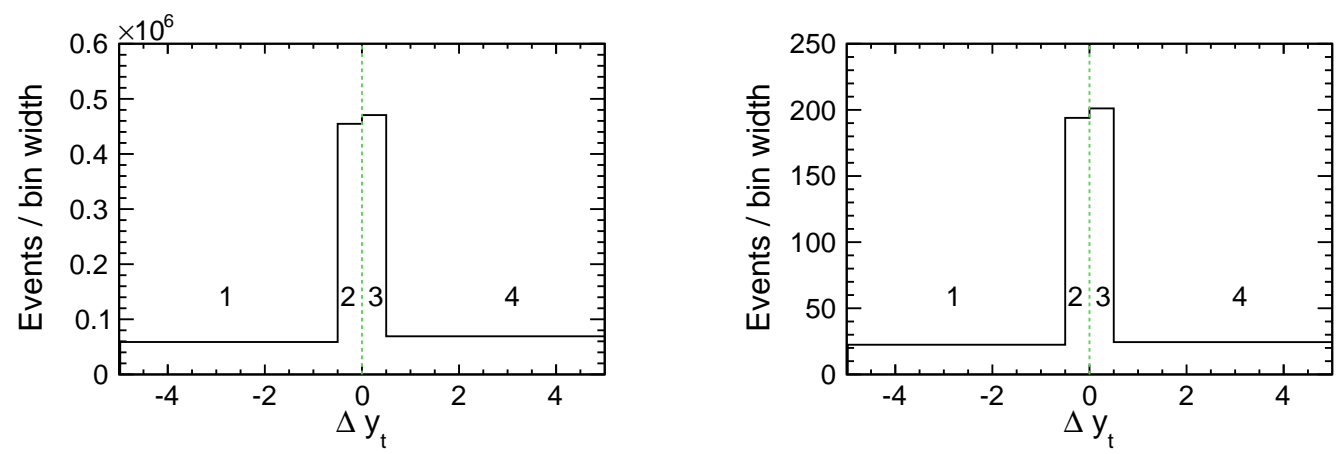

Figure 6.5: The bin-weighted $\Delta y_{t}$ distribution (a) from the nominal POWHEG at generator level before any event selection and (b) after reconstruction and baseline event selection. 
rected for in the unfolding process:

1. The limited acceptance and efficiency: This is due to the fact that not all particles fall in the detector coverage, and that not all signal events that do fall into the detector coverage pass the event selection.

2. The smearing effect: This is because that the properties of objects, like jets and $\vec{\not}_{T}$, measured by the detector are not identical to their true properties, and the $\Delta y_{t}$ values reconstructed by the top reconstruction are not identical to the true values.

The unfolding model is the current state-of-the art for the field and is subtle and depends on the assumed value of $A_{\mathrm{FB}}^{t \bar{t}}$. We incorporate it in a Bayesian model and use a Markov-chain Monte Carlo to scan the parameter space and map out the posterior probability density. The expected number of events in each bin, $N_{\exp }[r]$, for a set of candidate parton-level bin counts can be expressed as

$$
N_{\exp }[r]=\sum_{p=1}^{4} N_{\text {parton }}[p] \cdot \operatorname{Eff}[p]\left(A_{\mathrm{FB}}^{t \bar{t}}\right) \cdot \operatorname{Det}[p][r]
$$

where

- $N_{\text {parton }}[p]$ is the number of events in the $p$-th bin at parton level,

- the term $N_{\exp }[r]$ is the expected number of events in $r$-th bin, given a set of hypothetical $N_{\text {parton }}[p]$,

- the term $\operatorname{Eff}[p]\left(A_{\mathrm{FB}}^{t \bar{t}}\right)$ represents the bin-by-bin efficiency, to account for the acceptance imposed by the detector coverage and the efficiency caused by event selections (which depends on $A_{\mathrm{FB}}^{t \bar{t}}$, which is dependent on the current hypothesis of $\left.N_{\text {parton }}[p]\right)$, 
- and $\operatorname{Det}[p][r]$ represents the smearing matrix to account for the imperfect detector resolution and the top reconstruction procedure.

As shown below, this last term shows no substantive dependence on the $A_{\mathrm{FB}}^{t \bar{t}}$ input. The backgrounds will be incorporated later in Sec. 6.1.5.

Figure 6.6 shows the $\operatorname{Eff}[p]\left(A_{\mathrm{FB}}^{t \bar{t}}\right)$ as a function of $A_{\mathrm{FB}}^{t \bar{t}}$, as estimated using the reweighted MC samples, overlaid with a linear fit in the four $\Delta y_{t}$ bins. The linear fit results are used to interpolate the $\operatorname{Eff}[p]\left(A_{\mathrm{FB}}^{t \bar{t}}\right)$ value for each set of $N_{\text {parton }}[p]$ hypothesis. Figure 6.7 shows the detector smearing matrix, Det $[p][r]$, derived with the nominal POWHEG MC sample, where the smearing matrix represents the probability of an event with a parton-level $\Delta y_{t}$ in the $p$-th bin getting reconstructed in the $r$-th bin. The matrix is normalized so that the row-wise sum is equal to unity. Figure 6.8 shows that the $\operatorname{Det}[p][r]$ term is stable with different $A_{\mathrm{FB}}^{t \bar{t}}$. For this reason, we use the results from the nominal POWHEG sample in our unfolding procedure. The statistical uncertainties due to the limited MC size in deriving the $\operatorname{Eff}[p]\left(A_{\mathrm{FB}}^{t \bar{t}}\right)$ and $\operatorname{Det}[p][r]$ terms are taken into consideration in the Bayesian model, but only introduce negligible effects.

\subsubsection{Compound Poisson Statistics}

In this subsection we take a quick diversion to describe some important statistical issues that must be taken into account to get the correlation between the bins correct. With the model we defined in Eq.6.2, we compare the observed bin count from data, $N_{\text {obs }}[r]$, with the expectation $N_{\exp }[r]$ using a compound Poisson distribution, with correlations among bins taken into account. The compound Poisson distribution used here follows the same idea as in Ref. [57] in estimating the Legendre moments of the differential cross section of $t \bar{t}$ production in the lepton+jets final state. Here we provide a quick description of the model we use in this analysis. 

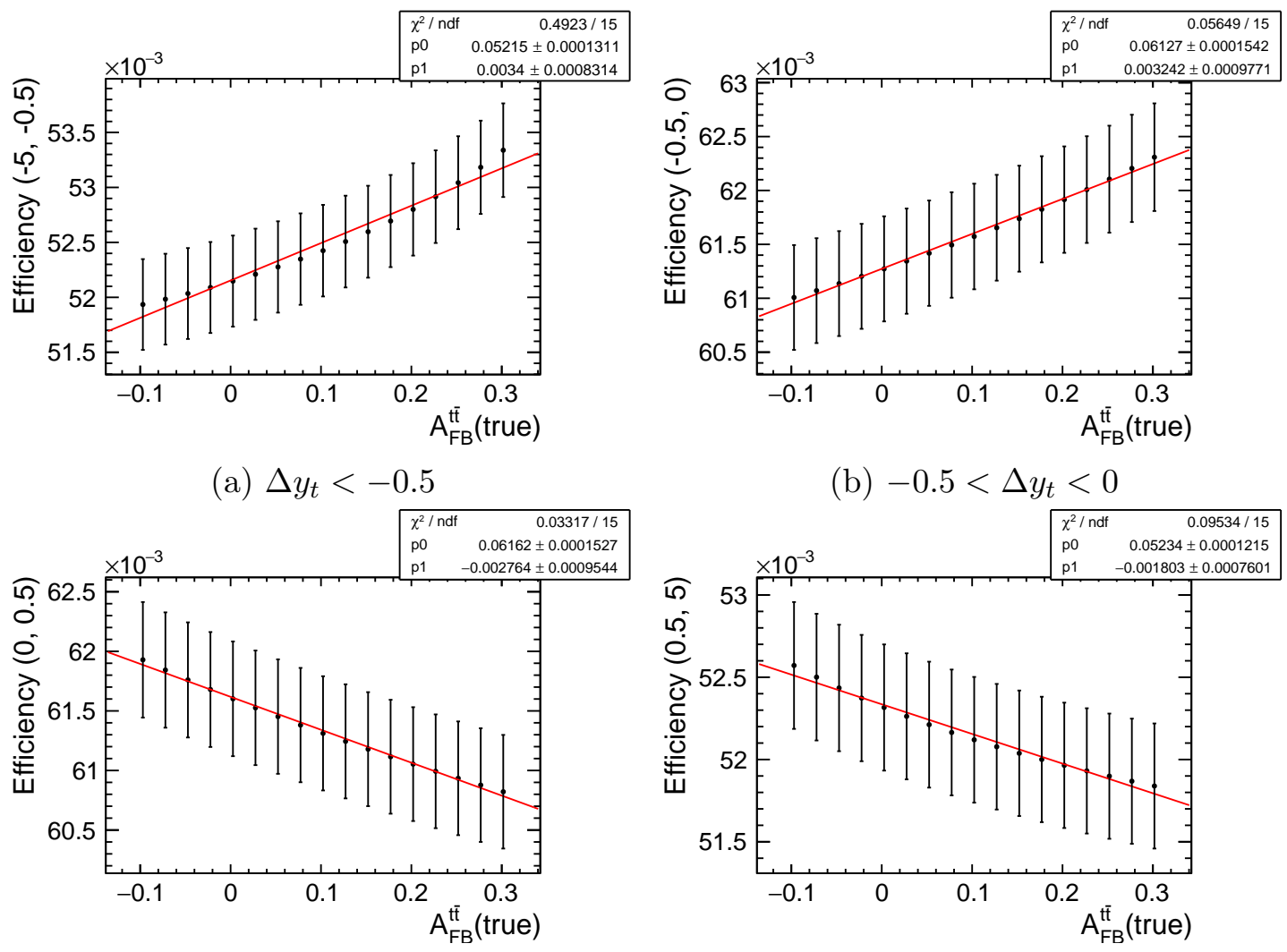

(c) $0<\Delta y_{t}<0.5$

(d) $\Delta y_{t}>0.5$

Figure 6.6: The value of $\operatorname{Eff}[p]\left(A_{\mathrm{FB}}^{t \bar{t}}\right)$ as a function of $A_{\mathrm{FB}}^{t \bar{t}}$ overlaid with a linear fit, shown in the four $\Delta y_{t}$ bins. Note that all the points in each of the figures are correlated. A final version after the optimization is shown in Fig. 6.25 and the fits are summarized in Fig. 6.26 . 


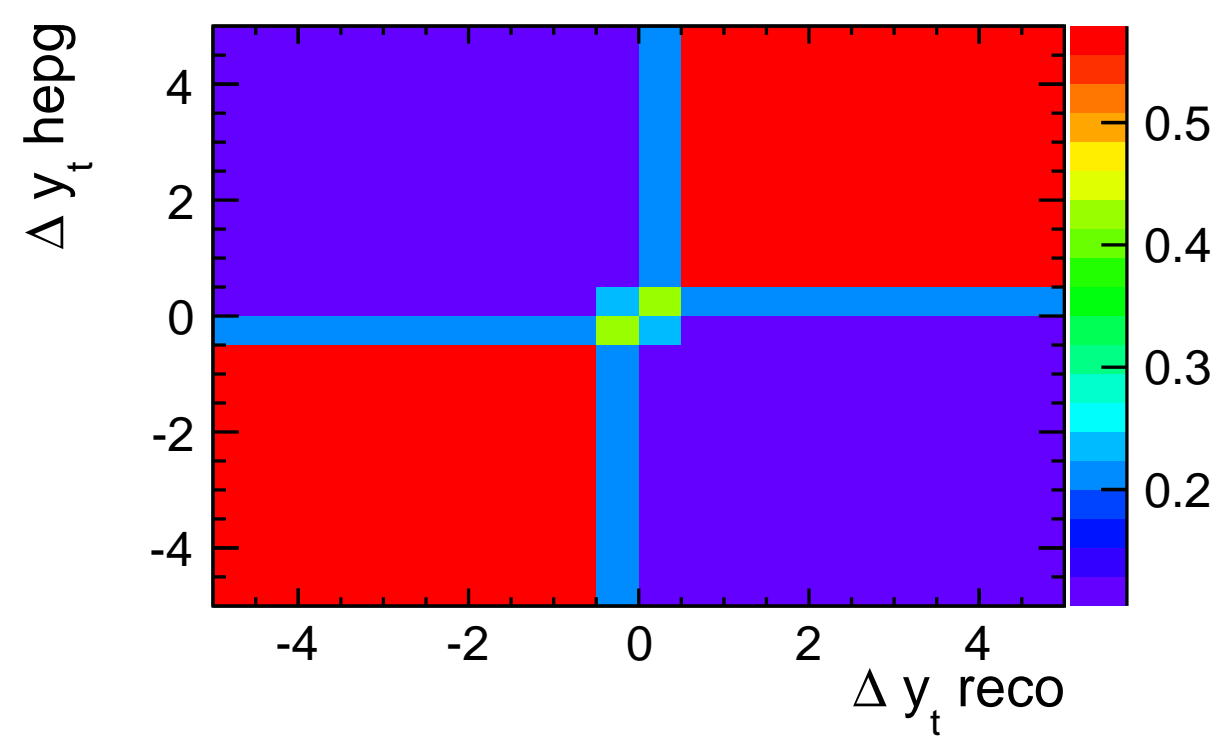

Figure 6.7: The detector smearing matrix with nominal POWHEG MC, which is the $\operatorname{Det}[p][r]$ term in our unfolding method. The final version is in Fig. 6.24b.

We note first that the output of the top reconstruction described in Sec. 5 is a set of probability density function of the parameter of interest, for example, the probability density function of $\Delta y_{t}$ as shown in Fig. 5.9a. With the binning specified in Sec. 6.1.2, each event has a certain probability of being in each of the 4 bins, and we refer to that probability as the "weight" of the event for that bin.

A compound Poission distribution can be used to describe the probability of observing a number of events, each with a specific weight. If 1) the weight of an event $(W)$ follows an (unspecified) distribution, 2) each event is independent of all the others, and 3) the number of events $(N)$ follows a Poisson distribution, then the sum of the weights $\left(Y=\sum_{i}^{N} W_{i}\right)$ follows the "compound Poisson distribution". The mean (E) and variance (Var) of the compound Poisson distribution can be estimated 

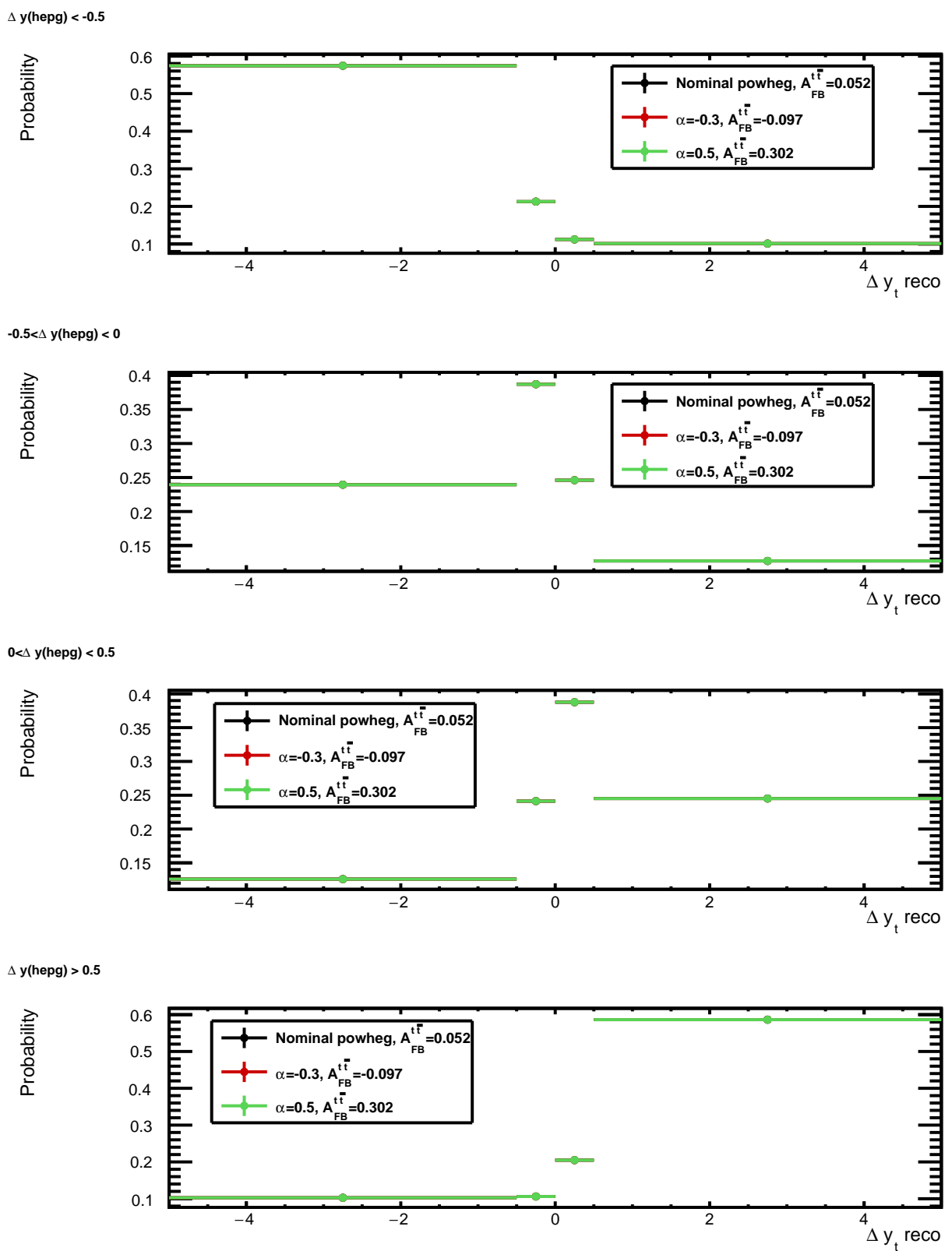

Figure 6.8: Row-slice of Fig. 6.7, with extra $a_{1}=-0.3,0$ and 0.5. The variations among the three samples are tiny. This shows that we do not need $\operatorname{Det}[p][r]$ to be a function of $A_{\mathrm{FB}}^{t \bar{t}}$, so we take the nominal POWHEG result. 
as described in Ref. [101] using

$$
\begin{aligned}
\mathrm{E}(Y) & =\mathrm{E}(N) \mathrm{E}(W), \\
\operatorname{Var}(Y) & =\mathrm{E}(N) \mathrm{E}\left(W^{2}\right) .
\end{aligned}
$$

In our analysis, we need to take the correlation among the four bins into consideration. We assume the events with parton-level (truth-level) $\Delta y_{t}$ in bin $p$ will be smeared into the four reconstructed-level bins (indexed with $r$ ) with probabilities $W_{r, p}$, where for each event $\sum_{r=1}^{4} W_{r, p}=1$. For events in the $p$-th parton-level bin (for a specific $p$ ), the distributions $W_{r, p}$ follows an (unspecified) distribution; events are independent of each other and the number of events $N_{p}$ follows a Poisson distribution, with the expectation value of $\mathrm{E}\left(N_{p}\right)=N_{\text {parton }}[p] \cdot \operatorname{Eff}[p]\left(A_{\mathrm{FB}}^{t \bar{t}}\right)$ following the first part of Eqn. 6.2. Thus, the distribution of the vector (4 bins in $r$ ) of the sum of the weights (a.k.a. bin content, $Y_{r}=\sum_{i=1}^{N_{p}} W_{i ; r, p}$ ) and the corresponding covariance matrix follows

$$
\begin{aligned}
\mathrm{E}\left(Y_{r, p}\right) & =\mathrm{E}\left(N_{p}\right) \mathrm{E}\left(W_{r, p}\right), \\
\operatorname{Cov}\left(Y_{r, r^{\prime}, p}\right) & =\mathrm{E}\left(N_{p}\right) \mathrm{E}\left(W_{r, p} \cdot W_{r^{\prime}, p}\right) .
\end{aligned}
$$

Note that $\mathrm{E}\left(W_{r, p}\right)$ is essentially the same as our definition of the detector smearing matrix $\operatorname{Det}[p][r]$, and Eqn. 6.4a is equivalent to Eqn. 6.2. The final covariance matrix 
$\left(\operatorname{Cov}\left(Y_{r, r^{\prime}}\right)\right)$ is obtained by summing Eqn 6.4b over the four truth-level bins:

$$
\begin{aligned}
\operatorname{Cov}\left(Y_{r, r^{\prime}}\right) & =\sum_{p=1}^{4} \operatorname{Cov}\left(Y_{r, r^{\prime}, p}\right) \\
& =\sum_{p=1}^{4} \mathrm{E}\left(N_{p}\right) \mathrm{E}\left(W_{r, p} \cdot W_{r^{\prime}, p}\right) \\
& =\sum_{p=1}^{4} N_{\text {parton }}[p] \cdot \operatorname{Eff}[p]\left(A_{\mathrm{FB}}^{t \bar{t}}\right) \cdot \mathrm{E}\left(W_{r, p} \cdot W_{r^{\prime}, p}\right) .
\end{aligned}
$$

Note that the covariance matrix depends on the current guess of the parton-level distribution $N_{\text {parton }}[p]$, which is a function of the inclusive $A_{\mathrm{FB}}^{t \bar{t}}$ guess.

Since we expect enough statistics in our data sample, we approximate the compound Poisson distribution with a multi-variable Gaussian distribution, with the terms in the covariance matrices estimated with POwHEG MC. The MCMC technique is used to fit for the best parameters that matches data, and the parton-level $A_{\mathrm{FB}}^{t \bar{t}}$ is extracted with marginalization techniques.

\subsubsection{Parameters and Signal-Only Uncertainty Expectations}

For better motivation of the priors of the parameters for use in our reconstruction, instead of doing our unfolding with the expected number of events in each bin, we do our unfolding using four alternative parameters. We re-parametrize with four parameters determining $N_{\text {parton }}[p]$, which allow us to easily use a Bayesian method with flat priors to determine the parton-level $A_{\mathrm{FB}}^{t \bar{t}}$. We choose:

1. $N_{\text {tot }}=\sum_{p=1}^{4} N_{\text {parton }}[p]$ is the total number of events, with a uniform non-negative prior distribution

2. $A_{\text {in }}=\frac{N_{\text {parton }}[3]-N_{\text {parton }}[2]}{N_{\text {parton }}[3]+N_{\text {parton }}[2]}=A_{\mathrm{FB}}^{t \bar{t}}\left(\left|\Delta y_{t}\right|<0.5\right)$ is the asymmetry of bins 2 and 3 , with a uniform prior in $(-1,1)$ 
3. $A_{\text {out }}=\frac{N_{\text {parton }}[4]-N_{\text {parton }}[1]}{N_{\text {parton }[4]+N_{\text {parton }}[1]}}=A_{\mathrm{FB}}^{t \bar{t}}\left(\left|\Delta y_{t}\right|>0.5\right)$ is the asymmetry of bins 1 and 4 , with a uniform prior in $(-1,1)$

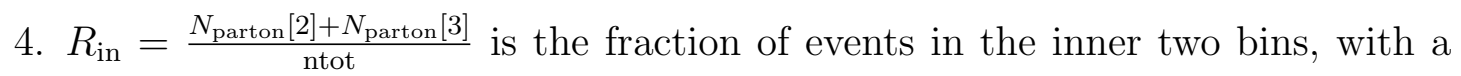
uniform prior in $(0,1)$.

With this new parametrization, the inclusive $A_{\mathrm{FB}}^{t \bar{t}}$ is

$$
A_{\mathrm{FB}}^{t \bar{t}}=R_{\text {in }} \cdot A_{\text {in }}+\left(1-R_{\text {in }}\right) \cdot A_{\text {out }},
$$

and the differential $A_{\mathrm{FB}}^{t \bar{t}}$ terms as a function of $\left|\Delta y_{t}\right|$ are given by $A_{\text {in }}$ and $A_{\text {out }}$.

We note here that this model is not readily applicable to the measurement of $A_{\mathrm{FB}}^{t \bar{t}}$ as a function of $m_{t \bar{t}}$, in which case an extra dimension of smearing effect is introduced. In addition, adding an extra dimension of binning would reduce the number of events in each bin to a degree that using a multi-variable Gaussian distribution to approximate the compound Poisson distribution will be invalid. For these reasons, we have not performed a measurement of the differential $A_{\mathrm{FB}}^{t \bar{t}}$ as a function of $m_{t \bar{t}}$ in the dilepton final state.

We again use BAT [97] to scan the parameter space and obtain the probability distributions of the parameters, including $A_{\mathrm{FB}}^{t \bar{t}}\left(\left|\Delta y_{t}\right|<0.5\right)$ and $A_{\mathrm{FB}}^{t \bar{t}}\left(\left|\Delta y_{t}\right|>0.5\right)$. The probability distribution of the inclusive $A_{\mathrm{FB}}^{t \bar{t}}$ is obtained by marginalizing out all other parameters. After making sure the MCMC chains converge and all posteriors have reasonable shapes, each $A_{\mathrm{FB}}^{t \bar{t}}$ measurement and its uncertainty is extracted by fitting a Gaussian function to the central region of the $A_{\mathrm{FB}}^{t \bar{t}}$ posterior distribution.

Figure 6.9 shows the posterior distribution of the inclusive value of $A_{\mathrm{FB}}^{t \bar{t}}$ with the full set of reconstructed events from the nominal POWHEG MC sample. The $A_{\mathrm{FB}}^{t \bar{t}}$ is extracted with a Gaussian fit to the central region of this distribution. The 
result $A_{\mathrm{FB}}^{t \bar{t}}=0.053 \pm 0.013$ is consistent with the generator-level value of 0.0524 , and the uncertainty reasonably reflects the $\sim 70 k$ reconstructed events in the POWHEG sample. The agreement is excellent and the posterior is very Gaussian. While more statistics could be generated, there is no reason to believe it would help the result.

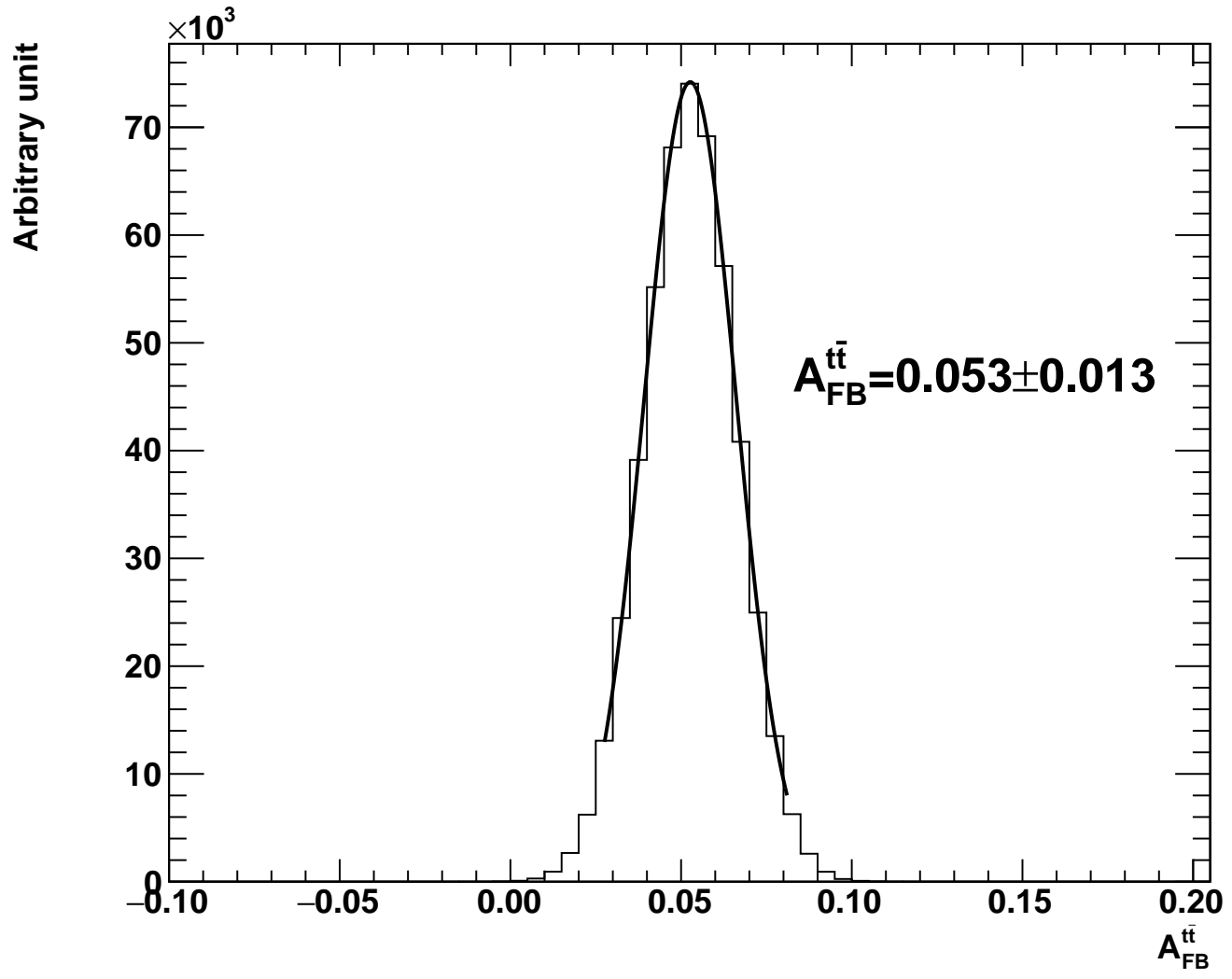

Figure 6.9: The posterior distribution of of the inclusive value of $A_{\mathrm{FB}}^{t \bar{t}}$ with the full set of reconstructed events from the POWHEG MC sample. The $A_{\mathrm{FB}}^{t \bar{t}}$ is extracted with a Gaussian fit to the central region of this distribution. The result $A_{\mathrm{FB}}^{t \bar{t}}=0.053 \pm 0.013$ is consistent with the generator-level value of 0.0524 .

We test the unfolding model with 5k pseudoexperiments (P.E.). Each P.E. is generated by randomly picking events from the nominal POWHEG MC sample with the number of events matching the expectation for data (408 events, see Table 3.3 . 
allowing the number of events in each P.E. to float according to the uncertainty on the expectation). Each P.E. is processed with the nominal unfolding procedure. The results of the unfolding procedures are collected and summarized in Fig 6.10. The pull distribution, which is the distribution of the difference between the result of one P.E. and the true $A_{\mathrm{FB}}^{t \bar{t}}$ divided by the uncertainty of the measured value, is consistent with a Gaussian distribution with a mean of zero and an RMS of one, which indicates the measurement methodology and its uncertainty estimation is not biased [102]. The expected signal-only statistical uncertainty, estimated as the RMS of Fig. 6.10a, is 0.107 .

We perform the same procedures for the two differential values of $A_{\mathrm{FB}}^{t \bar{t}}$ vs. $\left|\Delta y_{t}\right|$. As shown in Eq. 6.6. Ain and Aout, representing the two binned measurement of $A_{\mathrm{FB}}^{t \bar{t}}$ vs $\left|\Delta y_{t}\right|$, are two free parameters in our unfolding model, each with flat prior distributions in $(-1,1)$. Figure 6.11 shows the posterior distributions of $A_{\mathrm{FB}}^{t \bar{t}}\left(\left|\Delta y_{t}\right|<0.5\right)$ and $A_{\mathrm{FB}}^{t \bar{t}}\left(\left|\Delta y_{t}\right|>0.5\right)$ from POWHEG MC for signal only. The mean values $(0.02$, $0.08)$ can be compared with $(0.017,0.081)$ from the generator level. pseudoexperiments are again used to test the uncertainty estimation, as shown in Fig. 6.12. The pull distributions look good as well, i.e., have a mean of approximately zero, appear Gaussian, and have an RMS of approximately one. The expected signal-only statistical uncertainty is 0.28 for $A_{\mathrm{FB}}^{t \bar{t}}\left(\left|\Delta y_{t}\right|<0.5\right)$ due to large bin migration between the two central bins and 0.13 for $A_{\mathrm{FB}}^{t \bar{t}}\left(\left|\Delta y_{t}\right|>0.5\right)$.

\subsubsection{Adding Backgrounds to the Methodology}

The Bayesian model can be extended to include the background components in data with

$$
N_{\exp }[r]=\sum_{p=1}^{4} N_{\text {parton }}[p] \cdot \operatorname{Eff}[p]\left(A_{\mathrm{FB}}^{t \bar{t}}\right) \cdot \operatorname{Det}[p][r]+N_{\mathrm{bkg}}[r]
$$




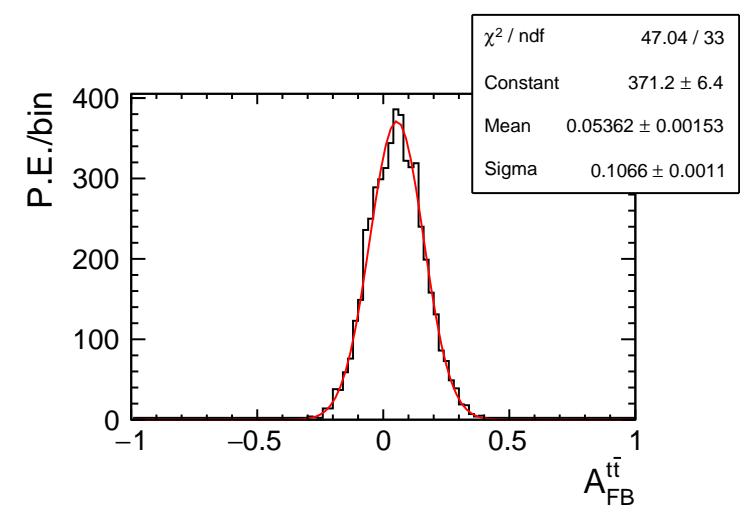

(a) $A_{\mathrm{FB}}^{t \bar{t}}$

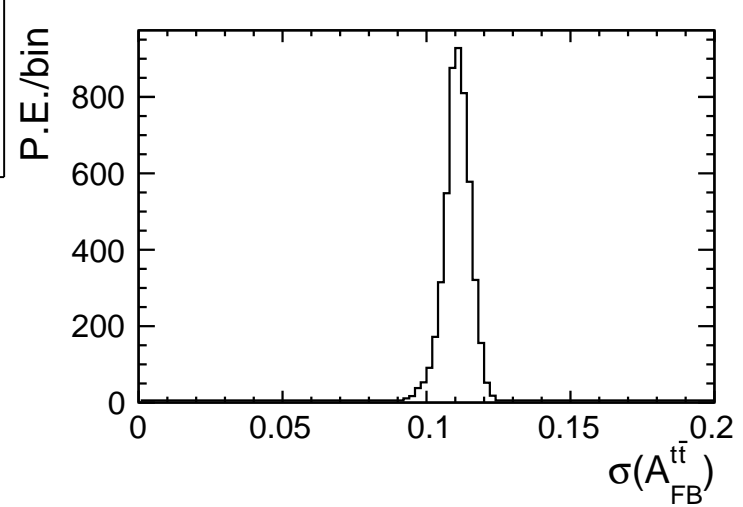

(b) $\sigma\left(A_{\mathrm{FB}}^{t \bar{t}}\right)$

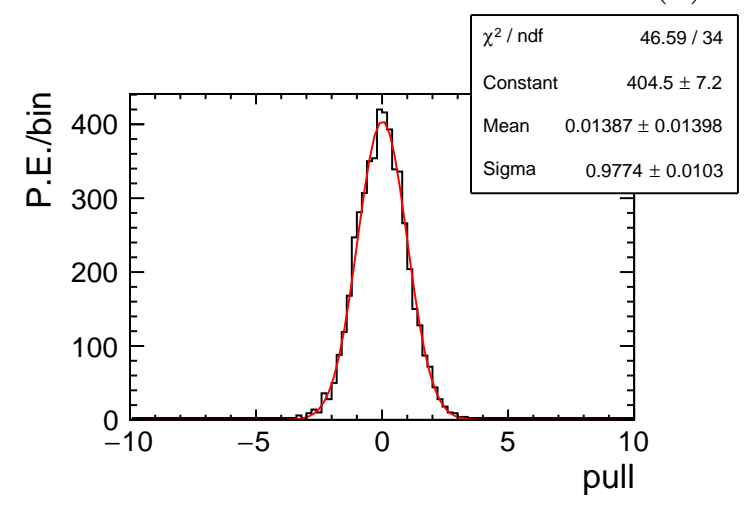

(c) $\operatorname{Pull}\left(A_{\mathrm{FB}}^{t \bar{t}}\right)$

Figure 6.10: The mean values of the inclusive $A_{\mathrm{FB}}^{t \bar{t}}$ measurements using pseudoexperiments(a), the expected statistical uncertainty (b) and the pull (c) from each pseudoexperiment with signal only. The pull distribution looks good. The expected signal-only statistical uncertainty is 0.107 , which is derived from the RMS of (a).

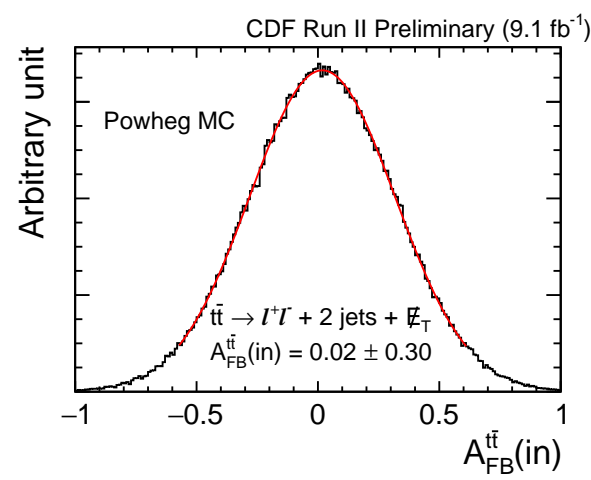

(a) $A_{\mathrm{FB}}^{t \bar{t}}\left(\left|\Delta y_{t}\right|<0.5\right)$

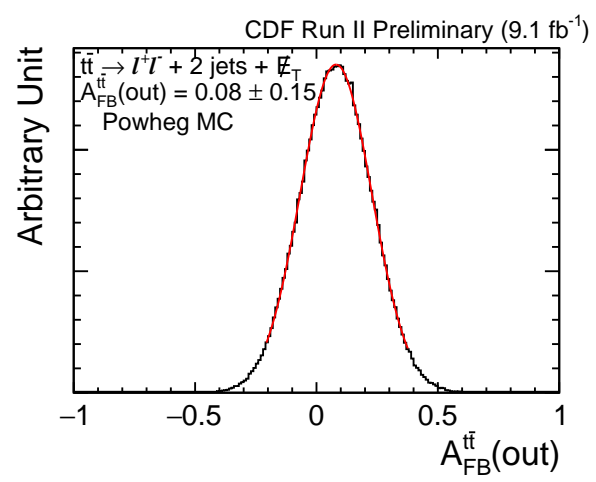

(b) $A_{\mathrm{FB}}^{t \bar{t}}\left(\left|\Delta y_{t}\right|>0.5\right)$

Figure 6.11: Posterior distributions of $A_{\mathrm{FB}}^{t \bar{t}}$ in the two $\left|\Delta y_{t}\right|$ bins with signal only, with Gaussian fits to extract their central values and uncertainties. 


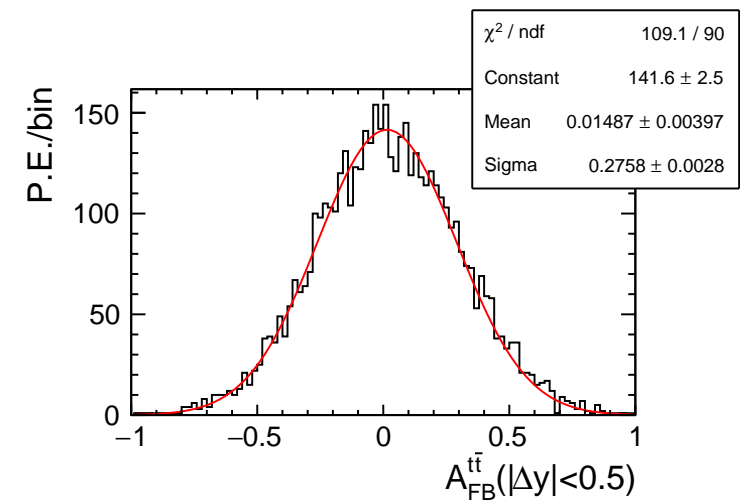

(a) $A_{\mathrm{FB}}^{t \bar{t}}\left(\left|\Delta y_{t}\right|<0.5\right)$, signal only

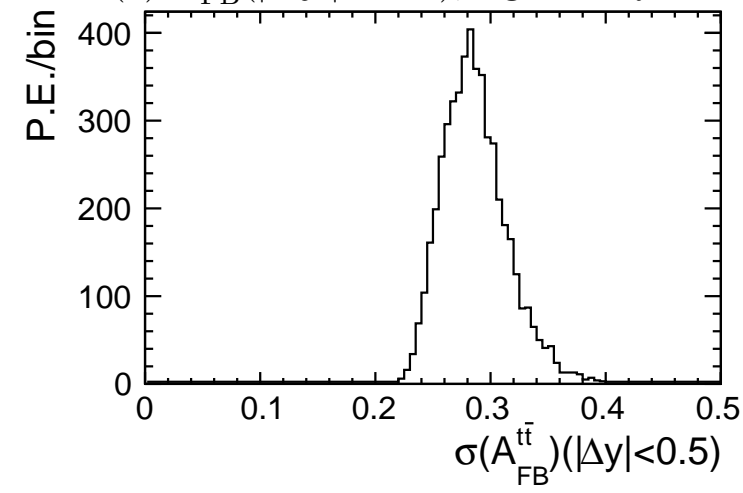

(c) $\sigma\left(A_{\mathrm{FB}}^{t \bar{t}}\right)\left(\left|\Delta y_{t}\right|<0.5\right)$, signal only

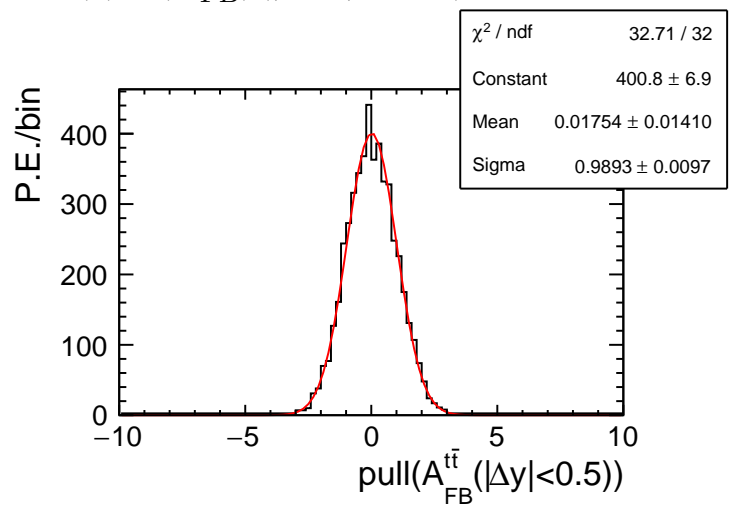

(e) $\operatorname{Pull}\left(A_{\mathrm{FB}}^{t \bar{t}}\right)\left(\left|\Delta y_{t}\right|<0.5\right)$, signal only

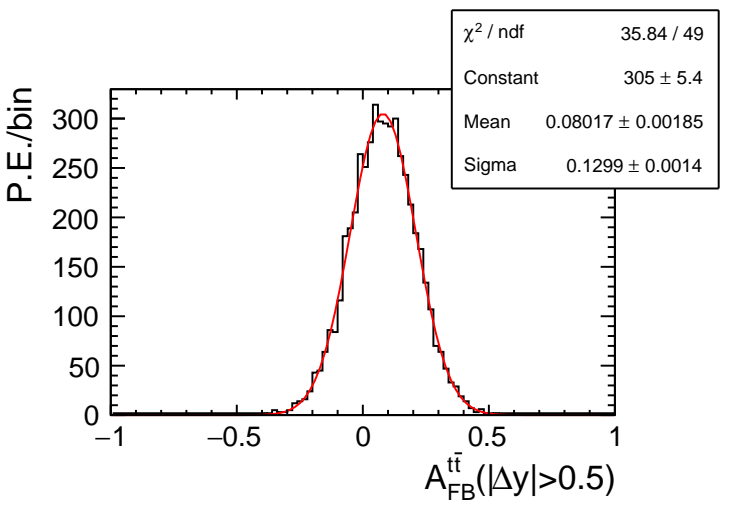

(b) $A_{\mathrm{FB}}^{t \bar{t}}\left(\left|\Delta y_{t}\right|>0.5\right)$, signal only

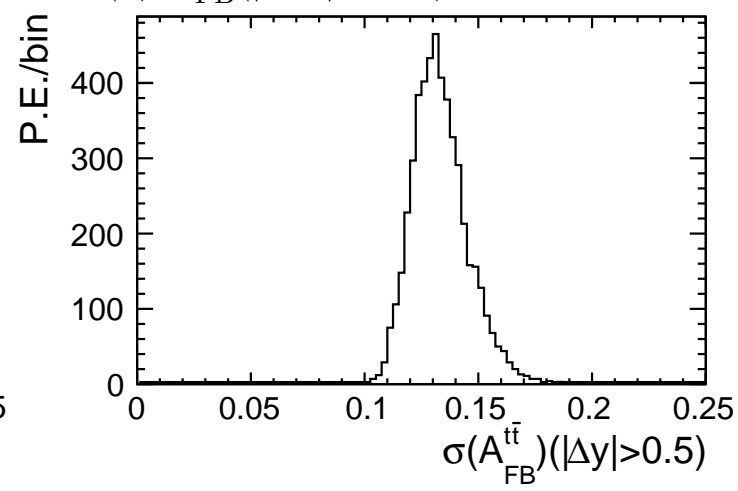

(d) $\sigma\left(A_{\mathrm{FB}}^{t \bar{t}}\right)\left(\left|\Delta y_{t}\right|>0.5\right)$, signal only

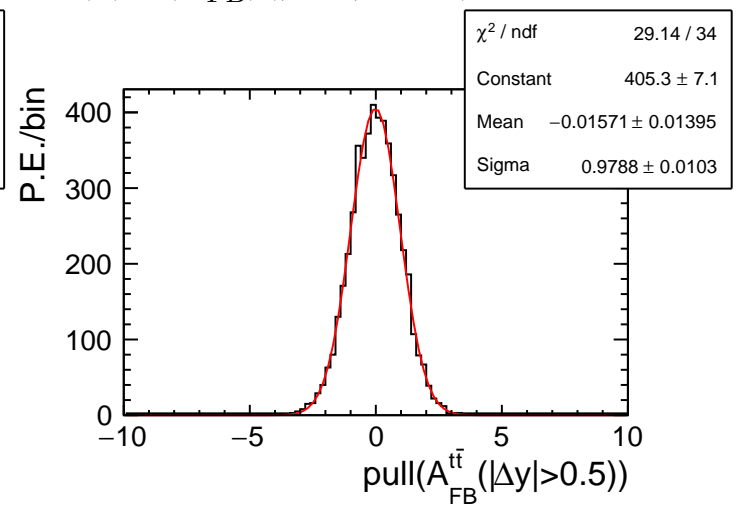

(f) $\operatorname{Pull}\left(A_{\mathrm{FB}}^{t \bar{t}}\right)\left(\left|\Delta y_{t}\right|>0.5\right)$, signal only

Figure 6.12: Results of the pseudoexperiments for $A_{\mathrm{FB}}^{t \bar{t}}\left(\left|\Delta y_{t}\right|<0.5\right)$ (LHS) and $A_{\mathrm{FB}}^{t \bar{t}}\left(\left|\Delta y_{t}\right|>0.5\right)$ (RHS), with signal only. The pull distributions look good. The expected statistical uncertainty with signal only is 0.28 for $A_{\mathrm{FB}}^{t \bar{t}}\left(\left|\Delta y_{t}\right|<0.5\right)$ and 0.13 for $A_{\mathrm{FB}}^{t \bar{t}}\left(\left|\Delta y_{t}\right|>0.5\right)$. 
where $N_{\mathrm{bkg}}[r]$ is the expected background contribution in $r$-th bin. The background contributions also follow a compound Poisson distribution, and the covariance matrices are estimated from the background samples with the formula:

$$
\begin{aligned}
\operatorname{Cov}\left(Y_{r, r^{\prime} ; b}\right) & =\mathrm{E}\left(N_{b}\right) \mathrm{E}\left(W_{r ; b} \cdot W_{r^{\prime} ; b}\right) \\
& =N_{\operatorname{Exp}, b} \cdot \mathrm{E}\left(W_{r ; b} \cdot W_{r^{\prime} ; b}\right)
\end{aligned}
$$

where the $b$ indexes each component of the background estimation, and $N_{\operatorname{Exp}, b}$ corresponds to the estimated background contributions (e.g. in Table 3.3 or the corresponding ones with additional optimization cuts to be described later).

The procedure, including the backgrounds, is also tested with pseudoexperiments. We again generated 5k P.E.s, each with randomly picked events from POWHEG sample as signal and background estimation samples (the same samples as in Sec. 4), with the number of events matching the expectations for data with the number of events from each allowed to float according to the uncertainties on the expectations. Each P.E. is again processed with the unfolding procedure. The results of the unfolding procedures are collected and summarized in Fig 6.13. The pull distribution looks good. The expected statistical uncertainty from data (namely the total statistical uncertainty), estimated as the RMS of Fig. 6.13a, is 0.122 . As this uncertainty will be larger than all the systematic uncertainties described in Sec. 6.4, this will be the dominant uncertainty. We also test the unfolding procedure with the BSM scenarios. The results will be shown after the optimization in Sec. 6.3.

Pseudoexperiments are again used to test the uncertainty estimation with signal + backgrounds for the differential $A_{\mathrm{FB}}^{t \bar{t}}$ vs. $\left|\Delta y_{t}\right|$, as shown in Fig. 6.14. The pull distributions look good. The expected total uncertainty is 0.34 for $A_{\mathrm{FB}}^{t \bar{t}}\left(\left|\Delta y_{t}\right|<0.5\right)$ due to large bin migration between the two central bins and 0.16 for $A_{\mathrm{FB}}^{t \bar{t}}\left(\left|\Delta y_{t}\right|>0.5\right)$. 
Since, as we will show, the uncertainty due to the background shapes will be the next dominant uncertainty we comment on it here and use it in the optimization procedure. To be conservative, and following the procedure in Sec. 4, we estimate the background systematic uncertainty to be equal to the difference between the total statistical uncertainty and the signal-only statistical uncertainty in quadrature $\left(\sigma_{\text {bkg }}^{2}=(\text { RMS of Fig. 6.13a })^{2}-(\text { RMS of Fig. 6.10a })^{2}\right)$. In Sec. 6.2 we will perform the optimization where we aim at minimizing the sum of the total statistical uncertainty and the background systematic uncertainty in quadrature. We will refer to this uncertainty as the expected uncertainty.

\subsection{Analysis Optimization}

The top reconstruction algorithm and the parton-level $A_{\mathrm{FB}}^{t \bar{t}}$ extraction procedures are designed to allow for an optimization strategy that minimizes the expected uncertainties. Besides the statistical uncertainty due to the limited data sample size and backgrounds, the uncertainty of the parton-level $A_{\mathrm{FB}}^{t \bar{t}}$ receives a significant contribution from the resolution of the reconstruction, especially the events with $\Delta y_{t}$ reconstructed far away from their parton-level values. For comparison the statistical uncertainty on the $A_{\mathrm{FB}}^{\ell \ell}$ measurement, which has negligible uncertainty due to the small lepton direction resolution, is 0.072 , so of the predicted value of 0.122 (from Fig. 6.10a) we currently have about 0.071 (estimated as $\sqrt{0.122^{2}-0.072^{2}}$ ) due to resolution effects. We next describe our optimization procedure.

We start by noting that the simplest analysis choice (chosen in the previous

dilepton analysis with $5.1 \mathrm{fb}^{-1}$ of the data), within the reconstruction, of picking the maximum-likelihood solution suffers from two primary problems: 1) the algorithm sometimes selects the wrong lepton-jet pairing and 2) the algorithm sometimes gives the highest likelihood values to a set of very wrong values of the parameter 


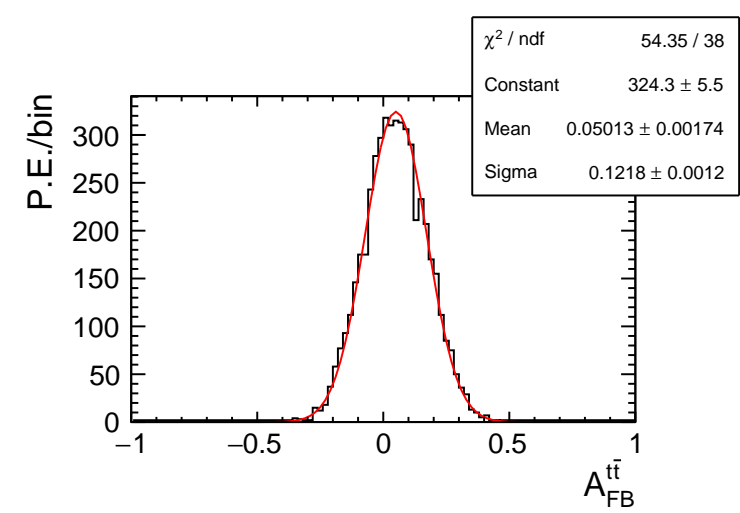

(a) $A_{\mathrm{FB}}^{t \bar{t}}$

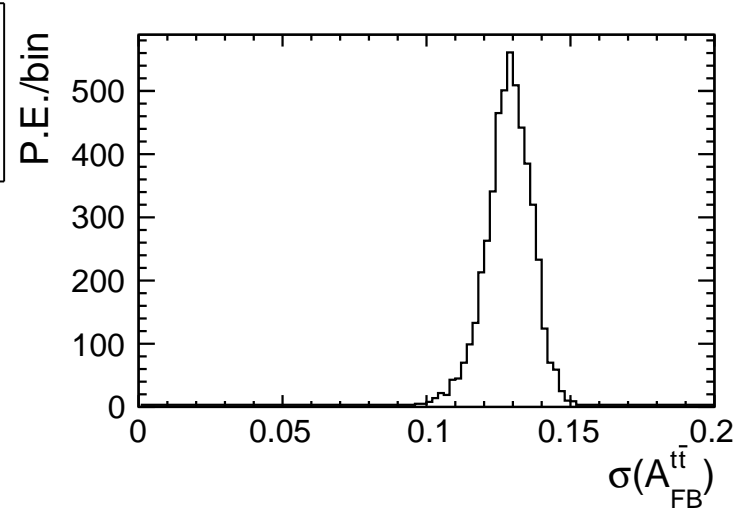

(b) $\sigma\left(A_{\mathrm{FB}}^{t \bar{t}}\right)$

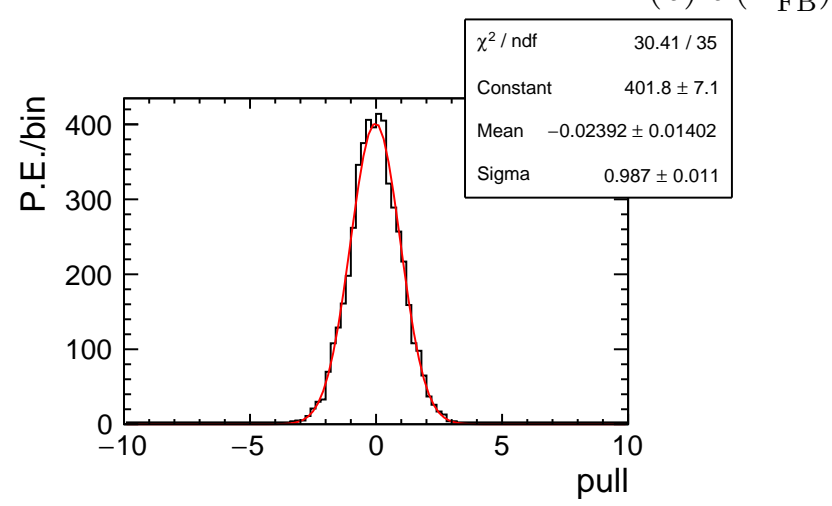

(c) $\operatorname{Pull}\left(A_{\mathrm{FB}}^{t \bar{t}}\right)$

Figure 6.13: The same as Fig. 6.10, but where we have included the backgrounds. The mean values of the inclusive values of $A_{\mathrm{FB}}^{t \bar{t}}$ as estimated using pseudoexperiments (a), the expected statistical uncertainty (b) and the pull (c). The pull distribution looks good. The expected signal+backgrounds statistical uncertainty is 0.122 . 

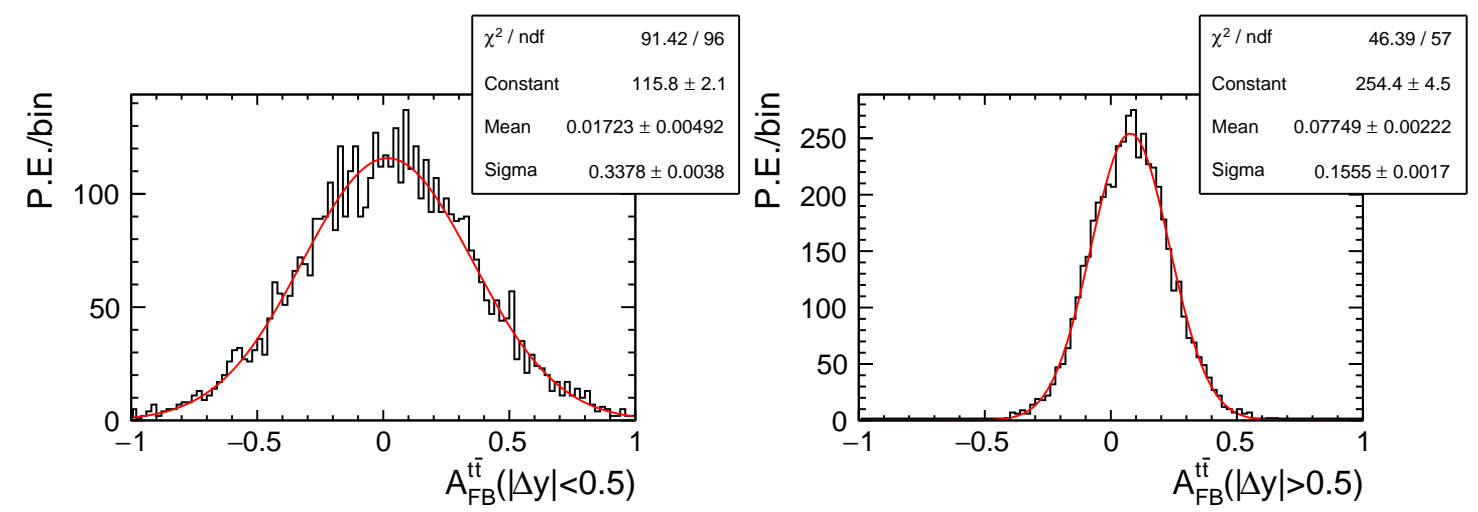

(a) $A_{\mathrm{FB}}^{t \bar{t}}\left(\left|\Delta y_{t}\right|<0.5\right)$, signal+backgrounds

(b) $A_{\mathrm{FB}}^{t \bar{t}}\left(\left|\Delta y_{t}\right|>0.5\right)$, signal+backgrounds
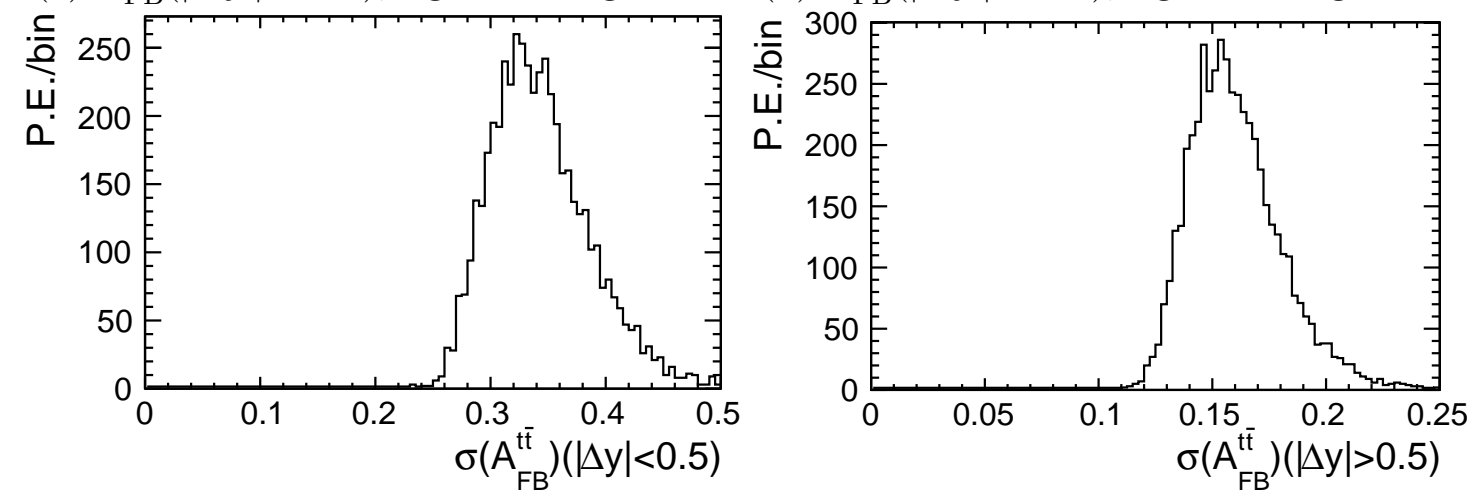

(c) $\sigma\left(A_{\mathrm{FB}}^{t \bar{t}}\right)\left(\left|\Delta y_{t}\right|<0.5\right)$, sig. + bkg.

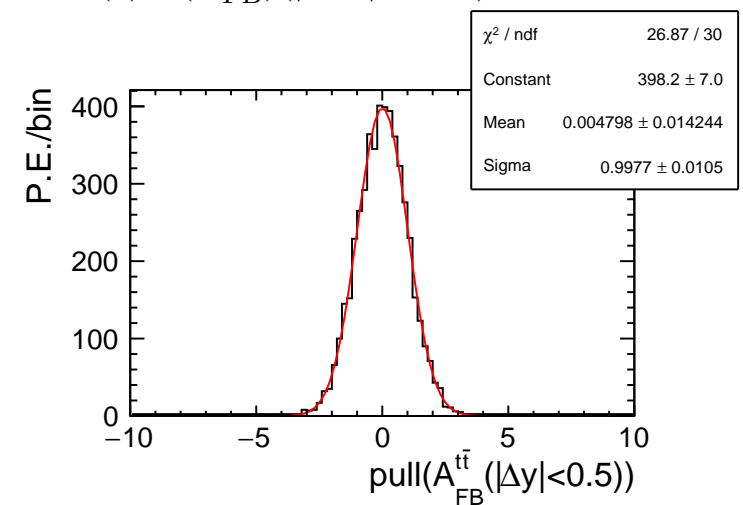

(d) $\sigma\left(A_{\mathrm{FB}}^{t \bar{t}}\right)\left(\left|\Delta y_{t}\right|>0.5\right)$, sig. + bkg.

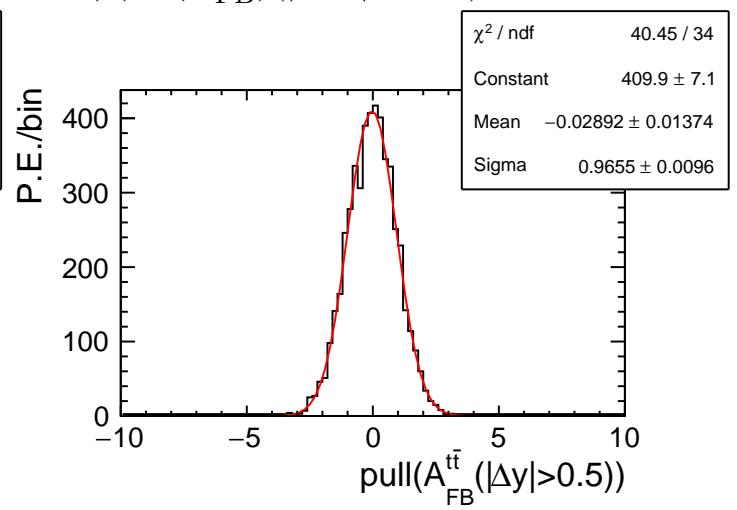

(e) $\operatorname{Pull}\left(A_{\mathrm{FB}}^{t \bar{t}}\right)\left(\left|\Delta y_{t}\right|<0.5\right)$, sig. + bkg.

(f) $\operatorname{Pull}\left(A_{\mathrm{FB}}^{t \bar{t}}\right)\left(\left|\Delta y_{t}\right|>0.5\right)$, sig. + bkg.

Figure 6.14: The same as Fig. 6.12, but with signal+background. The pull distributions look good. The expected total statistical uncertainties with signal + backgrounds are 0.34 for $A_{\mathrm{FB}}^{t \bar{t}}\left(\left|\Delta y_{t}\right|<0.5\right)$ and 0.16 for $A_{\mathrm{FB}}^{t \bar{t}}\left(\left|\Delta y_{t}\right|>0.5\right)$. 
choices within the right lepton-jet pairing. Each contributes significant tails to the $\Delta y_{t}($ reconstructed $)-\Delta y_{t}$ (generated $)$ distribution, which cause the significant additional measurement uncertainty. The reconstruction algorithm used in this analysis allows us to incorporate more information in the procedure to help us optimize the analysis. We will take advantage of this by using this new information, and additional tools, to:

1. Choose between the maximum-likelihood solution per event or using the full probability distribution

2. Pick the higher-likelihood lepton-jet pairing or weight both according to the best available information

3. Apply additional requirements to reject low-quality lepton-jet pairings, which rejects backgrounds or poorly reconstructed events

4. Add in more information about the jet charge to do better in choosing or weighting the lepton-jet pairings

To be more explicit, we note that for each lepton-jet pairing described in Sec. 5 we have the choice to either use the max-likelihood solution or keep the full probability distribution. We can also either choose the lepton-jet pairing that provides the better likelihood, or weight both according to the weights defined in Eqn. 5.10, Besides these, we can introduce quality cuts based on $\mathrm{jd}_{1,2}$ and $\Delta R_{\min }$ (lepton, jet), as well as incorporating extra information of track-weighted jet charge or the lepton-jet invariant mass $m_{l b}^{2}$.

In this section, we describe the expectations when we consider all these various possibilities with MC, and choose the algorithm that minimizes the expected total statistical + background systematic uncertainty before looking at the data. For now 
we ignore the non-background systematics since they are small and expected to not change much from choice to choice, and come back to them in Sec. 6.4.

For each optimization choice, we run 5k pseudoexperiments with signal only and with signal+background to determine the expected signal-only and total statistical uncertainty, which in turn gives us the systematic uncertainty due to the backgrounds, and thus an effective total uncertainty as described in the previous section. We will refer to this uncertainty as the "Total Statistical+Background Systematic Uncertainty" in the figures, but as the expected uncertainty in the text. The best optimization choice is made by minimizing the expected uncertainty.

\subsubsection{Max Likelihood vs. Full Probability and Weighting}

For each lepton-jet pairing, the MCMC-based top reconstruction produces a posterior probability distribution and likelihood for each parameter $\left(\mathrm{jd}_{1}, \mathrm{jd}_{2}, \phi_{1}, \phi_{2}\right.$ as well as any parameter of interest $y_{t}, y_{\bar{t}}, \Delta y_{t}$, etc.), as described in Sec. 5.4. Within each lepton-jet pairing, we have the choice of picking the max-likelihood solution, or using the full probability distribution. We can also pick the lepton-jet pairing with higher likelihood, or weight both pairings according to the weights defined in Eqn. 5.10. We have estimated the uncertainties for all 4 scenarios, and the different uncertainty numbers are summarized in Table 6.1. We find that keeping the full probability and weighting both lepton-jet pairings always gives the best expected

uncertainty for the inclusive $A_{\mathrm{FB}}^{t \bar{t}}$ measurement, so we choose these options. We note that while using the maximum-likelihood choice minimizes the background systematic, using the full probability optimizes the statistical uncertainty. Since that is the dominant uncertainty, ultimately that is the best strategy. It improves the expected uncertainty from 0.154 (as would be expected from the previous measurement style) to 0.136 . 
Table 6.1: The expected total statistical uncertainty, background systematic uncertainty and the sum of the two in quadrature (the expected uncertainty), for the choices of the max-likelihood solution or full probability, and the choice of the more likely lepton-jet pairing (L-J pairing) or weighting both. The best set of choices is with the full probability and weighting both lepton+jets pairings.

\begin{tabular}{|c|c|c|}
\hline Stat./Bkg./Stat.+Bkg uncertainty & Pick L-J pairing & Weight both \\
\hline Max-likelihood & $0.144 / 0.055 / 0.154$ & $0.137 / 0.054 / 0.147$ \\
\hline Full probability & $0.131 / 0.064 / 0.146$ & $\mathbf{0 . 1 2 2} / \mathbf{0 . 0 6 0 / 0 . 1 3 6}$ \\
\hline
\end{tabular}

\subsubsection{Optimization Based on Tunable Parameters}

We perform further optimization by adding in a few more selection criteria and tuning the weights for the lepton-jet pairings with more information. The additional selection criteria and the new information that will be added into the weights have a couple of tunable parameters, so that we can manipulate them to minimize the expected uncertainty of the $A_{\mathrm{FB}}^{t \bar{t}}$ measurement. The parameters are

1. The position of the peak of the $\mathrm{jd}_{1,2}$ probability distributions, $\mathrm{jd}$ peak

2. Track-weighted jet charge for each jet

3. The value of the square of the invariant mass of the charged lepton and the b-quark $\left(m_{l b}^{2}\right)$ for each lepton-jet pairing

4. The value of $\Delta R_{\min }$ (lepton, jet) for the smallest pairing between any lepton and any jet.

We will do a simultaneous optimization since many of these variables are correlated. We start with a description of the optimization based on the peak position of the $\mathrm{jd}_{1,2}$ distributions.

We can improve the resolution of the $A_{\mathrm{FB}}^{t \bar{t}}$ measurement by rejecting badly reconstructed lepton-jet pairings. We have considered many parameters that help 
achieve this goal, like the width or number of peaks in the probability distributions of $\phi_{1,2}$, the width of $\mathrm{jd}_{1,2}$, etc. While they are all heavily correlated, we find that the peak position of the probability distribution of $\mathrm{jd}_{1,2}$ captures most of the information. We saw in Fig. 5.6 that when the probability distribution of $\mathrm{jd}_{1,2}$ peaks at small value this can correspond to the correct lepton+jet pairing; large values of jd $\mathrm{d}_{\text {peak }}$ 's typically corresponds to an incorrect pairing. Figs. 6.15 and 6.16 show the distributions of the position of the mode of $\mathrm{jd}_{1,2}$ probability distributions $\left(\mathrm{jd}_{1,2 \text { peak }}\right)$ for the sample of POWHEG events that pass all the reconstruction requirements (we have used the generator level information to choose the correct pairing). It essentially shows how far the top reconstruction algorithm has to shift the jet $E_{\mathrm{T}}$ to get the best fit $t \bar{t}$ event-by-event. For the wrong lepton-jet pairing (or background events), the top reconstruction algorithm sometimes needs to pull the jet $E_{\mathrm{T}}$ far away from their measured valued to make a valid $t \bar{t}$, which results in large $\mathrm{jd}_{1,2}$. The region $\left|\mathrm{jd}_{1,2 \text { peak }}\right| \gtrsim 2$ is dominated by wrong lepton-jet pairings. For simplicity, we consider the estimate of the uncertainty when we reject any lepton-jet pairing with $\sqrt{\mathrm{jd}_{1, \text { peak }}^{2}+\mathrm{jd} \mathrm{d}_{2, \text { peak }}^{2}}>\Theta(\mathrm{jd})$, and reject the event if both lepton-jet pairings are rejected. We will optimize the $\Theta(\mathrm{jd})$ value based on the expected uncertainty.

The track-weighted jet charge is correlated with the charge of its original quark, as detailed in Ref. [98, and can be used to help reject wrong lepton-jet pairings. This technique was recently used to measure the $A_{\mathrm{FB}}$ of $b \bar{b}$ at high mass [103]. The jet charge, $Q_{\text {jet }}$, is defined as:

$$
Q_{\text {jet }}=\frac{\sum_{i}\left|\overrightarrow{p_{i}} \cdot \overrightarrow{P_{\text {jet }}}\right|^{0.5} q_{i}}{\sum_{i}\left|\overrightarrow{p_{i}} \cdot \overrightarrow{P_{\text {jet }}}\right|^{0.5}}
$$

where $\overrightarrow{p_{i}}$ and $q_{i}$ are the momentum and the charge of each good-quality track [98] associated with a jet, and $\vec{P}_{i}$ is the momentum of the reconstructed jet. If there are 


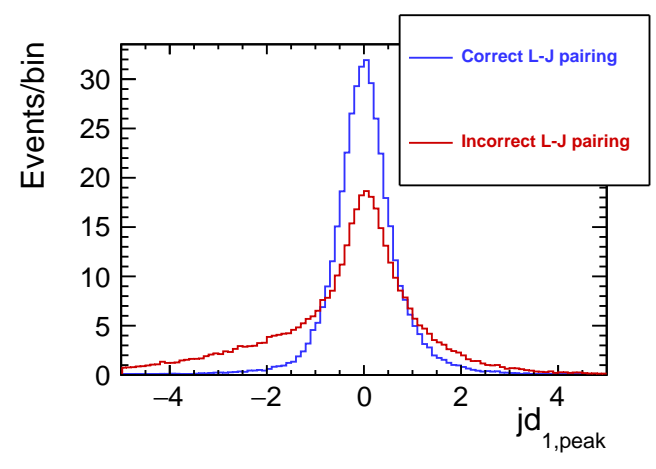

(a) $\mathrm{jd}_{1, \text { peak }}$

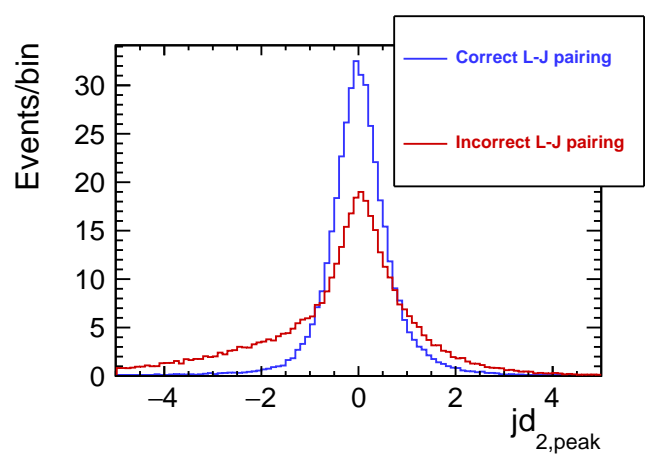

(b) $\mathrm{jd}_{2, \text { peak }}$

Figure 6.15: The $\mathrm{jd}_{\text {peak }}$ distributions for the correct and the incorrect lepton-jet pairings for the two jets.

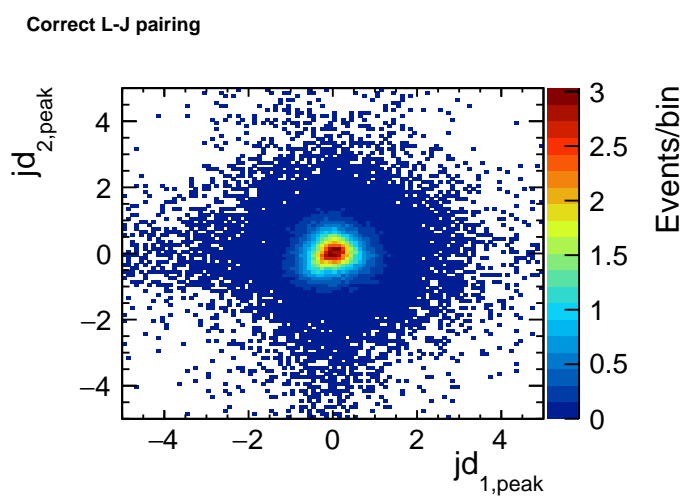

(a) Correct lepton-jet pairings
Incorrect L-J pairing

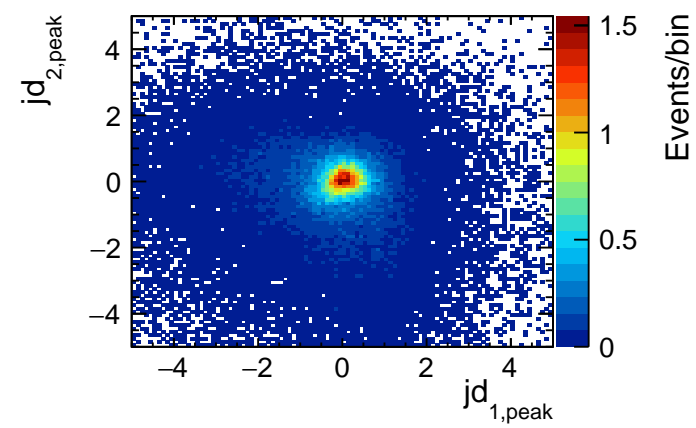

(b) Incorrect lepton-jet pairings

Figure 6.16: The contour plots for $\mathrm{jd}_{1, \text { peak }}$ vs. $\mathrm{jd}_{2, \text { peak }}$ for the correct and the incorrect lepton-jet pairings. 


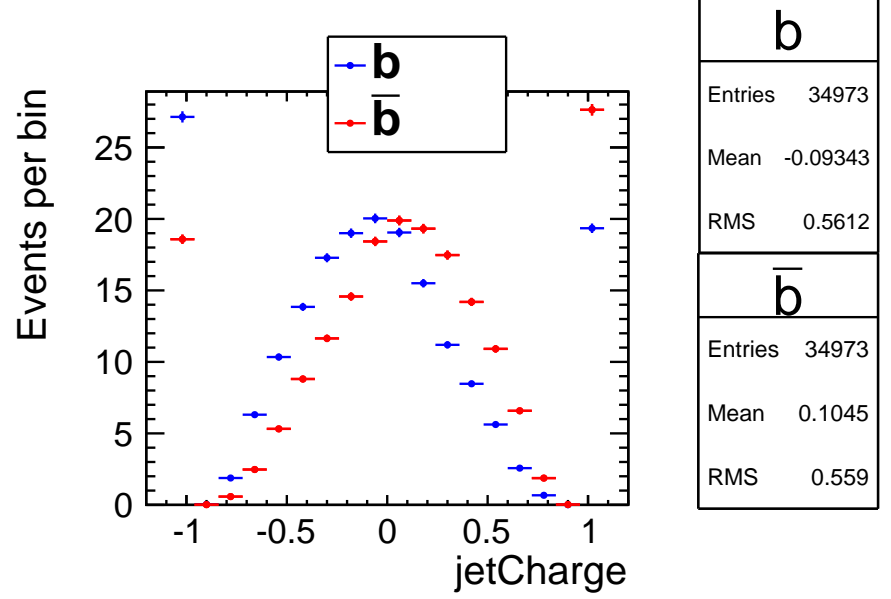

Figure 6.17: Distribution of the reconstructed jet charge, $Q_{\text {jet }}$, for jets originating from $b$ and $\bar{b}$ quarks as measured in our POWHEG sample passing all the dilepton selection criteria.

no good-quality tracks in a jet, we set $Q_{\text {jet }}=0$.

Figure 6.17 shows the distribution of the reconstructed jet charge, $Q_{\text {jet }}$, for jets originating from $b$ and $\bar{b}$ quarks in our POWHEG sample (which jet is originating from $b / \bar{b}$ quark is determined by looking at generator-level information). Only about $90 \%$ of the jets have valid jet charge reconstructed. The events piling up at \pm 1 are due to jets having only one good-quality track. The determination power of the track-weighted jet charge is not ideal, but useful.

We use the difference between the two jet charges $\delta Q=Q_{\text {jet1 }}-Q_{\text {jet2 }}$, where jet1 is the jet with larger $E_{\mathrm{T}}$ and jet2 is the other jet. The quantized jet charge $\Delta Q$ is determined by the sign of $\delta Q: \Delta Q=1$ if $\delta Q>0 ; \Delta Q=-1$ if $\delta Q<0$; and $\Delta Q=0$ otherwise. A value of $\Delta Q=1$ suggests that jet1 is from the $\bar{b}$-quark and jet2 from the $b$-quark, and $\Delta Q=-1$ suggests the opposite; a value of $\Delta Q=0$ indicates that the jet charge doesn't provide any power to distinguish between the $b / \bar{b}$ quarks. 
We introduce a tunable parameter, weight $w_{Q}$, to quantify the probability that the $\Delta Q$ gives the correct lepton-jet pairing, and amend the $L_{\max }$ of the two pairings used in Eqn. 5.10 to $L_{\max } * w_{Q}$ if $\Delta Q$ suggests this pairing and $L_{\max } *\left(1-w_{Q}\right)$ if $\Delta Q$ suggests otherwise, and proceed with Eqn. 5.10 in determining the weights of the two pairings. We optimize the value of $w_{Q}$ in the range of $(0.5,1.0)$ based on the expected uncertainty, where $w_{Q}=0.5$ is equivalent to ignoring the jet charge information, while $w_{Q}=1.0$ is equivalent to determining the lepton-jet pairing solely based on the jet charge information.

As suggested in Ref. [99], a large value of $m_{l b}^{2}$ is not likely to come from the correct lepton-jet pairing because of the heavy but finite top mass (i.e., $m_{l b}^{2}>m_{t}^{2}$ ). The distribution of $m_{l b}^{2}$ for the correct and incorrect lepton-jet pairings for events from POWHEG after all the selection requirements in Table 2.1 are shown in Fig. 6.18. While we could add the $m_{l b}^{2}$ information into our likelihood term in the overall likelihood, or in the pairing weighting, for simplicity, we use this information to motivate a cut. We reject the lepton-jet pairings with $m_{l b}^{2}>\Theta\left(m_{l b}^{2}\right)$, and reject the event if both lepton-jet pairings are rejected. We optimize $m_{l b}^{2}>\Theta\left(m_{l b}^{2}\right)$ based on the expected uncertainty.

We notice that events with a lepton that is very close to a jet in $\eta-\phi$ space do not reconstruct well or are likely to be background event (usually from $W+$ jets events where a $b$-jet fakes both a lepton and a jet). This effect is quantized as the minimum $\Delta R$ between any lepton and any jet $\left(\Delta R_{\min }\right.$ (lepton, jet $\left.)\right)$.

For visual purposes, to separate between well-reconstructed $t \bar{t}$ events and poorly reconstructed ones, we create two subsamples of events from our POWHEG sample. In the well-reconstructed sample we require both $y_{t}$ and $y_{\bar{t}}$ to be reconstructed within 0.1 of their generated values. The sample of badly reconstructed $t \bar{t}$ events is selected by requiring either $y_{t}$ or $y_{\bar{t}}$ to be reconstructed 1.5 away from their generated 


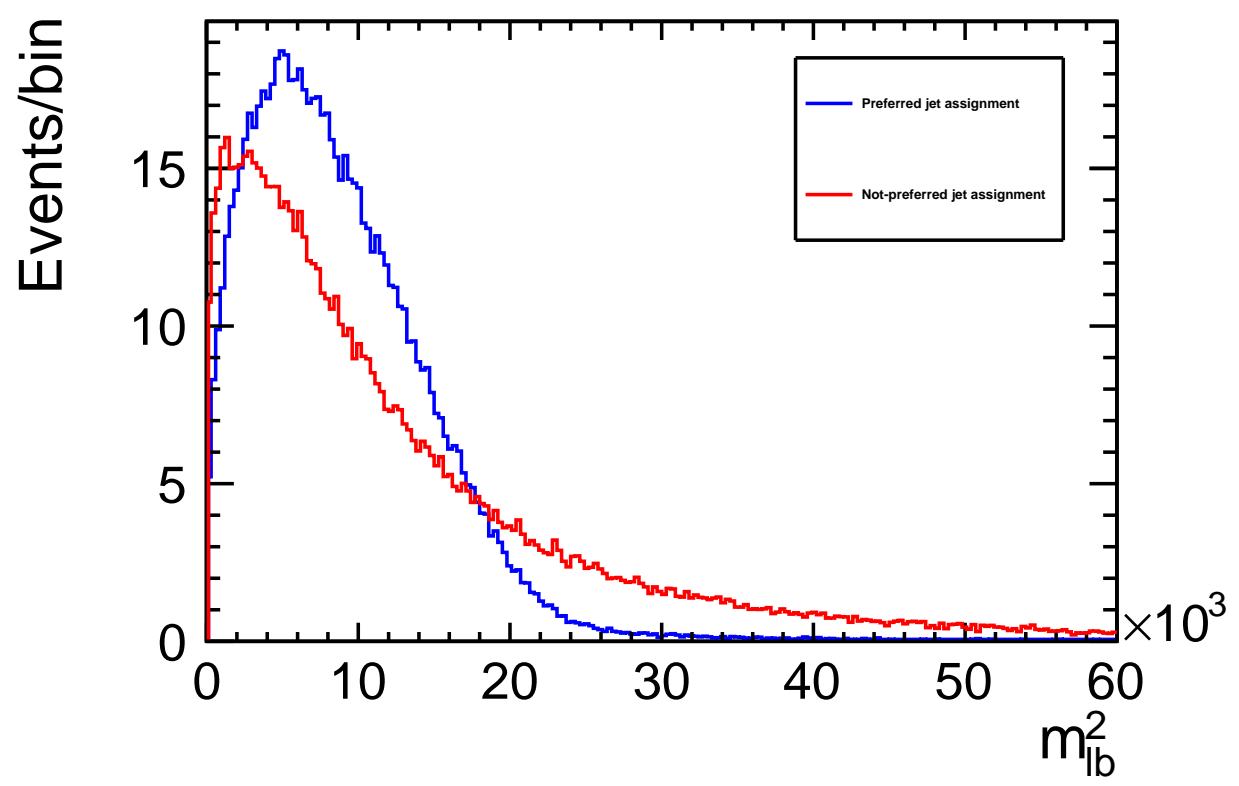

Figure 6.18: Distribution of $m_{l b}^{2}$ for the correct and incorrect lepton-jet pairings taken from the POWHEG sample. The blue curve is from the correct lepton-jet pairing, and the red curve is from the incorrect lepton-jet pairing. 
values. Figure 6.19 shows the $\Delta R_{\min }$ (lepton, jet) distributions of well reconstructed $t \bar{t}$, badly reconstructed $t \bar{t}$, as well as for background events (mostly $W+$ jets background). From this we expect a requirement on the minimal $\Delta R_{\min }\left(\Theta\left(\Delta R_{\min }\right)\right)$ would reject $W+$ jets background events without significantly hurting the number of the well reconstructed $t \bar{t}$ events. While this plot is illustrative, the two samples are not used determine the cut value. We optimize the cut value again based on the expected uncertainty.

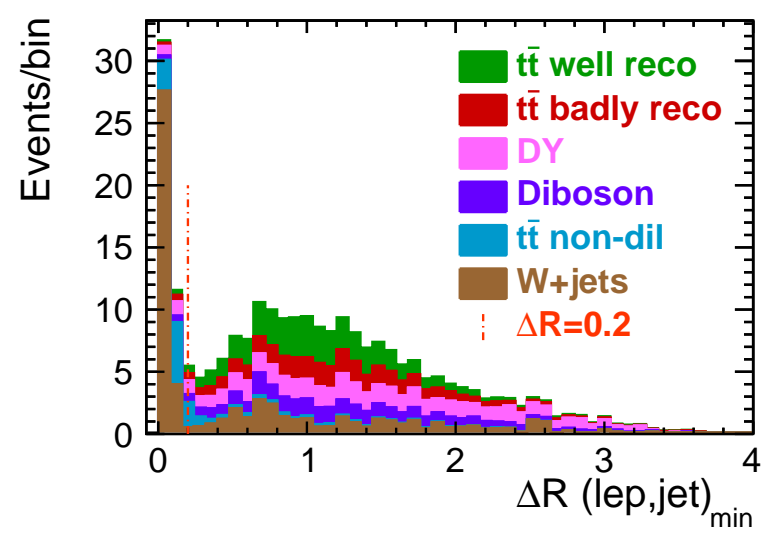

Figure 6.19: The distributions for $\Delta R_{\min }$ (lepton, jet) of well reconstructed $t \bar{t}$ events, badly reconstructed $t \bar{t}$ events, and the various backgrounds. The vertical dotdashed line shows $\Delta R_{\min }$ (lepton, jet) $=0.2$, which is the optimal value determined in Sec. 6.2.3.

\subsubsection{Final Optimization Values and Results}

The final selection of the parameters described in the previous subsection is based on minimizing the expected uncertainty, and with all four parameters varied simultaneously to find the global minimum. For each cut or weight value, we run 5k pseudoexperiments to obtain the expected uncertainty for that choice. Table 6.2 
shows the optimum values for all cuts and weights, and Figure 6.20 shows the expected uncertainties as functions of the cut and weight values with other values fixed at the optimum points. None of the individual parameter changes the result significantly, but with all of them, we are able to obtain a signal efficiency of $95 \%$ while rejecting $40 \%$ of the backgrounds after the baseline selection requirements. We find that the expected statistical uncertainty from signal-only is 0.106 (down from 0.107); the total statistical uncertainty from signal+backgrounds is 0.114 (down by $7 \%$ from 0.122); and the background systematic uncertainty is estimated to be 0.042 (down by $30 \%$ from 0.060 ). Overall, the total expected uncertainty is improved by $11 \%$ from 0.136 to 0.122 .

Table 6.2: Summary of the optimum cut and weight values used in the final analysis.

\begin{tabular}{cc} 
Optimum cut and weight \\
\hline$\Theta(\mathrm{jd})$ & 3.5 \\
$w_{Q}$ & 0.7 \\
$\Theta\left(m_{l b}^{2}\right)$ & $24000\left(\mathrm{GeV}^{2}\right)$ \\
$\Theta\left(\Delta R_{\min }\right)$ & 0.2 \\
\hline
\end{tabular}

\subsection{Validation of the Measurement Methodology}

With the optimal configuration of the top reconstruction quality cuts and the weights of the jet charge probability determined, in this section we show the validation of the background and signal modeling as well as for the unfolding algorithm. We will first show the validation of the signal and background modeling after the top reconstruction and optimization, then show tests of the unfolding procedure. 

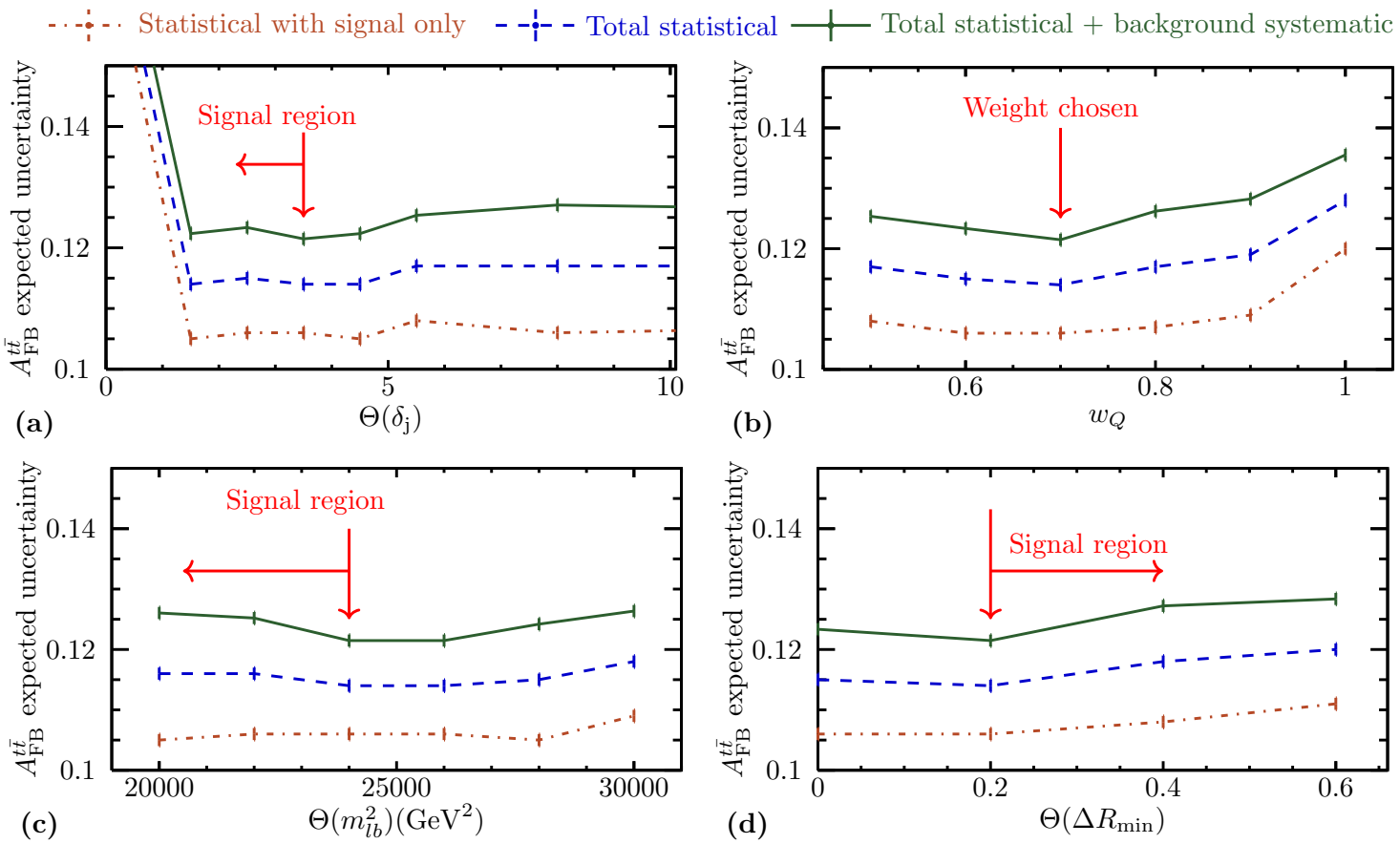

Figure 6.20: The expected statistical uncertainty with signal only (brown dot-dashed line), the total statistical uncertainty (with signal + background, blue dashed line), and the total statistical + background systematic uncertainty (the expected uncertainty in the text, green solid line), as functions of various cut and weight values. The optimum values are based on the minimum point of the green line, as marked with the red arrows on the plots, and summarized in Table 6.2. All other values are held at their optimal values for each plot. The expected uncertainty, after optimization, is 0.122 . 


\subsubsection{Additional Validation of Signal and Background Modeling}

The expected numbers of events in data passing all top dilepton event selections and top reconstruction quality selections, along with the observed number of events, are summarized in Table 6.3. The agreement between the prediction and the observation is good. We again note that compared with Table 3.3, the signal efficiency of the top reconstruction quality cut is $95 \%$ (408 vs. 386 expected) with a background rejection of $40 \%$ (160 vs. 96 expected); mostly we have rejected $W+$ jets events (from 64 to 28 ) and $Z / \gamma^{*}+$ jets events (from 50 to 37 ).

Table 6.3: Expected number of events passing all top dilepton event selections and top reconstruction quality selections, along with the observed number of events in data.

\begin{tabular}{lc}
\multicolumn{2}{c}{ CDF Run II Preliminary $\left(9.1 \mathrm{fb}^{-1}\right)$} \\
\hline \multicolumn{2}{c}{$\begin{array}{c}\text { Expected and observed events } \\
\left(t \bar{t} \rightarrow l^{+} l^{-}+2 \text { jets }+E_{T}\right)\end{array}$} \\
\hline \hline \multicolumn{1}{c}{ Source } & Events \\
\hline Diboson & $26 \pm 5$ \\
$Z / \gamma^{*}+$ jets & $37 \pm 4$ \\
$W+$ jets & $28 \pm 9$ \\
$t \bar{t}$ non-dilepton & $5.3 \pm 0.3$ \\
\hline Total background & $96 \pm 18$ \\
Signal $t \bar{t}(\sigma=7.4$ pb $)$ & $386 \pm 18$ \\
\hline Total SM expectation & $482 \pm 36$ \\
\hline Observed & 495 \\
\hline \hline
\end{tabular}

Figures 6.21 and 6.22 show the kinematic variables from data compared with the predictions. The agreement between data and the predictions is good. The reconstructed $\Delta y_{t}$ is shown in Fig. 6.23 compared with the sum of backgrounds and signal prediction from POWHEG. The $A_{\mathrm{FB}}^{t \bar{t}}$ will be extracted from this distribution. 


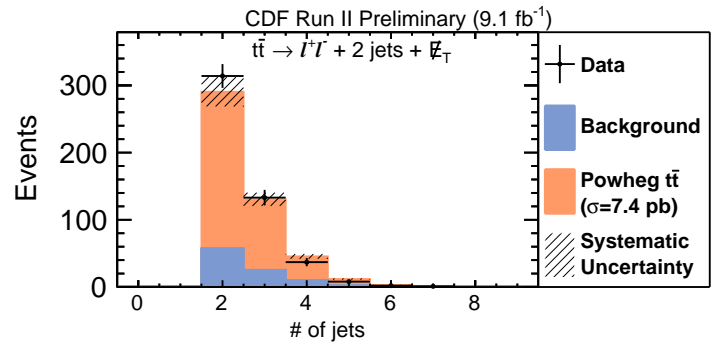

(a) Jet multiplicity

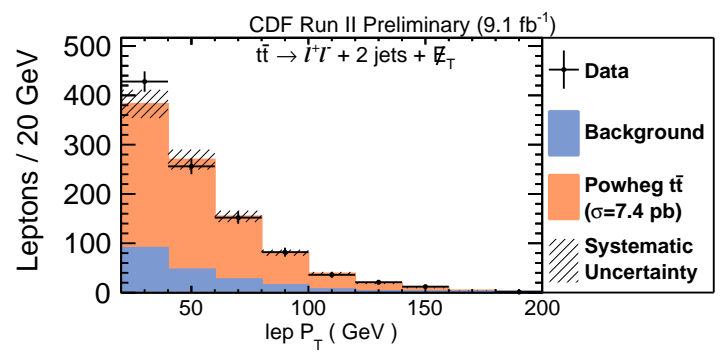

(c) $p_{T}$ of leptons

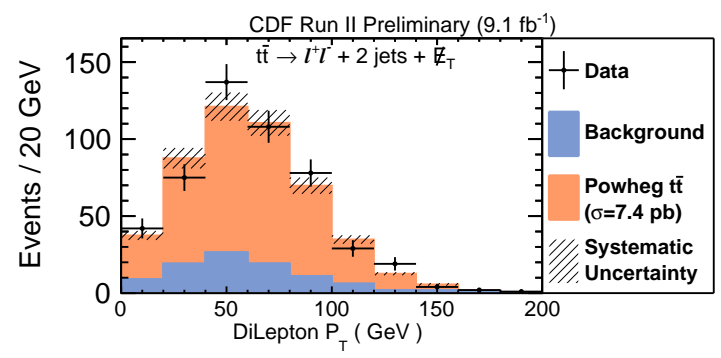

(e) $p_{T}$ of the dilepton system

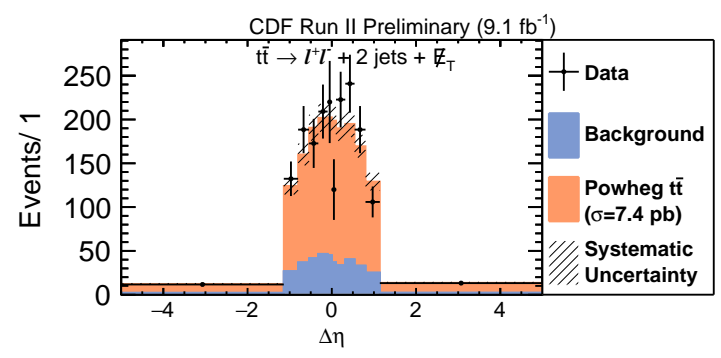

(g) $\Delta \eta$

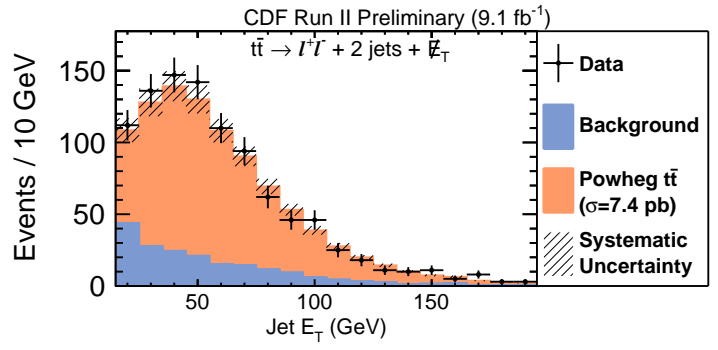

(b) Jet $E_{\mathrm{T}}$

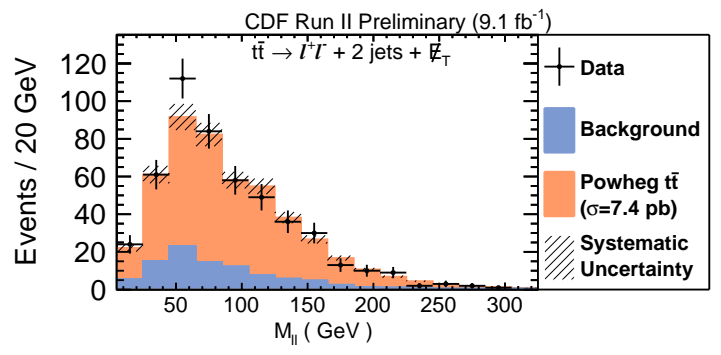

(d) $m_{l l}$

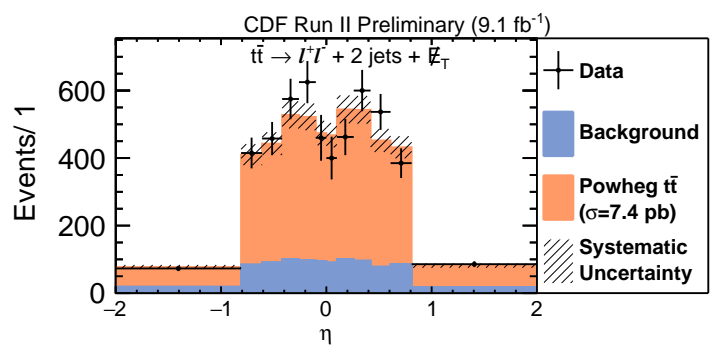

(f) $q_{\ell} \eta_{\ell}$

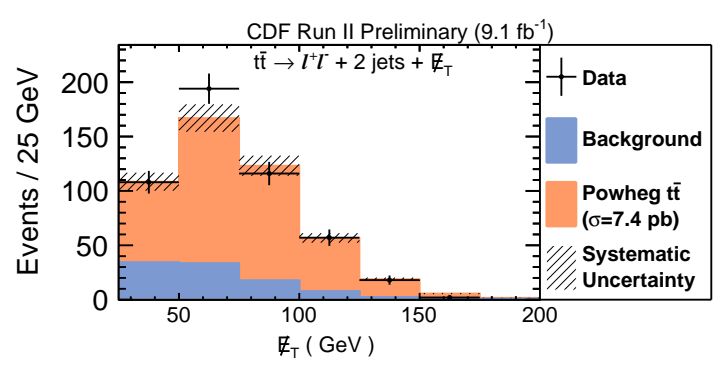

(h) $E_{T}$

Figure 6.21: Kinematic variables from data compared to predictions after all cuts in Tables 2.1 and 6.2 . 


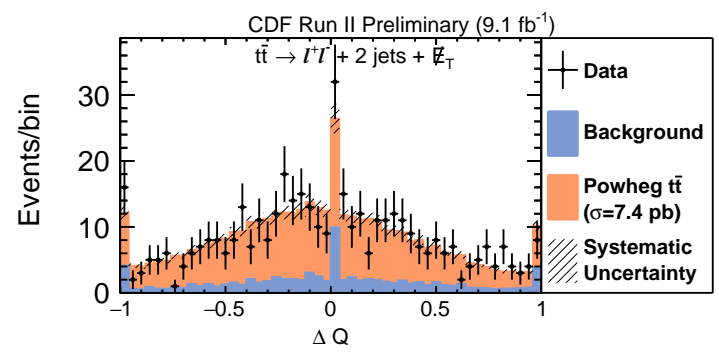

(a) $\Delta Q$

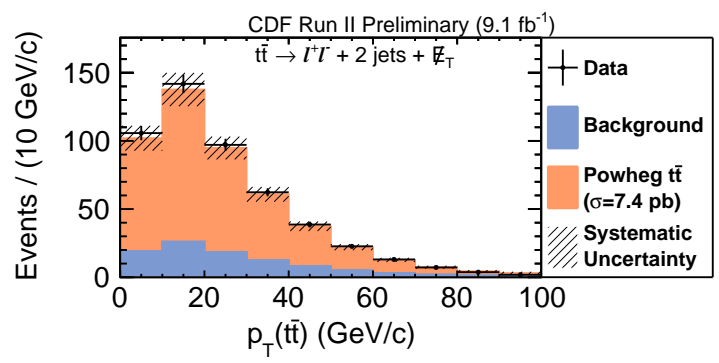

(c) $p_{T, t \bar{t}}$

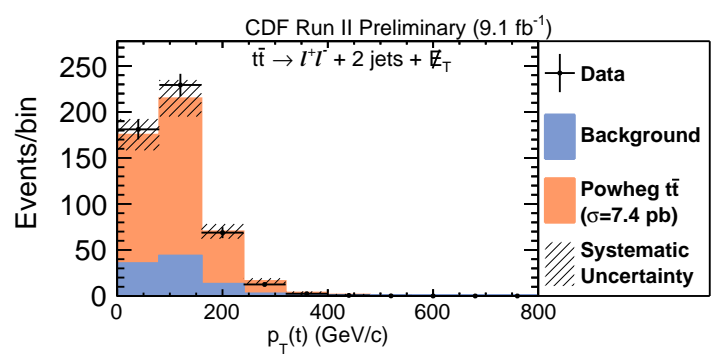

(e) $p_{T}(t)$

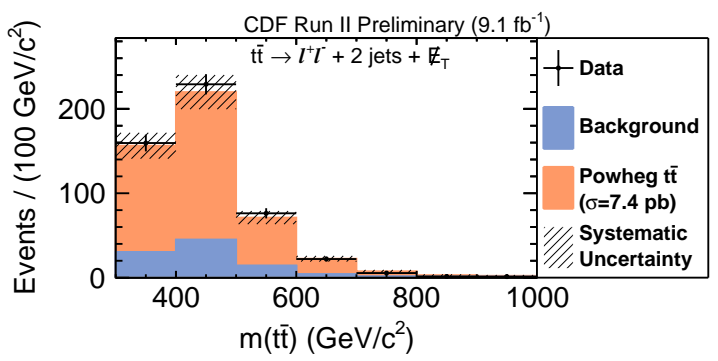

(b) $m_{t \bar{t}}$

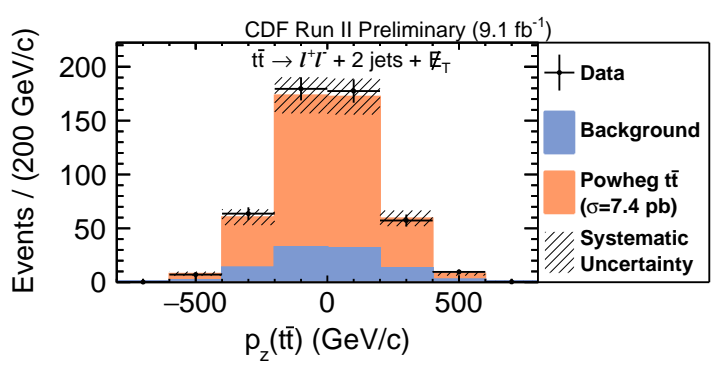

(d) $p_{z, t \bar{t}}$

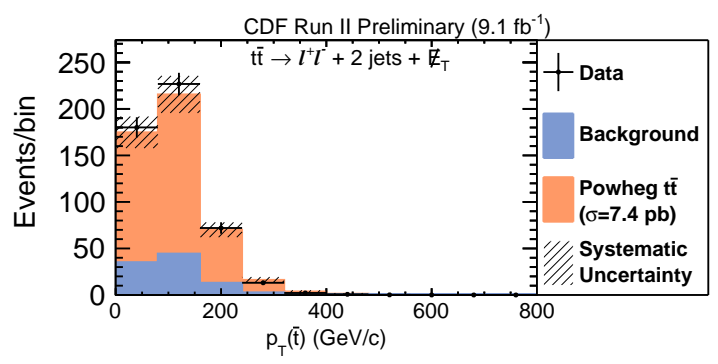

(f) $p_{T}(\bar{t})$

Figure 6.22: The same as Fig. 6.21, but with additional variables. 


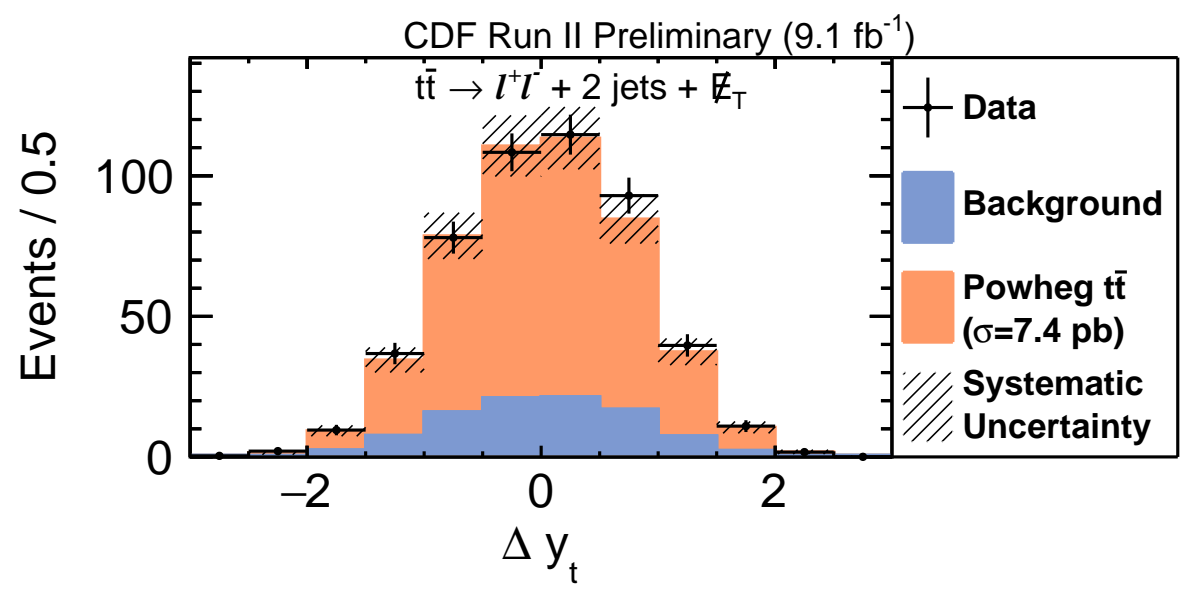

Figure 6.23: The final $\Delta y_{t}$ distribution from data compared with the SM expectations after all the event selection requirements and event reconstruction algorithms.

\subsubsection{Validation of the Inclusive $A_{F B}^{t \bar{t}}$ Measurement Methodology}

Figure 6.24 shows the reconstruction resolution $\left(\Delta y_{t}\right.$ (reconstructed) $-\Delta y_{t}$ (generated $))$ and the detector smearing matrix $\left(\Delta y_{t}\right.$ (generated) vs. $\Delta y_{t}($ reconstructed $\left.)\right)$ after the optimization. The center values and the uncertainties of the detector smearing matrix, Det $[p][r]$ in Eq. 6.7, are summarized in Table 6.4.

Figure 6.25 shows the efficiencies $\left(\operatorname{Eff}[p]\left(A_{\mathrm{FB}}^{t \bar{t}}\right)\right.$ in Eq. 6.7) in each $\left|\Delta y_{t}\right|$ bin as a function of $A_{\mathrm{FB}}^{t \bar{t}}$, fitted with linear functions. The best fit parameters are summarized in Table 6.5. The best fit functions are summarized in Fig. 6.26.

Figure 6.27 shows the results of the pseudoexperiments after the optimal requirements and weights, with signal only (LHS) and with signal+backgrounds (RHS). The pull distributions look good. The expected statistical uncertainty from signal-only is 0.106 , and the statistical uncertainty from signal+backgrounds is 0.114 , with the background systematic uncertainty estimated to be 0.037 by taking the difference of the two in quadrature as in Sec. 4 . 

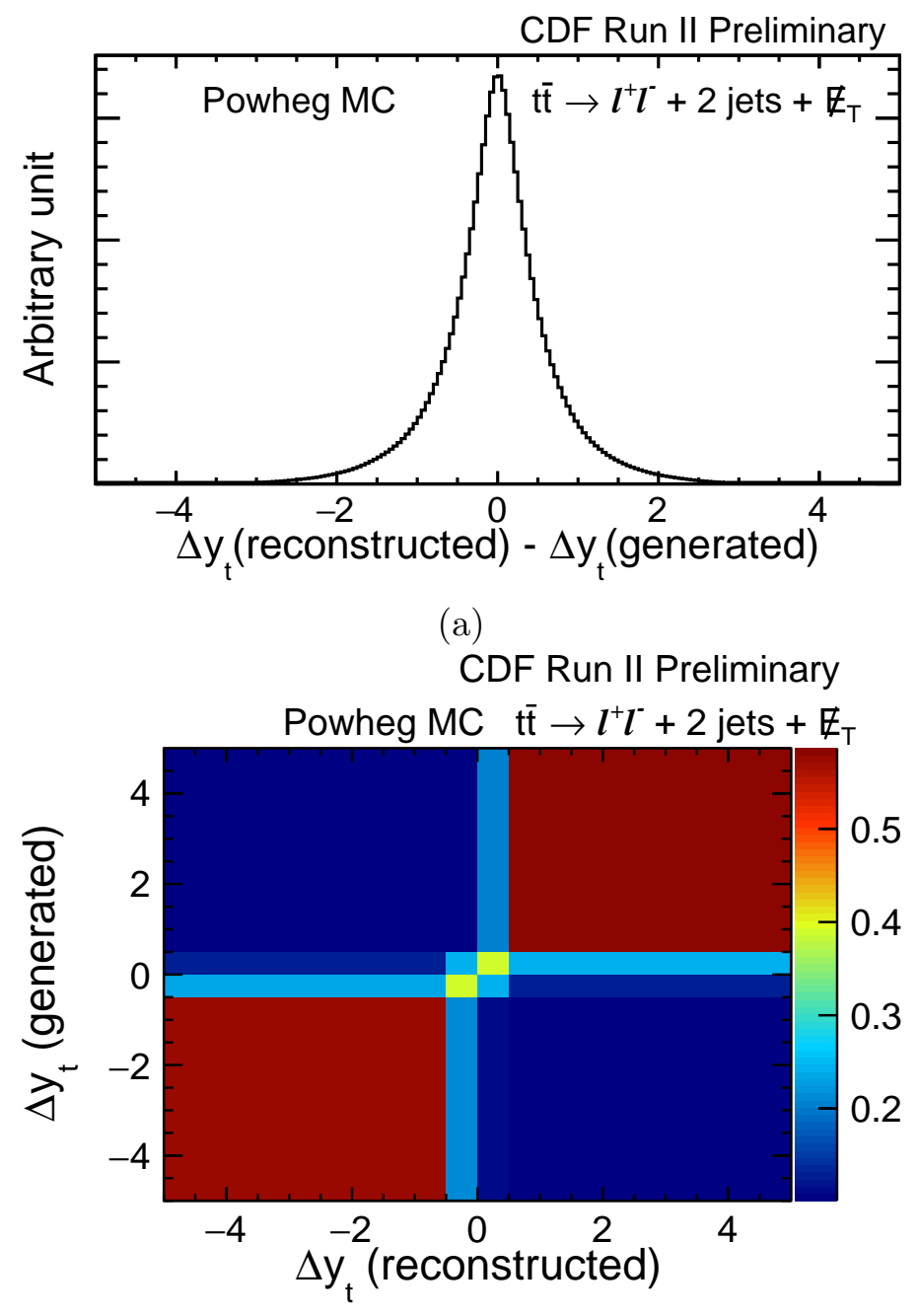

(b)

Figure 6.24: The same figures as Figs. 5.13 and 6.7, but after optimization. The numbers from (b) are summarized in Table 6.4. which corresponds to $\operatorname{Det}[p][r]$ in Eq. 6.7. 


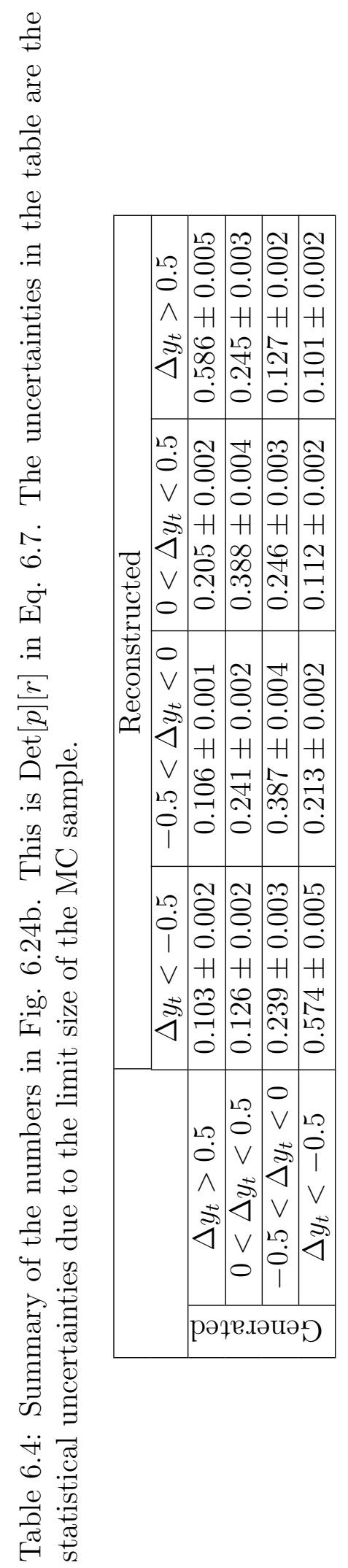




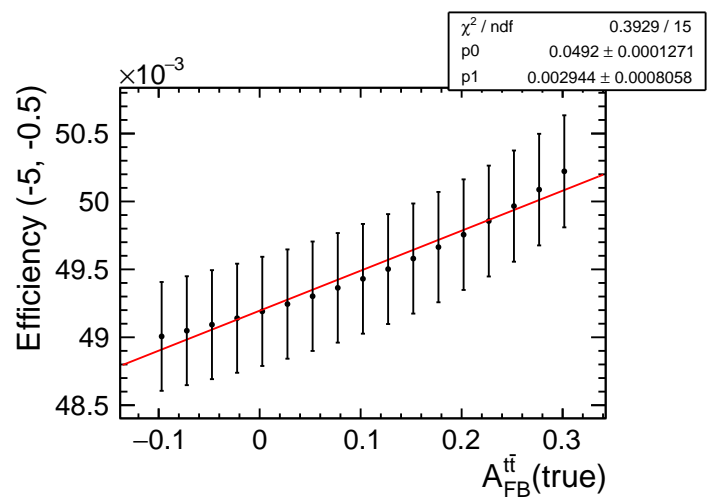

(a) $\Delta y_{t}<-0.5$

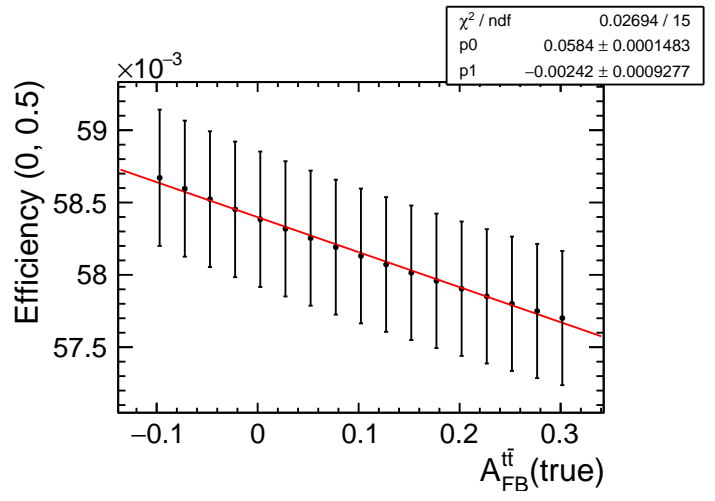

(c) $0<\Delta y_{t}<0.5$

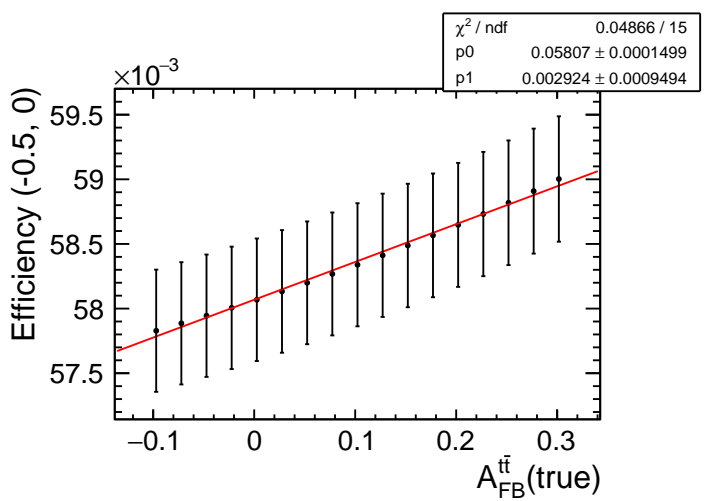

(b) $-0.5<\Delta y_{t}<0$

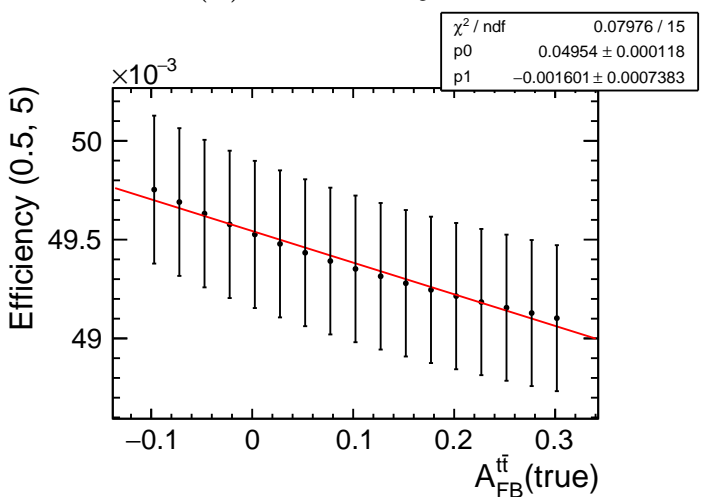

(d) $\Delta y_{t}>0.5$

Figure 6.25: The same figures as Fig. 6.6, but after the optimization. The best fit values are summarized in Table. 6.5 and shown in Fig. 6.26, and which corresponds to $\operatorname{Eff}[p]\left(A_{\mathrm{FB}}^{t \bar{t}}\right)$ in Eq. 6.7 .

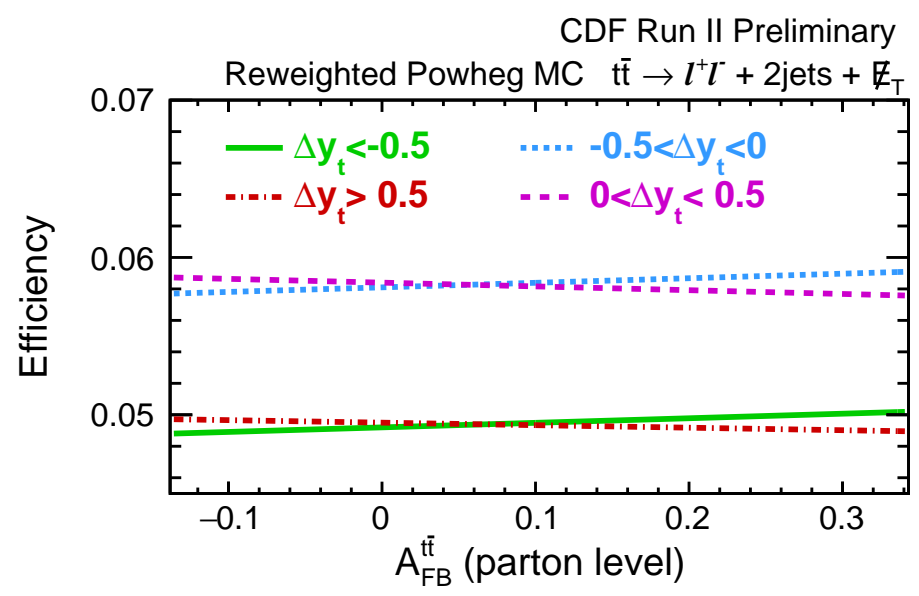

Figure 6.26: The best fit functions in Fig. 6.25. The best fit values are summarized in Table. 6.5, which corresponds to $\operatorname{Eff}[p]\left(A_{\mathrm{FB}}^{t t}\right)$ in Eq. 6.7. 


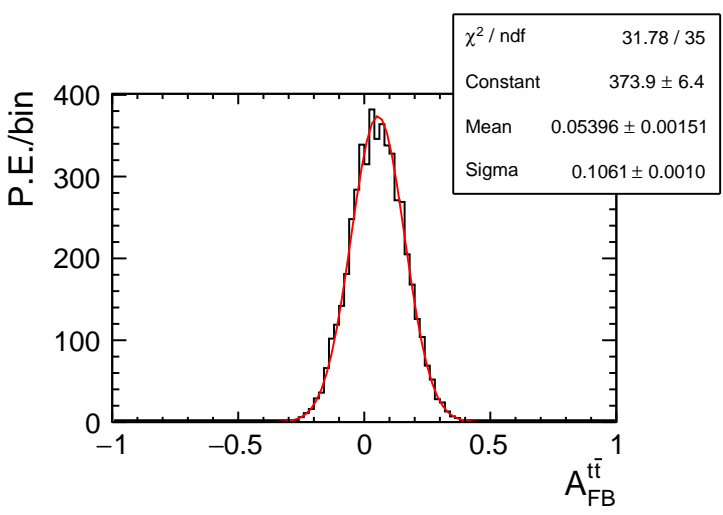

(a) $A_{\mathrm{FB}}^{t \bar{t}}$, signal only

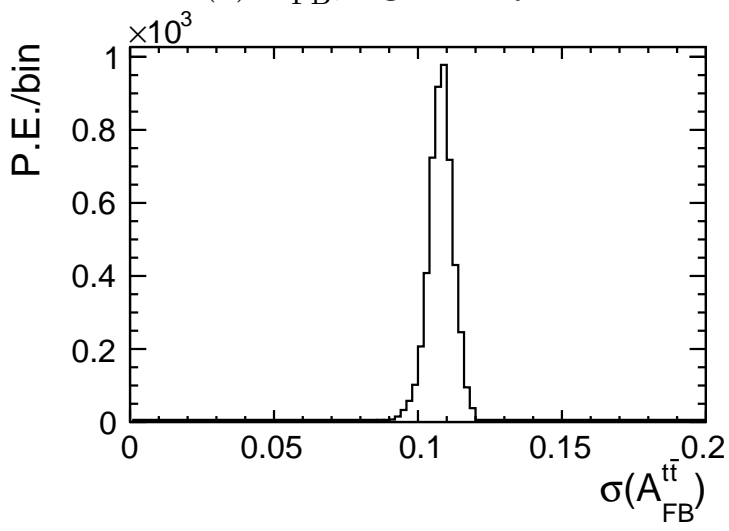

(c) $\sigma\left(A_{\mathrm{FB}}^{t \bar{t}}\right)$, signal only

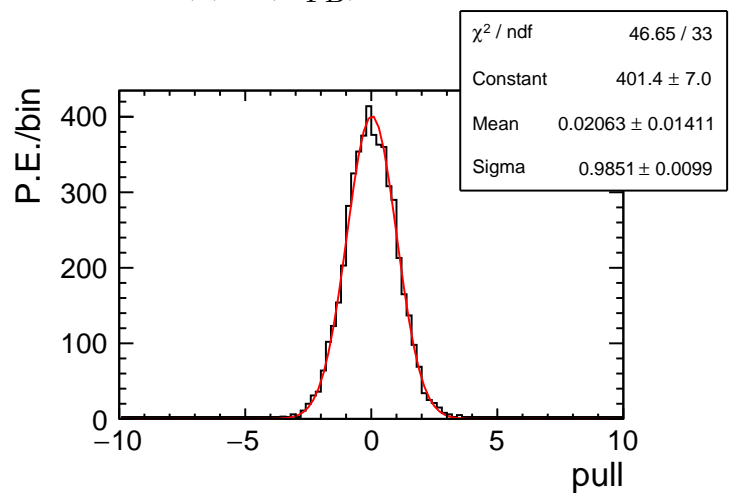

(e) $\operatorname{Pull}\left(A_{\mathrm{FB}}^{t \bar{t}}\right)$, signal only

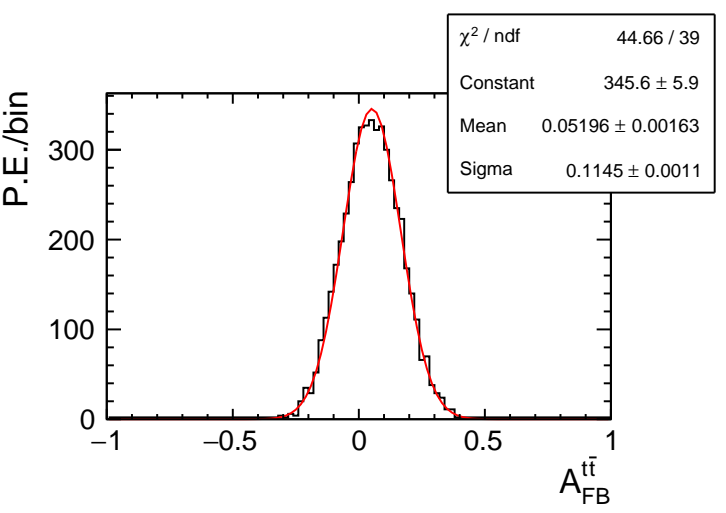

(b) $A_{\mathrm{FB}}^{t \bar{t}}$, signal+backgrounds

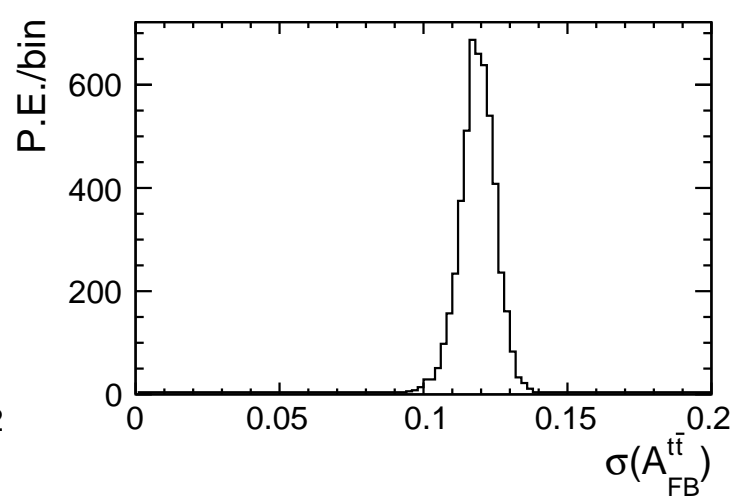

(d) $\sigma\left(A_{\mathrm{FB}}^{t \bar{t}}\right)$, signal+backgrounds

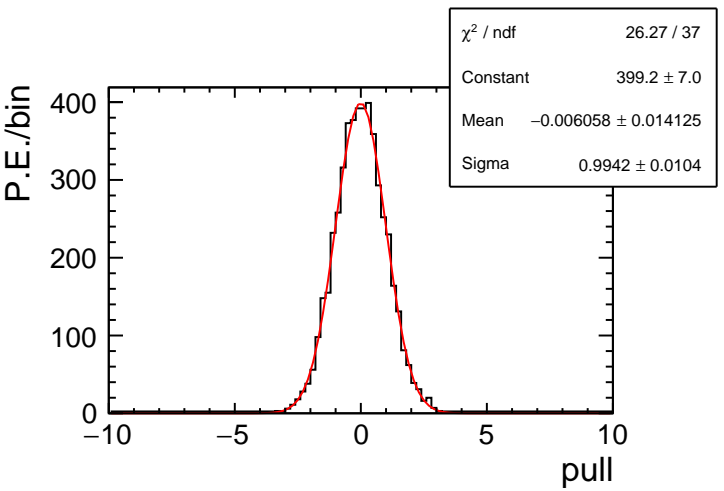

(f) $\operatorname{Pull}\left(A_{\mathrm{FB}}^{t \bar{t}}\right)$, signal+backgrounds

Figure 6.27: Inclusive $A_{\mathrm{FB}}^{t \bar{t}}$ measurement results of the pseudoexperiments with the optimal cuts and weights, with signal only (LHS) and with signal+backgrounds (RHS) for the measurement of the inclusive $A_{\mathrm{FB}}^{t \bar{t}}$. The pull distributions look good. The expected statistical uncertainty with signal only is 0.106 , and that with signal+backgrounds is 0.114 . These can be compared to Fig. 6.10 and 6.13. 
Table 6.5: Summary of the efficiencies in each $\left|\Delta y_{t}\right|$ bin as a linear function of $A_{\mathrm{FB}}^{t \bar{t}}$ taken from Fig. 6.25, after the optimization. This is $\operatorname{Eff}[p]\left(A_{\mathrm{FB}}^{t \bar{t}}\right)$ in Eq. 6.7. The uncertainties in the table are the statistical uncertainties caused by the limited size of the MC sample.

\begin{tabular}{|c|c|c|}
\multicolumn{3}{|c|}{ Eff $[\operatorname{Bin}]\left(A_{\mathrm{FB}}^{t \bar{t}}\right)=\mathrm{p} 0+\mathrm{p} 1 \cdot A_{\mathrm{FB}}^{t \bar{t}}($ true $)$} \\
\hline Generator-level bin & $\mathrm{p} 0$ & $\mathrm{p} 1$ \\
\hline$\Delta y_{t}<-0.5$ & $0.0492 \pm 0.0001$ & $0.0029 \pm 0.0008$ \\
\hline$-0.5<\Delta y_{t}<0$ & $0.0581 \pm 0.0001$ & $0.0029 \pm 0.0009$ \\
\hline $0<\Delta y_{t}<0.5$ & $0.0584 \pm 0.0001$ & $-0.0024 \pm 0.0009$ \\
\hline$\Delta y_{t}>0.5$ & $0.0495 \pm 0.0001$ & $-0.0016 \pm 0.0007$ \\
\hline
\end{tabular}

We test the unfolding procedure with the reweighted POWHEG samples described in Sec. 6.1.1 by comparing the measured value returned by the unfolding procedure to the true value from the generator level. The results are shown in Fig. 6.28. The error bars correspond to the statistical uncertainties based on the whole MC sample (10M events generated, $\sim 70 \mathrm{k}$ selected). There is no bias observed.

In addition, we test the unfolding algorithm with LO SM (PYTHIA [50], ALPGEN [51] and HERWIG [52]) as well as the BSM scenarios (t-channel Z' [56], $425 \mathrm{GeV}$ Axi [54], $200 \mathrm{Gev}$ AxiL/A/R [53], and 1.8/2.0 TeV Axi [19]). We take the difference between the generated $A_{\mathrm{FB}}^{t \bar{t}}$ and the measured $A_{\mathrm{FB}}^{t \bar{t}}$ with PYTHIA MC (0.02) as a systematic uncertainty for unfolding to cover the potential bias caused by the NLO SM assumption we made in the top reconstruction procedure and the unfolding procedure.

We do not anticipate the unfolding procedure to work perfectly with BSM scenarios because they are generated at LO and have non-SM kinematics, and most of them have already been ruled out. In particular, we do not expect the estimation of $A_{\mathrm{FB}}^{t \bar{t}}$ to have zero bias in all BSM scenarios, since the assumptions of the $p_{z, t \bar{t}}, p_{T, t \bar{t}}$, and $m_{t \bar{t}}$ distributions we made in top-momenta reconstruction will no longer hold, 


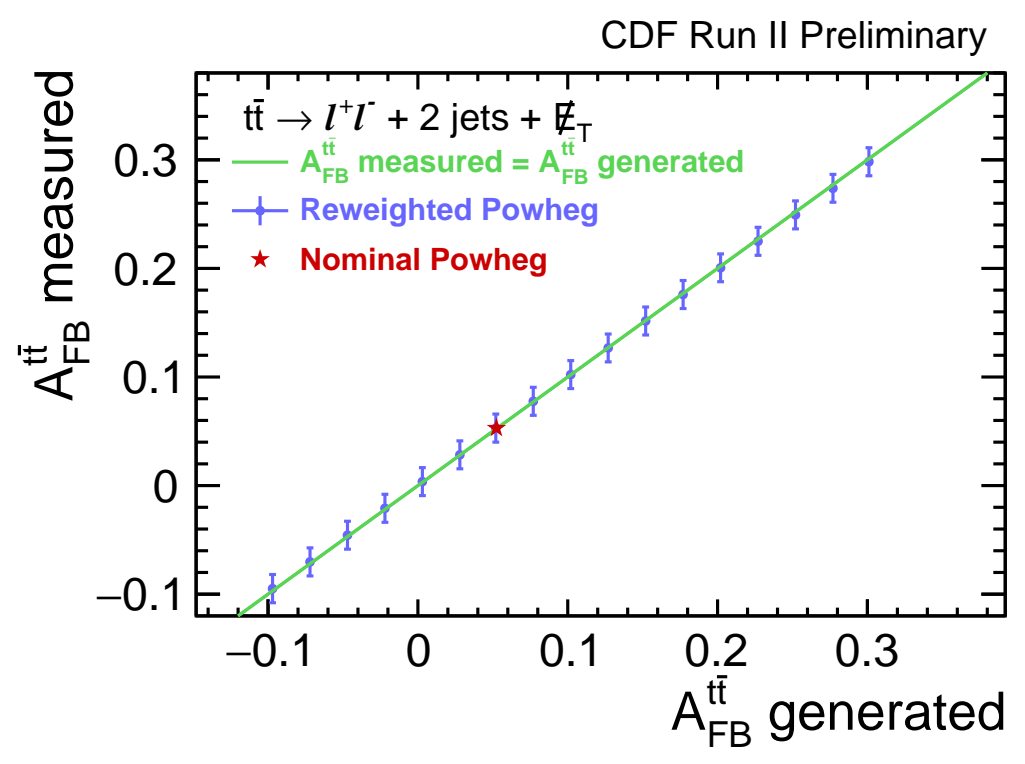

Figure 6.28: A comparison of the $A_{\mathrm{FB}}^{t \bar{t}}$ measured with the unfolding algorithm compared with the $A_{\mathrm{FB}}^{t \bar{t}}$ generated, for the reweighted POWHEG MC samples, with $-0.1<A_{\mathrm{FB}}^{t \bar{t}}<0.3$. No bias observed. We note that all the data points are correlated.

both due to the effect of the new physics and because the $t \bar{t}$ portion is only calculated at LO. For example, the $p_{T, t \bar{t}}$ spectrum calculated at LO shows deviation from data due to lack of higher-order diagrams with non-zero $p_{T, t \bar{t}}$, while the NLO calculation provides reasonable agreement [20]. The results can be found in Fig. 6.29. We note that the biggest deviation from expectation is 0.08 , but we confirm that if we correct for the non-realistic $p_{T, t \bar{t}}$ spectrum in the samples, we get back what we expect.

\subsubsection{Validation of the $A_{F B}^{t \bar{t}}$ vs. $\left|\Delta y_{t}\right|$ Measurement Methodology}

Figures 6.30 and 6.31 show the results of the pseudoexperiments for the measurements of $A_{\mathrm{FB}}^{t \bar{t}}\left(\left|\Delta y_{t}\right|<0.5\right)$ and $A_{\mathrm{FB}}^{t \bar{t}}\left(\left|\Delta y_{t}\right|>0.5\right)$ after the optimal cuts and weights, with signal only and with signal+backgrounds, respectively. The pull distributions look good. The expected statistical uncertainty from signal only is 0.27 and 0.13 for the two $\left|\Delta y_{t}\right|$ bins, and the statistical uncertainty from signal+backgrounds is 0.30 


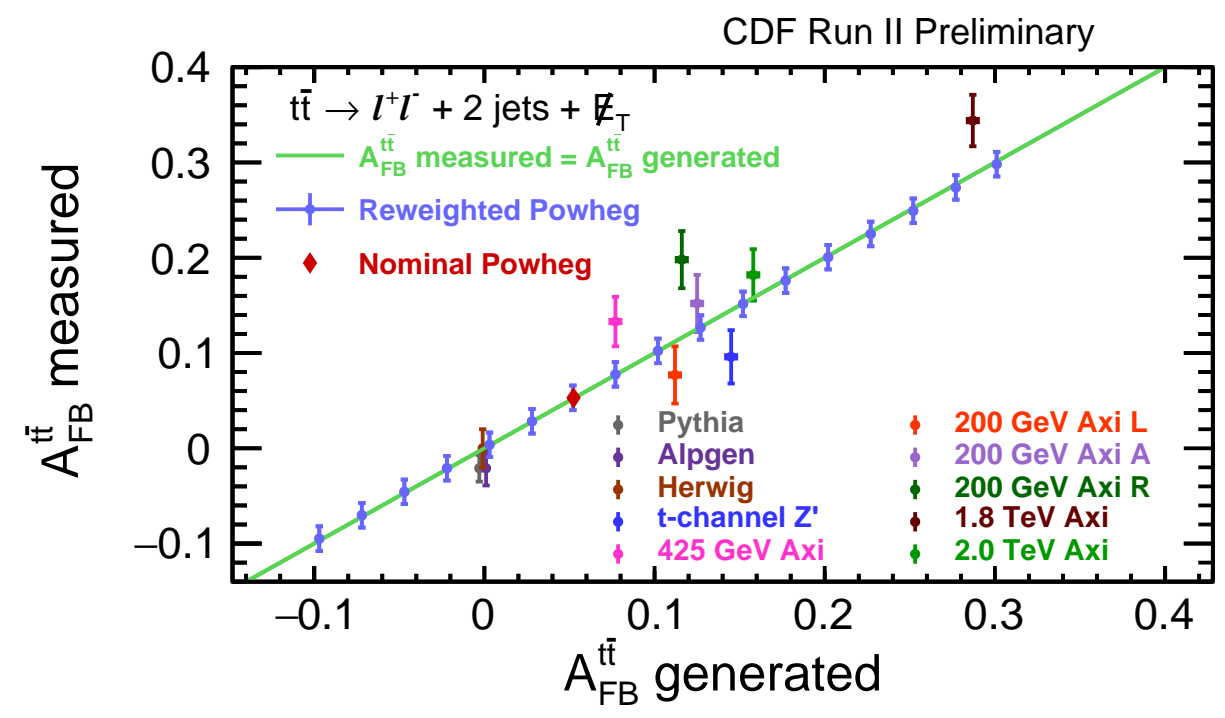

Figure 6.29: A comparison of the inclusive $A_{\mathrm{FB}}^{t \bar{t}}$ measured with the unfolding algorithm with the $A_{\mathrm{FB}}^{t \bar{t}}$ generated, for the reweighted POWHEG MC samples and benchmark BSM scenarios. We take the difference between the generated $A_{\mathrm{FB}}^{t \bar{t}}$ and the measured $A_{\mathrm{FB}}^{t \bar{t}}$ with PYTHIA MC (0.02) as an unfolding systematic uncertainty.

and 0.14 , with the background systematic uncertainty estimated at 0.13 and 0.06 in the two $\left|\Delta y_{t}\right|$ bins.

Figure 6.32 shows the differential $A_{\mathrm{FB}}^{t \bar{t}}$ measured with the unfolding algorithm compared with $A_{\mathrm{FB}}^{t \bar{t}}$ generated, for the reweighted POWHEG MC samples. The error bars corresponds to the statistical uncertainties with the full POWHEG MC sample (10M events in total, $\sim 70 \mathrm{k}$ selected). While there seems to be a small bias, we note that the deviation between the measured values and the generated values in the worst case is consistent with the statistical uncertainties, and negligible compared to the expected statistical uncertainties from data $\left(0.30\right.$ and 0.15 with $\left|\Delta y_{t}\right|<0.5$ and $\left|\Delta y_{t}\right|>0.5$, respectively). We elect not to attempt to fix this negligible bias and simply take the difference as systematic uncertainties according to Fig. 6.32, 


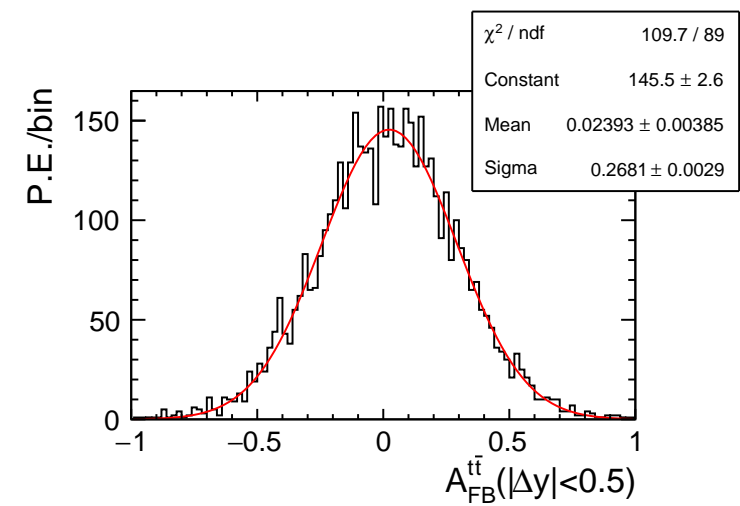

(a) $A_{\mathrm{FB}}^{t \bar{t}}\left(\left|\Delta y_{t}\right|<0.5\right)$, signal only

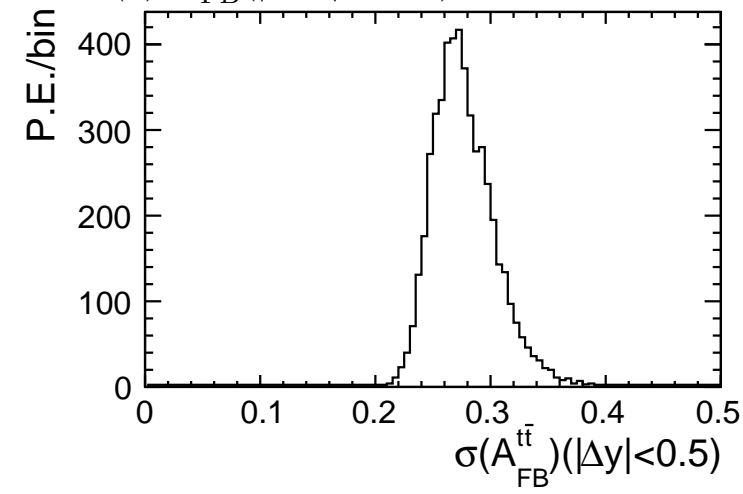

(c) $\sigma\left(A_{\mathrm{FB}}^{t \bar{t}}\right)\left(\left|\Delta y_{t}\right|<0.5\right)$, signal only

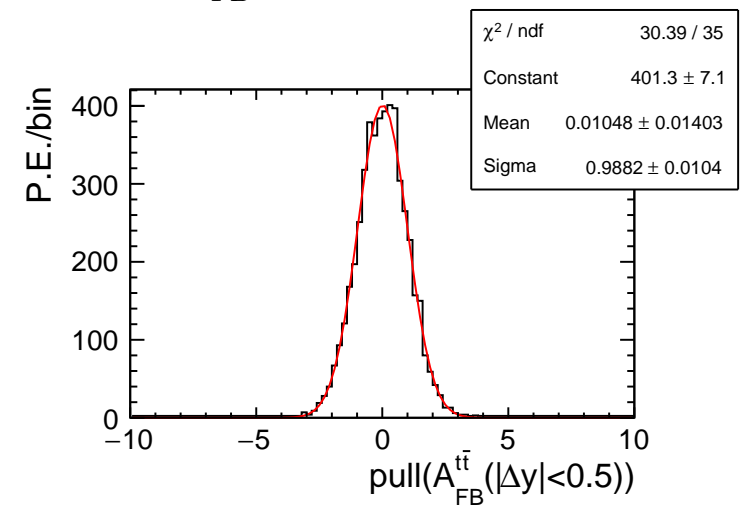

(e) $\operatorname{Pull}\left(A_{\mathrm{FB}}^{t \bar{t}}\right)\left(\left|\Delta y_{t}\right|<0.5\right)$, signal only

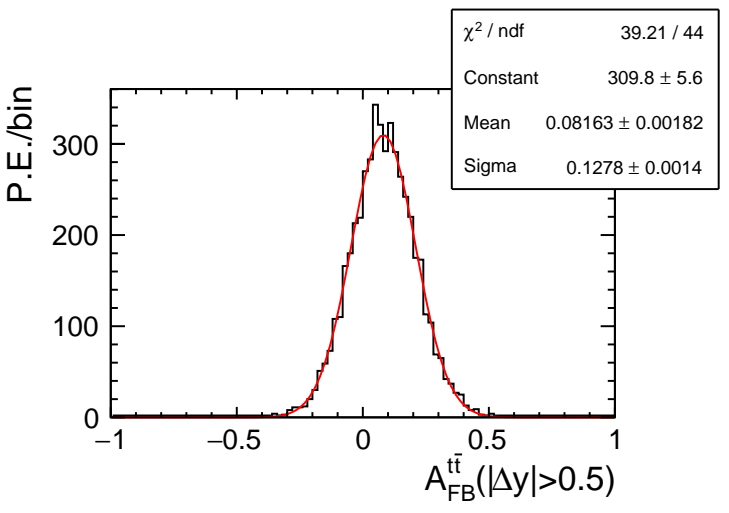

(b) $A_{\mathrm{FB}}^{t \bar{t}}\left(\left|\Delta y_{t}\right|>0.5\right)$, signal only

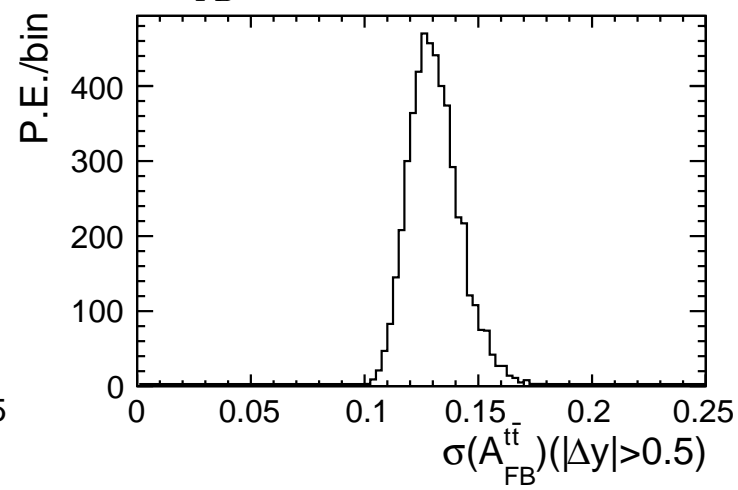

(d) $\sigma\left(A_{\mathrm{FB}}^{t \bar{t}}\right)\left(\left|\Delta y_{t}\right|>0.5\right)$, signal only

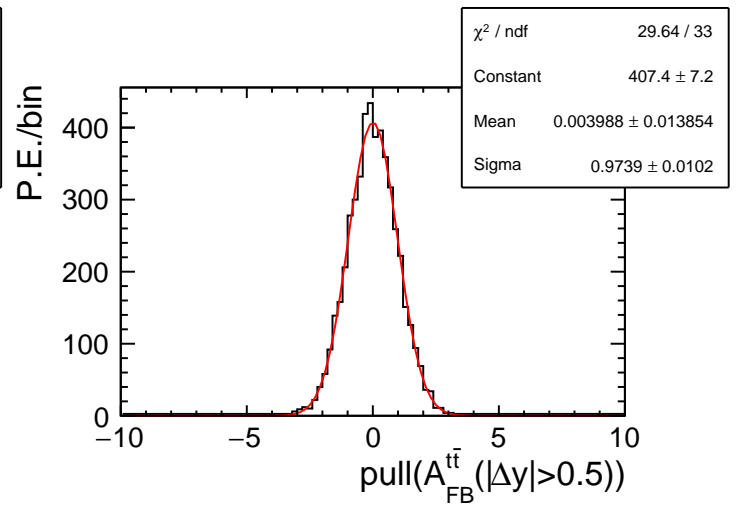

(f) $\operatorname{Pull}\left(A_{\mathrm{FB}}^{t \bar{t}}\right)\left(\left|\Delta y_{t}\right|>0.5\right)$, signal only

Figure 6.30: The same as Fig. 6.12 but after the final optimization requirements. The pull distributions look good. The expected statistical uncertainties with signal only are 0.27 for $A_{\mathrm{FB}}^{t \bar{t}}\left(\left|\Delta y_{t}\right|<0.5\right)$ and 0.13 for $A_{\mathrm{FB}}^{t \bar{t}}\left(\left|\Delta y_{t}\right|<0.5\right)$ 

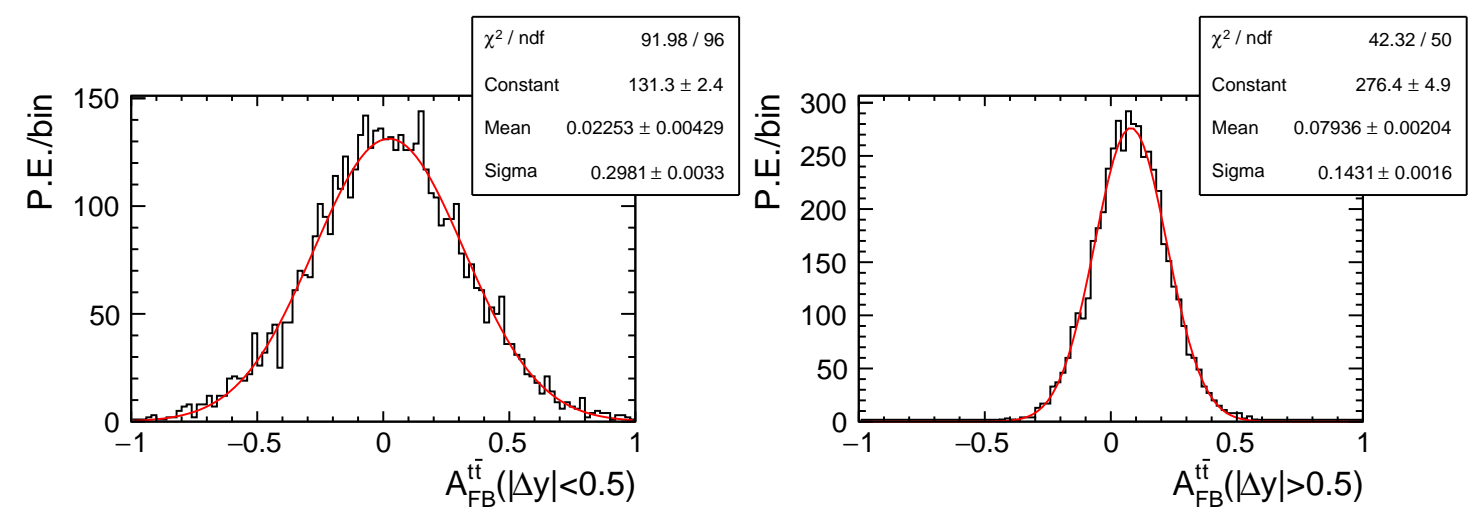

(a) $A_{\mathrm{FB}}^{t \bar{t}}\left(\left|\Delta y_{t}\right|<0.5\right)$, sig. + bkg.

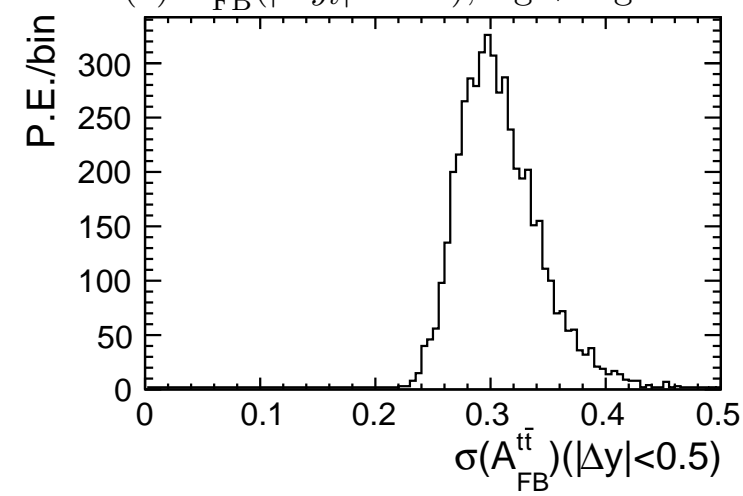

(b) $A_{\mathrm{FB}}^{t \bar{t}}\left(\left|\Delta y_{t}\right|>0.5\right)$, sig. + bkg.

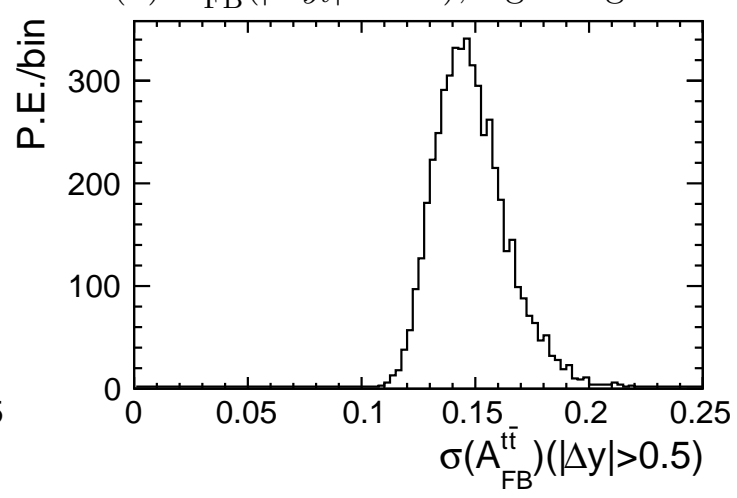

(c) $\sigma\left(A_{\mathrm{FB}}^{t \bar{t}}\right)\left(\left|\Delta y_{t}\right|<0.5\right)$, sig. + bkg.

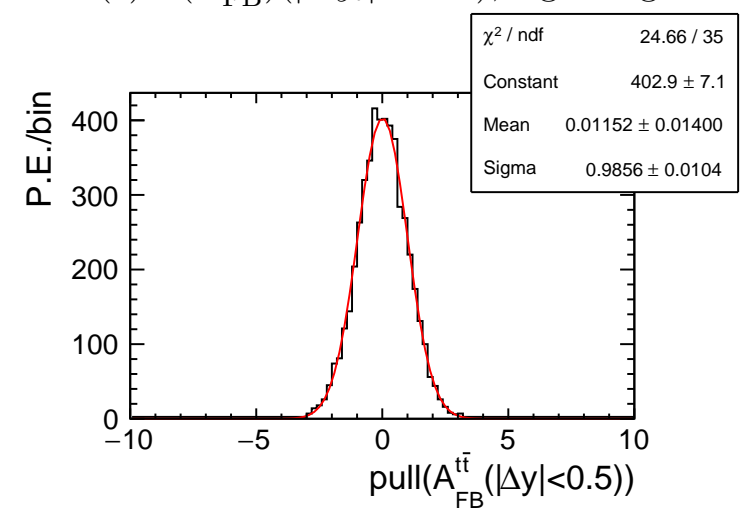

(e) $\operatorname{Pull}\left(A_{\mathrm{FB}}^{t \bar{t}}\right)\left(\left|\Delta y_{t}\right|<0.5\right)$, sig. + bkg.

(d) $\sigma\left(A_{\mathrm{FB}}^{t \bar{t}}\right)\left(\left|\Delta y_{t}\right|>0.5\right)$, sig. + bkg.

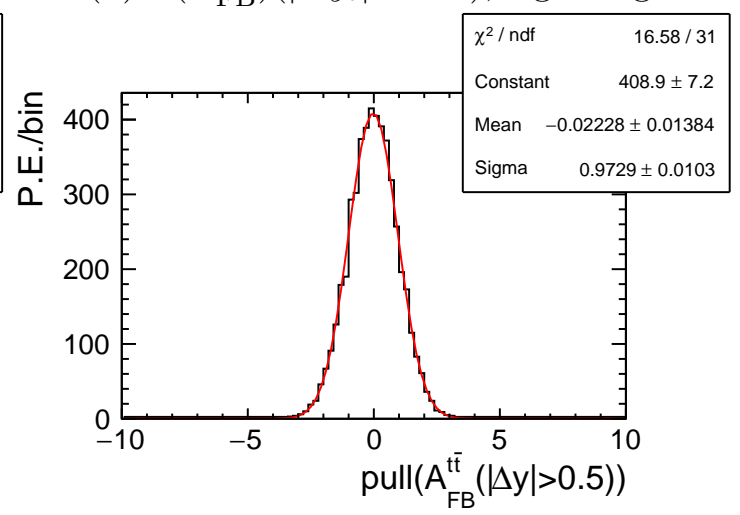

(f) $\operatorname{Pull}\left(A_{\mathrm{FB}}^{t \bar{t}}\right)\left(\left|\Delta y_{t}\right|>0.5\right)$, sig. + bkg.

Figure 6.31: The same as Fig. 6.14, but after the final optimization requirements. The pull distributions look good. The expected statistical uncertainties with signal+backgrounds are 0.30 for $A_{\mathrm{FB}}^{t \bar{t}}\left(\left|\Delta y_{t}\right|<0.5\right)$ and 0.14 for $A_{\mathrm{FB}}^{t \bar{t}}\left(\left|\Delta y_{t}\right|>0.5\right)$. 

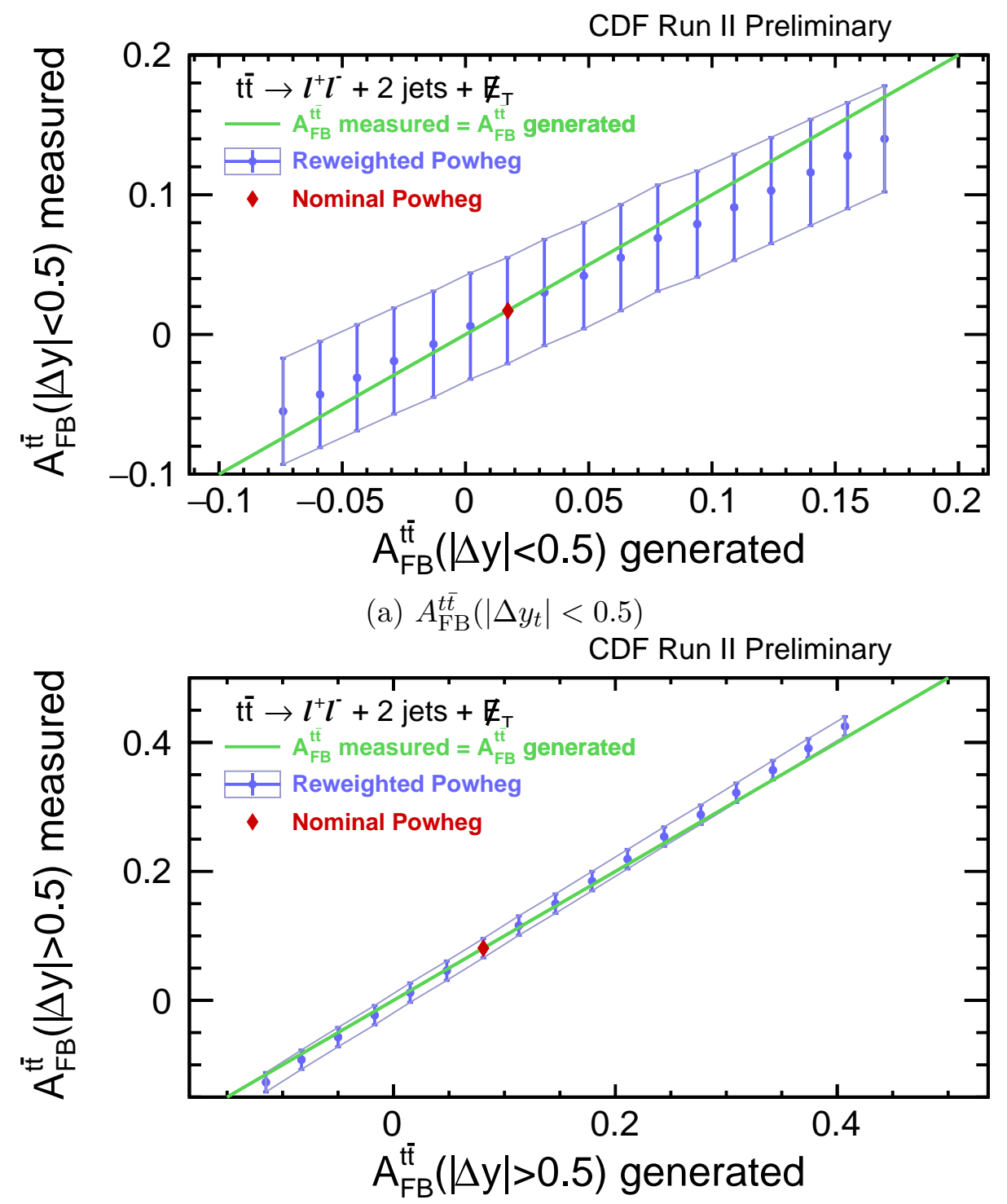

(b) $A_{\mathrm{FB}}^{t \bar{t}}\left(\left|\Delta y_{t}\right|>0.5\right)$

Figure 6.32: A comparison of the differential $A_{\mathrm{FB}}^{t \bar{t}}$ measured with the unfolding algorithm with the $A_{\mathrm{FB}}^{t \bar{t}}$ generated for the reweighted POwHEG MC samples. The two values are consistent with each other, but since there is some deviation which is in the worst case negligible compared with the expected statistical uncertainties $(0.30$ and 0.15 ), we take the deviations as systematic uncertainties. 


\subsection{Final Systematic Uncertainties and Measurement of the Top $A_{\mathrm{FB}}$ from Data}

In the previous section we focused on the statistical uncertainties as well as the systematic uncertainties from the backgrounds. In this section, we summarize the

remaining systematic uncertainty estimations and then finally extracting the $A_{\mathrm{FB}}^{t \bar{t}}$ values from data. The systematic uncertainties are estimated in the same way as in the leptonic $A_{\mathrm{FB}}$ measurement described in Sec. 4. Due to the limit of the size of the systematic MC samples, some of the systematic uncertainties have non-negligible statistical uncertainties from MC. In those cases, we add the statistical uncertainties from $\mathrm{MC}$ in quadrature with the systematic uncertainties to be conservative, although they do not contribute significantly because they are small compared to the dominant uncertainties.

\subsubsection{Measurement of the Inclusive $A_{F B}^{t \bar{t}}$ and Uncertainty Estimation}

In this subsection we first describe the estimation of the systematic uncertainties of the inclusive $A_{\mathrm{FB}}^{t \bar{t}}$ measurement, then apply the methodology to data to obtain the final result. The dominant systematic uncertainty is due to the uncertainties on the background, which is estimated with the pseudoexperiments as described in Sec 6.3.2. We quickly describe the remaining systematics and their estimation methods following the description in Sec. 4, which follows the standard CDF procedures [43].

- The jet energy scaling (JES) systematics is estimated by simultaneously shifting jet energy up and down $1 \sigma$, and taking the larger difference between the shifted $A_{\mathrm{FB}}^{t \bar{t}}$ values and the central value.

- The parton showering systematic is estimated as the difference between the $A_{\mathrm{FB}}^{t \bar{t}}$ values obtained with ALPGEN MC showered by PYTHIA and ALPGEN MC showered by HERWIG. 
- The color reconnection systematics is estimated as the difference between $A_{\mathrm{FB}}^{t \bar{t}}$ obtained with two MC samples with two different color reconnection models 104 .

- The initial- and final-state radiation (I/FSR) uncertainty is estimated as half of the difference between $A_{\mathrm{FB}}^{t \bar{t}}$ obtained with MC samples with "more" or "less" amount of I/FSR.

- The unfolding systematics is taken to be equal to the difference between the measured $A_{\mathrm{FB}}^{t \bar{t}}$ and the generated value from PYTHIA, as discussed in Sec. 6.3.2.

- The PDF uncertainty is estimated following the standard top-group guidelines, and the same as the one used in Sec. 4 .

For the systematic uncertainties due to parton showering, color reconnection, I/FSR and unfolding, the statistical uncertainties due to the limited size of the MC samples are added in quadrature to the differences stated above to be conservative. Table 6.6 summarizes the statistical and systematic uncertainties of the inclusive $A_{\mathrm{FB}}^{t \bar{t}}$ measurement. The total is derived assuming all are uncorrelated and taking each in quadrature.

The uncorrected $A_{\mathrm{FB}}^{t \bar{t}}$ from data only (counting the numbers with $\Delta y_{t}<0$ and $\Delta y_{t}>0$ in Fig. 6.23) is measured to be

$$
A_{\mathrm{FB}}^{t \bar{t}}(\text { uncorrected, data })=0.050 \pm 0.032 \text { (stat.) }
$$

After subtracting off the background contributions, the background-subtracted uncorrected $A_{\mathrm{FB}}^{t \bar{t}}$ is measured to be

$$
A_{\mathrm{FB}}^{t \bar{t}}(\text { uncorrected, data }-\mathrm{bkg})=0.060 \pm 0.043 \text { (stat.). }
$$


Table 6.6: Table of uncertainties for the $A_{\mathrm{FB}}^{t \bar{t}}$ measurement.

\begin{tabular}{ll}
$\begin{array}{l}\text { CDF Run II Preliminary } \\
\left(t \bar{t} \rightarrow l^{+} l^{-}+2 \text { jets }+\right.\end{array}$ & $\left(9.1 \mathrm{fb}_{T}\right)$ \\
\hline \begin{tabular}{c}
\multicolumn{1}{c}{ Source of uncertainty } \\
$A_{\mathrm{FB}}^{t \bar{t}}$
\end{tabular} & Value \\
\hline Statistical & 0.11 \\
\hline Background & 0.04 \\
Parton Showering & 0.03 \\
Color reconnection & 0.03 \\
I/FSR & 0.03 \\
JES & 0.02 \\
Unfolding & 0.02 \\
PDF & 0.01 \\
\hline Total systematic & 0.07 \\
\hline \hline Total uncertainty & 0.13 \\
\hline \hline
\end{tabular}

Note that the correlations among bins are taken into account when estimating the uncertainties.

To get our final answer, we apply the MCMC based unfolding procedure to the data in Fig. 6.23. Figure6.33 shows the posterior probability density for the inclusive $A_{\mathrm{FB}}^{t \bar{t}}$. A Gaussian function is fitted to the center of the distribution to extract the result. Including the systematic uncertainties summarized in Table 6.6, the partonlevel inclusive $A_{\mathrm{FB}}^{t \bar{t}}$ is measured to be

$$
A_{\mathrm{FB}}^{t \bar{t}}=0.12 \pm 0.11 \text { (stat.). } \pm 0.07 \text { (syst.) }=0.12 \pm 0.13 \text {. }
$$

The comparisons between this result and other results and SM predictions are summarized in Sec. 8 . 


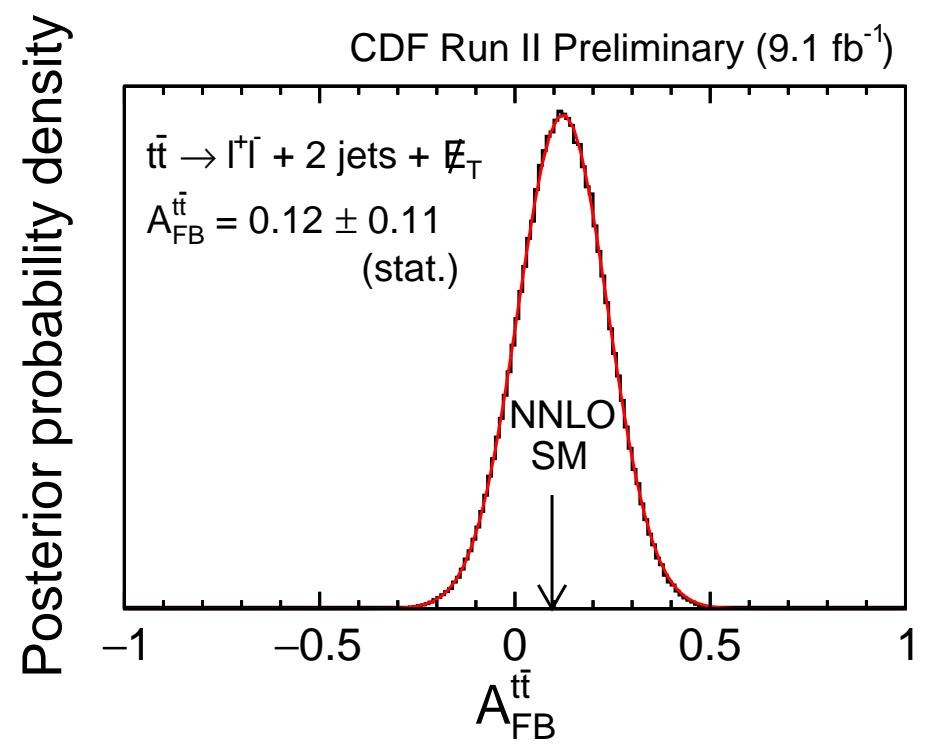

Figure 6.33: Posterior probability density of the inclusive $A_{\mathrm{FB}}^{t \bar{t}}$. A Gaussian function is fitted to the center of the distribution to extract the result.

\subsubsection{Measurement of $A_{F B}^{t \bar{t}}$ vs $\left|\Delta y_{t}\right|$ and Uncertainty Estimation}

In this subsection we again describe the estimation of the systematic uncertainties of the differential $A_{\mathrm{FB}}^{t \bar{t}}$ vs. $\left|\Delta y_{t}\right|$ measurement first, then apply the methodology to data to obtain the final result, as well as our estimation of the slope $\alpha$ of $A_{\mathrm{FB}}^{t \bar{t}}$ vs. $\left|\Delta y_{t}\right|$, where we assume a zero intercept at $\left|\Delta y_{t}\right|=0$. The systematics of the two bins for the differential $A_{\mathrm{FB}}^{t \bar{t}}$ measurements are estimated in the same way as in Sec. 6.4.1. The additional unfolding systematic uncertainties obtained from Fig. $6.32\left(0.02\right.$ for $\left|\Delta y_{t}\right|<0.5$ and 0.003 for $\left.\left|\Delta y_{t}\right|>0.5\right)$ are added in quadrature to the original unfolding systematics (0.04 and 0.004 , respectively). For the systematic uncertainties due to parton showering, color reconnection, I/FSR and unfolding, the statistical uncertainties due to the limited size of the MC samples are added in quadrature to the differences stated above. The results are summarized in Table 6.7. We did not run additional MC to help reduce them as it would not help the overall 
measurement.

Table 6.7: Table of uncertainties for the $A_{\mathrm{FB}}^{t \bar{t}}\left(\left|\Delta y_{t}\right|<0.5\right)$ and $A_{\mathrm{FB}}^{t \bar{t}}\left(\left|\Delta y_{t}\right|>0.5\right)$ measurements.

\begin{tabular}{lcc} 
& $\begin{array}{c}\text { CDF Run II Preliminary }\left(9.1 \mathrm{fb}^{-1}\right) \\
\left(t \bar{t} \rightarrow l^{+} l^{-}+2 \mathrm{jets}+\not_{T}\right)\end{array}$ \\
\hline \hline Source of uncertainty & $A_{\mathrm{FB}}^{t t}\left(\left|\Delta y_{t}\right|<0.5\right)$ & $A_{\mathrm{FB}}^{t t}\left(\left|\Delta y_{t}\right|>0.5\right)$ \\
\hline Statistical & 0.33 & 0.13 \\
\hline Background & 0.13 & 0.06 \\
Parton Showering & 0.07 & 0.06 \\
Color reconnection & 0.12 & 0.06 \\
I/FSR & 0.05 & 0.03 \\
JES & 0.02 & 0.02 \\
Unfolding & 0.06 & 0.02 \\
PDF & 0.01 & 0.01 \\
\hline Total systematic & 0.20 & 0.11 \\
\hline \hline Total uncertainty & 0.39 & 0.17 \\
\hline \hline
\end{tabular}

Figure 6.34 shows the posterior probability densities of $A_{\mathrm{FB}}^{t \bar{t}}\left(\left|\Delta y_{t}\right|<0.5\right)$ and $A_{\mathrm{FB}}^{t \bar{t}}\left(\left|\Delta y_{t}\right|>0.5\right)$ with Gaussian functions fitted to the center of the distributions to extract the results. Figure 6.35 shows the two-dimensional posterior probability density distribution of $A_{\mathrm{FB}}^{t \bar{t}}$ in the two $\left|\Delta y_{t}\right|$ bins, with the correlation between the two results measured from the posterior probability density distribution to be -0.44 . Including the systematic uncertainties summarized in Table 6.7, the parton-level $A_{\mathrm{FB}}^{t \bar{t}}$ vs $\left|\Delta y_{t}\right|$ values are measured to be

$$
\begin{aligned}
& A_{\mathrm{FB}}^{t \bar{t}}\left(\left|\Delta y_{t}\right|<0.5\right)=0.12 \pm 0.33(\text { stat. }) \pm 0.20 \text { (syst.) }=0.12 \pm 0.39 \\
& A_{\mathrm{FB}}^{t \bar{t}}\left(\left|\Delta y_{t}\right|>0.5\right)=0.13 \pm 0.13 \text { (stat.) } \pm 0.11 \text { (syst.) }=0.13 \pm 0.17
\end{aligned}
$$

where the two measurements are correlated both statistically and systematically. 
These results are consistent with the predictions of 0.017 and 0.081 from POWHEG, while no higher-order calculation is available.

Following the lead in the $A_{\mathrm{FB}}^{t \bar{t}}$ measurement in the lepton+jets final state [20], we quantize the differential $A_{\mathrm{FB}}^{t \bar{t}}$ vs. $\left|\Delta y_{t}\right|$ with a linear fit with zero intercept. The total covariance matrix of the measurements in the two $\left|\Delta y_{t}\right|$ regions is shown in Table 6.8. The fit for the slope is obtained by minimizing a $\chi^{2}$ term defined as

$$
\chi^{2}=\sum_{i=1}^{6} \sum_{j=1}^{6}\left(A_{\mathrm{FB}}^{t \bar{t}}[i]-\alpha \cdot\left|\Delta y_{t}\right|[i]\right) \cdot \mathrm{Cov}^{-1}[i][j] \cdot\left(A_{\mathrm{FB}}^{t \bar{t}}[j]-\alpha \cdot\left|\Delta y_{t}\right|[j]\right)
$$

where $\left|\Delta y_{t}\right|[i]$ represents the bin centroids of the two $\left|\Delta y_{t}\right|$ bins predicted by the POWHEG MC (0.24 and 1.01 respectively) and $A_{\mathrm{FB}}^{t \bar{t}}[i]$ is the $A_{\mathrm{FB}}^{t \bar{t}}\left(\left|\Delta y_{t}\right|\right)$ in the corresponding bin, $\mathrm{Cov}^{-1}[i][j]$ is the corresponding element of the inverse matrix of the covariance matrix shown in Table 6.8, and $\alpha$ is the slope we are fitting for. Using Minuit [93] to minimize the $\chi^{2}$, the slope is estimated to be $\alpha=0.14 \pm 0.15$, consistent with the result in the lepton + jets final state of $0.253 \pm 0.062$ [20] and the NNLO SM prediction of $0.114_{-0.012}^{+0.005}$ [46, 48], and displayed in Fig. 6.36 for comparison. The comparisons between this result and other results and SM predictions are summarized in Sec. 8 .

In the next section, we will combine the $A_{\mathrm{FB}}^{\ell}$ result described in Sec. 4 and the $A_{\mathrm{FB}}^{t \bar{t}}$ results described in this section with the corresponding results in the lepton + jets final state, to obtain the best determination from the CDF data.

Table 6.8: Covariance matrix of total uncertainty for differential $A_{\mathrm{FB}}^{t \bar{t}}$ vs. $\left|\Delta y_{t}\right|$.

\begin{tabular}{|c|c|c|}
\hline Total covariance of $A_{\mathrm{FB}}^{t t}\left(\left|\Delta y_{t}\right|\right)$ & $\left|\Delta y_{t}\right|<0.5$ & $\left|\Delta y_{t}\right|>0.5$ \\
\hline$\left|\Delta y_{t}\right|<0.5$ & 0.152 & -0.0223 \\
\hline$\left|\Delta y_{t}\right|>0.5$ & -0.0223 & 0.0295 \\
\hline
\end{tabular}



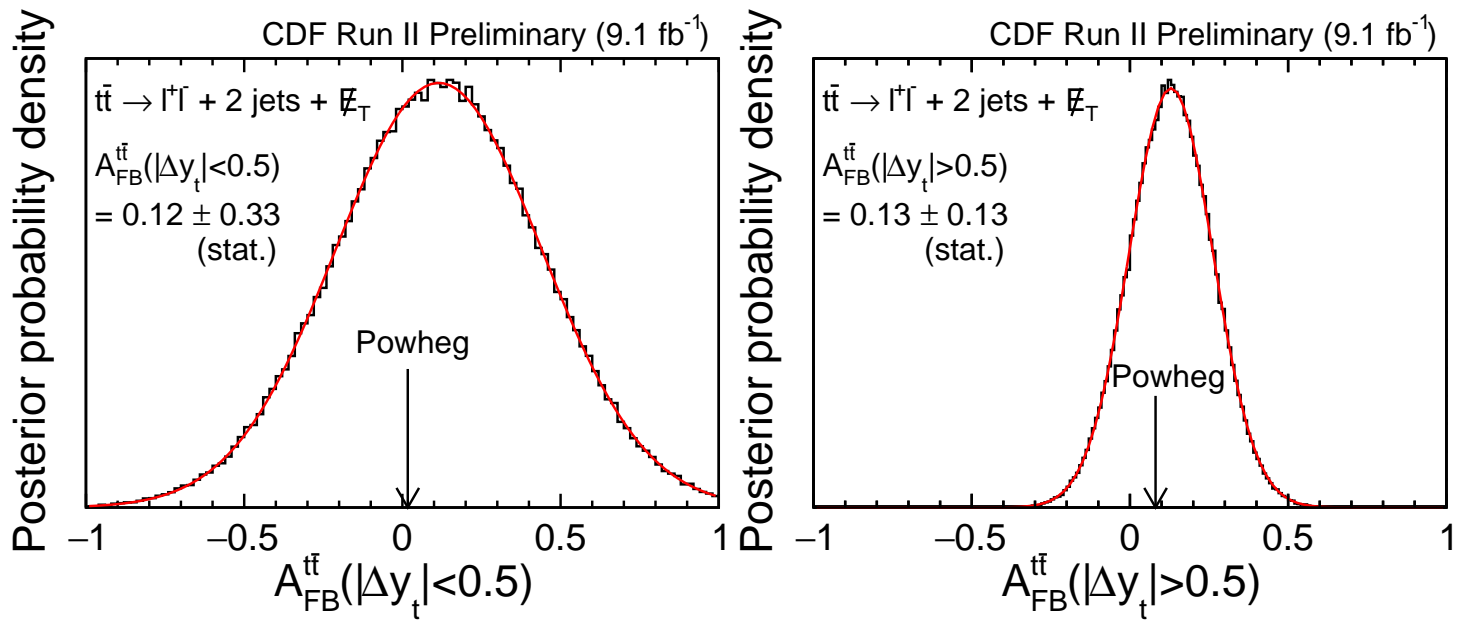

Figure 6.34: Posterior probability density distributions of $A_{\mathrm{FB}}^{t \bar{t}}\left(\left|\Delta y_{t}\right|<0.5\right)$ and $A_{\mathrm{FB}}^{t \bar{t}}\left(\left|\Delta y_{t}\right|>0.5\right)$ from data. Gaussian functions are fitted to the center of the distributions to extract the results.

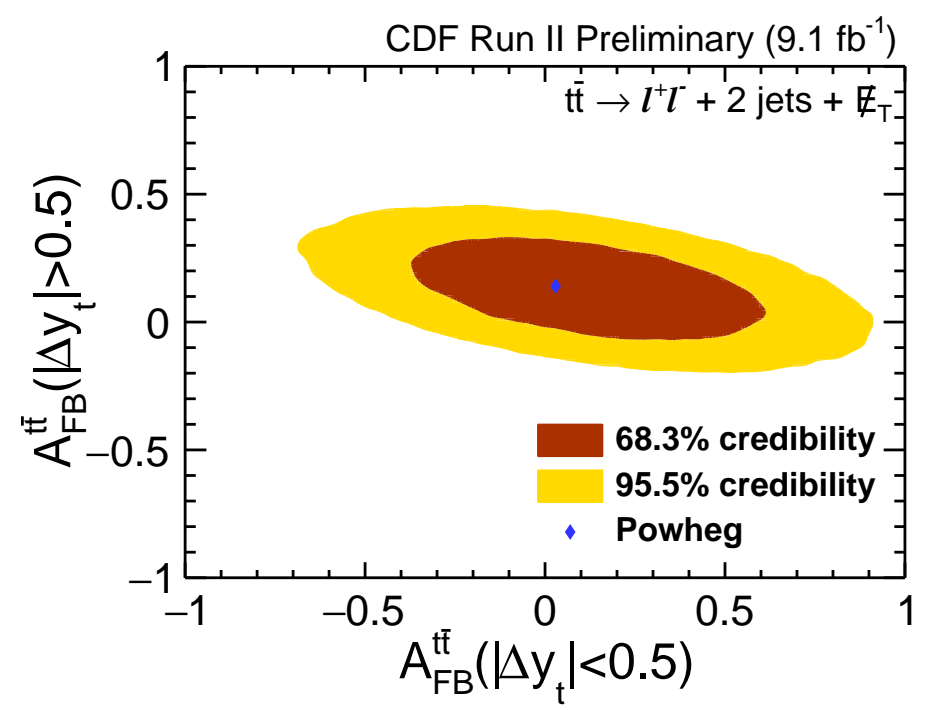

Figure 6.35: The 2D posterior probability density distribution of $A_{\mathrm{FB}}^{t \bar{t}}\left(\left|\Delta y_{t}\right|<0.5\right)$ vs. $A_{\mathrm{FB}}^{t \bar{t}}\left(\left|\Delta y_{t}\right|>0.5\right)$ from the data, with the result from the POWHEG simulation overlaid. The correlation of the two results is measured to be -0.44 . 


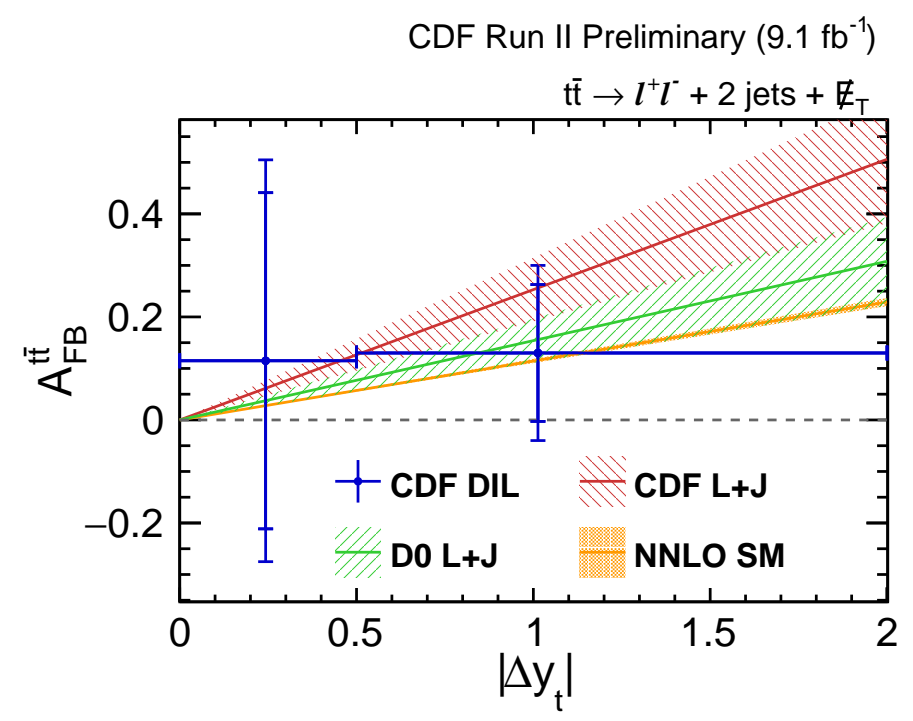

(a)

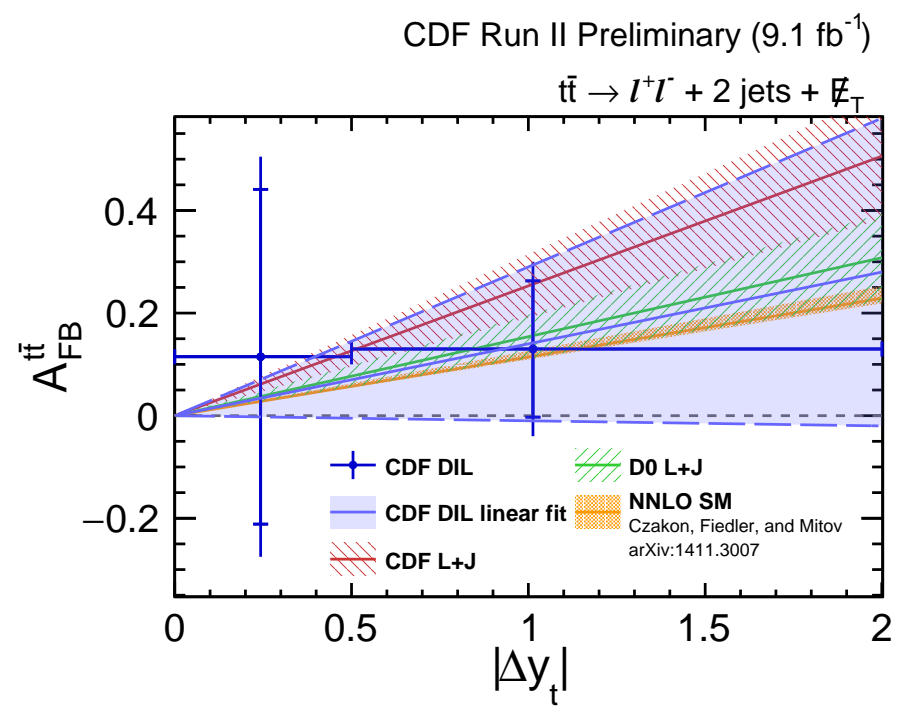

(b)

Figure 6.36: Comparison of $A_{\mathrm{FB}}^{t \bar{t}}$ vs. $\left|\Delta y_{t}\right|$ measurements of this measurement and other Tevatron measurements as well as the NNLO SM predictions. The linear fit for the dilepton measurement is also plotted in (b). 


\section{COMBINATION WITH PREVIOUS MEASUREMENTS*}

This section summarizes the CDF combinations of the top $A_{\mathrm{FB}}$ measurements in the lepton+jets final state and the dilepton final state. Three results are presented:

1. Inclusive $A_{\mathrm{FB}}^{\ell}$,

2. Inclusive $A_{\mathrm{FB}}^{t \bar{t}}$, and

3. Differential $A_{\mathrm{FB}}^{t \bar{t}}$ as a function of $\left|\Delta y_{t}\right|$.

The combinations of the inclusive measurements are based on the Best Linear Unbiased Estimates (BLUE) [105] method. In order to deal with the asymmetric uncertainties in the measurement, the approach of Asymmetric Iterative BLUE (AIB) [108] is utilized. The combination of the differential asymmetry is based on a simultaneous $\chi^{2}$ fit for all the data points measured.

\subsection{Outline of the BLUE Methodology and the AIB Technique}

In this section we outline the BLUE method and the AIB technique used in the combination of the top $A_{\mathrm{FB}}$ measurements. The BLUE method is based on the minimization of the $\chi^{2}$ to get the best estimate of the combination the two measurements $(\mu)$. The $\chi^{2}$ is defined as

$$
\chi^{2}(\mu)=\delta^{T}(\mu) \cdot \mathbf{S}^{-1} \cdot \delta(\mu)
$$

* Reprinted with permission from "Measurement of the inclusive leptonic asymmetry in topquark pairs that decay to two charged leptons at CDF" by the CDF Collaboration, 2014, Phys. Rev. Lett. 113, 042001. Copyright [2015] by the American Physical Society. 
where the column vector $\delta$ is defined as

$$
\delta=\left(\begin{array}{c}
m_{1}-\mu \\
m_{2}-\mu
\end{array}\right)
$$

in which $m_{i}$ is the result from the $i$-th measurement, the covariance matrix $\mathbf{S}$ is defined as

$$
\mathbf{S}=\sum_{e} \mathbf{S}_{\mathbf{e}}
$$

where

$$
\mathbf{S}_{\mathbf{e}}=\sigma_{e i} \cdot \sigma_{e j} \cdot \rho_{e(i, j)},
$$

where we sum over the covariance matrices for all types of uncertainties; $\sigma_{e i}$ is the $e$-th type of uncertainty on the $i$-th measurement; $\sigma_{e j}$ is the $e$-th type of uncertainty on the $j$-th measurement; and $\rho_{e(i, j)}$ is the correlation of the $e$-th uncertainty between measurements. After the covariance matrix is obtained, we can invert the covariance matrix to get the best estimate of $\mu, \mu_{\text {best }}$, and its uncertainty, $\sigma_{\mu}$, using

$$
\begin{aligned}
\operatorname{sum}_{w} & =\sum_{i} \sum_{j}\left(\mathbf{S}^{-\mathbf{1}}\right)_{i, j} \\
w_{i} & =\sum_{j} \frac{\left(\mathbf{S}^{-\mathbf{1}}\right)_{i, j}}{\operatorname{sum}_{w}} \\
\mu_{\text {best }} & =\sum_{i} w_{i} \cdot m_{i} \text { and } \\
\sigma_{\mu} & =\frac{1}{\sqrt{\operatorname{sum}_{w}}} .
\end{aligned}
$$

Due to the asymmetric values of some of the uncertainties, we employ the AIB technique, which uses a set of three BLUE combinations: a center BLUE combination, a upper-uncertainty combination, and a lower-uncertainty combination. The 
center BLUE combination is formed with the covariance matrix formed with the average values of the upper and lower uncertainties, and gives the center value of the combination as well as the symmetrized uncertainty. The upper and lower BLUE combinations are formed with the covariance matrix formed with the upper and lower uncertainties, respectively. The final uncertainty is obtained with the following formula:

$$
\begin{aligned}
\mathcal{R} & =\frac{\sigma_{\text {upper BLUE }}}{\sigma_{\text {upper BLUE }}+\sigma_{\text {lower BLUE }}} \\
\sigma_{\text {upper }} & =2 \cdot \mathcal{R} \cdot \sigma_{\text {center BLUE }} \\
\sigma_{\text {lower }} & =2 \cdot(1-\mathcal{R}) \cdot \sigma_{\text {center BLUE }},
\end{aligned}
$$

where $\sigma_{\text {upper/lower/center BLUE }}$ is the $\sigma_{\mu}$ from the upper-, lower-, and center uncertainty BLUE, respectively, and $\sigma_{\text {upper/lower }}$ are the upper and lower uncertainties of the final combination.

\subsection{CDF Combination of $A_{\mathrm{FB}}^{\ell}$ Measurements}

The $A_{\mathrm{FB}}^{\ell}$ measurement in the lepton+jets final state is documented in Ref. [43], while the same measurement in the dilepton final state is described in Sec. 4. The result in the lepton + jets final state is

$$
A_{\mathrm{FB}}^{\ell}=0.094 \pm 0.024(\text { stat. })_{-0.017}^{+0.022} \text { (syst.) }
$$

and the result in the dilepton final state is

$$
A_{\mathrm{FB}}^{\ell}=0.072 \pm 0.052 \text { (stat.) } \pm 0.030 \text { (syst.) }
$$

The table of the uncertainties are summarized in Tables 7.1, which also gives the correlations between the uncertainties in the two measurements. The two measurements are done in two orthogonal final states, thus the statistical uncertainties are 
uncorrelated. On the other hand, the two measurements are done basically with the same methodology, thus the correlations in the systematic uncertainties need to be taken into consideration. A brief description of each category of systematic uncertainty is listed below, as well as the reasons for treating the uncertainties as correlated or uncorrelated.

Table 7.1: Table of uncertainties for $A_{\mathrm{FB}}^{\ell}$ measurement in the lepton+jets $(\mathrm{L}+\mathrm{J})$ and the dilepton (DIL) final state. In the column of correlation, "0" indicates no correlation and "1" indicates fully positive correlation.

CDF Run II Preliminary

\begin{tabular}{|c|c|c|c|}
\hline Source of uncertainty & $\mathrm{L}+\mathrm{J}\left(9.4 \mathrm{fb}^{-1}\right)$ & $\operatorname{DIL}\left(9.1 \mathrm{fb}^{-1}\right)$ & Correlation \\
\hline Backgrounds & 0.015 & 0.029 & 0 \\
\hline $\begin{array}{c}\text { Recoil modelling } \\
\text { (Asymmetric modelling) }\end{array}$ & $\begin{array}{l}+0.013 \\
-0.000\end{array}$ & 0.006 & 1 \\
\hline Symmetric modelling & - & 0.001 & 0 \\
\hline Color reconnection & 0.0067 & 0.00007 & 1 \\
\hline Parton showering & 0.0027 & 0.0002 & 1 \\
\hline PDF & 0.0025 & 0.0002 & 1 \\
\hline JES & 0.0022 & 0.004 & 1 \\
\hline IFSR & 0.0018 & 0.00008 & 1 \\
\hline Total systematic & $\begin{array}{l}+0.022 \\
-0.017\end{array}$ & 0.030 & \\
\hline Statistics & 0.024 & 0.052 & 0 \\
\hline Total uncertainty & $\begin{array}{l}+0.032 \\
-0.029\end{array}$ & 0.060 & \\
\hline
\end{tabular}

- Backgrounds: this uncertainty is designed to cover the biases introduced when performing background subtraction from data. There are two sources of the uncertainties that are included here, one is due to the variation in the normalization of the background components, the other is due to the variation in shape of the $q_{\ell} \eta_{\ell}$ distribution. In both measurements with the lepton+jets final 
state and with the dilepton final state, the background uncertainty is estimated with pseudoexperiments, where the overall normalizations of each background components are fluctuated with Gaussian distribution, and the shape of $q_{\ell} \eta_{\ell}$ distribution is fluctuated with a Poisson distributed random number in each $q_{\ell} \eta_{\ell}$ bin. The uncertainty due to the variation of the overall normalizations is very small compared to the uncertainty caused by the variation in the shape. In principle, there should be some correlation of this uncertainty between the two measurements, since the two measurements partially share the same source of background, mostly from $\mathrm{W}+$ jets. However, due to the way this uncertainty is estimated, the uncertainties caused by the shape variation are uncorrelated between the two measurements, and the uncertainties caused by the variation of the normalizations are partially correlated, but much smaller than the dominant component. Due to these reasons, we take the background uncertainties in the two measurements as being uncorrelated.

\section{- Asymmetric modeling (Recoil modeling in lepton+jets final state} analysis): this uncertainty is designed to cover the potential biases introduced by the acceptance modelling and the potential biases introduced by the $a \cdot \tanh$ parametrization. We treat this uncertainty to be fully correlated between the two measurements.

- Symmetric modelling: this uncertainty is only estimated in the measurement with the dilepton final state. The uncertainty caused by this variation in the measurement with the lepton+jets final state is small, thus it's not included in the analysis, and is taken as 0 in the combination.

- Color reconnection: this uncertainty is estimated with the two $t \bar{t}$ signal MC samples with different color reconnection tunings as described in Ref. [104], 
and is done in the same way in the two measurements. It's treated as fully correlated.

- Parton showering: this uncertainty is estimated with two Alpgen $t \bar{t}$ signal MC samples showered each by PYThia and Herwig, and is done in the same way in the two measurements. It's treated as fully correlated.

- PDF: this uncertainty is estimated with the official prescription of the top group, and is done in the same way in the two measurements. It's treated as fully correlated.

- JES: This uncertainty is estimated by shifting the jet energy scaling up and down $1 \sigma$ simultaneously in all MC samples. It's treated as fully correlated.

- I/FSR: This uncertainty is estimated with two $t \bar{t}$ signal MC samples with more and less I/FSR. It's treated as fully correlated.

With the uncertainties specified in Table 7.1, we formed three covariance matrices corresponding to the central uncertainties as well as the upper and lower uncertainties. The three covariance matrices are summarized in Table 7.2. With the AIB procedure, the combined $A_{\mathrm{FB}}^{\ell}$ is

$$
A_{\mathrm{FB}}^{\ell}=0.090_{-0.026}^{+0.028}
$$

The weight of the measurement with the lepton+jets final state is $80 \%$, while the weight of the one with the dilepton final state is $20 \%$. The correlation between the two measurements is $2.6 \%$. The result is $2 \sigma$ higher than the NLO SM prediction of $0.038 \pm 0.003$ [42]. The comparison among the inclusive $A_{\mathrm{FB}}^{\ell}$, along with the $A_{\mathrm{FB}}^{\ell \ell}$ measurements for completeness, is shown in Sec. 8 . 
Table 7.2: Covariance matrices of the two measurements of the $A_{\mathrm{FB}}^{\ell}$.

\begin{tabular}{|c|c|}
\hline & Covariance Matrix $\left(\times 10^{-4}\right)$ \\
\hline \hline \multirow{2}{*}{ Central } & $\left(\begin{array}{cc}9.10 & 0.46 \\
0.46 & 35.91\end{array}\right)$ \\
\hline \multirow{2}{*}{ Upper } & $\left(\begin{array}{cc}10.36 & 0.85 \\
0.85 & 35.91\end{array}\right)$ \\
\hline \multirow{2}{*}{ Lower } & $\left(\begin{array}{cc}8.68 & 0.07 \\
0.07 & 35.91\end{array}\right)$ \\
\hline
\end{tabular}

\subsection{CDF Combination of Inclusive $A_{\mathrm{FB}}^{t \bar{t}}$ Measurements}

The measurement of the $A_{\mathrm{FB}}^{t \bar{t}}$ in the lepton+jets final state is documented in Ref. [20] and the measurement in the dilepton final states is given in Sec. 6. The inclusive value of $A_{\mathrm{FB}}^{t \bar{t}}$ measured in the lepton+jets final state is

$$
A_{\mathrm{FB}}^{t \bar{t}}=0.164 \pm 0.039 \text { (stat.) } \pm 0.026 \text { (syst.) }
$$

and the result in the dilepton final state is

$$
A_{\mathrm{FB}}^{t \bar{t}}=0.12 \pm 0.11 \text { (stat.) } \pm 0.07 \text { (syst.) }
$$

The uncertainties are summarized in Table 7.3 , which calls out the correlations between the uncertainties in the two measurements. The correlations are the same as those in the combination of the leptonic $A_{\mathrm{FB}}$ in the previous section. Some notes are in order: Again, the two measurements are done in orthogonal final states, thus the statistical uncertainties are uncorrelated. On the other hand, the two measurements are done basically with the same methodology, thus the correlations in the systematic uncertainties need to be taken into consideration. A brief description of each category of systematic uncertainty is listed below, as well as the reasons for the 
correlations we are taking.

Table 7.3: Table of uncertainties for the inclusive $A_{\mathrm{FB}}^{t \bar{t}}$ measurement in the lepton+jets $(\mathrm{L}+\mathrm{J})$ and the dilepton (DIL) final state. In the column of correlation, "0" indicates no correlation and "1" indicates fully positive correlation.

\begin{tabular}{cccc} 
& & CDF Run II Preliminary \\
\hline \hline Source of uncertainty & L+J $\left(9.4 \mathrm{fb}^{-1}\right)$ & DIL $\left(9.1 \mathrm{fb}^{-1}\right)$ & Correlation \\
\hline Background shape & 0.018 & 0.04 & 0 \\
Background normalization & 0.013 & 0.03 & 1 \\
Parton shower & 0.01 & 0.02 & 1 \\
Jet energy scale & 0.007 & 0.03 & 1 \\
Inital- and final-state radiation & 0.005 & 0.02 & 0 \\
Correction procedure & 0.004 & 0.03 & 1 \\
Color reconnection & 0.001 & 0.01 & 1 \\
Parton-distribution functions & 0.001 & 0.07 & 0 \\
\hline Total systematic & 0.026 & 0.11 & \\
Statistical & 0.039 & 0.13 & \\
\hline \hline Total uncertainty & 0.047 & & \\
\hline \hline
\end{tabular}

- Background shape/normalization: this uncertainty is designed to cover the biases introduced when modeling the background contributions in data. There are two sources of the uncertainties that are included here, one is due to the variation in the normalization of the background components, the other is due to the variation in the shape of the $\Delta y_{t}$ distribution. In the measurements with the lepton+jets final state, the background normalization uncertainty is due to the normalization uncertainty from Method-II, while the background shape uncertainty is estimated with two alternative templates (0-tagged template and $\mathrm{W}+$ jets template). In the dilepton analysis, the background uncertainty is estimated with pseudoexperiments, where the overall normalizations of each background components are fluctuated according to their expectations 
and uncertainties, and then randomly picking events according to the fluctuated normalizations. In principle, there should be some correlation of this uncertainty between the two measurements, since the two measurements partially share the same source of background, mostly from $\mathrm{W}+$ jets. However, due to the way this uncertainty is estimated, the uncertainties caused by the shape variation are uncorrelated between the two measurements, and the uncertainties caused by the variation of the normalizations are only partially correlated, but much smaller than the dominant component. Due to these reasons, we sum the background shape and background normalization uncertainties in the lepton+jets analysis in quadrature for the overall uncertainty due to backgrounds, and take that as uncorrelated with the background uncertainty in the dilepton analysis.

- Correction procedure: this uncertainty is to cover the potential bias introduced by the correction procedure, which is used to extract the parton-level $A_{\mathrm{FB}}^{t \bar{t}}$. The correction procedures in the two analyses are not the same. In the lepton+jets analysis, the correction procedure is based on a regularized singular value decomposition of the detector smearing matrix and a bin-by-bin correction for the acceptances and efficiencies [20]. The correction systematic is assigned as the difference between the generated $A_{\mathrm{FB}}^{t \bar{t}}$ from POWHEG and the after-reconstruction measured value of $A_{\mathrm{FB}}^{t \bar{t}}$, partially caused by the regularization introduced. In the dilepton analysis, the correction procedure is based on an Markov-chain Monte Carlo (MCMC) scan on a Bayesian model, which includes both the detector smearing matrix, as well as an $A_{\mathrm{FB}}^{t \bar{t}}$-dependent acceptance and efficiency correction. This method works well with the POWHEG sample. However, in the top reconstruction procedure in the dilepton analy- 
sis, templates of the kinematics of $t \bar{t}\left(p_{T, t \bar{t}}, p_{z, t \bar{t}}\right.$, and $\left.m_{t \bar{t}}\right)$ are introduced to help distinguish the better solutions and the less possible solutions, and the templates are derived from NLO SM MC POWHEG. A correction systematic is assigned as the difference between the generated and the measured $A_{\mathrm{FB}}^{t \bar{t}}$ from LO SM MC PYTHIA to cover the potential bias caused by the NLO assumption. Due to the way the two systematics are estimated in the two analyses, they are treated as uncorrelated.

- Parton shower, JES, I/FSR, Color reconnection, and PDF: theses are treated in the same way as in the $A_{\mathrm{FB}}^{\ell}$ combination.

With the uncertainties specified in Table 7.3 , we form a covariance matrix corresponding to the uncertainties, and proceed with the BLUE combination. We find

$$
A_{\mathrm{FB}}^{t \bar{t}}=0.160 \pm 0.045
$$

The weight of the measurement with the lepton+jets final state is $91 \%$, while the weight of the one with the dilepton final state is $9 \%$. The correlation between the two measurements is $10 \%$. It is worth noting that again the weight of the dilepton result is smaller than the weight of the lepton+jets result, and even smaller here than in the leptonic analysis [90] for a few reasons. The first is that the lepton+jets final state has a larger branching ratio, which means we have roughly six times more $t \bar{t}$ events after all selection requirements in the lepton+jets analysis than in the dilepton analysis. A second issue is that in the lepton+jets case, with only one neutrino, the top reconstruction is overly constrained, providing a reconstruction resolution of $\sim 0.25$; but with the dilepton final state there are two neutrinos so the kinematics are under-constrained, so the resolution is about 0.5 which further affects the final 
sensitivity. Finally, in the leptonic $A_{\mathrm{FB}}$ we are able to use both leptons as separate measurements, but in the full reconstruction case we only have one measurement per event. The combined $A_{\mathrm{FB}}^{t \bar{t}}$ result is $1.4 \sigma$ higher than the NNLO SM prediction of $0.095 \pm 0.007$ [46]. The comparison among the inclusive $A_{\mathrm{FB}}^{t \bar{t}}$ measurements is shown in Sec. 8.

\subsection{CDF Combination of the Differential $A_{\mathrm{FB}}^{t \bar{t}}$ vs. $\left|\Delta y_{t}\right|$}

The values of $A_{\mathrm{FB}}^{t \bar{t}}$ as a function of $\left|\Delta y_{t}\right|$ are measured at discrete values of $\left|\Delta y_{t}\right|$, in both the lepton+jets [20] and the dilepton final states, and have different numbers of measured points. In both two analyses, the sets of points are used to characterize the differential $A_{\mathrm{FB}}^{t \bar{t}}$ vs. $\left|\Delta y_{t}\right|$ with the slope of a linear function (with the assumption that the asymmetry goes to zero at $\left.\left|\Delta y_{t}\right|=0\right)$. Since the $A_{\mathrm{FB}}^{t \bar{t}}$ in the two analyses are measured at different values of $\left|\Delta y_{t}\right|$, we elect not to try to combine the individual points, and choose instead to report a best fit slope to the combined set of data points.

The combination is done with a simultaneous fit for all six data points (four from the lepton+jets final state and two from the dilepton final state) reported from the two measurements. The bin-centroids and the measured values of the $A_{\mathrm{FB}}^{t \bar{t}}$ for each data points are summarized in the LHS of Table 7.5. The correlations of the uncertainties between the individual data points in the two analyses are the same as described in the previous section. The total covariance matrix, which is obtained by summing up the all the contributed covariance matrices, is shown in Table 7.4 and Fig. 7.1. For an alternative format of presentation, the eigenvalues and the eigenvectors of the covariance matrix are shown on the RHS of Table 7.5. Everything needed to do the simultaneous linear fit is contained in this table. 


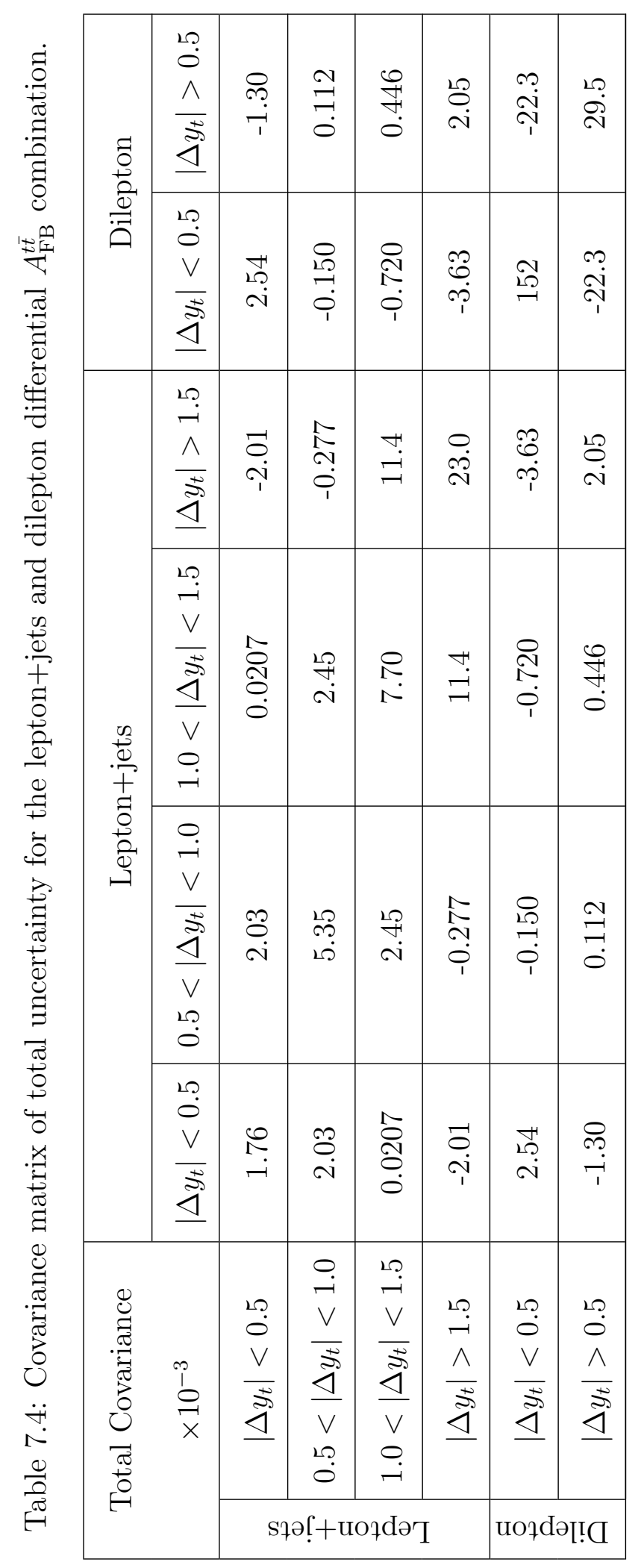




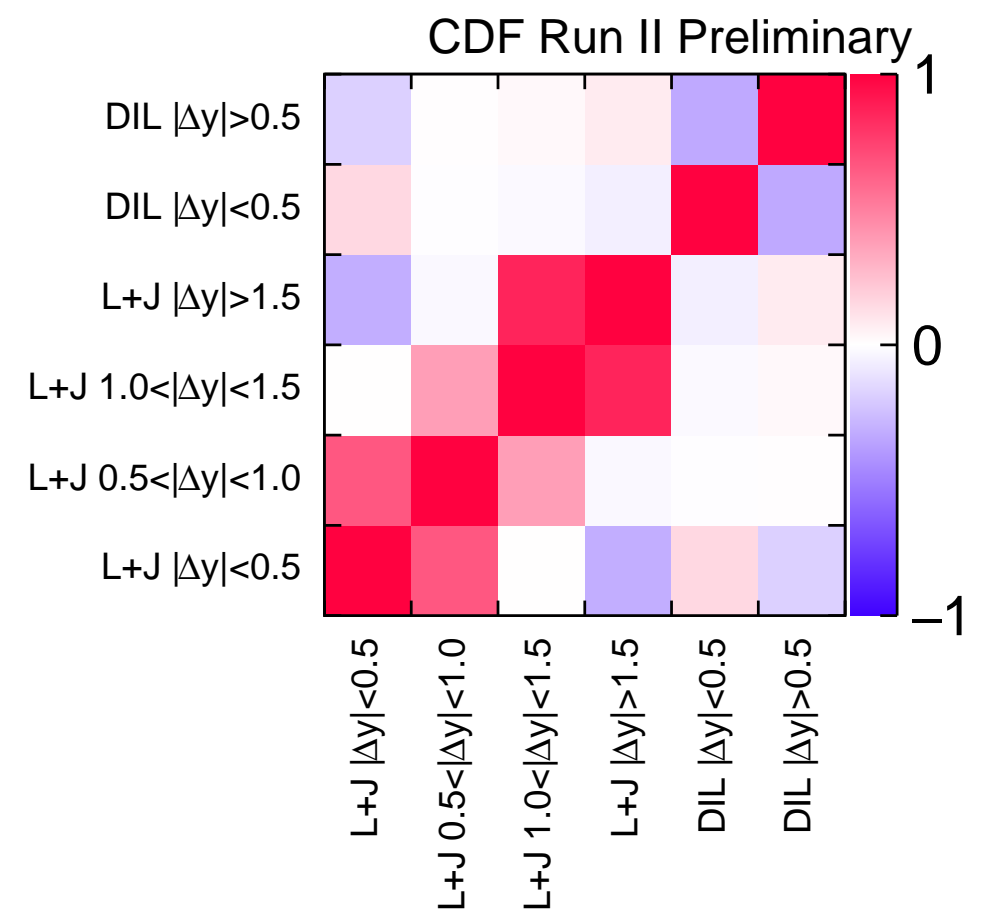

Figure 7.1: The correlation matrix of the differential $A_{\mathrm{FB}}^{t \bar{t}}$ in the lepton+jets and dilepton analyses. 


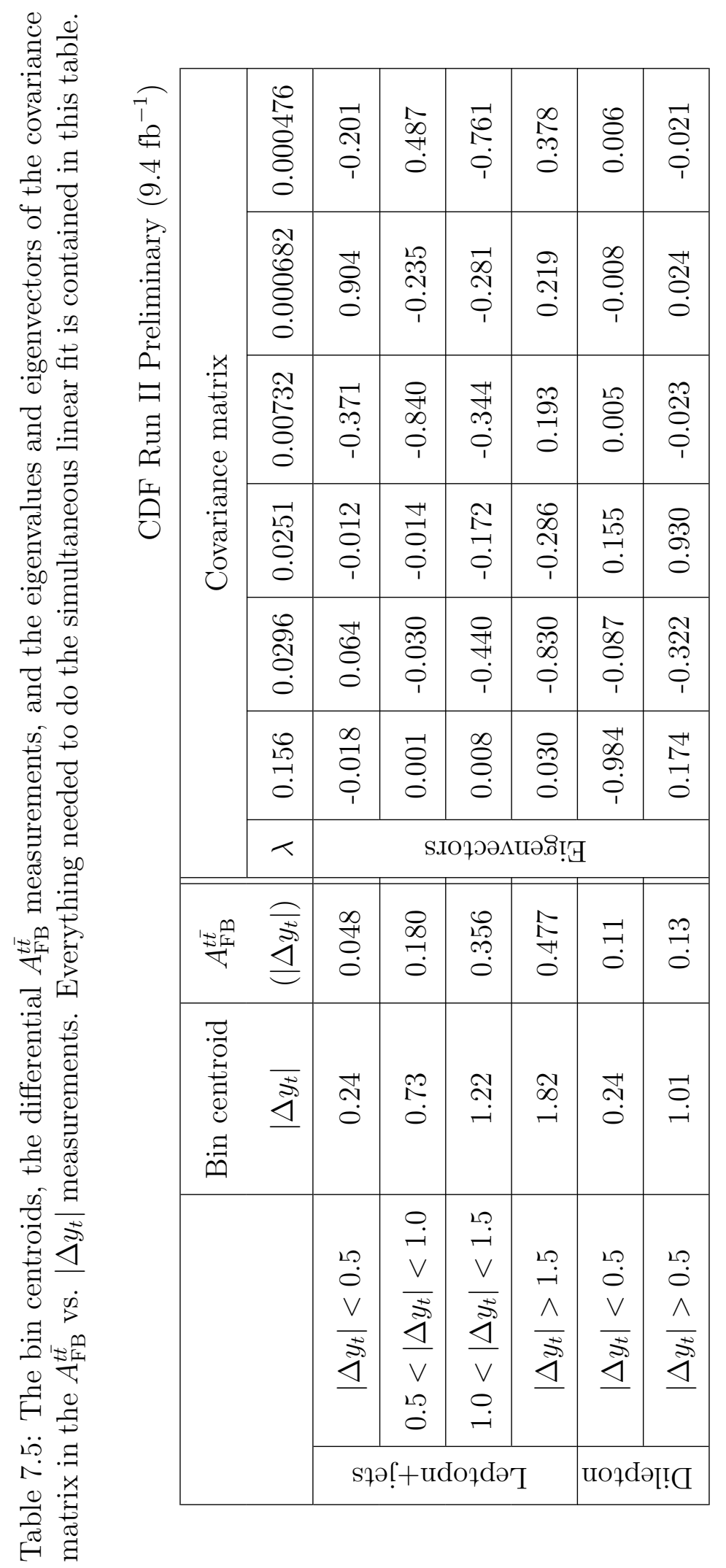


The simultaneous fit is obtained by minimizing a $\chi^{2}$ term defined as

$$
\chi^{2}=\sum_{i=1}^{6} \sum_{j=1}^{6}\left(A_{\mathrm{FB}}^{t \bar{t}}[i]-\alpha \cdot \Delta y_{t}[i]\right) \cdot \operatorname{Cov}^{-1}[i][j] \cdot\left(A_{\mathrm{FB}}^{t \bar{t}}[j]-\alpha \cdot \Delta y_{t}[j]\right)
$$

where $\Delta y_{t}[i]$ and $A_{\mathrm{FB}}^{t \bar{t}}[i]$ are the $i$-th bin centroids, the $A_{\mathrm{FB}}^{t \bar{t}}\left(\left|\Delta y_{t}\right|\right)$ are the measured asymmetry values (given in the LHS of Table 7.5 , $\mathrm{Cov}^{-1}[i][j]$ is the corresponding element of the inverse matrix of the covariance matrix shown in Table 7.4 , and $\alpha$ is the slope we are fitting for. We use Minuit [93] to find the slope $\alpha$ that minimizes $\chi^{2}$ and determines its corresponding uncertainty. The result is found to be

$$
\alpha=0.227 \pm 0.057
$$

which is between the two individual measurements of $0.253 \pm 0.062$ in the lepton + jets final state and $0.14 \pm 0.15$ in the dilepton final state. The $\alpha$ of the CDF combination is $2 \sigma$ higher than the NNLO SM prediction of $0.114_{-0.012}^{+0.005}$ [46, 48]. The comparison of the $\alpha$ measurements in different channels and NNLO SM prediction is shown in Fig 7.2 and in another format in Sec. 8 . 


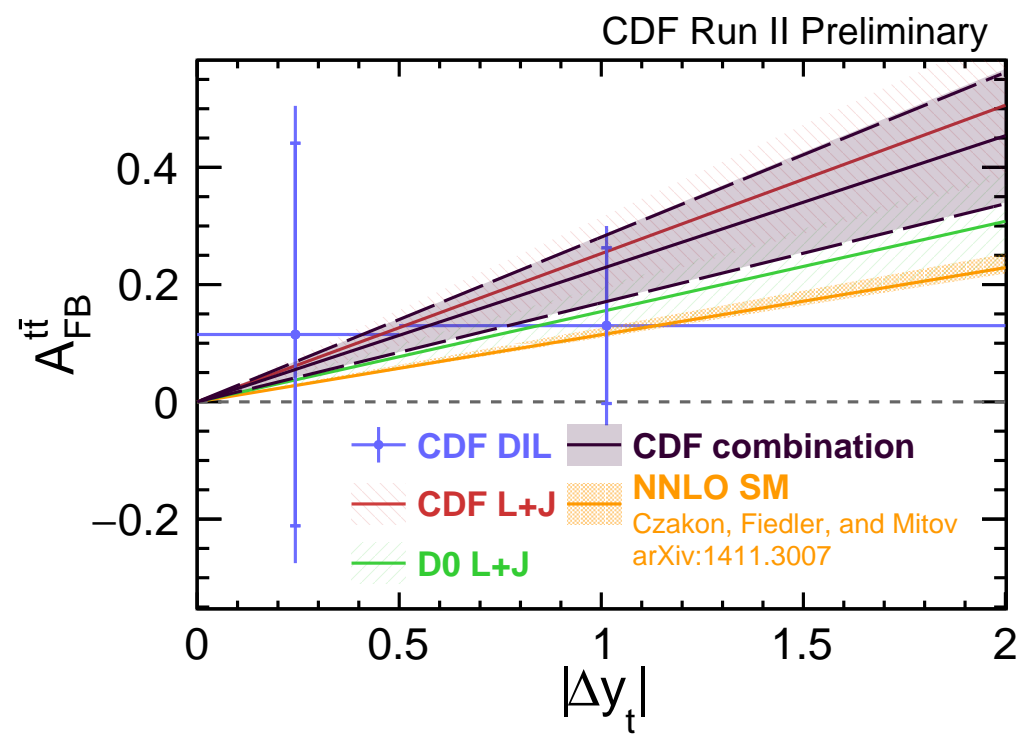

Figure 7.2: A comparison of the data points from the dilepton $A_{\mathrm{FB}}^{t \bar{t}}$ vs. $\left|\Delta y_{t}\right|$ result along with the slope from the CDF combination and other Tevatron measurements as well as the NNLO SM prediction [46, 48]. 


\section{CONCLUSIONS}

We have presented the final measurements of the forward-backward asymmetry $\left(A_{\mathrm{FB}}\right)$ of top quark-antiquark pair events $(t \bar{t})$ at the Fermilab Tevatron with the $\mathrm{CDF}$ detector. The inclusive $A_{\mathrm{FB}}^{\ell}, A_{\mathrm{FB}}^{\ell \ell}$, and $A_{\mathrm{FB}}^{t \bar{t}}$ are measured in the dilepton final state to be $0.072 \pm 0.060,0.076 \pm 0.081$, and $0.12 \pm 0.13$, respectively. The CDF combination of $A_{\mathrm{FB}}^{\ell}$ and $A_{\mathrm{FB}}^{t \bar{t}}$ are $0.090_{-0.026}^{+0.028}$, and $0.160 \pm 0.045$, respectively. Figure 8.1 summarizes the leptonic $A_{\mathrm{FB}}\left(A_{\mathrm{FB}}^{\ell}\right.$ and $\left.A_{\mathrm{FB}}^{\ell \ell}\right)$ measurements from the $\mathrm{CDF}$ and D0 collaborations, comparing with the SM predictions. Figure 8.2 summarizes the inclusive reconstructed top $A_{\mathrm{FB}}\left(A_{\mathrm{FB}}^{t \bar{t}}\right)$ measurements from the CDF and D0 collaborations, along with the SM predictions. In Figure 8.3, we summarize in another format the comparison of all Tevatron inclusive $A_{\mathrm{FB}}^{\ell}, A_{\mathrm{FB}}^{\ell \ell}$, and $A_{\mathrm{FB}}^{t \bar{t}}$ measurements and the NLO/NNLO SM predictions. All results are consistent with the SM predictions [42, 46].

The differential $A_{\mathrm{FB}}^{t \bar{t}}$ values as a function of $\left|\Delta y_{t}\right|$ measured in the dilepton final state are $A_{\mathrm{FB}}^{t \bar{t}}\left(\left|\Delta y_{t}\right|<0.5\right)=0.12 \pm 0.33$ (stat.) \pm 0.20 (syst.) $=0.12 \pm 0.39$, and $A_{\mathrm{FB}}^{t \bar{t}}\left(\left|\Delta y_{t}\right|>0.5\right)=0.13 \pm 0.13$ (stat.) \pm 0.11 (syst.) $=0.13 \pm 0.17$. A linear fit with zero intercept to the differential $A_{\mathrm{FB}}^{t \bar{t}}$ as a function of $\left|\Delta y_{t}\right|$ yields a slope of $\alpha=0.14 \pm 0.15$. The CDF combination of the slope yields $\alpha=0.227 \pm 0.057$, which is $2.0 \sigma$ higher than the NNLO SM prediction [46, 48]. Figure 8.4 summarizes the slope measurements of $A_{\mathrm{FB}}^{t \bar{t}}$ as a function of $\left|\Delta y_{t}\right|$.

We find that all measurement results are consistent with the current state-of-theart SM predictions. However, we do find that all the results are higher than the corresponding SM predictions. The final best "legacy" answers from the Tevatron experiments, combinations of results from both the CDF and the D0 collaborations, 
is underway.

Only a few years ago it appeared that we may have hints of new physics. Many channels showed larger-than-expected asymmetries and especially the previous $A_{\mathrm{FB}}^{t \bar{t}}$ measurement in the dilepton final state at CDF with half of the final dataset [17]. It appeared to be true in lepton+jets and dilepton; it appeared to be true in the total asymmetry and the leptonic asymmetries; and it appeared to be true at both CDF and D0. However, with better measurements and better predictions of the SM, the current data no longer support this. Our best understanding is that there is no evidence for any anomalies suggesting for physics beyond the SM. While this is disappointing, we note that the chase has been exciting. In the meanwhile, during the chase we have learned a great deal about the production of the heaviest known fundamental particle, not the least of which is that the production of $t \bar{t}$ is not well described by the LO predictions, as originally thought. In addition, the tools for the measurements have changed significantly. We have come a long way, and left an excellent legacy from the Tevatron which will help pave the way for the next round of results from the LHC. 


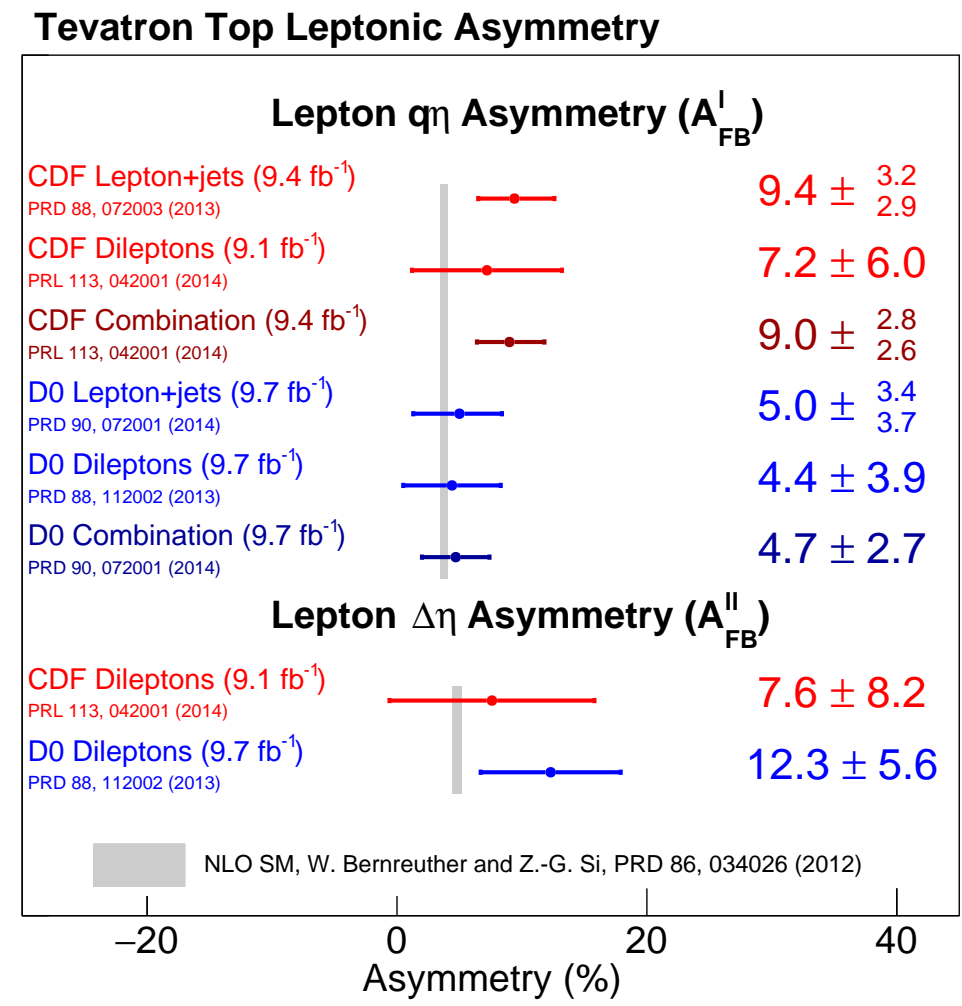

Figure 8.1: Comparison of the inclusive $A_{\mathrm{FB}}^{\ell}$ measurements with lepton+jets and dilepton final states from CDF [43], D0 [59, 61], and the NLO [42] SM calculations, as well as the CDF combination. 


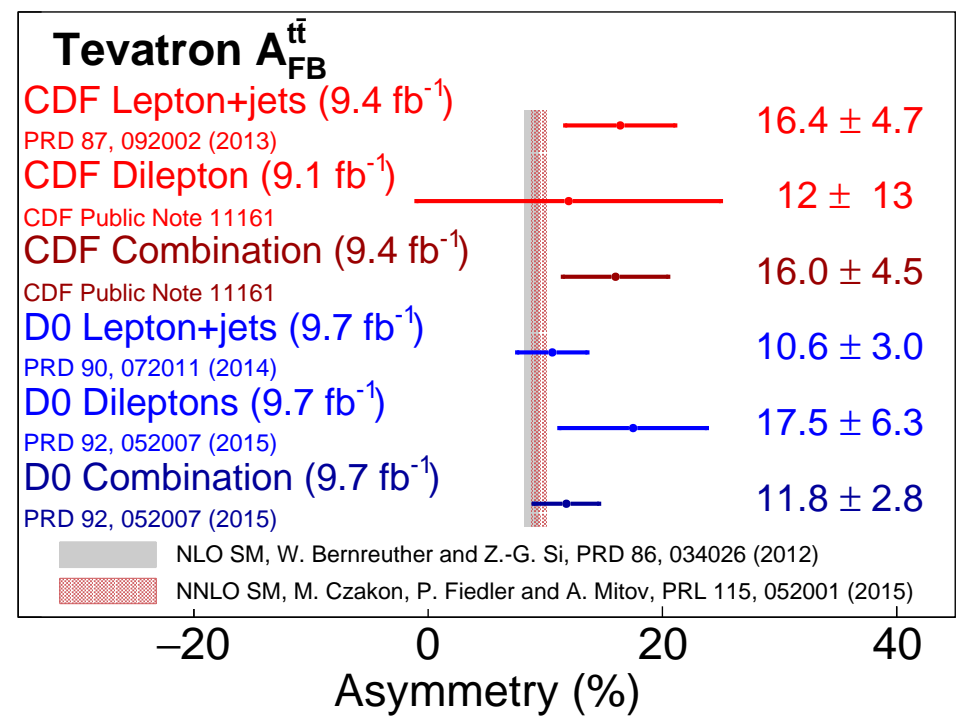

Figure 8.2: Comparison of the inclusive $A_{\mathrm{FB}}^{t \bar{t}}$ measurements with lepton + jets and dilepton final states from CDF [20], D0 [60, 62, the NLO [42] and the NNLO [46] SM calculations, as well as the CDF combination. 


\section{Tevatron Top Asymmetry}

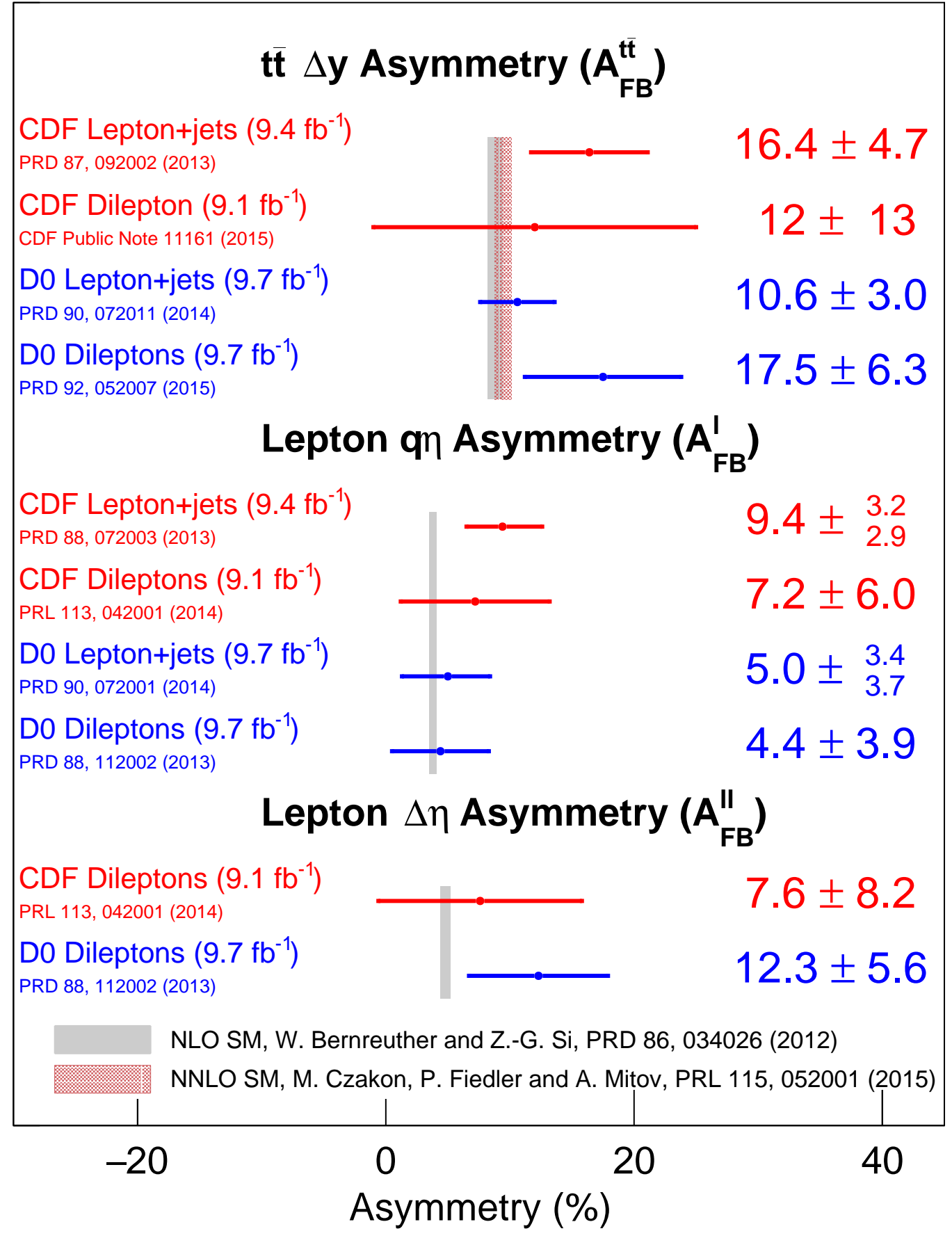

Figure 8.3: Comparison of all inclusive top $A_{\mathrm{FB}}$ results from the Tevatron with the NLO and NNLO SM predictions. 


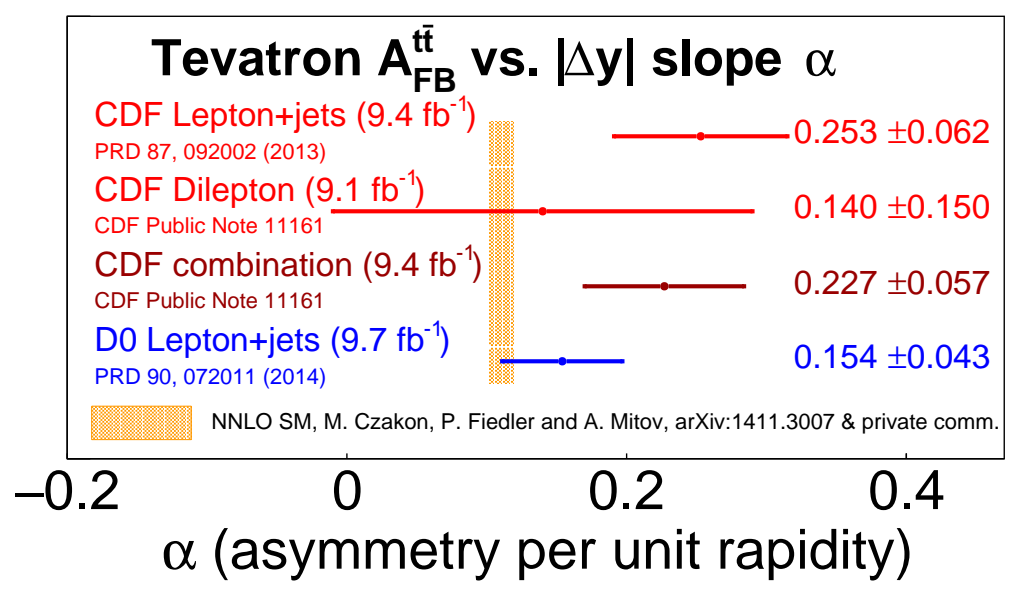

Figure 8.4: Comparison of the individual and combined slope $\alpha$ values of $A_{\mathrm{FB}}^{t \bar{t}}$ vs. $\left|\Delta y_{t}\right|$ from CDF [20], along with the corresponding result in the lepton+jets final state from D0 [60, and the SM calculation at NNLO [46, 48]. 


\section{REFERENCES}

[1] S. Glashow, Nucl. Phys. 22, 579 (1961).

[2] S. Weinberg, Phys. Rev. Lett. 19, 1264 (1967).

[3] A. Salam, in Elementary Particle Theory, p. 367, edited by N. Svartholm (Almqvist and Wiksell, Stockholm, 1968).

[4] P. Ade et al. (Planck Collaboration), arXiv:1502.01589 [astro-ph] .

[5] M. Baak, M. Goebel, J. Haller, A. Hoecker, D. Kennedy, R. Kogler, K. Moenig, M. Schott, and J. Stelzer, Eur. Phys. J. C 72, 2205 (2012),

[6] F. Abe et al. (CDF Collaboration), Phys. Rev. Lett. 74, 2626 (1995).

[7] S. Abachi et al. (D0 Collaboration), Phys. Rev. Lett. 74, 2632 (1995).

[8] L. Evans and P. Bryant, JINST 3, S08001 (2008).

[9] K. Olive et al. (Particle Data Group), Chin. Phys. C 38, 090001 (2014).

[10] J. H. Kühn and G. Rodrigo, Phys. Rev. D 59, 054017 (1999).

[11] J. H. Kühn and G. Rodrigo, Phys. Rev. Lett. 81, 49 (1998).

[12] O. Antunano, J. H. Kühn, and G. Rodrigo, Phys. Rev. D 77, 014003 (2008).

[13] J. M. Campbell and R. K. Ellis, Phys. Rev. D 60, 113006 (1999).

[14] S. Frixione, P. Nason, and B. R. Webber, J. High Energy Phys. 08 (2003) 007.

[15] T. Aaltonen et al. (CDF collaboration), Phys. Rev. Lett. 101, 202001 (2008).

[16] V. Abazov et al. (D0 Collaboration), Phys. Rev. Lett. 100, 142002 (2008).

[17] T. Aaltonen et al. (CDF Collaboration), CDF Public Note 10436 (2011).

[18] V. Abazov et al. (D0 Collaboration), Phys. Rev. D 84, 112005 (2011).

[19] T. Aaltonen et al. (CDF Collaboration), Phys. Rev. D 83, 112003 (2011).

[20] T. Aaltonen et al. (CDF Collaboration), Phys. Rev. D 87, 092002 (2013).

[21] J. A. Aguilar-Saavedra, D. Amidei, A. Juste, and M. Pérez-Victoria, Rev. Mod. 
Phys. 87, 421 (2015).

[22] P. W. Higgs, Phys. Rev. 145, 1156 (1966).

[23] G. Aad et al. (ATLAS collaboration), Phys. Lett. B 716, 1 (2012).

[24] S. Chatrchyan et al. (CMS collaboration), Phys. Lett. B 716, 30 (2012).

[25] T. Aaltonen et al. (CDF and D0 collaborations), Phys. Rev. D 88, 052014 (2013).

[26] Wikipedia, "Elementary particle — wikipedia, the free encyclopedia," (2015), [Online; accessed 13-November-2015].

[27] D. Buttazzo, G. Degrassi, P. P. Giardino, G. F. Giudice, F. Sala, A. Salvio, and A. Strumia, J. High Energy Phys. 12 (2013) 089.

[28] Tevatron Electroweak Working Group (CDF and D0 Collaborations), arXiv:1407.2682 [hep-ex] .

[29] L. H. Orr, Phys. Rev. D 44, 88 (1991).

[30] T. Aaltonen et al. (CDF Collaboration), Phys. Rev. Lett. 111, 202001 (2013).

[31] V. Abazov et al. (D0 Collaboration), Phys. Rev. D 85, 091104 (2012).

[32] Figures taken from http://www-cdf.fnal.gov/internal/physics/top/ feynman.shtml.

[33] T. Aaltonen et al. (CDF and D0 Collaborations), Phys. Rev. D 89, 072001 $(2014)$.

[34] M. Czakon and A. Mitov, Comput. Phys. Commun. 185, 2930 (2014).

[35] M. Czakon, P. Fiedler, and A. Mitov, Phys. Rev. Lett. 110, 252004 (2013).

[36] W. Hofmann, Nucl. Phys. B 3, 671 (1988).

[37] Figures taken from http://www-d0.fnal.gov/Run2Physics/top/top_ public_web_pages/top_feynman_diagrams.html.

[38] T. Schwarz, Ph.D. thesis, University of Michigan, [Report No. FERMILABTHESIS-2006-51]. 
[39] W. Hollik and D. Pagani, Phys. Rev. D 84, 093003 (2011).

[40] A. V. Manohar and M. Trott, Phys. Lett. B 711, 313 (2012).

[41] J. Kühn and G. Rodrigo, J. High Energy Phys. 01 (2012) 063.

[42] W. Bernreuther and Z.-G. Si, Phys. Rev. D 86, 034026 (2012).

[43] T. Aaltonen et al. (CDF Collaboration), Phys. Rev. D 88, 072003 (2013).

[44] Figure taken from http://www-d0.fnal.gov/Run2Physics/top/top_ public_web_pages/top_public.html.

[45] J. A. Aguilar-Saavedra and M. Perez-Victoria, J. High Energy Phys. 09 (2011) 097 .

[46] M. Czakon, P. Fiedler, and A. Mitov, Phys. Rev. Lett. 115, 052001 (2015).

[47] N. Kidonakis, Phys. Rev. D 91, 071502 (2015).

[48] M. Czakon, P. Fiedler, and A. Mitov, Private communication.

[49] S. Frixione, P. Nason, and G. Ridolfi, J. High Energy Phys. 09 (2007) 126.

[50] T. Sjostrand, S. Mrenna, and P. Z. Skands, J. High Energy Phys. 05 (2006) 026.

[51] M. L. Mangano, M. Moretti, F. Piccinini, R. Pittau, and A. D. Polosa, J. High Energy Phys. 07 (2003) 001.

[52] M. Bahr et al., Eur.Phys.J. C 58, 639 (2008),

[53] A. Falkowski, M. L. Mangano, A. Martin, G. Perez, and J. Winter, Phys. Rev. D 87, 034039 (2013).

[54] G. Marques Tavares and M. Schmaltz, Phys. Rev. D 84, 054008 (2011).

[55] S. Chatrchyan et al. (CMS Collaboration), CMS-PAS-EXO-15-001 (2015).

[56] S. Jung, A. Pierce, and J. D. Wells, Phys. Rev. D 83, 114039 (2011).

[57] T. Aaltonen et al. (CDF Collaboration), Phys. Rev. Lett. 111, 182002 (2013).

[58] J. Alwall et al., J. High Energy Phys. 09 (2007) 028.

[59] V. Abazov et al. (D0 Collaboration), Phys. Rev. D 90, 072001 (2014). 
[60] V. Abazov et al. (D0 Collaboration), Phys. Rev. D 90, 072011 (2014).

[61] V. Abazov et al. (D0 Collaboration), Phys. Rev. D 88, 112002 (2013).

[62] V. Abazov et al. (D0 Collaboration), Phys. Rev. D 92, 052007 (2015).

[63] F. T. Cole, E. L. Goldwasser, and R. R. Wilson, "National Accelerator Laboratory design report," (1968).

[64] D. Crawford (Fermilab Accelerator Division), "Tevatron rookie book," (2009).

[65] P. T. Lukens (CDF collaboration), "The CDF IIb detector Technical design report," (2003).

[66] R. Blair et al. (CDF collaboration), "The CDF-II detector: Technical design report," (1996).

[67] T. Aaltonen et al. (CDF Collaboration), Phys.Rev. D 88, 091103 (2013).

[68] D. Barak, B. Harrison, and A. Watts (Fermilab Accelerator Division), "Concepts rookie book," (2010).

[69] B. Worthel (Fermilab Accelerator Division), "Linac rookie book," (2004).

[70] B. Worthel (Fermilab Accelerator Division), "Booster rookie book," (2009).

[71] T. Asher and S. Baginski (Fermilab Accelerator Division), "Main injector rookie book," (2003).

[72] Figure taken from http://www.fnal.gov/pub/tevatron/ tevatron-accelerator.html.

[73] B. Worthel (Fermilab Accelerator Division), "Antiproton source rookie book," (2000).

[74] T. Aaltonen et al. (CDF Collaboration), Nucl. Instrum. Meth. A 729, 153 (2013).

[75] A. A. Affolder et al. (CDF Collaboration), Nucl. Instrum. Meth. A 526, 249 (2004).

[76] L. Balka et al. (CDF Collaboration), Nucl. Instrum. Meth. A 267, 272 (1988). 
[77] S. Bertolucci et al. (CDF Collaboration), Nucl. Instrum. Meth. A 267, 301 (1988).

[78] G. Ascoli, L. E. Holloway, I. Karliner, U. E. Kruse, R. D. Sard, V. J. Simaitis, D. A. Smith, and T. K. Westhusing, Nucl. Instrum. Meth. A 268, 33 (1988).

[79] T. Dorigo et al. (CDF Collaboration), Nucl. Instrum. Meth. A 461, 560 (2001).

[80] Figure taken from http://hep-www.px.tsukuba.ac.jp/ doraemon/misc/ misc.html.

[81] A. Bhatti et al. (CDF Collaboration), Nucl. Instrum. Meth. A 566, 375 (2006).

[82] D. Acosta et al. (CDF Collaboration), Phys. Rev. D 71, 052003 (2005).

[83] A. Abulencia et al. (CDF collaboration), Journal of Physics G: Nuclear and Particle Physics 34, 2457 (2007).

[84] Figure taken from http://www-cdf.fnal.gov/events/CDFPictures.html.

[85] B. L. Winer, Particles and fields. Proceedings, Meeting, DPF 2000, Columbus, USA, August 9-12, 2000, Int. J. Mod. Phys. A16S1C, 1169 (2001).

[86] K. Anikeev et al. (CDF Collaboration), Comput. Phys. Commun. 140, 110 (2001).

[87] S. Agostinelli et al., Nucl. Instrum. Meth. A 506, 250 (2003).

[88] E. Gerchtein and M. Paulini, eConf C0303241, TUMT005 (2003).

[89] U. Baur, T. Han, and J. Ohnemus, Phys. Rev. D 48, 5140 (1993).

[90] T. Aaltonen et al. (CDF Collaboration), Phys. Rev. Lett. 113, 042001 (2014).

[91] R. Edgar, Ph.D. thesis, University of Michigan,.

[92] Z. Hong, R. Edgar, S. Henry, D. Toback, J. S. Wilson, and D. Amidei, Phys. Rev. D 90, 014040 (2014).

[93] F. James, in Proceedings of the 1972 CERN Computing and Data Processing School, Pertisau, Austria, 10-24 Septempber, 1972 (CERN 72-21) (1972).

[94] P. Nason, J. High Energy Phys. 11 (2004) 040. 
[95] S. Frixione, P. Nason, and C. Oleari, J. High Energy Phys. 11 (2007) 070.

[96] S. Alioli, P. Nason, C. Oleari, and E. Re, J. High Energy Phys. 06 (2010) 043.

[97] A. Caldwell, D. Kollr, and K. Krninger, Computer Physics Communications 180, 2197 (2009).

[98] T. Aaltonen et al. (CDF Collaboration), Phys. Rev. D 88, 032003 (2013).

[99] J. Antos, Acta Phys.Slov. 58, 155 (2008).

[100] N. Metropolis et al., The Journal of Chemical Physics 21, 1087 (1953).

[101] Wikipedia, "Compound poisson distribution — wikipedia, the free encyclopedia," (2015), [Online; accessed 6-May-2015].

[102] L. Demortier and L. Louis (CDF Collaboration), CDFNote 5776 (2013).

[103] T. Aaltonen et al. (CDF Collaboration), Phys. Rev. D 92, 032006 (2015).

[104] T. Aaltonen et al. (CDF Collaboration), Phys. Rev. D 81, 031102 (2010).

[105] L. Lyons, D. Gibaut, and P. Clifford, Nucl. Instrum. Meth. A 270, 110 (1988).

[106] L. Lyons, A. J. Martin, and D. H. Saxon, Phys. Rev. D 41, 982 (1990).

[107] A. Valassi, Nucl. Instrum. Meth. A 500, 391 (2003).

[108] R. Group, C. Ciobanu, K. Lannon, and C. Plager (CDF Collaboration), in Proceedings of the 34th International Conference in High Energy Physics (ICHEP08), Philadelphia, 2008, eConf C080730 (2008).

[109] After communication with Dr. Michelangelo Mangano, we realized that this shape may not have a first-principle analytical explanation, but rather be a combined effect from the behavior of the PDFs, the matrix element and the top decay kinematics. There is some evidence that the charge weighted rapidity distribution of the top quark is actually Gaussian distributed, so the second Gaussian may be just the boosts as part of the decay processes of the top and the $W$ boson. 


\section{APPENDIX A}

\section{LEPTON IDENTIFICATION REQUIREMENTS}

In this appendix, we describe briefly the lepton identification used for the analyses in this dissertation, but give the full set of requirements using the technical notation for completeness. These lepton identification techniques and requirements are standardized at CDF [83] and have been used for many years in many analyses, including almost all the studies and measurements of the top quark.

The portions of the detector used for lepton identification are shown in Figs. 2.2 and 2.3. The electrons detected by the barrel part of the detector, $|\eta|<1.1$, are denoted as the "CEM" (Central ElectroMagnetic calorimeter) electrons, and those detected by the plug part, $1.1<|\eta|<2.0$, are denoted as the "PHX" (PHoeniX) electrons for historical reasons. There are three muon systems that are used together for muon identification. They are the "CMU" (Central MUon chambers), the "CMP" (Central Muon uPgrade chambers) and the "CMX" (Central Muon eXtension chambers). Since there is significant, but not complete, overlap between the CMU and CMP, many muons will go through both and we refer to such muons as "CMUP" muons. The muons leaving signals in the CMX detector are denoted as the "CMX" muons. The muon coverage in this analysis is $|\eta|<1.1$.

All the leptons are categorized into a "tight" category or a "loose" category, and further into "isolated" leptons or "non-isolated" leptons [83, as described in Chapter 2, The "tight" leptons are those which satisfy all the object identification criteria and thus have a higher probability of originating from a real lepton, while the "loose" leptons are those which satisfy only a specific subset of the criteria (but not including the tight leptons). The two categories are set up so that in the 
event selection criteria we can choose to apply either the tight or the loose object identification, depending on whether we want the selected sample to have a higher purity or we want a better selection efficiency. A CEM electron can fall in either the tight or the loose category, depending on whether it satisfies all or only a subset of the requirements. All PHX electrons belong to the tight category, so do the CMUP and CMX muons. Muons that leave signals only in the CMU or CMP portion of the muon sub-detectors, but not both, are denoted as "CMU" or "CMP" muons, and can only contribute to the loose lepton category. A special category of muons which pass all the selection cuts except the requirement of a signal in the muon detectors, but from geometric considerations are not expected to hit the muon detectors, are called the "CMIO" (Central Minimal IOnized particle) muons. These are also in the category of loose leptons.

For completeness, we list all the technical requirements here. Tables A.1, A.2, A.3, and A.4 list the identification requirements of each of the lepton categories. Note that since these these technical terms are standard in CDF, we refer to Ref. [83] for the technical terms quoted in these tables as well as more details regarding the identification requirements and how the values of each requirement were selected. 
Table A.1: The requirements used to select electrons in the Central ElectroMagnetic calorimeter (CEM). Tight CEM electrons ("CEM") are required to pass all the criteria. Nonisolated CEM electrons ("nisoCEM") pass all of the cuts except for Isolation (marked with an *). Loose, but isolated, CEM electrons ("looseCEM") are required to pass only the criteria above the line. Nonisolated loose CEM electrons (nisolooseCEM) pass all of the looseCEM cuts except isolation. We refer to Ref. [83] for the technical terms quoted in these tables as well as more details regarding the identification requirements.

\begin{tabular}{|l||r|}
\hline Variable & Requirement \\
\hline Region & CEM \\
Fiducial & Fiducial TRUE \\
$E_{\mathrm{T}}$ & $\geq 20 \mathrm{GeV}$ \\
Track $Z_{0}$ & $\leq 60 \mathrm{~cm}$ \\
Track $p_{T}$ & $\geq 10 \mathrm{GeV} / \mathrm{c}$ \\
COT Axial Seg. & $\geq 3$ \\
COT Stereo Seg. & $\geq 2$ \\
Conversion & $\neq 1$ \\
Had/em & $\leq(0.055+(0.00045 \times E))$ \\
Isolation* & $\leq 0.1$ \\
\hline LshrTrk & $\leq 0.2$ \\
$E / P$ & $\leq 2.0$ unless $p_{T} \geq 50 \mathrm{GeV} / \mathrm{c}$ \\
CES $\Delta Z$ & $\leq 3.0 \mathrm{~cm}$ \\
Signed CES $\Delta X$ & $-3.0 \leq q \times \Delta X \leq 1.5$ \\
CES StripChi2 & $\leq 10.0$ \\
\hline
\end{tabular}


Table A.2: The requirements to identify electrons in the plug electromagnetic calorimeter (PEM, and the electrons are identified with tracking package named PHENIX, thus denoted as "PHX"). All the PHX electrons belong to the tight category. We refer to Ref. [83] for the technical terms quoted in these tables as well as more details regarding the identification requirements.

\begin{tabular}{|l||r|}
\hline Variable & Requirement \\
\hline$E_{\mathrm{T}}$ & $\geq 20 \mathrm{GeV}$ \\
Pes2DEta & $\leq|\eta| \leq 2.8$ \\
Had/Em & $\leq 0.05$ \\
Pem3x3FitTow & $\neq 0$ \\
Pem3x3Chisq & $\leq 10$ \\
Pes5by9U & $\geq 0.65$ \\
Pes5by9v & $\geq 0.65$ \\
Isolation & $\leq 0.1$ \\
$\Delta R$ & $\leq 3.0 \mathrm{~cm}$ \\
PhxMatch & TRUE \\
$N_{\text {hits }}^{S i}$ & $\geq 3$ \\
$\left|Z_{0}^{P H X}\right|$ & $\leq 60 \mathrm{~cm}$ \\
\hline
\end{tabular}

Table A.3: The muon identification selection requirements for candidates in the CMUP systems. Tight muons must pass all the requirements in the table. Note that loose muons "CMU" ("CMP") are defined by not requiring the requirement below the line on the "CMP" ("CMU") counterpart. We refer to Ref. 83] for the technical terms quoted in these tables as well as more details regarding the identification requirements.

\begin{tabular}{|l||r|}
\hline Cosmic tag & FALSE \\
$p_{T}$ & $>20 \mathrm{GeV} / \mathrm{c}$ \\
$E_{E M}$ & $<2+\max (0,(p-100) \cdot 0.0115) \mathrm{GeV}$ \\
$E_{H A D}$ & $<6+\max (0,(p-100) \cdot 0.028) \mathrm{GeV}$ \\
$E_{T}^{i s o l .} / p_{T}$ & $<0.1$ \\
$\#$ of axial SL with $\geq 5$ hits & $\geq 3$ \\
$\#$ of stereo SL with $\geq 5$ hits & $\geq 2$ \\
$\left|z_{0}\right|$ & $<60 \mathrm{~cm}$ \\
Tracks w/ no silicon hits: $\left|d_{0}\right|$ & $<0.2 \mathrm{~cm}$ \\
Tracks w/ silicon hits: $\left|d_{0}\right|$ & $<0.02 \mathrm{~cm}$ \\
\hline Fiducial for CMU & TRUE \\
$\left|\Delta x_{C M U}\right|$ & $<7 \mathrm{~cm}$ \\
Fiducial for CMP & TRUE \\
$\left|\Delta x_{C M P}\right|$ & $<5 \mathrm{~cm}$ \\
\hline
\end{tabular}


Table A.4: The muon identification selection requirements for candidates in the CMX systems. All CMX muons fall in the tight category. We refer to Ref. 83] for the technical terms quoted in these tables as well as more details regarding the identification requirements.

\begin{tabular}{|l||r|}
\hline Cosmic tag & FALSE \\
Fiducial & TRUE \\
$p_{T}$ & $>20 \mathrm{GeV} / \mathrm{c}$ \\
$E_{E M}$ & $<2+\max (0,(p-100) \cdot 0.0115) \mathrm{GeV}$ \\
$E_{H A D}$ & $<6+\max (0,(p-100) \cdot 0.028) \mathrm{GeV}$ \\
$E_{T}^{i s o l} / p_{T}$ & $<0.1$ \\
$\#$ of axial SL with $\geq 5$ hits & $\geq 3$ \\
$\#$ of stereo SL with $\geq 5$ hits & $\geq 2$ \\
$\left|z_{0}\right|$ & $<60 \mathrm{~cm}$ \\
Tracks w/ no silicon hits: $\left|d_{0}\right|$ & $<0.2 \mathrm{~cm}$ \\
Tracks w/ silicon hits: $\left|d_{0}\right|$ & $<0.02 \mathrm{~cm}$ \\
$\left|\Delta x_{C M X}\right|$ & $<6 \mathrm{~cm}$ \\
Run & $>1501445$ \\
\hline
\end{tabular}




\section{APPENDIX B}

\section{ESTIMATION OF THE DRELL-YAN BACKGROUNDS IN THE DILEPTON+DIJET + H $_{T}$ FINAL STATE}

Drell-Yan (DY) production of lepton pairs through a $Z$ and an off-shell photon $\left(Z / \gamma^{*}\right)$ production, in association with jets, is one of the dominant backgrounds in this analysis. Since DY has very different kinematic distributions from $t \bar{t}$, it has the potential to systematically bias all our measurements. For these reasons we want to get a robust estimation of the DY background, both in terms of the total number of expected events as well as in the distributions of the kinematic variables in each of the possible final states it produces.

In this appendix we describe the estimation of the contamination of the DY+jets process in the data after the event selection described in Table 2.1. We note that this estimation is only used to estimate the expected number of events; the distributions of the kinematic variables will be estimated purely with the $\mathrm{MC}$ simulations after normalizing the number of events to the estimated numbers from this appendix.

We first describe the reason why a simple estimation with $\mathrm{MC}$ method is not good enough for our needs, and why a sophisticated method is required. This will be done in Sec. B.1. The estimation method used in the previous CDF analyses, for example the $t \bar{t}$ cross section measurement in the dilepton final state, is given in Sec. B.2. We note that this estimation method can only be applied to the scenario where the $Z / \gamma^{*}$ decays to a pair of electrons or muons, and are reconstructed as a pair of electrons or muons in the detector (denoted as DY $\rightarrow e e / \mu \mu \rightarrow e e / \mu \mu$ ). As explained below, it cannot be applied to the scenarios where the $Z / \gamma^{*}$ decays to a pair of electrons or muons, but are reconstructed as an electron plus a muon in 
the detector. We note that this usually happens when a muon bremsstrahlungs a photon which is collinear with the original muon, thus the energy deposition in the calorimeter by the photon and the muon track combined make an electron candidate. Thus, this scenario is denoted as DY $\rightarrow e e / \mu \mu \rightarrow e_{\text {fake }} \mu$. Finally, the original estimation method does not apply to the scenario where the $Z / \gamma^{*}$ decays to a pair of $\tau$ leptons, and the $\tau$ leptons further decay leptonically into the $e e, e \mu$, or $\mu \mu$ final states. This scenario is denoted as DY $\rightarrow \tau \tau$, which includes all three final states $(\mathrm{DY} \rightarrow \tau \tau \rightarrow e e / e \mu / \mu \mu)$. In Sec. B.3 we describe the updated estimation method, which is consistent with the method described in Sec. B.2, but is applicable to the final states of DY $\rightarrow e e / \mu \mu \rightarrow e_{\text {fake }} \mu$ and $\mathrm{DY} \rightarrow \tau \tau$.

\section{B.1 Motivation for a Sophisticated DY Estimation Method}

While it is true that we have MC event generators to simulate the production and decay of DY, it is well known that they are not good enough for use by themselves [67]. We quickly summarizes the issues. One is that the additional jets must be produced, and that isn't done well by the MC. The dominant issue, however, is that in the case of the DY $\rightarrow e e / \mu \mu \rightarrow e e / \mu \mu$ final state the $\mathscr{H}_{T}$ measured in the event is due to instrumental energy mis-measurement, and our detector simulation is known to be not good enough to simulate very large mis-measurement correctly enough for our purposes. Thus, we use data to help us properly estimate: 1) the cross section of DY production with two or more associated jets and 2) the modeling of $\not_{T}$ where all the $\mathscr{H}_{T}$ comes from mis-measurements from the detector.

In the previous $t \bar{t}$ cross section measurement in this final state, the normalization for $\mathrm{DY} \rightarrow e e / \mu \mu \rightarrow e e / \mu \mu$ employed a sophisticated data-MC hybrid method where data is used to correct the $\mathrm{MC}$ for the rate in the high- $\not_{T}$ regime [67], based on normalizing the MC simulated cross sections to that observed in data in a region 
where $\mathrm{DY} \rightarrow e e / \mu \mu$ dominates. However, in the $t \bar{t}$ cross section measurement, the contribution of DY $\rightarrow e e / \mu \mu$ process which is mis-identified as the $e \mu$ final state $\left(\mathrm{DY} \rightarrow e e / \mu \mu \rightarrow e_{\text {fake }} \mu\right)$, as well as the contribution of DY $\rightarrow \tau \tau$ process, are estimated only based on MC predictions normalized to theoretical predicted cross sections, which are now known to be not an accurate assumption. Since the $\tau$ leptons are not observable in the detector as they are not stable, and the DY $\rightarrow e e / \mu \mu \rightarrow$ $e_{\text {fake }} \mu$ scenario is caused by a mis-identification, there is no region in data where these two scenarios dominate, thus there is no apparent way to apply the same data-MC hybrid method in these scenarios. Because of this, an updated estimation method is needed for these two scenarios.

In the following sections, we describe the estimation method used for DY $\rightarrow$ $e e / \mu \mu \rightarrow e e / \mu \mu$ in the previous analyses in Sec. B.2, and then introduce the new estimation method, which is consistent with the previous one, but can be extended to the final states of DY $\rightarrow e e / \mu \mu \rightarrow e_{\text {fake }} \mu$ and DY $\rightarrow \tau \tau \rightarrow e e / e \mu / \mu \mu$ in Sec. B.3.

\section{B.2 The Data-MC Hybrid Estimation Method for the ee and $\mu \mu$ Final States}

Here we describe the data-MC hybrid method for estimating the DY $\rightarrow e e / \mu \mu \rightarrow$ $e e / \mu \mu$ backgrounds, which is detailed in Ref. [67]. We note before we start that the description in this section is restricted to the $e e$ and $\mu \mu$ final states; we will come back to the $e \mu$ final state in the next section. In addition, we will be focusing on estimating the contribution from DY $\rightarrow e e / \mu \mu$ where the events are observed as dielectron or di-muon events in the detector. While we will consider the contribution from $\mathrm{DY} \rightarrow \tau \tau \rightarrow e e / \mu \mu$ as a background to the estimation of $\mathrm{DY} \rightarrow e e / \mu \mu$, the estimation of DY $\rightarrow \tau \tau$ itself in all three dilepton flavors $(e e, \mu \mu$, and $e \mu)$ will be described in the next section.

The idea of the data-MC hybrid method is to measure the contribution from 
$\mathrm{DY} \rightarrow e e / \mu \mu$ in data, using samples that are not the signal region but where DY $\rightarrow$ $e e / \mu \mu$ is the dominant source (control regions), and use an extrapolation factor (from the control regions to the signal regions) derived from MC samples to obtain the expected contribution in data in the signal regions. We note here that this dataMC hybrid method is functionally the same as normalizing the number of events in the $\mathrm{MC}$ estimation in control regions to the number of events observed in data in the same regions, which successfully avoids the mis-modeling of the inclusive crosssection and the $\mathscr{E}_{T}$ distribution in the MC samples. The assumption in this method is that the amount of mismodeling (which causes the deviation between the MCbased predictions and the observations in data in the control regions) is the same in both the control regions and the corresponding signal regions. To accomplish this task, we define a couple of orthogonal regions which are our control regions and signal regions.

As described in Table 2.1, the event selection criteria are different for 1) events with same lepton flavor and with the dilepton invariant mass consistent with the $Z$ boson mass (we will define "inside the $Z$-window" as the mass regime $76 \mathrm{GeV}<\mathrm{m}_{l l}<$ $106 \mathrm{GeV}$ ), with an extra requirement of high METSig (METSig $>4 \sqrt{\mathrm{GeV}}$ ) and 2) events outside the window or with different lepton flavors, without any requirement on METSig. Because of this, we select the set of events with the ee and $\mu \mu$ final states, passing all the "minimal requirements" in Table 2.1 except the requirements on METSig and $\mathrm{m}_{11}$, and break them into one of the four regions shown in Table B.1. The four regions are defined separately with jet multiplicity of 0,1 , and $\geq 2$, and again separately with $e e$ and $\mu \mu$ events, for a total of six versions of the table.

We note that the combined Regions 1, 2, and 4 form the sample that passes the minimal requirement defined in Table 2.1, but further requirements corresponding to the final "signal requirements" (oppositely charged leptons, $\geq 2$ jets, and high $H_{T}$ ) 
Table B.1: Definition of signal and control regions for DY estimation. Note that this table is only for the events in the $e e$ and $\mu \mu$ final states (not for the $e \mu$ final state). The events in all regions defined here are after all the "minimal requirements" in Table 2.1 except the requirements on METSig and $\mathrm{m}_{l l}$. The estimations in Regions 1, 2, and 4, after applying the efficiency for the "signal requirements", are the final estimations in the signal region of this analysis. Note that there are six different versions of this table, one for each of the $e e$ and $\mu \mu$ final states, and each with a jet multiplicity of 0,1 , and $\geq 2$.

\begin{tabular}{|c|c|c|}
\hline $\begin{array}{l}\text { METSig }>4 \sqrt{\mathrm{GeV}} \\
\text { (High METSig) }\end{array}$ & Region 1 & Region 2 \\
\hline $\begin{array}{l}\text { METSig }<4 \sqrt{\mathrm{GeV}} \\
\text { (Low METSig) }\end{array}$ & Region 3 & Region 4 \\
\hline & $\begin{array}{c}76 \mathrm{GeV}<\mathrm{m}_{\mathrm{ll}}<106 \mathrm{GeV} \\
(\mathrm{In} Z \text {-window })\end{array}$ & $\begin{array}{c}\mathrm{m}_{\mathrm{ll}}<76 \mathrm{GeV} \text { or }>106 \mathrm{GeV} \\
\text { (Outside } Z \text {-window) }\end{array}$ \\
\hline
\end{tabular}

are needed to get us to the predictions for the set of events passing the full set of the requirements in Table 2.1. These further requirements are incorporated into our estimation technique by deriving and applying the efficiencies of these requirements to the number of events in Regions 1, 2, and 4. These efficiencies for the signal requirements are derived from the $\mathrm{DY} \rightarrow e e / \mu \mu \mathrm{MC}$ samples, and applied to the estimations in Regions 1, 2, and 4, to obtain the estimation of the contamination from the DY $\rightarrow e e / \mu \mu \rightarrow e e / \mu \mu$ process in the final signal sample. Note that the estimations with 0 or 1 jet are used for cross checks to build confidence in this estimation method.

With these two ideas in mind, the data-MC hybrid method is:

1. For regions where DY $\rightarrow e e / \mu \mu$ is expected to be the dominant source (Regions 3 , and Region $1+3$ combined), measure the contribution from DY $\rightarrow e e / \mu \mu$ in data (after subtracting off non-DY $\rightarrow e e / \mu \mu$ contributions). We will refer to these estimations as $\left(N_{\text {Region 3 }}^{\text {Data }}-N_{\text {Region 3 }}^{\text {Non-DY }}\right)$ and $\left(N_{\text {Region } 1+3}^{\text {Data }}-N_{\text {Region 1+3 }}^{\text {Non-DY }}\right)$. 
2. Measure the relative ratios of $\frac{N_{\text {Region }}^{\mathrm{DY} M C}}{N_{\text {Region } 3}^{\mathrm{DY}} 3}$ and $\frac{N_{\text {Region } 2+4}^{\mathrm{DY} M C}}{N_{\text {Region } 1+3}^{\mathrm{DY} M}}$ from the DY $\rightarrow e e / \mu \mu$ MC samples.

3. Use the numbers measured in (1) and ratios in (2) to calculate the contributions of DY $\rightarrow e e / \mu \mu$ in Regions 1, 2, and 4 (equations given below), corresponding to the samples after the minimal cuts. These estimations are also used as cross checks.

4. Derive the efficiency of the "signal requirements" in Table $2.1\left(\epsilon^{\text {Signal cut }}\right)$ from the $\mathrm{DY} \rightarrow e e / \mu \mu \mathrm{MC}$ samples.

5. Apply the $\epsilon^{\text {Signal cut }}$ to the estimated numbers in (3) with $\geq 2$ jets, for the final estimation of $\mathrm{DY} \rightarrow e e / \mu \mu \rightarrow e e / \mu \mu$ in the signal region.

To display the estimation method in a mathematical way, we list the equations used to estimate the number of DY $\rightarrow e e / \mu \mu \rightarrow e e / \mu \mu$ events passing the minimal requirements in Table 2.1 $\left(N_{\text {Minimal cut }}^{\text {DY Estimat }}\right)$ and eventually calculate the final estimates $\left(N_{\text {Signal cut }}^{\text {DY Estion }}\right)$. We note that the equations are applied to regions specified in Table B.1, with 0,1 , and $\geq 2$ jets separately, and again separately to the final states of $e e$ and $\mu \mu$ ( $e \mu$ final state will be discussed in the next section). For briefness, the notation "DY" in the equations refers exclusively to DY $\rightarrow e e / \mu \mu$, and the notation "Non-DY" refers to all SM sources except DY $\rightarrow e e / \mu \mu$ (to be explicit, DY $\rightarrow \tau \tau$ is included in Non-DY category). The equations are:

$$
N_{\text {Minimal cut }}^{\text {DY Estimation }}=N_{\text {Region 2+4 }}^{\text {DY Estimation }}+N_{\text {Region 1 }}^{\text {DY Estimation }},
$$


where we estimate the contributions from each using

$$
\begin{array}{ll}
N_{\text {Region 2+4 }}^{\mathrm{DY} \text { Estimation }} & =\frac{N_{\text {Region 2+4 }}^{\mathrm{DY} \text { MC }}}{N_{\text {Region 1+3 }}^{\mathrm{DY} \mathrm{MC}}} \cdot\left(N_{\text {Region 1+3 }}^{\text {Data }}-N_{\text {Region 1+3 }}^{\text {Non-DY }}\right) \\
N_{\text {Region 1 }}^{\text {DY Estimation }} & =\frac{N_{\text {Region 1 }}^{\mathrm{DY} \mathrm{MC}}}{N_{\text {Region 3 }}^{\text {DY MC }}} \cdot\left(N_{\text {Region 3 }}^{\text {Data }}-N_{\text {Region 3 }}^{\text {Non-DY }}\right)
\end{array}
$$

where all $N$ parameters indicate the number of events in the corresponding category. The numbers with the superscript "Data" are measured from data, with the corresponding requirements indicated by the subscript; the numbers with the superscript "Non-DY" refers to the expected number of events from all sources except DY $\rightarrow e e / \mu \mu$; the numbers with the superscript DY MC are measured in the $\mathrm{DY} \rightarrow e e / \mu \mu \mathrm{MC}$ samples, which are used to estimate the ratio of the number of events in Region $2+4$ over that in Region $1+3$, and also the ratio in Region 1 vs. Region 3. Note that we use this set of equations separately for jet multiplicities of 0,1 , and $\geq 2$, and each in the $e e$ and $\mu \mu$ final states separately. The inputs and outputs of Eqs. B.2a and B.2b are collected in Tables B.2 and B.3 respectively. The output numbers are the estimated number of events passing the minimal requirement in Table 2.1 in the corresponding category.

As the final step, the efficiencies of the signal requirements defined in Table 2.1 are estimated with $\mathrm{DY} \rightarrow e e / \mu \mu \mathrm{MC}$ samples. The final estimation of the DY $\rightarrow$ $e e / \mu \mu \rightarrow e e / \mu \mu$ contribution to our signal (passing both the minimal requirements and the signal requirements) is calculated as

$$
\begin{aligned}
N_{\text {Signal cut }}^{\mathrm{DY} \text { Estimation }}=\sum_{\ell \ell=e e, \mu \mu} & \left\{N_{\text {Region 1 }}^{\mathrm{DY} \text { Estimation }}(\ell \ell) \cdot \epsilon_{\text {Region } 1}^{\text {Signal cut }}(\ell \ell)\right. \\
& \left.+N_{\text {Region 2,4 }}^{\text {DY Estimation }}(\ell \ell) \cdot \epsilon_{\text {Region 2,4 }}^{\text {Signal cut }}(\ell \ell)\right\} .
\end{aligned}
$$

The results are collected in Table B.4. This is the estimated contamination of DY $\rightarrow$ 
$e e / \mu \mu \rightarrow e e / \mu \mu$ in the final data sample used in the asymmetry analyses.

Despite its success, this method cannot be directly applied to the $e \mu$ final state, nor can it be applied to estimate the contribution from DY $\rightarrow \tau \tau$, due to the lack of the control regions where these two processes dominate. In the next section, we will describe a new estimation method, which is consistent with the one described in this section, but can be applied to the final state of DY $\rightarrow e e / \mu \mu \rightarrow e_{\text {fake }} \mu$ and $\mathrm{DY} \rightarrow \tau \tau$. 


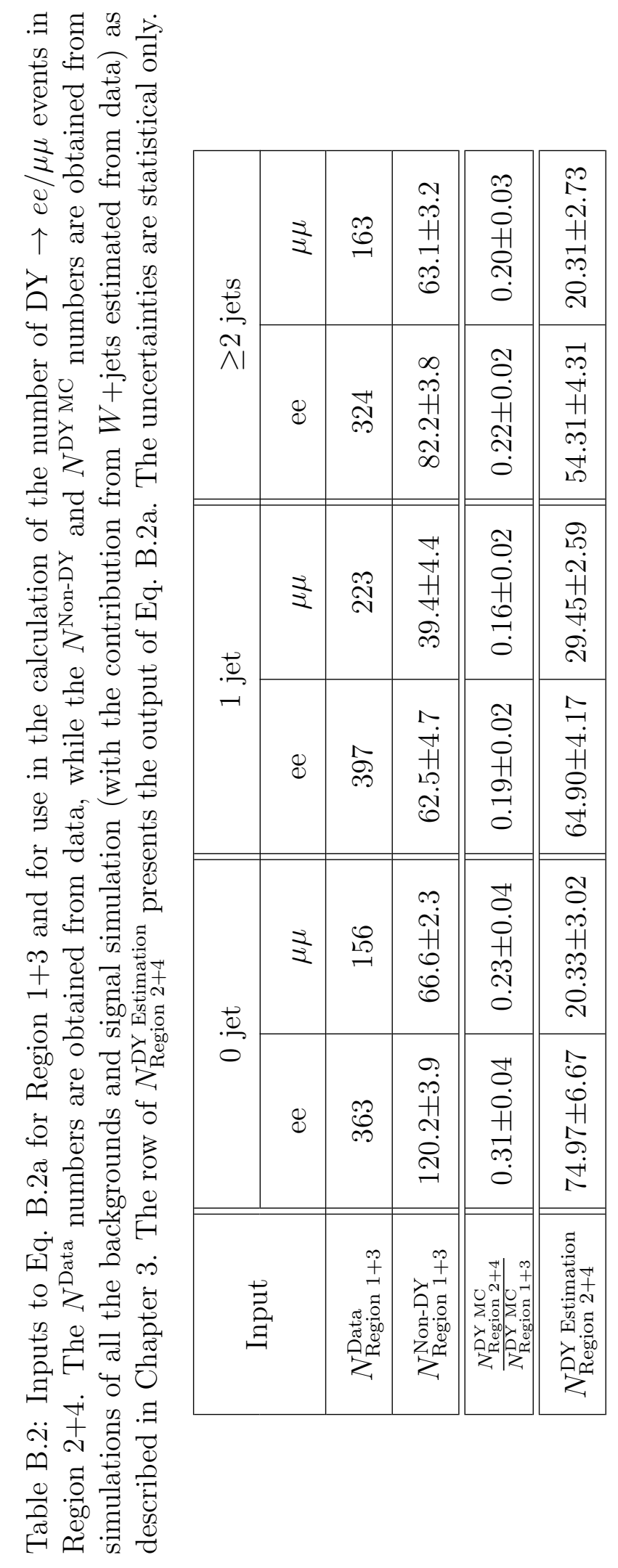




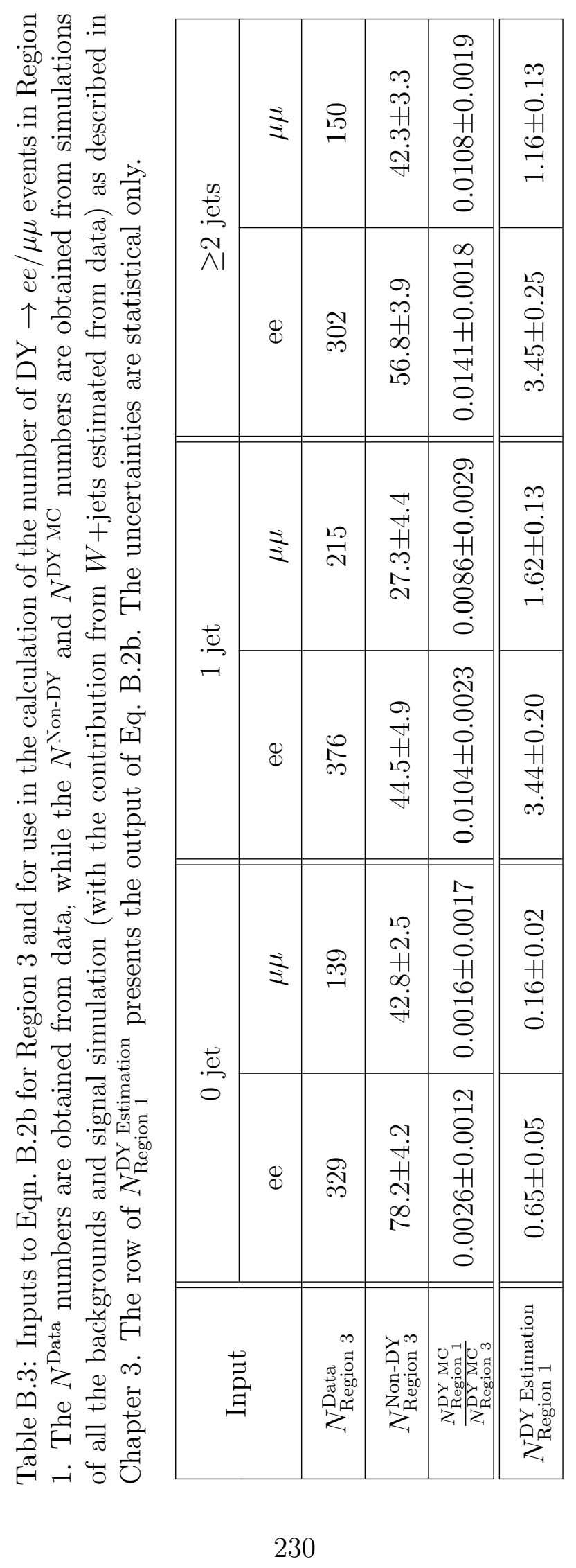


Table B.4: Efficiencies of the signal requirements with $\geq 2$ jets $\left(\epsilon^{\text {Signal cut }}\right)$. This is the efficiencies for the opposite charged leptons and $H_{T}>200 \mathrm{GeV}$ requirements, to be applied to the outputs with $\geq 2$ jets in Tables $\mathrm{B} .2$ and $\mathrm{B} .3$ using Eq. B.3.

\begin{tabular}{|c|c|c|}
\hline$\epsilon^{\text {Signal cut }}$ & $e e$ & $\mu \mu$ \\
\hline For $N_{\text {Region 1 }}^{\text {DY Estimation }}$ & $0.303 \pm 0.024$ & $0.457 \pm 0.057$ \\
\hline For $N_{\text {Region 2 }+4}^{\text {DY Estimation }}$ & $0.957 \pm 0.016$ & $0.992 \pm 0.010$ \\
\hline
\end{tabular}




\section{B.3 Scale Factors for the $e \mu$ and $\tau \tau$ Final States}

While the data-MC hybrid method described in the previous section can be used to model DY $\rightarrow e e / \mu \mu \rightarrow e e / \mu \mu$ backgrounds, the background from DY $\rightarrow e e / \mu \mu \rightarrow$ $e_{\text {fake }} \mu$ and DY $\rightarrow \tau \tau \rightarrow e e / e \mu / \mu \mu$ cannot be estimated using the same techniques because there are not good control samples. In the previous analysis, the rates were estimated by simulating events and normalizing to the predicted LO cross section with K-factors [67]. We now introduce a better modeling of these two categories based on the assumption that the DY production cross section for the $e e, \mu \mu$ and $\tau \tau$ final states are identical. This estimation is accomplished by deriving two sets of scaling factors $(\mathrm{SF})$ for 1 ) the overall DY normalization $\left(\mathrm{SF}_{\mathrm{DY}}\right)$ and 2$)$ the $\mathscr{E}_{T}$ distribution $\left(\mathrm{SF}_{\mathrm{DY}} \not_{T}\right)$. We note from the beginning that following the naming convention in the previous section, the ultimate estimation of the DY $\rightarrow e e / \mu \mu$ and $\mathrm{DY} \rightarrow \tau \tau$ contributions $\left(N_{\text {Signal cut }}^{\text {DY Estion }}\right)$ in the signal region or any control region is calculated with

$$
N_{\text {Signal cut }}^{\mathrm{DY} \text { Estimation }}=N_{\text {Signal cut }}^{\mathrm{DY} \mathrm{MC}} \cdot \mathrm{SF}_{\mathrm{DY}} \cdot \mathrm{SF}_{\mathrm{DY}} E_{T} \cdot
$$

The first set of scale factors is to correct for the discrepancy between the estimation of $\mathrm{DY} \rightarrow e e / \mu \mu$ from $\mathrm{MC}$ and the observation in data, in a region that is heavily dominated by the DY $\rightarrow e e / \mu \mu$ process. This is thus a set of scale factors for the production cross section of the DY process for ee and $\mu \mu$ separately, as well as for 0,1 and $\geq 2$ jets separately. To obtain these scale factors, we first apply a set of event selection criteria aiming to select a sample dominated by the DY $\rightarrow e e / \mu \mu$ process. The event selection criteria are: 1) two reconstructed charged leptons, 2) the invariant mass of the two charged leptons being consistent with the $Z$-boson mass $\left(76 \mathrm{GeV}<\mathrm{m}_{11}<106 \mathrm{GeV}\right)$. This region is denoted as "Region Z" for short. (This Region $\mathrm{Z}$ is different from the Region $1+3$ defined in the previous section since Region 
$1+3$ also requires the events to pass the $E_{T}$ requirements.) Following the notations in the previous section, we use the following equations to obtain the scale factors for the DY production cross section $\left(\mathrm{SF}_{\mathrm{DY}}\right)$ with 0,1 , and $\geq 2$ jets separately, and with $e e$ and $\mu \mu$ final states separately:

$$
\mathrm{SF}_{\mathrm{DY}}=\frac{N_{\text {Region Z }}^{\text {Data }}-N_{\text {Region Z }}^{\text {Non-DY }}}{N_{\text {Region Z }}^{\mathrm{DY} M C}} .
$$

Table B.5 shows the numbers used in the calculation of the $\mathrm{SF}_{\mathrm{DY}}$. The average between $e e$ and $\mu \mu$ categories in each jet multiplicity is used to scale the DY $\rightarrow$ $e e / \mu \mu \rightarrow e_{\text {fake }} \mu$ and all DY $\rightarrow \tau \tau$ components with the corresponding jet multiplicity, as will be shown in Table B.7.

The second set of scale factors is used to correct for the not-well-estimated $\ddot{B}_{T}$ distribution in the MC simulations. In the $\mathrm{DY} \rightarrow e e / \mu \mu$ category, $\not_{T}$ is caused by instrumental mis-measurement. It is known that the MC simulation does not model the $E_{T}$ spectrum for large $E_{T}^{\prime}$ from instrumental mis-measurement well. This set of DY $\not_{T}$ scale factors $\left(\mathrm{SF}_{\mathrm{DY}} \not_{T}\right)$ is designed to correct for such mis-modeling in MC simulations. To calculate this set of scale factors, we use the previously defined region "Region $1+3$ ", but note that it is essentially the set of events in the Region Z, but satisfying two additional criteria: 1) $\left.E_{T}^{\prime}>25 \mathrm{GeV}, 2\right) E_{T}>50 \mathrm{GeV}$ when there is any lepton or jet within $20^{\circ}$ of the direction of $E_{T}^{\prime}$. We name this region as "Region $\mathrm{Z}+E_{T}$ " to be more intuitive. The ratio of the number of events in Region $\mathrm{Z}+E_{T}$ over that in Region $\mathrm{Z}$ is defined as the selection efficiency of the $\mathbb{H}_{T}$ requirement $\left(\epsilon_{E_{T}}\right)$. The $\epsilon_{E_{T}}$ can be estimated with two approaches: 1) estimating with data after subtracting off Non-DY contributions, and 2) estimating with DY MC samples. 


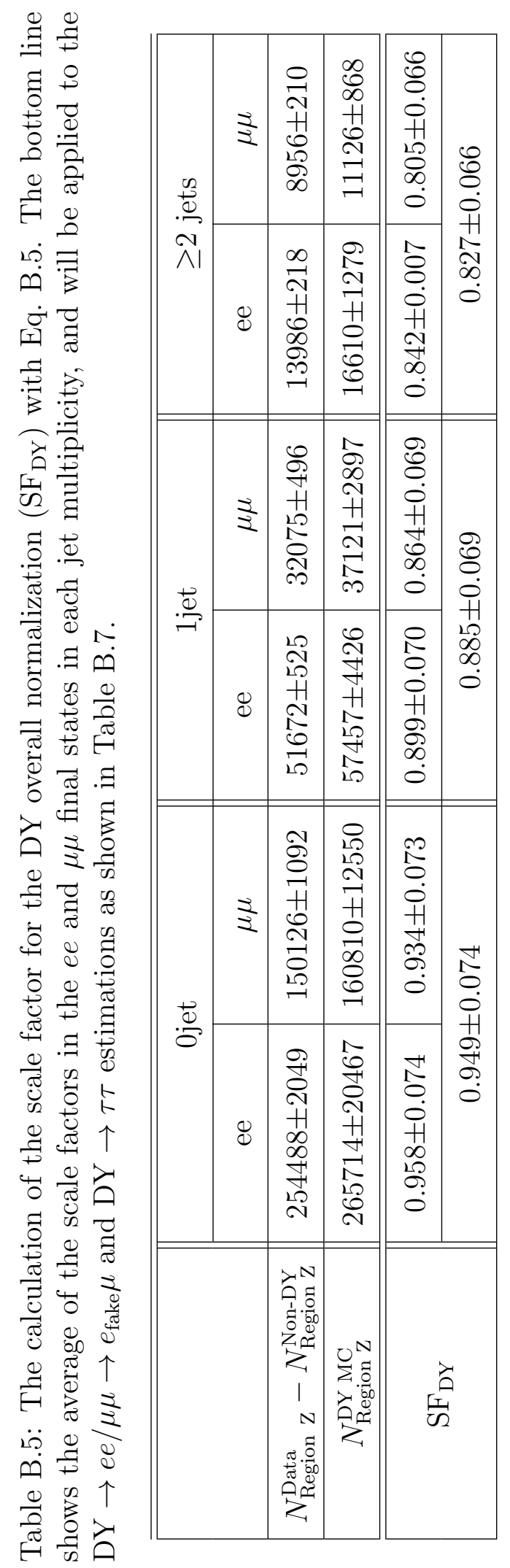


The equations for this process are:

$$
\begin{aligned}
& \epsilon_{E_{T}^{\prime}}^{\text {Data }}=\frac{N_{\text {Region Z+ }+\hbar_{T}^{\text {Data }}}^{\text {Degion }}-N_{\text {Region Z }+E_{T}^{\prime}}^{\text {Non-DY }}}{N_{\text {Region Z }}^{\text {Data }}-N_{\text {Region Z }}^{\text {Non-DY }}} \text {, and } \\
& \epsilon_{E_{T}^{\mathrm{DY}} \mathrm{MC}}^{\mathrm{D}}=\frac{N_{\text {Region Z }+E_{T}}^{\mathrm{DY}}}{N_{\text {Region Z }}^{\mathrm{DY} \mathrm{MC}}}
\end{aligned}
$$

The $\mathrm{SF}_{\mathrm{DY}} \not_{T}^{\prime}$ is estimated as the ratio of the two efficiencies as:

$$
\mathrm{SF}_{\mathrm{DY}} \mathscr{H}_{T}=\frac{\epsilon_{\mathscr{E}_{T}^{\mathrm{Data}}}^{\mathrm{C}}}{\epsilon_{\text {HY }_{T}^{\mathrm{DC}}}} .
$$

The $\mathrm{SF}_{\mathrm{DY}}{\not C_{T}}_{T}$ is also estimated separately with 0,1 , and $\geq 2$ jets, and again separately with $e e$ and $\mu \mu$ final states. Table B.6 shows the calculation of the scale factors. The $\mathrm{SF}_{\mathrm{DY}} E_{T}$ in the $e e$ and $\mu \mu$ final states are quite different, indicating different levels of mis-modeling in $\ddot{H}_{T}$ in the detector simulation (due to different ways the electrons and muons depositing energy with the detector). The average of the SF ${ }_{\mathrm{DY}} E_{T}$ in the $e e$ and $\mu \mu$ categories is applied to the estimation of DY $\rightarrow e e / \mu \mu \rightarrow e_{\text {fake }} \mu$ component, and the difference between $\mathrm{SF}_{\mathrm{DY}}{\not \phi_{T}}_{T}$ values in the ee and $\mu \mu$ categories is taken as a systematic uncertainty of the averaged scale factor conservatively. Since the $E_{T}$ in the DY $\rightarrow \tau \tau$ process is physical and there is a decent fraction of events beyond $\not_{T}>50 \mathrm{GeV}$ (Fig. B.1), the mis-modeling of $\not_{T}$ is presumably small. A $\mathrm{SF}_{\mathrm{DY}} E_{T}$ of 1.0 is applied to $\mathrm{DY} \rightarrow \tau \tau$, and a systematic uncertainty of 0.1 is taken conservatively, as $10 \%$ is more than enough to cover the potential bias even if $\mathscr{H}_{T}$ is mis-measured by $\sim 5 \mathrm{GeV}$. 


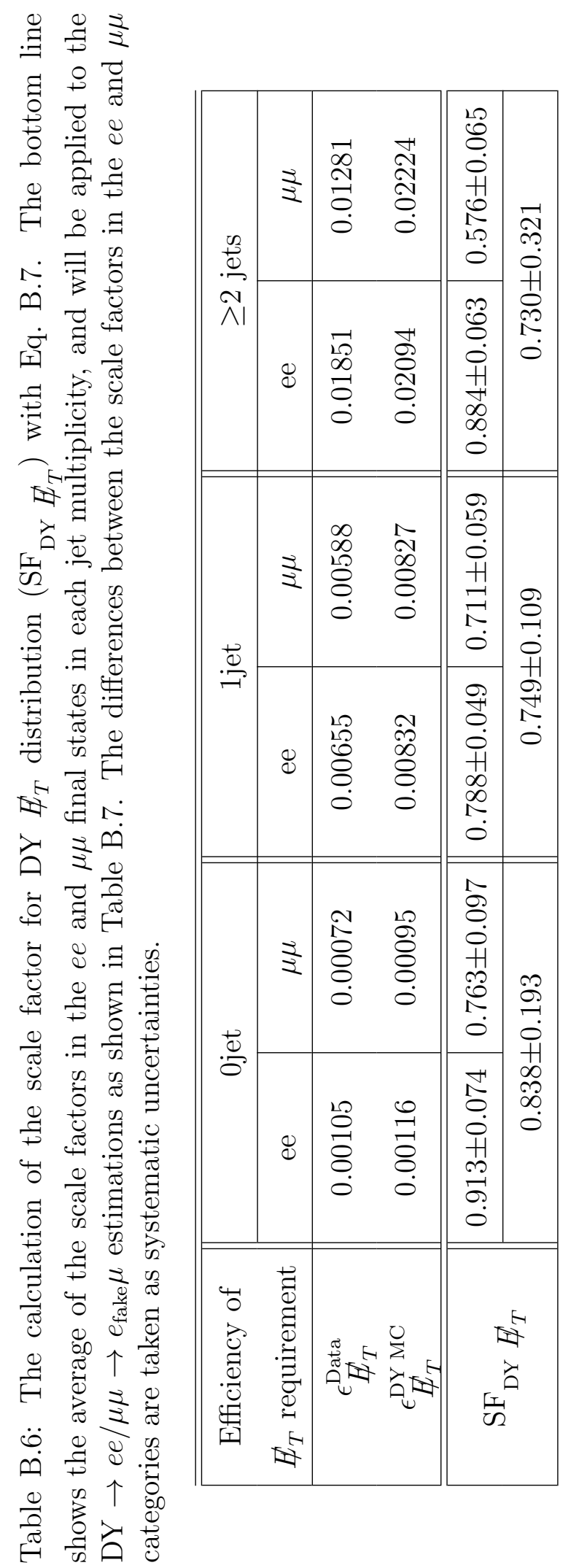




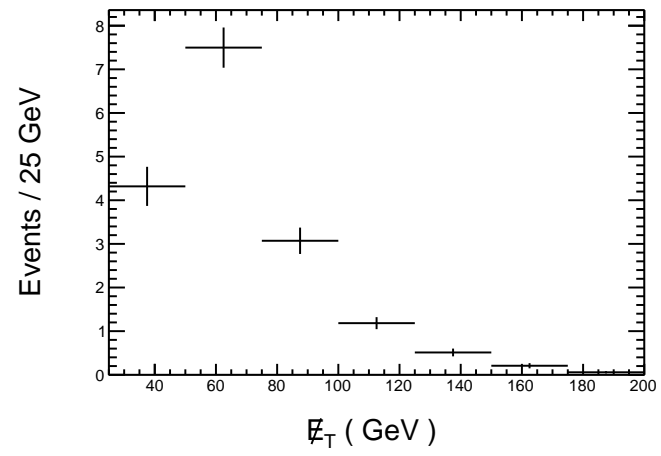

Figure B.1: The $\mathbb{E}_{T}$ distribution of DY $\rightarrow \tau \tau$ in the signal region as estimated from the POWHEG MC.

\section{B.4 Performance of the New DY Estimation Method}

In this section we describe a check of the updated DY estimation method by comparing the expected number of DY $\rightarrow e e / \mu \mu \rightarrow e e / \mu \mu$ events with the dataMC hybrid method with a pure MC estimate but including the scale factors derived in the previous section. In Table B.7 we show the comparison in the signal region (passing both the minimal requirements and the signal requirements in Table 2.1) as well as in several control regions (passing only the minimal requirements and the requirement of 0,1 , and $\geq 2$ jets). The expected numbers in DY $\rightarrow e e / \mu \mu \rightarrow$ $e e / \mu \mu$ using two methods agree with each other within uncertainties. This gives us confidence that we can apply the two scale factors to the DY $\rightarrow e e / \mu \mu \rightarrow e_{\text {fake }} \mu$ and DY $\rightarrow \tau \tau$ categories. The estimation numbers in DY $\rightarrow e e / \mu \mu \rightarrow e_{\text {fake }} \mu$ and $\mathrm{DY} \rightarrow \tau \tau$ categories are also listed in Table B.7. We note that ultimately in this analysis, we use the result from the Data-MC hybrid method when applicable, and the expectation after correction in other DY categories. 


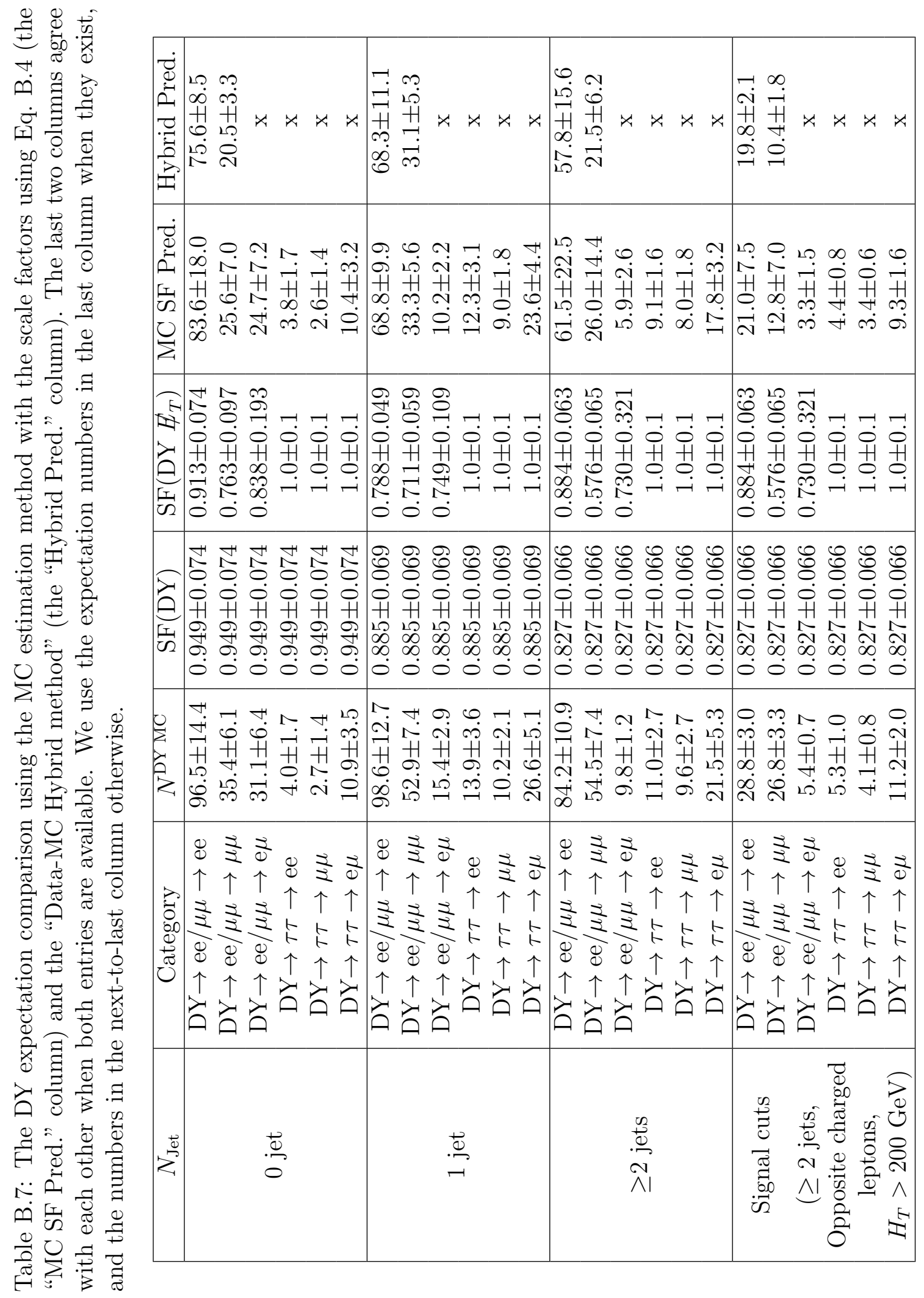




\section{APPENDIX C}

\section{BACKGROUND AND SIGNAL VALIDATION IN THE 0-JET AND 1-JET CONTROL REGIONS}

In Chapter 3 we discussed the modeling of the $t \bar{t}$ signal and background for the signal region, with dileptons + dijets $+\not_{T}$ signature, passing all the requirements in Table 2.1. In this appendix, we describe the validation of the modeling of the $t \bar{t}$ signal and background using events in the 0 -jet and 1-jet control regions to help give us confidence that our data is well modeled in the signal region. These two control regions are defined with the minimal requirements described in Table 2.1, with additional requirement of exactly zero or one jet with $E_{\mathrm{T}}>15 \mathrm{GeV}$ within $|\eta|<2.5$. The contributions from the $t \bar{t}$ are not significant in these two control regions, thus each control region is well suited for validating the estimations of the background contributions. The 0-jet control region is dominated by diboson (especially $W W$ ) production, while the 1 -jet control region is dominated by $W+$ jets and DY + jets productions.

The expected number of events from each background component, as estimated using the techniques described in Chapter 3 , as well as from $t \bar{t}$ dilepton production compared with the observed numbers in 0 -jet and 1-jet control regions are summa-

rized in Tables C.1 and C.2. The observed numbers are in good agreement with the expectations.

As shown in Table 3.3, we have multiple background sources, several of them have non-negligible contribution to the signal region. It is critical to get a robust modeling of the kinematics, especially the lepton $q_{\ell} \eta_{\ell}$ spectrum, for the background components. As a check, Fig. C.1 shows the comparison of the $q_{\ell} \eta_{\ell}$ and $\Delta \eta$ distri- 


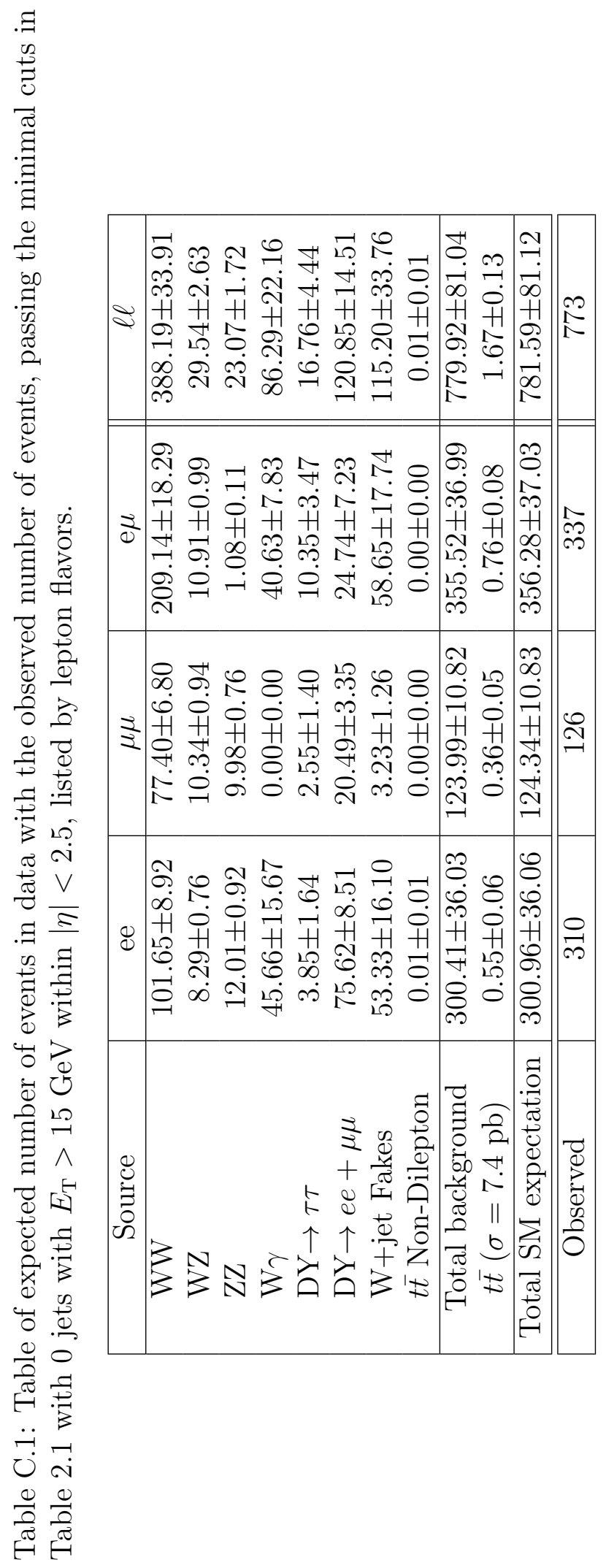




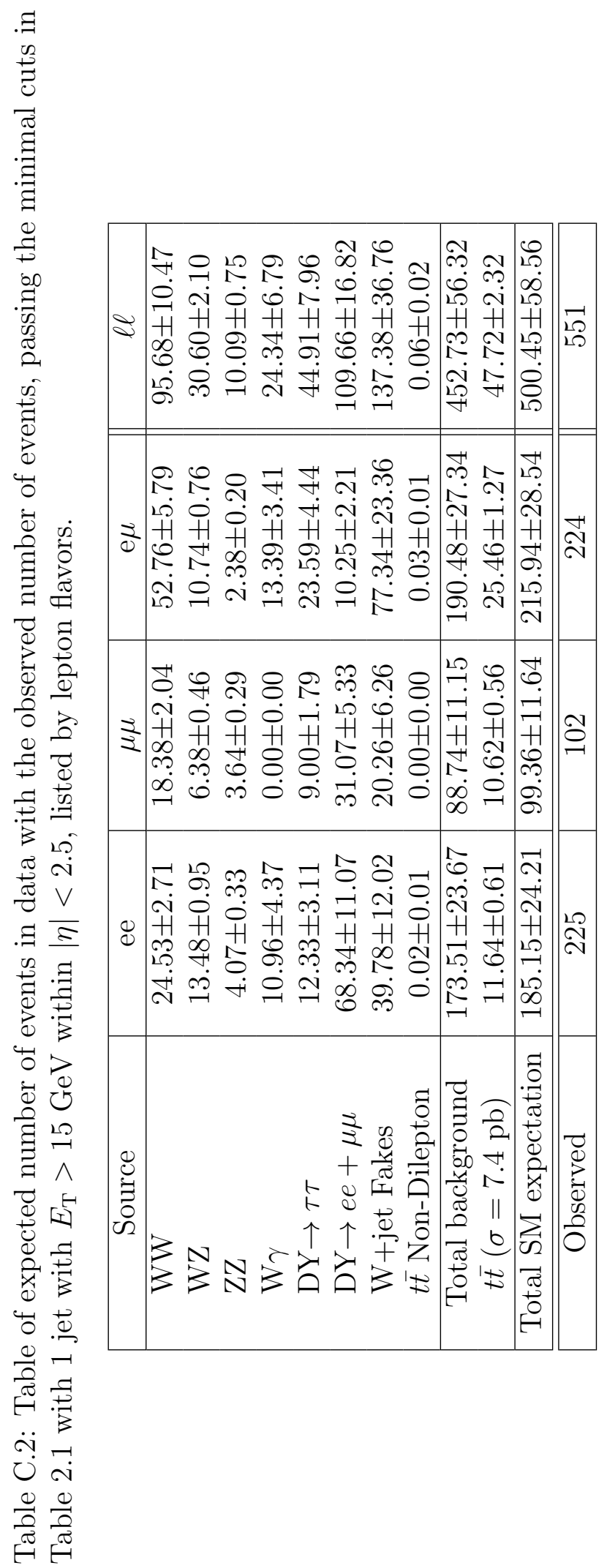


bution in the 0 and 1 jet control regions overlaid with data, and Table C.3 lists the "uncorrected" $A_{\mathrm{FB}}^{\ell}$ and $A_{\mathrm{FB}}^{\ell \ell}$, calculated by simply counting the events in the forward and backward regions, from data and from SM expectation in the two control regions. The values from data and SM estimation are consistent with each other. All the checks described in this appendix yield good agreement between the data and the expectations, indicating that our modelling of background asymmetry is robust.
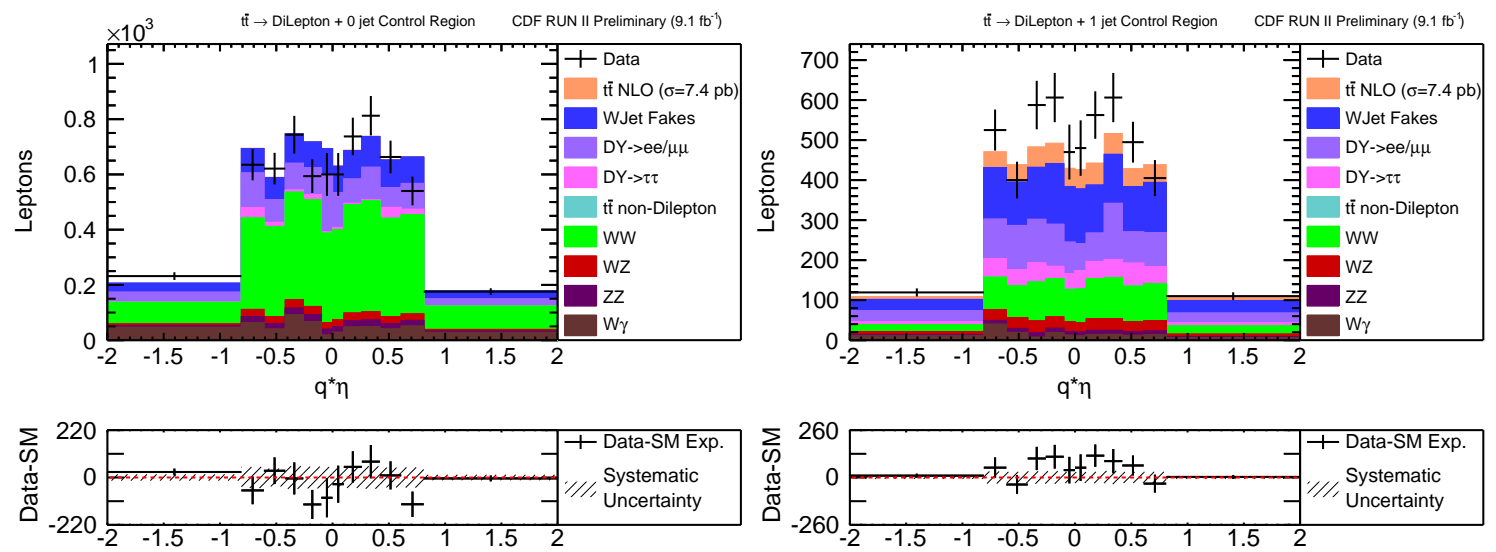

(a) 0 jet

(b) 1 jet
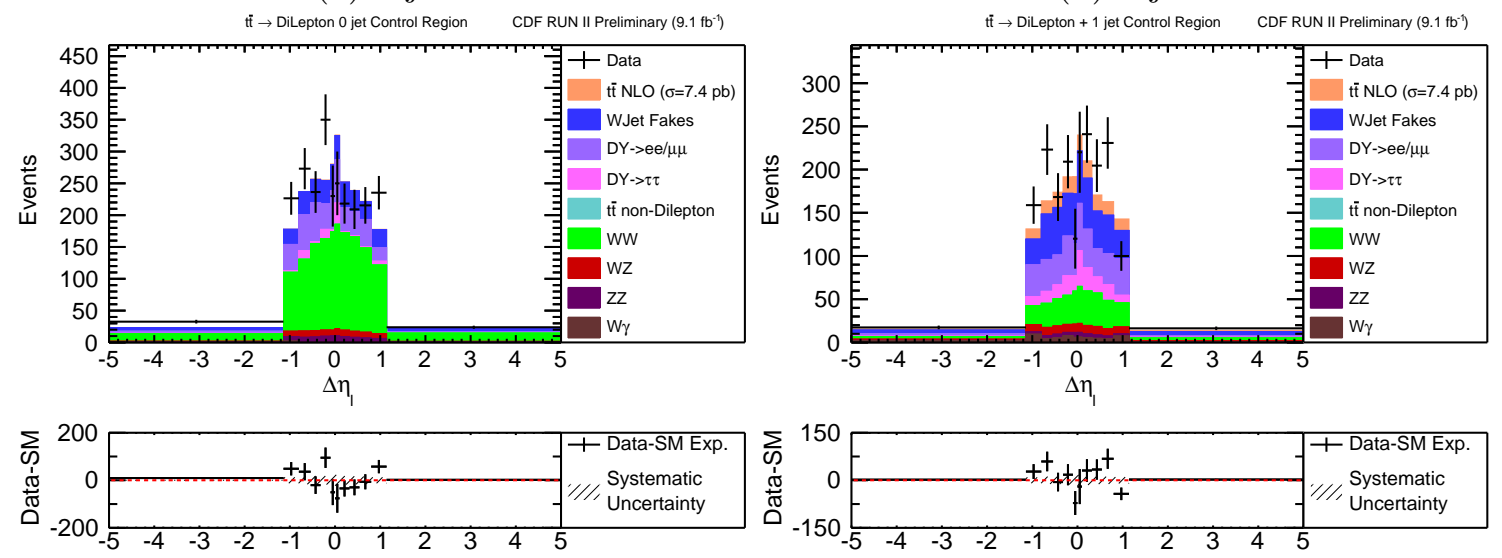

(c) 0 jet

(d) 1 jet

Figure C.1: The distributions of $q_{\ell} \eta_{\ell}$ and $\Delta \eta$ in the 0 and 1 jet control regions. 
Table C.3: The uncorrected $A_{\mathrm{FB}}^{\ell}$ and $A_{\mathrm{FB}}^{\ell \ell}$ calculated by counting the number of events in the forward and backward regions from data and SM expectation in the 0 and 1 jet control regions. The uncertainties are statistical only.

\begin{tabular}{cccc}
\hline & Sample & Uncorrected $A_{\mathrm{FB}}^{\ell}$ & Uncorrected $A_{\mathrm{FB}}^{\ell \ell}$ \\
\hline \hline \multirow{2}{*}{0 jet } & Data & $-0.027 \pm 0.025$ & $-0.102 \pm 0.036$ \\
& SM Expectation & $-0.024 \pm 0.012$ & $-0.044 \pm 0.017$ \\
\hline \multirow{2}{*}{1 jet } & Data & $-0.018 \pm 0.030$ & $0.005 \pm 0.043$ \\
& SM Expectation & $-0.014 \pm 0.013$ & $0.012 \pm 0.018$ \\
\hline
\end{tabular}




\section{APPENDIX D}

\section{BACKGROUND AND SIGNAL VALIDATION WITH B-TAGGING REQUIREMENT}

In this appendix, we show a validation of the modeling of the $t \bar{t}$ signal and backgrounds by using events in the signal region, passing all the requirements in Table 2.1, but with an additional requirement of at least one jet being $b$-tagged. The requirement of a SECVTX $b$-tagged jet rejects most of the backgrounds, but with the cost of significant loss of $t \bar{t}$ events. We lose events both due to the inefficiency of the $b$-jet selection requirements (the efficiency of the $b$-jet tagging is approximately $50 \%)$ as well as the requirement of silicon detector to be working. The good run list used with $b$-tagging corresponds to an integrated luminosity of $8.7 \mathrm{fb}^{-1}$, as opposed to $9.1 \mathrm{fb}^{-1}$ without the requirement. Since this measurement is statistics limited, the sample after $b$-jet tagging isn't a better sample, but can provide an important cross check.

Table D.1 shows the expected and observed number of events in each lepton flavor after all event selection with at least one SECVTX $b$-tag. The purity goes from $72 \%$ before $b$-tagging to $88 \%$ after, while the number of events surviving the requirements drops from 569 to 246 (we predict that the number of $t \bar{t}$ events is reduced from 408 to 225). Figures D.1, D.2, D.3, D.4, D.5, and D.6 show the distributions of expected and observed kinematic variables after the $b$-tagging requirement and show that each is well modeled, giving us further confidence in our background and signal estimation methods. 


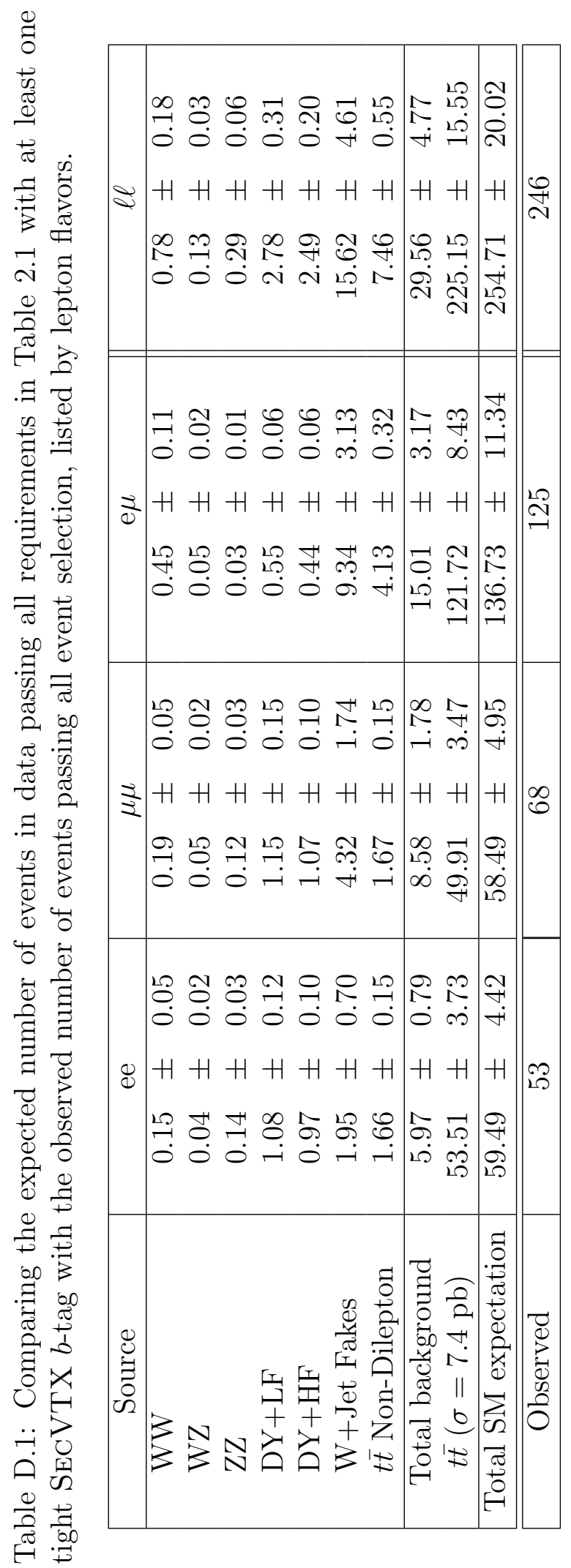



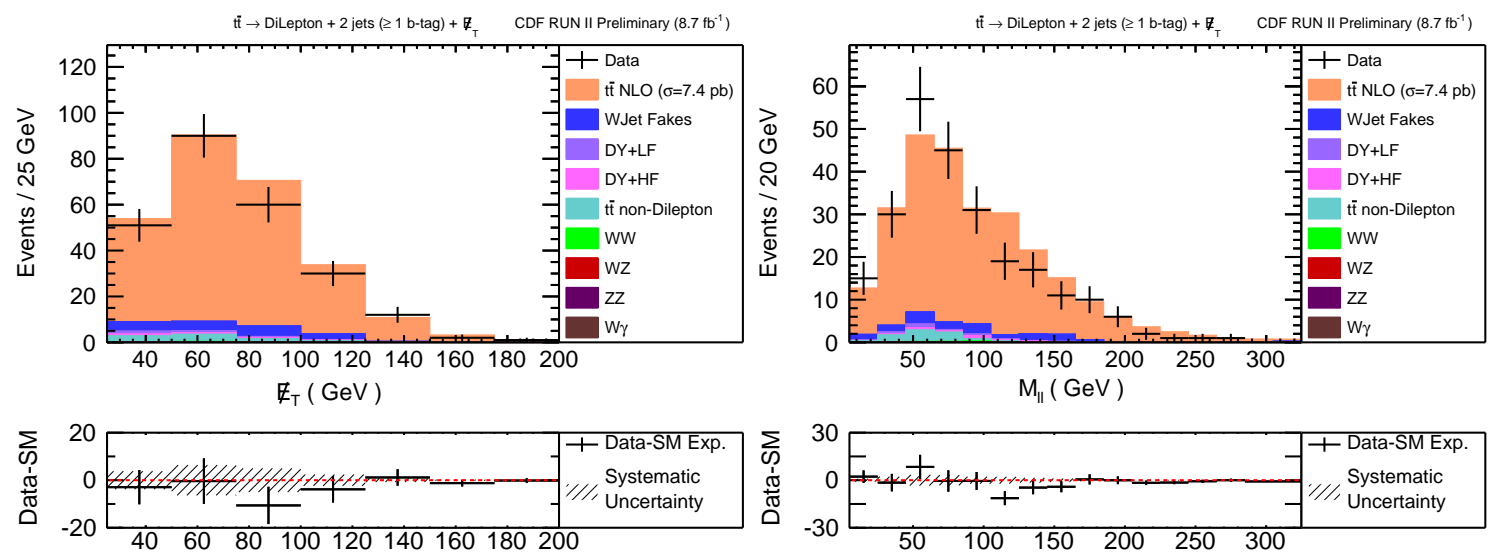

(a) $E_{T}$

(b) $\mathrm{m}_{11}$
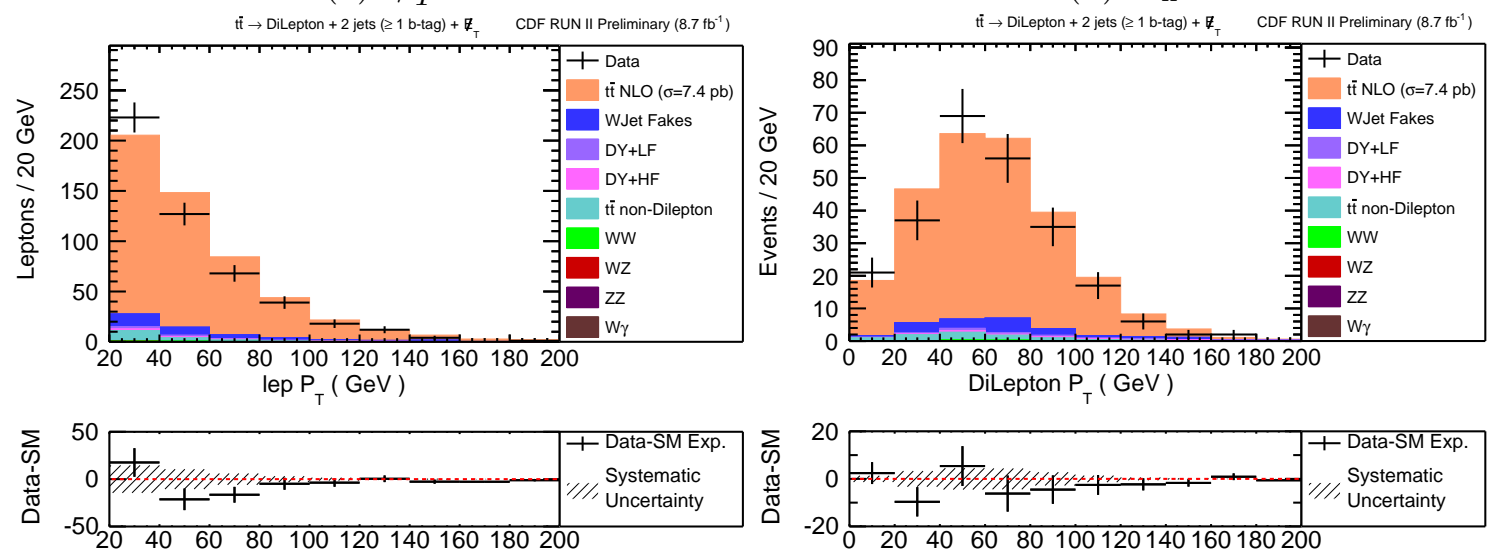

(c) $p_{T}$ for both leptons

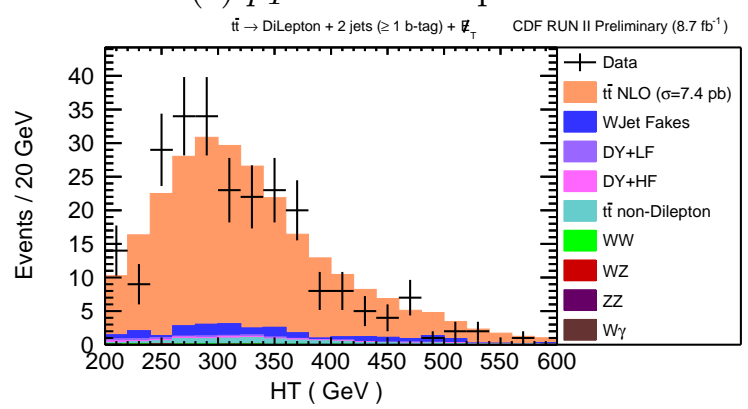

(d) DiLepton $p_{T}$
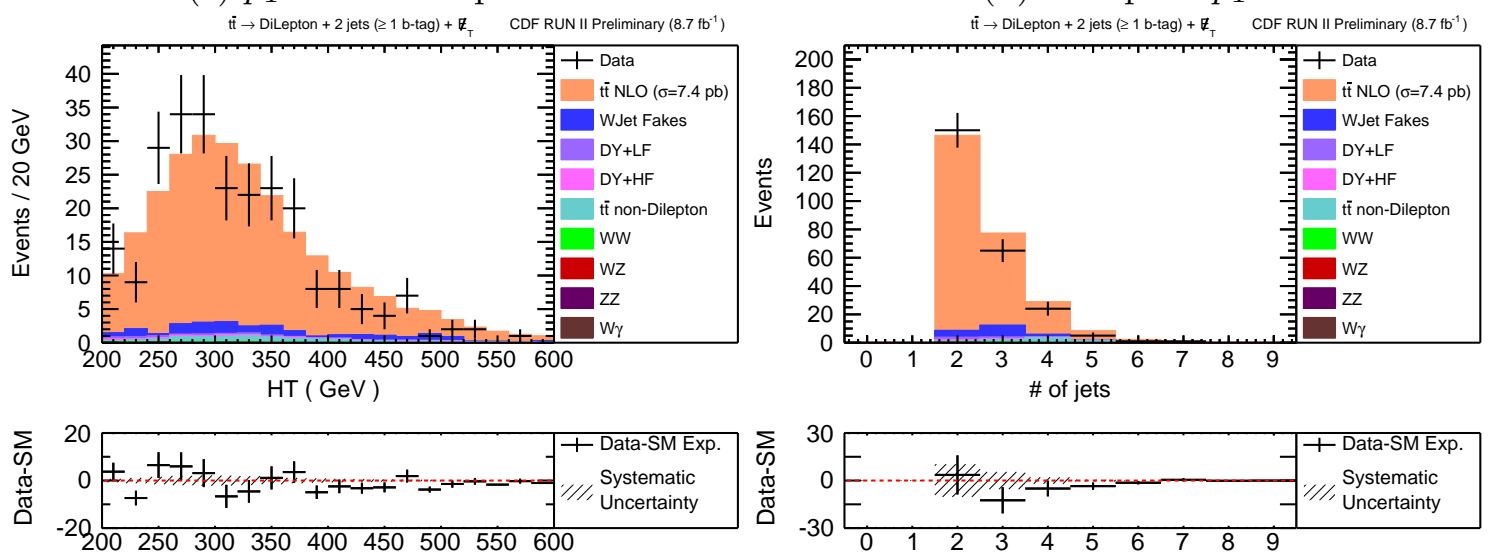

(e) $\mathrm{H}_{\mathrm{T}}$

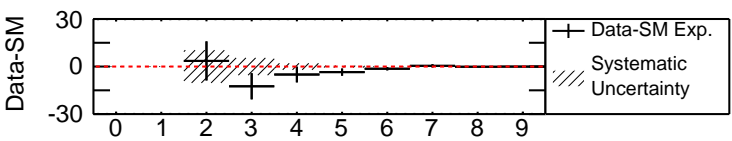

(f) Jet Multiplicity

Figure D.1: The same plots as Fig. 3.1, but with at least one SECVTX $b$-tagged jet. 

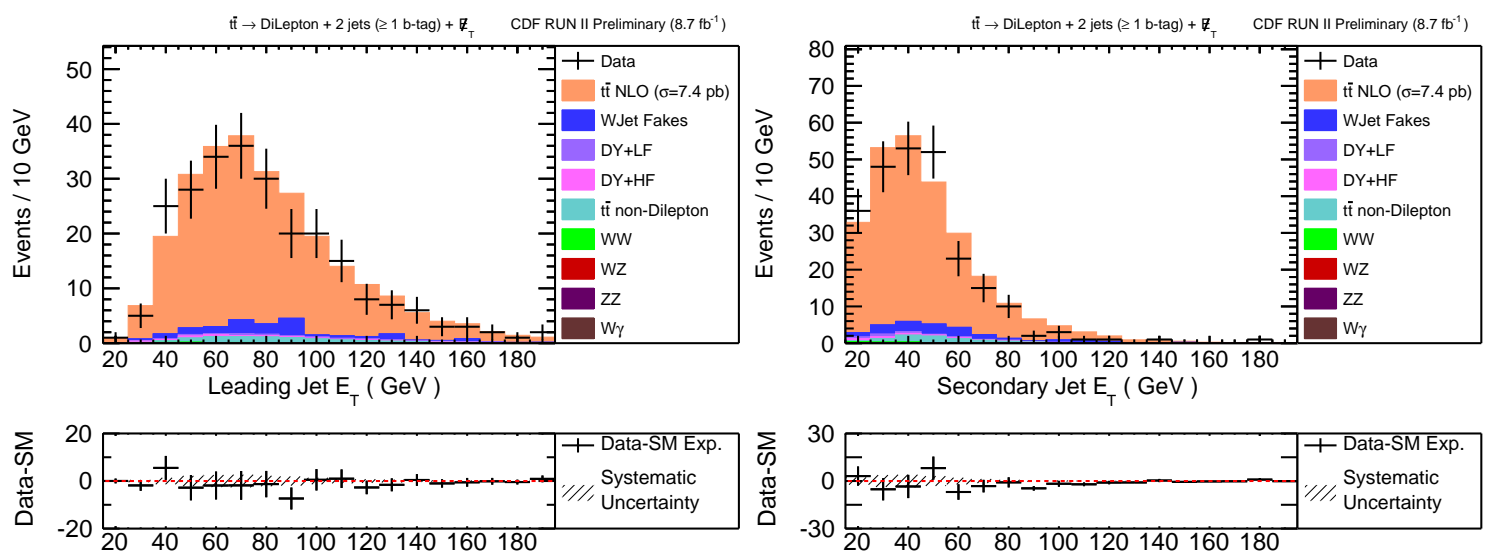

(a) Leading jet $E_{\mathrm{T}}$

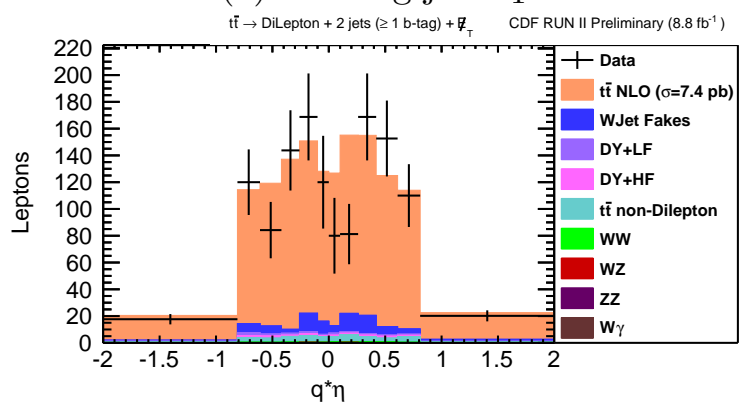

(b) Secondary jet $E_{\mathrm{T}}$
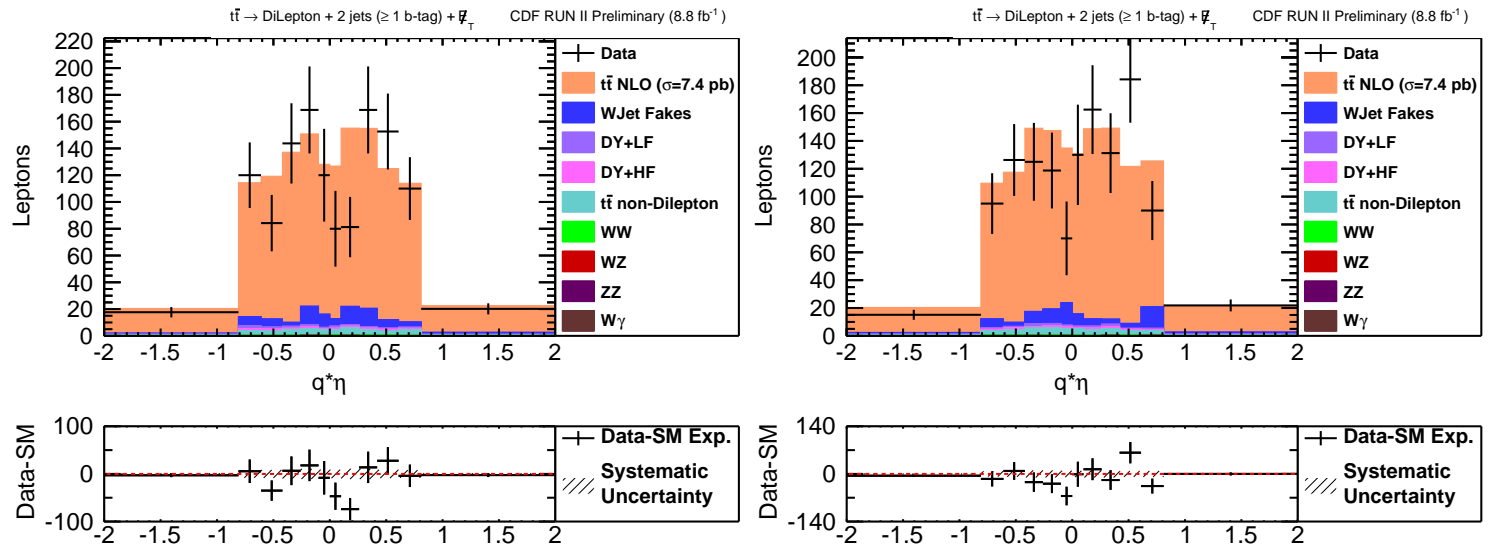

(c) Positive Lepton $q_{\ell} \eta_{\ell}$

$\mathrm{tf} \rightarrow$ DiLepton +2 jets $(\geq 1 \mathrm{~b}$-tag $)+\#_{T} \quad$ CDF RUN $I I$ Preliminary $\left(8.7 \mathrm{fb}^{-1}\right)$

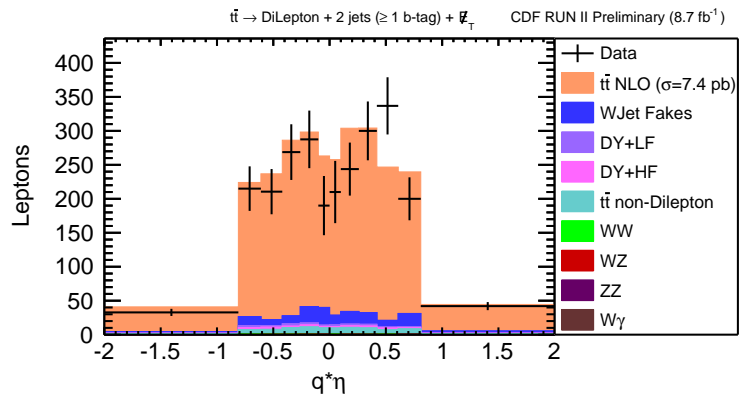

(d) Negative Lepton $q_{\ell} \eta_{\ell}$
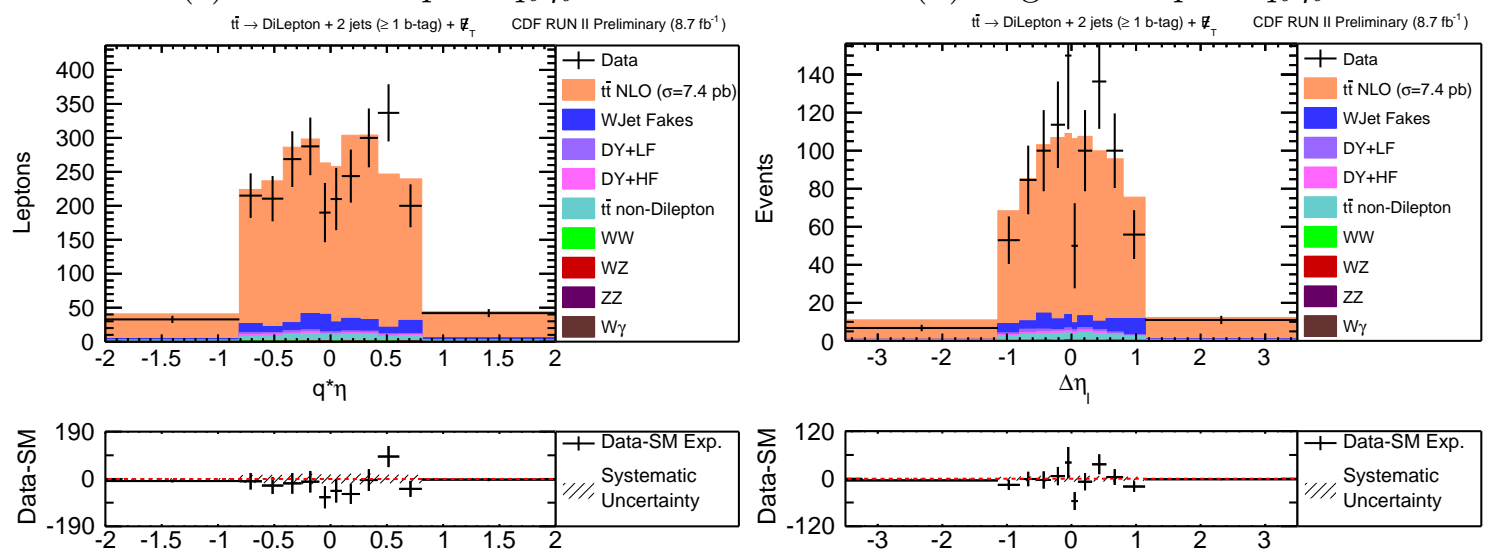

(e) Both Lepton $q_{\ell} \eta_{\ell}$

(f) $\Delta \eta$

Figure D.2: The same plots as Fig. 3.2, but with at least one SECVTX b-tagged jet. 

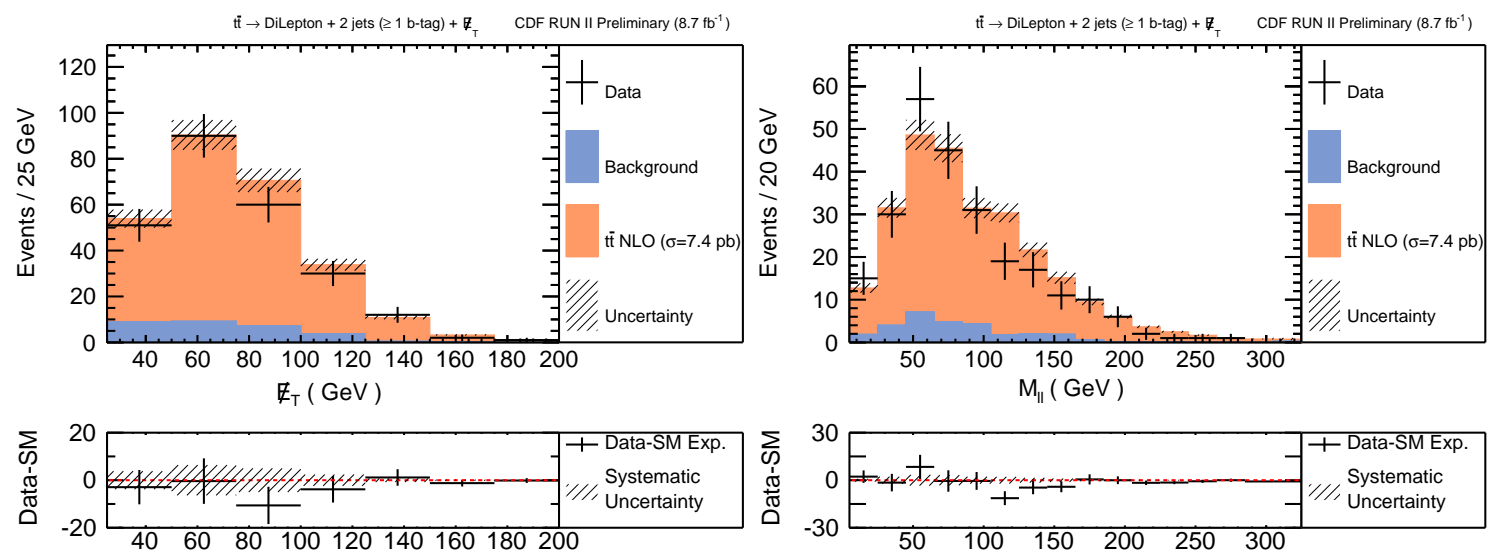

(a) $\ddot{H}_{T}$

(b) $\mathrm{m}_{11}$
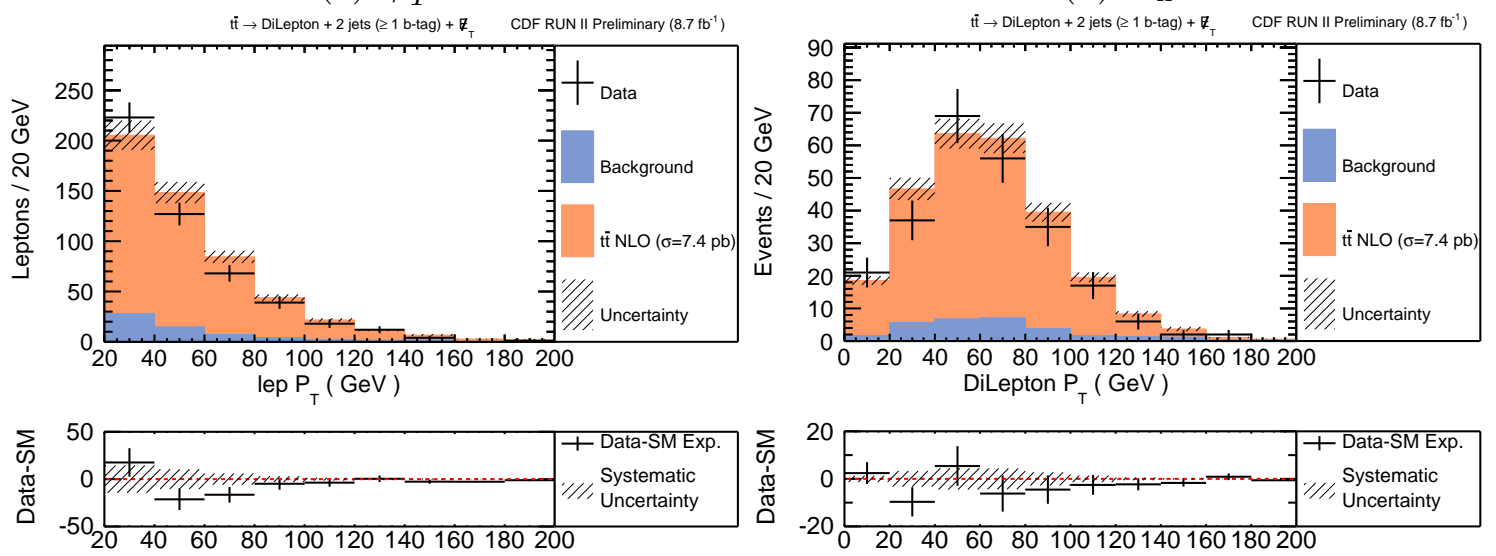

(c) $p_{T}$ for both leptons

(d) DiLepton $p_{T}$

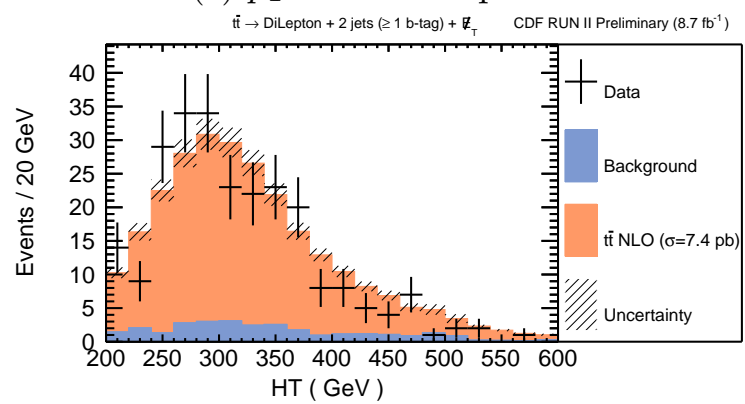

$\mathrm{f} \rightarrow$ DiLepton +2 jets $(\geq 1 \mathrm{~b}$-tag $)+\sharp_{T} \quad$ CDF RUN II Preliminary $\left(8.7 \mathrm{fb}^{-1}\right)$
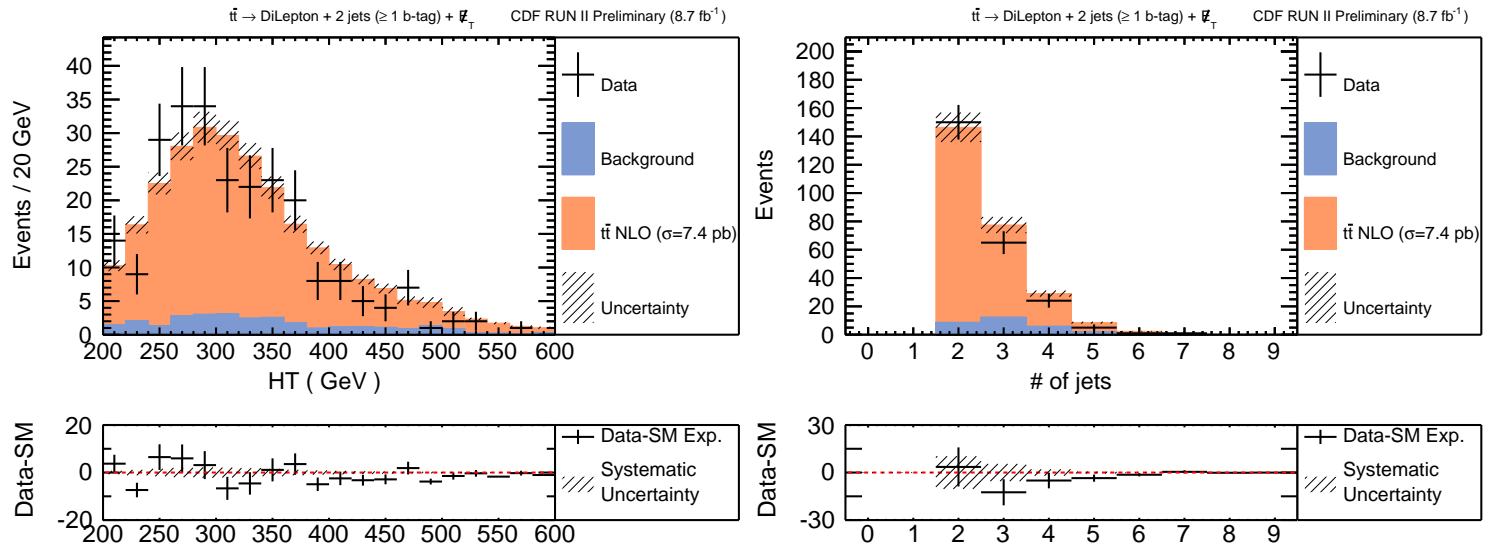

(e) $\mathrm{H}_{\mathrm{T}}$

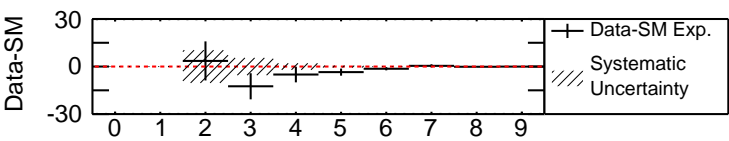

(f) Jet Multiplicity

Figure D.3: The same plots as Fig. 3.3, but with at least one SECVTX $b$-tagged jet. 

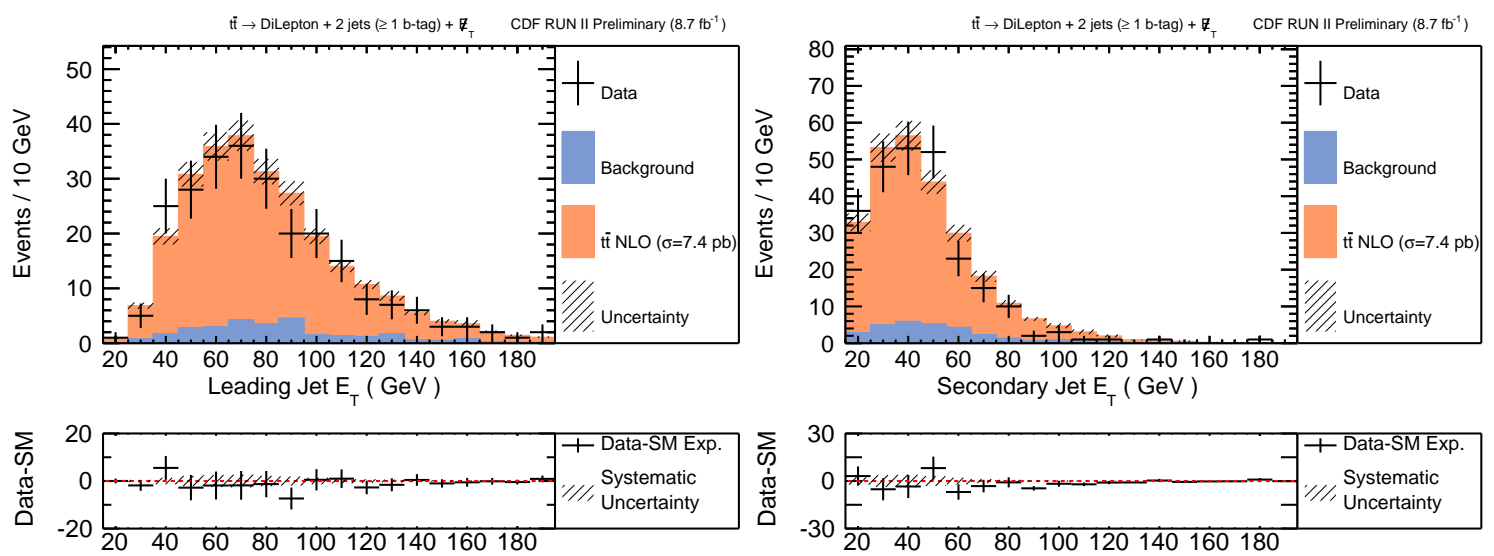

(a) Leading jet $E_{\mathrm{T}}$

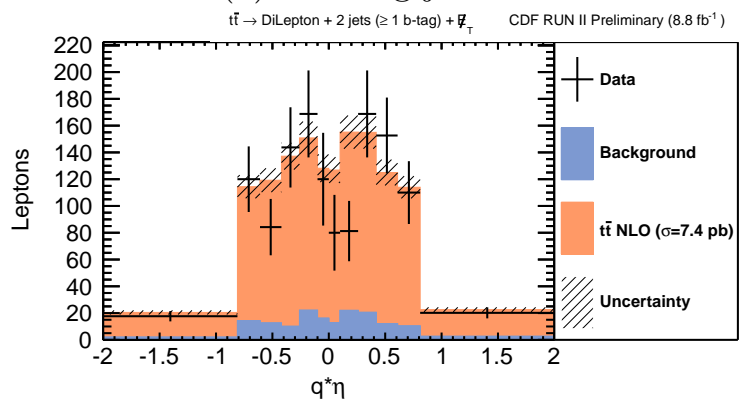

(b) Secondary jet $E_{\mathrm{T}}$
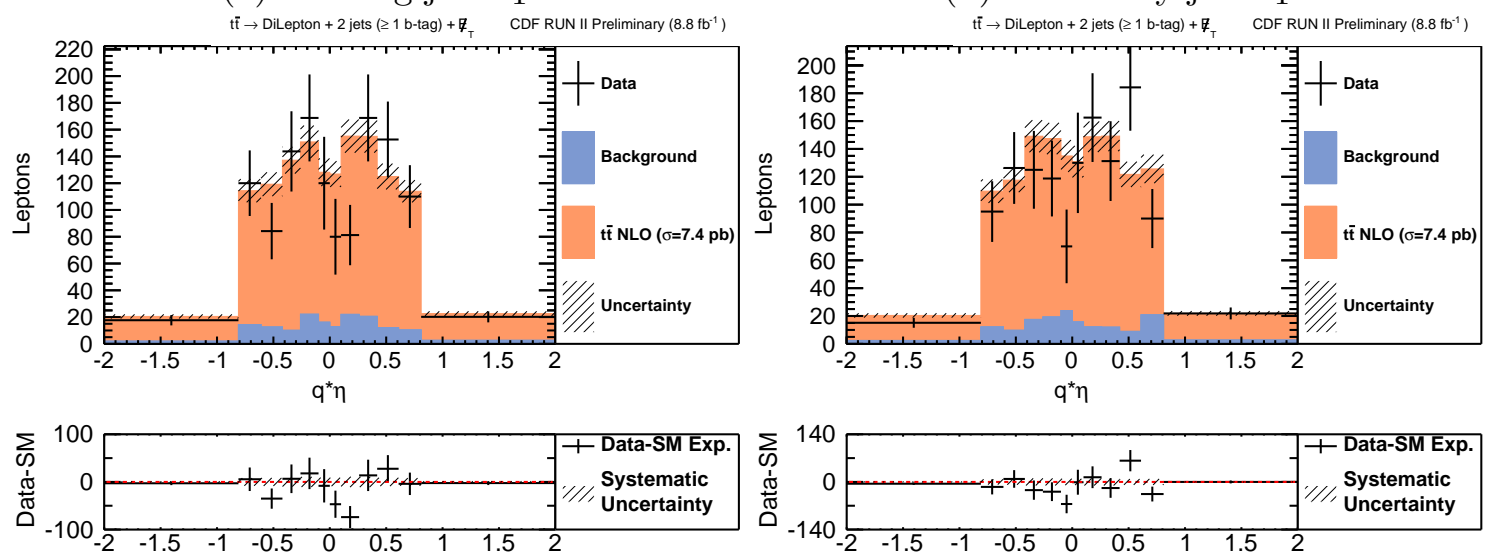

(c) Positive Lepton $q_{\ell} \eta_{\ell}$

$\mathrm{ft} \rightarrow$ DiLepton +2 jets $(\geq 1 \mathrm{~b}$-tag $)+\nabla_{T} \quad$ CDF RUN II Preliminary $\left(8.7 \mathrm{fb}^{-1}\right)$

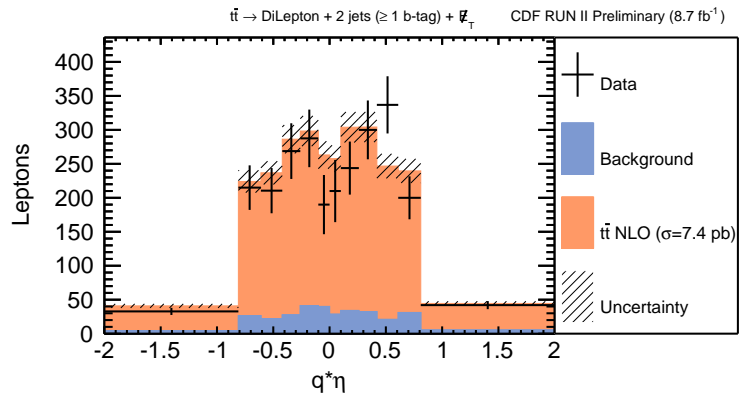

(d) Negative Lepton $q_{\ell} \eta_{\ell}$
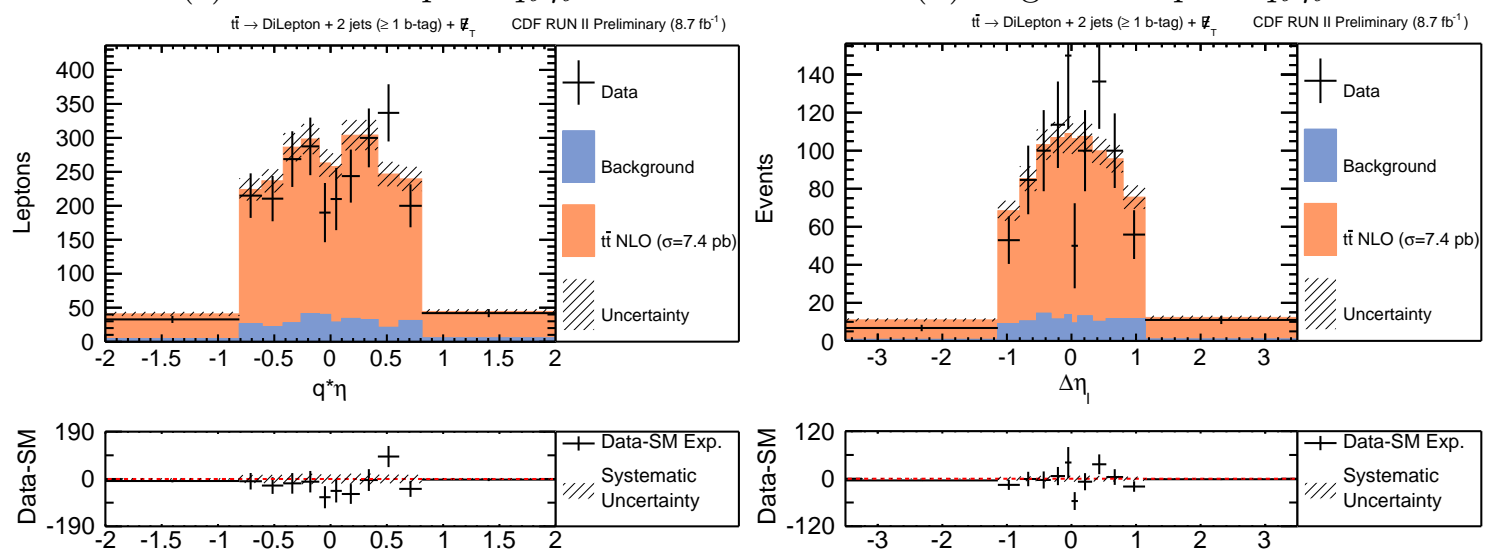

(e) Both Lepton $q_{\ell} \eta_{\ell}$

(f) $\Delta \eta$

Figure D.4: The same plots as Fig. 3.4 but with at least one SECVTX $b$-tagged jet. 

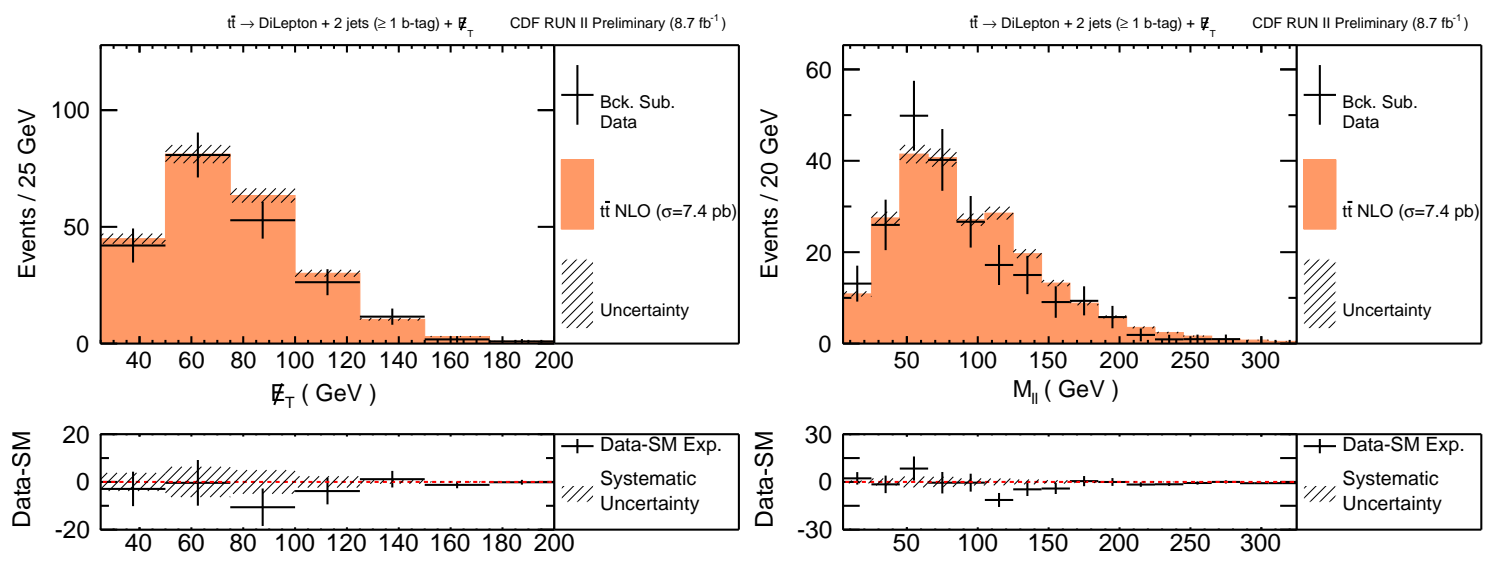

(a) $\ddot{H}_{T}$

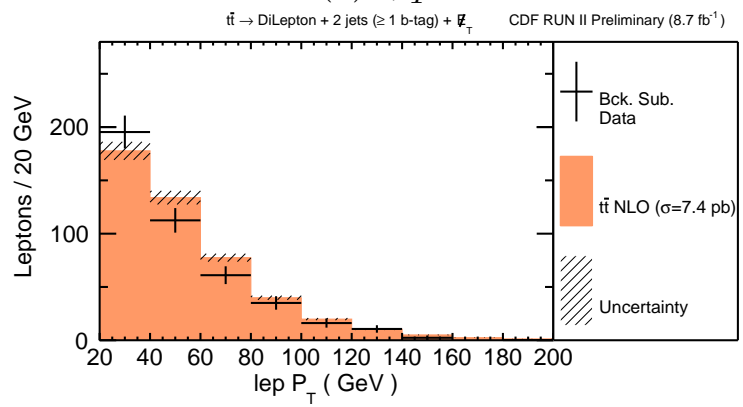

(b) $\mathrm{m}_{11}$
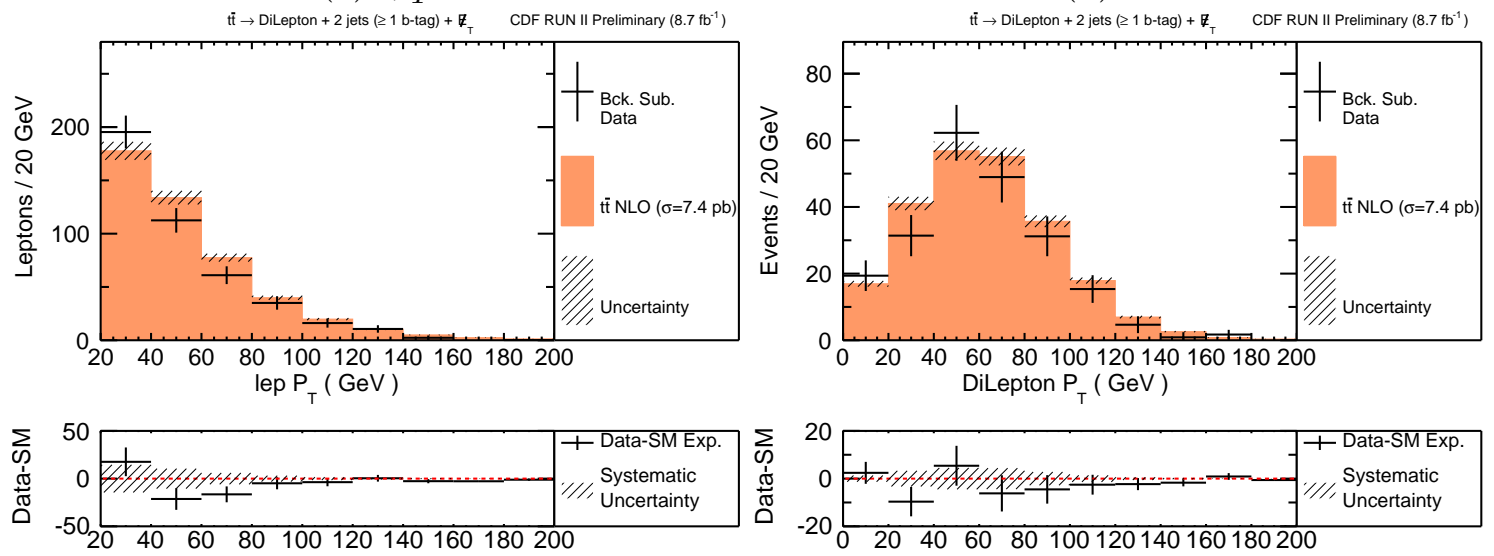

(c) $p_{T}$ for both leptons

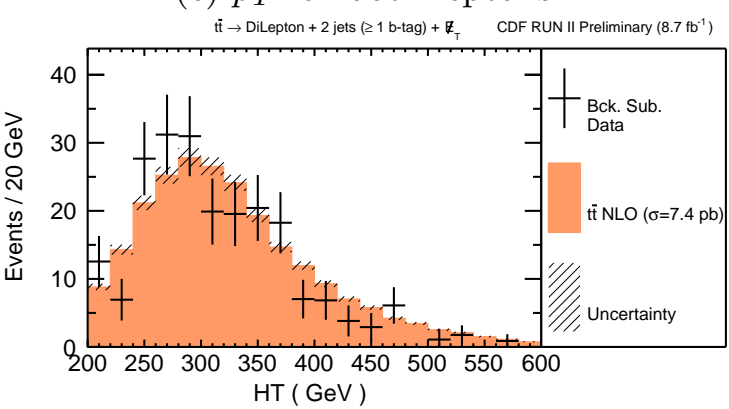

(d) DiLepton $p_{T}$
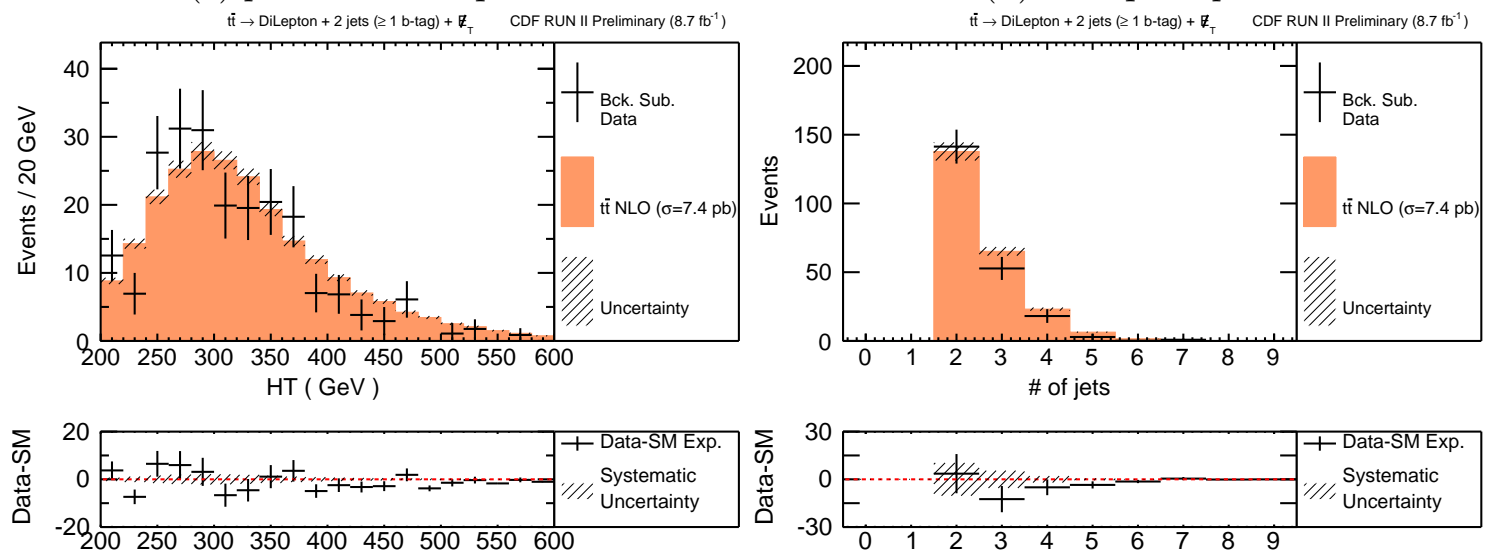

(e) $\mathrm{H}_{\mathrm{T}}$

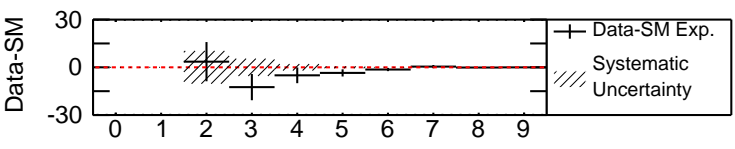

(f) Jet Multiplicity

Figure D.5: The same plots as Fig. 3.5, but with at least one SECVTX $b$-tagged jet. 

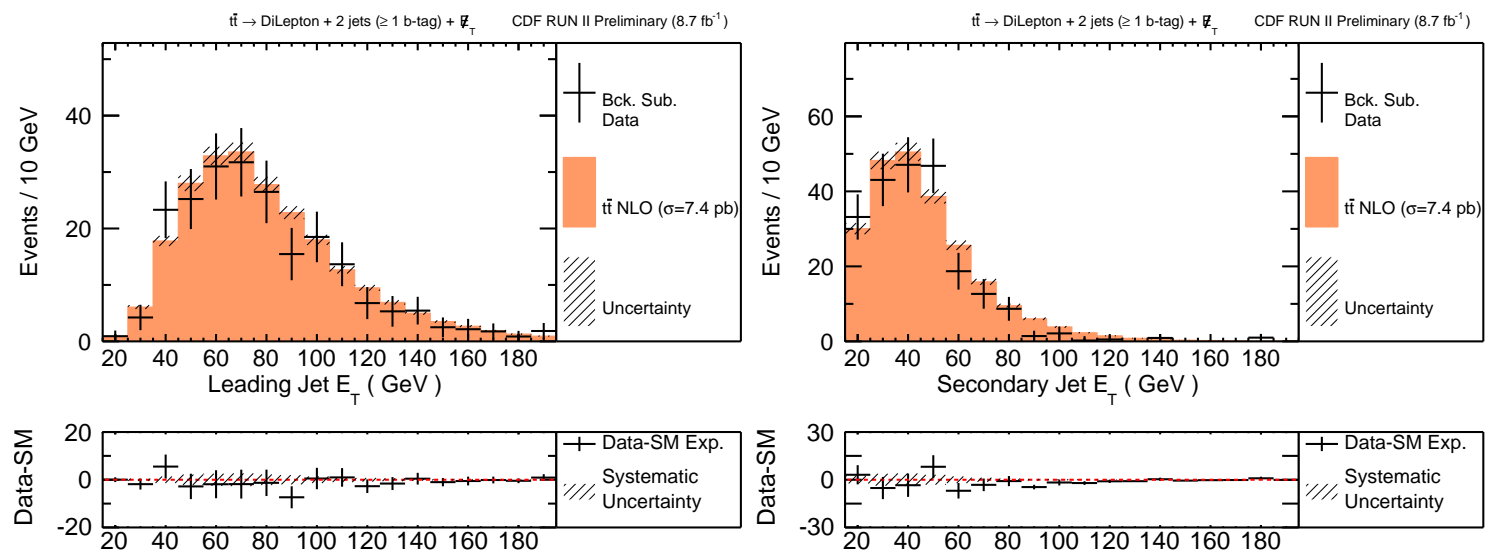

(a) Leading jet $E_{\mathrm{T}}$

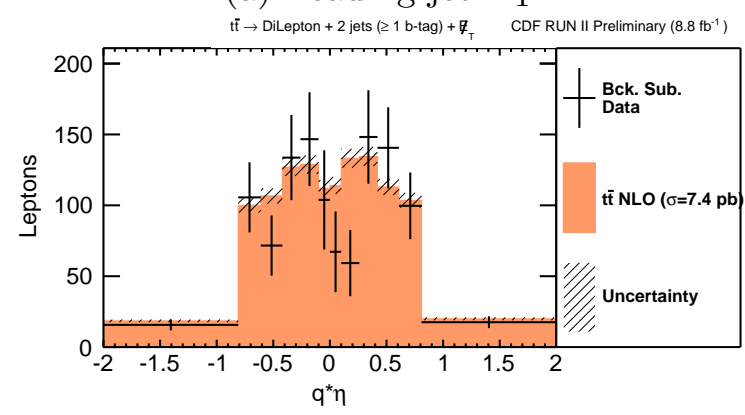

(b) Secondary jet $E_{\mathrm{T}}$
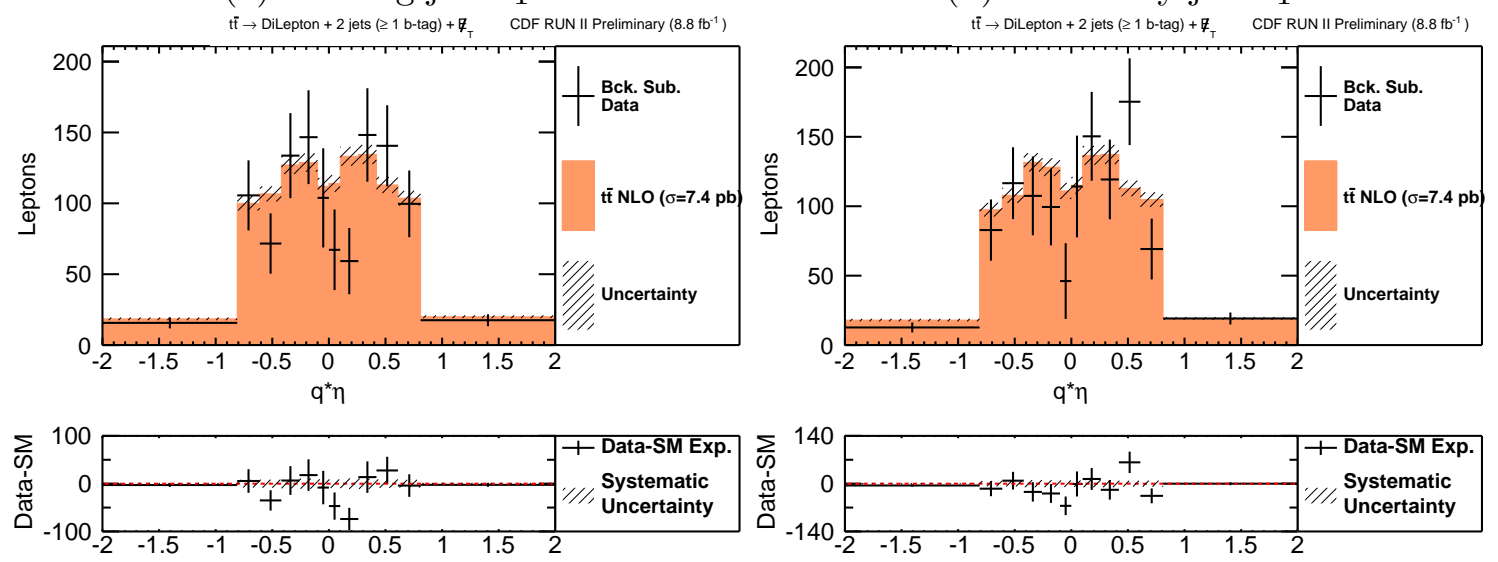

(c) Positive Lepton $q_{\ell} \eta_{\ell}$

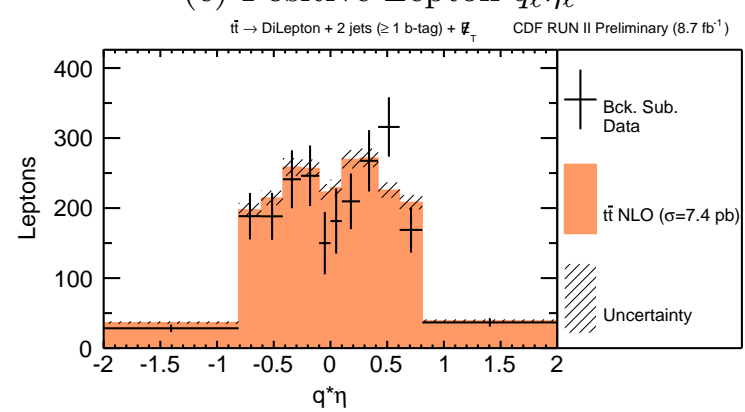

(d) Negative Lepton $q_{\ell} \eta_{\ell}$
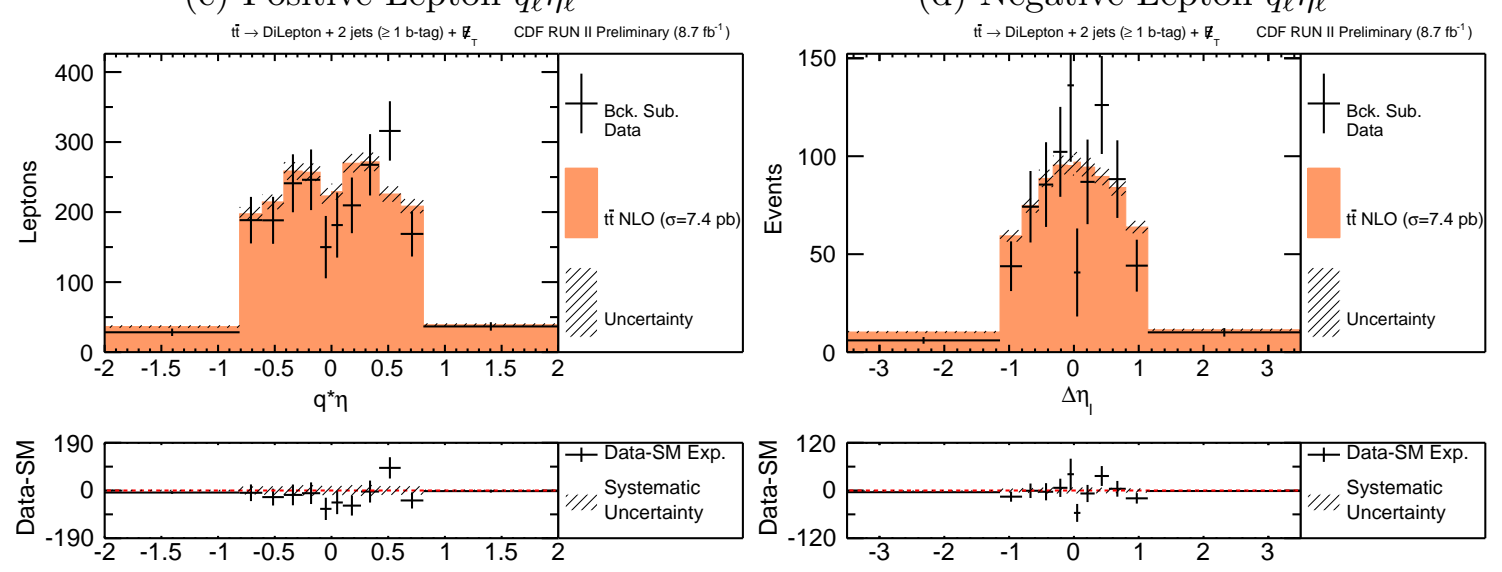

(e) Both Lepton $q_{\ell} \eta_{\ell}$

(f) $\Delta \eta$

Figure D.6: The same plots as Fig. 3.6. but with at least one SECVTX $b$-tagged jet. 


\section{APPENDIX E}

\section{A DESCRIPTION OF WHY THE METHODOLOGY OF $A_{\mathrm{FB}}^{\ell}$ MEASUREMENT WORKS}

In Chapter 4, we presented the measurement methodology of $A_{\mathrm{FB}}^{\ell}$. In particular, we showed the asymmetric decomposition of the $q_{\ell} \eta_{\ell}$ spectrum and found that the shape was closely correlated with the $A_{\mathrm{FB}}^{\ell}$ of the sample. Previous results had found that the $a \cdot \tanh$ formulation worked well, especially if $\left|q_{\ell} \eta_{\ell}\right|<2.0$, but did not present any justification for this choice other than showing it worked beautifully, as shown in Fig. 4.3. In this Appendix, we provide a partial explanation of where the hyperbolic tangent functional form comes from as well as a better parametrization and what the new understanding leads us to. This text summarizes the work published in Ref. [92].

\section{E.1 Single and Double Gaussian Modeling}

The $q_{\ell} \eta_{\ell}$ distributions in Fig. 4.1 appear to be roughly Gaussian distributed with a non-zero mean. Figure E.1 shows the $q_{\ell} \eta_{\ell}$ distribution at parton level from the POWHEG $t \bar{t}$ sample with a fit to a Gaussian function, but with the fit restricted to $\left|q_{\ell} \eta_{\ell}\right|<1.4$. Note that the fit is decent, but clearly not good for $\left|q_{\ell} \eta_{\ell}\right|>1.4$. This simple model is clearly insufficient.

Before moving on to a better model, we use this simple Gaussian model to illustrate the methodology of where the functional form of tanh comes from. We note that the number of events in a single bin of data (for example each of the bins in Fig.E.1, 
corresponding to the interval of $\left.\left(q_{\ell} \eta_{\ell}, q_{\ell} \eta_{\ell}+\delta\left(q_{\ell} \eta_{\ell}\right)\right)\right)$ can be readily calculated using

$$
\begin{aligned}
\mathcal{N}\left(q_{\ell} \eta_{\ell}, q_{\ell} \eta_{\ell}+\delta\left(q_{\ell} \eta_{\ell}\right)\right) & =\int_{q_{\ell} \eta_{\ell}}^{q_{\ell} \eta_{\ell}+\delta\left(q_{\ell} \eta_{\ell}\right)} \mathrm{d} x C \cdot \operatorname{Exp}\left(-\frac{(x-\mu)^{2}}{2 \sigma^{2}}\right) \\
& =C \cdot \operatorname{Exp}\left(-\frac{\left(q_{\ell} \eta_{\ell}-\mu\right)^{2}}{2 \sigma^{2}}\right) \delta\left(q_{\ell} \eta_{\ell}\right), \text { when } \delta\left(q_{\ell} \eta_{\ell}\right) \rightarrow 0
\end{aligned}
$$

where $C$ is a normalization constant, $\mu$ is the mean of the distribution and $\sigma$ is the width of the distribution. We can then calculate $\mathcal{A}\left(q_{\ell} \eta_{\ell}\right)$ with this function:

$$
\begin{aligned}
\mathcal{A}\left(q_{\ell} \eta_{\ell}\right) & =\lim _{\delta\left(q_{\ell} \eta_{\ell}\right) \rightarrow 0} \frac{\mathcal{N}\left(q_{\ell} \eta_{\ell}, q_{\ell} \eta_{\ell}+\delta\left(q_{\ell} \eta_{\ell}\right)\right)-\mathcal{N}\left(-q_{\ell} \eta_{\ell}-\delta\left(q_{\ell} \eta_{\ell}\right),-q_{\ell} \eta_{\ell}\right)}{\mathcal{N}\left(q_{\ell} \eta_{\ell}, q_{\ell} \eta_{\ell}+\delta\left(q_{\ell} \eta_{\ell}\right)\right)+\mathcal{N}\left(-q_{\ell} \eta_{\ell}-\delta\left(q_{\ell} \eta_{\ell}\right),-q_{\ell} \eta_{\ell}\right)} \\
& =\frac{\operatorname{Exp}\left(-\frac{\left(q_{\ell} \eta_{\ell}-\mu\right)^{2}}{2 \sigma^{2}}\right)-\operatorname{Exp}\left(-\frac{\left(-q_{\ell} \eta_{\ell}-\mu\right)^{2}}{2 \sigma^{2}}\right)}{\operatorname{Exp}\left(-\frac{\left(q_{\ell} \eta_{\ell}-\mu\right)^{2}}{2 \sigma^{2}}\right)+\operatorname{Exp}\left(-\frac{\left(-q_{\ell} \eta_{\ell}-\mu\right)^{2}}{2 \sigma^{2}}\right)} \\
& =\tanh \left(\frac{\mu \cdot q_{\ell} \eta_{\ell}}{\sigma^{2}}\right)
\end{aligned}
$$

We note that it has the form of a hyperbolic tangent function, but with the parameter inside the function argument, not an overall scaling factor as in Eq. 4.3.

Since the single Gaussian function works only in the small $\left|q_{\ell} \eta_{\ell}\right|$ region, we tried a more sophisticated model, and found that the sum of two Gaussian functions with a common mean works very well at describing the data, even at $\left|q_{\ell} \eta_{\ell}\right|>2.0$. We have not uncovered an a priori explanation why this should be so, but it appears to be true for all the models we considered [109]. We use the functional form:

$$
\frac{\mathrm{d} \mathcal{N}\left(q_{\ell} \eta_{\ell}\right)}{\mathrm{d}\left(q_{\ell} \eta_{\ell}\right)}=C \cdot\left(\operatorname{Exp}\left(-\frac{\left(q_{\ell} \eta_{\ell}-\mu\right)^{2}}{2 \sigma_{1}^{2}}\right)+r \cdot \operatorname{Exp}\left(-\frac{\left(q_{\ell} \eta_{\ell}-\mu\right)^{2}}{2 \sigma_{2}^{2}}\right)\right)
$$

where $C$ is a normalization constant, $r$ is a multiplicative factor that covers the relative normalization of the two components and $\sigma_{1}$ and $\sigma_{2}$ are the widths of the two different distributions. Fig. E.2 shows a comparison between the best fit and the parton level data. This functional form works well for all our benchmark signal 
samples; the two $\sigma$ terms and the $r$ term are very consistent as shown in Fig. E.3. We find $\sigma_{1}=0.91, \sigma_{2}=1.61$ and $r=0.11$. More importantly, the mean $(\mu)$ varies significantly from one sample to another, and appears to be linear with $A_{\mathrm{FB}}^{\ell}$. From here on, we assume the two $\sigma$ terms and the $r$ term have the best fit values from the benchmark samples for further studies.

The double-Gaussian modeling allows for closed form calculations of the $\mathcal{S}\left(q_{\ell} \eta_{\ell}\right)$ and the $\mathcal{A}\left(q_{\ell} \eta_{\ell}\right)$ terms as well as the inclusive $A_{\mathrm{FB}}^{\ell}$ using just the $\mu, \sigma_{1}, \sigma_{2}$ and $r$ parameters. We find the $\mathcal{S}\left(q_{\ell} \eta_{\ell}\right)$ term and the $\mathcal{A}\left(q_{\ell} \eta_{\ell}\right)$ term have the functional forms of

$$
\begin{aligned}
& \mathcal{S}\left(q_{\ell} \eta_{\ell}\right)=\frac{C}{2} \cdot\left(e^{-\frac{\left(q_{\ell} \eta_{\ell}-\mu\right)^{2}}{2 \sigma_{1}^{2}}}+e^{-\frac{\left(q_{\ell} \eta_{\ell}+\mu\right)^{2}}{2 \sigma_{1}^{2}}}+r \cdot e^{-\frac{\left(q_{\ell} \eta_{\ell}-\mu\right)^{2}}{2 \sigma_{2}^{2}}}+r \cdot e^{-\frac{\left(q_{\ell} \eta_{\ell}+\mu\right)^{2}}{2 \sigma_{2}^{2}}}\right), \text { and } \\
& \mathcal{A}\left(q_{\ell} \eta_{\ell}\right)=\frac{e^{-\frac{\left(q_{\ell} \eta_{\ell}-\mu\right)^{2}}{2 \sigma_{1}^{2}}}-e^{-\frac{\left(q_{\ell} \eta_{\ell}+\mu\right)^{2}}{2 \sigma_{1}^{2}}}+r \cdot e^{-\frac{\left(q_{\ell} \eta_{\ell}-\mu\right)^{2}}{2 \sigma_{2}^{2}}}-r \cdot e^{-\frac{\left(q_{\ell} \eta_{\ell}+\mu\right)^{2}}{2 \sigma_{2}^{2}}}}{e^{-\frac{\left(q_{\ell} \eta_{\ell}-\mu\right)^{2}}{2 \sigma_{1}^{2}}}+e^{-\frac{\left(q_{\ell} \eta_{\ell}+\mu\right)^{2}}{2 \sigma_{1}^{2}}}+r \cdot e^{-\frac{\left(q_{\ell} \eta_{\ell}-\mu\right)^{2}}{2 \sigma_{2}^{2}}}+r \cdot e^{-\frac{\left(q_{\ell} \eta_{\ell}+\mu\right)^{2}}{2 \sigma_{2}^{2}}}}
\end{aligned}
$$

It is not clear how to simplify these. However, the inclusive $A_{\mathrm{FB}}^{\ell}$ from Eq. 4.2 can be simplified to

$$
A_{\mathrm{FB}}^{\ell}=\frac{\sigma_{1} \cdot \operatorname{erf}\left(\frac{\mu}{\sqrt{2} \sigma_{1}}\right)+r \cdot \sigma_{2} \cdot \operatorname{erf}\left(\frac{\mu}{\sqrt{2} \sigma_{2}}\right)}{\sigma_{1}+r \cdot \sigma_{2}} .
$$

This functional form is shown in Fig. E.4, and, in the limit of $\mu \ll \sigma_{1}$, which corresponds to $\left|A_{\mathrm{FB}}^{\ell}\right| \lesssim 0.2$, in Fig. E.4a, we find that $A_{\mathrm{FB}}^{\ell}=1.22 \cdot \mu$ which approximates the data well.

The SM prediction and most models of new physics all have values of $\left|A_{\mathrm{FB}}^{\ell}\right|<0.2$, so this can have a significant impact in simplifying the measurements. We can show the distribution of the $\mathcal{S}\left(q_{\ell} \eta_{\ell}\right)$ and $\mathcal{A}\left(q_{\ell} \eta_{\ell}\right)$ from Eq. E.4 with $\mu=-0.1,0.02$ and 0.2 in Fig. E.5. The $\mathcal{S}\left(q_{\ell} \eta_{\ell}\right)$ term is largely unchanged except for small values of $q_{\ell} \eta_{\ell}$ as 


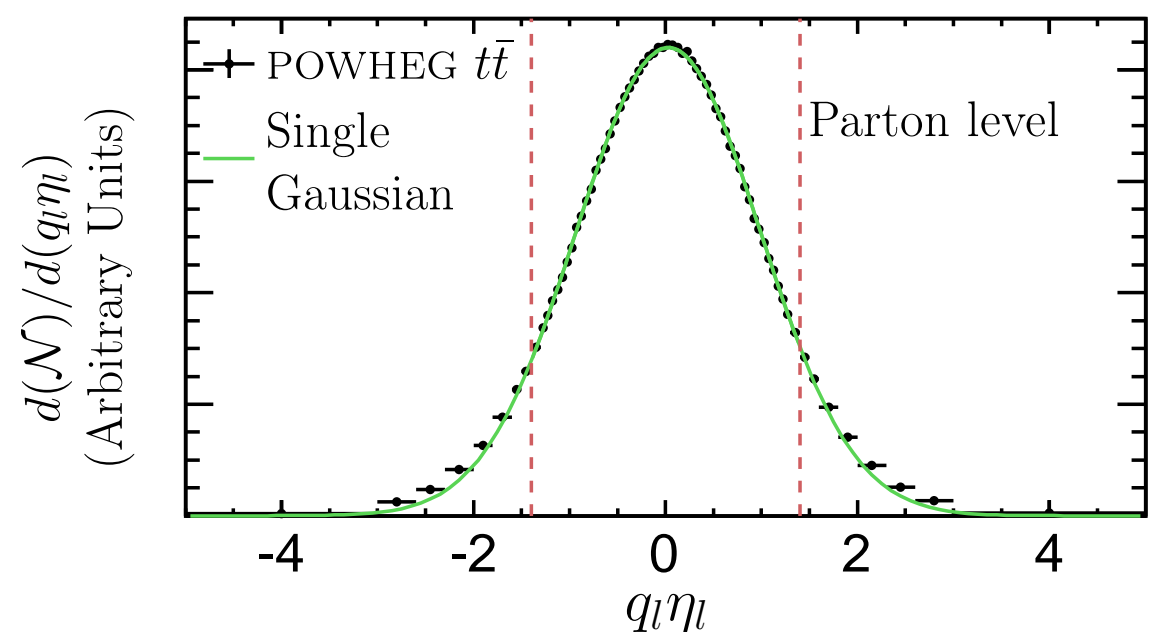

(a)

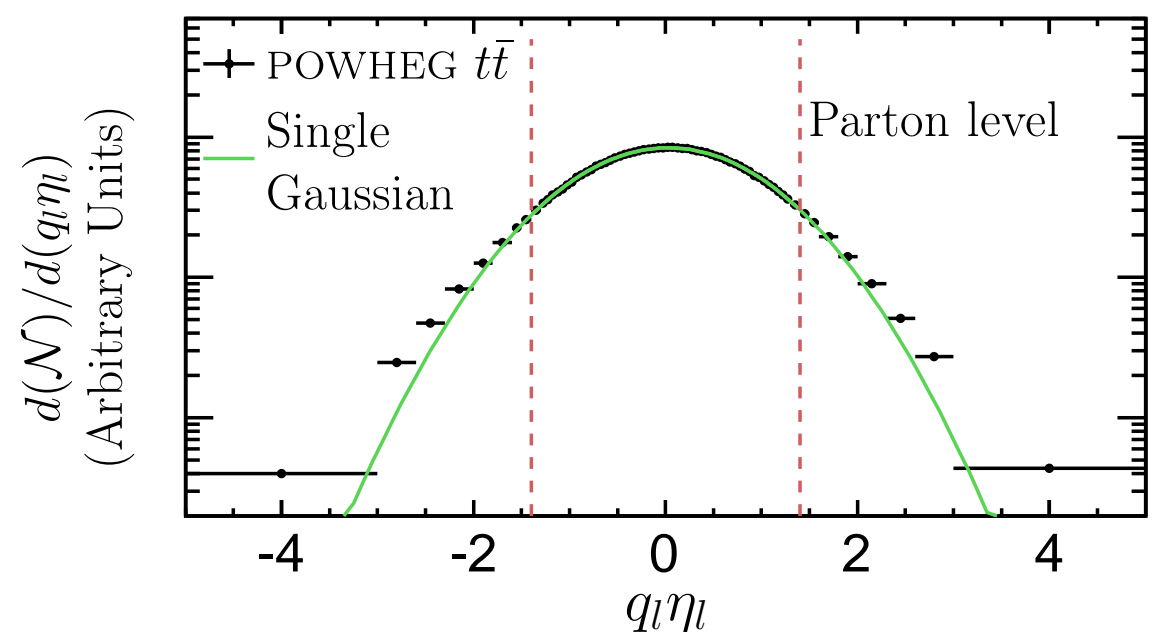

(b)

Figure E.1: The $q_{\ell} \eta_{\ell}$ distribution from the POWHEG $t \bar{t}$ sample at parton level, with a fit to a single Gaussian function in the region $\left|q_{\ell} \eta_{\ell}\right|<1.4$ (indicated by the dashed lines). Note that the agreement is not good for $\left|q_{\ell} \eta_{\ell}\right|>1.4$. 


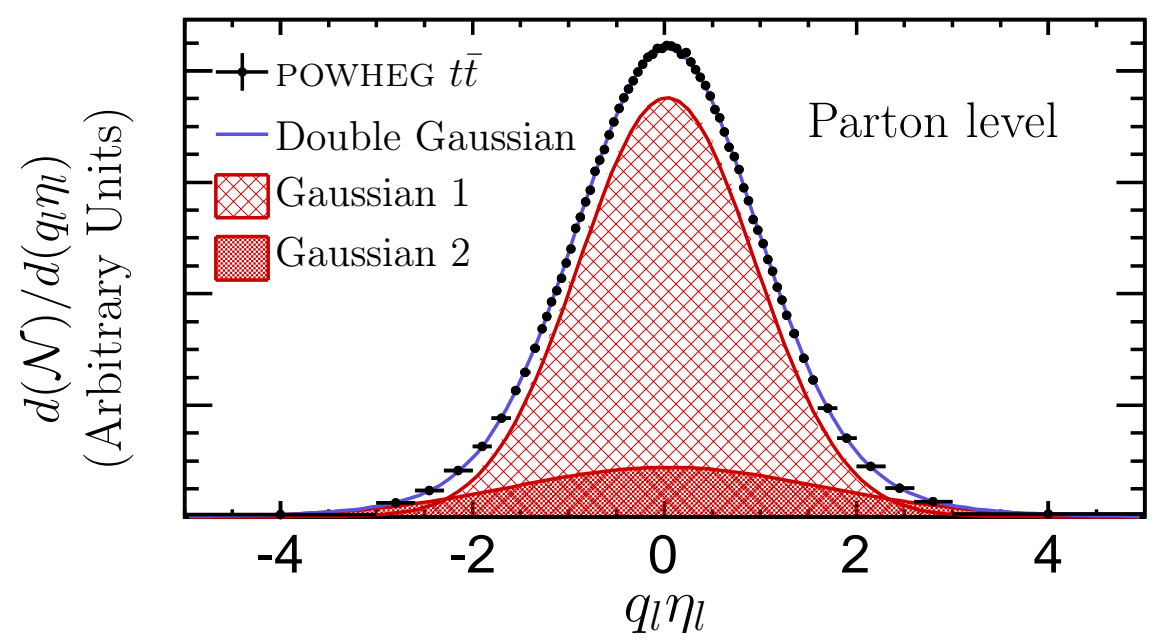

(a)

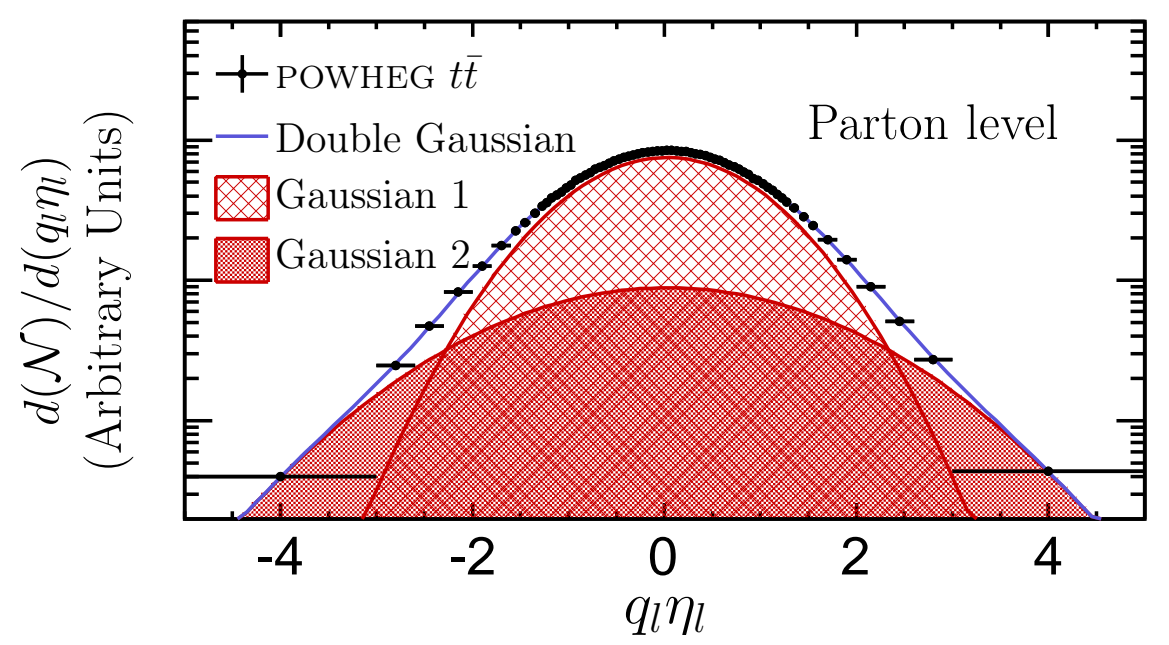

(b)

Figure E.2: The $q_{\ell} \eta_{\ell}$ distribution from the POWHEG $t \bar{t}$ sample at parton level, overlaid with the double-Gaussian fit. Note that both the tails and the central part of the distribution are well described. 


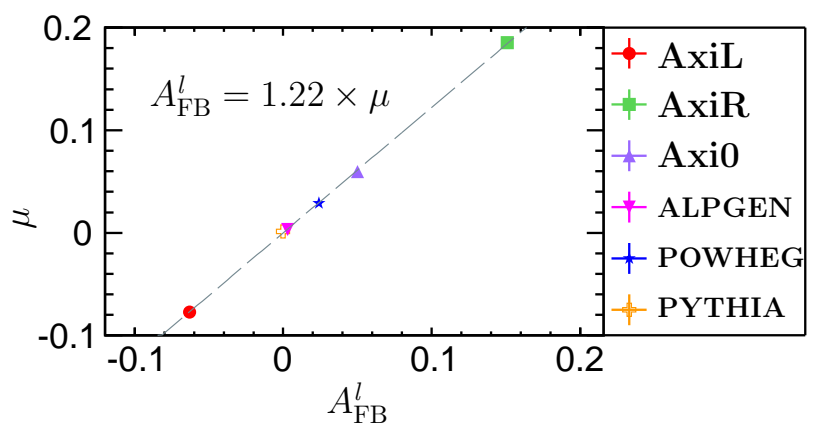

(a)

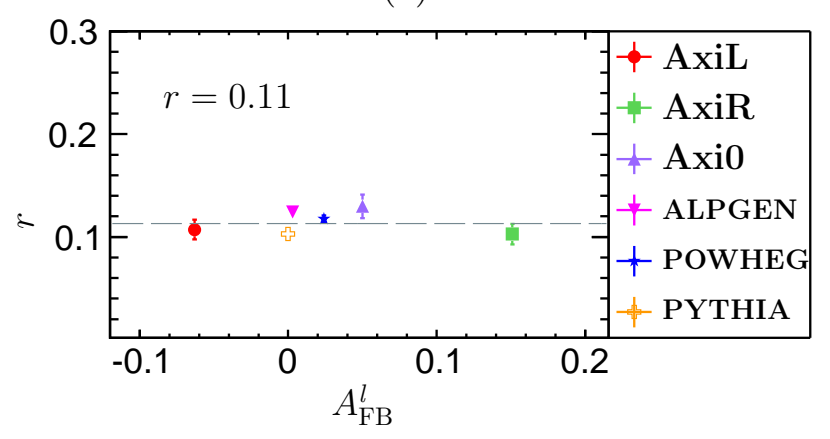

(b)

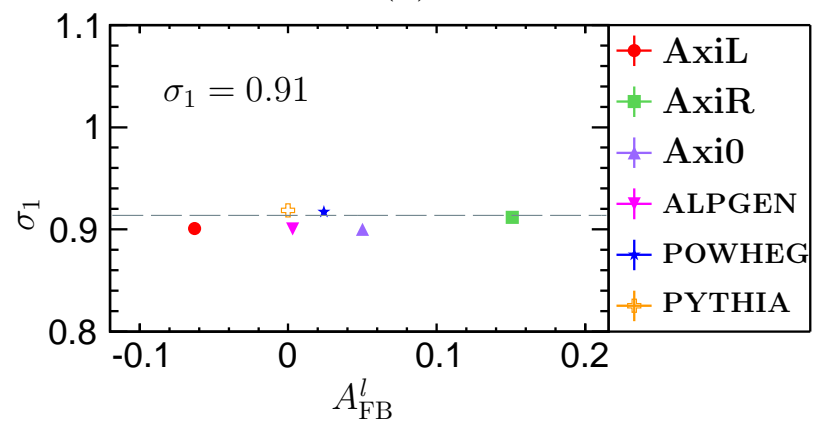

(c)

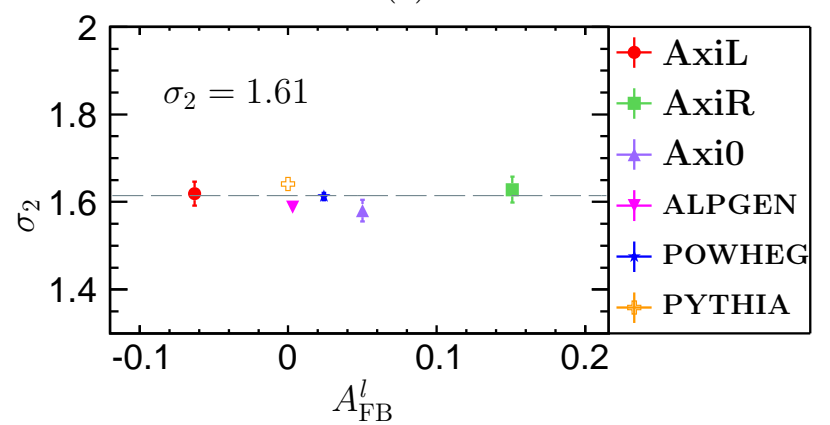

(d)

Figure E.3: Fit parameters from our benchmark samples as a function of $A_{\mathrm{FB}}^{\ell}$. 


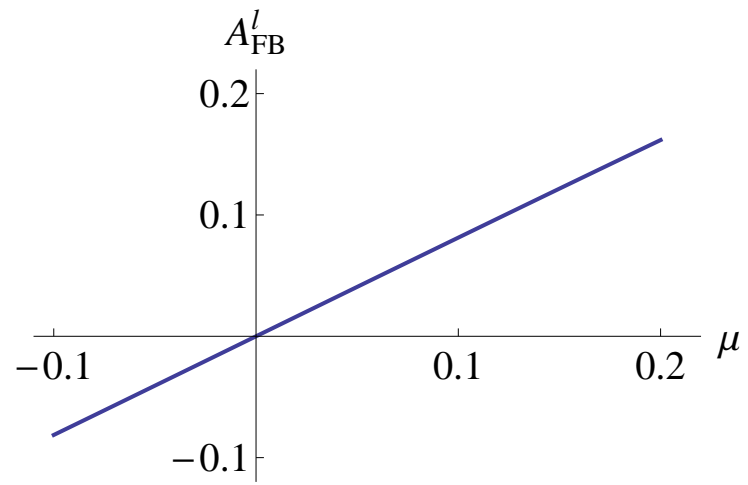

(a)

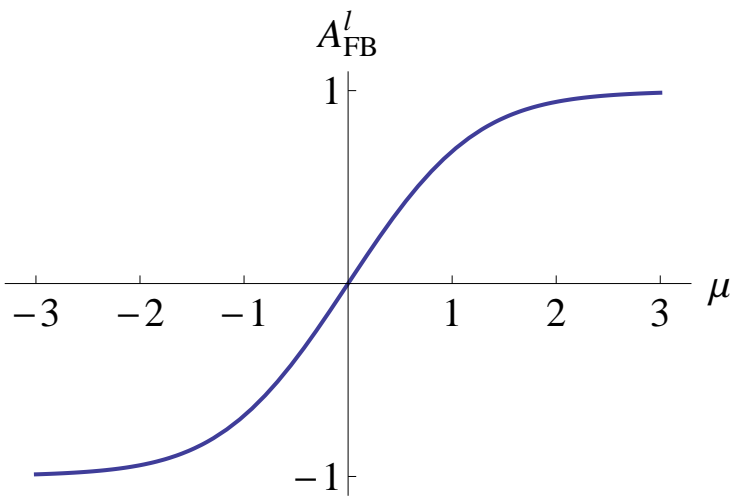

(b)

Figure E.4: With the double-Gaussian modeling, and constraining the two $\sigma$ values and the $r$ to the best estimated values from the benchmark simulations, $A_{\mathrm{FB}}^{\ell}$ appears to be linear as a function of the mean of the double-Gaussian function in the small $A_{\mathrm{FB}}^{\ell}$ region. In a larger region, $A_{\mathrm{FB}}^{\ell}$ asymptotes to \pm 1 .

previously noted, and the $\mathcal{A}\left(q_{\ell} \eta_{\ell}\right)$ term varies significantly. We also note that the distribution looks like a hyperbolic tangent function for $q_{\ell} \eta_{\ell}<2$, but has different structures for larger values of $q_{\ell} \eta_{\ell}$.

A second set of important results comes from a description of of how much contribution there is to the total asymmetry as a function of $q_{\ell} \eta_{\ell}$ (the differential contribution). It can be calculated as

$$
\frac{\mathcal{S}\left(q_{\ell} \eta_{\ell}\right) \cdot \mathcal{A}\left(q_{\ell} \eta_{\ell}\right)}{\int_{0}^{\infty} \mathcal{S}(x) \mathrm{d} x}
$$

where the denominator normalizes the area under the curve to be the total asymetry. The results are shown in Fig. E.6a for the same three $\mu$ values. In some ways the three curves look very different, but they do share some common features. While the area under the curve is strongly dependent on $\mu$, the shape of the distribution looks remarkably similar for all three curves. To see the similarity, we plot the normalized shape by rewriting Eq. E.6 such that the integral under the curve is equal to unity. 
Specifically:

$$
\frac{\mathcal{S}\left(q_{\ell} \eta_{\ell}\right) \cdot \mathcal{A}\left(q_{\ell} \eta_{\ell}\right)}{\int_{0}^{\infty} \mathcal{S}(x) \cdot \mathcal{A}(x) \mathrm{d} x}
$$

The results are shown in Fig. E.6b and we note that the shape of the differential contribution stays remarkably stable.

We are now able to make a number of further observations. First, the dominant contribution to the overall asymmetry comes from the region around $\left|q_{\ell} \eta_{\ell}\right|=1$, which is the place where the detectors have excellent coverage and resolution. We can also see why the slight mismodeling in the vicinity of $q_{\ell} \eta_{\ell}=0$ in the $\mathcal{S}\left(q_{\ell} \eta_{\ell}\right)$ term, as shown in Fig. 4.2a, and the mismodeling from the $a \cdot$ tanh description in the region where $q_{\ell} \eta_{\ell}>2.5$ in the $\mathcal{A}\left(q_{\ell} \eta_{\ell}\right)$ term would only introduce small biases in the overall measurement compared to the dominant uncertainties. Specifically, even though most of the events have $\left|q_{\ell} \eta_{\ell}\right|<0.1$, the contribution to $A_{\mathrm{FB}}^{\ell}$ from this region is $\sim 2 \%$. Similarly, the $q_{\ell} \eta_{\ell}$ region where there is no detector coverage at CDF or D0, $\left|q_{\ell} \eta_{\ell}\right|>2.0$, contributes $\sim 11 \%$ to the inclusive $A_{\mathrm{FB}}^{\ell}$; conversely, the region where the $a$ - tanh fit performs poorly, $\left|q_{\ell} \eta_{\ell}\right|>2.5$, contributes only $4 \%$. In addition, the constancy of the shape of the differential contribution provides an explanation for why the extrapolation technique from the measured $A_{\mathrm{FB}}^{\ell}$ to the inclusive $A_{\mathrm{FB}}^{\ell}$ is robust. The fraction of the $A_{\mathrm{FB}}^{\ell}$ within certain $\left|q_{\ell} \eta_{\ell}\right|$ ranges are shown in Fig. E.7. and some interesting numbers corresponding to typical lepton coverages at CDF and D0 are listed in Table E.1.

Table E.1: Fraction of $A_{\mathrm{FB}}^{\ell}$ within typical $q_{\ell} \eta_{\ell}$ coverage at CDF and D0.

\begin{tabular}{cc}
\hline \hline$q_{\ell} \eta_{\ell}$ Coverage & $A_{\mathrm{FB}}^{\ell}$ Fraction \\
\hline 1.25 & 0.73 \\
1.5 & 0.82 \\
2.0 & 0.93 \\
\hline \hline
\end{tabular}




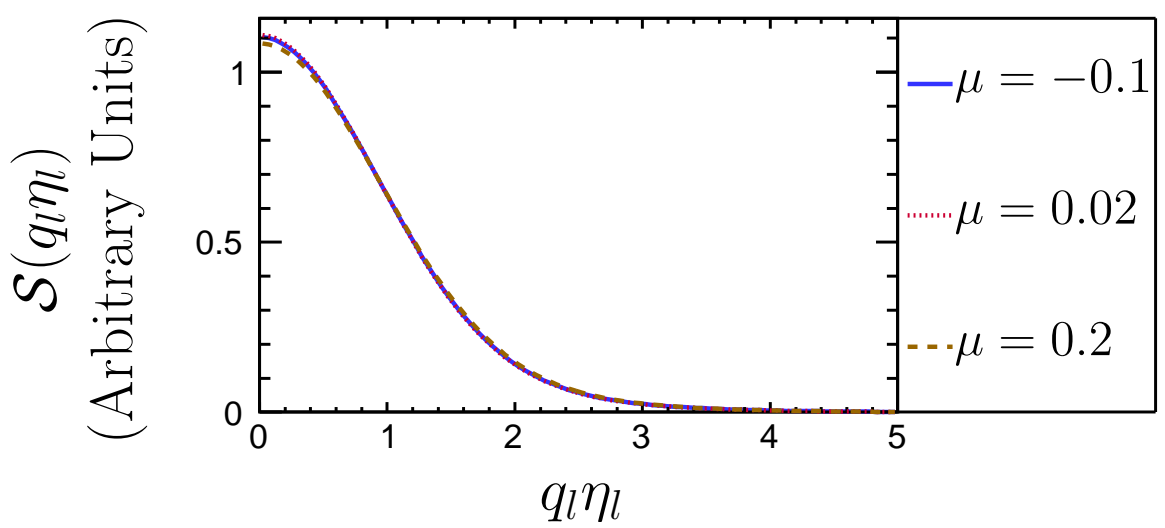

(a)

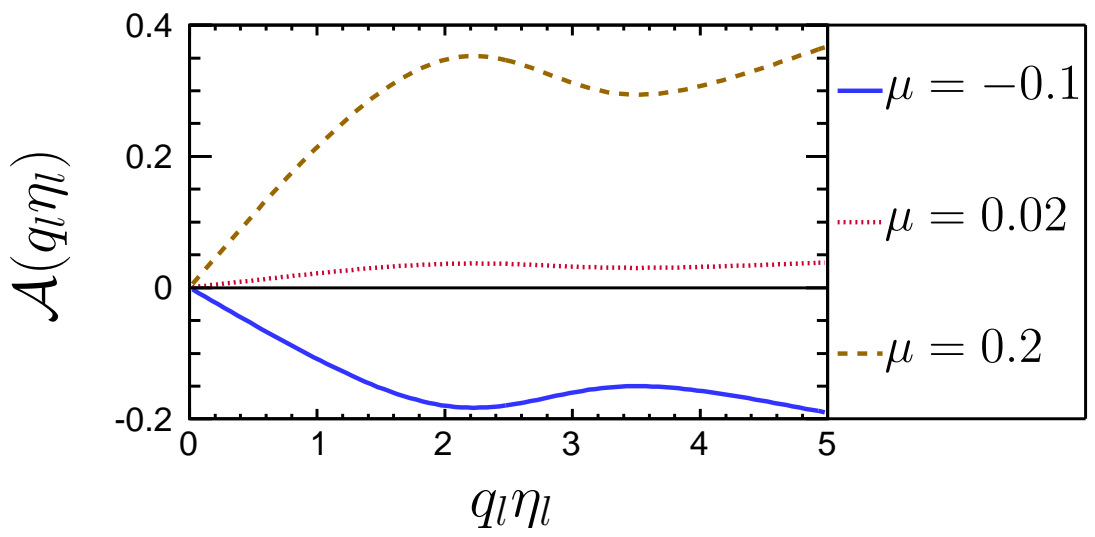

(b)

Figure E.5: The $\mathcal{S}\left(q_{\ell} \eta_{\ell}\right)$ term and the $\mathcal{A}\left(q_{\ell} \eta_{\ell}\right)$ term from the double-Gaussian model, with the $\mu$ parameter varied. 


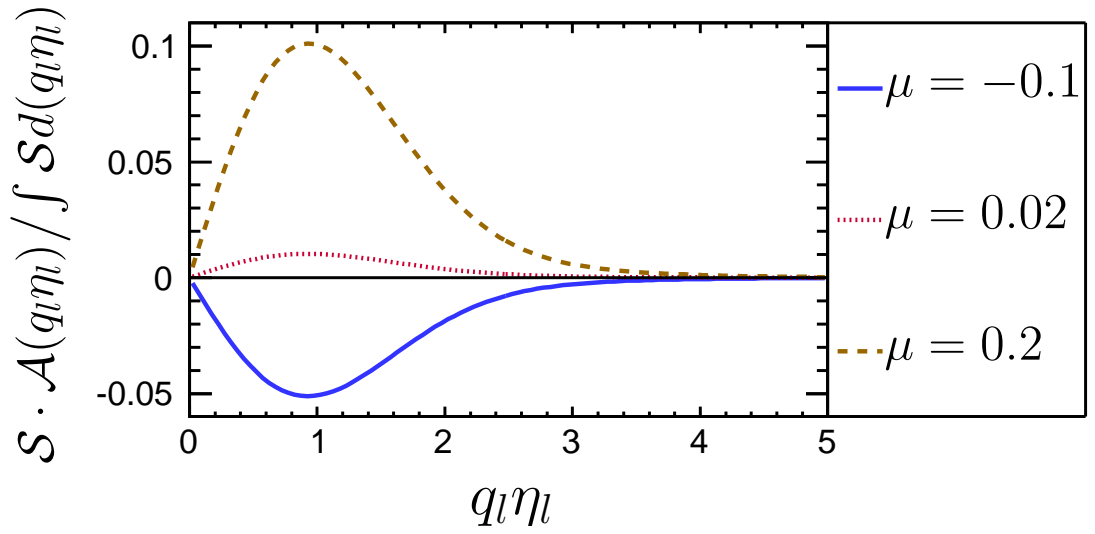

(a)

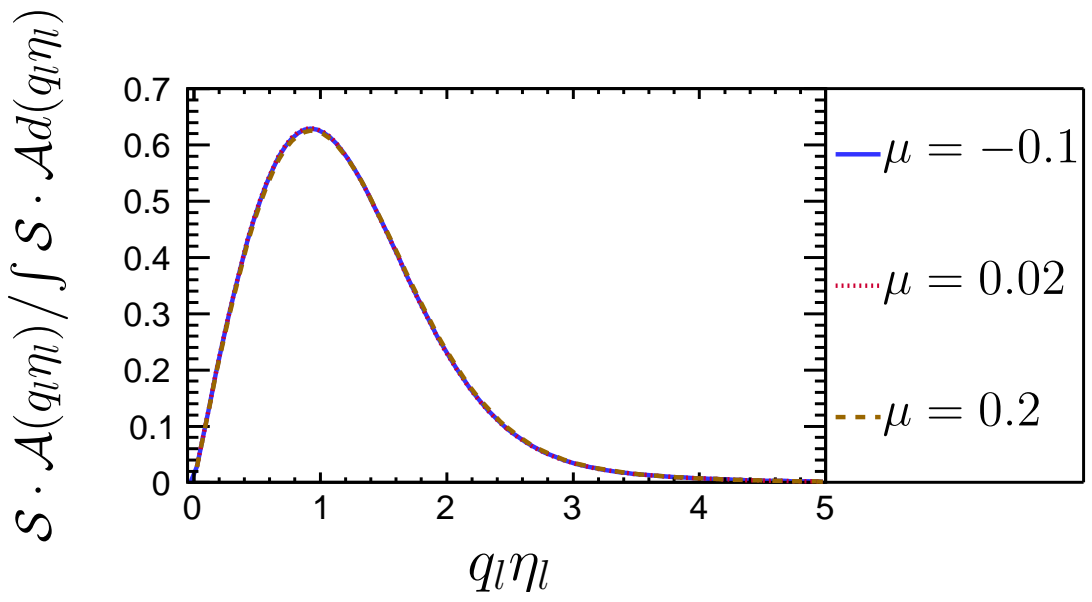

(b)

Figure E.6: Figures showing the differential contribution to the total asymmetry as a function of $q_{\ell} \eta_{\ell}$ using the double-Gaussian model. This is estimated using the $\mathcal{S}\left(q_{\ell} \eta_{\ell}\right)$ term times the $\mathcal{A}\left(q_{\ell} \eta_{\ell}\right)$ term, with the $\mu$ parameter varied, with different overall normalizations. (a) The curves are normalized so that $\int \mathcal{S}\left(q_{\ell} \eta_{\ell}\right) \mathrm{d} q_{\ell} \eta_{\ell}=1$ as in Eq. E.6. In this case, the areas under the curves give the inclusive asymmetry. (b) The curves are normalized to $\int \mathcal{S}\left(q_{\ell} \eta_{\ell}\right) \cdot \mathcal{A}\left(q_{\ell} \eta_{\ell}\right) \mathrm{d} q_{\ell} \eta_{\ell}=1$ as in Eq. E.7. In this case, we can see that the differential contribution to the asymmetry as a function of $q_{\ell} \eta_{\ell}$ is largely independent of the value of $\mu$ for small values of $\mu$. 


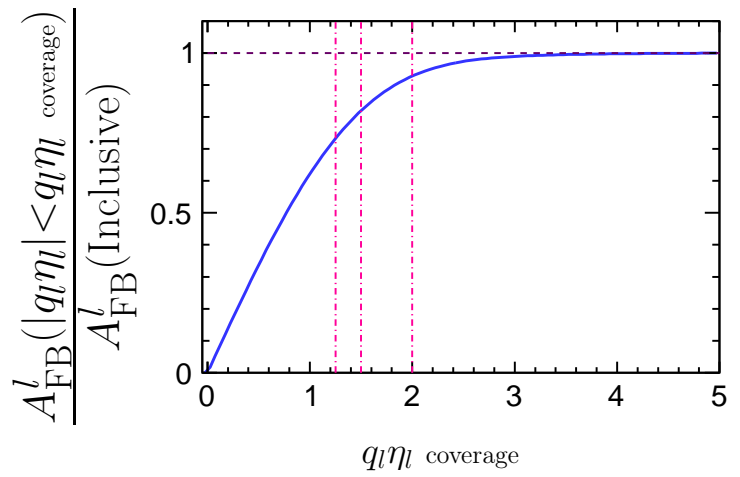

Figure E.7: Fraction of $A_{\mathrm{FB}}^{\ell}$ within a certain $q_{\ell} \eta_{\ell}$ coverage. The vertical lines show $q_{\ell} \eta_{\ell}=1.25,1.5$ and 2.0 corresponding to the typical detector coverages at CDF and D0. The numbers are given in Table E.1. The horizontal line indicates that the fraction asymptotes to one as the $q_{\ell} \eta_{\ell}$ coverage goes to infinity.

\section{E.2 Comparing the Sensitivity of the $a \cdot \tanh$, Single-Gaussian and Double-Gaussian Models}

We compare the sensitivity of the three possible measurement techniques in a number of ways. First we compare them visually, then we consider how well the different measurement techniques would work. Fig E.8 shows the $\mathcal{A}\left(q_{\ell} \eta_{\ell}\right)$ term and the differential contribution to the inclusive $A_{\mathrm{FB}}^{\ell}$ as a function of $q_{\ell} \eta_{\ell}$ from the POWHEG sample, overlaid with the best fit from the $a \cdot \tanh$ model, the singleGaussian model and the double-Gaussian model described, when we only consider events with $\left|q_{\ell} \eta_{\ell}\right|<2.0$. All three models fit this $q_{\ell} \eta_{\ell}$ region well. Since the region $\left|q_{\ell} \eta_{\ell}\right|<2.0$ is where most of the contribution to $A_{\mathrm{FB}}^{\ell}$ comes from, all three models (including the single-Gaussian model) get back to the inclusive $A_{\mathrm{FB}}^{\ell}$ of the sample reasonably well. The double-Gaussian model fits the asymmetric part better in the $q_{\ell} \eta_{\ell}$ region above 2.0 than the tanh model, thus the differential contribution predicted by the double-Gaussian model lines up with the POWHEG predicted points marginally 
better. However, as stated earlier, the improvement is in the region where the contribution to the inclusive $A_{\mathrm{FB}}^{\ell}$ is small, thus the improvement in the resultant $A_{\mathrm{FB}}^{\ell}$ using the double-Gaussian model is very small. Fig. E.9 shows the double-Gaussian model fit to the $\mathcal{A}\left(q_{\ell} \eta_{\ell}\right)$ distribution for all the six benchmark samples at parton level. A comparison with Fig. 4.2b shows that the double-Gaussian model matches all the simulated samples better than the $a$-tanh model, although the differences are mostly in the high- $q_{\ell} \eta_{\ell}$ region where the contribution to the inclusive $A_{\mathrm{FB}}^{\ell}$ is small, and there is no data from the experiments in this region.

We next compare how well the various methods would work for simulated data by considering just the set of POWHEG simulated events within $\left|q_{\ell} \eta_{\ell}\right|<2.0$ and employing different methodologies to see how well each reproduces the inclusive $A_{\mathrm{FB}}^{\ell}$ of 0.0236. We performed 10,000 pseudoexperiments by varying the $\mathrm{d} \mathcal{N}\left(q_{\ell} \eta_{\ell}\right) / \mathrm{d}\left(q_{\ell} \eta_{\ell}\right)$ distribution with statistical fluctuations for about 1 million simulated events. We then measured $A_{\mathrm{FB}}^{\ell}$ for each pseudoexperiment using each of the four methods:

1. A pure counting of the number of events with positive and negative $q_{\ell} \eta_{\ell}$ values, with a correction for the limited detector coverage using the correction factor of 0.93 (see Table E.1) to extrapolate to the inclusive value

2. Fitting the $a \cdot \tanh$ model to the $\mathcal{A}\left(q_{\ell} \eta_{\ell}\right)$ term of the distribution for the parameter $a$ and calculating the inclusive $A_{\mathrm{FB}}^{\ell}$ using the $\mathcal{S}\left(q_{\ell} \eta_{\ell}\right)$ distribution and Eq. 4.2

3. Fitting the asymmetric part of the double-Gaussian model to the $\mathcal{A}\left(q_{\ell} \eta_{\ell}\right)$ term of the distribution for the parameter $\mu$ and calculating the inclusive $A_{\mathrm{FB}}^{\ell}$ with Eq. E.5

4. Fitting the double-Gaussian model to the $q_{\ell} \eta_{\ell}$ distribution itself for the param- 


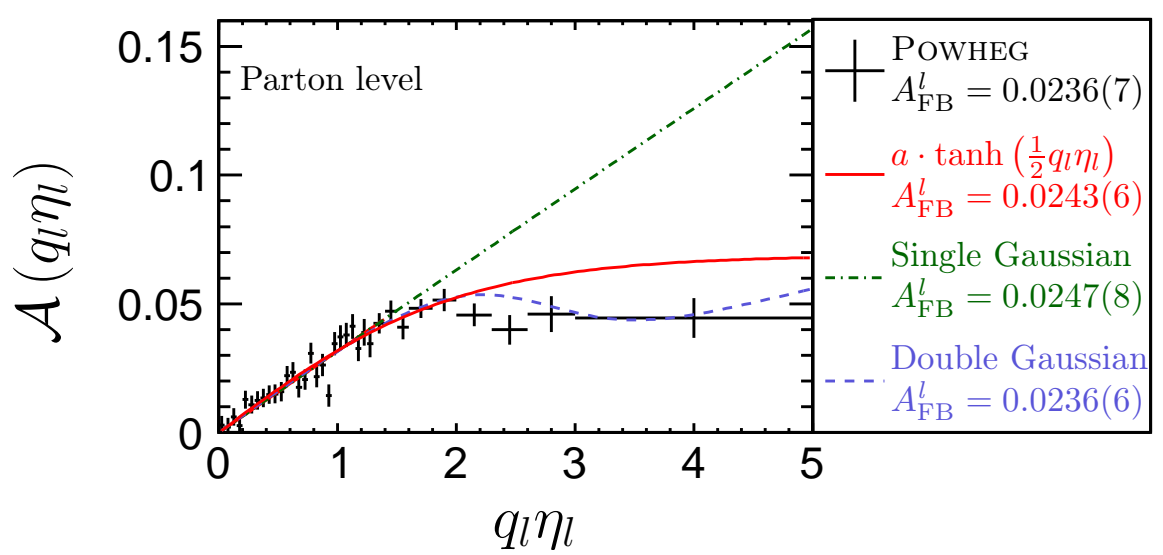

(a)

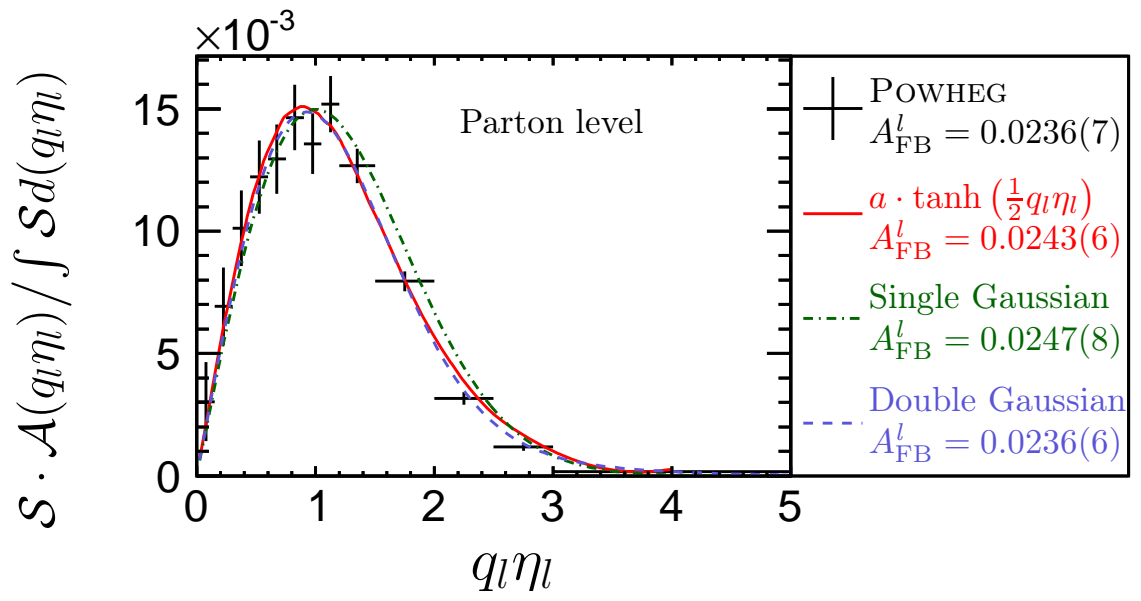

(b)

Figure E.8: Comparison among the $a \cdot$ tanh model, the single-Gaussian model, the double-Gaussian model and the POWHEG simulation. (a) shows the best fits of the $\mathcal{A}\left(q_{\ell} \eta_{\ell}\right)$ distribution (done only using events with $\left|q_{\ell} \eta_{\ell}\right|<2.0$ ), while (b) shows the differential contribution to the $A_{\mathrm{FB}}^{\ell}$ as a function of $q_{\ell} \eta_{\ell}$ from different models. 


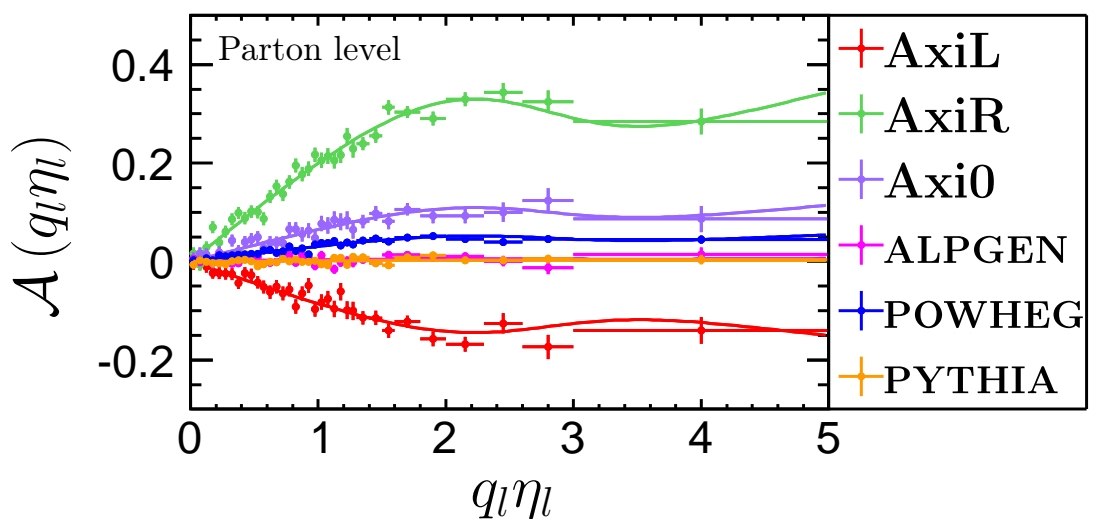

Figure E.9: Best fit of double-Gaussian model to the $\mathcal{A}\left(q_{\ell} \eta_{\ell}\right)$ distribution for various $t \bar{t}$ samples at generator level. This figure can be compared directly to Fig. $4.2 \mathrm{~b}$ where we fit the same data, but using the $a \cdot \tanh$ function.

eter $\mu$ and calculating the inclusive $A_{\mathrm{FB}}^{\ell}$ again with Eq. E.5

The results of the pseudoexperiments are shown in Table E.2.

The average of the pseudoexperiments for each method is always within one standard deviation of the input $A_{\mathrm{FB}}^{\ell}$ value, indicating none have noticeable bias. As expected, the pure counting method has the largest uncertainty, as the fits incorporate the additional shape information to reduce the uncertainties. While there does not seem to be much difference in the sensitivity of the fitting methods, we note that the fit on the $\mathcal{A}\left(q_{\ell} \eta_{\ell}\right)$ term has the systematic advantage over the pure fit to the mean, $\mu$, of the full $\mathrm{d} \mathcal{N}\left(q_{\ell} \eta_{\ell}\right) / \mathrm{d}\left(q_{\ell} \eta_{\ell}\right)$ distribution as most of the systematic uncertainties due to the acceptance of the detector are expected to cancel out [43]. Thus, we favor the use of the fit on the $\mathcal{A}\left(q_{\ell} \eta_{\ell}\right)$ distribution over the simple counting for resolution reasons, and over the fit on the full distribution for robustness reasons. Between the two fits on the $\mathcal{A}\left(q_{\ell} \eta_{\ell}\right)$ term, we see that the $a \cdot$ tanh formulation is easier to work with, but further checks to see if there are other effects due to detector 
response should be considered.

Table E.2: Results of pseudoexperiments using the different methods to reproduce $A_{\mathrm{FB}}^{\ell}$ of the POWHEG simulation (0.0236), but only using events with $\left|q_{\ell} \eta_{\ell}\right|<2.0$. Note that the uncertainties listed are statistical only and are due to the size of the simulated data sample.

\begin{tabular}{cccc}
\hline Method & Mean & Mean-Expected & Uncertainty \\
\hline \hline Counting & 0.0241 & 0.0004 & 0.0008 \\
$a \cdot \tanh \mathcal{A}\left(q_{\ell} \eta_{\ell}\right)$ Fit & 0.0243 & 0.0006 & 0.0006 \\
Double Gaussian $\mathcal{A}\left(q_{\ell} \eta_{\ell}\right)$ Fit & 0.0236 & -0.0001 & 0.0006 \\
Double Gaussian Direct Fit & 0.0238 & 0.0002 & 0.0006 \\
\hline
\end{tabular}

We next test how well the $a \cdot$ tanh and the double-Gaussian methods reproduce the inclusive $A_{\mathrm{FB}}^{\ell}$ values for all 6 simulated samples with only events within $\left|q_{\ell} \eta_{\ell}\right|<2.0$. A comparison of results is given in Table E.3. Table E.4 lists the fit parameter $(a)$ for the $a \cdot$ tanh model for comparison with the same fit with detector effects taken into account in the next section. Though the double-Gaussian model works better in the high $q_{\ell} \eta_{\ell}$ region, the impact on the $A_{\mathrm{FB}}^{\ell}$ measurement is negligible compared to the dominant uncertainties in the measurement $(\sim 0.02$ in the lepton + jets final state [43] and $\sim 0.05$ in the dilepton final state 90 ]

\section{E.3 Conclusions of $A_{\mathrm{FB}}^{\ell}$ Measurement Methodology Study}

The measurement methodology with an empirical functional form of $a \cdot \tanh$ to extrapolate from the limited detector $\eta$ coverage to an inclusive parton-level estimate is fortuitous but robust. The $a \cdot \tanh$ parametrization is an approximation that is only good for values of $\left|q_{\ell} \eta_{\ell}\right|<2.5$, but it is more than good enough for the Tevatron experiments. Our studies show that a more sophisticated empirical function, which takes the form of the sum of two Gaussian functions with a common mean, and 
Table E.3: Comparison of the predicted $A_{\mathrm{FB}}^{\ell}$ values and the corresponding measured $A_{\mathrm{FB}}^{\ell}$ values with the $a \cdot$ tanh model and the double-Gaussian model. The uncertainties are statistical only and are always small compared to the expected statistical uncertainty in data collected by the CDF and D0 experiments.

\begin{tabular}{cccc}
\hline Model & True $A_{\mathrm{FB}}^{\ell}$ & $\begin{array}{c}\text { Measured } A_{\mathrm{FB}}^{\ell} \\
(a \cdot \text { tanh model })\end{array}$ & $\begin{array}{c}\text { Measured } A_{\mathrm{FB}}^{\ell} \\
(\text { Double-Gaussian model })\end{array}$ \\
\hline \hline AxiL & $-0.063(2)$ & $-0.064(2)$ & $-0.064(2)$ \\
AxiR & $0.151(2)$ & $0.148(2)$ & $0.150(2)$ \\
Axi0 & $0.050(2)$ & $0.048(2)$ & $0.048(2)$ \\
ALPGEN & $0.003(1)$ & $-0.004(1)$ & $0.002(1)$ \\
PYTHIA & $0.001(1)$ & $-0.005(1)$ & $0.001(1)$ \\
POWHEG & $0.023(1)$ & $0.024(1)$ & $0.023(1)$ \\
\hline
\end{tabular}

Table E.4: Hyperbolic tangent fit on generator level asymmetric part

\begin{tabular}{|c|c|c|}
\hline Model & $A_{\mathrm{FB}}^{\ell}($ Generator Level $)$ & Fit Parameter(a) \\
\hline AxiL & $-0.0626 \pm 0.0024$ & $-0.182 \pm 0.006$ \\
\hline AxiR & $0.1513 \pm 0.0024$ & $0.419 \pm 0.005$ \\
\hline Axi0 & $0.0497 \pm 0.0024$ & $0.137 \pm 0.006$ \\
\hline ALPGEN & $0.0032 \pm 0.0013$ & $0.007 \pm 0.003$ \\
\hline PYTHIA & $0.0001 \pm 0.0008$ & $0.003 \pm 0.002$ \\
\hline POWHEG & $0.0236 \pm 0.0007$ & $0.065 \pm 0.002$ \\
\hline
\end{tabular}


with empirically determined values of the two $\sigma$ and $r$ parameters, describes the $q_{\ell} \eta_{\ell}$ distribution better at all $q_{\ell} \eta_{\ell}$ values. This functional form has not yielded a simple closed form for the $\mathcal{A}\left(q_{\ell} \eta_{\ell}\right)$ term. While the double-Gaussian parametrization is better in principle, in practice using it does not provide additional useful measurement sensitivity and it is more cumbersome to use. On the other hand, this better understanding of the expected shapes lead to some interesting and useful conclusions in addition to the confidence we now have in the methods previously being employed. First, it is advantageous to think of the asymmetry as coming from the shift of the mean of the $q_{\ell} \eta_{\ell}$ distribution. To a good degree of approximation, measuring the $A_{\mathrm{FB}}^{\ell}$ is equivalent to measuring the mean, $\mu$, in the limit of small $A_{\mathrm{FB}}^{\ell}$; measuring the $\mathcal{A}\left(q_{\ell} \eta_{\ell}\right)$ term of the distribution is one of a number of ways to do so, which also takes advantage of the cancelling of the systematic uncertainties caused by the detector response in the measurement. Ultimately, we now understand that the dominant contribution to the inclusive $A_{\mathrm{FB}}^{\ell}$ comes from the region within the CDF detector which are best covered, and that the extrapolation procedures allow for a robust measurement. 


\section{APPENDIX F}

\section{CORRELATION BETWEEN TWO LEPTONS IN EACH EVENT}

In each $t \bar{t} \rightarrow \ell^{+} \ell^{\prime-} \nu \bar{\nu}^{\prime} b \bar{b}$ candidate event there are two reconstructed charged leptons. Since we include both leptons in our leptonic $A_{\mathrm{FB}}$ measurement to get better statistics, as done in Chapter 4, we describe in this appendix that positive and negative leptons follow the same $q_{\ell} \eta_{\ell}$ distribution and are not correlated in a way that would mislead us into thinking that the statistical uncertainty is smaller than it should be, or that we might bias the result in a significant way.

Figure F.1 shows the distribution of $q_{\ell} \eta_{\ell}$ for positive and negative leptons separately from the POWHEG $t \bar{t}$ MC sample at generator level. The two distributions agree with each other within statistics. This gives us confidence that by mixing the two leptons together we are still measuring the same distribution.

To get a sense of the correlation between the two leptons in each event, we test it with pseudoexperiments (P.E.s) from Powheg $t \bar{t} \mathrm{MC}$ sample in the following way. There are over $70 \mathrm{k}$ candidate events after reconstruction in the $\mathrm{MC}$ sample. In each P.E., we randomly pick 410 candidate events, which is the expected number of signal events in data, to mimic the final event data sample size. In each P.E., a raw $A_{\mathrm{FB}}$ (calculated by directly counting the number of forward and backward leptons) is calculated for positive lepton only, negative lepton only, and using both leptons. All the $A_{\mathrm{FB}}^{\ell}$ values from each P.E. are filled into a histogram, and fitted with a Gaussian function. The mean of the Gaussian function serves as an estimate of the true raw $A_{\mathrm{FB}}$ in the MC sample, and the RMS from the fit function gives an estimate of the statistical uncertainty of $A_{\mathrm{FB}}$.

In addition, a set of P.E.s with 820 candidate events (twice as many independent 


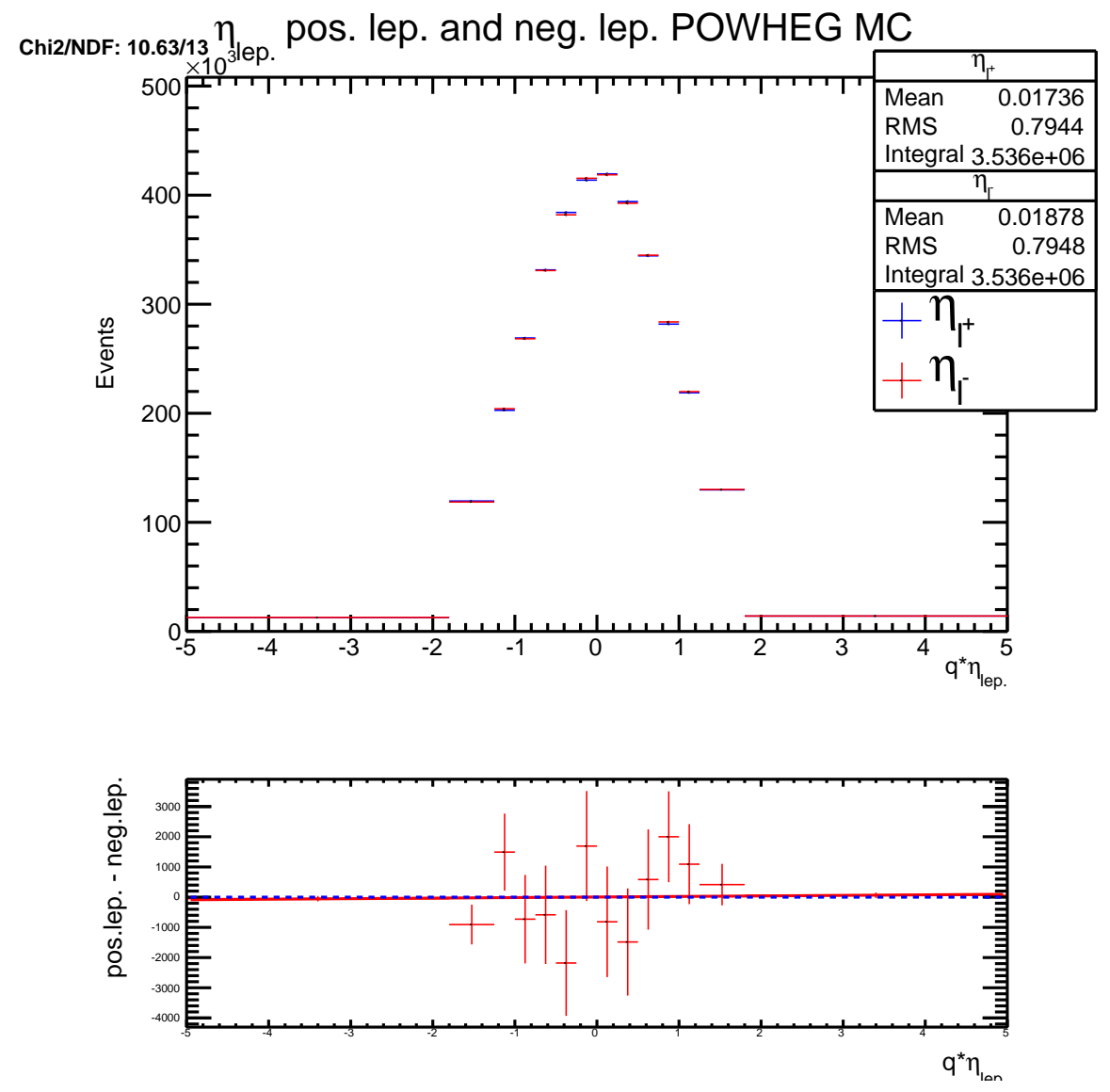

Figure F.1: The $q_{\ell} \eta_{\ell}$ distribution for positive and negative leptons from Powheg MC at generator level. 
events as in data) in each P.E. is generated to see the expected statistical uncertainty if the two leptons are fully uncorrelated. Fig. F.2 shows the result of the P.E.s described above. The means of the four situations shown in the figures are consistent with each other, indicating that no bias is introduced when using both leptons in an event for measuring leptonic $A_{\mathrm{FB}}$. With 410 independent entries, the statistical uncertainty is $\sim 0.049$. With 820 independent entries, the statistical uncertainty is $\sim 0.035$. With 410 independent events, two entries per event, the statistical uncertainty turns out to be 0.036 , slightly larger than the statistical uncertainty with 820 independent entries, indicating a correlation between the two leptons that is very small, and introduces a tiny effect compared to the expected resolution of the $A_{\mathrm{FB}}^{\ell}$ measurement. From these results we concluded that in the $A_{\mathrm{FB}}^{\ell}$ analysis, we can safely use both leptons to measure leptonic $A_{\mathrm{FB}}$ as it increases our statistical precision. 

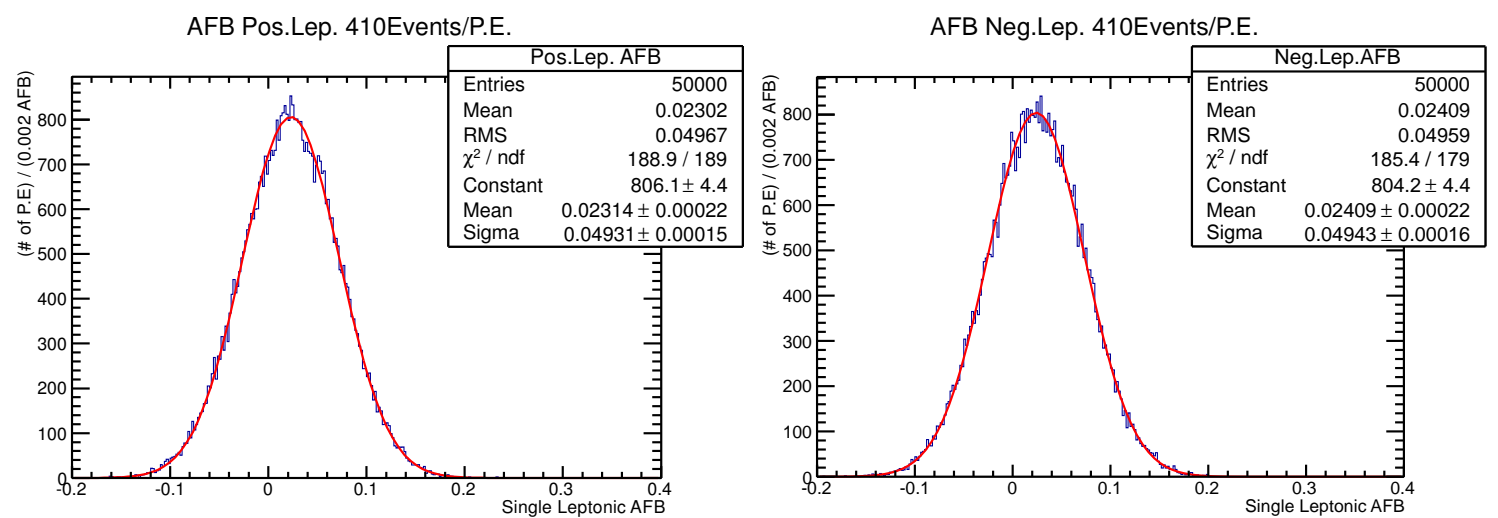

(a) $A_{\mathrm{FB}}$ with only positive leptons, 410 events per P.E.

AFB Pos.\&Neg.Lep. 410Events/P.E.

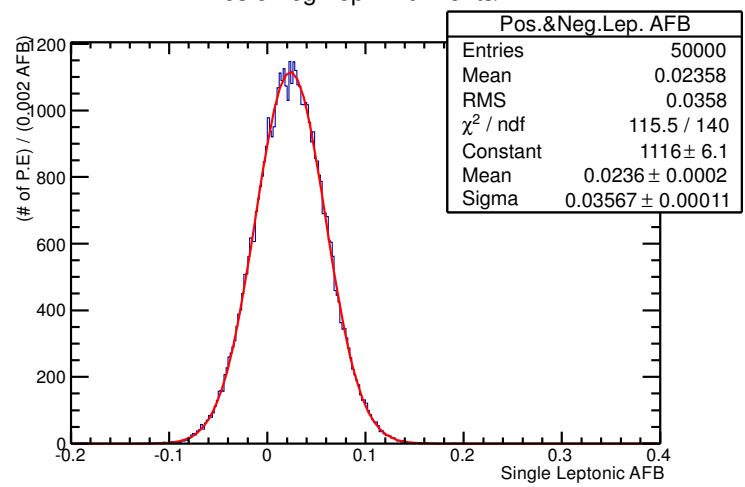

$A_{\mathrm{FB}}$ with only negative leptons, 410 events per P.E.

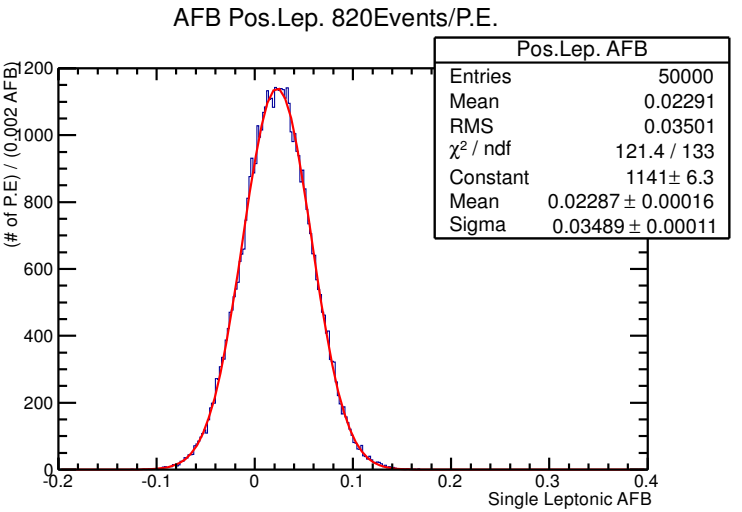

(c) $A_{\mathrm{FB}}$ with both positive and negative lep- (d) $A_{\mathrm{FB}}$ with only positive leptons, 820 tons, 410 events per P.E. events per P.E.

Figure F.2: Pseudoexperiments to test correlation between two leptons in an event. 